\title{
PRECIPITATION, STREAMFLOW, AND WATER- QUALITY DATA FROM SELECTED SITES IN THE CITY OF CHARLOTTE AND MECKLENBURG COUNTY, NORTH CAROLINA, 1995-97
}

By J.B. Robinson, W.F. Hazell, and R.G. Garrett

\section{U.S. GEOLOGICAL SURVEY}

Open-File Report 98-67

Prepared in cooperation with the City of Charlotte, Mecklenburg County, and Charlotte-Mecklenburg Utility Department

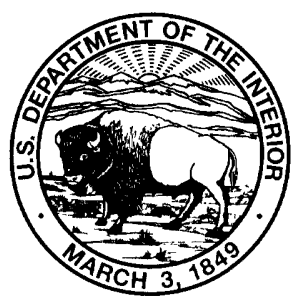




\section{U.S. DEPARTMENT OF THE INTERIOR \\ BRUCE BABBITT, Secretary}

U.S. GEOLOGICAL SURVEY

Thomas J. Casadevall, Acting Director

The use of firm, trade, and brand names in this report is for identification purposes only and does not constitute endorsement by the U.S. Geological Survey.

For additional information write to:

Copies of this report can be purchased from:

District Chief

U.S. Geological Survey

3916 Sunset Ridge Road

Raleigh, North Carolina 27607
U.S. Geological Survey

Information Services

Box 25286, Federal Center

Denver, CO 80225 


\section{CONTENTS}

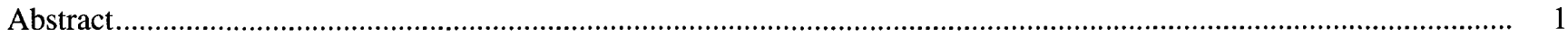

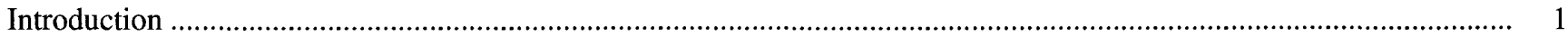

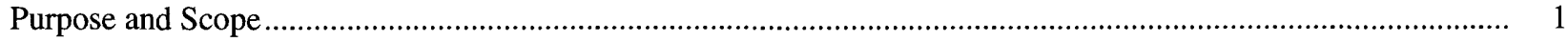

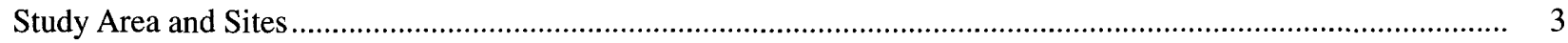

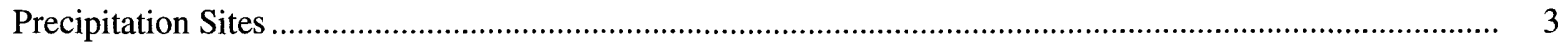

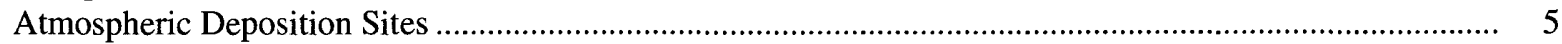

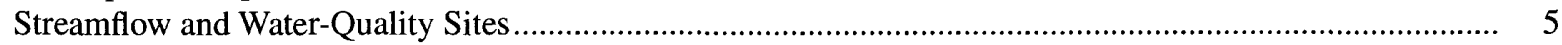

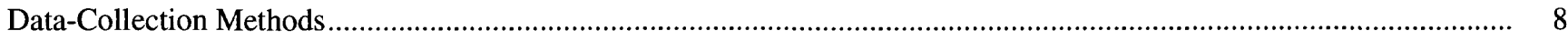

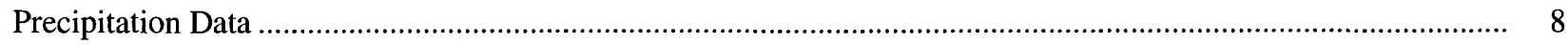

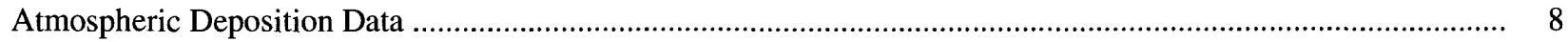

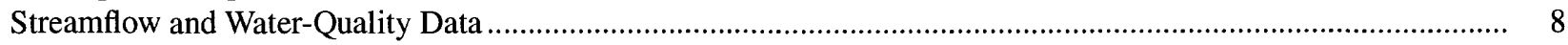

Quality-Assurance Procedures ................................................................................................................. 9

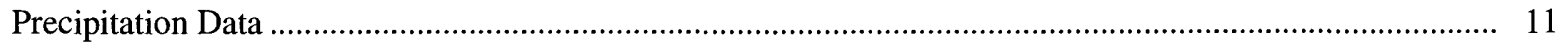

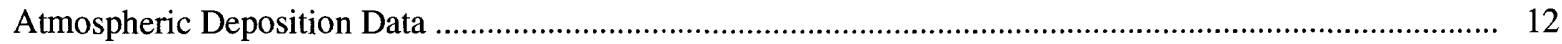

Streamflow and Water-Quality Data ………………………………………………………………. 12

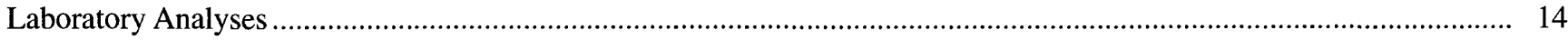

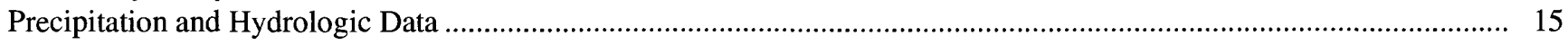

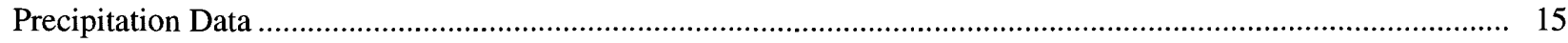

Atmospheric Deposition Data ……………………………………………………………………………. 16

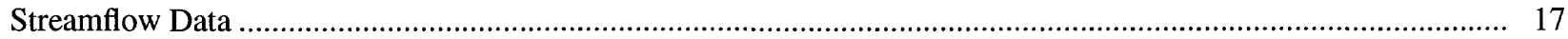

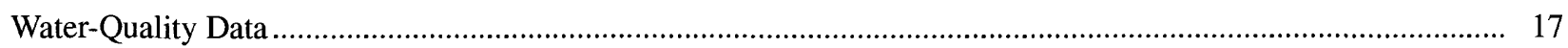

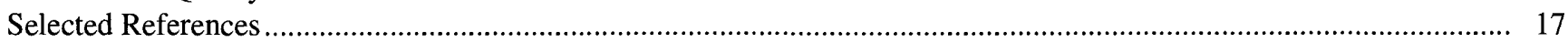

\section{FIGURES}

1. Map showing city of Charlotte and Mecklenburg County data-collection network, 1995-97 .......................... 2

2. Flow chart showing raingage locations, by basin, in Charlotte and Mecklenburg County .................................. 5

3. Map showing annual rainfall distribution in Mecklenburg County for July 1996 through June 1997 ................. 15

4. Map showing rainfall recurrence intervals for the city of Charlotte for August 26-27, $1995 \ldots \ldots \ldots \ldots \ldots \ldots \ldots \ldots \ldots \ldots . . . \ldots \ldots$

\section{TABLES}

1. Precipitation network sites located in Mecklenburg County, October 1988 through June 1997 .......................... 3

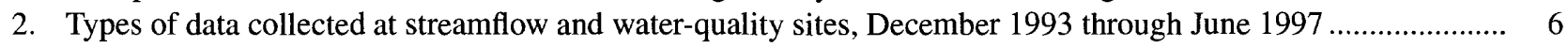

3. Land-use percentage distribution for study site drainage areas .................................................................. 7

4. Containers, container treatment, and preservation procedures required for samples collected at the streamflow and water-quality study sites and analyzed by the U. S. Geological Survey National Water-Quality Laboratory, May 1994 through August 1995.

5. Containers, container treatment, and preservation procedures required for samples collected at the streamflow and water-quality study sites and analyzed by the Mecklenburg County Department of Environmental Protection Laboratory, September 1995 through June 1997.

6. Automatic pumping sampler intake locations and number of manual suspended-sediment samples collected, May 1994 through June 1997

7. Analytical procedures and method detection limits for chemical constituents in water analyzed by the U.S. Geological Survey National Water-Quality Laboratory, May 1994 through August 1995.

8. Analytical procedures and method detection limits for chemical constituents in water analyzed by the Mecklenburg County Department of Environmental Protection Laboratory, September 1995 through June 1997. 
9-54. Daily rainfall totals at:

9. Site 3 (CRN10), July 1995 through June 1997

10. Site 5 (CRN06), July 1995 through June 1997

11. Site 6 (CRN18), July 1995 through June 1997

12. Site 13 (CRN01), July 1995 through June 1997

13. Site 14 (CRN02), July 1995 through June 1997

14. Site 15 (CRN03), July 1995 through June 1997

15. Site 16 (CRN04), July 1995 through June 1997

16. Site 17 (CRN05), July 1995 through June 1997

17. Site 18 (CRN07), July 1995 through June 1997

18. Site 19 (CRN08), July 1995 through June 1997

19. Site 20 (CRN09), July 1995 through June 1997

20. Site 21 (CRN11), July 1995 through June 1997

21. Site 22 (CRN12), July 1995 through June 1997

22. Site 23 (CRN13), July 1995 through June 1997

23. Site 24 (CRN14), July 1995 through June 1997

24. Site 25 (CRN15), July 1995 through June 1997

25. Site 26 (CRN16), July 1995 through June 1997

26. Site 27 (CRN17), July 1995 through June 1997

27. Site 28 (CRN19), July 1995 through June 1997

28. Site 29 (CRN20), July 1995 through June 1997

29. Site 30 (CRN21), July 1995 through June 1997

30. Site 31 (CRN22), July 1995 through June 1997

31. Site 32 (CRN23), July 1995 through June 1997

32. Site 33 (CRN25), July 1995 through June 1997

33. Site 34 (CRN24), July 1995 through June 1997

34. Site 35 (CRN26), July 1995 through June 1997

35. Site 36 (CRN27), July 1995 through June 1997

36. Site 37 (CRN28), July 1995 through June 1997

37. Site 44 (CRN41), November 1996 through June 1997

38. Site 45 (CRN29), February 1996 through June 1997.

39. Site 46 (CRN30), February 1996 through June 1997

40. Site 47 (CRN31), February 1996 through June 1997

41. Site 48 (CRN32), February 1996 through June 1997.

42. Site 49 (CRN33), December 1995 through June 1997

43. Site 50 (CRN34), February 1996 through June 1997

44. Site 51 (CRN35), January 1996 through June 1997

45. Site 52 (CRN36), February 1996 through June 1997

46. Site 53 (CRN37), February 1996 through June 1997

47. Site 54 (CRN38), February 1996 through June 1997

48. Site 55 (CRN39), February 1996 through June 1997

49. Site 56 (CRN40), February 1996 through June 1997

50. Site 57 (CRN42), January through June 1997

51. Site 58 (CRN43), January through June 1997

52. Site 59 (CRN44), January through June 1997

53. Site 60 (CRN45), January through June 1997

54. Site 61 (CRN46), January through June 1997.

55. Streamflow statistics at the streamflow and water-quality study sites, December 1993 through June $1997 \ldots \ldots . . . .120$

56-64. Daily mean discharge at:

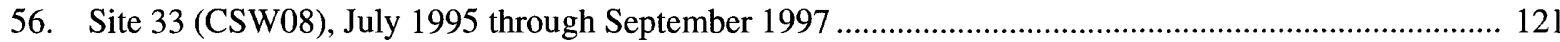

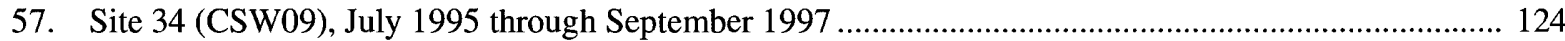

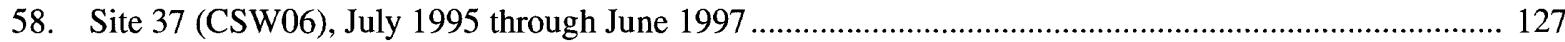

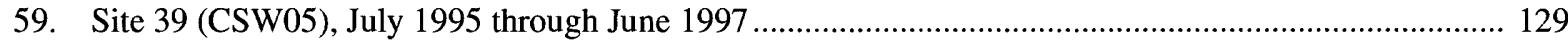

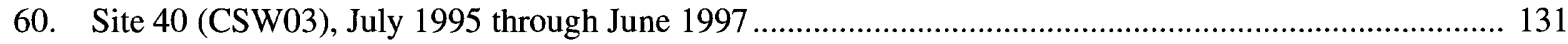

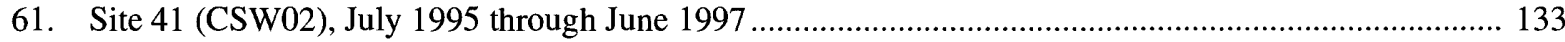




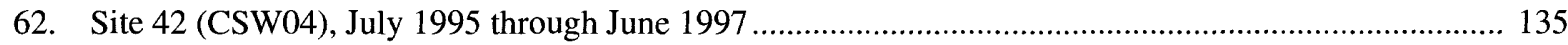

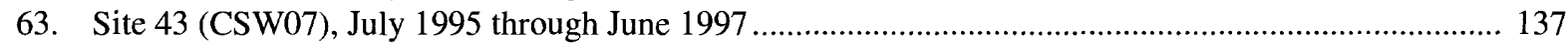

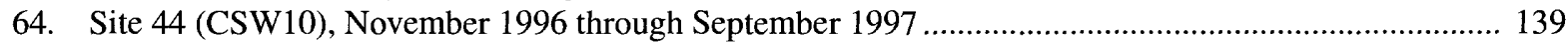

65. Maximum, minimum, and median specific conductance and maximum and minimum water temperature recorded by monitors at the streamflow and water-quality study sites, October 1994 through

September 1997

66-74. Statistical summary of water-quality data at:

66. Site 33 (CSW08), June 1994 through September 1997 .................................................................... 141

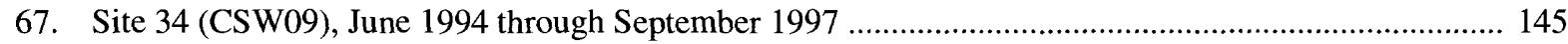

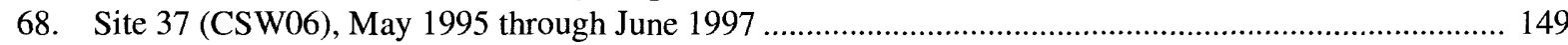

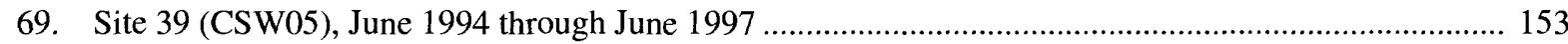

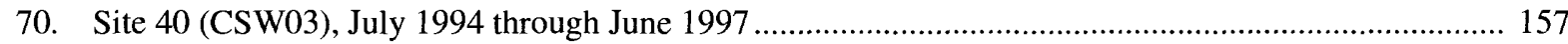

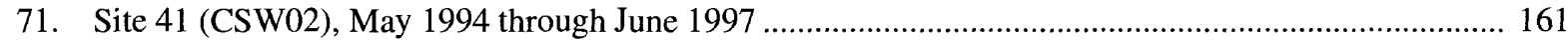

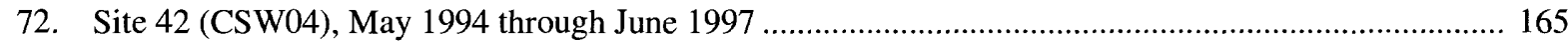

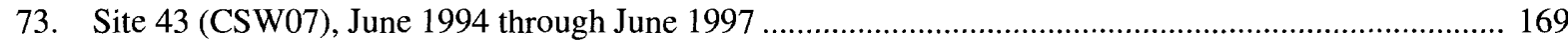

74. Site 44 (CSW10), November 1996 through September 1997 ............................................................. 173

75-83. Water-quality data at:

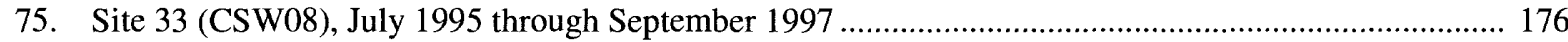

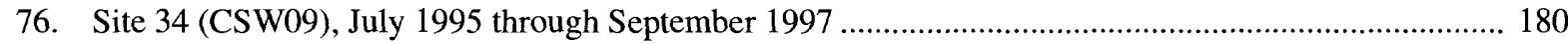

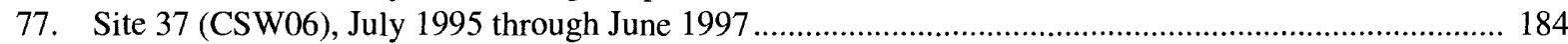

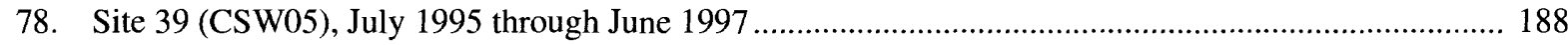

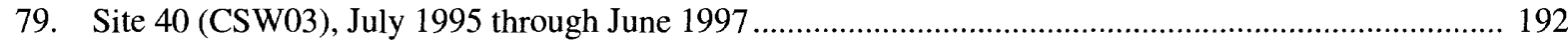

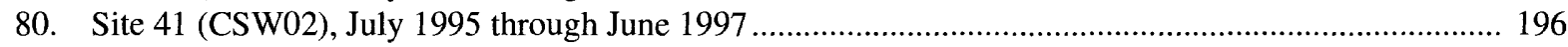

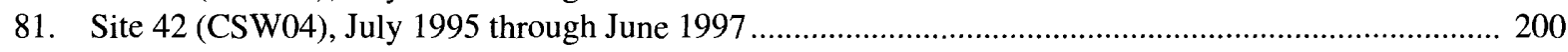

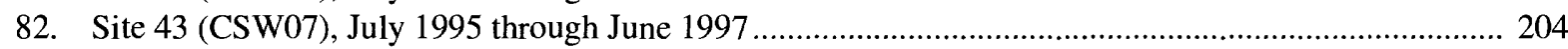

83. Site 44 (CSW10), November 1996 through July 1997 .................................................................... 208

84-92. Rainfall and streamflow characteristics for the monitored storms at:

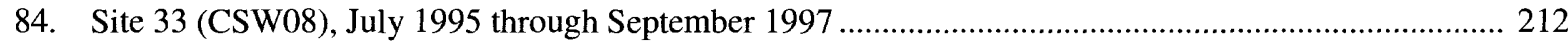

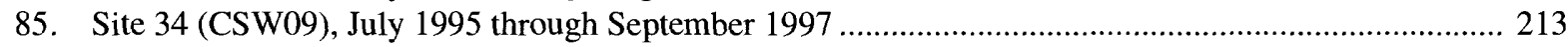

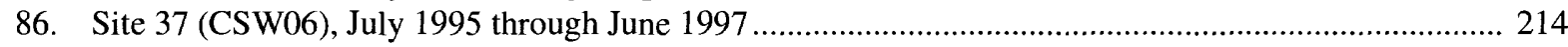

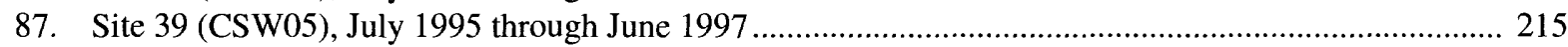

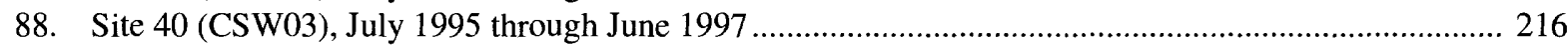

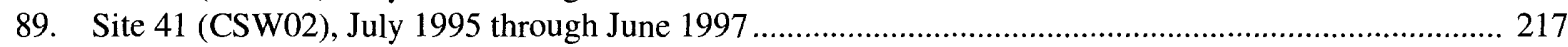

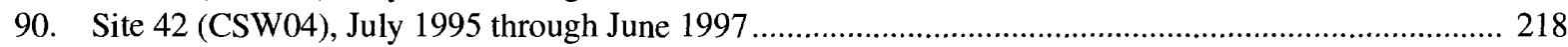

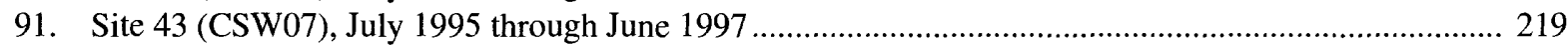

92. Site 44 (CSW10), November 1996 through September 1997 ......................................................... 220

CONVERSION FACTORS, VERTICAL DATUM, SPECIFIC CONDUCTANCE, AND TEMPERATURE

\begin{tabular}{rcl}
\hline Multiply & By & To obtain \\
\hline inch (in.) & 25.4 & millimeter \\
foot $(\mathrm{ft})$ & 0.3048 & meter \\
square $\mathrm{mile}\left(\mathrm{mi}^{2}\right)$ & 2.59 & square kilometer \\
inch per year $(\mathrm{in} / \mathrm{yr})$ & 25.4 & millimeter per year \\
\hline
\end{tabular}

Sea level: In this report "sea level" refers to the National Geodetic Vertical Datum of 1929 (NGVD of 1929) - A geodetic datum derived from a general adjustment of the first-order level nets of both the United States and Canada, formerly called Sea Level Datum of 1929.

Specific conductance is given in microsiemens per centimeter at 25 degrees Celsius $\left(\mu \mathrm{S} / \mathrm{cm}\right.$ at $\left.25^{\circ} \mathrm{C}\right)$.

Equations for temperature conversion between degrees Celsius $\left({ }^{\circ} \mathrm{C}\right)$ and degrees Fahrenheit $\left({ }^{\circ} \mathrm{F}\right)$ :

$$
\begin{aligned}
& { }^{\circ} \mathrm{C}=5 / 9\left({ }^{\circ} \mathrm{F}-32\right) \\
& { }^{\circ} \mathrm{F}=1.8\left({ }^{\circ} \mathrm{C}\right)+32
\end{aligned}
$$




\title{
Precipitation, Streamflow, and Water-Quality Data from Selected Sites in the City of Charlotte and Mecklenburg County, North Carolina, 1995-97
}

\author{
By J.B. Robinson, W.F. Hazell, and R.G. Garrett
}

\section{ABSTRACT}

Precipitation data were collected at 46 precipitation sites and 3 atmospheric deposition sites, and hydrologic data were collected at 9 stream sites in the vicinity of Charlotte and Mecklenburg County, North Carolina, from July 1995 through June 1997. Data were collected to identify the type, concentration, and amount of nonpoint-source stormwater runoff within the area. The data collected include measurements of precipitation; streamflow; physical characteristics, such as water temperature, $\mathrm{pH}$, specific conductance, biochemical oxygen demand, oil and grease, and suspended sediment concentrations; and concentrations of nutrients, metals and minor constituents, and organic compounds.

These data should provide valuable information needed for (1) planned watershed simulation models, (2) estimates of nonpointsource constituent loadings to the Catawba River, and (3) characterization of water quality in relation to basin conditions. Streamflow and rainfall data have been used to provide early warning of possible flooding.

\section{INTRODUCTION}

During 1992-94, the U.S. Geological Survey (USGS), in cooperation with the Western Piedmont Council of Governments, began an investigation of water quality in the upper Catawba River Basin from the headwaters to Lookout Shoals Dam. The objectives of the study were to collect and interpret water-quality data from streams and reservoirs in the region and to develop an unsteady circulation and transport model for the reservoirs in the area.

In October 1993, the USGS, in cooperation with the City of Charlotte, Mecklenburg County, and Charlotte-Mecklenburg Utility Department (CMUD), began a similar study in the Catawba River Basin between Lookout Shoals Dam and Lake Wylie Dam. Study efforts for the City of Charlotte were focused on characterizing stormwater quantity and quality from selected land uses, information on nonpoint-source loadings to the Catawba River, and installation and operation of a precipitation network. Study efforts for Mecklenburg County and CMUD were focused on Mountain Island Lake and included inflow sampling from two basins, outflow sampling, and reservoir monitoring.

The South Carolina District of the USGS is conducting an investigation of water quality in the Catawba River Basin downstream from Lake Wylie. The Catawba River Basin also is part of the USGS National Water-Quality Assessment (NAWQA) Programs's Santee-Coastal Basin study unit. These four studies are providing consistent methods of data collection, interpretation, and modeling techniques for the Catawba River Basin.

\section{Purpose and Scope}

The purpose of this report is to summarize the precipitation and hydrologic data collected in Charlotte and Mecklenburg County from July 1995 through June 1997. Summary statistics are presented for the entire period of record (May 1994 through June 1997). The data collected include measurements of precipitation; streamflow; physical characteristics, such as water 
temperature, $\mathrm{pH}$, specific conductance, biochemical oxygen demand, oil and grease, and suspended sediment concentrations; and concentrations of nutrients, metals and minor constituents, and organic compounds. This report also describes the field and laboratory methods used to collect and analyze these data. A similar report documenting data collected during October 1993-June 1995 was published in 1996 (Robinson and others).
The data-collection network that was initiated in October 1993 with the City of Charlotte, Mecklenburg County, and CMUD consists of 46 precipitation sites, 3 atmospheric deposition sites, and 9 stream sites, which are needed to determine the effects of land development on water quality and to evaluate the effectiveness of control measures (fig. 1). Six of the sites define runoff characteristics from streams with differing land-use characteristics within the city, and

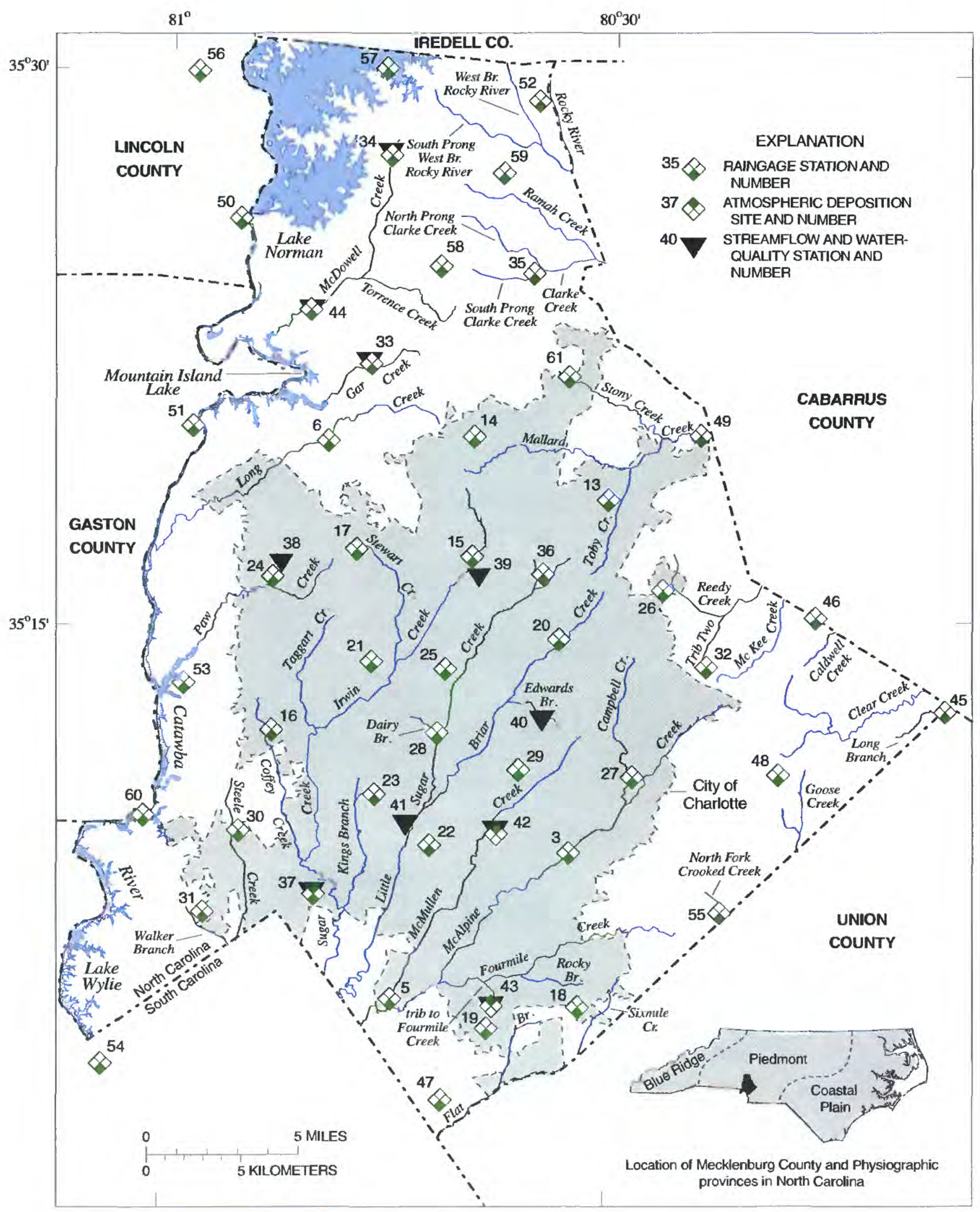

Figure 1. City of Charlotte and Mecklenburg County data-collection network, 1995-97. 
three of the sites define runoff characteristics from streams located within the county. These data should provide valuable information needed for stormwater management, estimates of nonpoint-source constituent loadings to the Catawba River, and information needed to calibrate watershed models necessary for evaluating stormwater management options.

\section{Study Area and Sites}

Mecklenburg County is located in south-central North Carolina within the southern Piedmont Province and encompasses an area of 528 square miles $\left(\mathrm{mi}^{2}\right)$. The county is bounded on the west by the Catawba River and its reservoirs, Lake Norman, Mountain Island Lake, and Lake Wylie (fig. 1). The Catawba River drains approximately 75 percent of the county. The remaining 25 percent of the county is drained by the Rocky River and its tributaries in the Pee Dee River Basin (McCachren, 1980). Lake Norman is the major water-supply reservoir for several municipalities in northern Mecklenburg County. Mountain Island Lake supplies Charlotte and several other municipalities in Mecklenburg and surrounding counties.

Charlotte is the principal municipality in Mecklenburg County and the largest city in North Carolina with a 1997 population of 513,000 in the metropolitan area-an increase of approximately 55,000 persons since 1994. An additional 97,000 people live within Mecklenburg County outside the city limits of Charlotte (Steve Patterson, City of Charlotte Planning Office, oral commun., 1997). Recent annexation has increased the city area from $213 \mathrm{mi}^{2}$ in 1996 to $234 \mathrm{mi}^{2}$, or approximately 44 percent of the county. Most of the urban area is drained by four large creeks-Irwin, Little Sugar, McAlpine, and Briar (fig. 1). Irwin, Little Sugar, and McAlpine Creeks receive effluent from Charlotte wastewater-treatment plants, as well as effluent from smaller dischargers.

The climate of the study area is characterized by hot, humid summers, moderate but short winters, and long growing seasons. The mean monthly temperature ranges from about $41^{\circ} \mathrm{F}$ in January to about $79^{\circ} \mathrm{F}$ in July. Precipitation in the study area averages about 43 inches per year (in/yr) (McCachren, 1980). The topography of the area is characterized by broad, gently rolling interstream areas and by steeper slopes along the drainageways. The elevation of the study area has a range of 520 feet $(\mathrm{ft})$ above mean sea level at the State line south of Pineville, N.C., to about $830 \mathrm{ft}$ in the extreme northern portion of the county (McCachren, 1980). The area is predominately underlain by granite with some slate in the southeast (LeGrand and Mundorff, 1952). The soils in the study area are described as well drained sandy loams with a clayey subsoil (McCachren, 1980).

\section{Precipitation Sites}

Since 1963, the USGS has collected precipitation data at various locations throughout Charlotte and Mecklenburg County. Eighteen raingages installed between December 1995 and January 1997 combined with 28 existing raingages provide precipitation data for this report (fig. 1; table 1). These sites have previously been referred to using CRN numbers; those CRN numbers are included in this report for reference. The primary criterion for site selection of raingage locations was to provide good areal coverage of Charlotte and Mecklenburg County.

Table 1. Precipitation network sites located in Mecklenburg County, October 1988 through June 1997

[Shaded rows indicate collection well sites. All others are tipping bucket sites. WWTP, wastewater-treatment plant]

\begin{tabular}{rcclcr}
\hline $\begin{array}{c}\text { Site } \\
\text { no. } \\
\text { (fig. 1) }\end{array}$ & Station no. $^{\text {a }}$ & Latitude & Longitude & $\begin{array}{c}\text { Period of } \\
\text { record }\end{array}$ \\
\hline 3 & 02146600 & $35^{\circ} 08^{\prime} 14^{\prime \prime}$ & $80^{\circ} 46^{\prime} 05^{\prime \prime}$ CRN10, McAlpine Cr. at Sardis Road nr Charlotte, N.C. & $11 / 92-6 / 97$ \\
5 & 02146750 & $35^{\circ} 03^{\prime} 59^{\prime \prime}$ & $80^{\circ} 52^{\prime} 12^{\prime \prime}$ & CRN06, McAlpine Cr. below McMullen Cr. nr Pineville, N.C. & $5 / 93-6 / 97$ \\
6 & 02142900 & $35^{\circ} 19^{\prime} 42^{\prime \prime}$ & $80^{\circ} 54^{\prime} 35^{\prime \prime}$ CRN18, Long Cr. nr Paw Cr., N.C., at Oakdale Rd. & $3 / 93-6 / 97$ \\
13 & 351812080445545 & $35^{\circ} 18^{\prime} 12^{\prime \prime}$ & $80^{\circ} 44^{\prime} 55^{\prime \prime}$ CRN01, Fire Station 27, 111 Ken Hoffman Dr. & $10 / 92-6 / 97$ \\
14 & 351954080493445 & $35^{\circ} 19^{\prime} 54^{\prime \prime}$ & $80^{\circ} 49^{\prime} 34^{\prime \prime}$ CRN02, Fire Station 28, 8013 Old Statesville Rd. & $10 / 92-6 / 97$ \\
15 & 0214620760 & $35^{\circ} 16^{\prime} 32^{\prime \prime}$ & $80^{\circ} 49^{\prime} 35^{\prime \prime}$ CRN03, Irwin Cr. at Starita Road at Charlotte, N.C. & $10 / 92-6 / 97$ \\
\hline
\end{tabular}

\footnotetext{
aStation number is assigned by the U.S. Geological Survey and is based on geographic location. The "downstream order number" system is used for streamflow sites, and the "latitude-longitude" system is used for well sites.

${ }^{b}$ Precipitation data collection is ongoing at date of publication.
} 
Table 1. Precipitation network sites located in Mecklenburg County, October 1988 through June 1997-Continued [Shaded rows indicate collection well sites. All others are tipping bucket sites. WWTP, wastewater-treatment plant]

\begin{tabular}{|c|c|c|c|c|}
\hline $\begin{array}{c}\text { Site } \\
\text { no. } \\
\text { (fig. 1) }\end{array}$ & Station no. ${ }^{a}$ & Latitude & Longitude & $\begin{array}{l}\text { Period of } \\
\text { record }^{\mathrm{b}}\end{array}$ \\
\hline 16 & 351132080562345 & $35^{\circ} 11^{\prime} 32^{\prime \prime}$ & $80^{\circ} 56^{\prime 23 "}$ CRN04, Fire Station 30, 4707 Belle Oaks Rd. & $10 / 92-6 / 97$ \\
\hline 17 & 351642080533445 & $35^{\circ} 16^{\prime} 42^{\prime \prime}$ & 8053'34" CRN05, CMUD Admin. Bldg., 5100 Brookshire Blvd. & $10 / 92-6 / 97$ \\
\hline 18 & 350351080454145 & $35^{\circ} 03^{\prime} 51^{\prime \prime}$ & $80^{\circ} 45^{\prime} 41^{\prime \prime}$ CRN07, Fire Station 9, 4529 McKee Rd. & $10 / 92-6 / 97$ \\
\hline 19 & 350314080484945 & $35^{\circ} 03^{\prime} 14^{\prime \prime}$ & $80^{\circ} 48^{\prime} 49^{\prime \prime}$ CRN08, 11515 Elm Lane at intersection of Providence Rd. Wesf & $10 / 92-6 / 97$ \\
\hline 20 & 351414080463245 & $35^{\circ} 14^{\prime} 14^{\prime \prime}$ & $80^{\circ} 46^{\prime} 32^{\prime \prime}$ CRN09, Fire Station 15, 3617 Frontenac Ave. & $11 / 92-6 / 97$ \\
\hline 21 & 351331080525945 & $35^{\circ} 13^{\prime} 31^{\prime \prime}$ & $80^{\circ} 52^{\prime} 59^{\prime \prime}$ CRN11, Fire Station 10, 2135 Remount Rd. & $11 / 92-6 / 97$ \\
\hline 22 & 350823080505345 & $35^{\circ} 08^{\prime} 23^{\prime \prime}$ & $80^{\circ} 50^{\prime} 53^{\prime \prime}$ CRN12, Fire Station 16, 6623 Park South Dr. & 3/93-6/97 \\
\hline 23 & 350947080524945 & $35^{\circ} 09^{\prime} 47^{\prime \prime}$ & $80^{\circ} 52^{\prime} 49^{\prime \prime}$ CRN13, USGS Office, 810 Tyvola Rd. & $3 / 93-6 / 97$ \\
\hline 24 & 351553080562645 & $35^{\circ} 15^{\prime} 53^{\prime \prime}$ & $80^{\circ} 56^{\prime} 26^{\prime \prime}$ CRN14, Fire Station 21, 1023 Little Rock Rd. & 3/93-6/97 \\
\hline 25 & 351320080502645 & $35^{\circ} 13^{\prime} 20^{\prime \prime}$ & 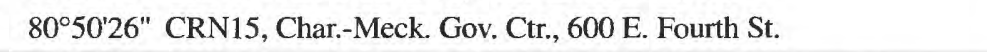 & $3 / 93-6 / 97$ \\
\hline 26 & 351540080430045 & $35^{\circ} 15^{\prime} 40^{\prime \prime}$ & $80^{\circ} 43^{\prime} 00^{\prime \prime}$ CRN16, Reedy Cr. Park Envir. Ctr., 2900 Rocky River Rd. & 3/93-6/97 \\
\hline 27 & 351023080435745 & $35^{\circ} 10^{\prime} 23^{\prime \prime}$ & $80^{\circ} 43^{\prime} 57^{\prime \prime}$ CRN17, Piney Grove Elementary School, 8801 Eaglewind Dr. & 3/93-6/97 \\
\hline 28 & 351132080504145 & $35^{\circ} 11 ' 32^{\prime \prime}$ & $80^{\circ} 50^{\prime} 41^{\prime \prime}$ CRN19, Freedom Park, Cumberland Dr. & 9/93-6/97 \\
\hline 29 & 351032080475245 & $35^{\circ} 10^{\prime} 32^{\prime \prime}$ & $80^{\circ} 47^{\prime} 52^{\prime \prime}$ CRN20, Fire Station 14,114 N. Sharon Amity Rd. & 9/93-6/97 \\
\hline 30 & 350842080572801 & $35^{\circ} 08^{\prime} 42^{\prime \prime}$ & $80^{\circ} 57^{\prime 2} 28^{\prime \prime}$ CRN21, Kennedy Jr. High, 4000 Gallant Lane & 9/90-6/97 \\
\hline 31 & 350623080583801 & $35^{\circ} 06^{\prime} 23^{\prime \prime}$ & 80 58'38" CRN22, Walker Branch Basin, Choate Circle & 9/90-6/97 \\
\hline 32 & 351302080412701 & $35^{\circ} 13^{\prime} 02^{\prime \prime}$ & $80^{\circ} 41^{\prime} 27^{\prime \prime}$ CRN23, Harrisburg Road Landfill, 7817 Harrisburg Rd. & $10 / 88-6 / 97$ \\
\hline 33 & 0214266075 & $35^{\circ} 21^{\prime} 55^{\prime \prime}$ & $80^{\circ} 53^{\prime} 12^{\prime \prime}$ CRN25, Gar Cr. at SR2120 (McCoy Rd.) nr Oakdale, N.C. & $4 / 94-6 / 97$ \\
\hline 34 & 02142651 & $35^{\circ} 27^{\prime} 49^{\prime \prime}$ & 80 52'36" CRN24, McDowell Cr. at Westmoreland Rd. nr Cornelius, N.C. & $5 / 94-6 / 97$ \\
\hline 35 & 352432080473745 & $35^{\circ} 24^{\prime} 32^{\prime \prime}$ & $80^{\circ} 47^{\prime} 37^{\prime \prime}$ CRN26, Bradford Airfield, Huntersville-Concord Rd. & 6/94-6/97 \\
\hline 36 & 351604080470845 & $35^{\circ} 16^{\prime} 04^{\prime \prime}$ & $80^{\circ} 47^{\prime} 08^{\prime \prime}$ CRN27, Hidden Valley Elem. Sch., 5100 Snow White Lane & 10/94-6/97 \\
\hline 37 & 0214635212 & $35^{\circ} 06^{\prime} 57^{\prime \prime}$ & $80^{\circ} 54^{\prime} 49^{\prime \prime}$ CRN28, Unnamed tributary to Sugar Cr. at Crompton St. & 4/95-6/97 \\
\hline 44 & 0214266000 & $35^{\circ} 23^{\prime} 22^{\prime \prime}$ & $80^{\circ} 55^{\prime} 16^{\prime \prime}$ CRN41, McDowell Creek near Charlotte & $11 / 96-6 / 97$ \\
\hline 45 & 351218080331345 & $35^{\circ} 12^{\prime} 18^{\prime \prime}$ & 80³3'13" CRN29, Clear Creek Boy Scout Camp, 9408 Belt Rd. & $2 / 96-6 / 97$ \\
\hline 46 & 351455080374445 & $35^{\circ} 14^{\prime} 55^{\prime \prime}$ & $80^{\circ} 37^{\prime} 44^{\prime \prime}$ CRN30, Rhyne Farm, 3600 Peach Orchard Rd. & 2/96-6/97 \\
\hline 47 & 350110080502045 & $35^{\circ} 01^{\prime} 10^{\prime \prime}$ & $80^{\circ} 50^{\prime} 20^{\prime \prime}$ CRN31, Elon Homes, 11401 Ardrey-Kell Rd. & 2/96-6/97 \\
\hline 48 & 351028080385545 & $35^{\circ} 10^{\prime} 28^{\prime \prime}$ & 80³8'55" CRN32, Bain Elementary School, 11524 Bain School Rd. & 2/96-6/97 \\
\hline 49 & 352000080414645 & $35^{\circ} 20^{\prime} 00^{\prime \prime}$ & $80^{\circ} 41^{\prime} 46^{\prime \prime}$ CRN33, Mallard Creek WWTP, 12400 Hwy. 29 North & $12 / 95-6 / 97$ \\
\hline 50 & 352555080574445 & $35^{\circ} 25^{\prime} 55^{\prime \prime}$ & $80^{\circ} 57^{\prime} 44^{\prime \prime}$ CRN34, Cowans Ford Dam area, 257 Duke Lane & $2 / 96-6 / 97$ \\
\hline 51 & 0214267600 & $35^{\circ} 20^{\prime} 02^{\prime \prime}$ & $80^{\circ} 59^{\prime} 12^{\prime \prime}$ CRN35, Catawba River at Mountain Island Dam & $1 / 96-6 / 97$ \\
\hline 52 & 352921080473245 & $35^{\circ} 29^{\prime} 21^{\prime \prime}$ & $80^{\circ} 47^{\prime} 32^{\prime \prime}$ CRN36, West Fork substation, 20801 Shearer Rd. & $2 / 96-6 / 97$ \\
\hline 53 & 351247080592745 & $35^{\circ} 12^{\prime} 47^{\prime \prime}$ & 8059'27" CRN37, Berryhill Elemen. Sch., 10501 Walkers Ferry Rd. & $2 / 96-6 / 97$ \\
\hline 54 & 350200081020345 & $35^{\circ} 02^{\prime} 00^{\prime \prime}$ & $81^{\circ} 02^{\prime} 03^{\prime \prime}$ CRN38, Tega Cay city offices, 7000 Tega Cay Drive & $2 / 96-6 / 97$ \\
\hline 55 & 350634080405245 & $35^{\circ} 06^{\prime} 34^{\prime \prime}$ & $80^{\circ} 40^{\prime} 52^{\prime \prime}$ CRN39, Phillips Farm, 2248 Mount Harmony Church Rd. & 2/96-6/97 \\
\hline 56 & 353003080591745 & $35^{\circ} 30^{\prime} 03^{\prime \prime}$ & $80^{\circ} 59^{\prime} 17^{\prime \prime}$ CRN40, Westport Golf Course ${ }^{\mathrm{d}}$ & $2 / 96-6 / 97$ \\
\hline 57 & 353014080524945 & $35^{\circ} 30^{\prime} 14^{\prime \prime}$ & 8052'49" CRN42, Horton pool house, 21509 Norman Shores Dr. & $1 / 97-6 / 97$ \\
\hline 58 & 352440080505045 & $35^{\circ} 24^{\prime} 40^{\prime \prime}$ & $80^{\circ} 50^{\prime} 50^{\prime \prime}$ CRN43, Huntersville Elementary School, 200 Gilead Rd. & $1 / 97-6 / 97$ \\
\hline 59 & 352718080484345 & $35^{\circ} 27^{\prime} 18^{\prime \prime}$ & 8048'43" CRN44, Knox Farm, 13516 Mayes Rd. & $1 / 97-6 / 97$ \\
\hline 60 & 350903081004545 & $35^{\circ} 09^{\prime} 03^{\prime \prime}$ & $81^{\circ} 00^{\prime} 45^{\prime \prime}$ CRN45, 12700 Withers Cove Rd. & $1 / 97-6 / 97$ \\
\hline 61 & 352135080462045 & $35^{\circ} 21^{\prime} 35^{\prime \prime}$ & 8046'20" CRN46, Oehler Farm, 3491 Johnston-Oehler Rd. & $1 / 97-6 / 97$ \\
\hline
\end{tabular}

aStation number is assigned by the U.S. Geological Survey and is based on geographic location. The "downstream order number" system is used for streamflow sites, and the "latitude-longitude" system is used for well sites.

${ }^{b}$ Precipitation data collection is ongoing at date of publication.

'Prior to August 4, 1994, located at McAlpine Creek Elementary School, 9100 Carswell Lane, station number 350458080493245.

'Prior to June 4, 1996, located at Lake Norman Volunteer Fire Department, 1206 Brawley School Road, station number 353402080543145. 
Consideration also was given to providing optimum precipitation data for water-quality sampling events and combining installations with existing streamgaging locations. Four raingages were installed with streamflow and water-quality sites-sites $33,34,37$, and 44 . Three raingages were installed at existing USGS stream-gaging stations-sites 3, 5, and 6 (fig. 1; table 1).

Forty-one named stream basins are covered by the raingage locations, including all major stream basins in Charlotte and Mecklenburg County (fig. 1). Raingages located in specific stream subbasins are shown in figure 2 . Four raingages, sites $14,18,46$, and 59 , are located on basin divides and, therefore, represent rainfall coverage in multiple headwater basins.

\section{Atmospheric Deposition Sites}

Atmospheric deposition sites were located in basins with existing streamflow and water-quality datacollection sites. Atmospheric data collection occurred at sites 37, 42, and 43, which represent different land uses within Charlotte (fig. 1; table 2). A detailed description of these sites is given in the following section. Atmospheric deposition samples were collected weekly.

\section{Streamflow and Water-Quality Sites}

Streamflow and water-quality site selection was based on the size of the drainage areas and the type of land use. The land-use information presented in this report was obtained from the City of Charlotte and is based on data classified from 1990 aerial photographs and reconnaissance conducted by USGS personnel.
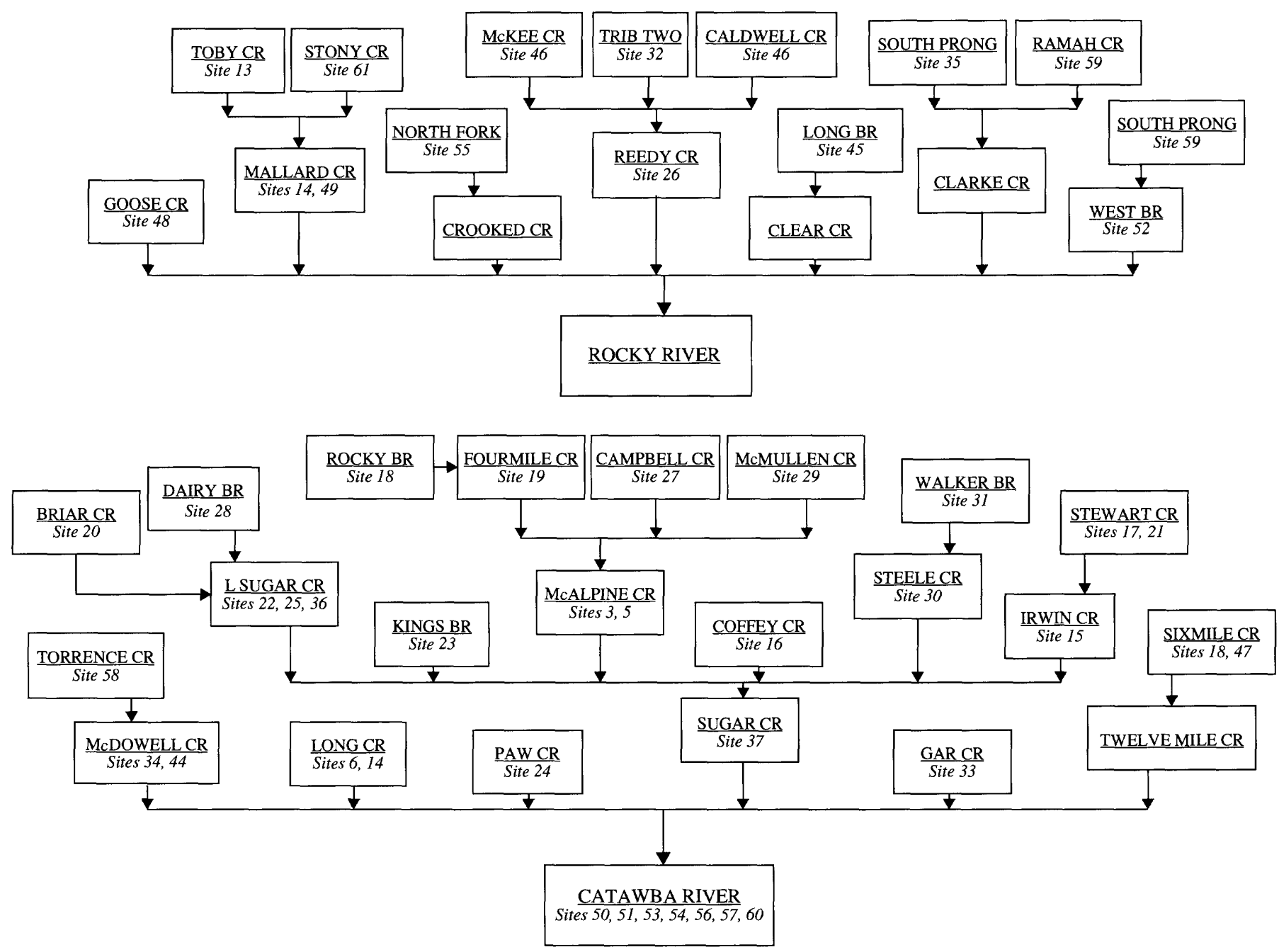

Figure 2. Raingage locations, by basin, in Charlotte and Mecklenburg County, North Carolina. 


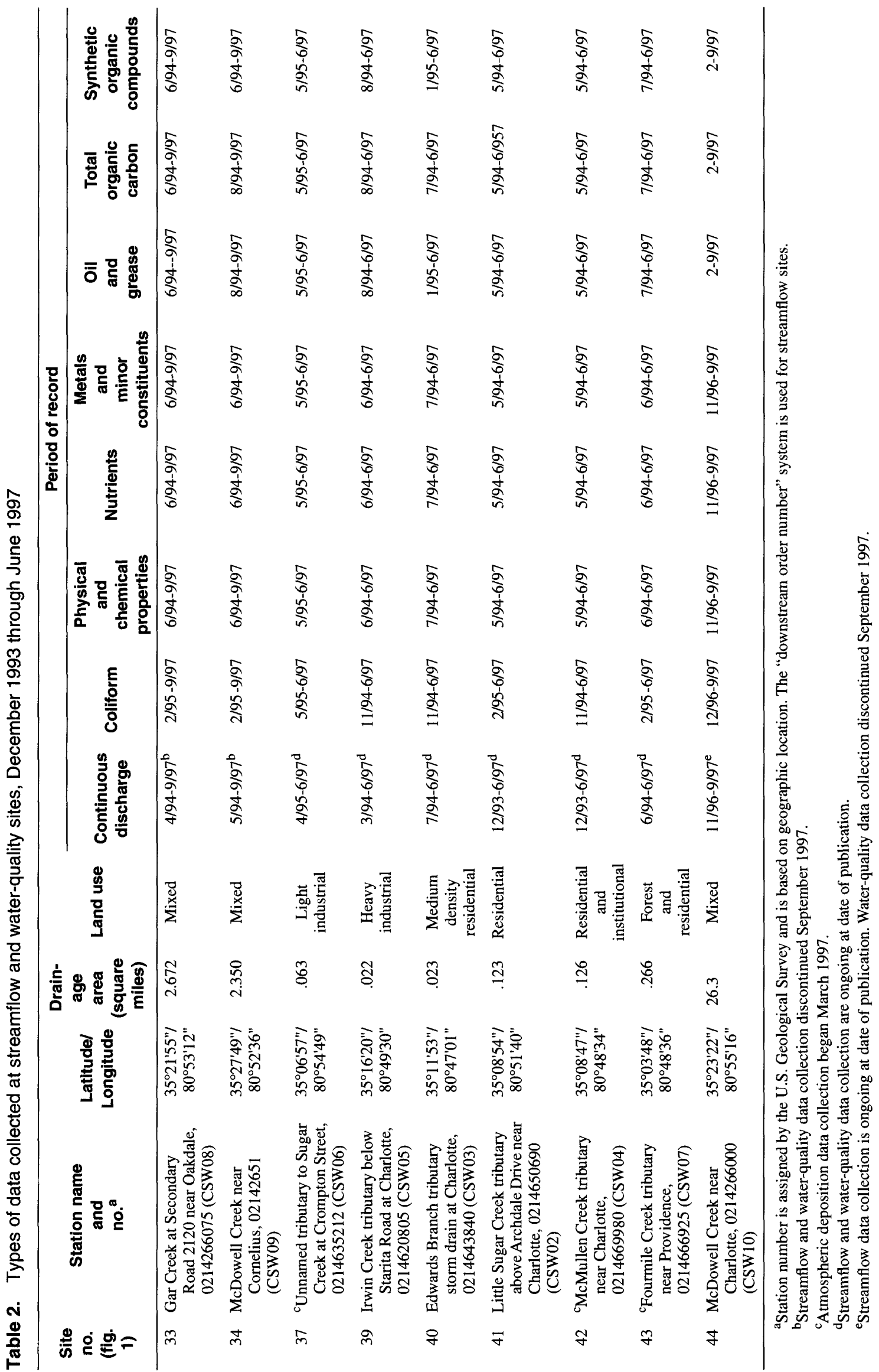

6 Precipitation, Streamflow, and Water-Quality Data for Charlotte and Mecklenburg County, N.C., 1995-97 
Basin land-use maps were previously published (Robinson and others, 1996) except for site 44. Six sites were chosen within the city limits of Charlotte, sites $37,39,40,41,42$, and 43 , and three sites were chosen in the northern part of the county, sites 33, 34, and 44 (fig. 1; table 2). These sites have previously been referred to using CSW numbers; those CSW numbers are included in this report for reference. An additional site within Charlotte, site 38, was constructed and data collection began in April 1996. Unforeseen changes in the basin diverted runoff away from this location and the site was abandoned in September 1996. The amount of data collected at this site is limited and thus not included in this report. Each site within the city drains into one of the four major streams carrying runoff from the metropolitan area. Sites 33, 34, and 44 all drain directly into Mountain Island Lake water-supply reservoir. All sites have continuous records of stage and discharge, water temperature, and specific conductance. Water-quality samples were collected seasonally, during runoff events.

Site 33 is located on Gar Creek, a major tributary to Mountain Island Lake (fig. 1). The drainage area has mixed land use-primarily woods or brush and residential (table 3)-and encompasses $2.67 \mathrm{mi}^{2}$. The area classified as residential (greater than 2 acres) may include some agricultural land uses.

Site 34 is located on McDowell Creek, also a major tributary to Mountain Island Lake (fig. 1). The drainage area of $2.35 \mathrm{mi}^{2}$ is bisected by Interstate
Highway I-77. Land-use types are mixed and include residential, woods or brush, commercial, industrial, and institutional (table 3).

Site 37 is located on a tributary to Sugar Creek (fig. 1). The drainage area encompasses $0.063 \mathrm{mi}^{2}$ and consists of light industrial, light commercial, and some woods or brush (table 3 ). A small portion of an active railroad is also within the basin.

Site 39 is located on a tributary to Irwin Creek (fig. 1). Land use is entirely heavy industrial with a drainage area of $0.022 \mathrm{mi}^{2}$ (table 3).

Site 40 is located in a storm drain to a tributary of Edwards Branch which flows into Briar Creek (fig. 1). Land use is almost entirely medium density residential (table 3) with a drainage area of $0.023 \mathrm{mi}^{2}$. A very small portion of the basin includes some light industry as well as an elementary school.

Site 41 is located on a tributary to Little Sugar Creek (fig. 1) and has a multi-use drainage area of $0.123 \mathrm{mi}^{2}$. Residential housing is the primary land use. The basin also includes a portion of a large chemical research laboratory, an elementary school, and some light commercial activity (table 3 ).

Site 42 is located on a tributary to McMullen Creek (fig. 1) and has a drainage area of $0.126 \mathrm{mi}^{2}$. Land use within the basin is residential and institutional (a private school). Some light commercial activity is also present (table 3 ).

Site 43 is located on a tributary to Fourmile Creek (fig. 1). At the time of site selection, land use was considered to be pre-development. Much of the

Table 3. Land-use percentage distribution for study site drainage areas

[Values are in percent. ---, no land-use data for this category]

\begin{tabular}{|c|c|c|c|c|c|c|c|c|c|c|c|c|}
\hline \multirow[b]{2}{*}{$\begin{array}{c}\text { Site no. } \\
\text { (fig. 1) }\end{array}$} & \multirow[b]{2}{*}{$\begin{array}{l}\text { Woods/ } \\
\text { Brush }\end{array}$} & \multicolumn{4}{|c|}{ Residential } & \multirow[b]{2}{*}{$\begin{array}{l}\text { Institu- } \\
\text { tional }\end{array}$} & \multicolumn{2}{|c|}{ Industrial } & \multicolumn{2}{|c|}{ Commercial } & \multirow[b]{2}{*}{$\begin{array}{l}\text { Stand- } \\
\text { ing } \\
\text { water }\end{array}$} & \multirow[b]{2}{*}{$\begin{array}{c}\text { Trans- } \\
\text { porta- } \\
\text { tion }\end{array}$} \\
\hline & & $\begin{array}{c}\text { Greater } \\
\text { than } \\
2 \text { acres }\end{array}$ & $\begin{array}{c}\text { Greater } \\
\text { than } \\
1 / 2 \text { to } \\
2 \text { acres }\end{array}$ & $\begin{array}{c}\text { Greater } \\
\text { than } \\
1 / 4 \text { to } \\
1 / 2 \text { acre }\end{array}$ & $\begin{array}{l}\text { Less } \\
\text { than } \\
\text { or } \\
\text { equal } \\
\text { to } 1 / 4 \\
\text { acre }\end{array}$ & & Light & Heavy & Light & Heavy & & \\
\hline 33 [CSW08] & 58.1 & 29.3 & 9.9 & 1.3 & $-\cdots$ & 0.5 & 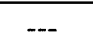 & 0.5 & $-\cdots$ & -- & 0.4 & -- \\
\hline 34 [CSW09] & 39.9 & 23.2 & 13.6 & 8.7 & 0.3 & .9 & 0.7 & --- & 6.9 & 1.5 & .1 & 4.2 \\
\hline 37 [CSW06] & 10.3 & .1 & $-\cdots$ & -.- & $\cdots$ & $\cdots$ & 63.5 & $\cdots$ & 26.1 & $\cdots$ & -- & --- \\
\hline 39 [CSW05] & .1 & -- & -- & -- & -- & -- & -- & 99.8 & .1 & -- & -- & -- \\
\hline 40 [CSW03] & -- & --- & 2.1 & 96.8 & -- & -- & 1.1 & $\cdots$ & --- & -- & -- & -- \\
\hline 41 [CSW02] & 1.7 & -- & $\cdots$ & 57.7 & -- & 5.8 & -- & 22.9 & 11.9 & -- & -- & --- \\
\hline 42 [CSW04] & -- & -- & 7.9 & 19.4 & 31.3 & 40.6 & --- & -- & .8 & -- & -- & -- \\
\hline 43 [CSW07] & 17.2 & --- & 2.5 & 33.0 & --- & 3.2 & -- & --- & 44.1 & --- & -- & --- \\
\hline 44 [CSW10] & 42.6 & 35.7 & 9.3 & 4.5 & .2 & .6 & .8 & .6 & 1.7 & .3 & .3 & 3.4 \\
\hline
\end{tabular}


drainage area is now residential (both single and multifamily) with ongoing new construction as well as some woods or brush. Some light commercial land use, a large church, and a rest home are also present (table 3). The drainage area is $0.266 \mathrm{mi}^{2}$.

Site 44 is also located on McDowell Creek approximately 6 miles downstream from site 34 (fig. 1). Land-use types are mixed, predominately woods or brush and residential, but include some commercial, industrial, and institutional (table 3 ). The drainage area is $26.3 \mathrm{mi}^{2}$.

\section{DATA-COLLECTION METHODS}

All sites are equipped with electronic dataloggers for instrument operation and data collection. Storage modules with independent, internal batteries and nonvolatile memory also store programs and data for backup. Modems at the sites allow remote communication and interaction with the dataloggers. Software was developed to automatically retrieve and process data daily. Remote interaction also allows users to monitor, test, and activate peripheral devices from any offsite location.

\section{Precipitation Data}

Two types of raingages were installed in the study area-tipping bucket raingage or 3-inch (in.) diameter collection well with water-level sensor. The type of rainfall measuring equipment installed was determined on a site-by-site basis. Thirteen sites initially were installed with collection well pipes in less secure areas or where rooftop tipping buckets were not feasible. Site 17 was converted to a tipping bucket site on Dec. 29, 1994. As of June 1997, there were 34 tipping bucket sites and 12 collection well sites (table 1).

All sites record rainfall amounts at 5-minute intervals. The raingages located at water-quality sampling sites also record rainfall at 1-minute intervals when rainfall is detected. Neither type of raingage is designed to measure rainfall equivalency during periods of frozen precipitation. Melting of this frozen precipitation by sunlight, warming temperatures, or artificial heat source may provide these rainfall equivalent data for a daily or monthly total.

\section{Atmospheric Deposition Data}

Collection of atmospheric deposition samples began in March 1997 at sites 37, 42, and 43 (fig. 1; table 2). Hydrologic data include quantity of wet deposition and analysis of wet deposition samples for specific conductance, $\mathrm{pH}$, nutrients, selected metals, chloride, and sulfate.

Wet deposition samples were collected using an automatic wet/dry sampler equipped with a plastic sample-collection container and powered by a 12-volt battery. This device has a motorized protective lid which keeps the sample-collection container covered during periods of no precipitation. When the moisture sensor detects precipitation, the lid is mechanically moved to allow wet deposition to be collected in the sample container. When the precipitation stops, the lid is mechanically returned to the protective position. Samples were retrieved on Mondays of each week.

Samples were weighed in the USGS Charlotte

Field Office using an analytical balance. Once weighed, the precipitation amount was computed in inches equivalent. Samples were then decanted directly from the collection container into the appropriate subsample containers and preserved.

\section{Streamflow and Water-Quality Data}

Data collection began in December 1993 at sites 41 and 42, March 1994 at site 39, April 1994 at site 33, May 1994 at site 34, June 1994 at site 43, July 1994 at site 40, April 1995 at site 37, and November 1996 at site 44. Hydrologic data include measurements of streamflow, coliform bacteria, physical and chemical properties, nutrients, concentrations of metals and minor constituents, oil and grease, organic compounds in water (table 2), and suspended sediment. Streamflow and water-quality data collection were discontinued September 1997 at sites 33 and 34. Water-quality data collection was discontinued September 1997 at site 44 .

Instrumentation at each site includes sensors for the collection of water temperature, specific conductance, $\mathrm{pH}$, and water level. Water-quality samples were collected using an automatic refrigerated sampler. All equipment is housed in a walk-in shelter with alternating current (a.c.). Sites 33, 34, 37, and 44 have tipping bucket raingages for the collection of precipitation data.

Continuous record gages were established at each site. Because of the almost instantaneous response 
of streamflow to precipitation in small urban basins, water levels were recorded every 5 minutes. Water levels were recorded every minute when stream stage rose above a predetermined threshold and during water-quality sampling events. At the beginning of the study (December 1993), sites 41 and 42 had collection intervals of 15 minutes.

Instantaneous water level, or stage, was recorded and streamflow, or discharge, was measured on an asneeded basis following procedures outlined by Rantz and others (1982). Stage-discharge relations were subsequently developed and utilized to quantify streamflow at each recorded 5-minute interval. The absence of a suitable measuring location at site 40 required the stage-discharge relation to be based on flow through a weir at low stages and a mathematical culvert flow determination at higher stages. Similar conditions at site 39 required the use of a computed weir stage-discharge rating to determine flow over the weir. Periodic current-meter measurements were made when possible.

Water temperature and specific conductance were measured every 5 minutes with an in situ probe. Initially, these data also were collected at 1-minute intervals when flow was above the predetermined threshold and during water-quality sampling events. Review of these data indicated that water temperature and specific conductance did not vary significantly at 1-minute intervals. Thus, the collection interval was increased to 5 minutes in June 1995.

$\mathrm{pH}$ was measured during water-quality sampling events. Due to its fragile nature, the $\mathrm{pH}$ probe was mounted in a flowthrough cell connected to a water pump rather than being placed in situ. Collection of $\mathrm{pH}$ data was initiated simultaneously with water-quality sample collection and continued for a period of 10 minutes. Data were recorded every minute during the 10 -minute period. Collection of continuous $\mathrm{pH}$ data during water-quality sampling events was discontinued January 1996. Subsequently, raw water samples collected by the automatic samplers, as well as grab samples collected during an event, were used for $\mathrm{pH}$ determinations.

Water samples were collected at each study site during runoff events on a seasonal basis. The criteria, provided by the cooperators, used to determine if the sampled event met the requirements of the project were that (1) the minimum period between sampled events was at least 21 days, (2) the rainfall duration was between 3 and 13 hours, (3) the rainfall amount was between 0.2 and 0.8 in., and (4) there had been less than $0.1 \mathrm{in}$. of rainfall in the 72 hours prior to the sampled event. The rainfall amount could exceed $0.8 \mathrm{in}$. and (or) the duration could be longer than 13 hours as long as the total rainfall amount during the first 3 hours was less than 0.8 in. Every effort was made to adhere to these criteria, but there were times when all criteria were not met.

Generally, three discrete samples were collected during increasing, near peak, and receding streamflows associated with the runoff event. Specific conductance and $\mathrm{pH}$ of each sample were measured as the sample was processed. Samples were analyzed for a broad range of constituents.

Water samples for inorganic analysis were collected using an automatic refrigerated sampler. Each discrete sample consisted of two raw water samples collected in 1.9-liter glass bottles. The two bottles were composited in a polycarbonate churn splitter, and processed and preserved as described by Horowitz and others (1994) (table 4). Beginning in September 1995, the Mecklenburg County Department of Environmental Laboratory began analyzing the samples that were collected for inorganic constituents. These samples were preserved as required by the Mecklenburg County laboratory (table 5). Total organic carbon (TOC) samples were taken from the discrete samples prior to placement in the churn splitter. Samples for the analysis of dissolved constituents were filtered through a 0.45 -micron poresize capsule filter using a peristaltic pump.

Samples for most organic analyses were collected using an automatic refrigerated sampler with methanol cleaned Teflon tubing or by hand as a grab sample. Pesticide samples were collected during the spring and were decanted directly from the glass collection bottles to the appropriate sample containers.

Grab samples included oil and grease and volatile organic compounds (VOCs), which were collected during the first 20-30 minutes of the runoff event. Bacteria samples were collected manually during increasing, near peak, and receding streamflows. TOC also was collected as a grab sample in the spring when the automatic sampler was equipped with methanol cleaned tubing.

\section{Quality-Assurance Procedures}

Quality-assurance procedures for precipitation, atmospheric deposition, streamflow, and water-quality 
Table 4. Containers, container treatment, and preservation procedures required for samples collected at the streamflow and water-quality study sites and analyzed by the U.S. Geological Survey National Water-Quality Laboratory, May 1994 through August 1995

$\left[{ }^{\circ} \mathrm{C}\right.$, degrees Celsius; $\mathrm{mL}$, milliliter; <, less than; $N$, normality of solution; $>$, greater than; $\mathrm{L}$, liter]

\begin{tabular}{|c|c|c|c|}
\hline $\begin{array}{l}\text { Compounds, elements, } \\
\text { or } \\
\text { properties analyzed }\end{array}$ & $\begin{array}{l}\text { Container } \\
\text { size }\end{array}$ & Container type & $\begin{array}{l}\text { Container treatment } \\
\text { and } \\
\text { sample preservation }\end{array}$ \\
\hline \multicolumn{4}{|c|}{ Physical and chemical properties } \\
\hline Dissolved solids, residue at $180^{\circ} \mathrm{C}$ & $250 \mathrm{~mL}$ & Polyethylene & $\begin{array}{l}\text { Filter through a disposable capsule filter with } 0.45 \text {-micron pore } \\
\text { size; use filtered sample to rinse containers. }\end{array}$ \\
\hline $\mathrm{pH}$, specific conductance, alkalinity & $500 \mathrm{~mL}$ & Polyethylene & Unfiltered; use unfiltered sample to rinse containers. \\
\hline Volatile suspended solids & $500 \mathrm{~mL}$ & Polyethylene & Unfiltered; use unfiltered sample to rinse containers. \\
\hline Chemical oxygen demand & $125 \mathrm{~mL}$ & Glass & $\begin{array}{l}\text { Bottle baked at } 450{ }^{\circ} \mathrm{C} \text {. Acidify collected sample with } \mathrm{H}_{2} \mathrm{SO}_{4} \text { to } \\
\mathrm{pH}<2 \text {; chill and maintain sample at } 4{ }^{\circ} \mathrm{C} \text {. }\end{array}$ \\
\hline Biochemical oxygen demand ${ }^{a}$ & $500 \mathrm{~mL}$ & Polyethylene & Unfiltered, chill and maintain sample at $4{ }^{\circ} \mathrm{C}$. \\
\hline Coliform $^{a}$ & $200 \mathrm{~mL}$ & Glass & Sterile, chill and maintain sample at $4{ }^{\circ} \mathrm{C}$. \\
\hline \multicolumn{4}{|c|}{ Nutrients } \\
\hline Dissolved nutrients & $125 \mathrm{~mL}$ & Brown polyethylene & $\begin{array}{l}\text { Filter through a disposable capsule filter with } 0.45 \text {-micron pore } \\
\text { size; use filtered sample to rinse containers. Chill and maintain } \\
\text { sample at } 4^{\circ} \mathrm{C} \text {. }\end{array}$ \\
\hline Total nutrients & $125 \mathrm{~mL}$ & Brown polyethylene & $\begin{array}{l}\text { Unfiltered; use unfiltered sample to rinse containers. Chill and } \\
\text { maintain sample at } 4{ }^{\circ} \mathrm{C} \text {. }\end{array}$ \\
\hline \multicolumn{4}{|c|}{ Metals and minor constituents } \\
\hline $\mathrm{Sb}, \mathrm{Be}, \mathrm{Cr}, \mathrm{Cu}, \mathrm{Cd}, \mathrm{Pb}, \mathrm{Ni}, \mathrm{Ag}, \mathrm{Zn}$ & $500 \mathrm{~mL}$ & Polyethylene, acid rinsed & $\begin{array}{l}\text { Unfiltered; use unfiltered sample to rinse containers. Acidify } \\
\text { collected sample with nitric acid to } \mathrm{pH}<2 \text {. }\end{array}$ \\
\hline As, $\mathrm{Se}$ & $250 \mathrm{~mL}$ & Polyethylene, acid rinsed & $\begin{array}{l}\text { Unfiltered; use unfiltered sample to rinse containers. Acidify } \\
\text { collected sample with nitric acid to } \mathrm{pH}<2 \text {. }\end{array}$ \\
\hline $\mathrm{Hg}$ & $250 \mathrm{~mL}$ & Glass, acid rinsed & $\begin{array}{l}\text { Unfiltered; use unfiltered sample to rinse containers. Acidify } \\
\text { collected sample with nitric acid/potassium dichromate to } \mathrm{pH} \\
<2 \text {. }\end{array}$ \\
\hline $\mathrm{Cn}$ & $250 \mathrm{~mL}$ & Polyethylene & $\begin{array}{l}\text { Unfiltered; use unfiltered sample to rinse containers. Add to } \\
\text { sample, } 5 \mathrm{~N} \text { sodium hydroxide to } \mathrm{pH}>12 \text {. Chill and maintain } \\
\text { sample at } 4{ }^{\circ} \mathrm{C} \text {. }\end{array}$ \\
\hline \multicolumn{4}{|c|}{ Organic compounds } \\
\hline Pesticides and herbicides ${ }^{b}$ & $1 \mathrm{~L}$ & Glass, amber & $\begin{array}{l}\text { Bottle baked at } 450^{\circ} \mathrm{C} \text {. Do not rinse container in field. Chill and } \\
\text { maintain sample at } 4{ }^{\circ} \mathrm{C} \text {. }\end{array}$ \\
\hline Total organic carbon ${ }^{b}$ & $125 \mathrm{~mL}$ & Glass, amber & $\begin{array}{l}\text { Bottle baked at } 450^{\circ} \mathrm{C} \text {. Do not rinse container in field. Chill and } \\
\text { maintain sample at } 4{ }^{\circ} \mathrm{C} \text {. }\end{array}$ \\
\hline Volatile organic compounds ${ }^{b}$ & $40 \mathrm{~mL}$ & Glass septum vial, amber & $\begin{array}{l}\text { Do not rinse container in field. Exclude all air bubbles in sample } \\
\text { by completely filling vial. Protect sample from sunlight. Chill } \\
\text { and maintain sample at } 4{ }^{\circ} \mathrm{C} \text {. }\end{array}$ \\
\hline Oil and grease & $1 \mathrm{~L}$ & Glass, amber & $\begin{array}{l}\text { Bottle baked at } 450{ }^{\circ} \mathrm{C} \text {. Do not rinse container in field. Leave } \\
\text { small air space. Add to sample, } 2.0 \mathrm{~mL} \mathrm{H}_{2} \mathrm{SO}_{4} \text { to } \mathrm{pH}<2 \text {. Chill } \\
\text { and maintain sample at } 4{ }^{\circ} \mathrm{C} \text {. }\end{array}$ \\
\hline \multicolumn{4}{|c|}{ Sediment } \\
\hline $\begin{array}{l}\text { Suspended sediment and volatile } \\
\text { suspended solids }{ }^{c}\end{array}$ & 1 pint & Glass & None. \\
\hline
\end{tabular}

${ }^{a}$ Analyses performed by the Mecklenburg County Department of Environmental Protection Laboratory.

${ }^{b}$ Analyses performed by the U.S. Geological Survey Water-Quality Laboratory, May 1994 through June 1997.

${ }^{c}$ Analyses performed by the U.S. Geological Survey Sediment Laboratory, May 1994 through June 1997. 
Table 5. Containers, container treatment, and preservation procedures required for samples collected at the streamflow and water-quality study sites and analyzed by the Mecklenburg County Department of Environmental Protection Laboratory, September 1995 through June 1997

$\left[{ }^{\circ} \mathrm{C}\right.$, degrees Celsius; $\mathrm{mL}$, milliliter; $N$, normality of solution]

\begin{tabular}{|c|c|c|c|}
\hline $\begin{array}{c}\text { Compounds, elements, } \\
\text { or } \\
\text { properties analyzed }\end{array}$ & $\begin{array}{l}\text { Container } \\
\text { size }\end{array}$ & Container type & $\begin{array}{l}\text { Container treatment } \\
\text { and } \\
\text { sample preservation }\end{array}$ \\
\hline \multicolumn{4}{|c|}{ Physical and chemical properties } \\
\hline Dissolved solids, residue at $180^{\circ} \mathrm{C}$ & $500 \mathrm{~mL}$ & $\begin{array}{l}\text { Polyethylene, red cap, } \\
\text { disposable }\end{array}$ & $\begin{array}{l}\text { Filter through a disposable capsule filter with } 0.45 \text {-micron pore } \\
\text { size; use filtered sample to rinse containers. }\end{array}$ \\
\hline $\mathrm{pH}$, specific conductance, alkalinity & $1,000 \mathrm{~mL}$ & $\begin{array}{l}\text { Polyethylene, blue cap, } \\
\text { disposable }\end{array}$ & Unfiltered; use unfiltered sample to rinse containers. \\
\hline Volatile suspended solids & $1,000 \mathrm{~mL}$ & $\begin{array}{l}\text { Polyethylene, blue cap, } \\
\text { disposable }\end{array}$ & Unfiltered; use unfiltered sample to rinse containers. \\
\hline Chemical oxygen demand & $250 \mathrm{~mL}$ & $\begin{array}{l}\text { Polyethylene, orange cap, } \\
\text { disposable }\end{array}$ & $\begin{array}{l}\text { Acidify collected sample with } 1.0 \mathrm{~mL} \mathrm{H}_{2} \mathrm{SO}_{4} \text {; chill and maintain } \\
\text { sample at } 4{ }^{\circ} \mathrm{C} \text {. }\end{array}$ \\
\hline Biochemical oxygen demand ${ }^{a}$ & $1,000 \mathrm{~mL}$ & $\begin{array}{l}\text { Polyethylene, blue cap, } \\
\text { disposable }\end{array}$ & Unfiltered, chill and maintain sample at $4{ }^{\circ} \mathrm{C}$. \\
\hline Coliform $^{\mathrm{a}}$ & $200 \mathrm{~mL}$ & Glass & Sterile, chill and maintain sample at $4^{\circ} \mathrm{C}$. \\
\hline \multicolumn{4}{|c|}{ Nutrients } \\
\hline Dissolved nutrients & $250 \mathrm{~mL}$ & $\begin{array}{l}\text { Polyethylene, green cap, } \\
\text { disposable }\end{array}$ & $\begin{array}{l}\text { Filter through a disposable capsule filter with } 0.45 \text {-micron pore } \\
\text { size; use filtered sample to rinse containers. Add } 1.0 \mathrm{~mL} \\
\mathrm{H}_{2} \mathrm{SO}_{4} \text {; chill and maintain sample at } 4{ }^{\circ} \mathrm{C} \text {. }\end{array}$ \\
\hline Total nutrients & $250 \mathrm{~mL}$ & $\begin{array}{l}\text { Polyethylene, orange cap, } \\
\text { disposable }\end{array}$ & $\begin{array}{l}\text { Unfiltered; use unfiltered sample to rinse containers. Add } 1.0 \mathrm{~mL} \\
\mathrm{H}_{2} \mathrm{SO}_{4} \text {; chill and maintain sample at } 4{ }^{\circ} \mathrm{C} \text {. }\end{array}$ \\
\hline \multicolumn{4}{|c|}{ Metals and minor constituents } \\
\hline $\begin{array}{l}\text { As, } \mathrm{Se}, \mathrm{Hg}, \mathrm{Sb}, \mathrm{Be}, \mathrm{Cr}, \mathrm{Cu}, \mathrm{Cd}, \mathrm{Pb} \\
\quad \mathrm{Ni}, \mathrm{Ag}, \mathrm{Zn}\end{array}$ & $500 \mathrm{~mL}$ & $\begin{array}{l}\text { Polyethylene, acid rinsed, } \\
\text { yellow cap, disposable }\end{array}$ & $\begin{array}{l}\text { Unfiltered; use unfiltered sample to rinse containers. Add } \\
1.25 \mathrm{~mL} \text { of } \mathrm{HNO}_{3} \text {. }\end{array}$ \\
\hline $\mathrm{Cn}$ & $1,000 \mathrm{~mL}$ & $\begin{array}{l}\text { Polyethylene, acid rinsed, } \\
\text { blue cap, disposable }\end{array}$ & $\begin{array}{l}\text { Unfiltered; use unfiltered sample to rinse containers. Add } 10 \mathrm{~mL} \\
\text { of } 5 \mathrm{~N} \text { sodium hydroxide. Chill and maintain sample at } 4{ }^{\circ} \mathrm{C} \text {. }\end{array}$ \\
\hline \multicolumn{4}{|c|}{ Organic compounds } \\
\hline Oil and grease & $2,500 \mathrm{~mL}$ & Glass & Surface skim, unfiltered. Add $5.0 \mathrm{~mL} \mathrm{HCl}$. \\
\hline
\end{tabular}

${ }^{a}$ Analyses performed by the Mecklenburg County Department of Environmental Protection Laboratory, May 1994 through June 1997.

data collection and processing are presented in the following sections. All procedures followed standard USGS guidelines as documented in each section. Detailed quality-assurance procedures were prepared and are documented in a USGS administrative report.

\section{Precipitation Data}

Tipping bucket raingages were delivered from the factory with documented calibration. Factory calibration consists of pouring a known amount of water into the bucket at a fixed rate and comparing the recorded amount with the known rainfall equivalency. Collection well raingages were designed and constructed according to generally accepted standards.

All sites were field calibrated from April 1997 to June 1997. Tipping buckets were calibrated using a technique similar to that applied in the factory. At collection well sites, catchment dimensions were measured and a surface area computed. A known amount of water was poured into the catchment, and the rainfall total recorded was compared to the rainfall equivalent of the known volume. Measured precipitation for 34 raingages was within 5 percent of the actual amount, and 45 of the raingages recorded precipitation within 10 percent of the actual amount. Rainfall was under-recorded at site 61 by 12 percent. The equipment was adjusted and a correction was applied to the data.

Sites are visited on an average of every 6 to 8 weeks. Initial readings of time and rainfall are recorded. Catchment, funnel, and tubing are inspected for blockage, and conditions are noted. Catchments and funnels are wiped clean and rinsed free of debris. Tubing is reamed, rinsed, and brushed clean. Battery voltage is measured with an external voltmeter, and the reading is compared to that of the datalogger. Freshly 
charged batteries are installed when needed. The installation and phone lines are inspected for vandalism or tampering.

Tipping bucket pivots are oiled, and buckets are inspected for freedom of movement and assurance of interaction with the datalogger. After draining a collection well, a small amount of water is returned to the well. Inspections include visibly watching the float wheel turn and physically checking the response of the float wheel.

Final readings of time and rainfall are recorded before leaving the site. After completion of the site visit but before leaving the area of the site, contact is made with the datalogger using a cellular phone to assure that all phone connections are working properly.

Data are automatically retrieved daily via modem and phone line. Daily summary printouts available for inspection include: daily rainfall total, accumulated rainfall total since last service, and battery voltage. A location map of the raingages with corresponding totals for the previous day also is available. This allows for early identification and correction of problems. Rainfall plots are printed from the USGS database for the 5 previous days to check the data and assure that these data have been entered into the database.

Data are inspected for signs of drifting float wheels. This drift is easily spotted, and any accumulated rainfall amounts resulting from the drift are removed from the database. Rainfall data during and after site visits are inspected and compared to field notes to assure proper readings. Daily totals are compared with data from surrounding sites to check for reasonable agreement.

During periods of sub-freezing air temperature or suspected frozen precipitation, data are inspected for signs of improper recording of precipitation. Incremental data for periods of apparent frozen precipitation are deleted from the database. When possible, daily or monthly totals are estimated based on readings recorded as the snow and ice melts.

\section{Atmospheric Deposition Data}

Installation and operation of the automatic wet/ dry samplers were in accordance with protocols established by the National Atmospheric Deposition Program (NADP) (Bigelow, 1984; Bigelow and Dossett, 1988) with the exception that samples were retrieved on Mondays rather than Tuesdays. Samplers were equipped with polycarbonate protective lids and
Teflon coated arms to prevent metal contamination of samples collected for metals and minor constituents.

Plastic sample-collection containers were prepared by washing with a nonphosphate detergent and soaking in a 5-percent hydrochloric acid solution as described by Horowitz and others (1994). Equipment was assigned to each site to prevent possible cross-contamination between sites.

Quality-assurance samples comprise approximately 20 percent of the samples analyzed. Equipment blanks using inorganic blank water provided by the USGS laboratory were prepared and analyzed for nutrients and metals and minor constituents to validate the cleaning procedures as well as to ensure no contaminants were leaching from the sample-collection container. The quality-assurance blank analysis for nutrients was done using the lowlevel automated-segment flow method (ASF), and the metals and minor constituents were analyzed using the Inductively Coupled Plasma-Mass Spectrometry method (ICP-MS). In addition, split samples were analyzed periodically for each site when sample volume allowed.

\section{Streamflow and Water-Quality Data}

Installation and operation of the continuous record gages were in accordance with USGS standards described in the Techniques of Water-Resources Investigations (TWRI) series of manuals published by the USGS. Measurement of streamflow and computation of discharge record from stage were also done according to TWRI specifications.

Discharge measurements were made as needed at each site to develop stage-discharge relation curves. Periodic check measurements of the rating were made when warranted by extreme or unstable conditions. Variable stage-discharge shifts were generally applied for periods when the absolute difference between the measured discharge and the expected discharge from the rating curve exceeded 5 percent.

Site visits were routinely conducted every 4-6 weeks. Corrections to gage height record were made when the absolute difference between the reference gage observations and the water-level sensor exceeded $0.015 \mathrm{ft}$.

Data were automatically retrieved daily using a modem and phone line. Plots of stage for the 4 previous days were generated and reviewed daily. This allowed quick detection and reconciliation of potential problems due to instrumentation malfunctions. 
All sensors used for measuring water temperature, specific conductance, and $\mathrm{pH}$ were tested prior to being placed in the field. Thereafter, sensors were routinely calibrated every 4-6 weeks. This procedure began with an initial check of the probe in its current state. The probes were then thoroughly cleaned and calibrated using several standards. Adjustments to the sensor readings were applied over time and $\mathrm{pH}$ range, as needed, based on calibration records. Sensors were calibrated as soon as possible following sampling events to minimize any potential problems with drift.

All data were retrieved daily using a modem and phone line, and plots for the 4 previous days were generated daily. Review of these plots allows potential problems with sensors to be detected.

The water temperature sensor was calibrated by using either an American Bureau of Standards mercury thermometer or an electronic thermistor that had been previously calibrated. The thermometer or thermistor was placed in the stream and allowed to equilibrate prior to disturbing the temperature sensor. All readings were recorded on the calibration sheet. The temperature sensor was then removed, cleaned, returned to the stream, and allowed to equilibrate. All readings were then recorded a second time. As needed, adjustments to the data were time corrected based on observed versus actual readings.

The specific conductance probe was calibrated using five standards ranging from 20 to 500 microsiemens per centimeter at 25 degrees Celsius $\left(\mu \mathrm{S} / \mathrm{cm}\right.$ at $\left.25^{\circ} \mathrm{C}\right)$. The three standards that best bracketed the typically observed specific conductance readings were used to apply any needed adjustments to the data. The probe was rinsed with deionized water, sequentially immersed in each standard, and allowed to equilibrate. Readings were recorded on the calibration sheet with the actual standard value. The probe was then thoroughly cleaned using a special scrub brush and deionized water to remove any accumulation of dirt and algae. The probe was then calibrated once more using the same procedure. This allowed for adjustments to the data with time and range in the event of probe degradation.

The $\mathrm{pH}$ probe was checked using standards of $4.0,7.0$, and $10.0 \mathrm{pH}$ units. The probe was removed from the flowthrough cell, rinsed with deionized water, placed in each standard, and allowed to equilibrate. Readings were recorded on the calibration sheet. Following this initial check, the probe was cleaned using methanol and a cotton swab. The electrode gel was checked and filled to capacity if needed. Because the probe utilizes a single-point calibration method, it was placed in the $7.0 \mathrm{pH}$ standard unit and calibrated to this value. The probe was then placed in the 4.0 and 10.0 standard units, and all readings were recorded on the calibration sheet. The probe was then returned to the flowthrough cell, which was filled with 4.0 buffer. This maintained the probe in a storage state and minimized drift until the probe was needed. A test of the flowthrough cell was performed yearly with readings recorded every minute during a 10 -minute period. These readings were then compared to an in situ $\mathrm{pH}$ reading taken directly from the stream to determine any effects due to pumping with the flowthrough cell. Adjustments to the data were applied with time and $\mathrm{pH}$ range as indicated by the calibration record. After January 1996, $\mathrm{pH}$ values were determined in the USGS, Charlotte Field Office from raw water or grab samples. The $\mathrm{pH}$ meter was calibrated with standards of $4.0,7.0$, and $10.0 \mathrm{pH}$ units prior to each use, and calibrations were documented in the meter log book.

All equipment used to collect water-quality samples was prepared by washing with a nonphosphate detergent and soaking in a 5-percent hydrochloric acid solution as described by Horowitz and others (1994). Equipment was assigned to each site to prevent crosscontamination between sites. Blanks were run on each piece of sampling equipment at each site on a yearly basis and analyzed for nutrients and metals and minor constituents using inorganic blank water prepared by the USGS laboratory.

The Teflon lined tubing on all automatic samplers was replaced with new tubing yearly. Between sampling events, this tubing was field-cleaned using the above procedure. In addition, the tubing was rinsed with methanol and the sample-collection bottles were baked at $450{ }^{\circ} \mathrm{C}$ prior to the spring collection of organic constituents. An equipment blank for the analysis of pesticides and herbicides was performed yearly at one randomly chosen site using organic free water purchased from a scientific supply company. Sample-collection volume was checked and calibrated at least yearly or when problems were suspected.

Sample processing equipment assigned to each site was prepared with the cleaning procedure described above. Samples for the analysis of organic constituents were decanted directly from the glass collection bottles into the appropriate glass sample containers, then set aside and chilled. The remaining water was then placed in a polycarbonate churn splitter 
to remove homogenous subsamples for inorganic and sediment analyses. Samples for the analysis of dissolved constituents were filtered using silicone tubing prepared with the previously described cleaning procedure and a disposable 0.45 -micron pore-size capsule filter (table 4). Samples collected for inorganic analyses were preserved using USGS protocols as described by Horowitz and others (1994). Beginning in September 1995, samples collected for inorganic analyses were analyzed by the Mecklenburg County Department of Environmental Protection Laboratory and were preserved as required by the Mecklenburg County laboratory (table 5).

Churn splitters were field-cleaned with deionized water and 5-percent hydrochloric solution between each discrete sample collected at each site during an event. Blanks were processed on these fieldcleaned churns for the analysis of nutrients and metals and minor constituents with a frequency of one blank per site per event. Lab blanks were run at the same frequency to check for contamination due to atmospheric deposition in the sample processing area.

Approximately 10 percent of all samples analyzed were quality-assurance samples. These included the previously mentioned blanks as well as split, duplicate, and blank samples for all constituents analyzed. The quality-assurance blanks for nutrients were analyzed using the low-level ASF method, and the metals and minor constituents were analyzed using the ICP-MS method.

Concurrent manual sampling using USGS approved methods was conducted to compare crosssectionally averaged samples with point samples (table 6). Automatically collected point samples were assumed to represent the cross-sectionally averaged sediment concentrations.

\section{LABORATORY ANALYSES}

Samples collected during the period, May 1994 through August 1995, were analyzed by the USGS National Water-Quality Laboratory (NWQL) in Denver, Colorado (table 7, p. 20-31). The analytical methods used by the USGS laboratory are documented in Wershaw and others (1987), Britton and Greeson (1989), Fishman and Friedman (1989), and Fishman (1993). Beginning in September 1995, samples collected for inorganic constituents were analyzed by the Mecklenburg County Department of Environmental Protection Laboratory (table 8, p. 32-33). Organic constituents continue to be analyzed by the NWQL.
Table 6. Automatic pumping sampler intake locations and number of manual suspended-sediment samples collected, May 1994 through June 1997

[EWI, equal-width increment]

\begin{tabular}{|c|c|c|c|c|}
\hline $\begin{array}{c}\text { Site } \\
\text { no. } \\
\text { (fig. 1) }\end{array}$ & $\begin{array}{l}\text { Streambed } \\
\text { type }\end{array}$ & $\begin{array}{c}\text { Sampler } \\
\text { intake } \\
\text { (feet } \\
\text { above } \\
\text { bottom) }\end{array}$ & $\begin{array}{l}\text { Width of } \\
\text { stream } \\
\text { (in feet) } \\
\text { [Location of } \\
\text { sampler } \\
\text { intake in } \\
\text { stream cross } \\
\text { section] }^{\mathrm{a}}\end{array}$ & $\begin{array}{c}\text { Number of } \\
\text { manual } \\
\text { suspended- } \\
\text { sediment } \\
\text { samples } \\
\text { collected } \\
\text { using the } \\
\text { EWI } \\
\text { technique }\end{array}$ \\
\hline 33 [CSW08] $^{\mathrm{b}}$ & sand & 0.8 & $\begin{array}{c}13.5 \\
\text { [midstream] }\end{array}$ & 10 \\
\hline 34 [CSW09] $^{\mathbf{b}}$ & sand & 1.1 & $\begin{array}{c}8.0 \\
\text { [midstream] }\end{array}$ & 11 \\
\hline 37 [CSW06] & concrete/gravel & .5 & $\begin{array}{c}12.6 \\
\text { [midstream] }\end{array}$ & 8 \\
\hline 39 [CSW05] & cobble/sand & .3 & $\begin{array}{c}2.4 \\
\text { [midstream] }\end{array}$ & 10 \\
\hline 40 [CSW03] & concrete & .1 & $\begin{array}{c}2.0 \\
\text { [midstream] }\end{array}$ & 5 \\
\hline 41 [CSW02] & clay/sand & 1.1 & $\begin{array}{c}9.1 \\
\text { [midstream] }\end{array}$ & 8 \\
\hline 42 [CSW04] & clay/cobble/sand & .4 & $\begin{array}{c}6.7 \\
\text { [midstream] }\end{array}$ & 12 \\
\hline 43 [CSW07] & silt/clay & .4 & $\begin{array}{c}3.8 \\
\text { [midstream] }\end{array}$ & 13 \\
\hline $44\left[^{C S W 10}\right]^{c}$ & sand & $1.0-3.0$ & $\begin{array}{c}22.0 \\
\text { [midstream] }\end{array}$ & 8 \\
\hline
\end{tabular}

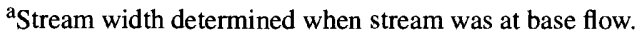

beriod of record May 1994 through September 1997.

${ }^{c}$ Period of record November 1996 through September 1997.
}

Suspended-sediment concentrations were determined during the study period by the USGS sediment laboratories located in Raleigh, N.C., Baton Rouge, La., and Louisville, Ky., by using methods and procedures documented by Guy (1969). Analytical procedures and method detection limits for chemical constituents in water analyzed by the USGS NWQL are listed in table 7. Analytical procedures and method detection limits for the Mecklenburg County Department of Environmental Protection Laboratory are listed in table 8.

Method detection limits (MDL's) are a statistical estimate of a property of the analytical method used to measure the compound concentration. MDL's for the 88 dissolved pesticide organic compounds (table 7) were revised by the USGS laboratory April 15, 1996, based on detailed method performance tests. MDL's were generally lowered by one-half to an order of magnitude from values previously published in Robinson and others (1996). The USGS water-quality database was updated in late 1996. Hence, 
concentrations of dissolved organic compounds reported in statistical summary tables may be different from previously published values.

\section{PRECIPITATION AND HYDROLOGIC DATA}

Precipitation and hydrologic data from 46 precipitation sites and 9 stream sites collected during July 1995 through June 1997 are discussed in the following sections. Rainfall and streamflow characteristics for the monitored storms at the stream sites also are summarized.

\section{Precipitation Data}

Daily and monthly rainfall totals at the 46 rainfall sites (fig. 1) are presented in tables 9-54 (p. 34-119). The distribution of annual rainfall in parts of Mecklenburg County, based on the data from the 29 rainfall sites for July 1996 through June 1997, ranged from approximately 35 in. to 50 in. (fig. 3). The

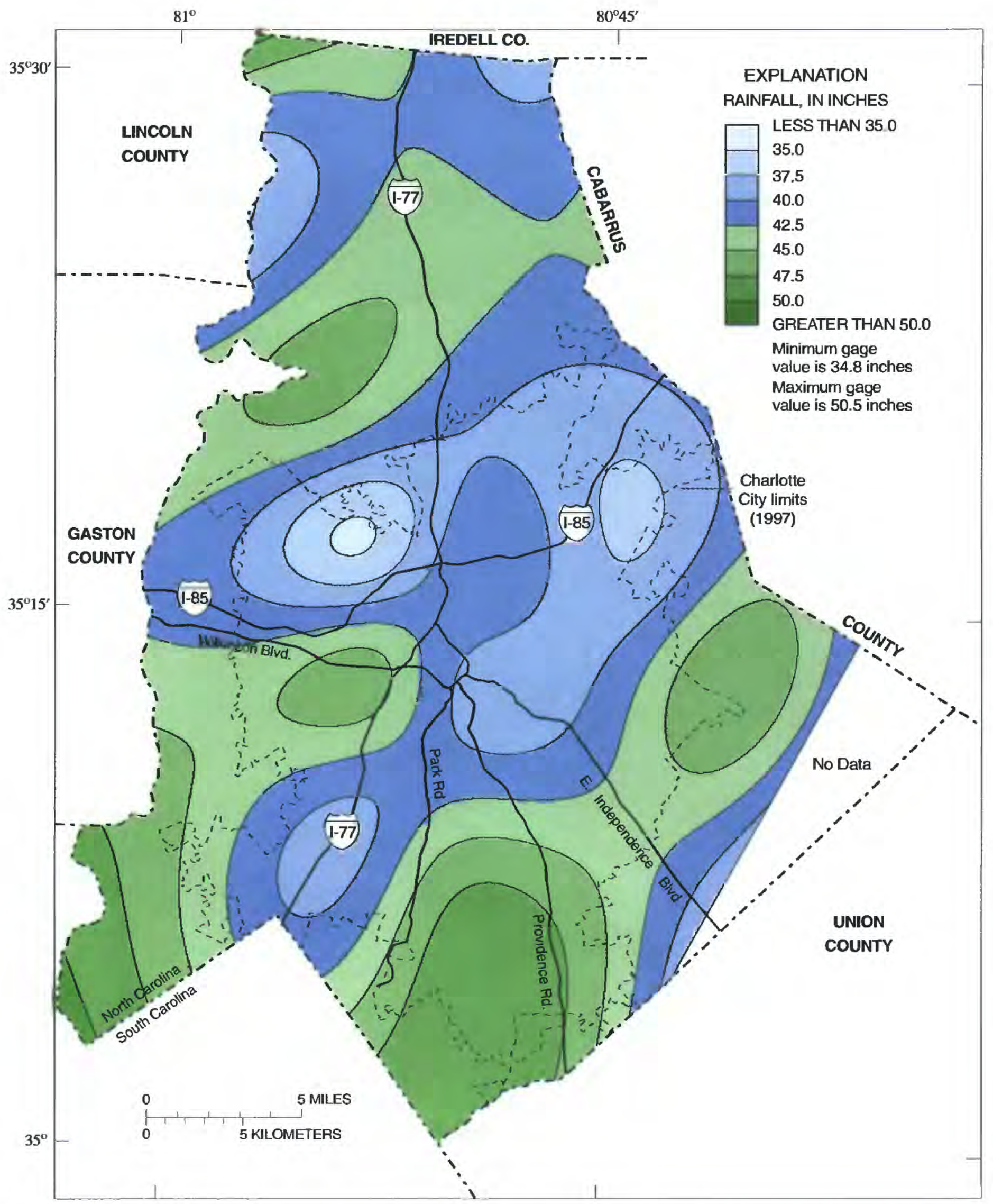

Figure 3. Annual rainfall distribution in Mecklenburg County for July 1996 through June 1997. 
distribution of recurrence intervals for a 24-hour rainfall duration in the city of Charlotte for the storm of August 26-27, 1995 (fig. 4), based on data from 24 raingages, ranged from less than 2 years to greater than 100 years as published by Hershfield (1961) and also in USGS Fact Sheet FS-052-97 (Hazell and Bales, 1997).

\section{Atmospheric Deposition Data}

Beginning in March 1997 atmospheric deposition data were collected at sites 37,42 , and 43 (fig. 1; table 2). Analyses for $\mathrm{pH}$, specific conductance, sulfate, chloride, nutrients, and concentrations of

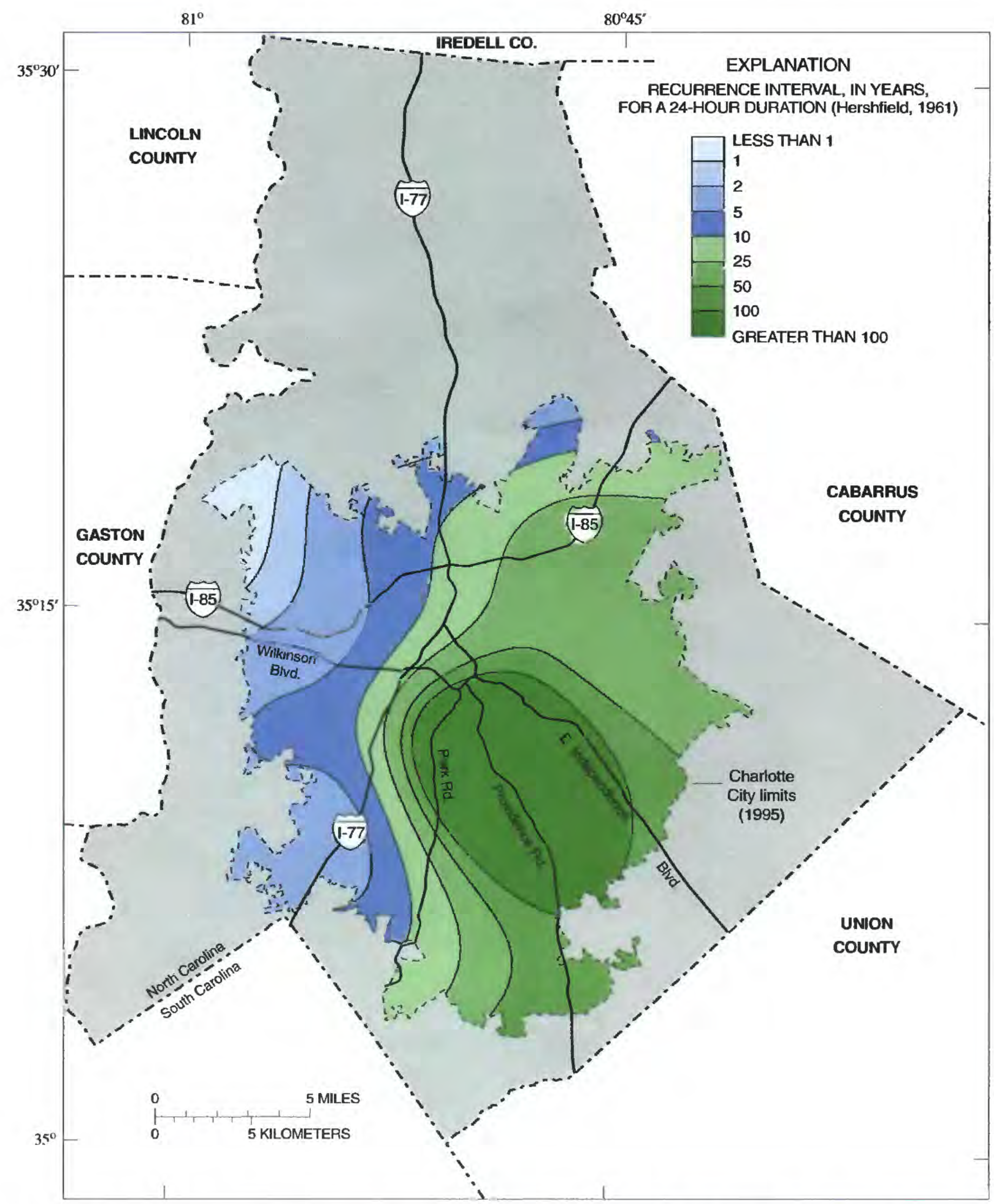

Figure 4. Rainfall recurrence intervals for the city of Charlotte for August 26-27, 1995 (revised from Hazell and Bales, 1997). 
metals and minor constituents were performed weekly on samples if sufficient rainfall occurred.

The atmospheric deposition data collection is scheduled to be completed in March 1998. These data are not included in this report but are planned to be published in an interpretive report.

\section{Streamflow Data}

Streamflow statistics for December 1993 through June 1997 are presented in table 55 (p. 120). Daily mean discharge data at the nine streamflow sites (fig. 1) are presented in tables 56-64 (p. 121-139). During periods of missing record, a daily mean discharge was estimated based on rainfall and computed streamflow for other gages in the area. The minimum instantaneous discharge for the period of record at site 34 was indeterminate due to an unstable stage-discharge relation for low stages.

\section{Water-Quality Data}

Continuous specific conductance and water temperature data were collected at 5-minute intervals at the streamflow sites. These data are available from the USGS District Office in Raleigh, N.C. Continuous specific conductance and water temperature statistics for the nine streamflow sites are presented in table 65 (p. 140). Continuous measurements of $\mathrm{pH}$ were made during sampled storm events during May 1995 through January 1996. Statistical summary tables of approximately 250 chemical constituents include measurements of coliform, physical and chemical properties, nutrients, concentrations of metals and minor constituents, oil and grease, suspended sediment, and organic compounds in water (tables 66-74, p. 141175). Samples taken on April 3, 1996, and May 7, 1997 , were collected during periods of no rainfall runoff to determine background levels of selected constituents. These data are included in the statistical summaries.

The statistical summaries were prepared using programs developed by the USGS (Maddy and others, 1992). If the total number of observations above and below the method detection limit is greater than 1 but less than or equal to 5, only the maximum and minimum values are reported in the tables. If only one observation is available, the value is reported as the maximum value. Percentiles are not shown for values greater than 5 . The statistical summaries are for the period of record, May 1994-June 1997, unless otherwise noted.

The instantaneous discharges reported in the statistical summary tables and discrete sample tables are associated with the individual water-quality samplecollection dates. Water-quality data for discrete samples were collected during July 1995 through June 1997 , unless otherwise noted (tables 75-83, p. 176-211). These data include measurements of coliform, physical and chemical properties, nutrients, metals and minor constituents, oil and grease, total organic carbon, and suspended sediment. The organic compounds were not included in these tables because of the small number of samples with concentrations greater than the MDL and the large number of organic constituents. Several values for biochemical oxygen demand (BOD) listed in the discrete sample tables are reported as "greater than" because of uncertainties in the results.

Rainfall and streamflow characteristics for the monitored storms at the stream sites are presented in tables 84-92 (p. 212-220). Total accumulated rainfall is reported as the total rainfall for the duration of each storm event.

\section{SELECTED REFERENCES}

Bigelow, D.S., 1984, Instruction manual, NADP/NTN site selection and installation: National Atmospheric Deposition Program, 23 p.

Bigelow, D.S., and Dossett, S.R., 1988, Instruction manual, NADP/NTN site operation: National Atmospheric Deposition Program, $40 \mathrm{p}$.

Britton, L.J., and Greeson, P.E., eds., 1989, Methods for collection and analyses of aquatic biological and microbiological samples: U.S. Geological Survey Techniques of Water-Resources Investigations, book 5 , chap. A4, 363 p.

Edwards, T.K., and Glysson, G.D., 1988, Field methods for measurements of fluvial sediment: U.S. Geological Survey Open-File Report 86-531, 118 p.

Fishman, M.J., ed., 1993, Methods of analysis by the U.S. Geological Survey National Water-Quality Laboratory-Determination of inorganic and organic constituents in water and fluvial sediments: U.S. Geological Survey Open-File Report 93-125, 217 p.

Fishman, M.J., and Friedman, L.C., eds., 1989, Methods for determination of inorganic substances in water and fluvial sediments: U.S. Geological Survey Techniques of Water-Resources Investigations, book 5, chap. A1, $545 \mathrm{p}$. 
Guy, H.P., 1969, Laboratory theory and methods for sediment analyses: U.S. Geological Survey Techniques of Water-Resources Investigations, book 5, chap. C1, 58 p.

Hazell, W.F., and Bales, J.D., 1997, Real-time rainfall measurement in the city of Charlotte and Mecklenburg County, North Carolina: U.S. Geological Survey Fact Sheet FS-052-97, 4 p.

Hershfield, D.M., 1961, Rainfall frequency atlas of the United States: Washington, D.C., Department of Commerce, Weather Bureau, Technical Paper No. 40, $114 \mathrm{p}$.

Horowitz, A.J., Demas, C.R., Fitzgerald, K.K., Miller, T.L., and Rickert, D.A., 1994, U.S. Geological Survey protocol for the collections and processing of surfacewater samples for the subsequent determination of inorganic constituents in filtered water: U.S. Geological Survey Open-File Report 94-539, 57 p.

Legrand, H.E., and Mundorff, M.J., 1952, Geology and groundwater in the Charlotte area, North Carolina: North Carolina Department of Conservation and Development, Bulletin 63, 88 p.

Maddy, D.V., Lopp, L.E., Jackson, D.L., Coupe, R.H., Schertz, T.L., and Garcia, K.T., 1992, National water information system user's manual, v. 2, chapter 2, water-quality system, p. 5-14-5-17.

McCachren, C.M., 1980, Soil survey of Mecklenburg County, North Carolina: U.S. Department of Agriculture, Soil Conservation Service, 97 p.

Outlaw, G.S., Hoos, A.B., and Pankey, J.T., 1994, Rainfall, streamflow, and water-quality data for five small watersheds, Nashville, Tenn., 1990-92: U.S. Geological Survey Open-File Report 94-68, 43 p.
Rantz, S.E., and others, 1982, Measurements and computation of streamflow-Volume 1, Measurement of stage and discharge, and Volume 2, Computation of discharge: U.S. Geological Survey Water-Supply Paper $2175,631 \mathrm{p}$.

Robinson, J.B., Hazell, W.F., and Garrett, R.G., 1996, Precipitation, streamflow, and water-quality data from selected sites in the city of Charlotte and Mecklenburg County, North Carolina, 1993-95: U.S. Geological Survey Open-File Report 96-150, 136 p.

Stanley, D.W., 1994, An evaluation of pollutant removal by a demonstration of an urban stormwater detention pond: Institute for Coastal and Marine Resources, and Department of Biology, East Carolina University, $112 \mathrm{p}$.

Timme, P.J., 1995, National Water-Quality Laboratory 1995 services catalog: U.S. Geological Survey Open-File Report 95-352, 120 p.

U.S. Environmental Protection Agency, 1990, National pollutant regulations for storm water discharges: U.S. Federal Register, v. 55, No. 222, p. 47990-48091.

Ward, J.R., and Harr, C.A., eds., 1990, Methods for collection and processing of surface-water and bedmaterial samples for physical and chemical analyses: U.S. Geological Survey Open-File Report 90-140, 71 p.

Wershaw, R.L., Fishman, M.J., Grabbe, R.R., and Lowe, L.E., 1987, Methods for the determination of organic substances in water and fluvial sediments: U.S. Geological Survey Techniques of Water-Resources Investigations, book 5, chap. A3, $80 \mathrm{p}$. 


\section{ABBREVIATIONS USED IN DATA TABLES 7-92}

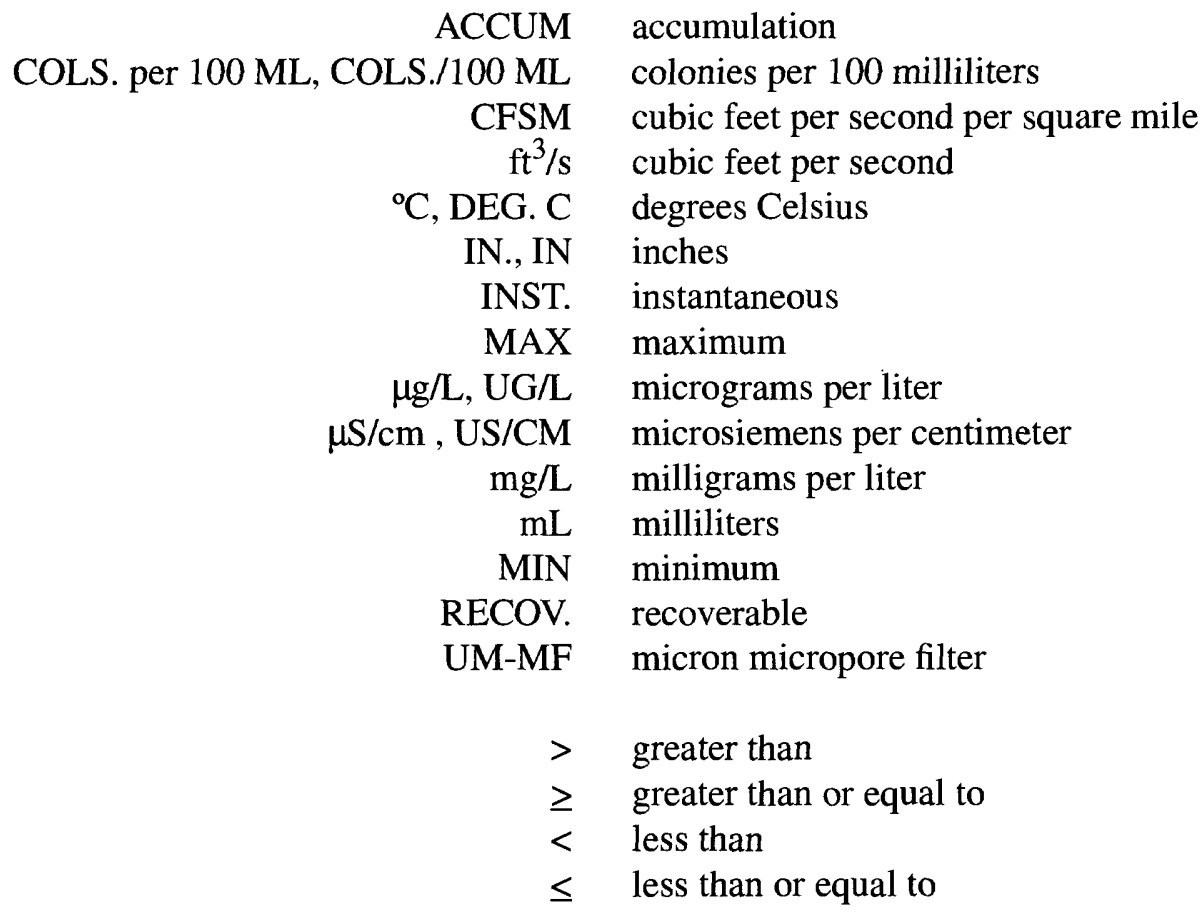




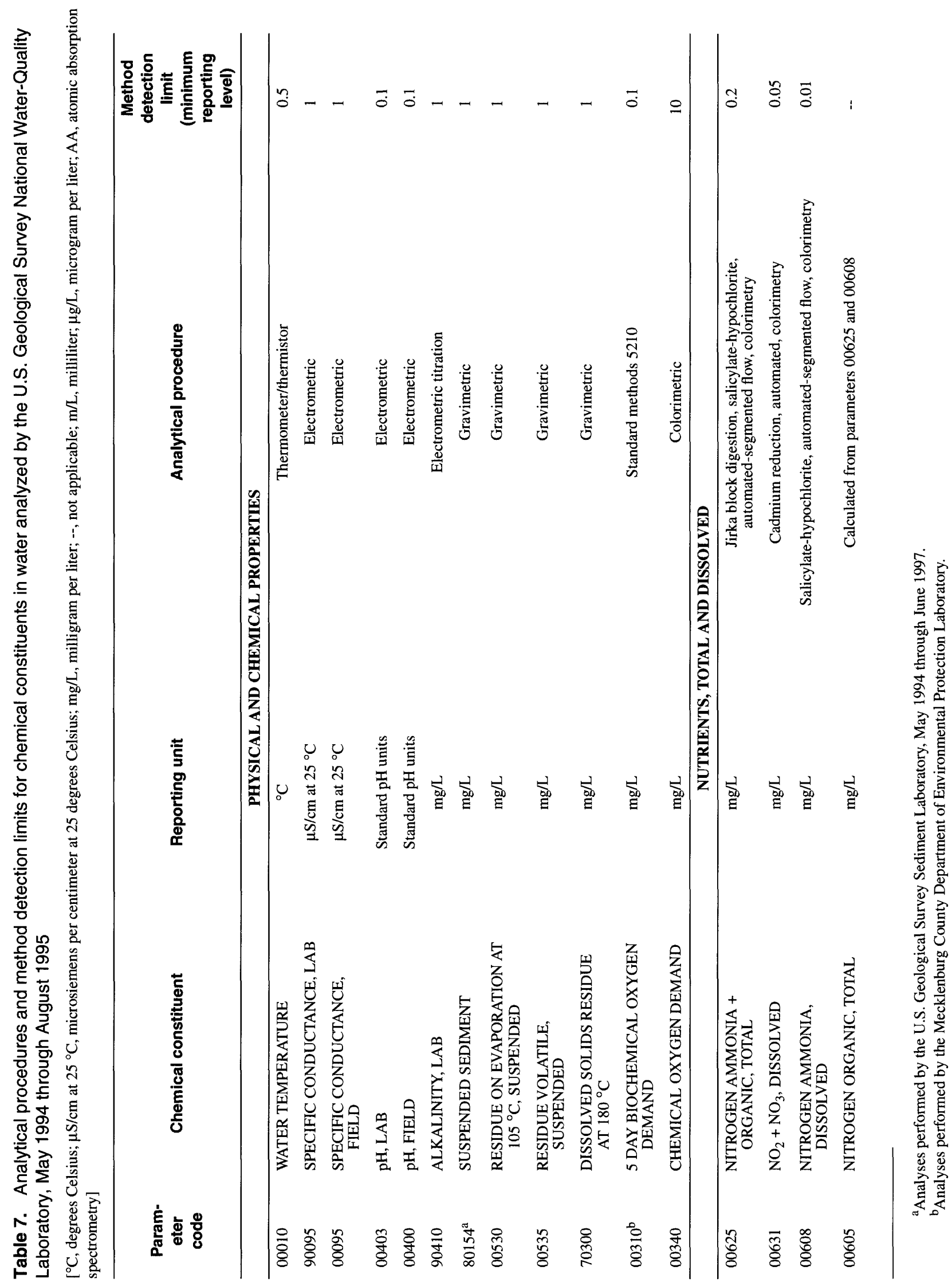




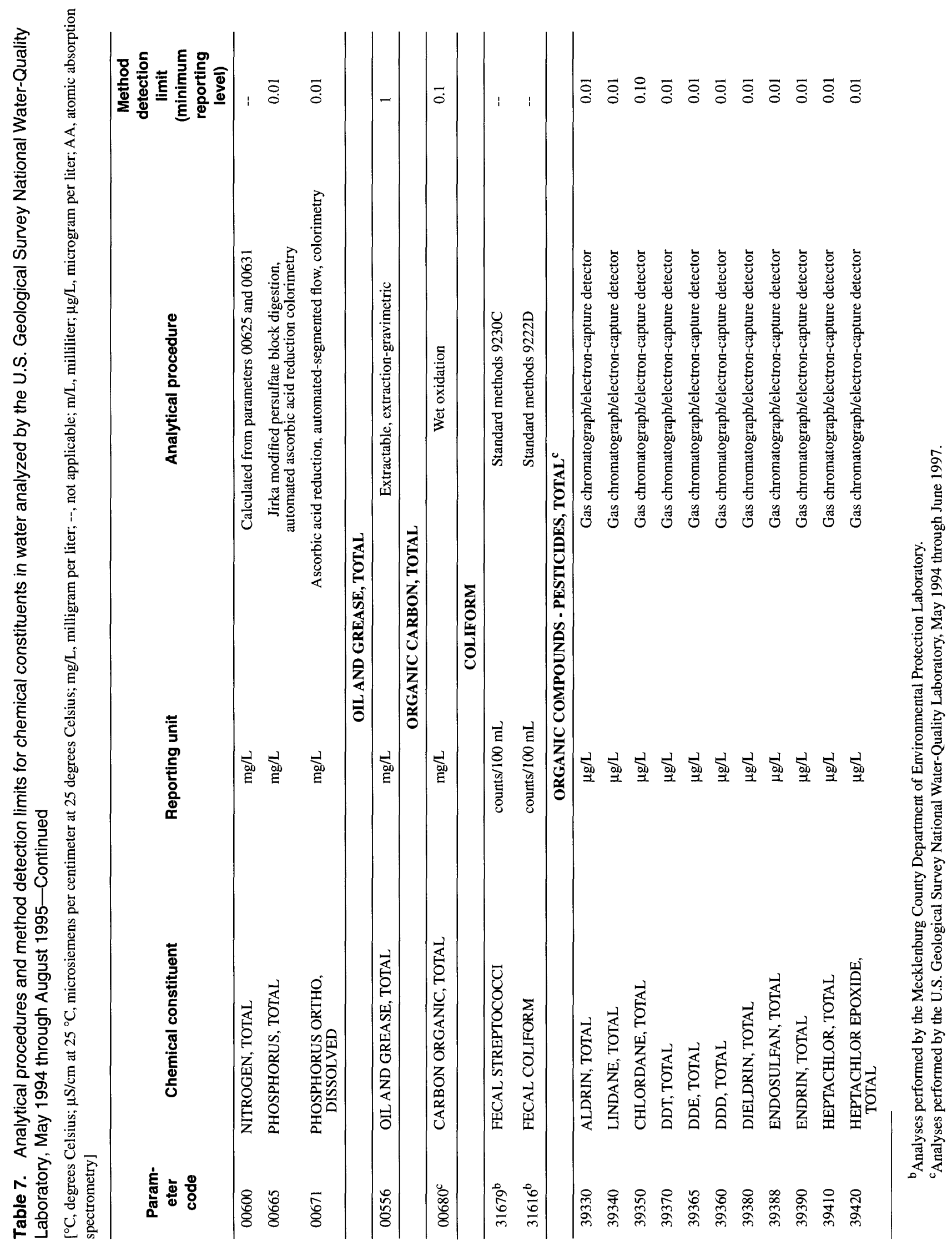




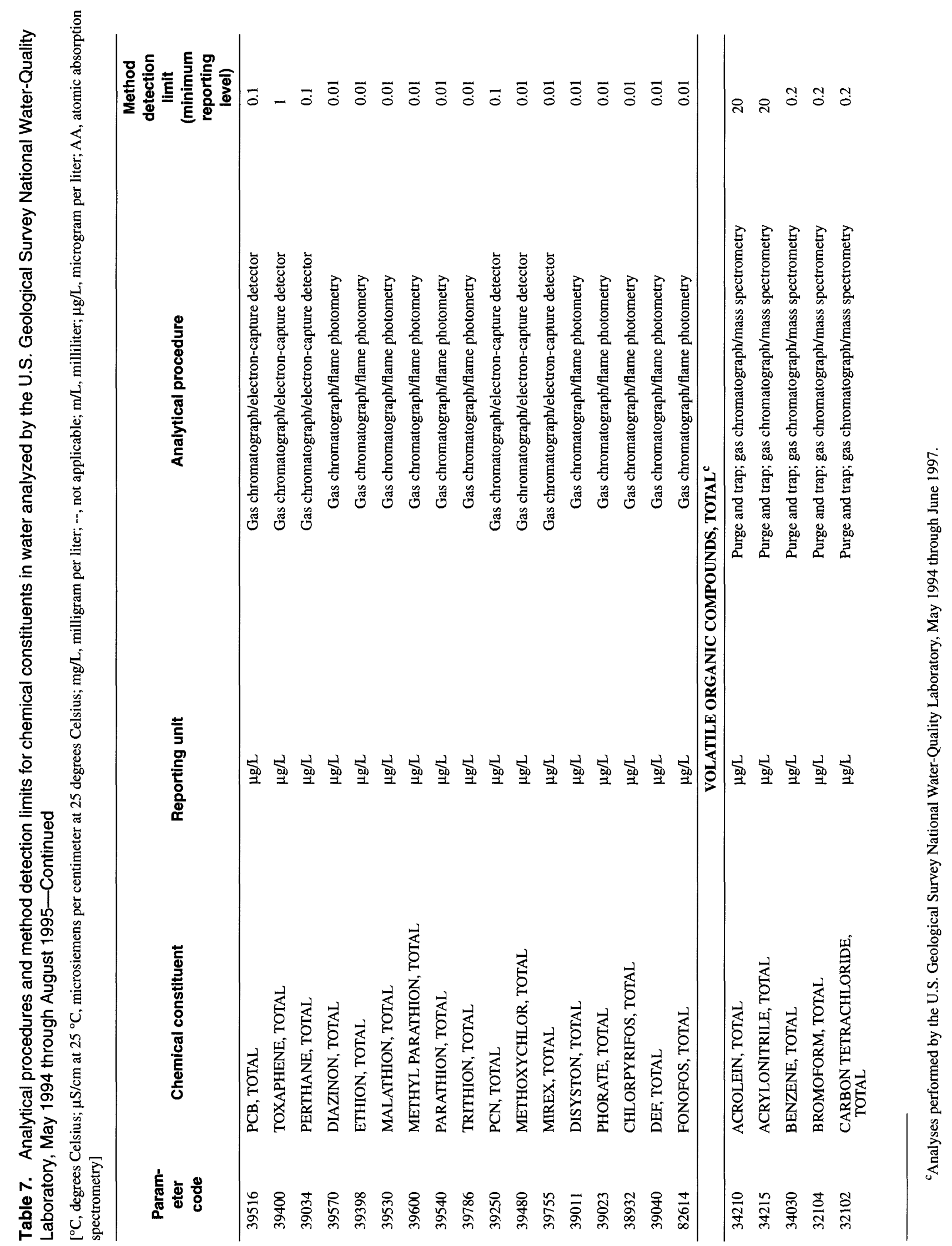




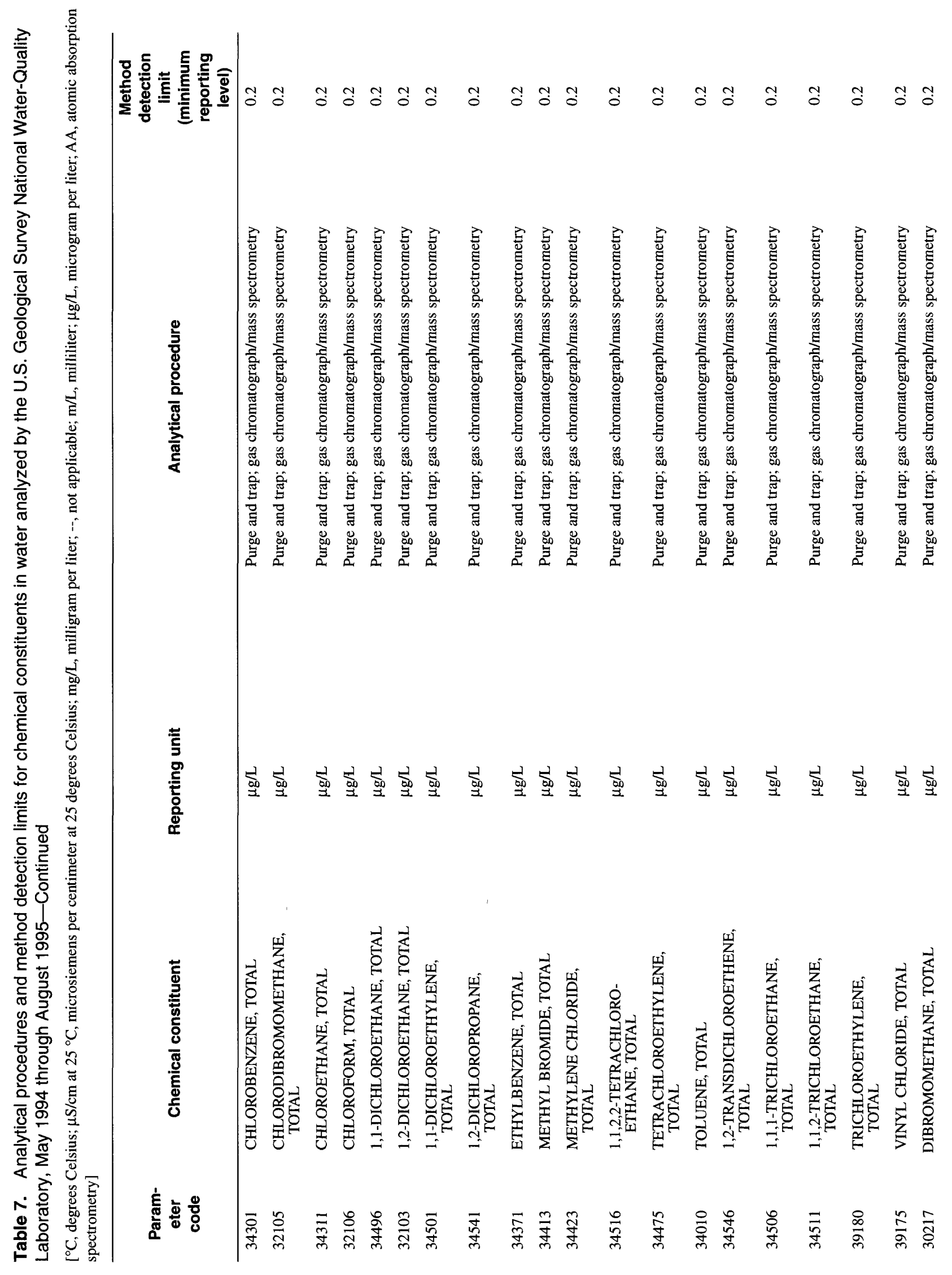




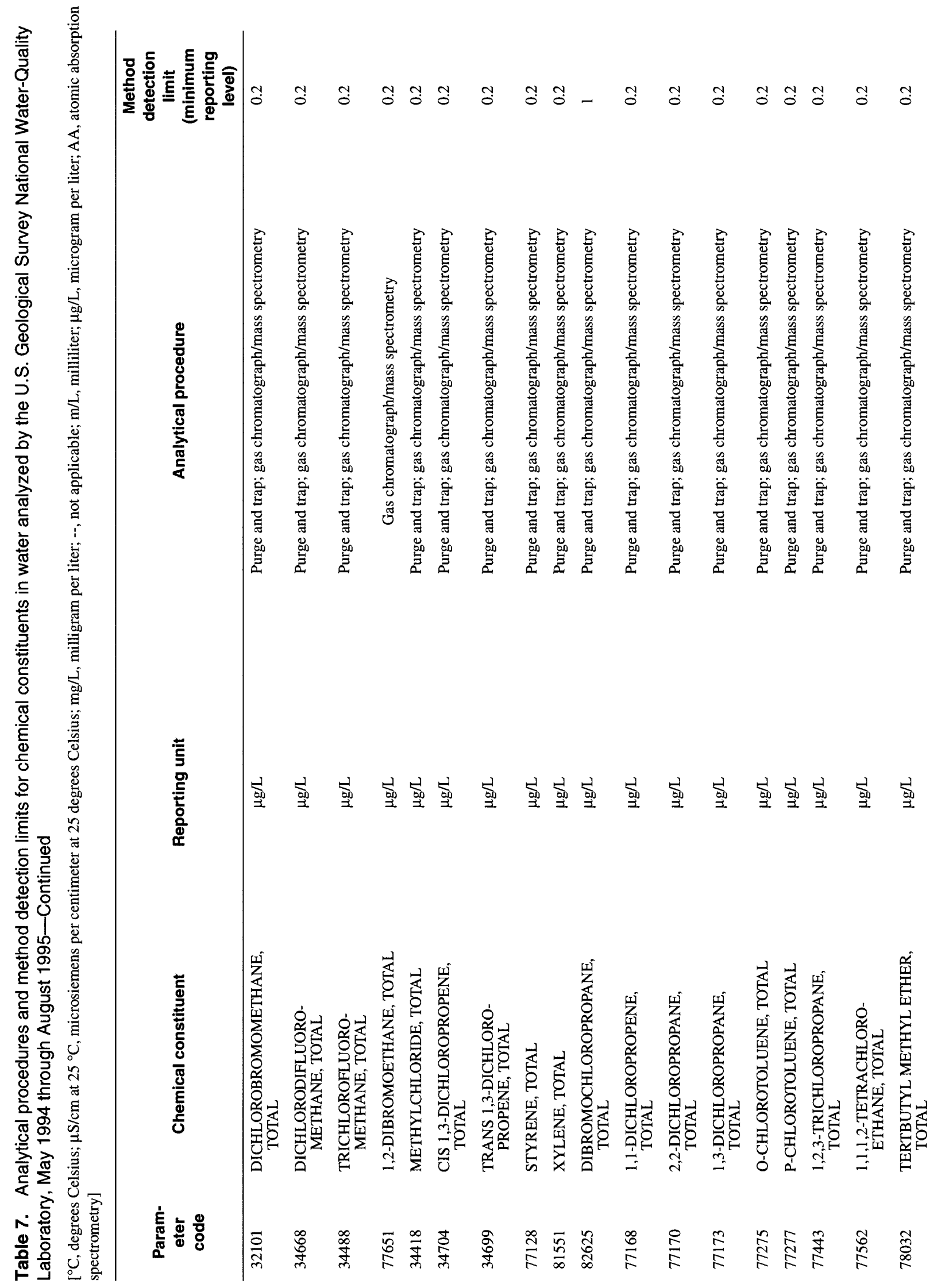




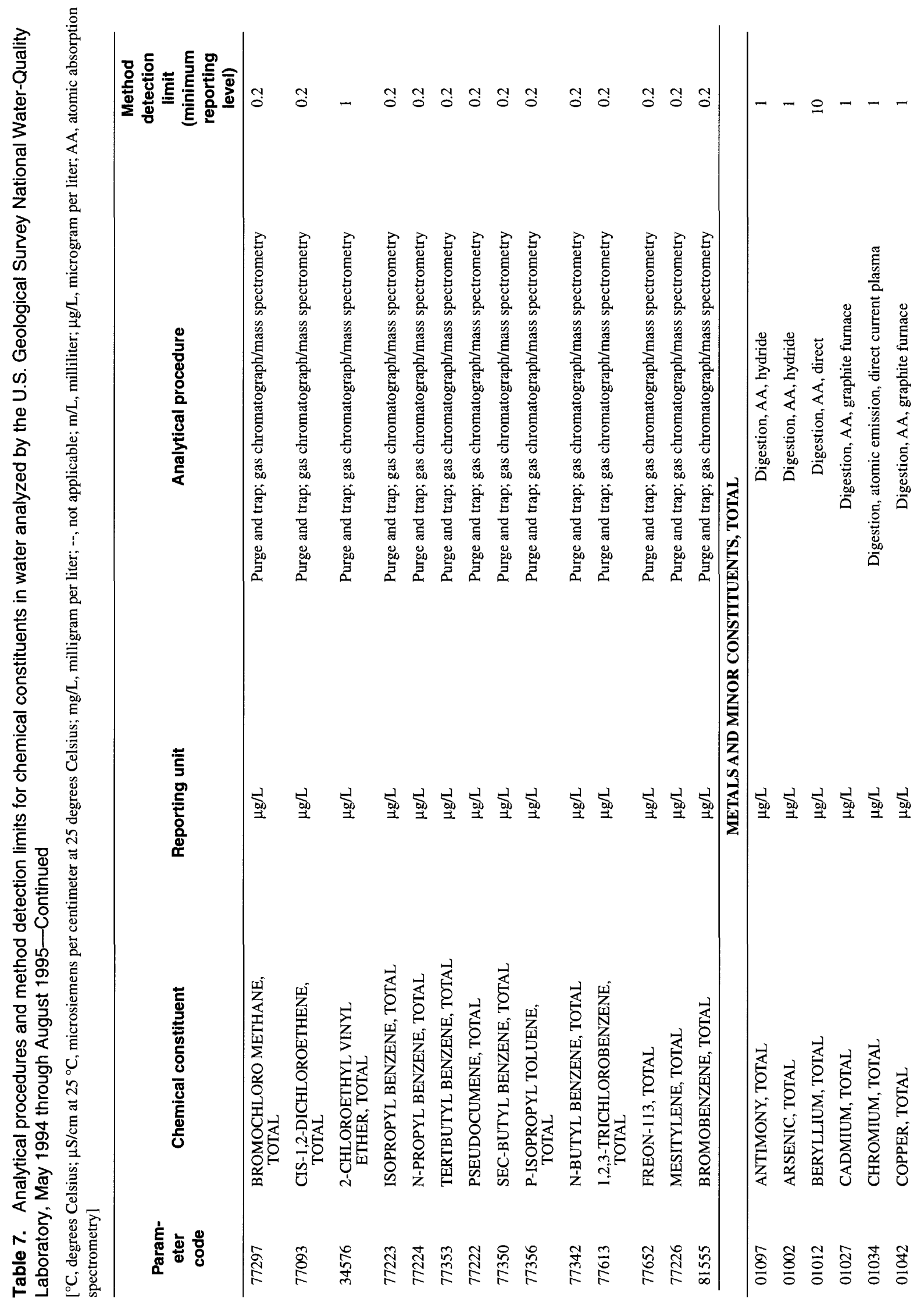




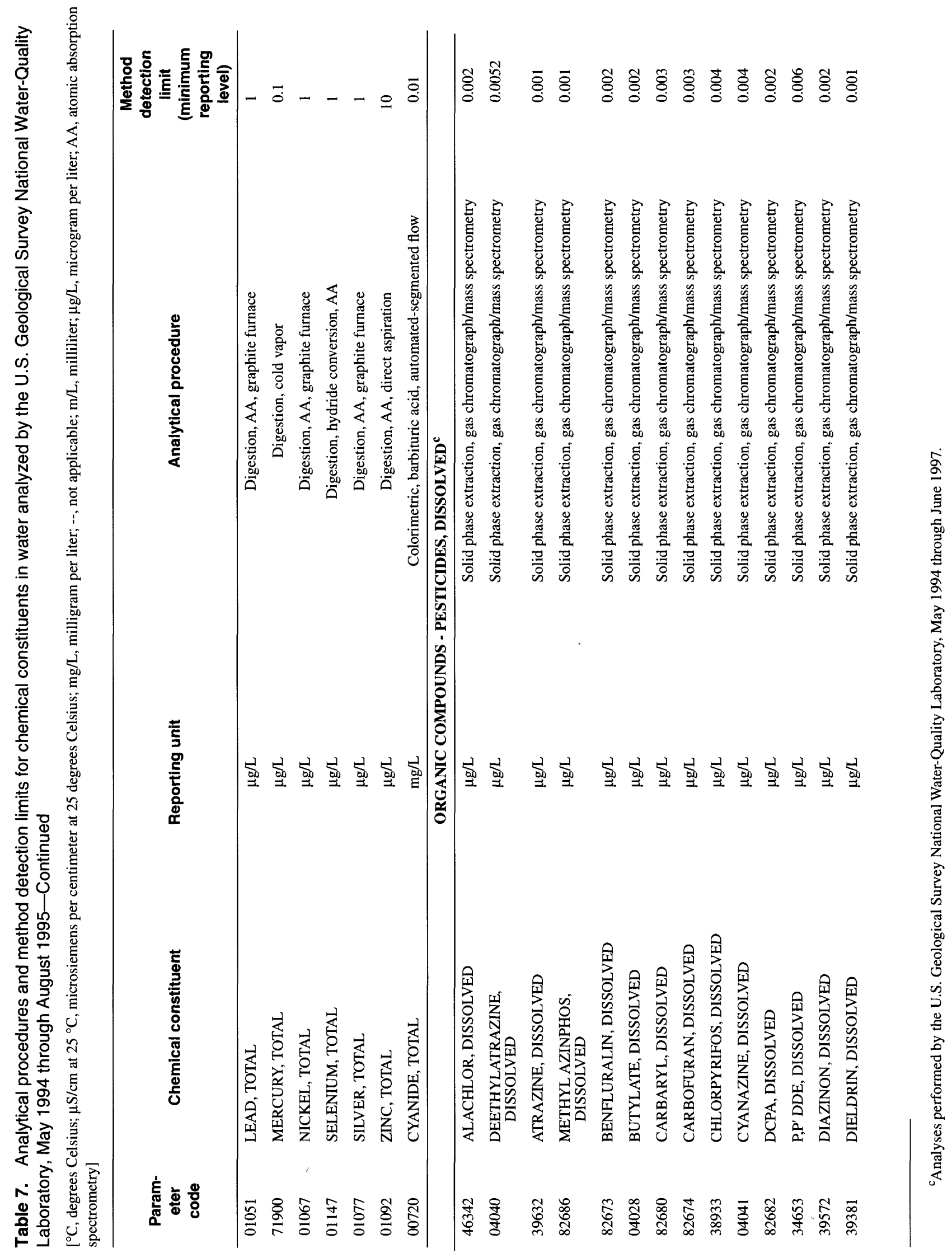




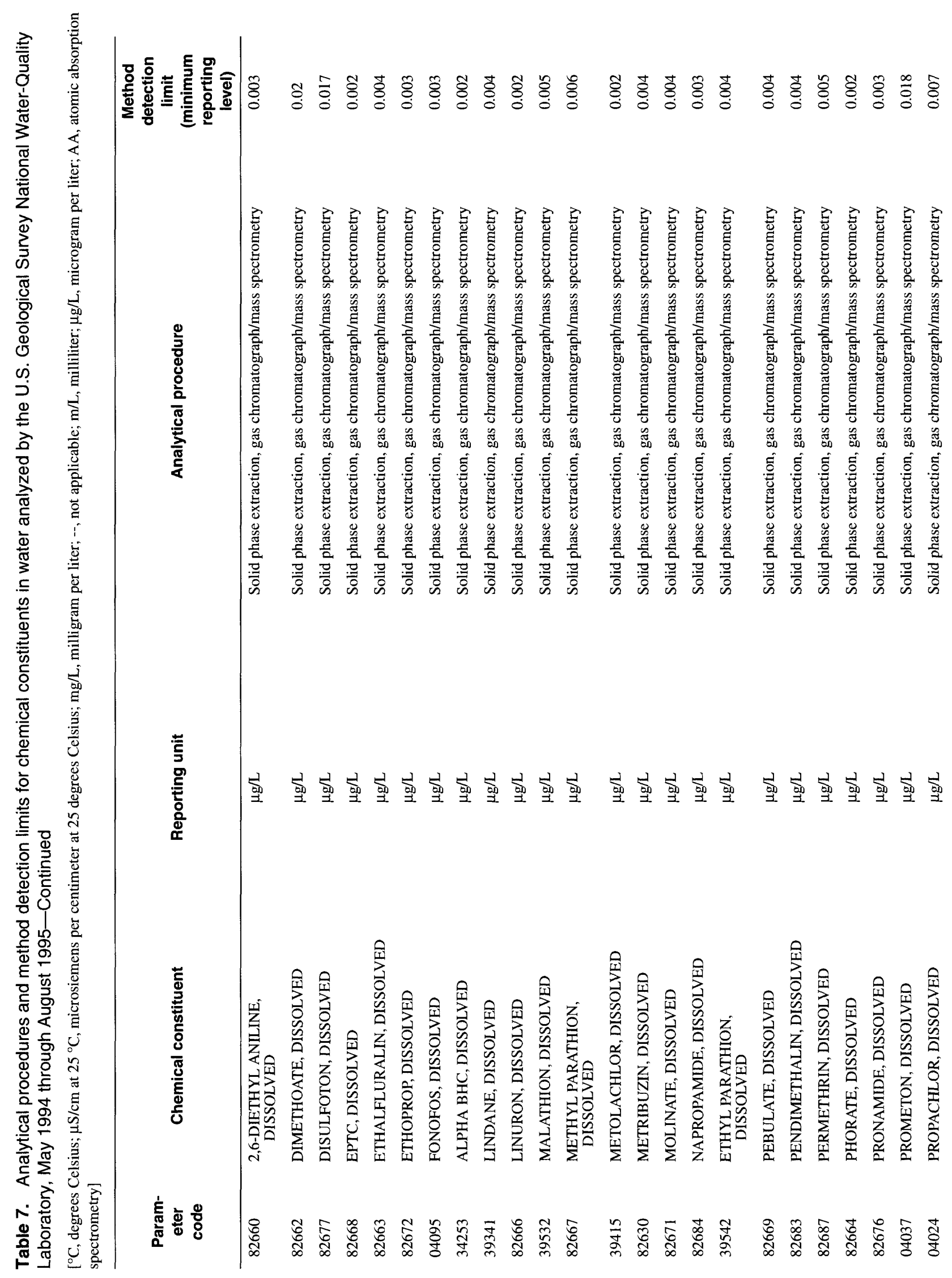




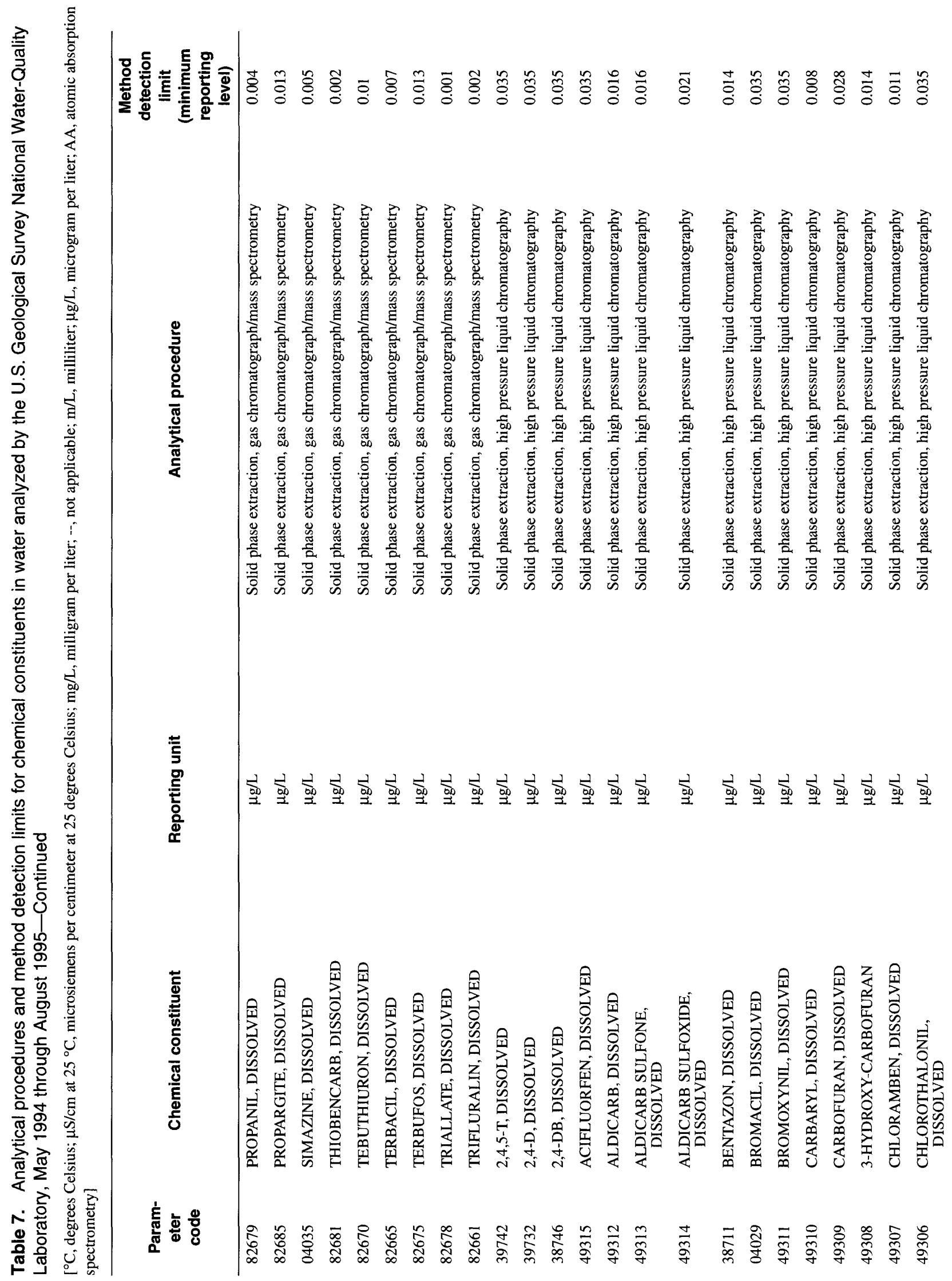




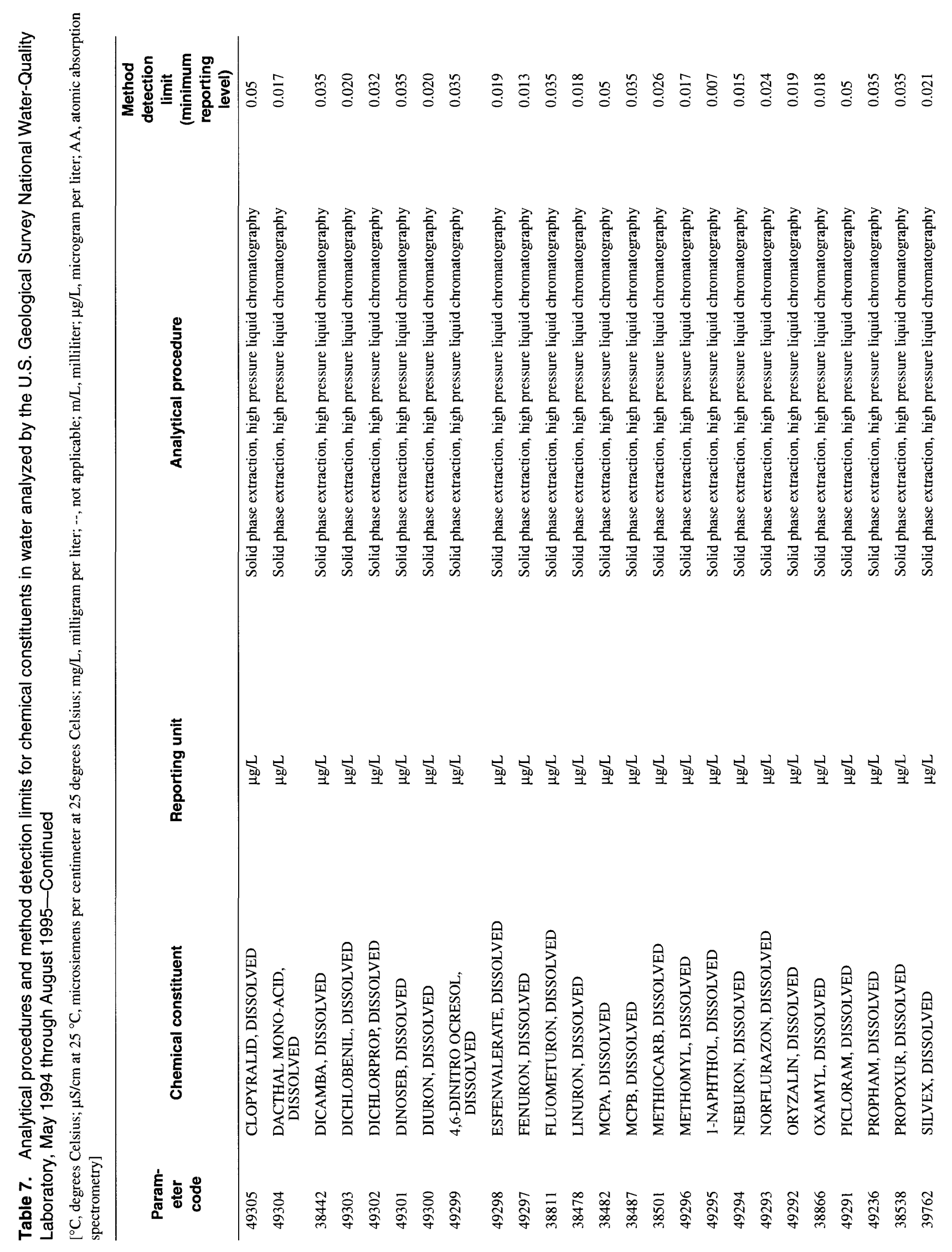




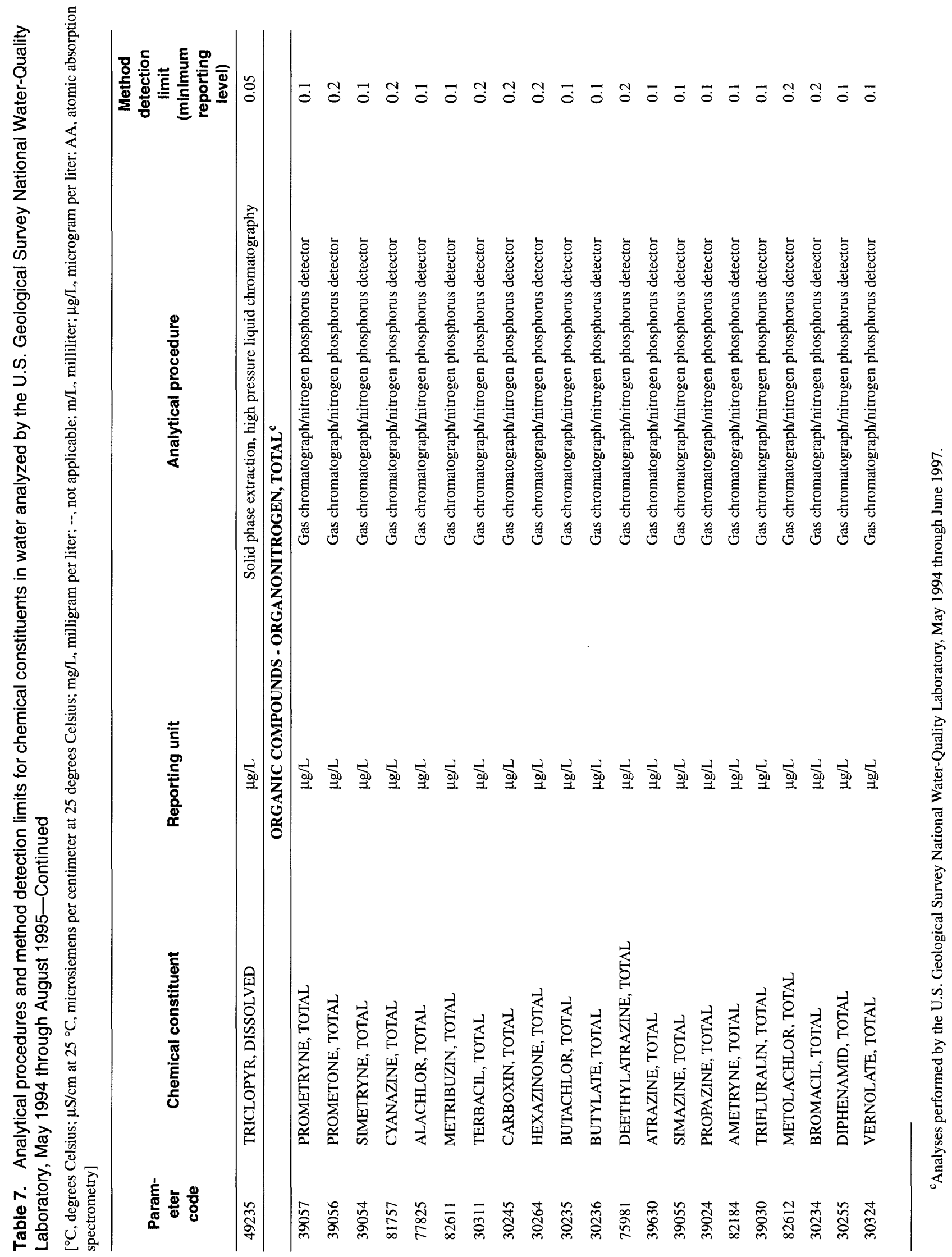




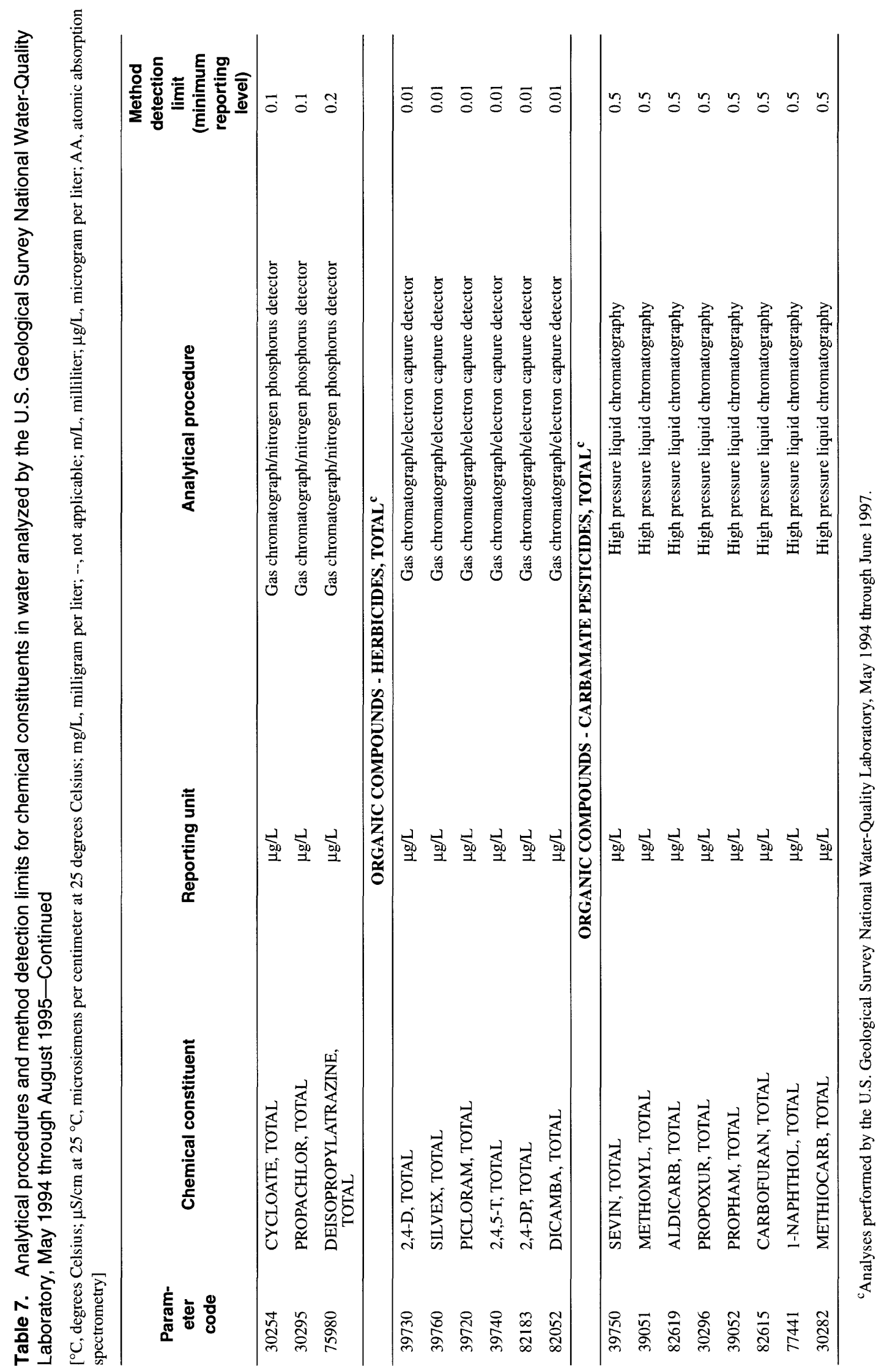




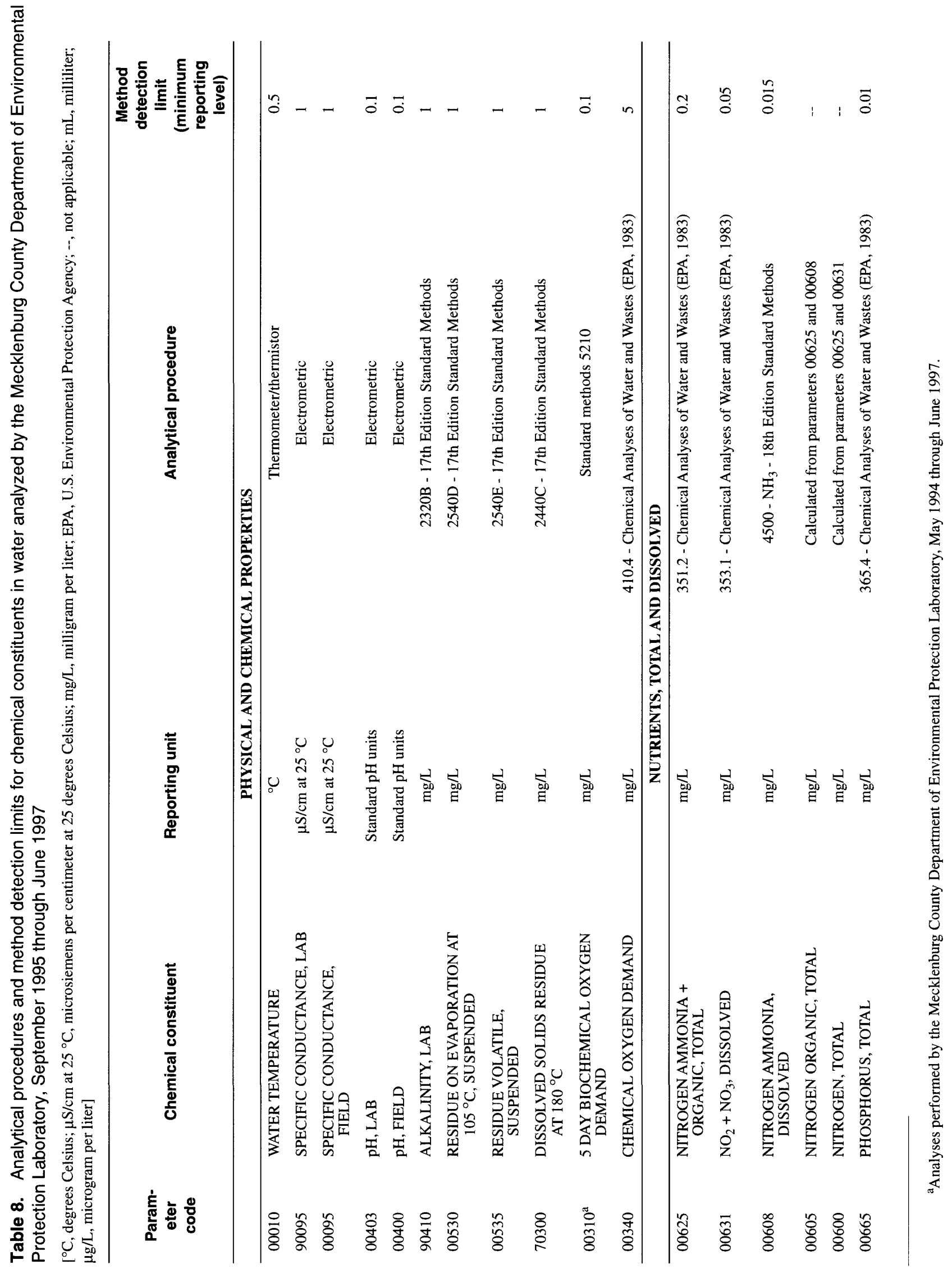




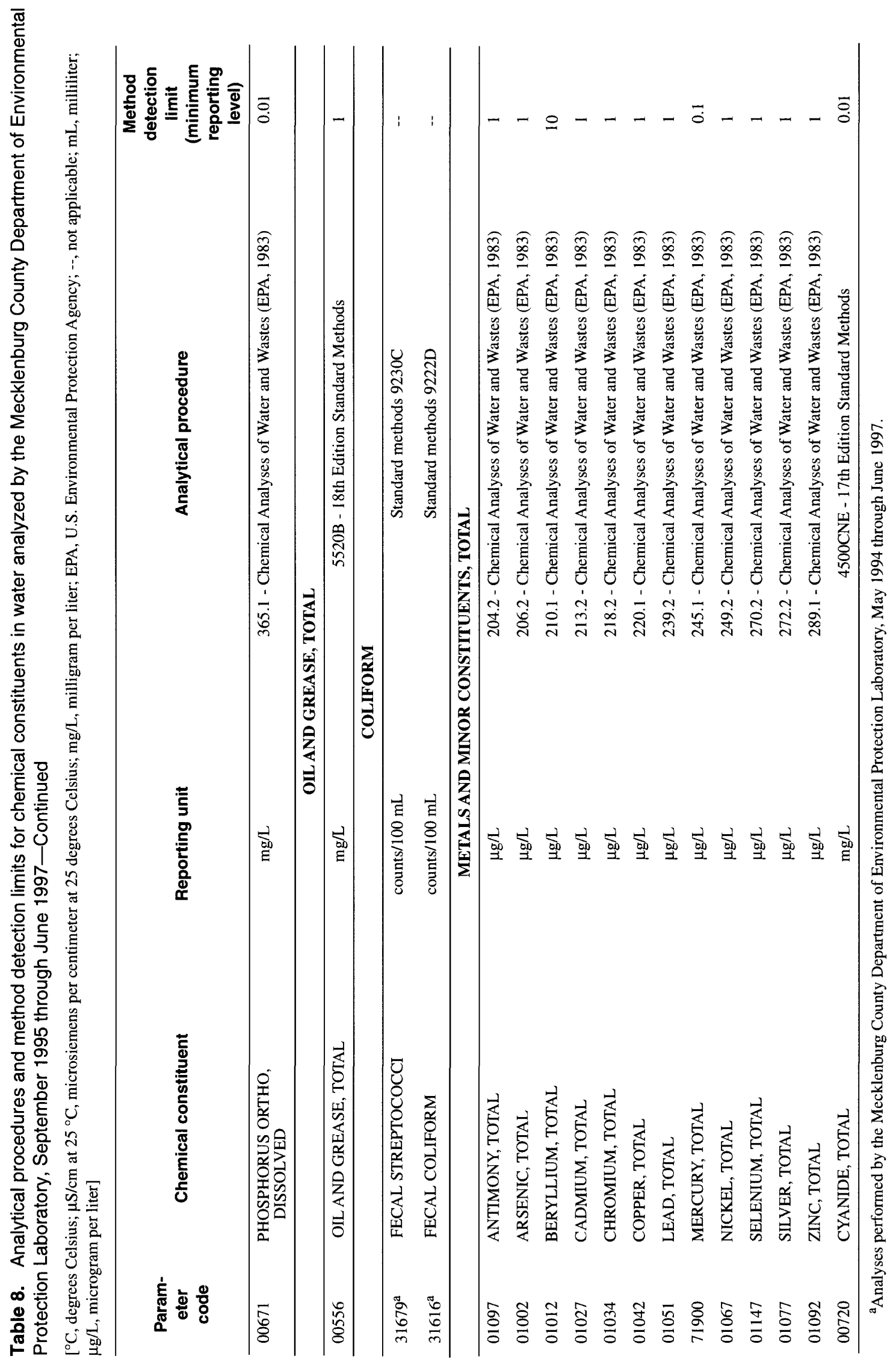




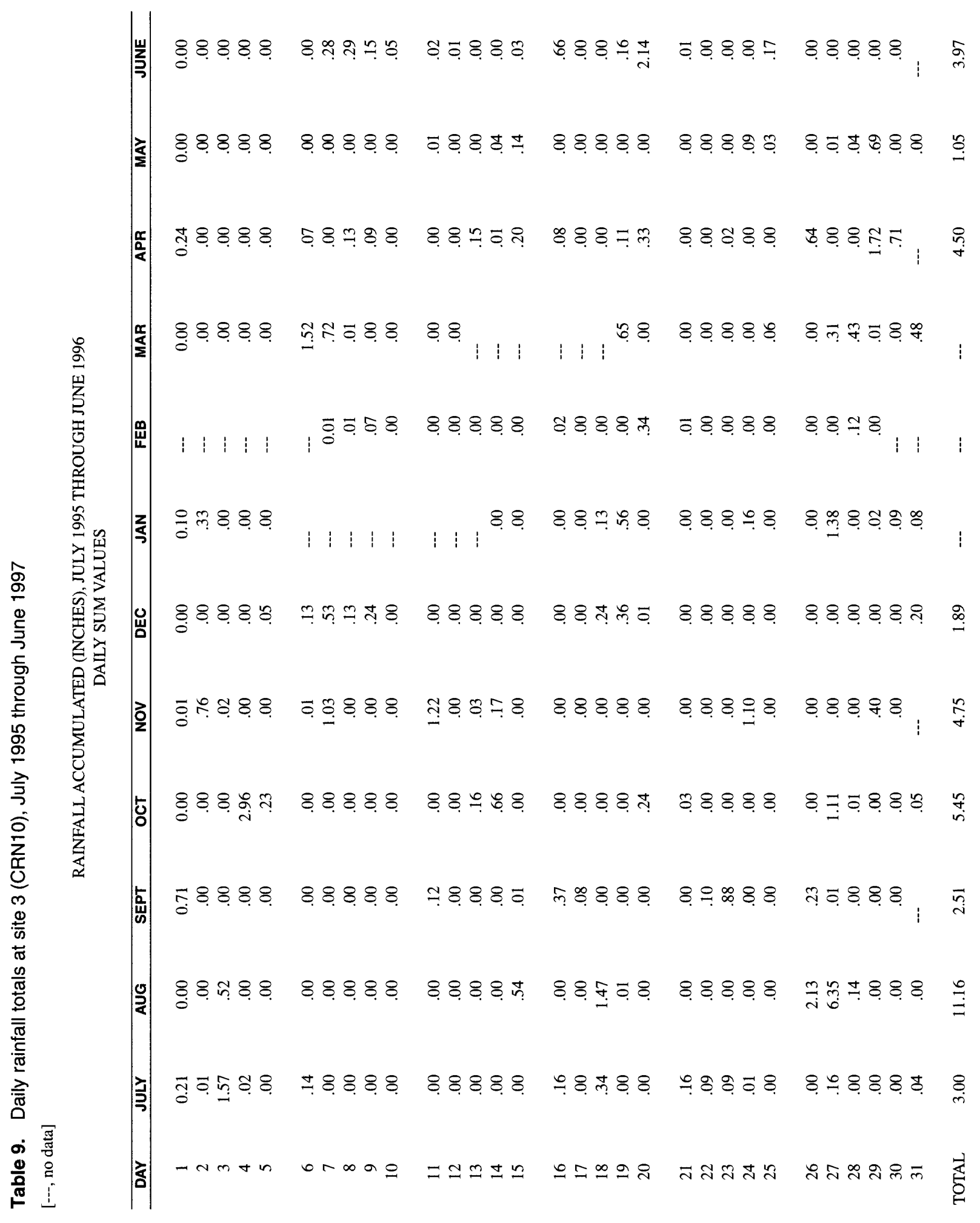




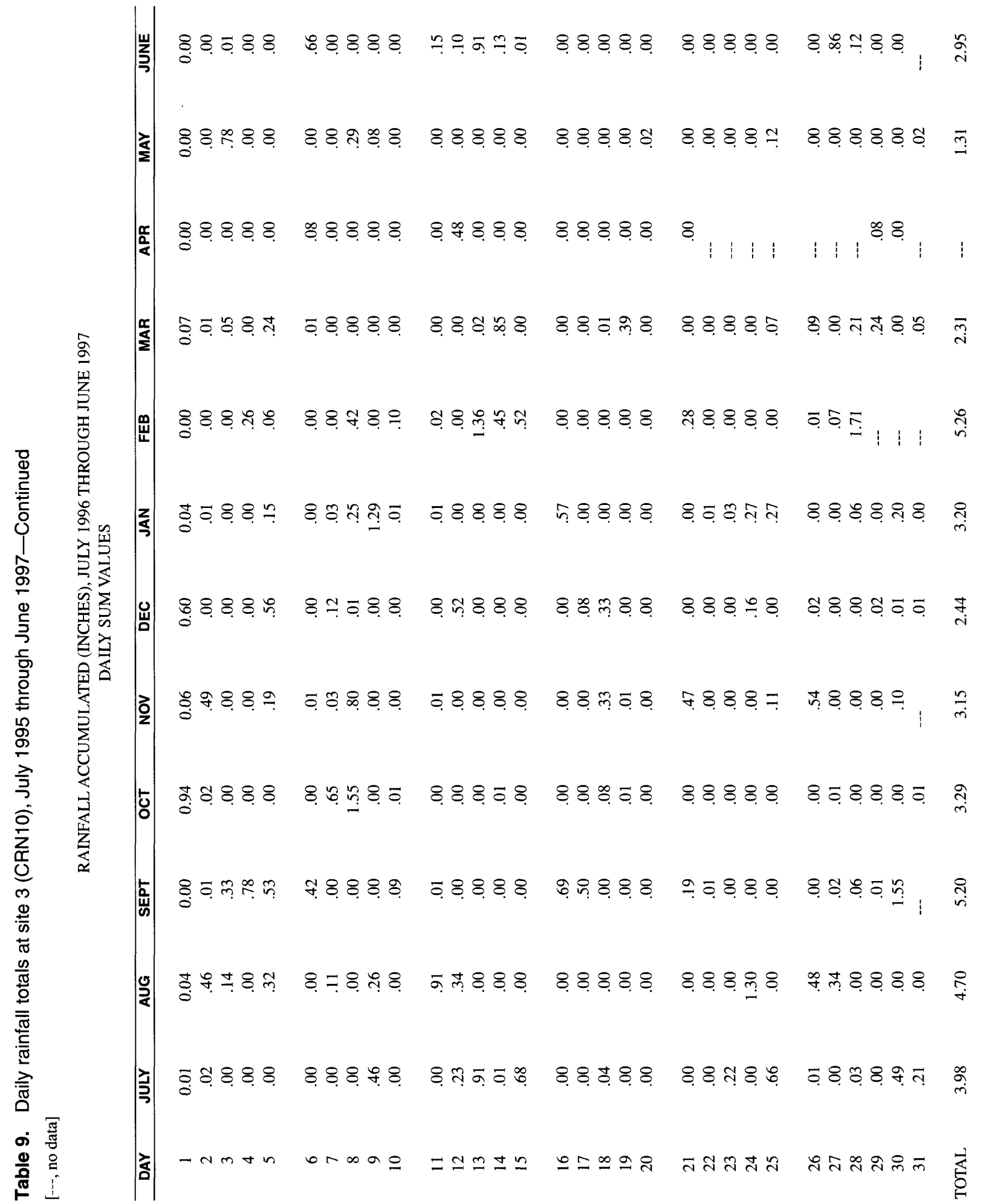




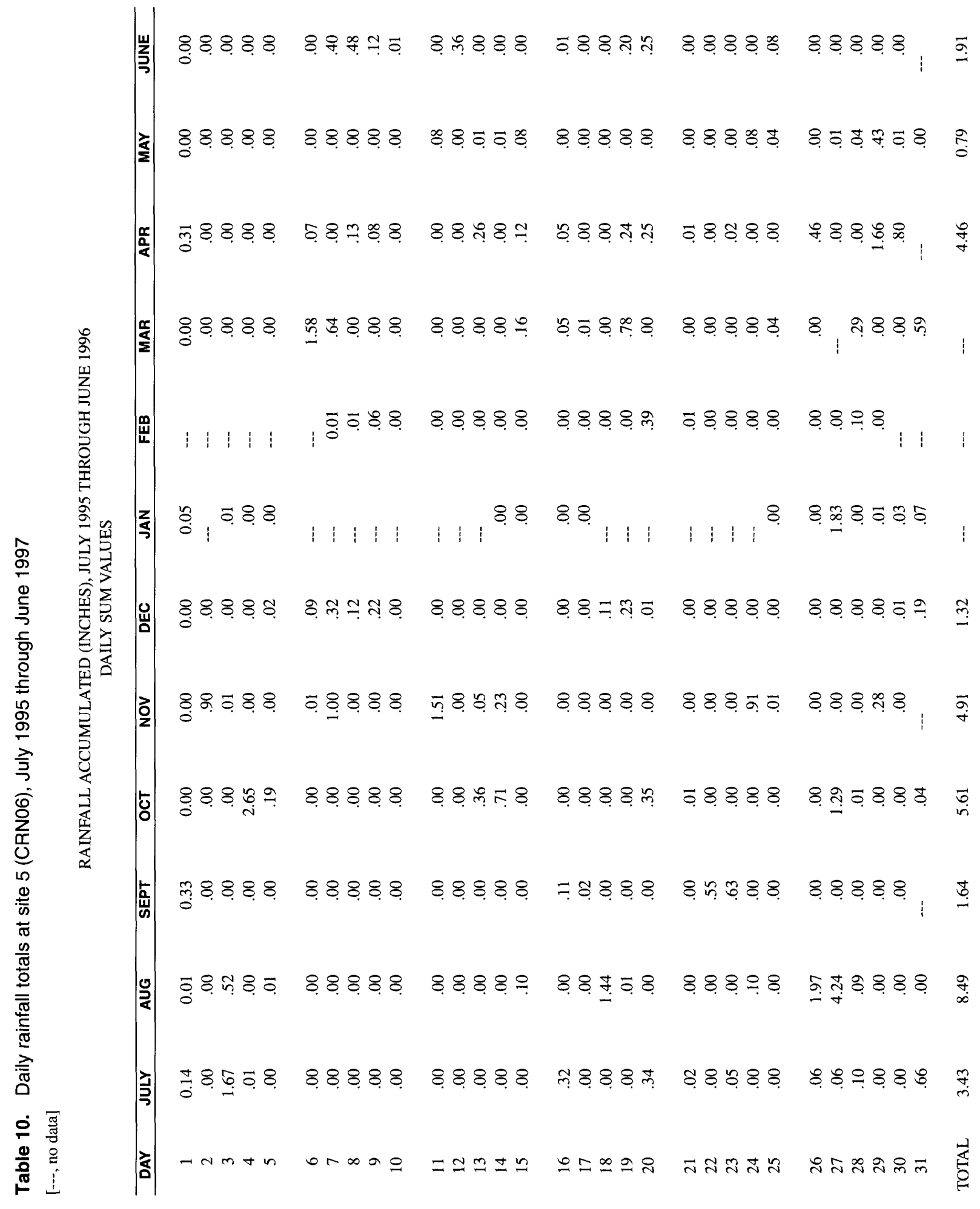




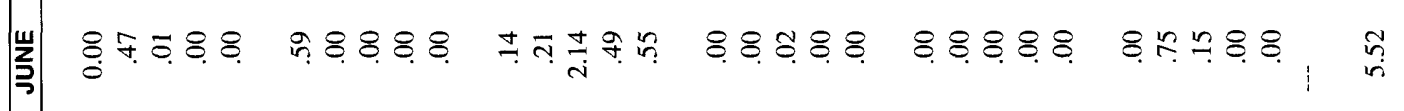

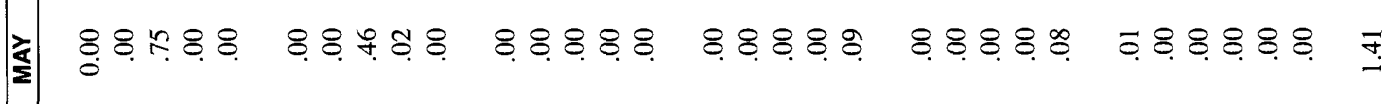

$\frac{\pi}{4}$

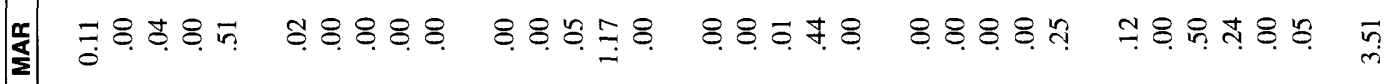

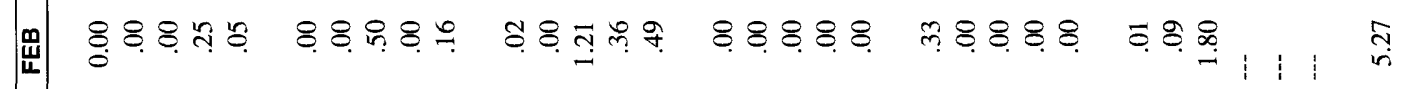

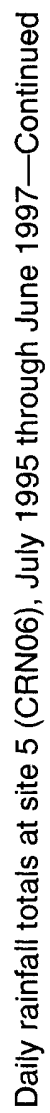

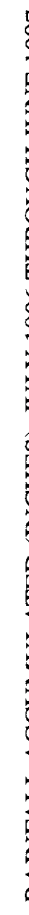

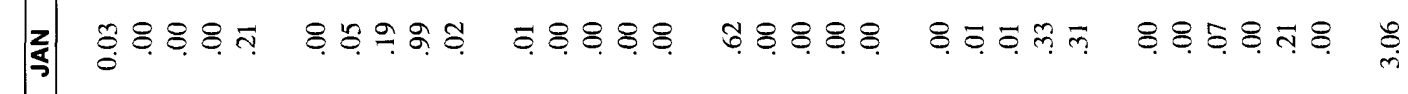

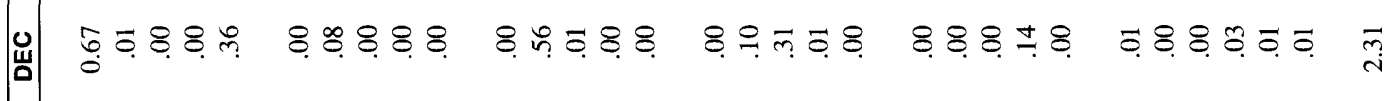

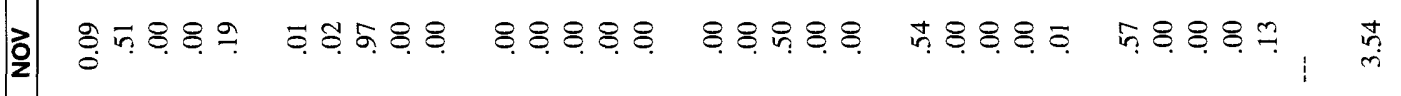

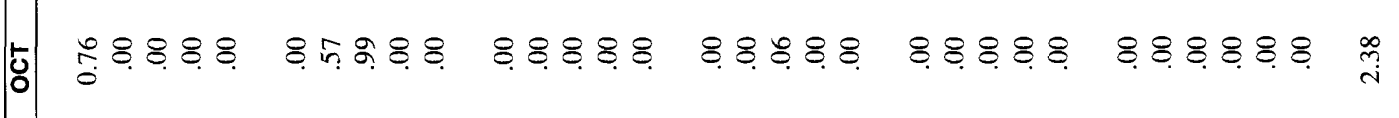

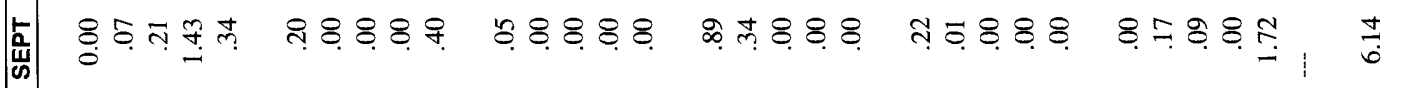

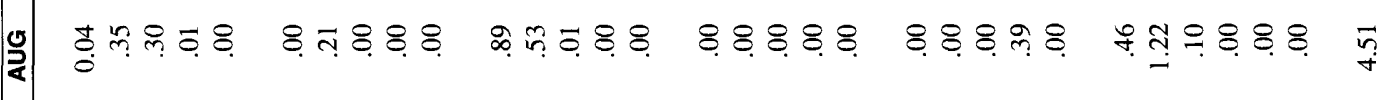

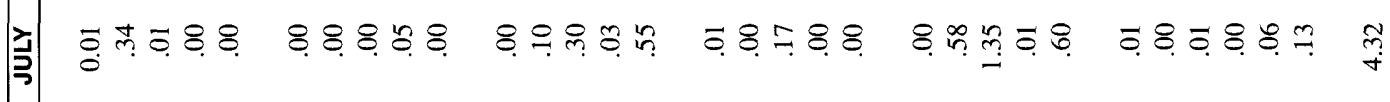

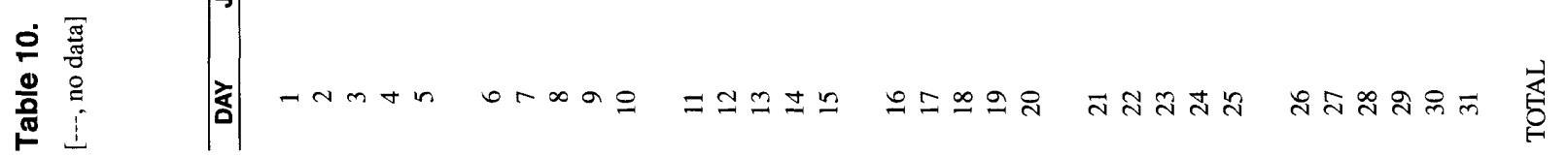




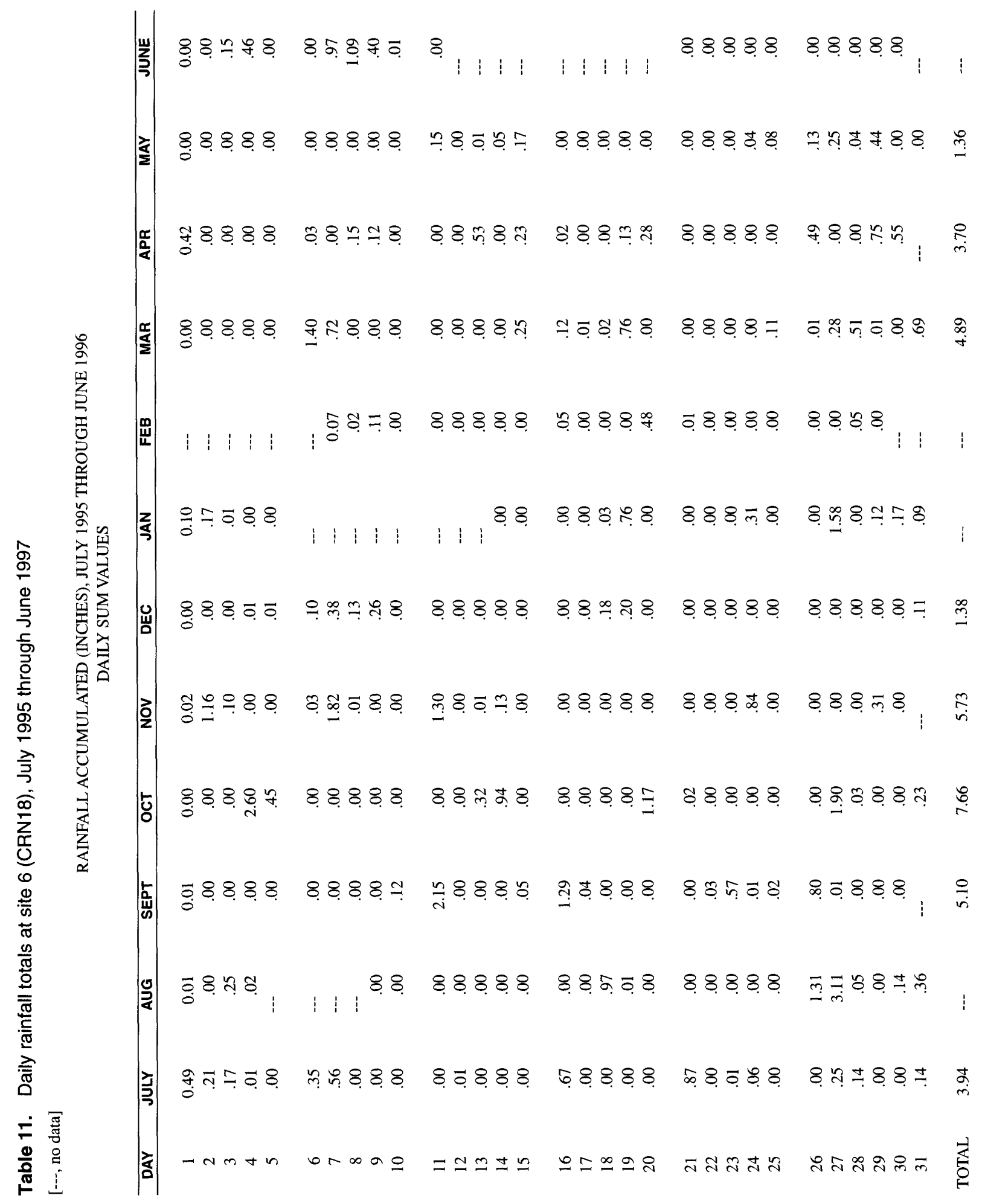


แ్

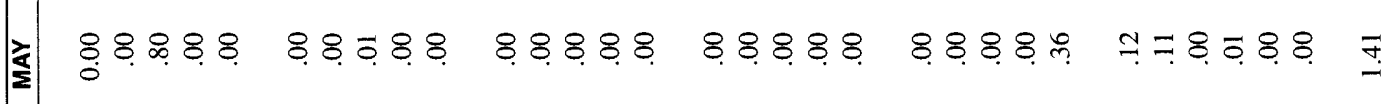

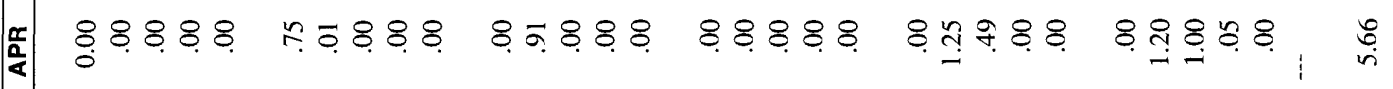

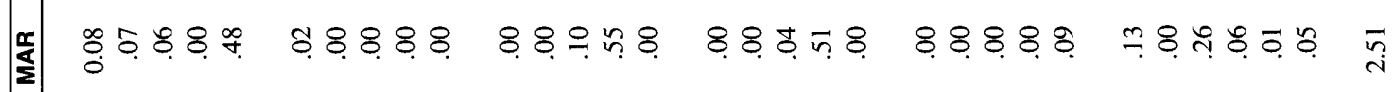

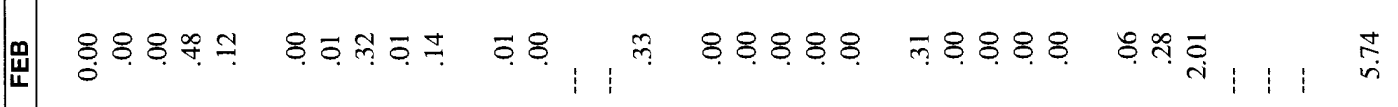

द ०.

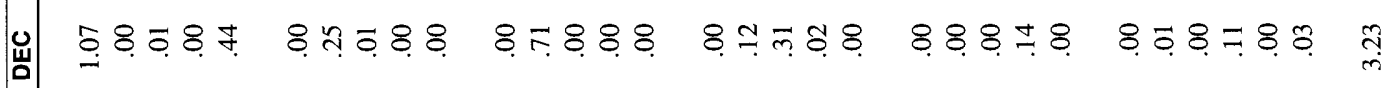

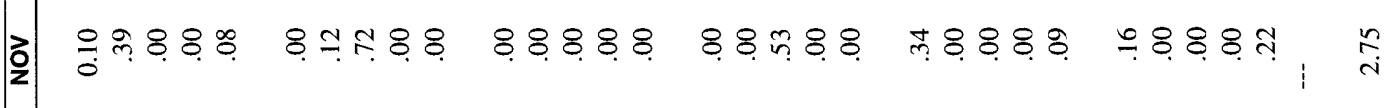

เ

:

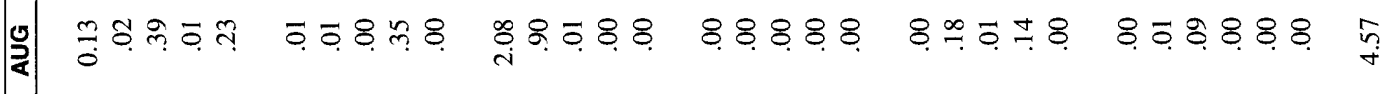

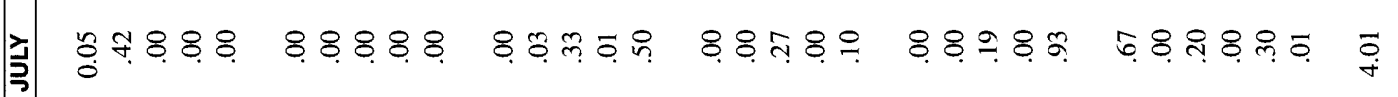

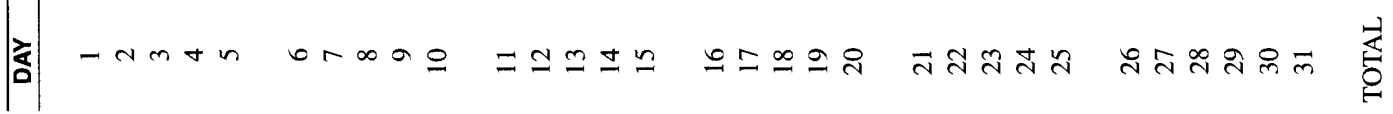




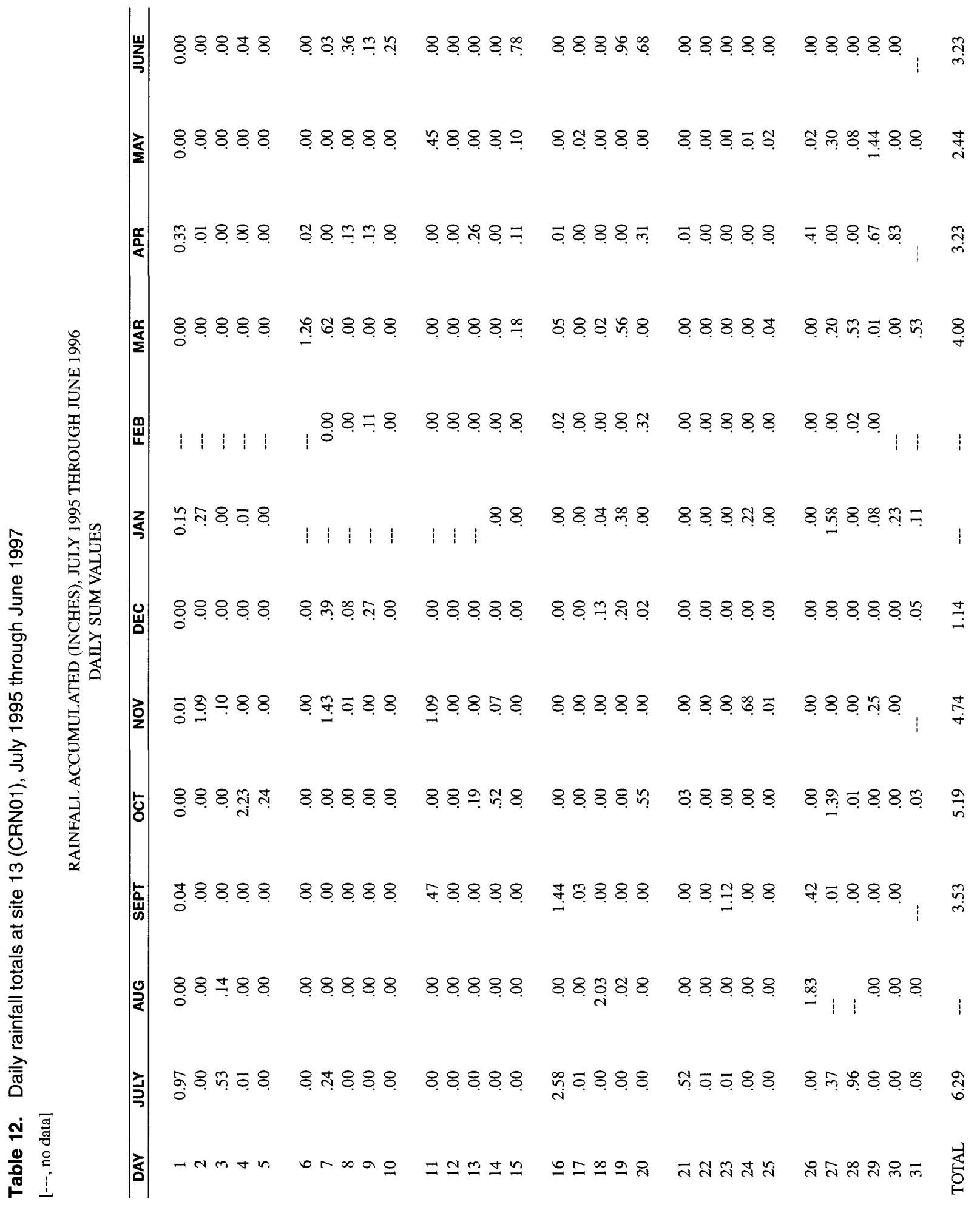




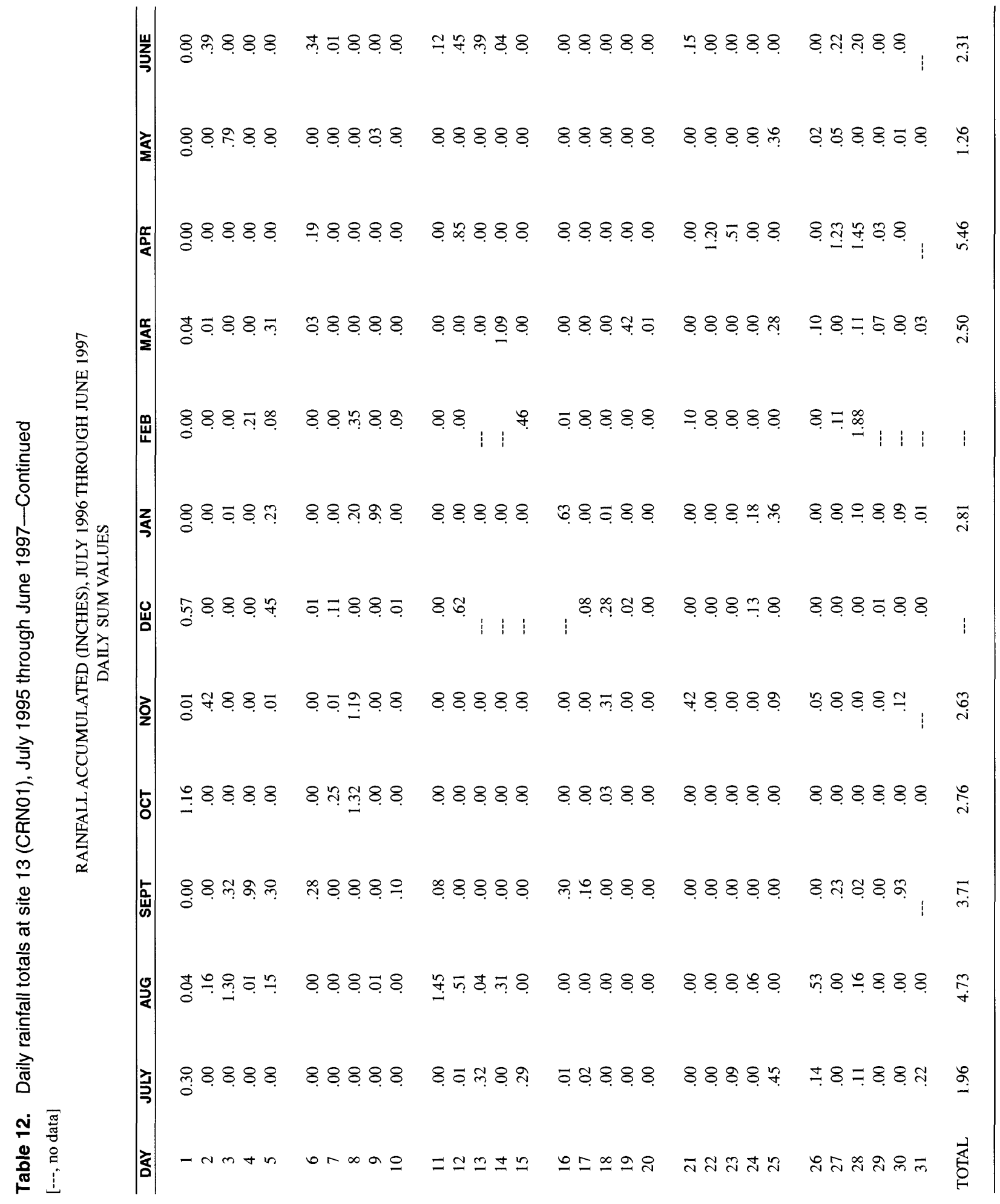




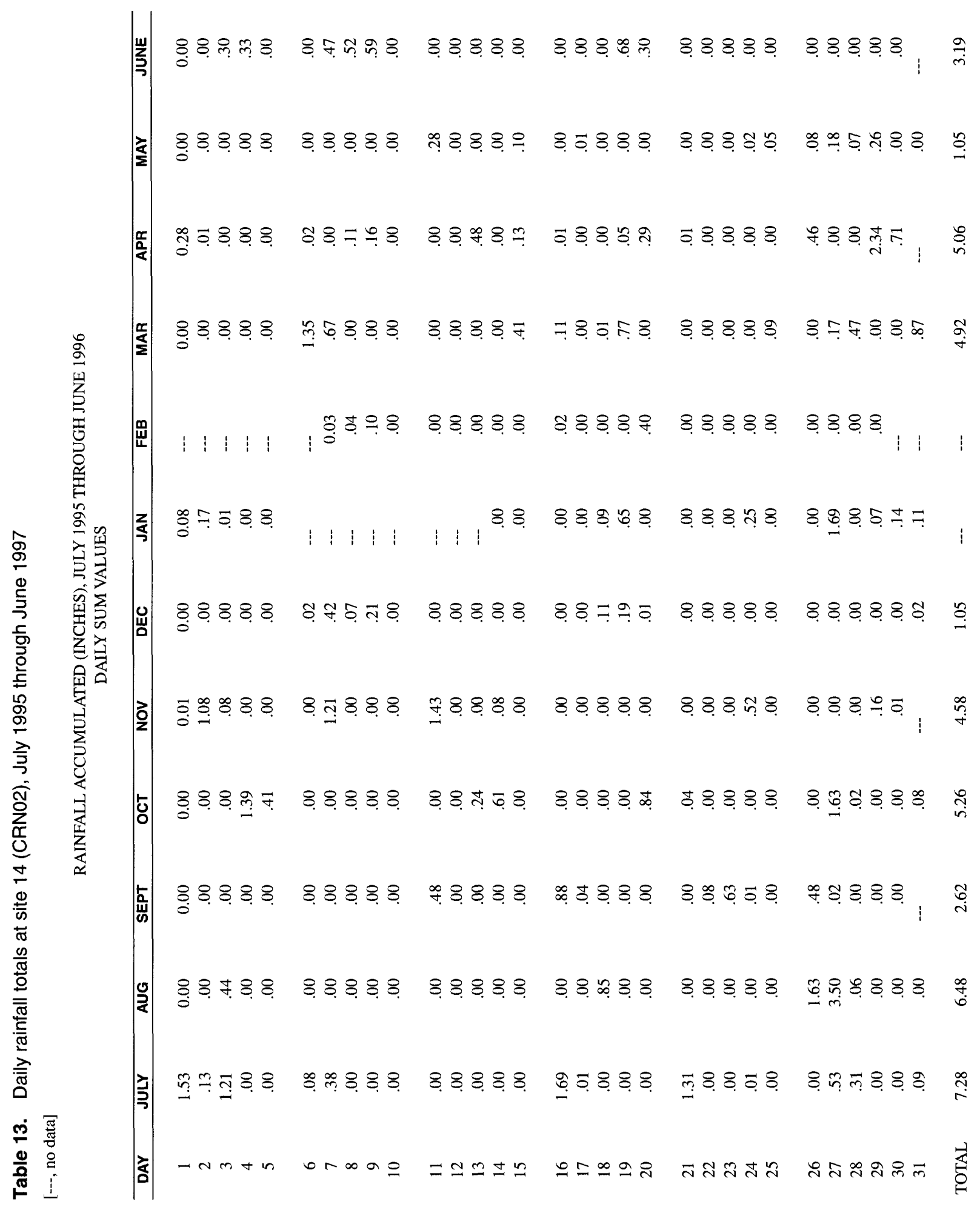




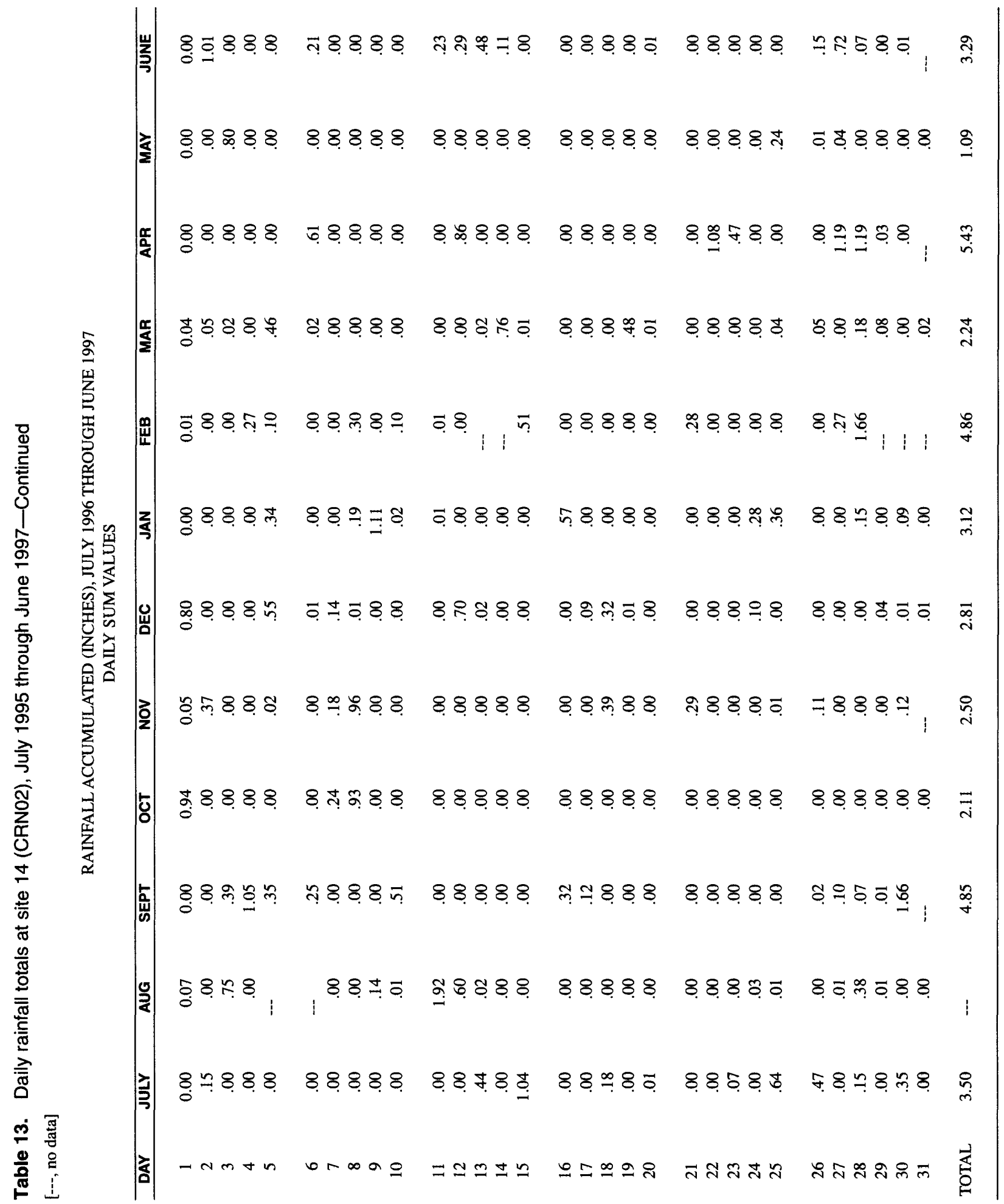




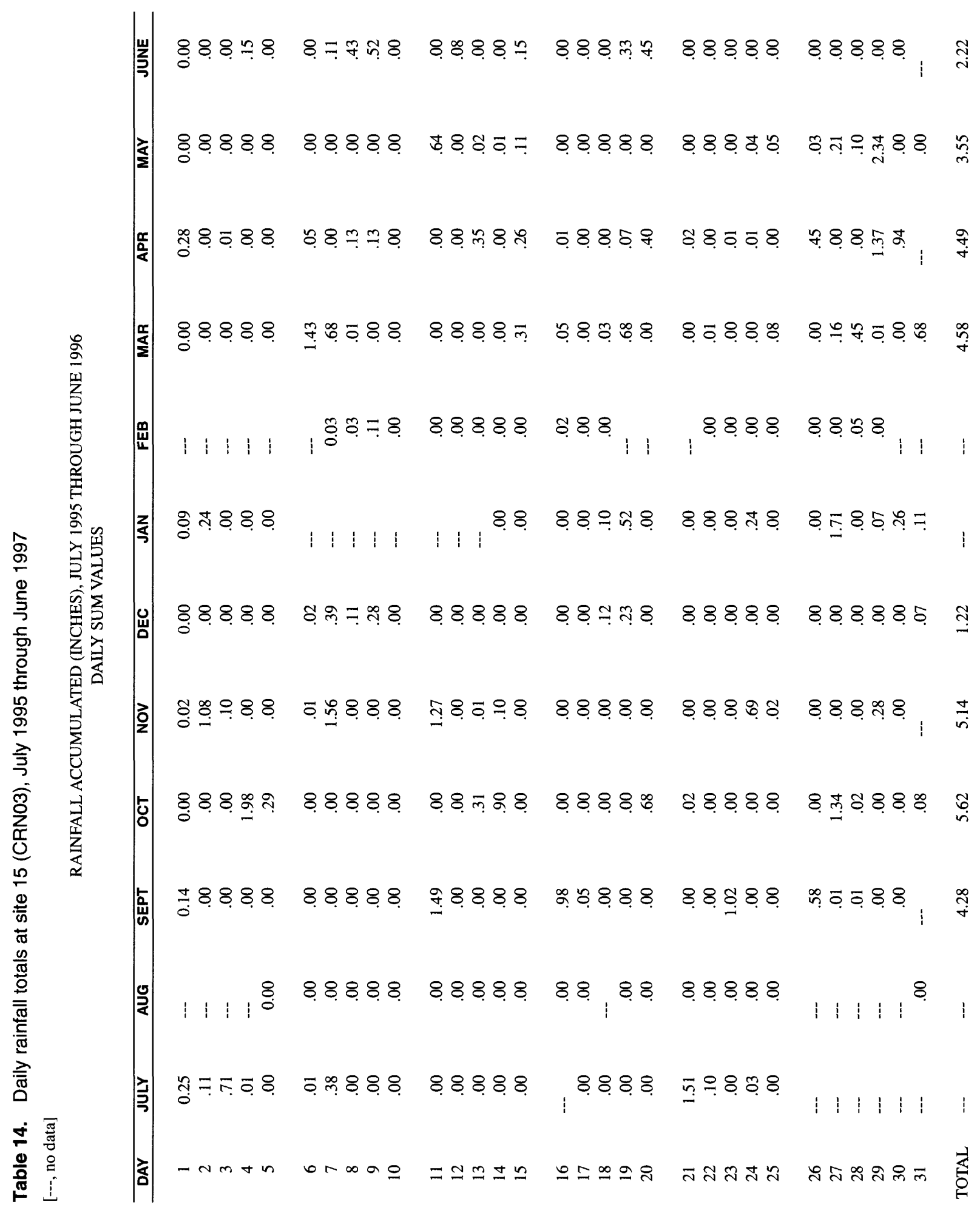




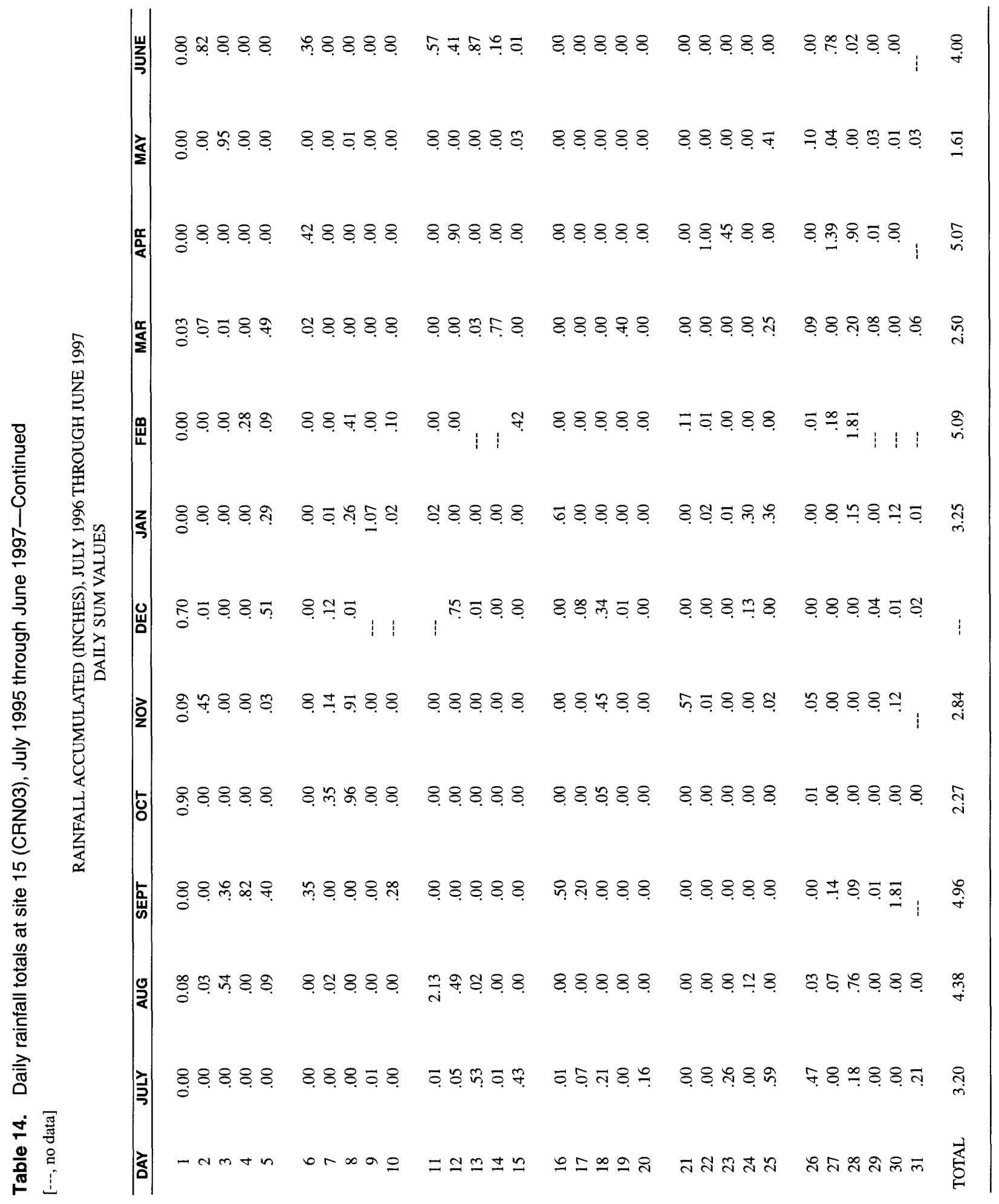




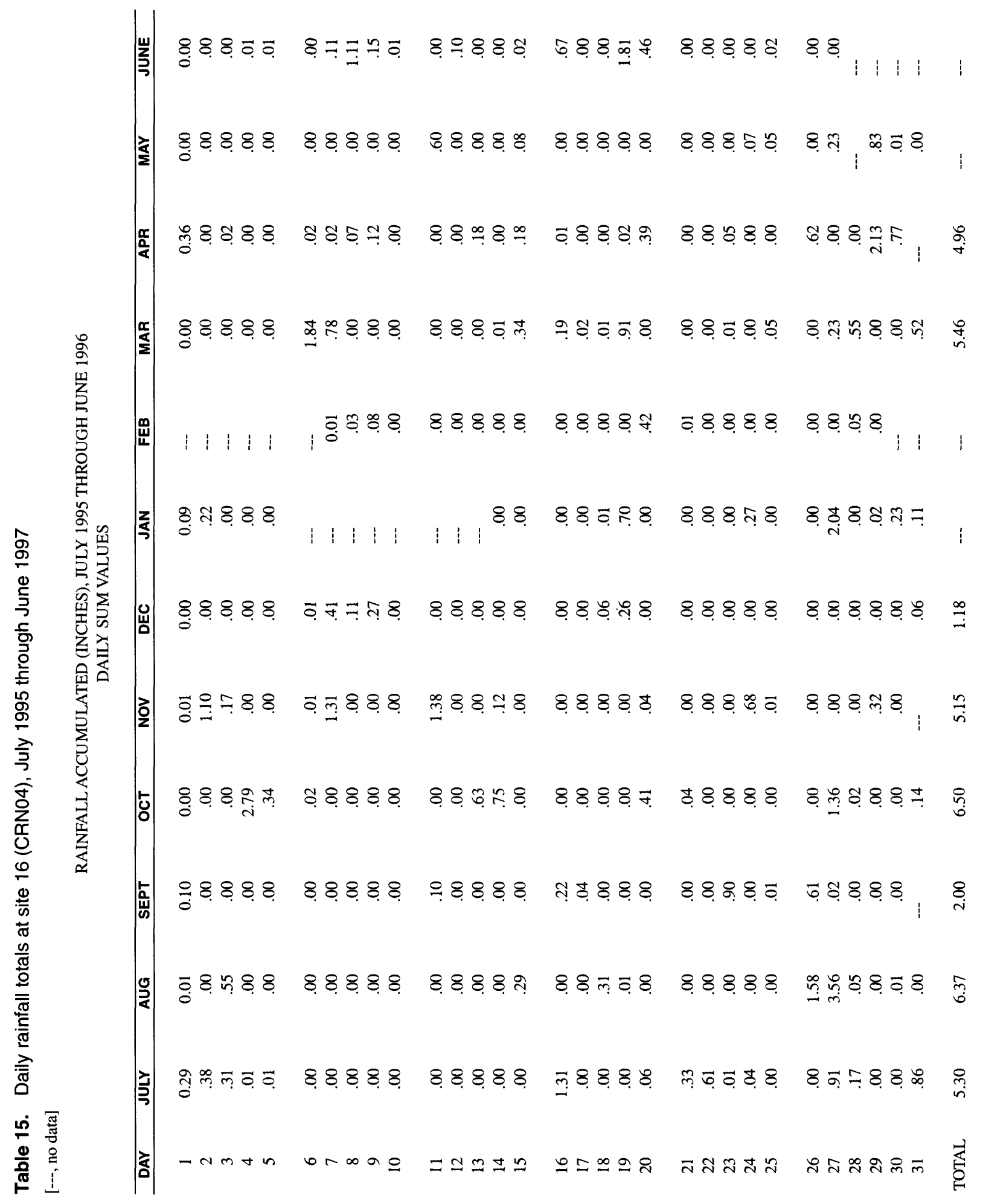




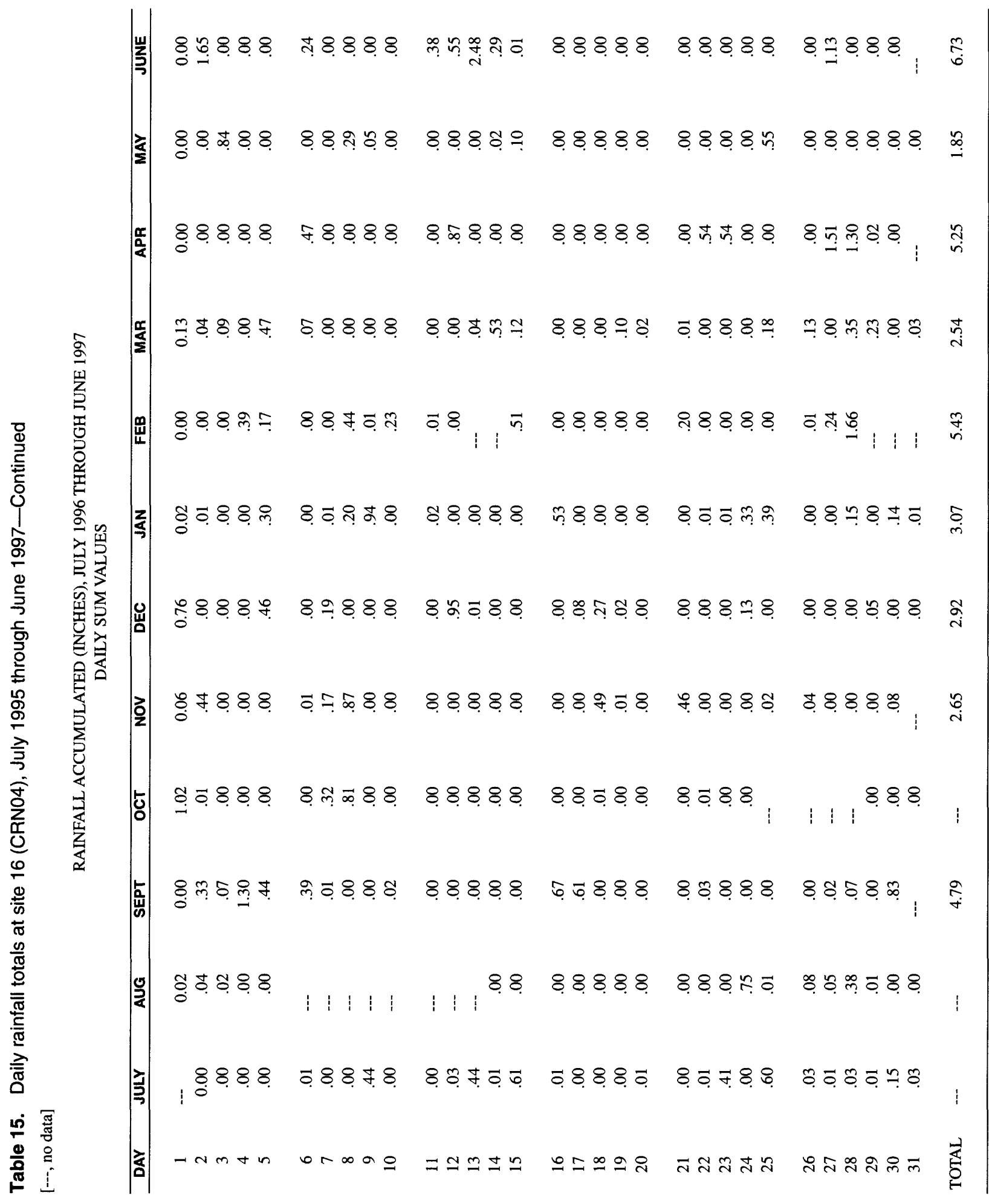




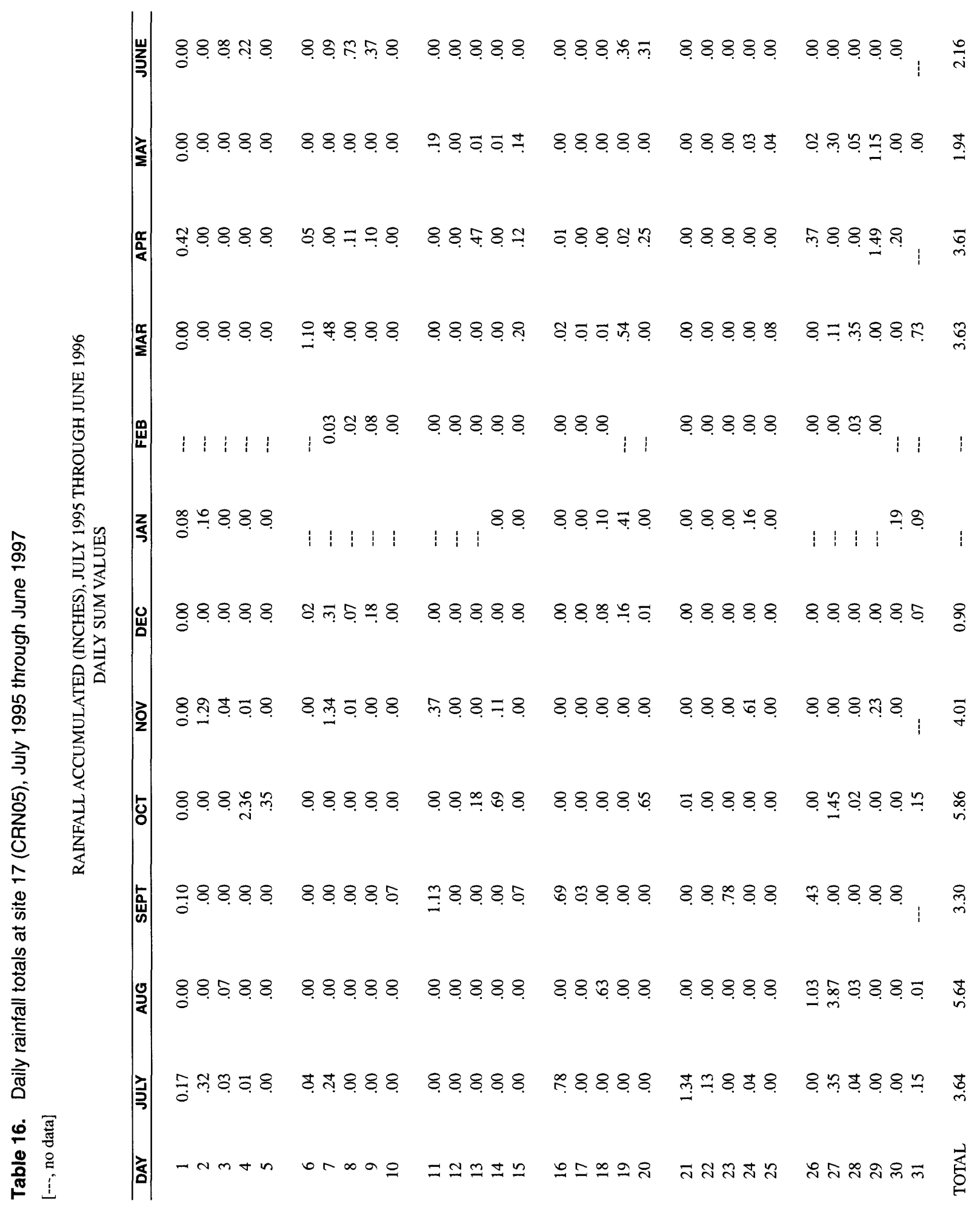




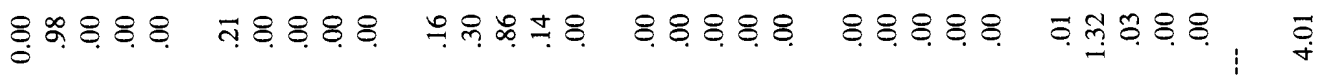

웅 8.

急

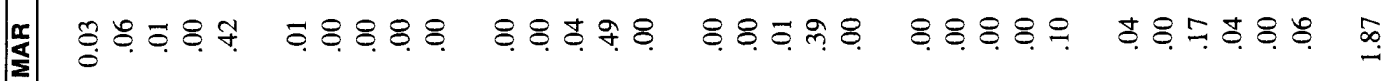

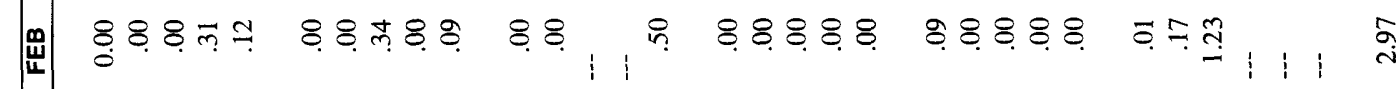

ว 8.8.

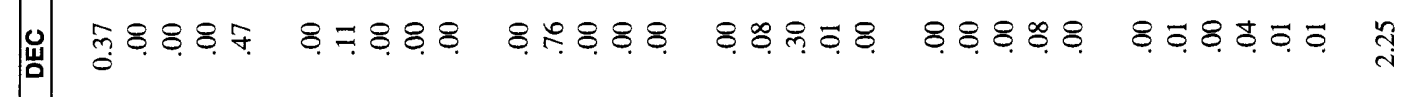

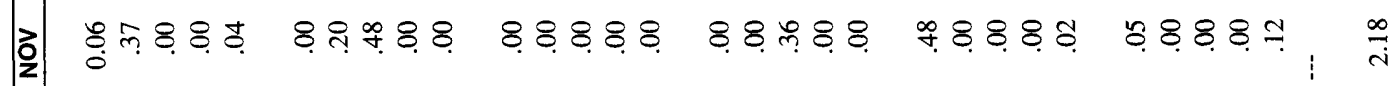

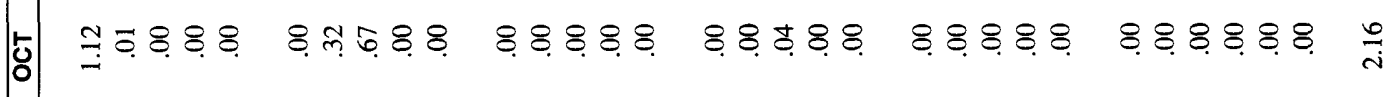

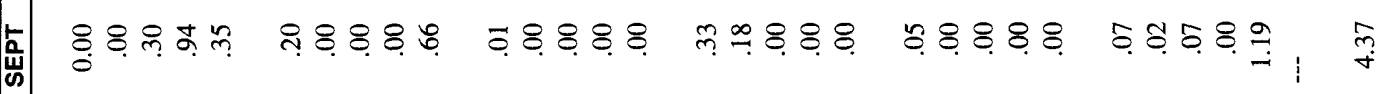

ํำ

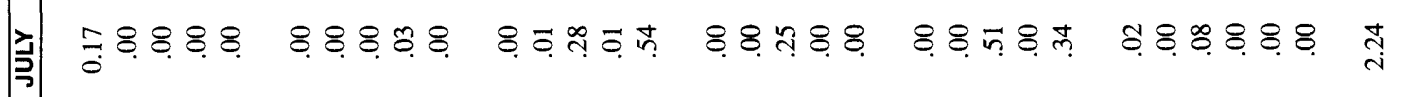




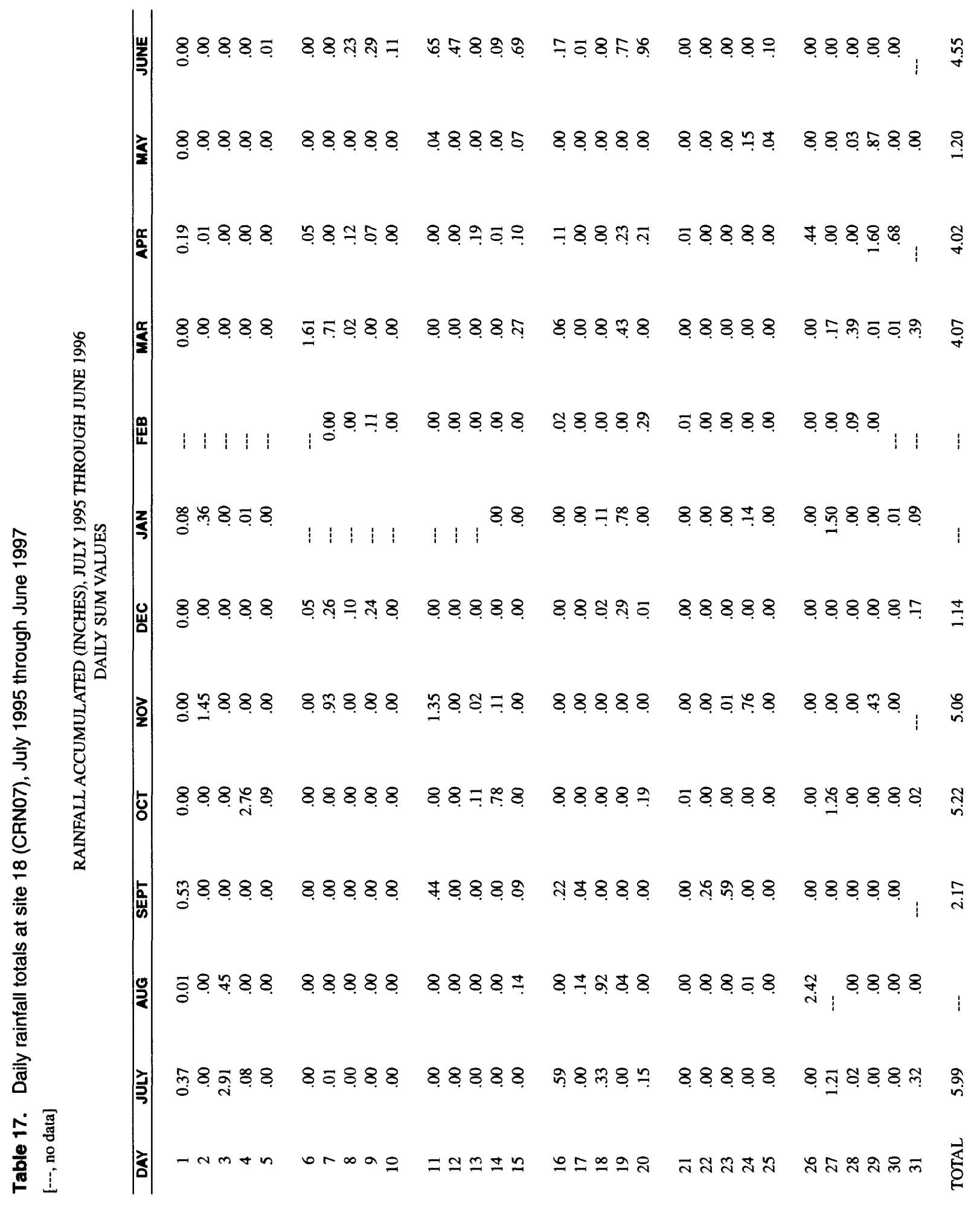




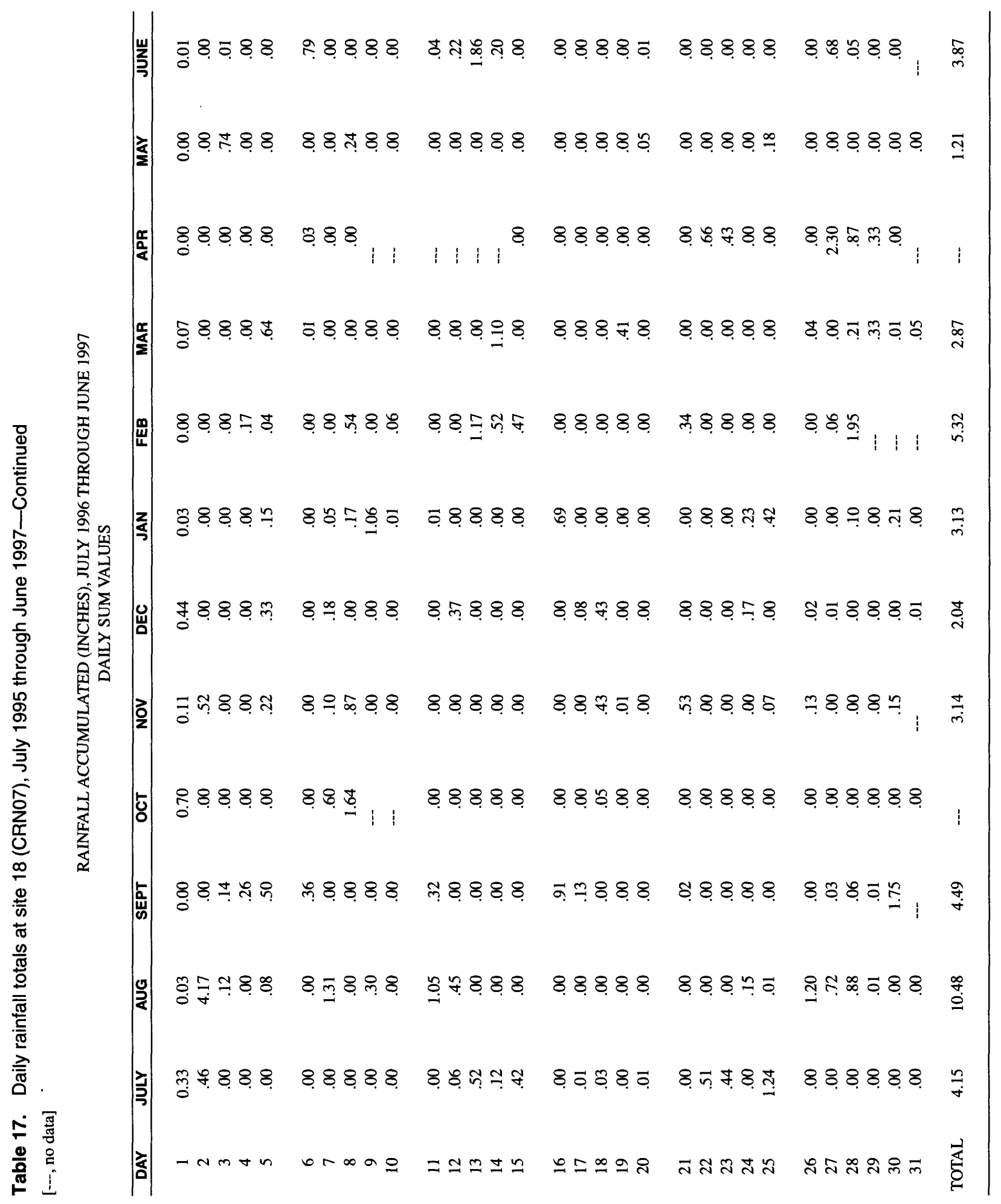




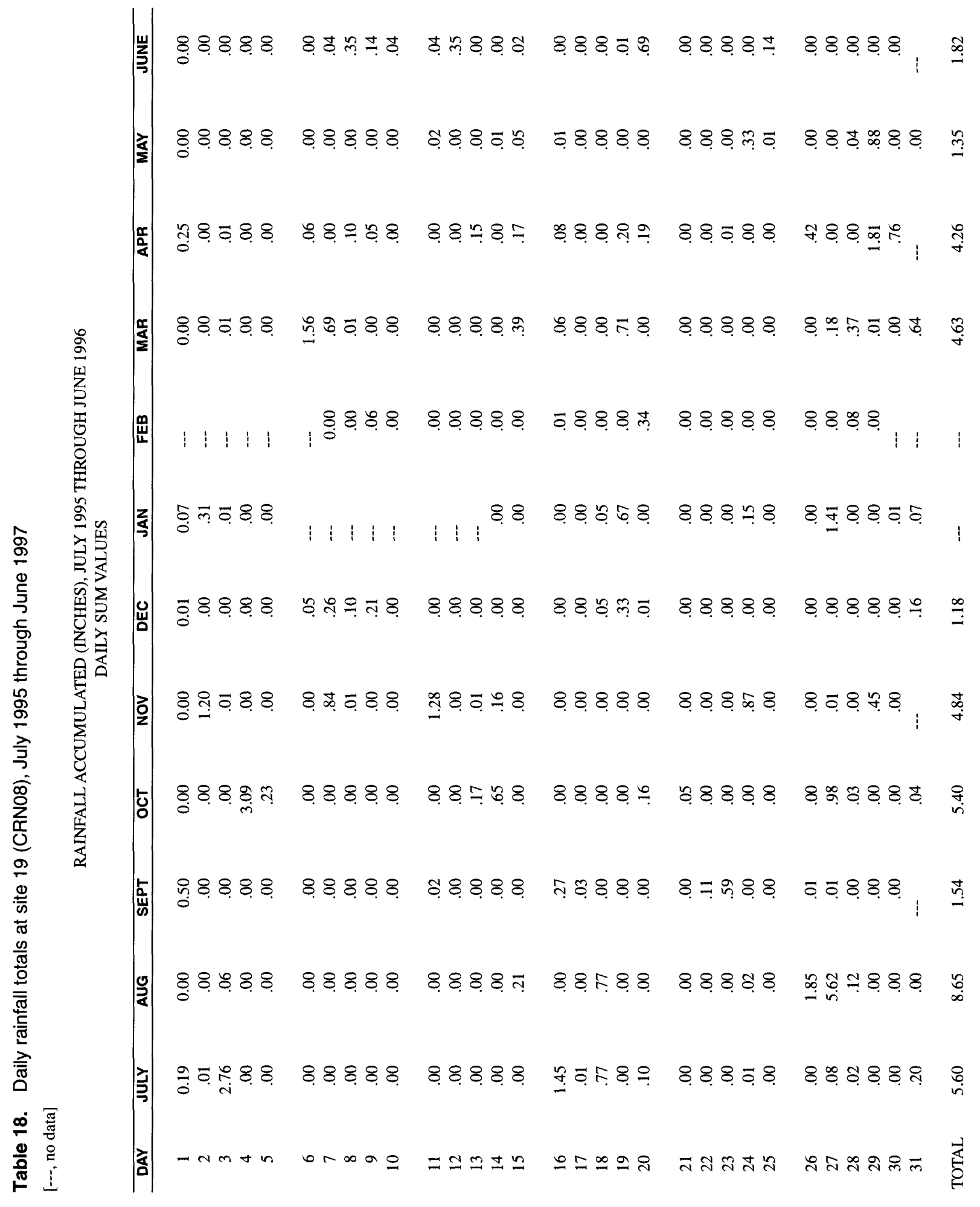




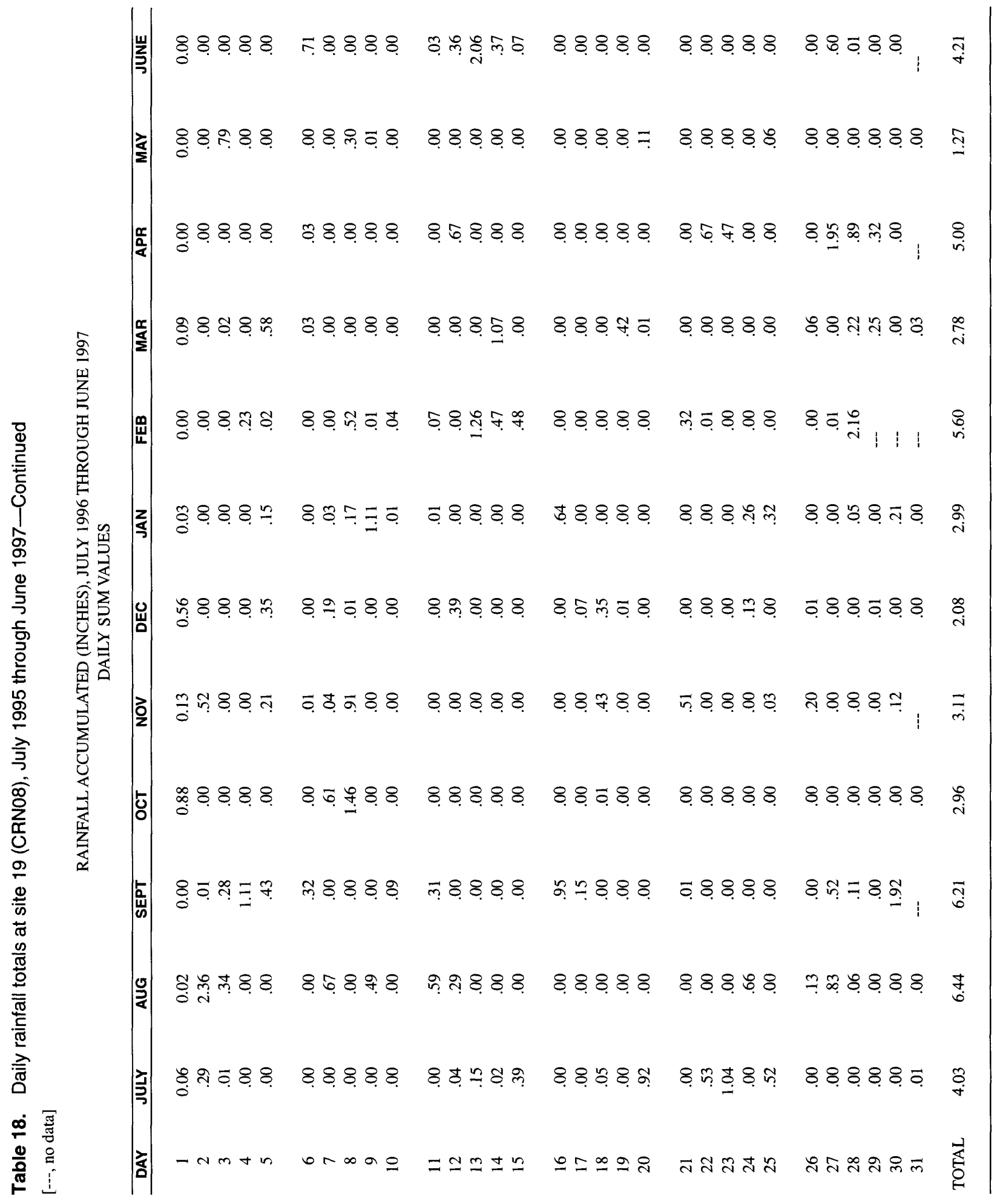




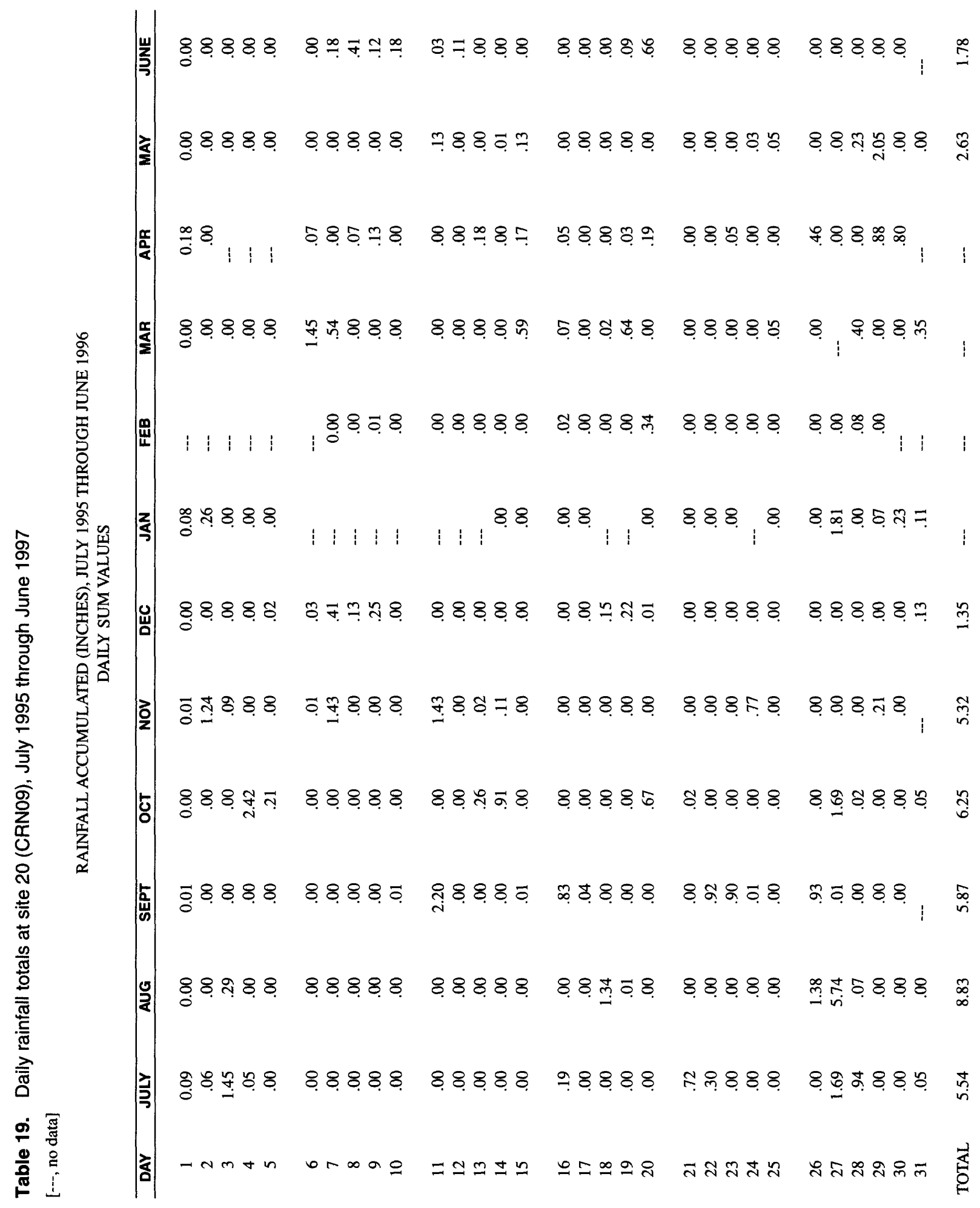




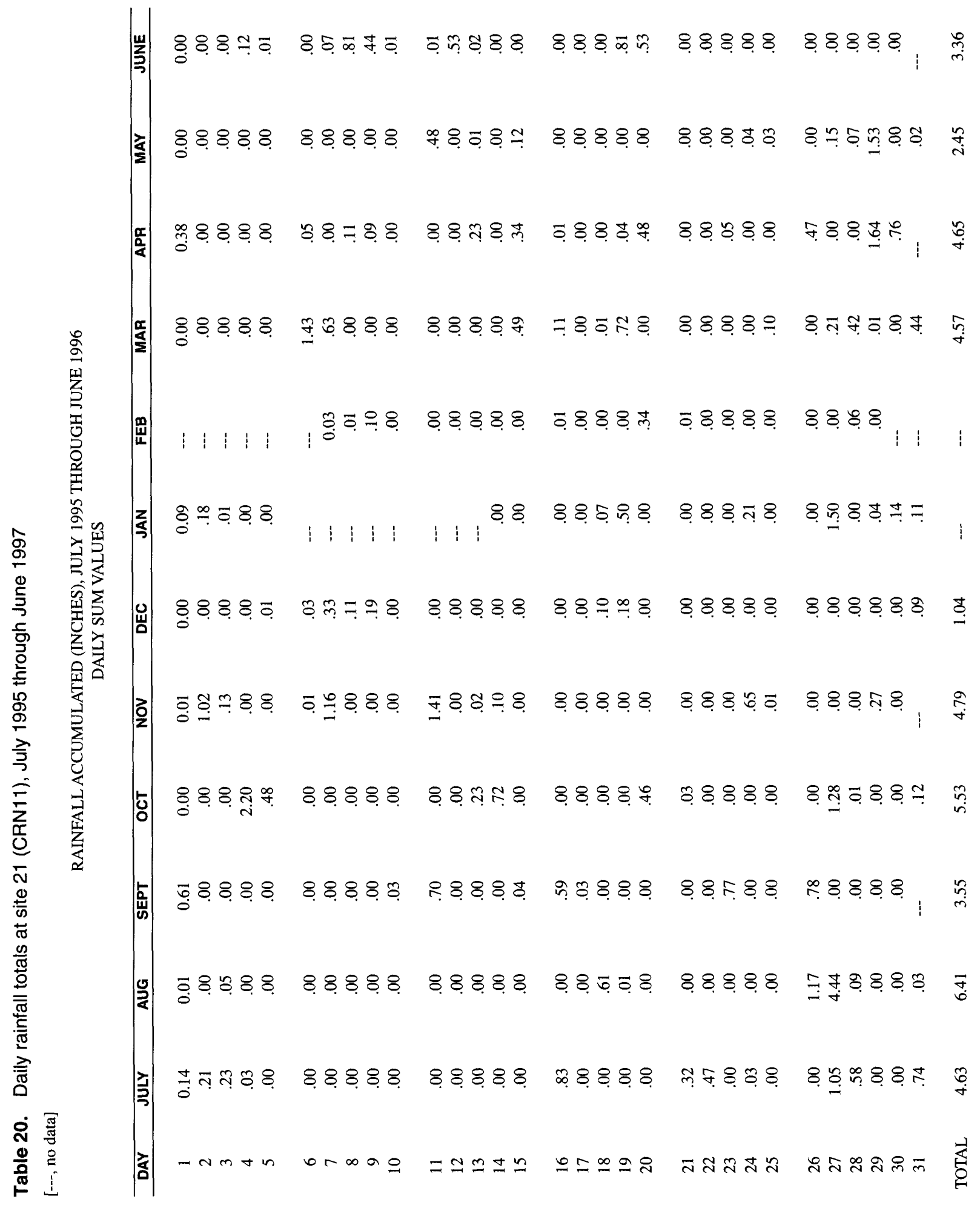




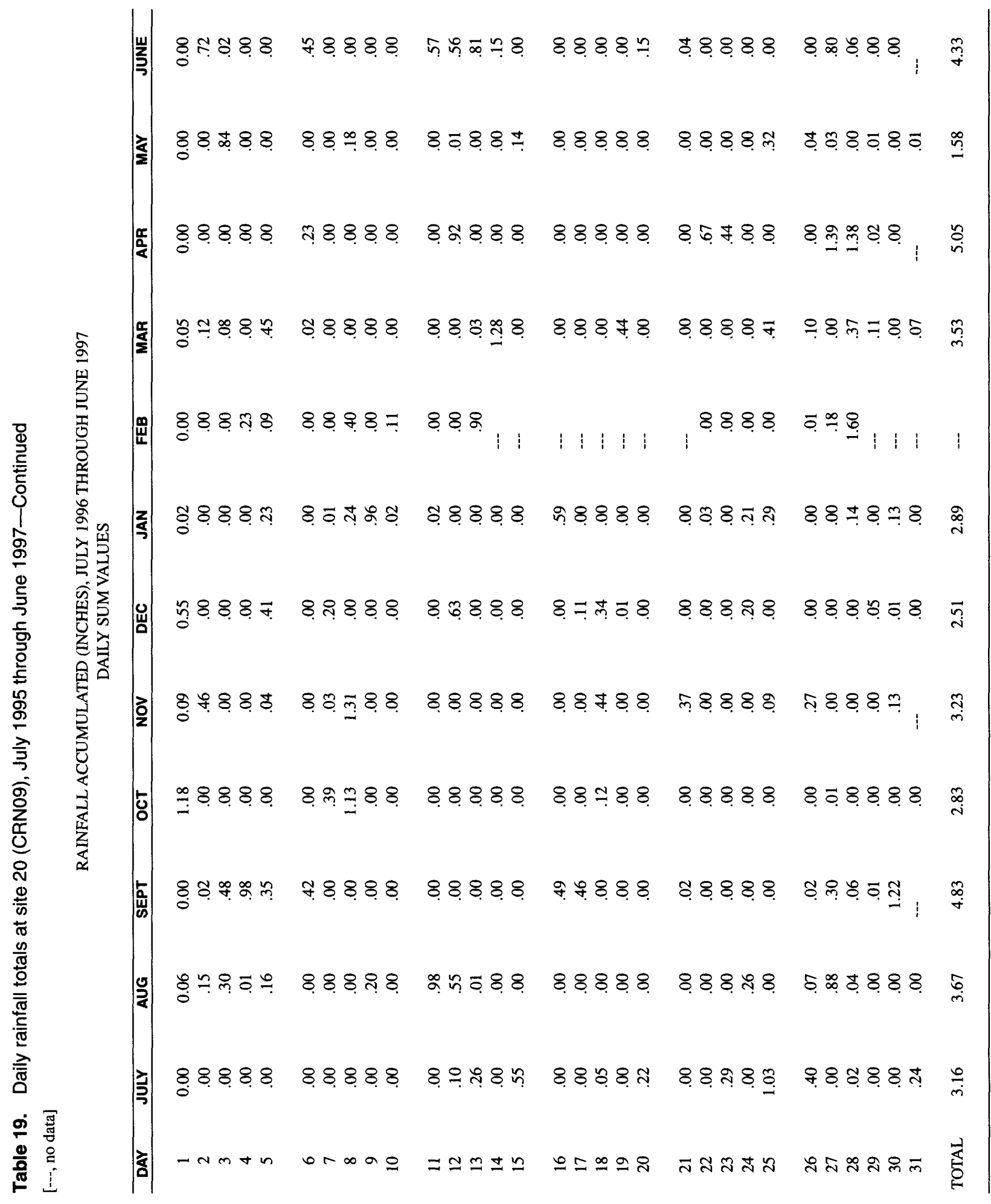




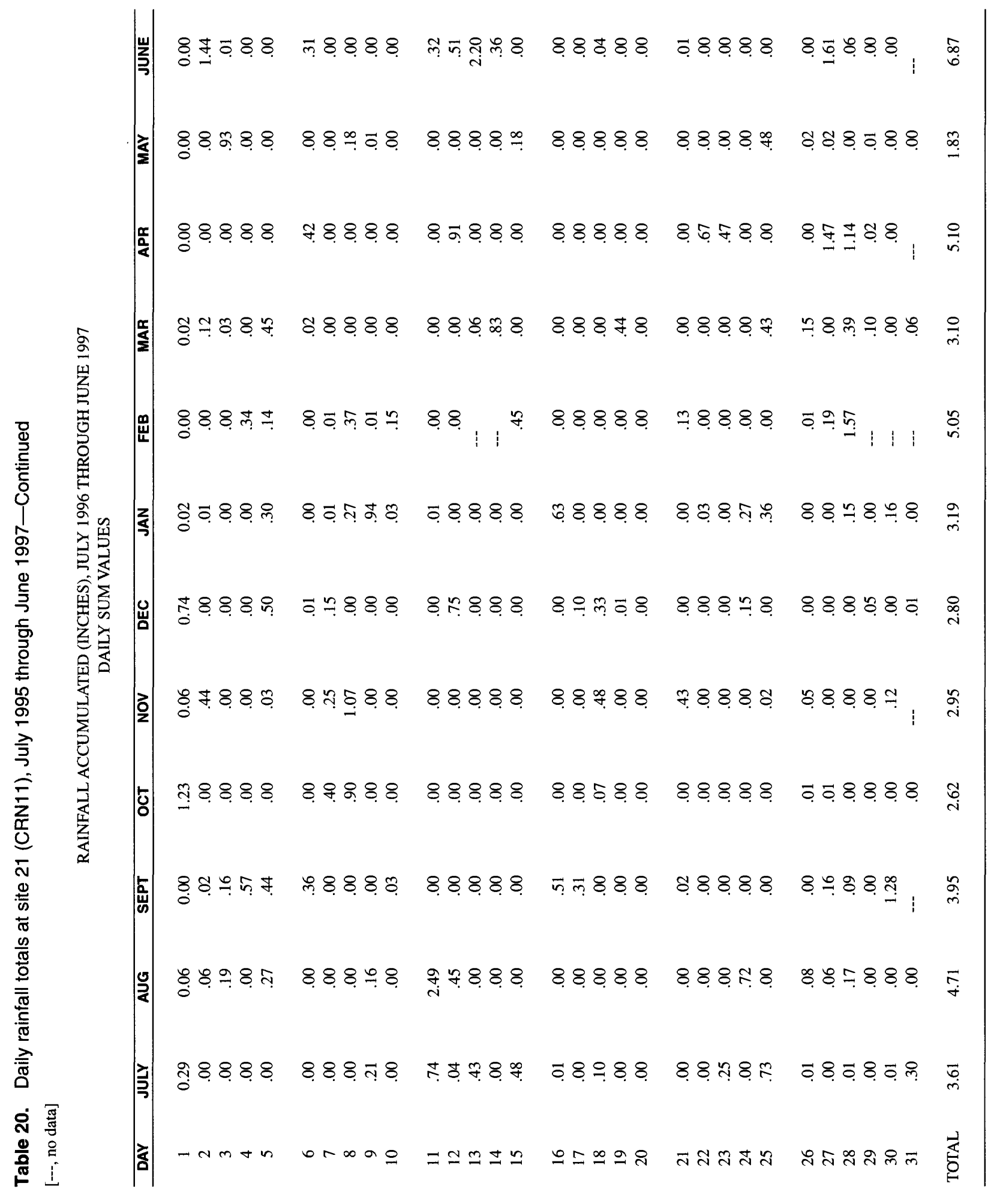




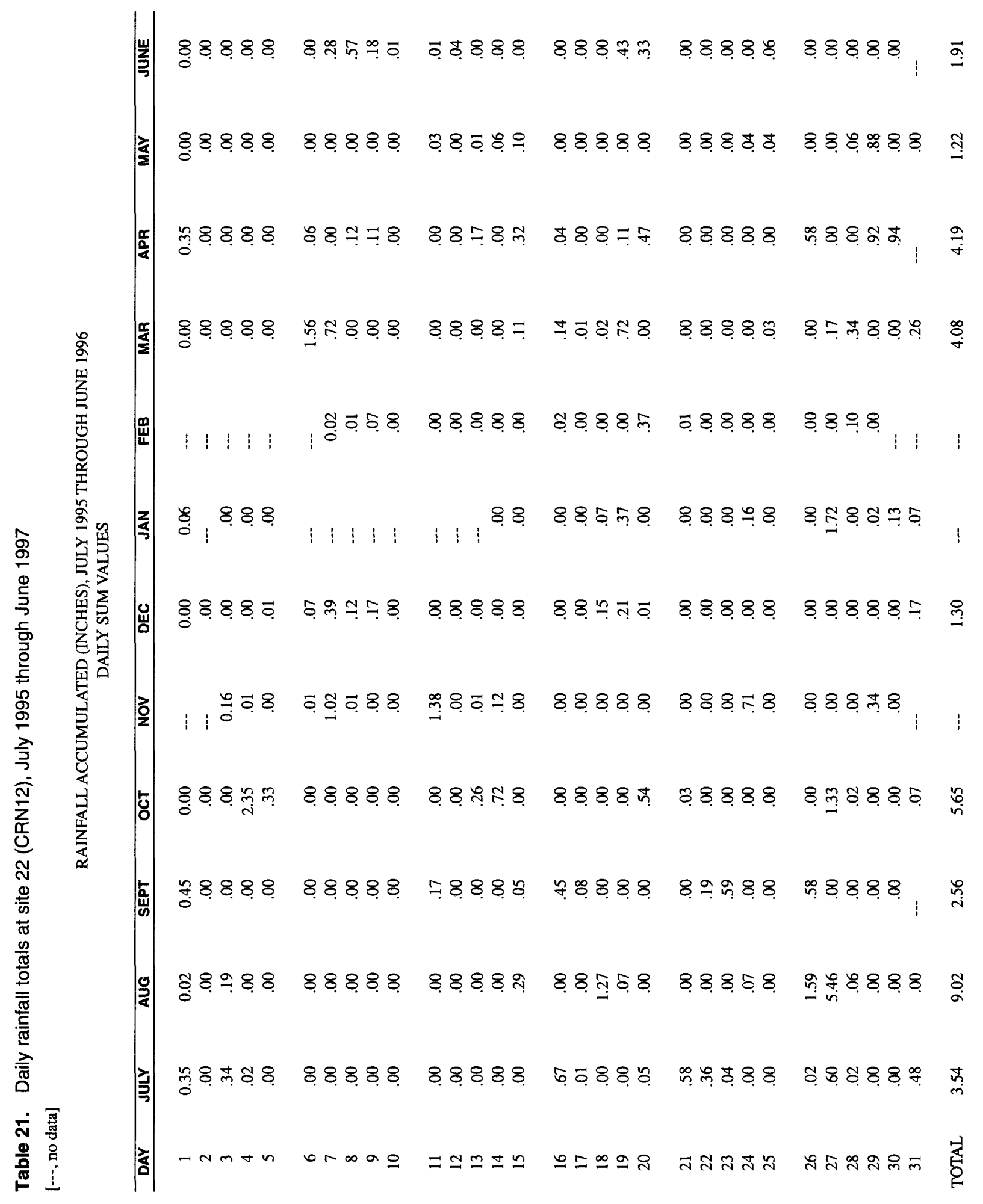




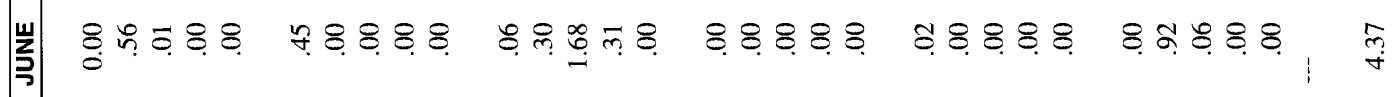

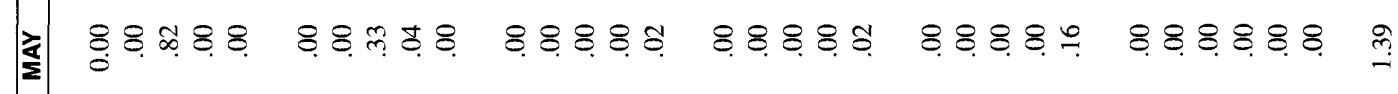

|

赵

m

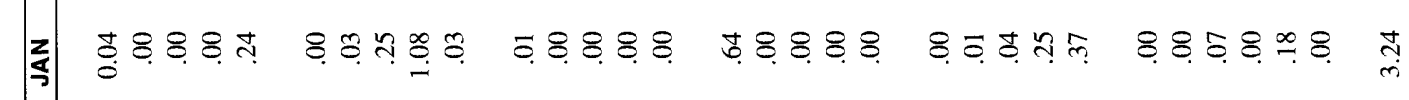

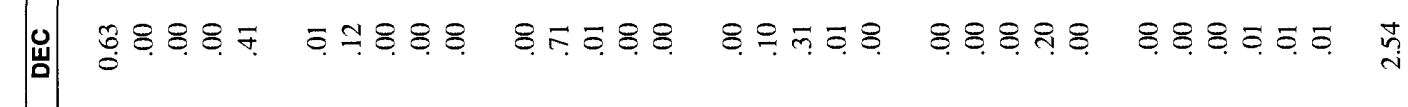

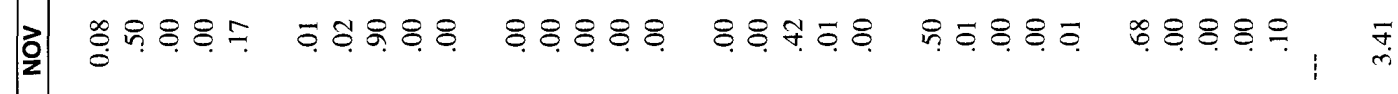

용

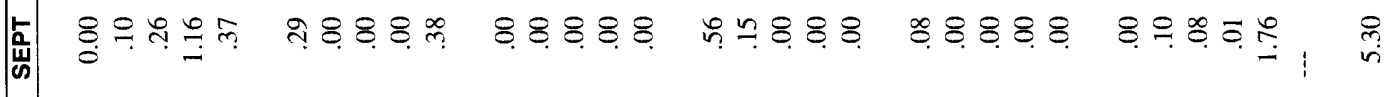

난

ไู่

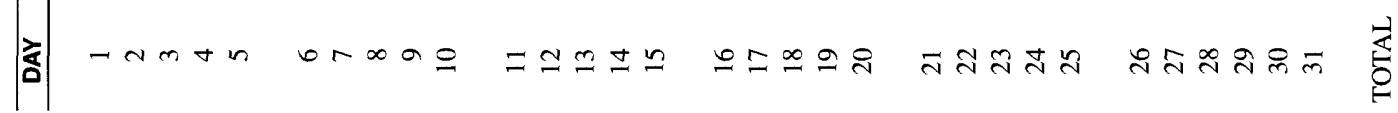




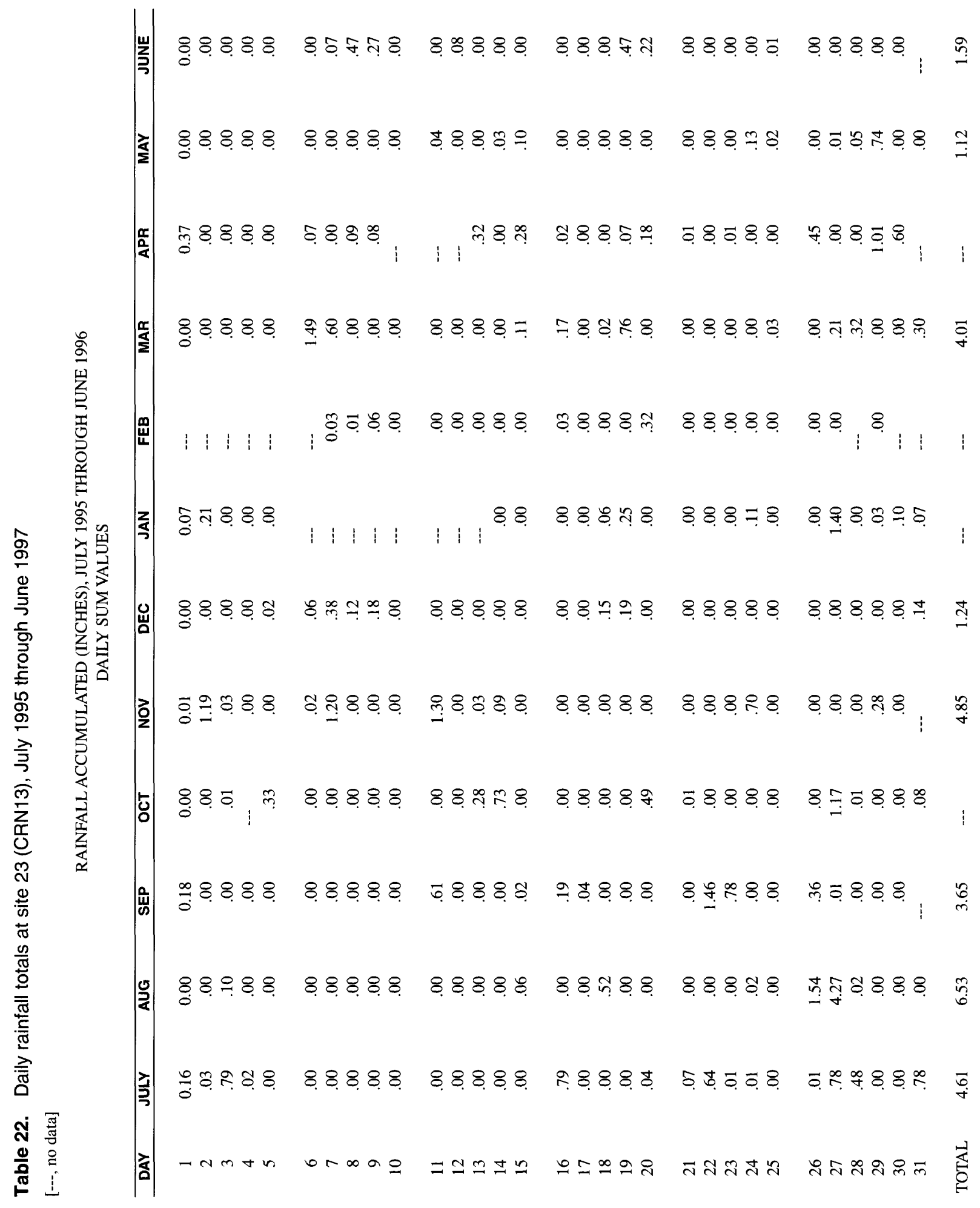




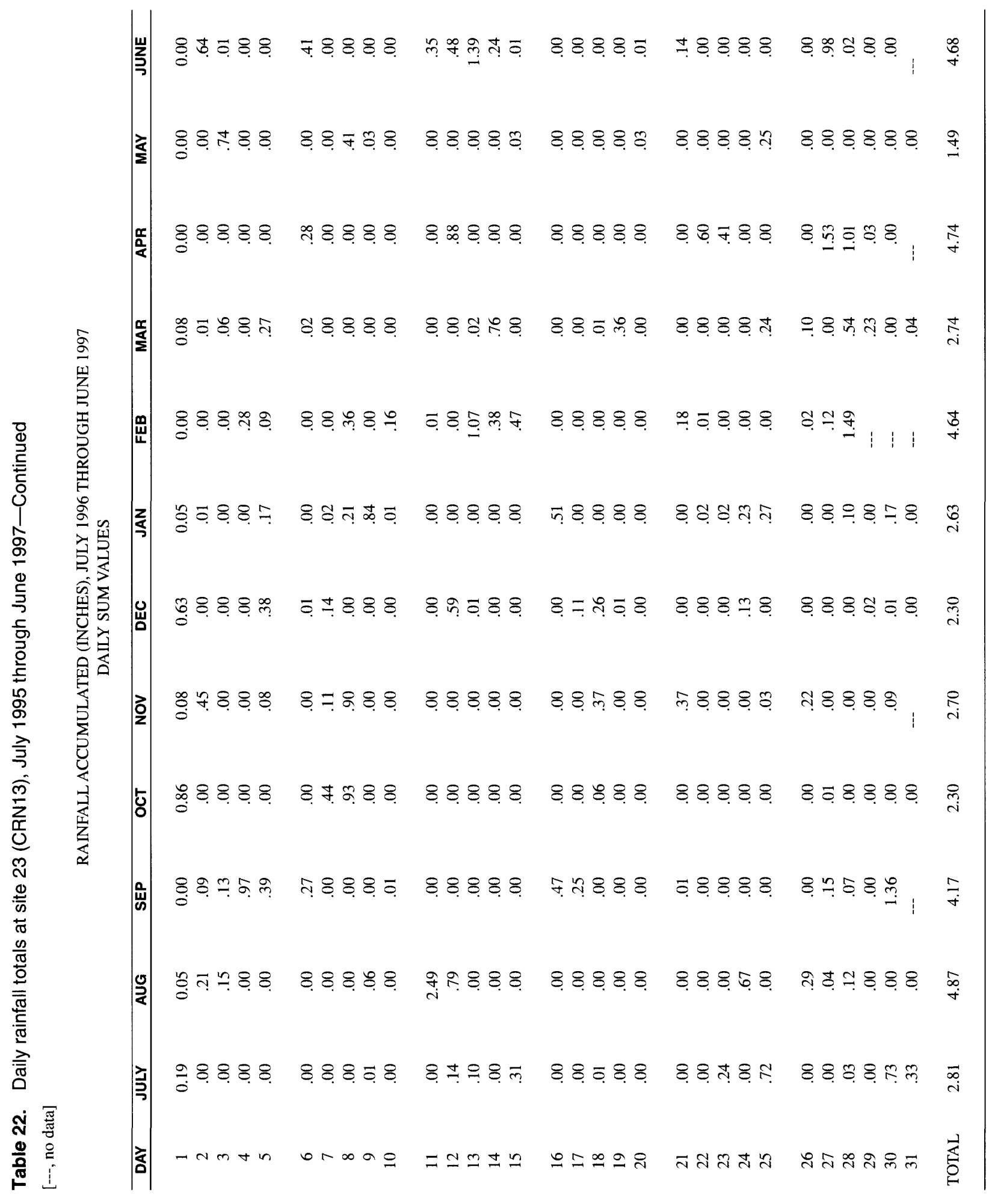




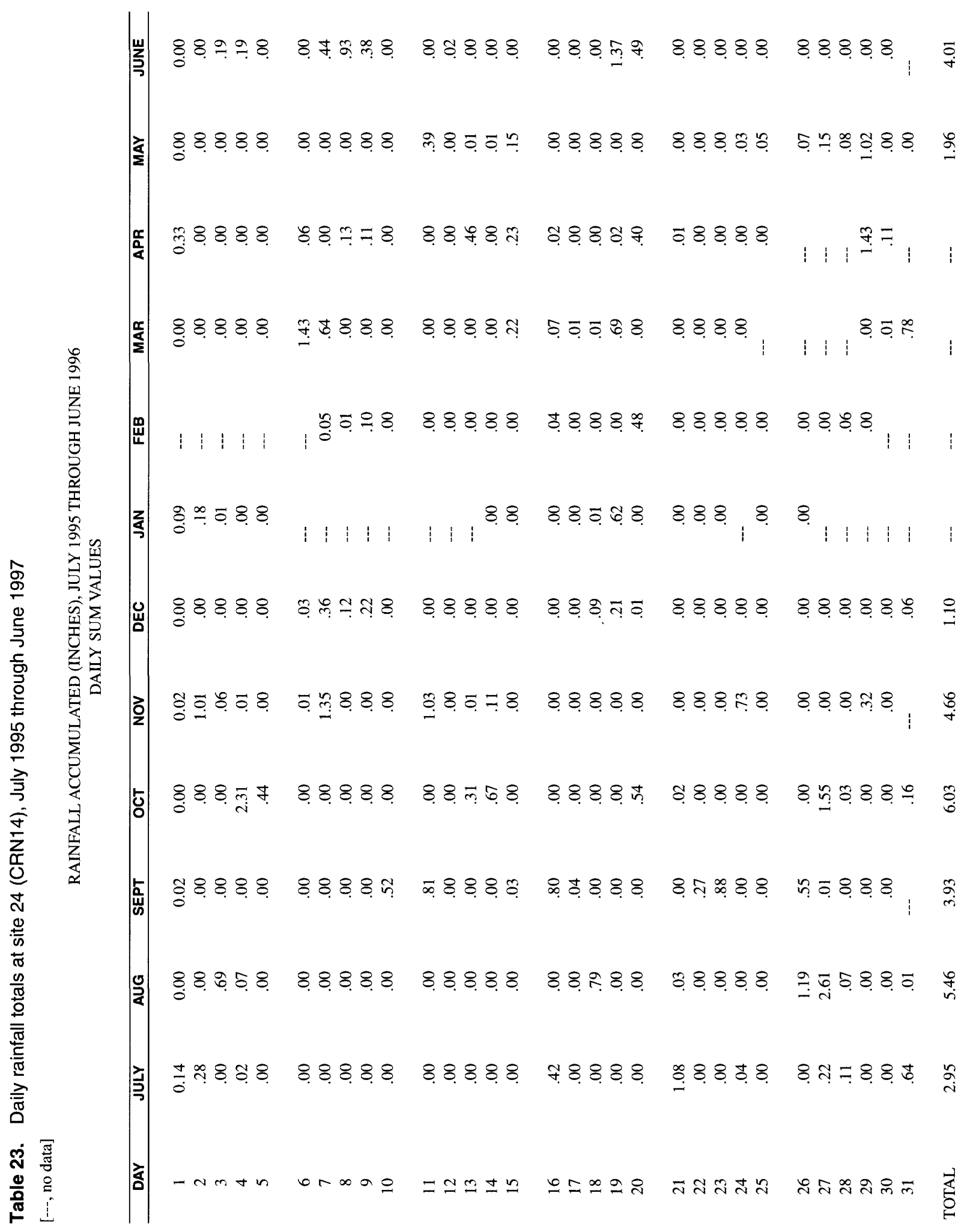




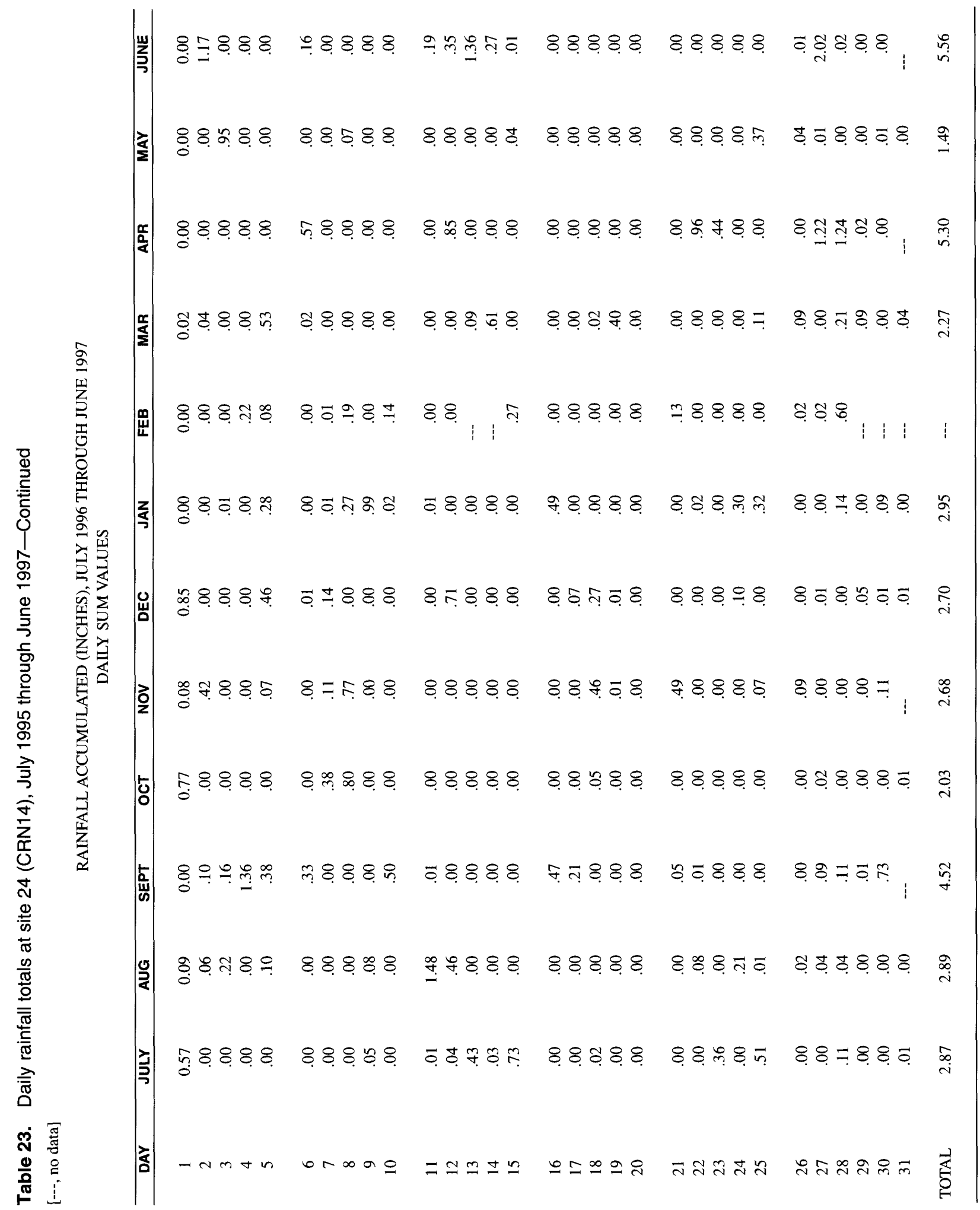




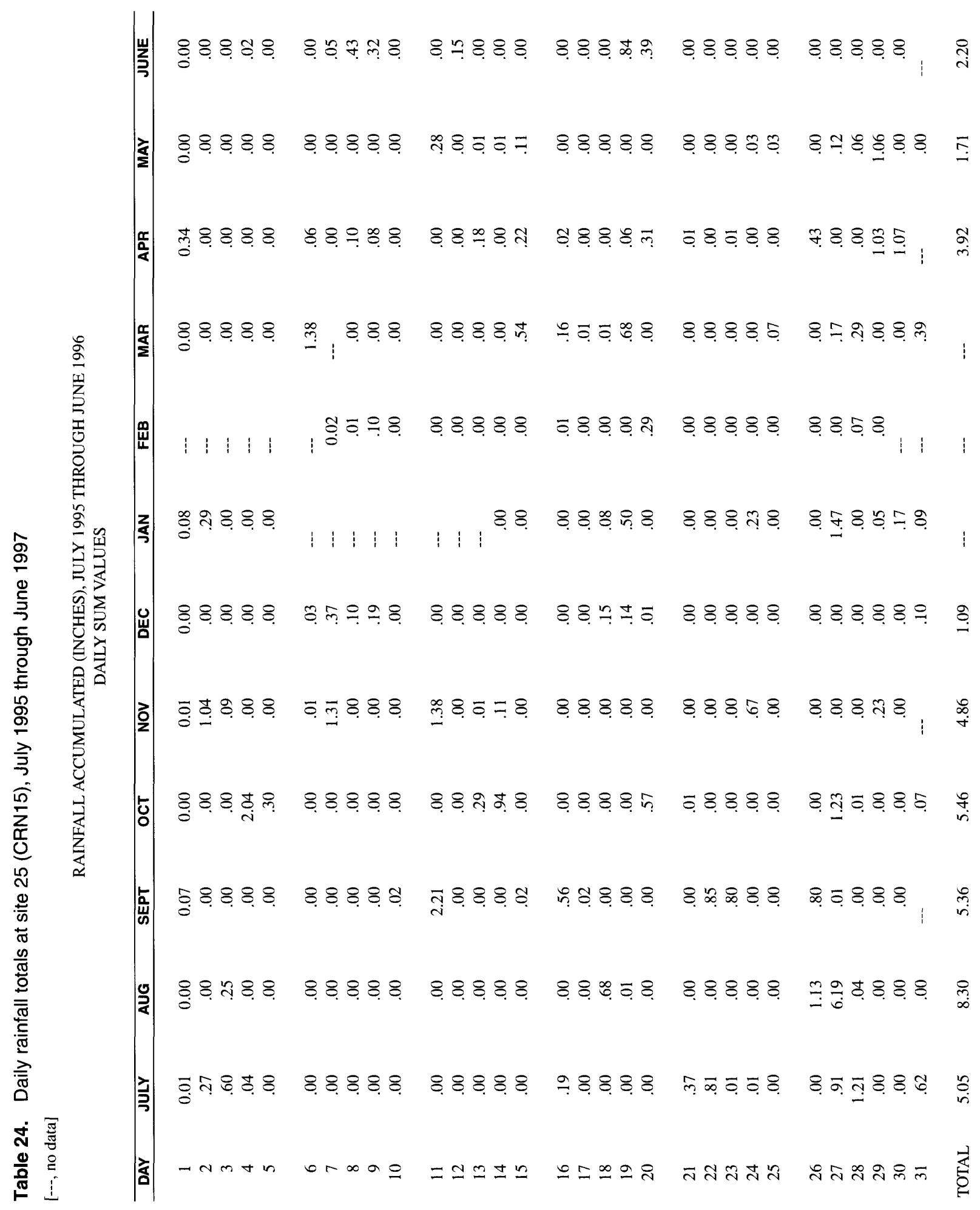




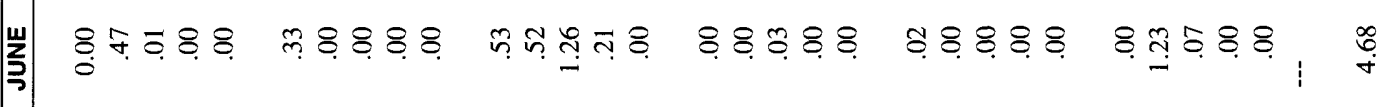

\&8.

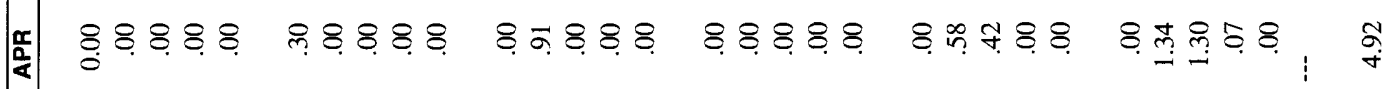

造

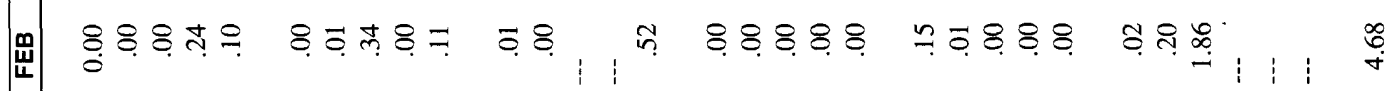

₹

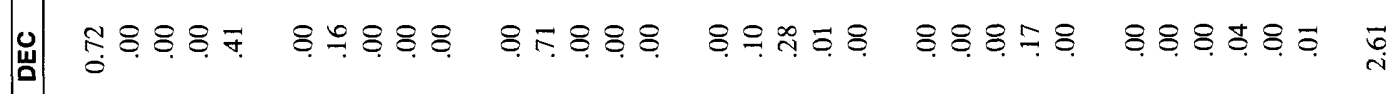

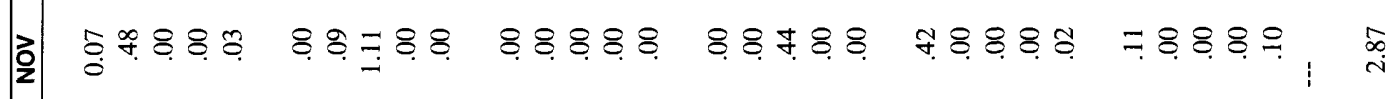

능

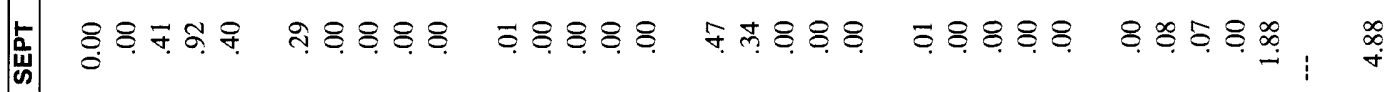

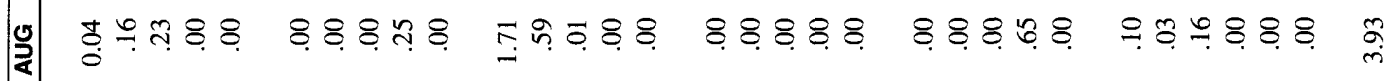

产

䇾

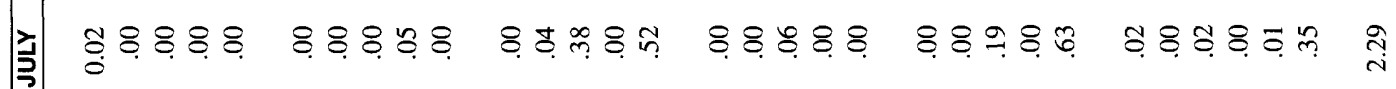

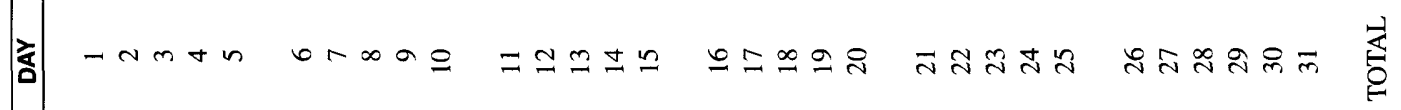




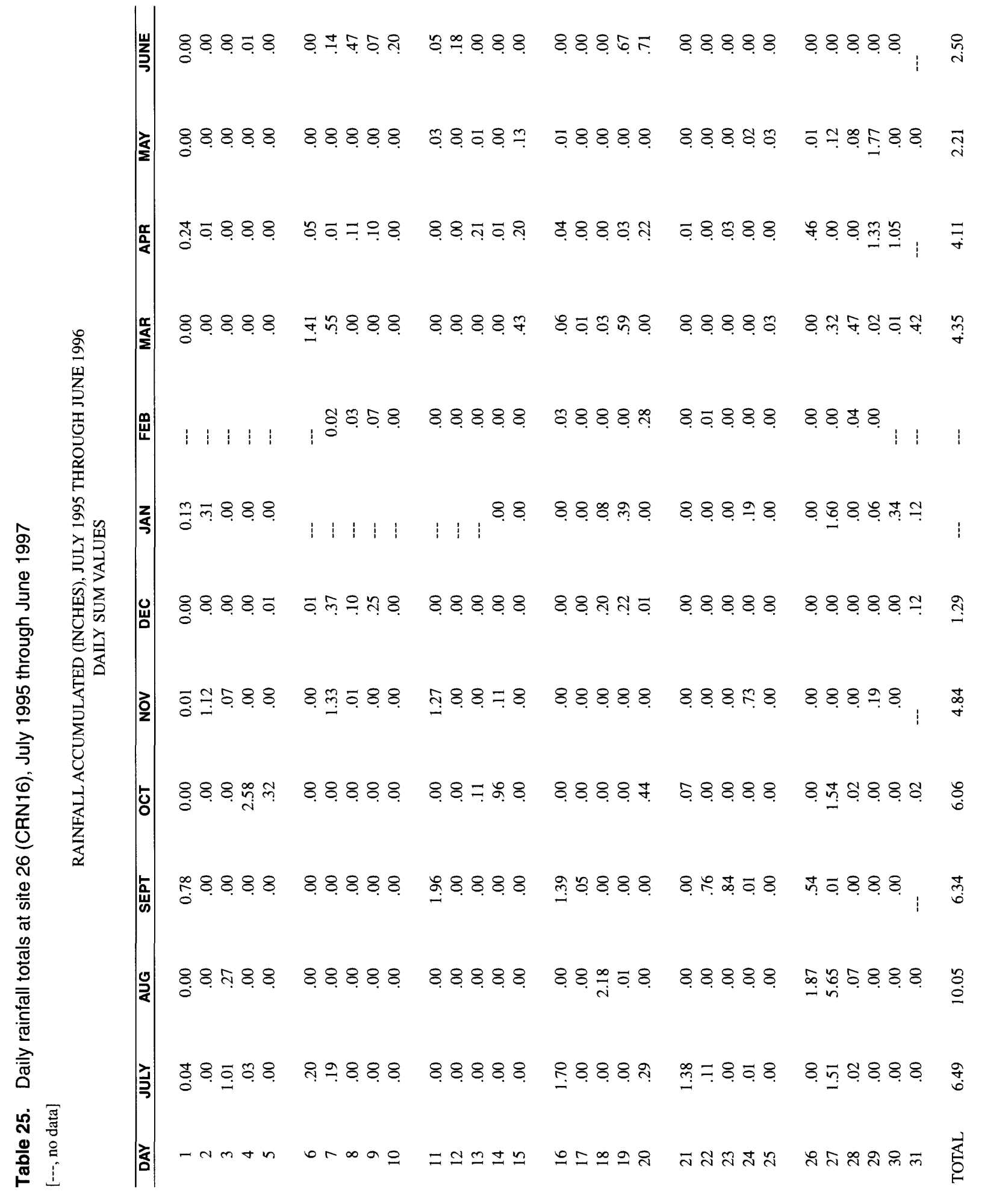




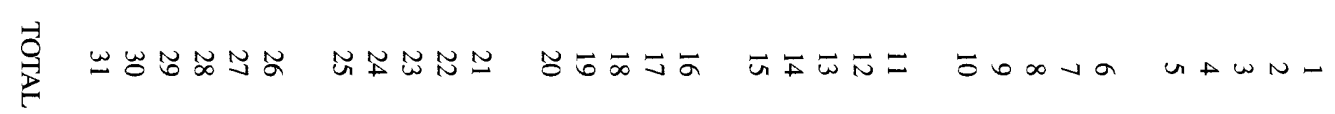
岀 용요 பூ $\underset{\omega}{\infty} \quad$ 88888888888 88요 $88888 \quad$ 岕出 888 㟒 苫 花 8ப் 苟 苛 芯 Е

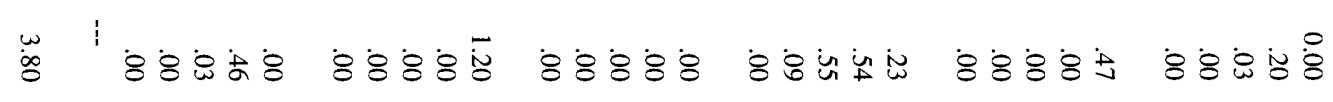




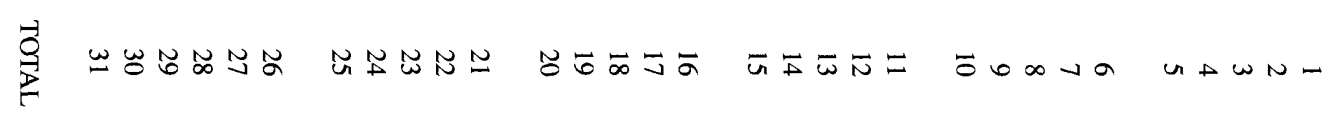
商 888888 88

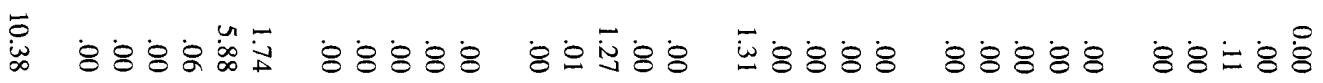
Ш

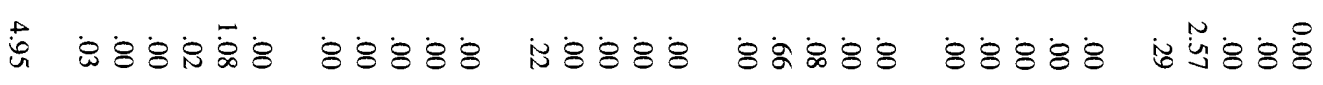
行 活

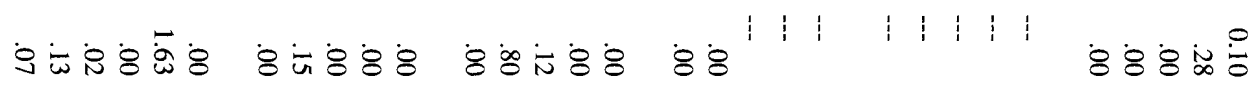
8

莽

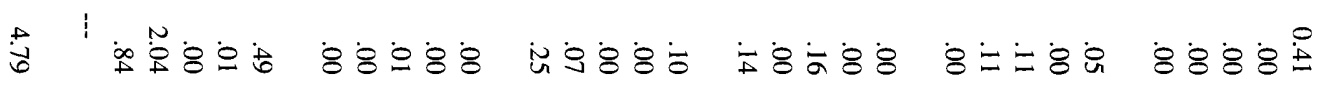
\&

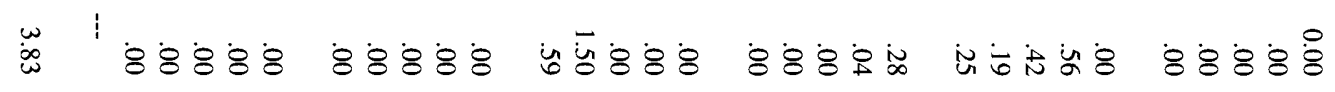




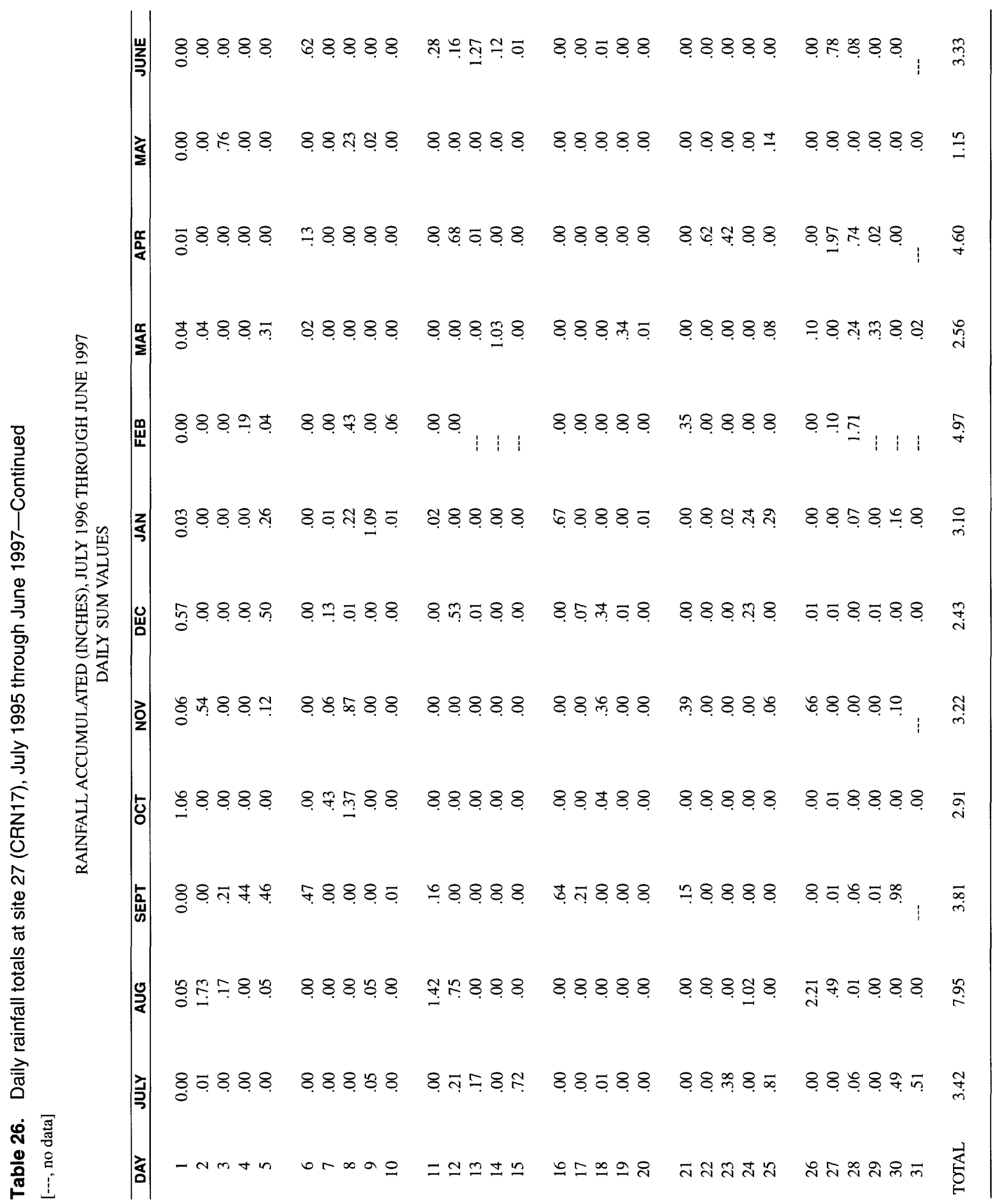




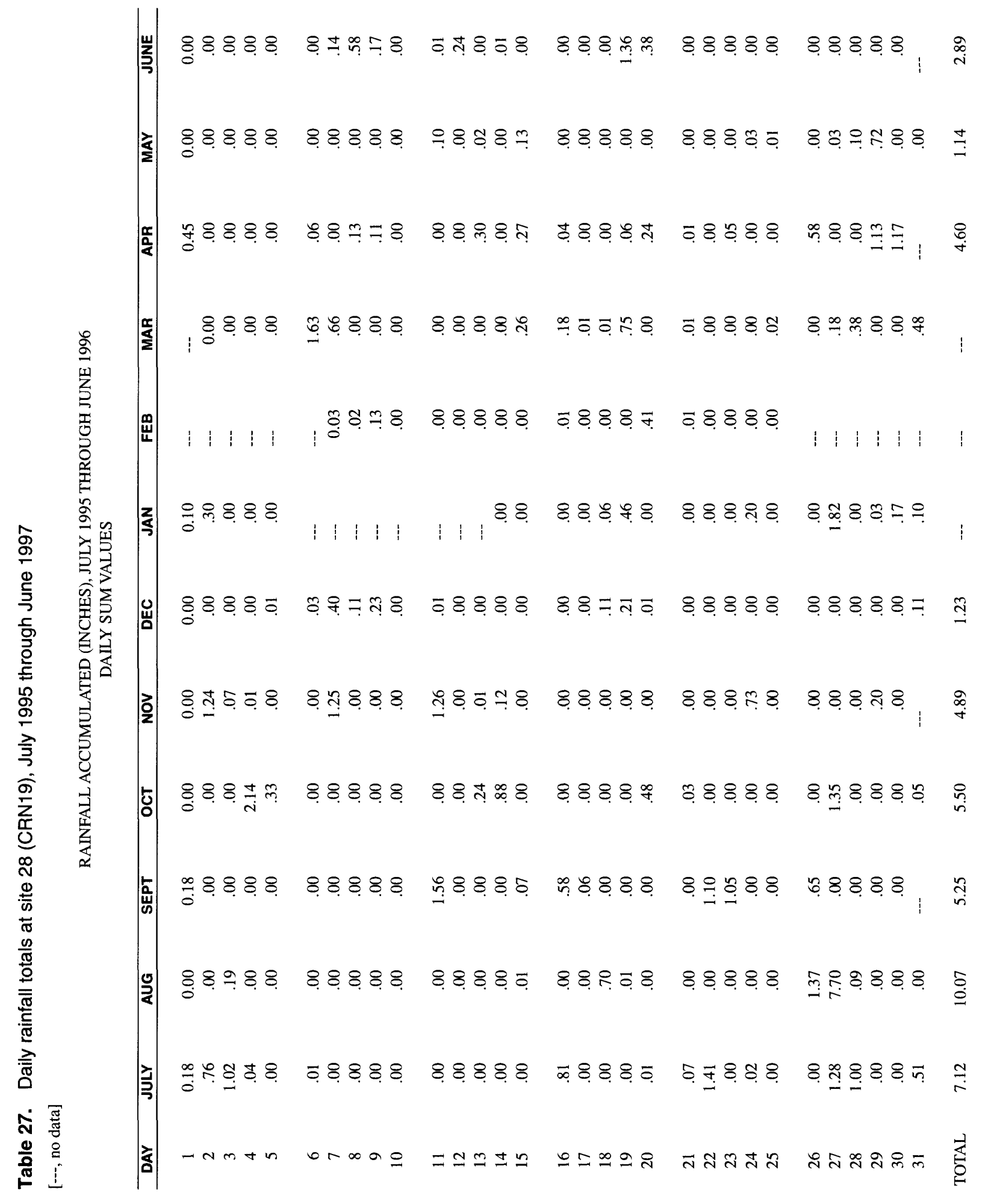


岁 8 :

888.4988 .88 .88

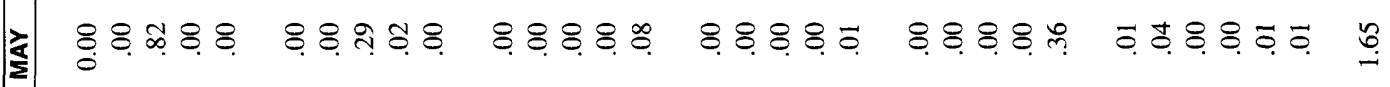

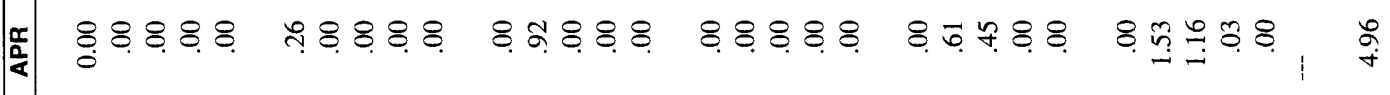

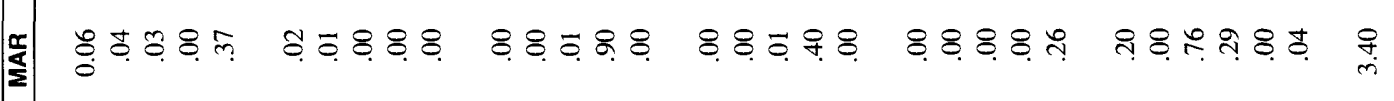

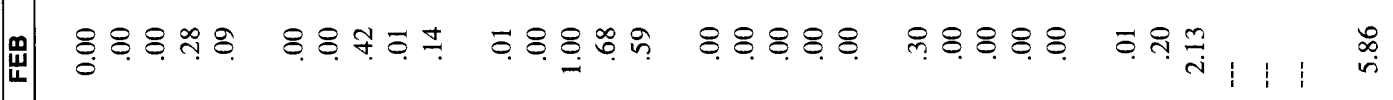

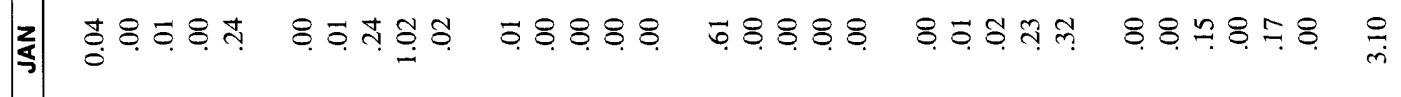

แั

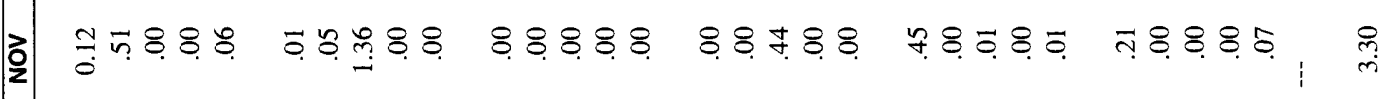

|

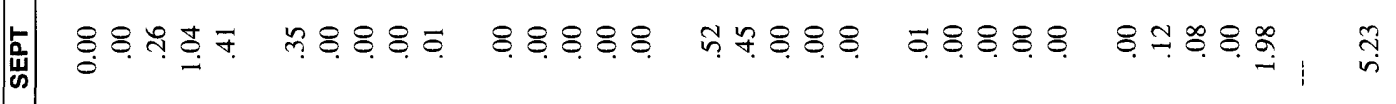

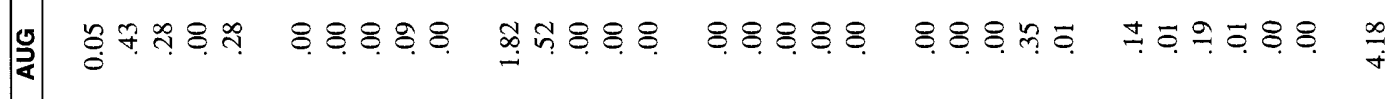

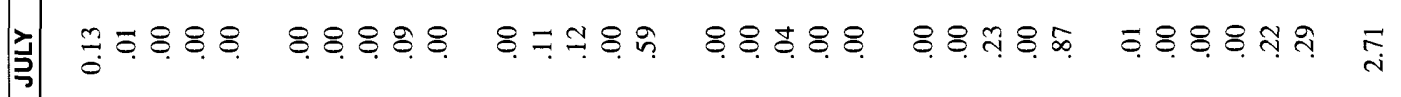

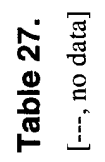

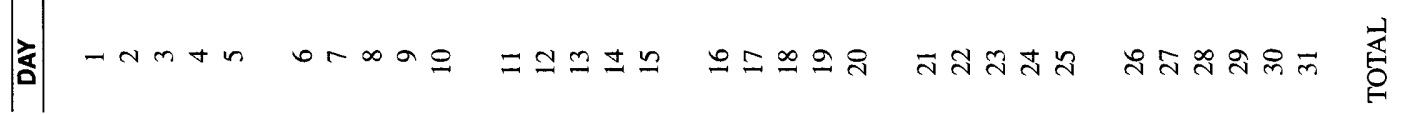




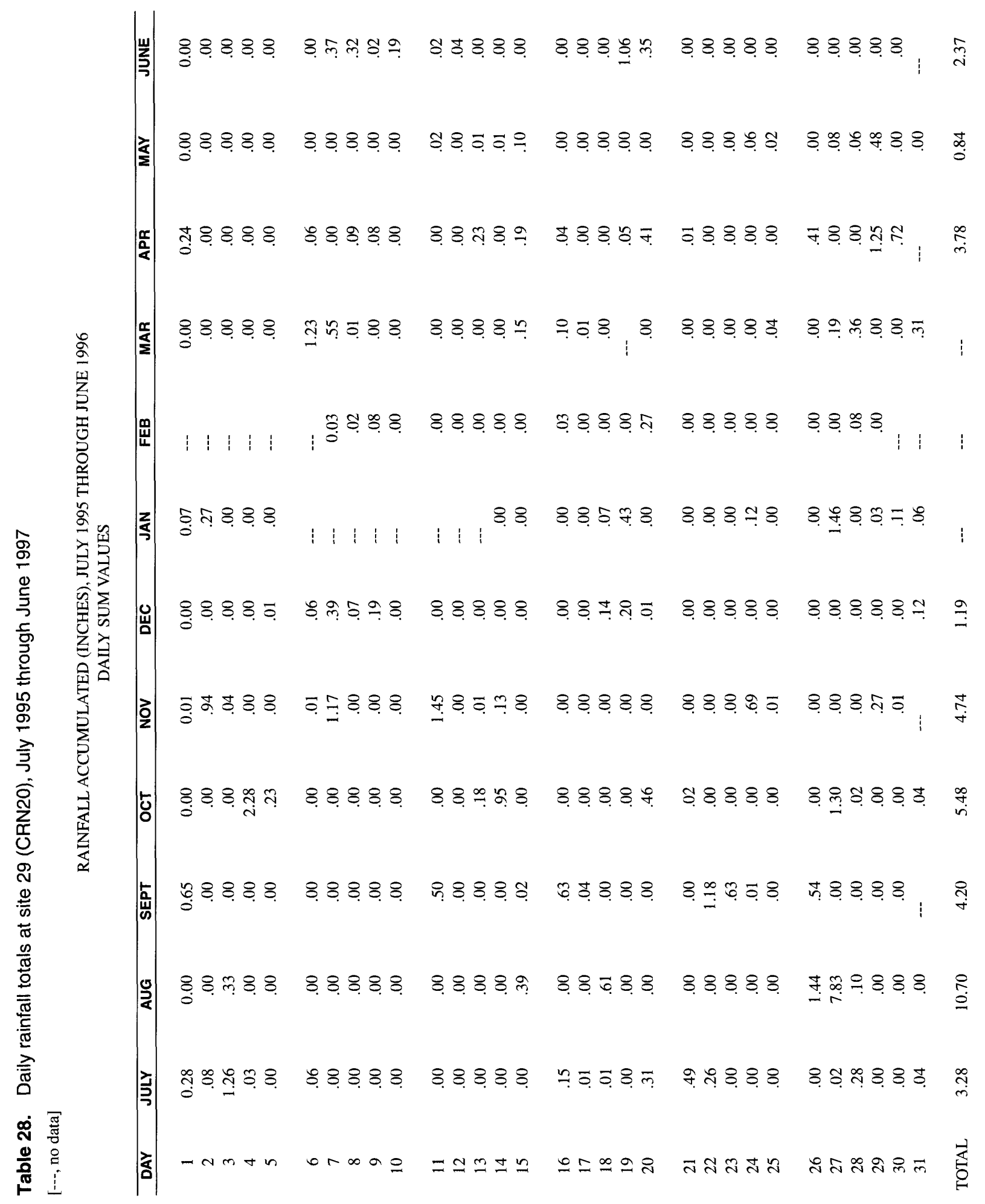




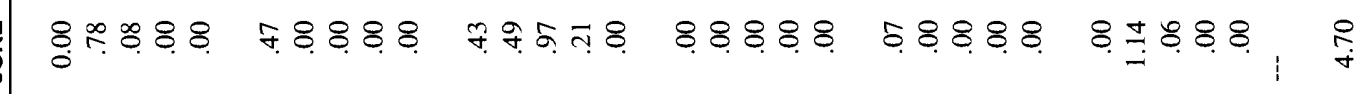

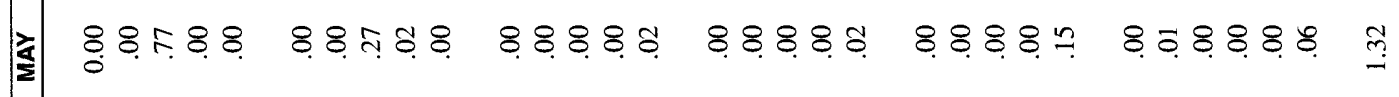

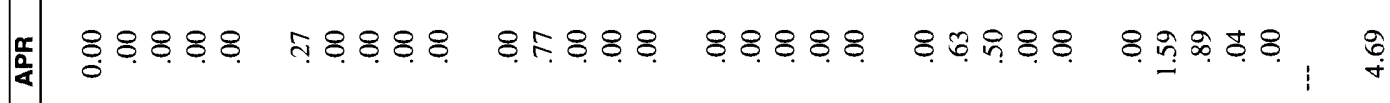

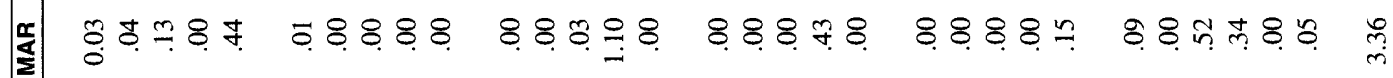

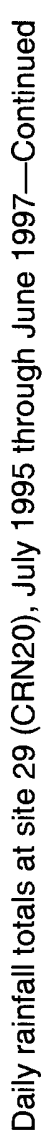

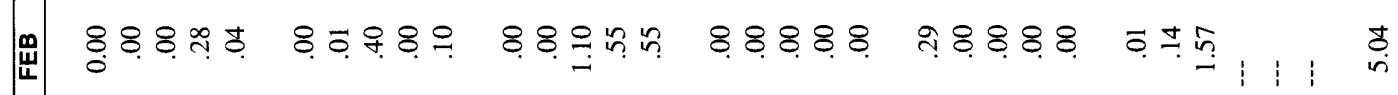

Ұ

뱅

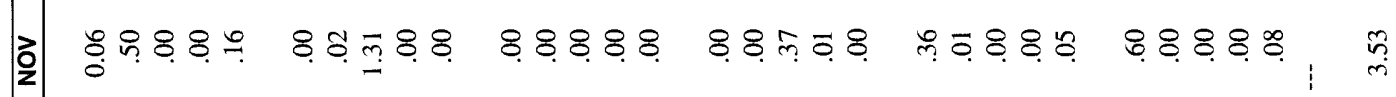

형

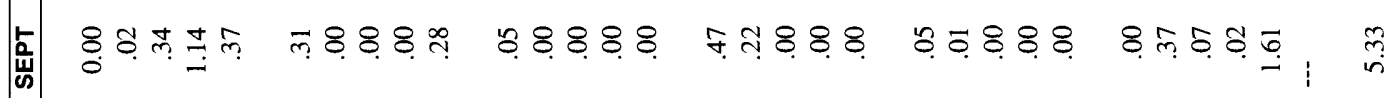

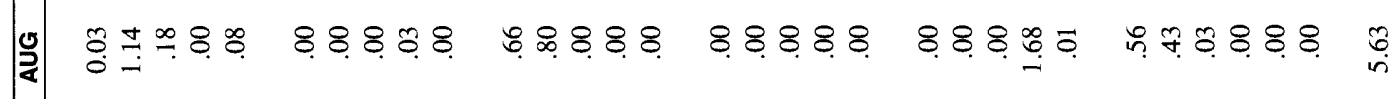

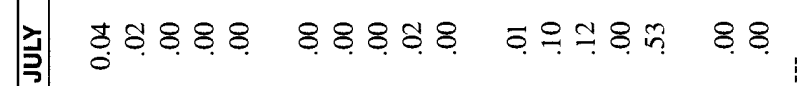

8. प. 8. ळ. ले

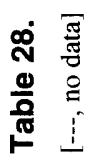

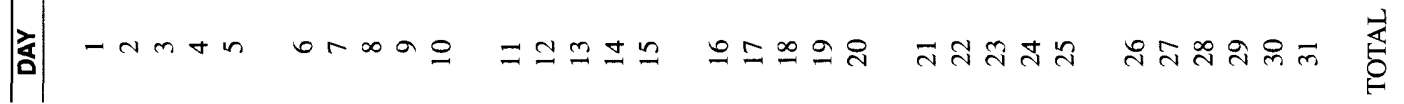




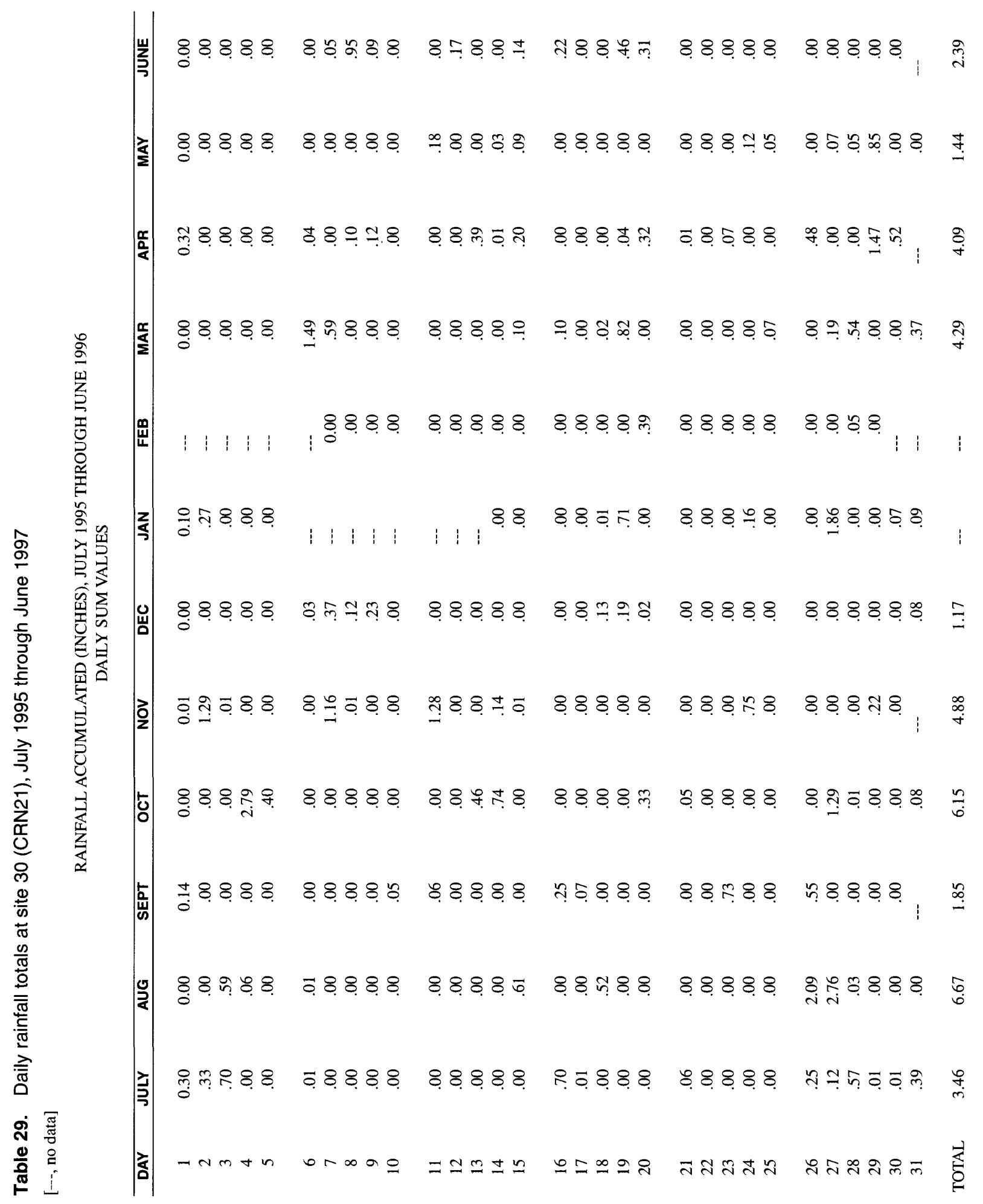




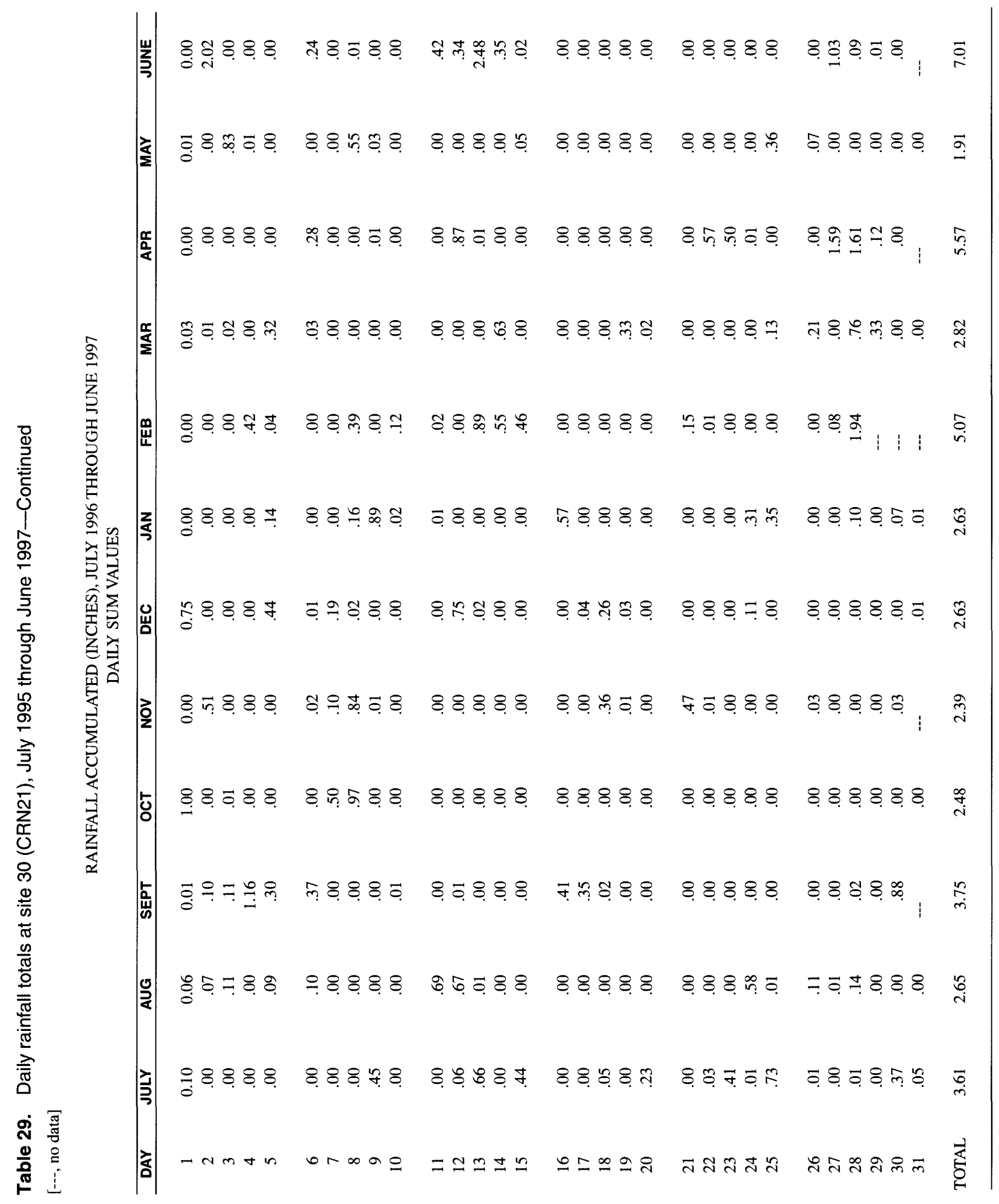




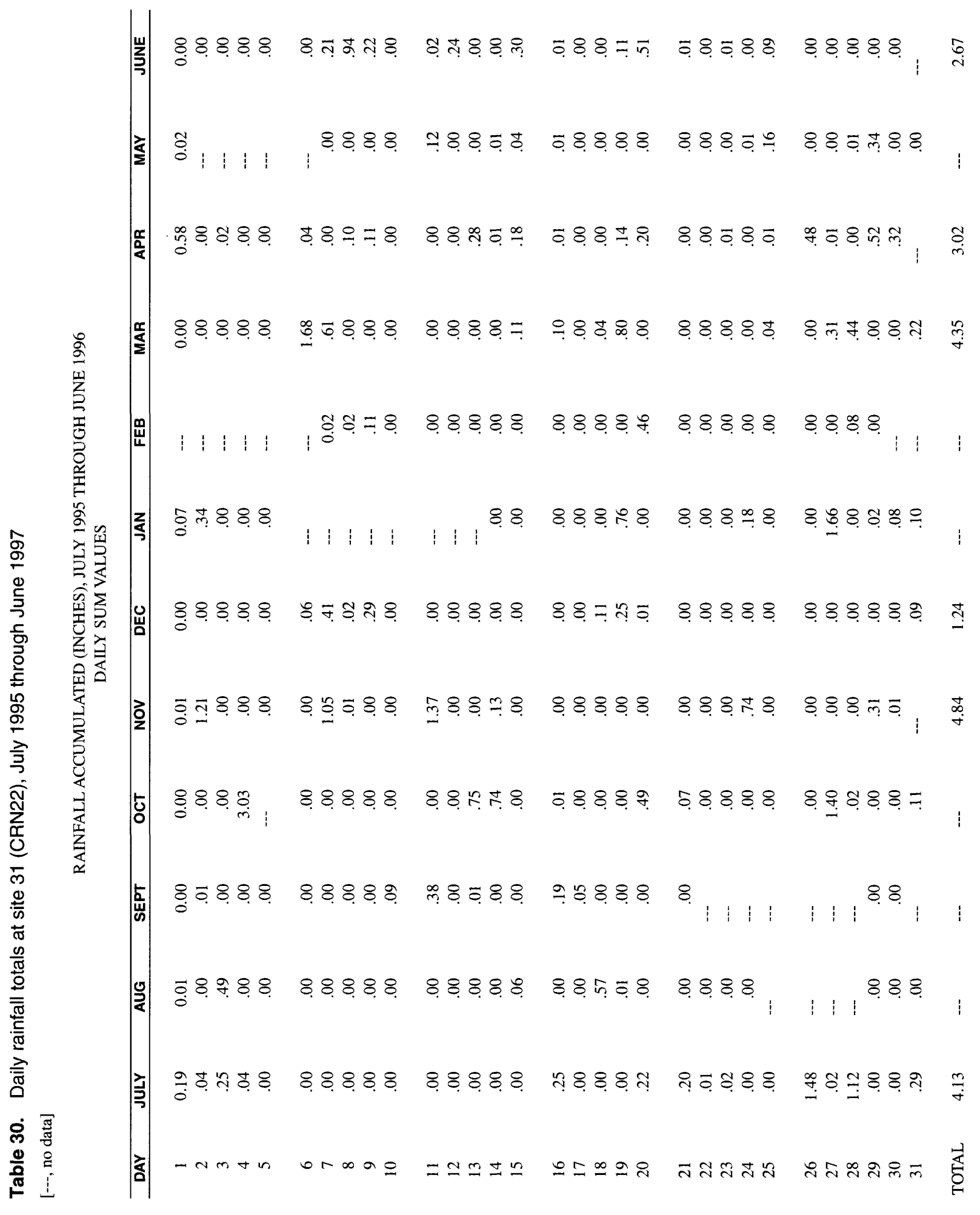




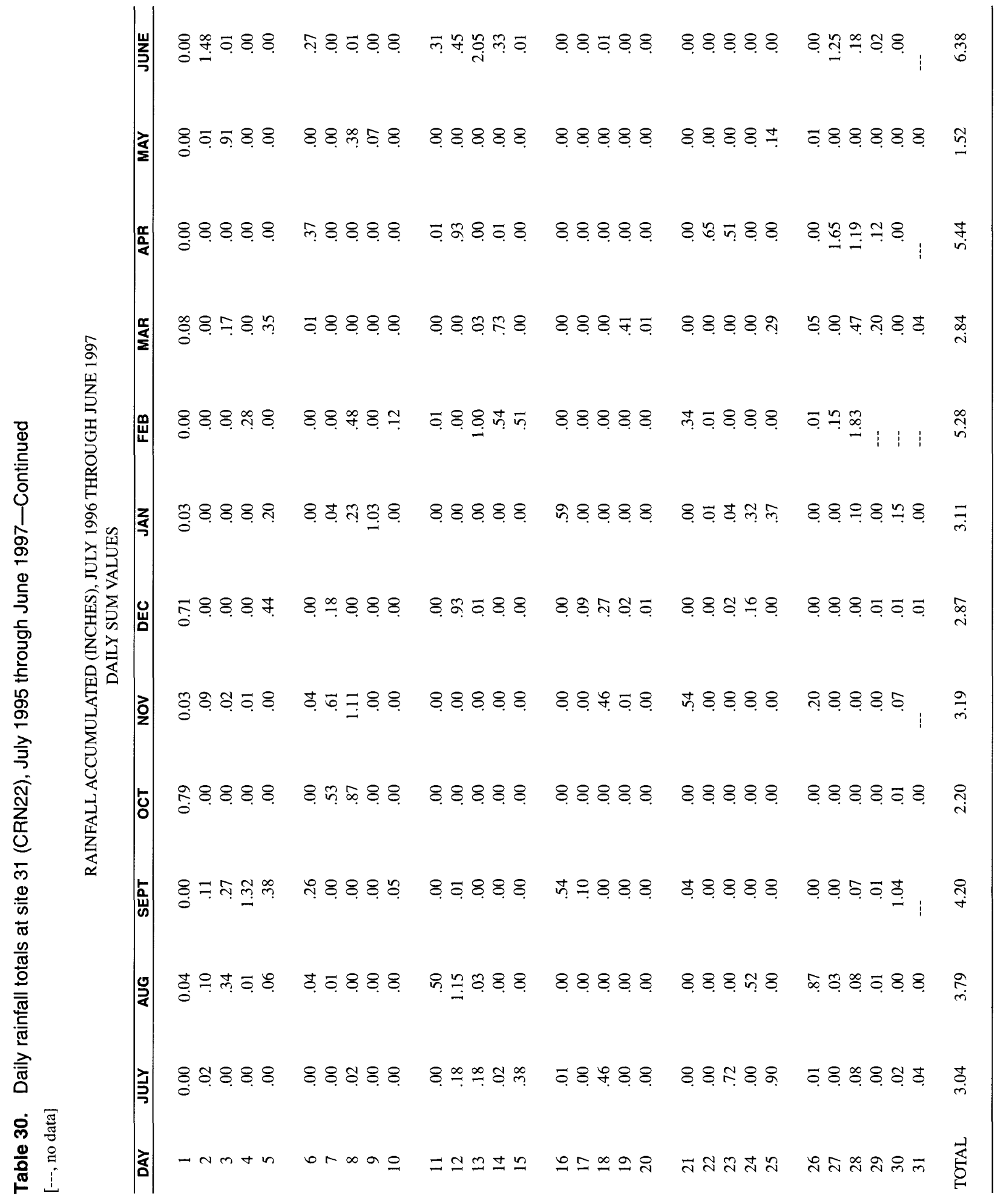




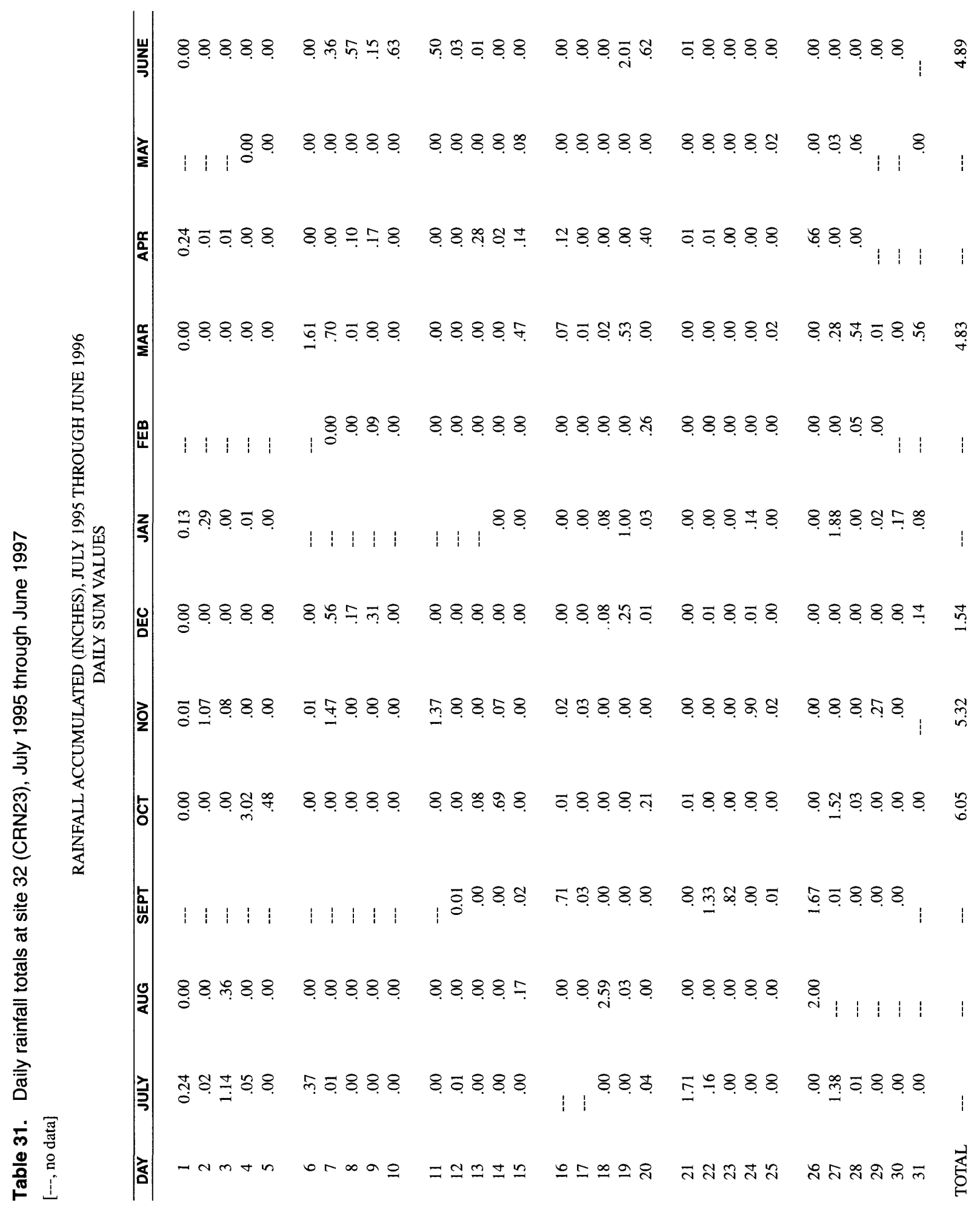




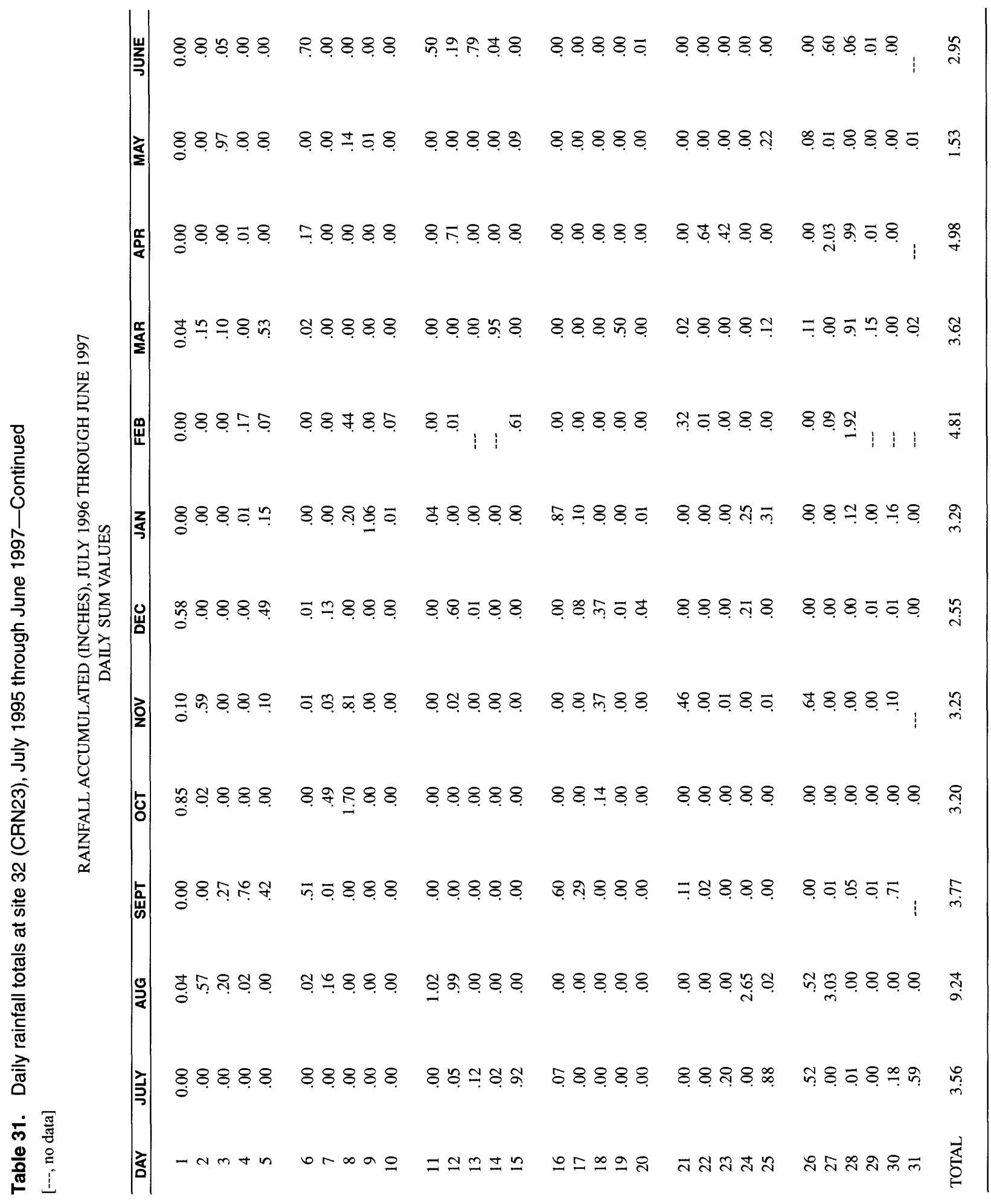




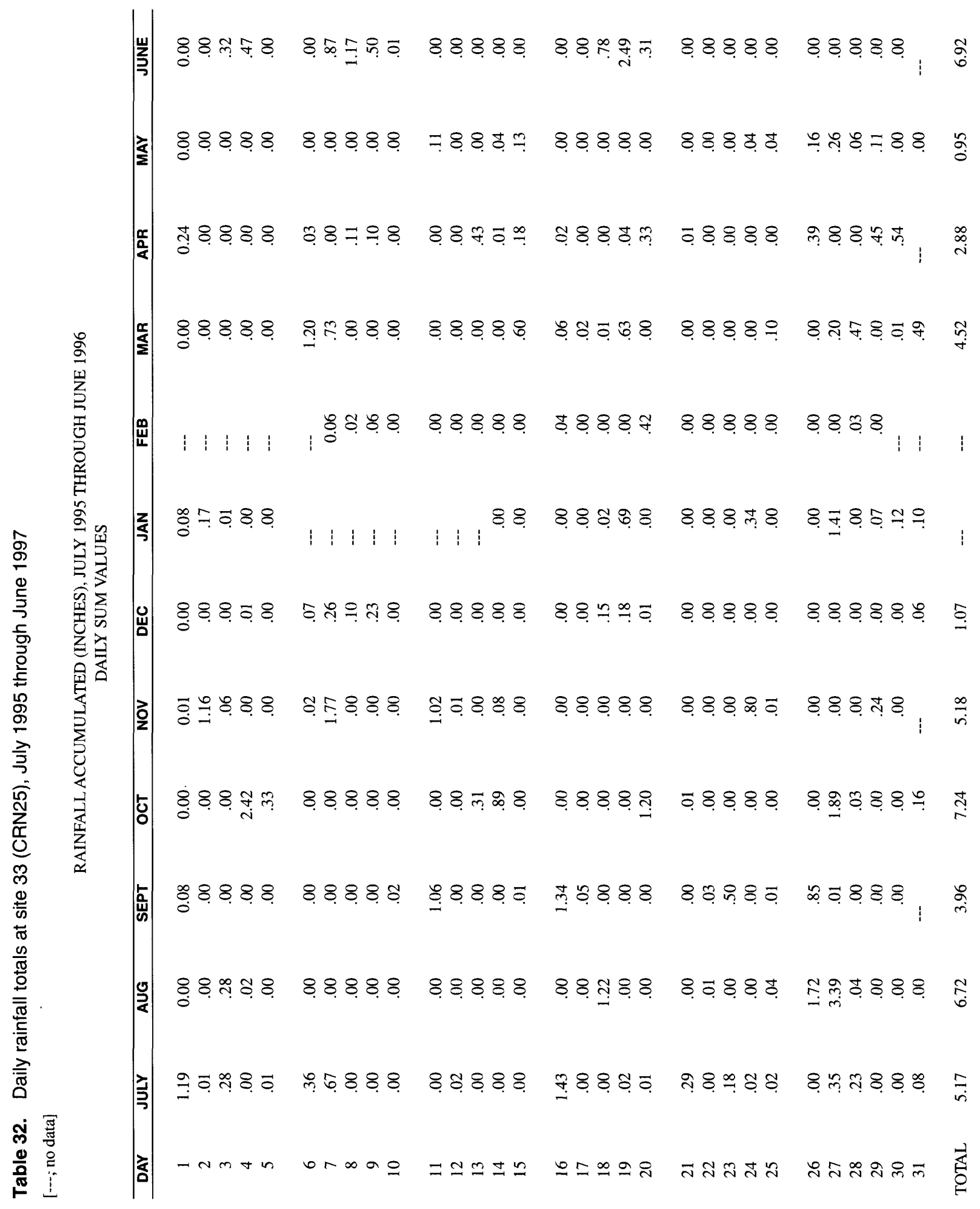


س

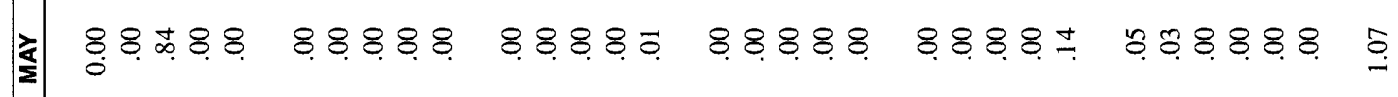

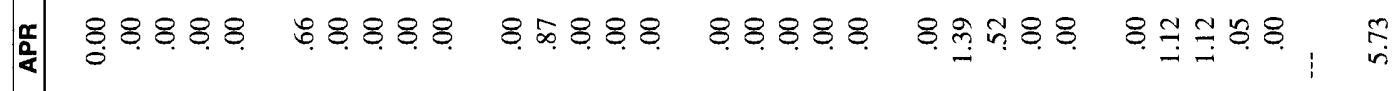

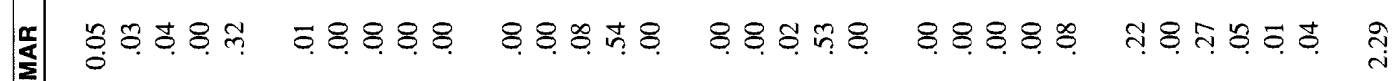

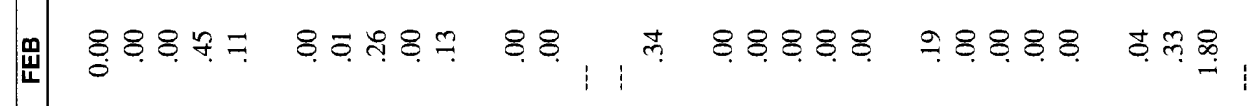

z

냄

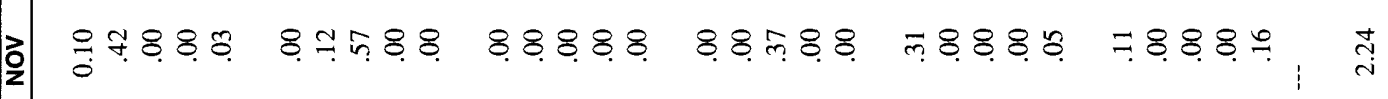

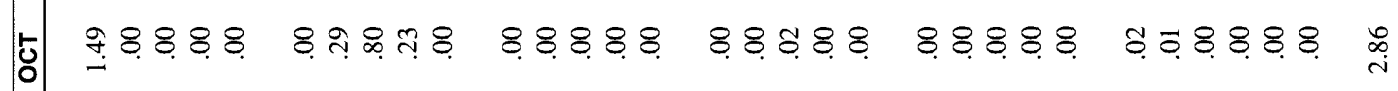

点 8.

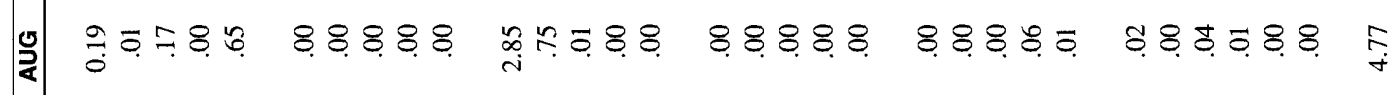

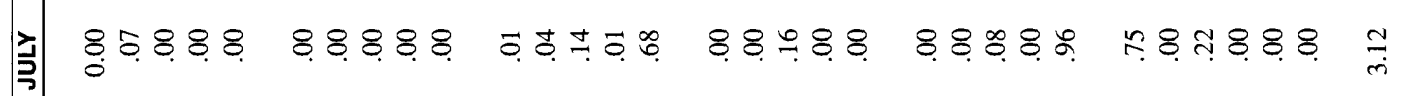

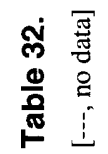

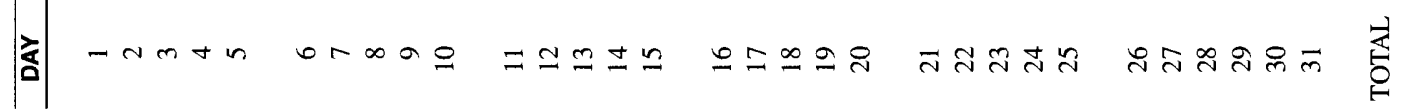




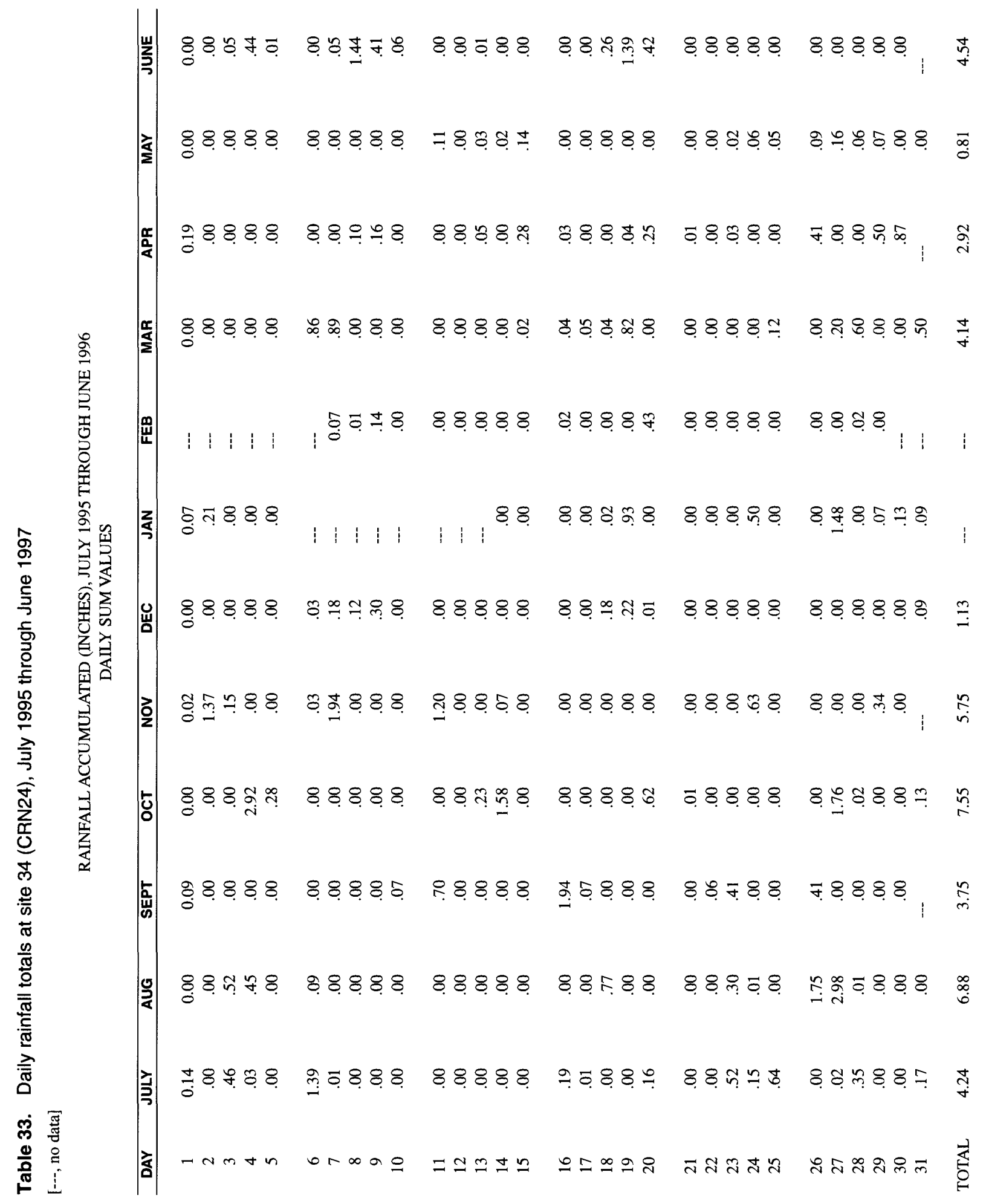




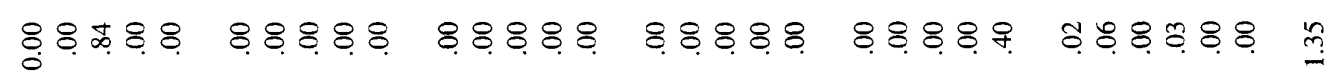

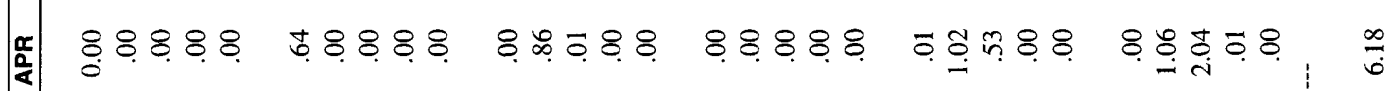

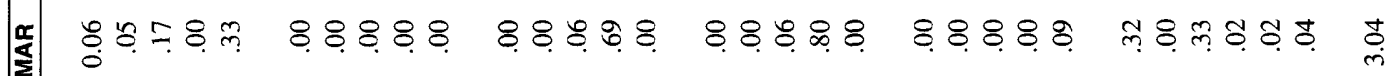

还

ऊ ०.8.8\%

=

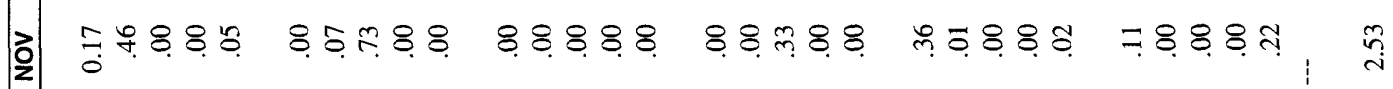

ป

占

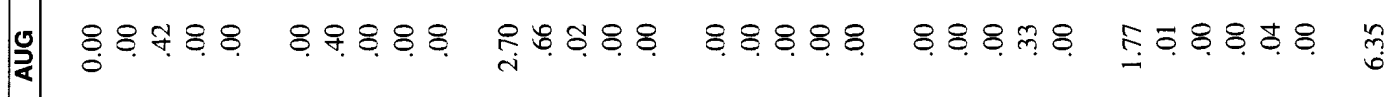

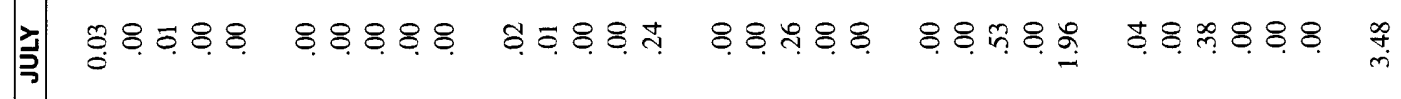

| - 


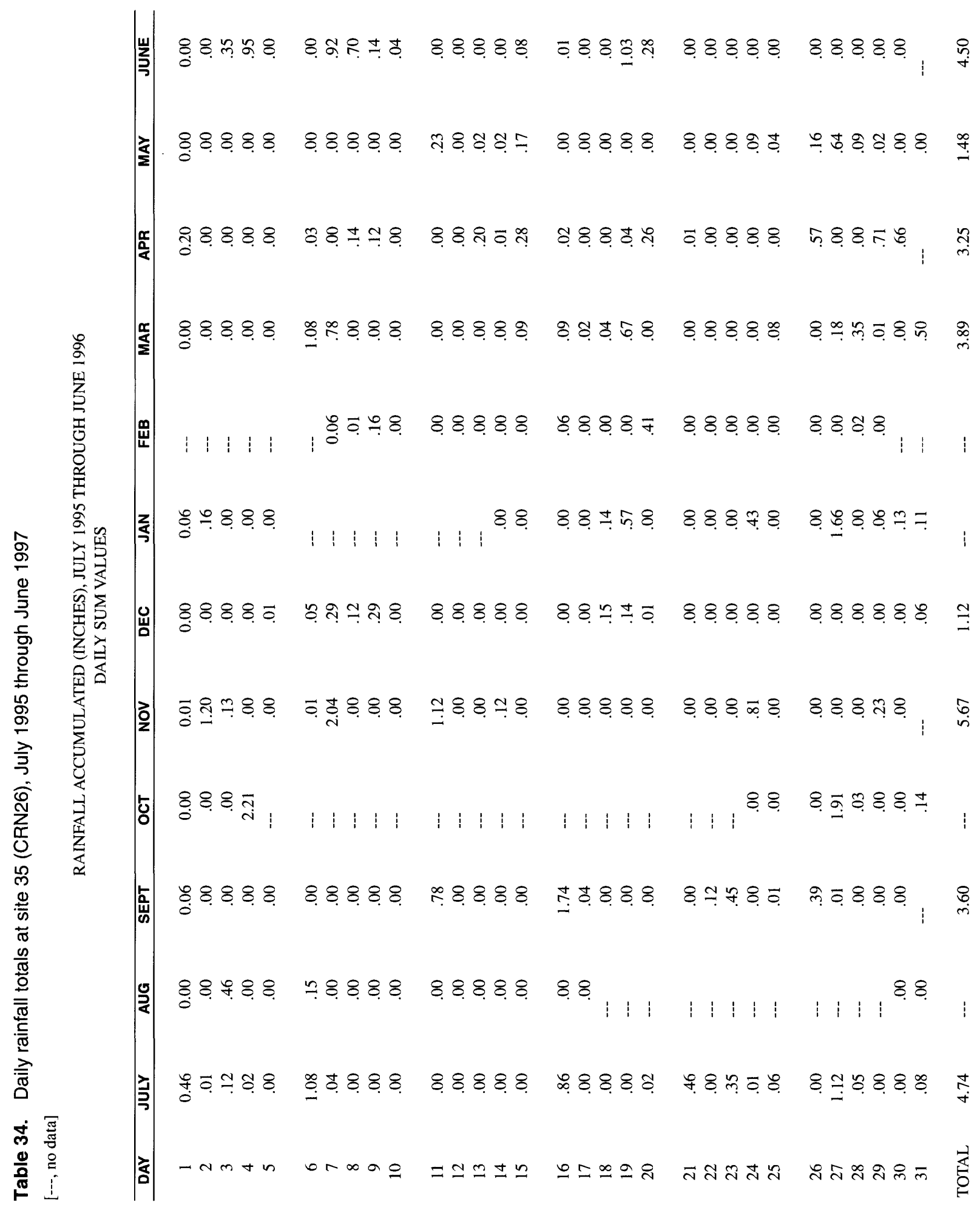




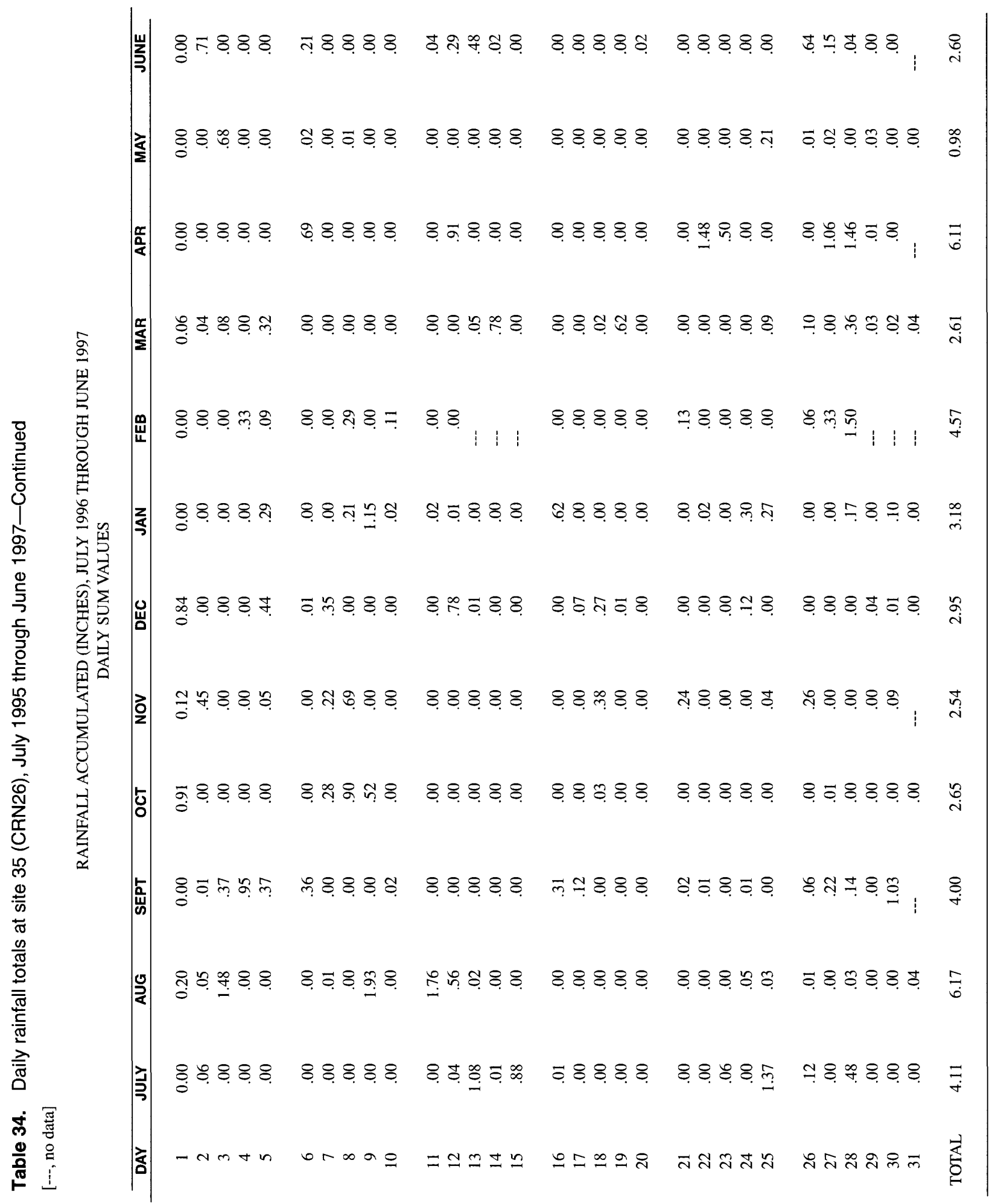




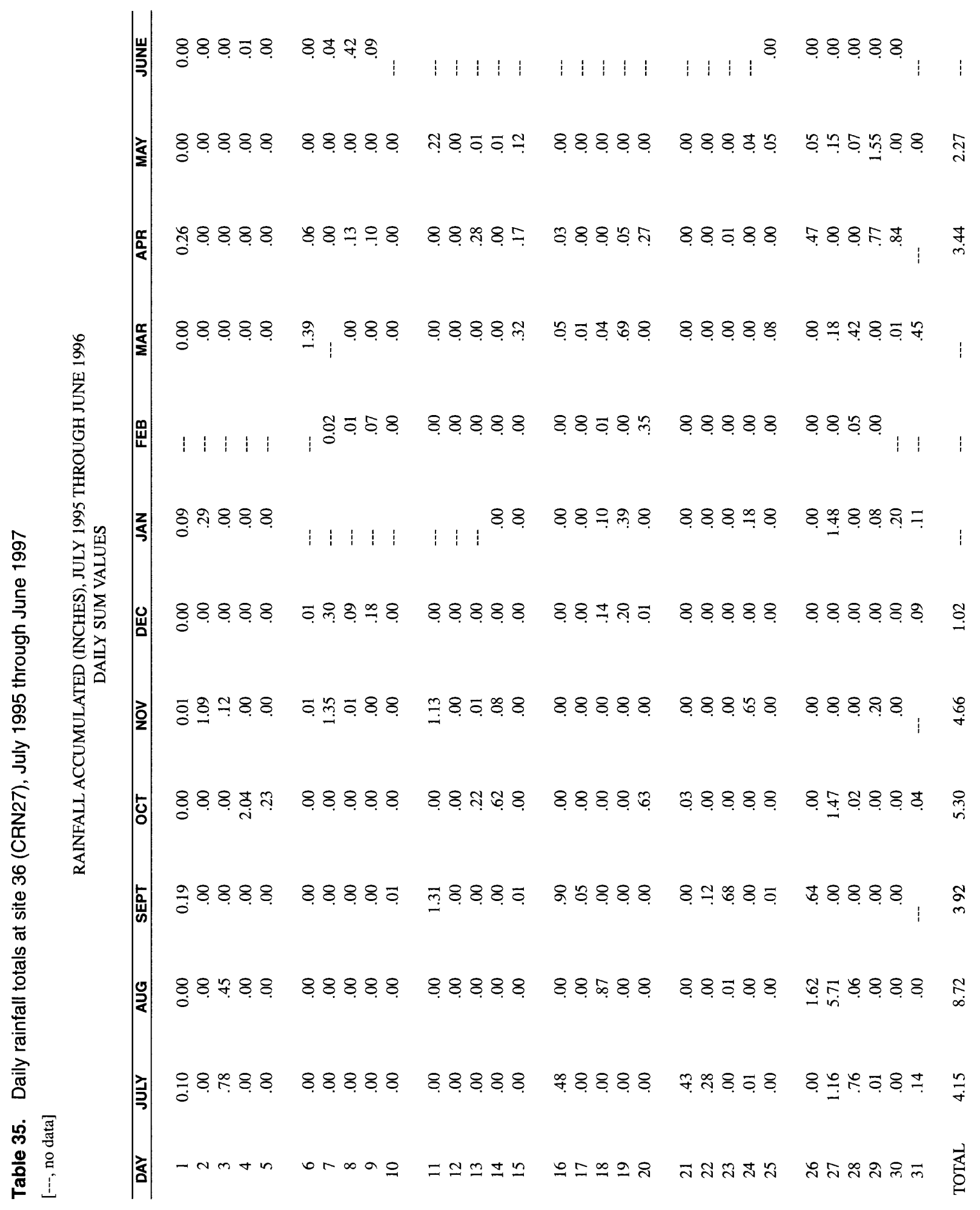




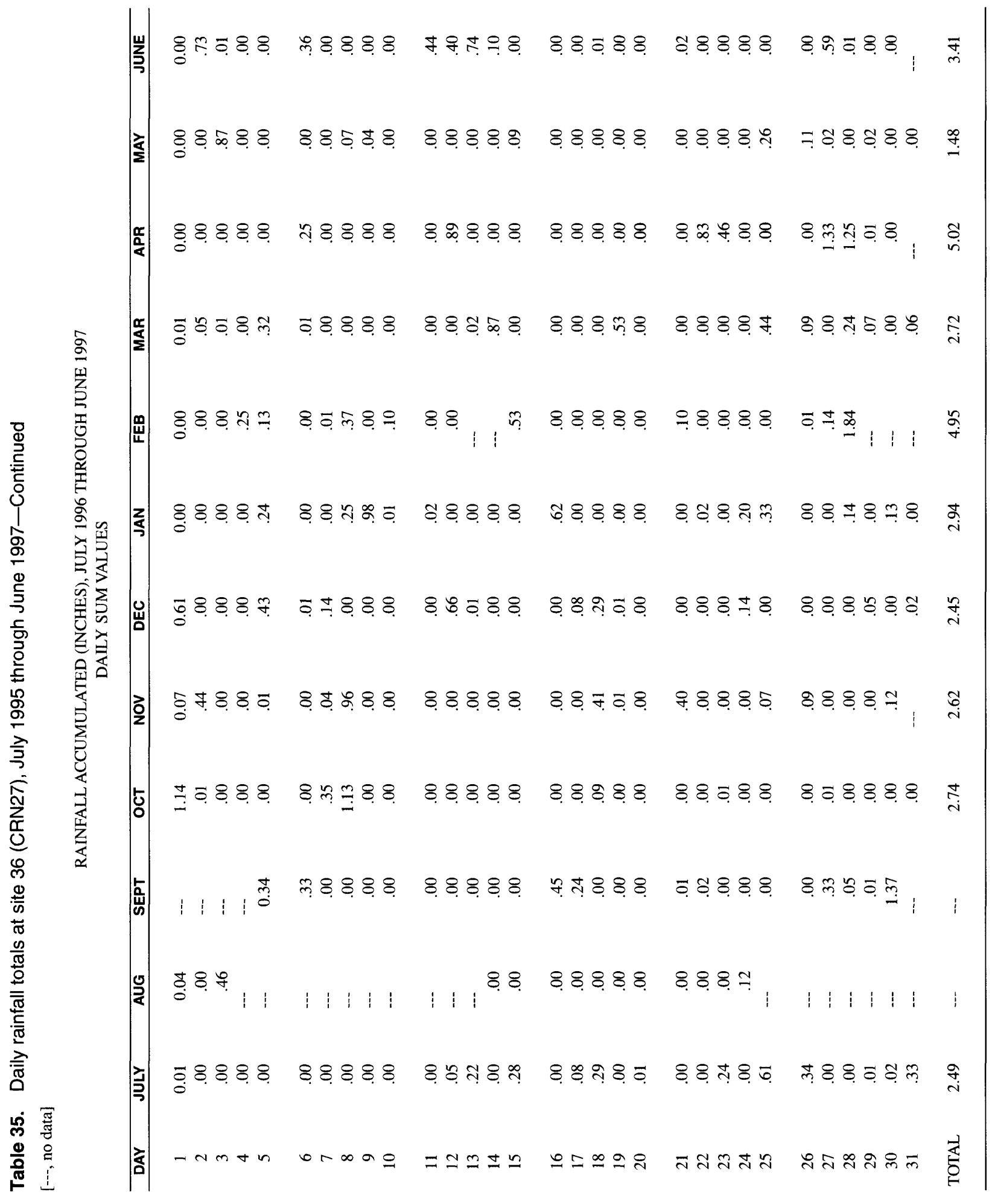




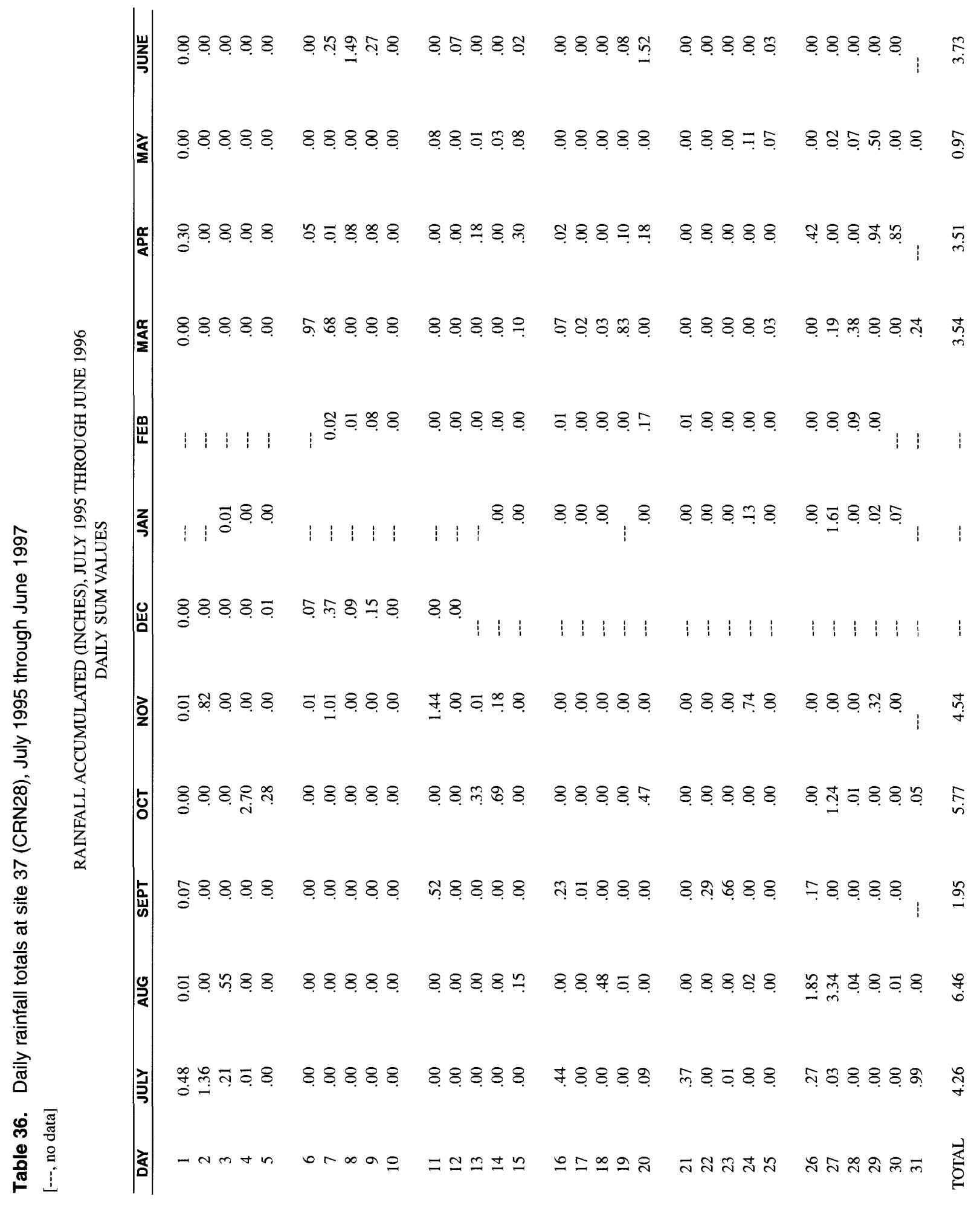




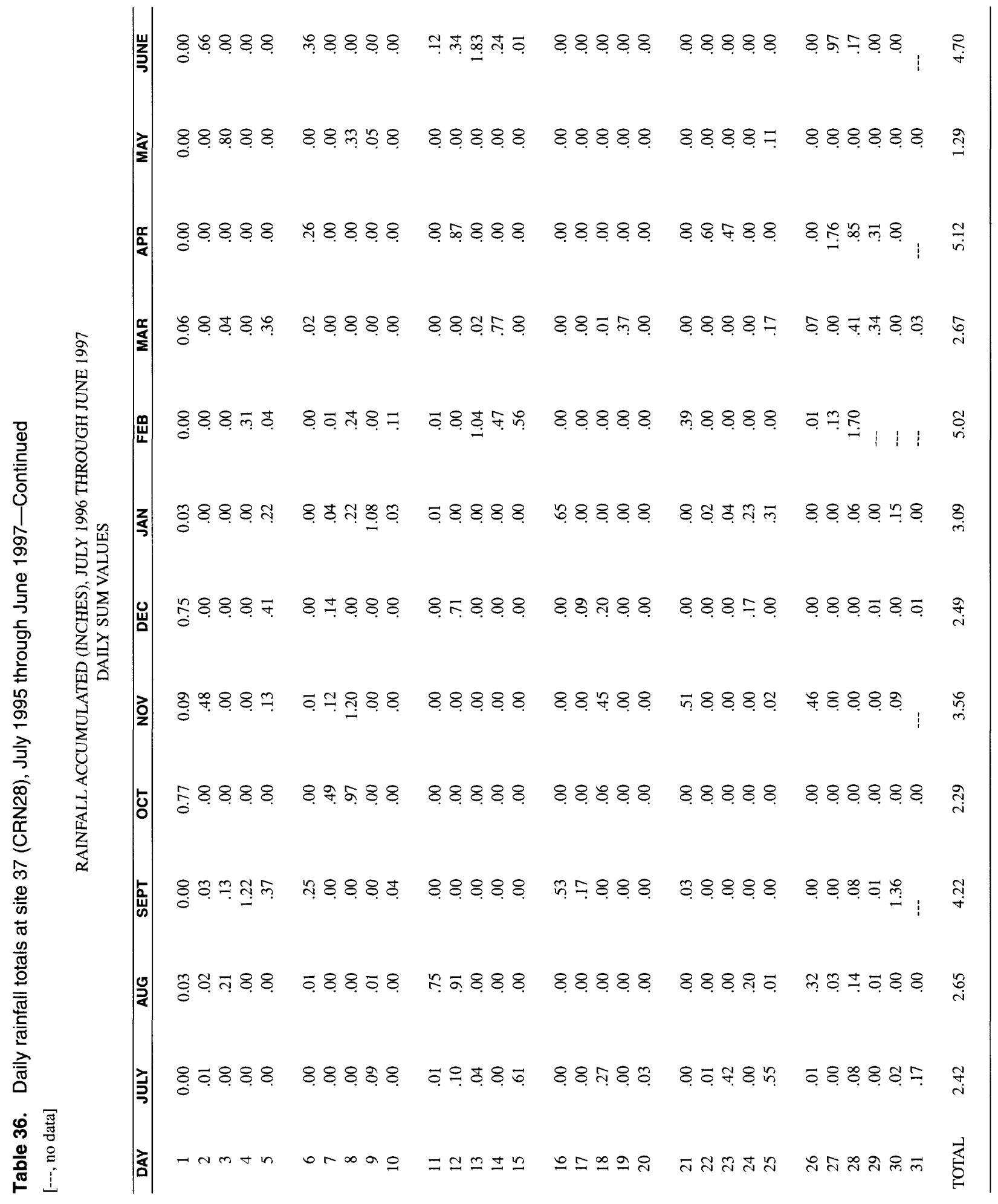




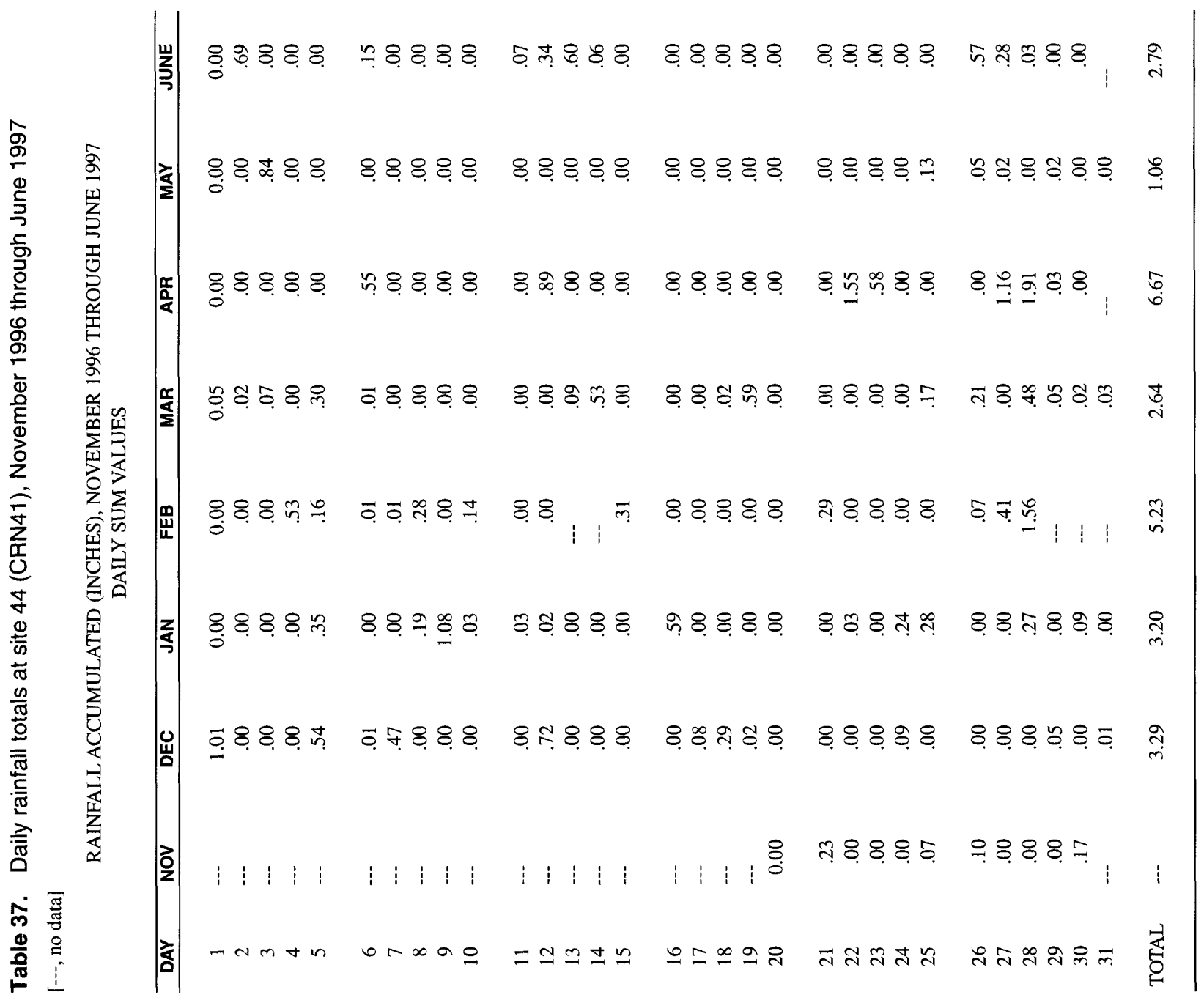




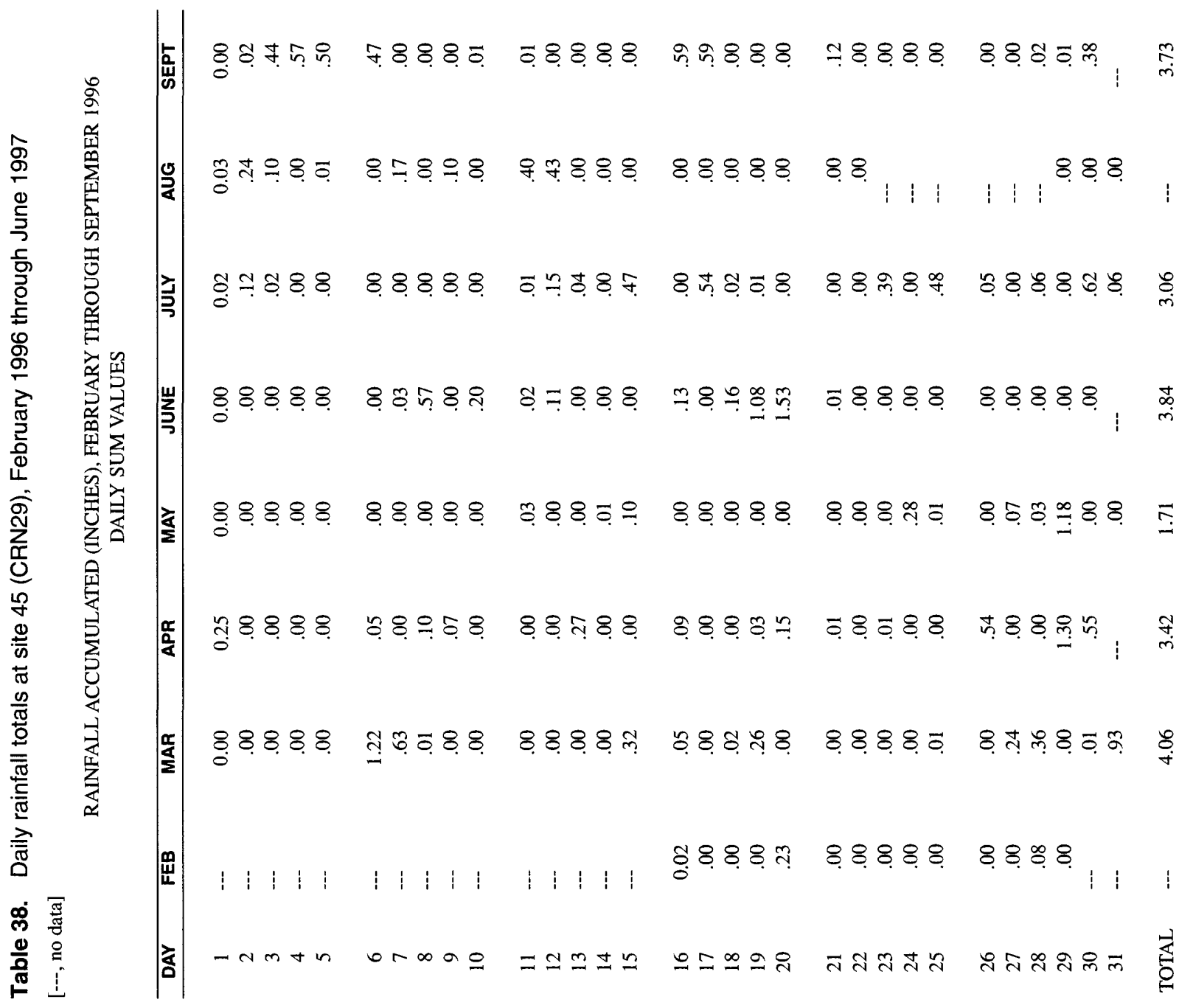




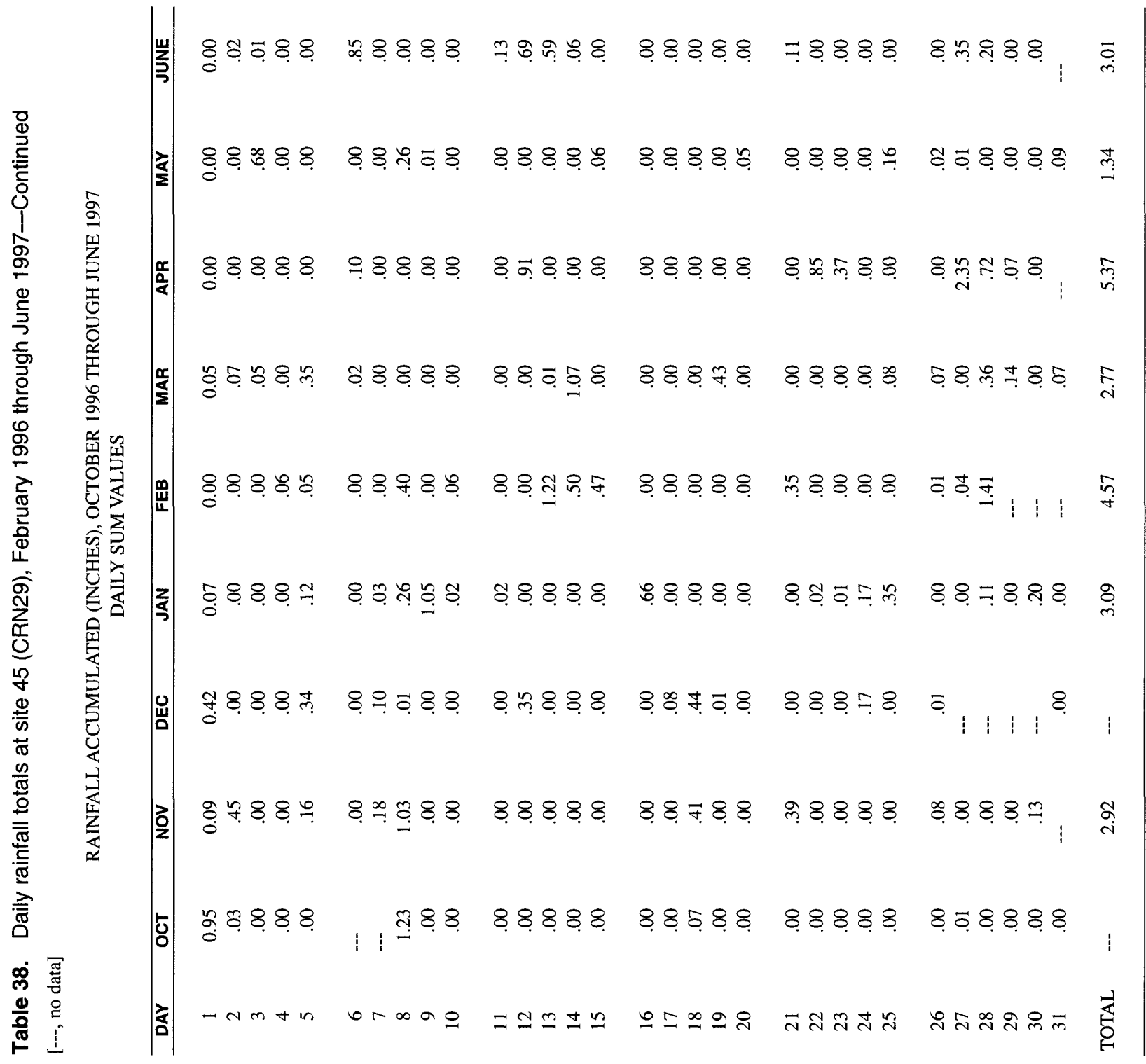




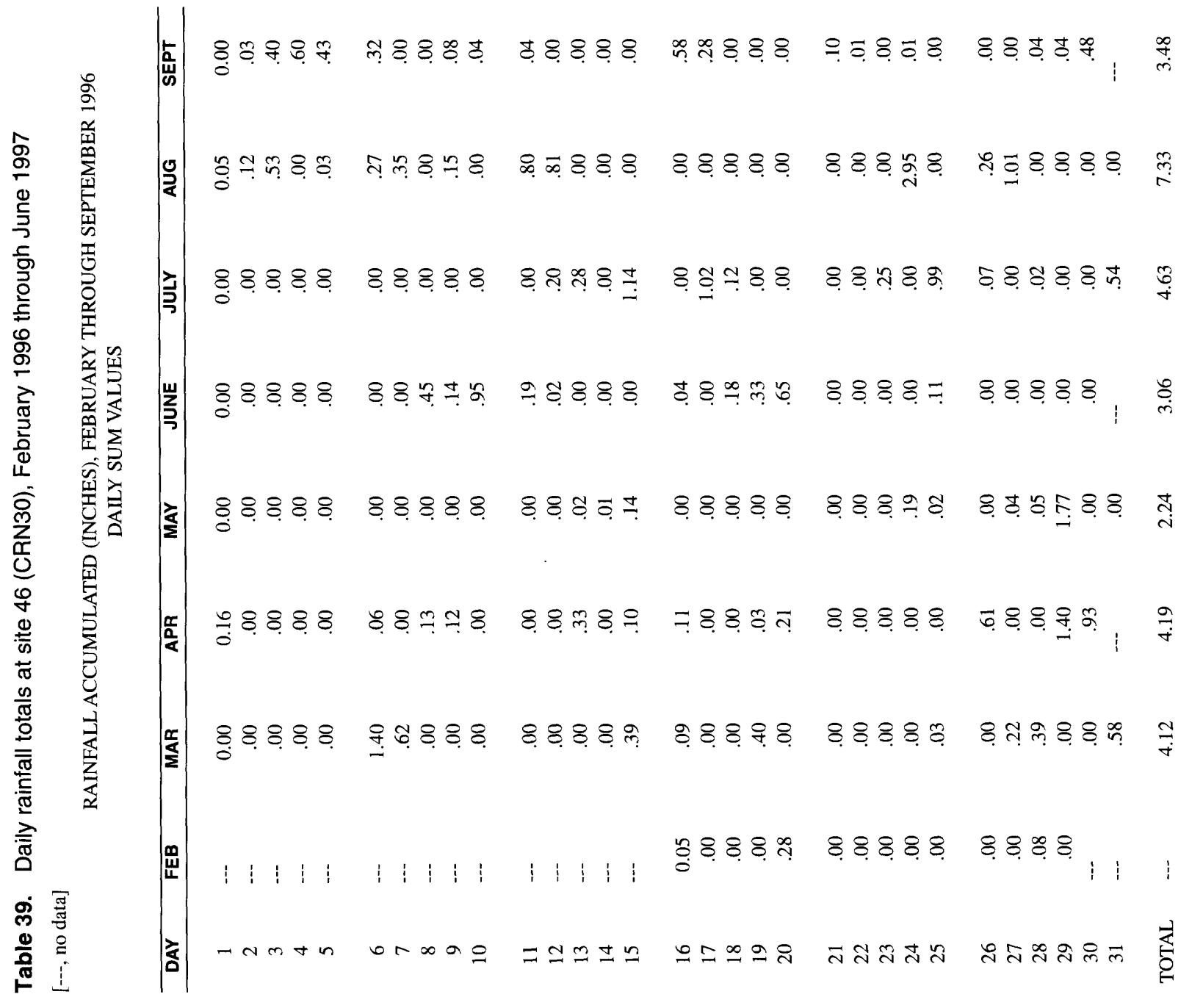




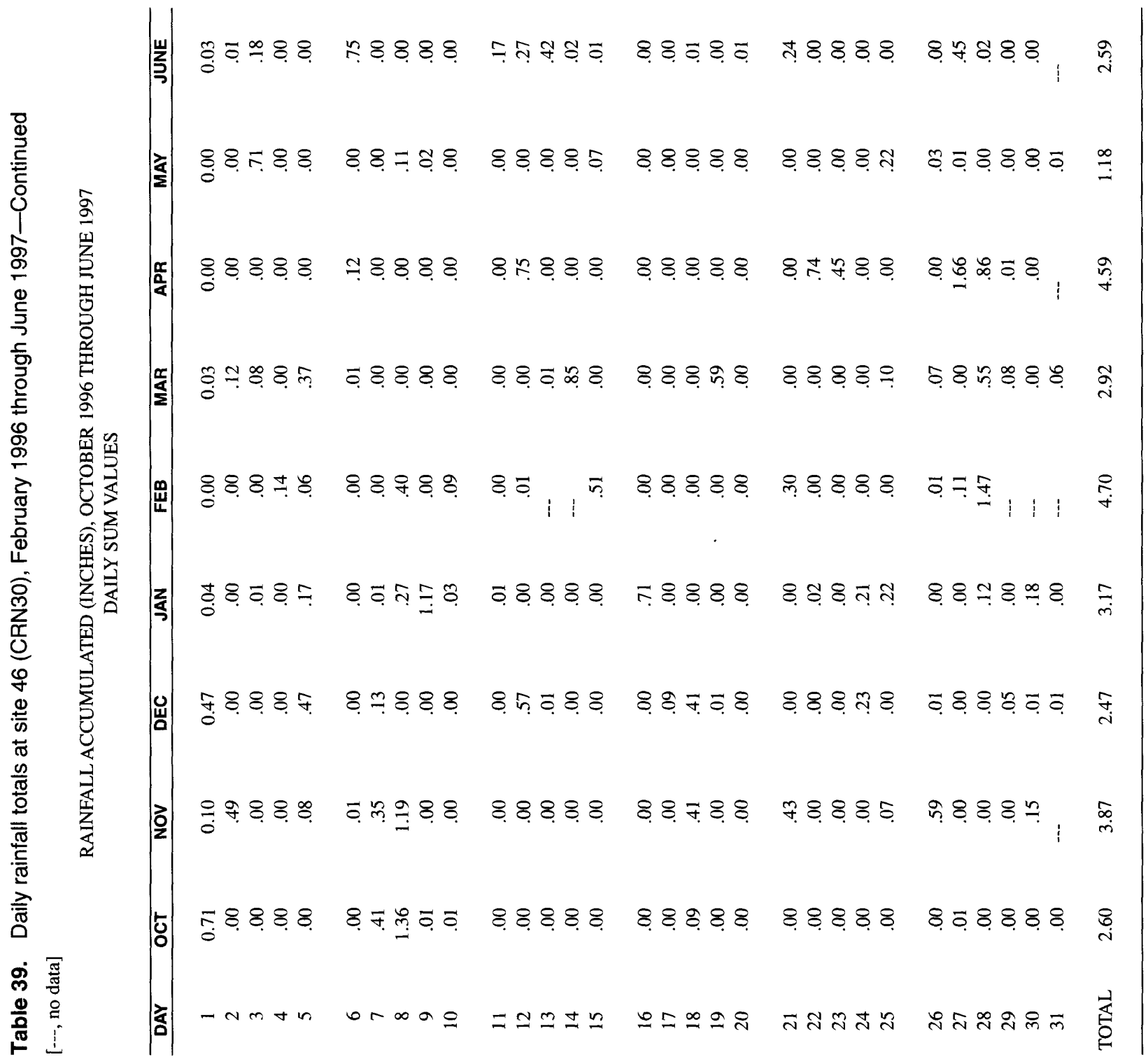




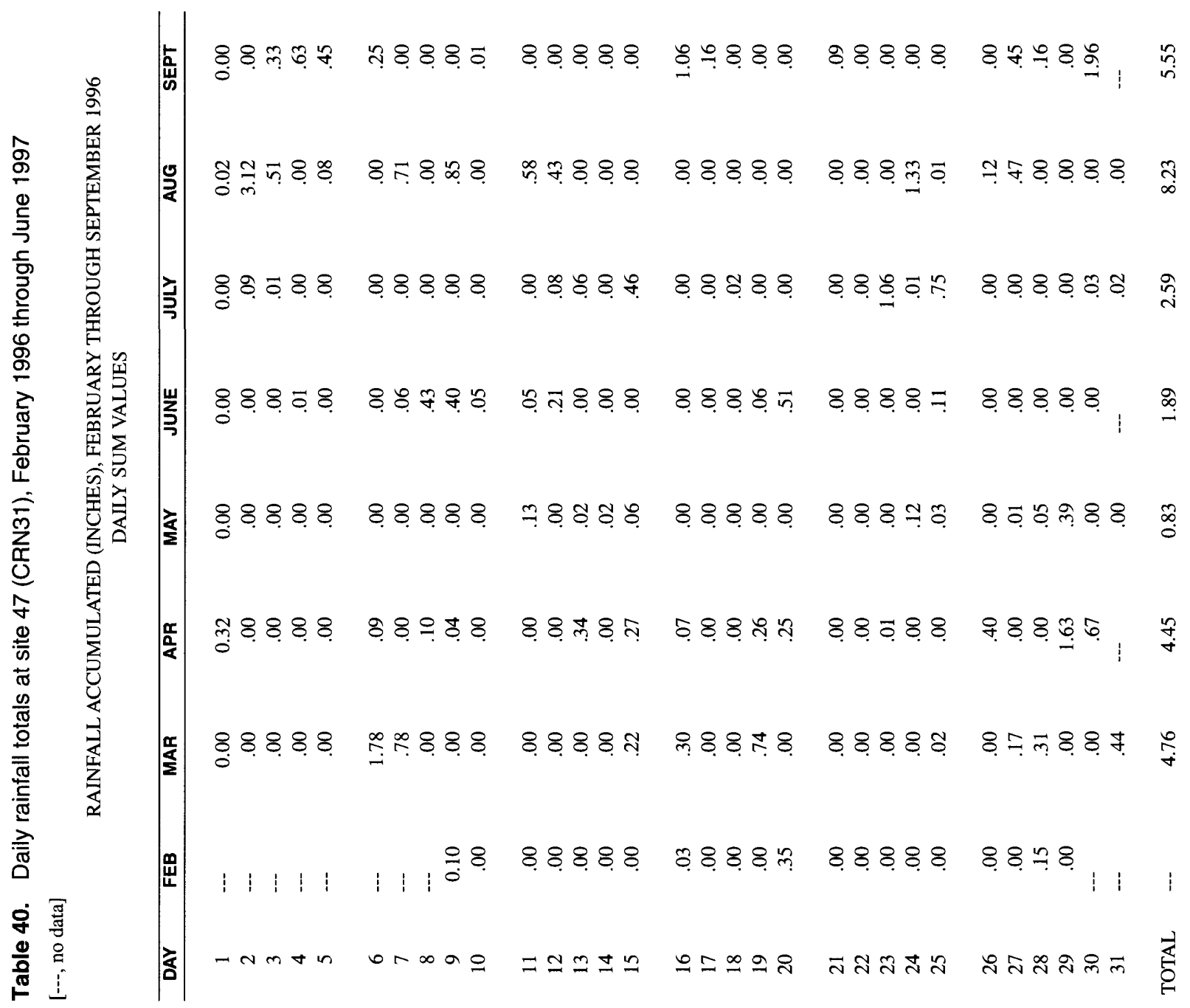




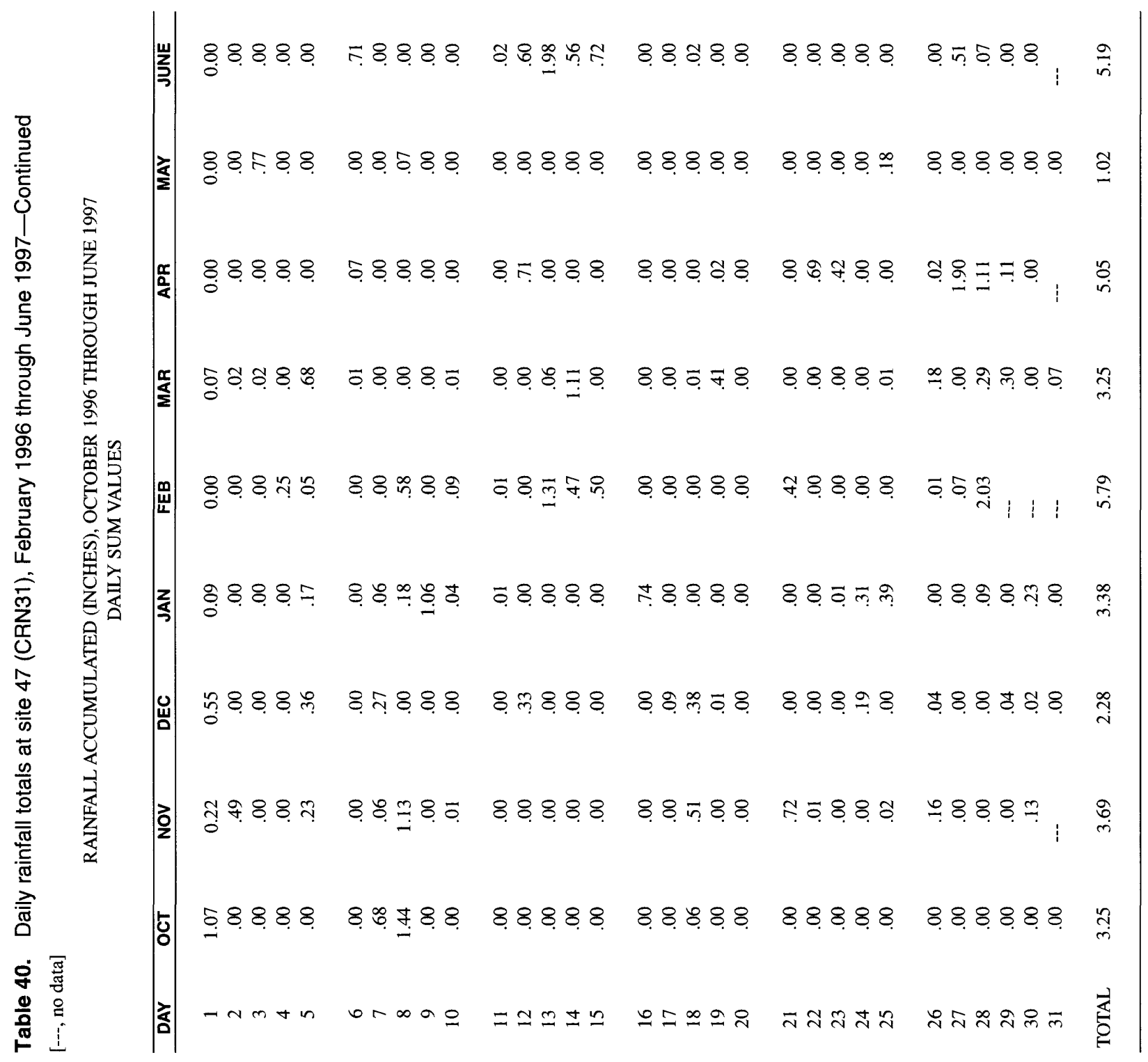




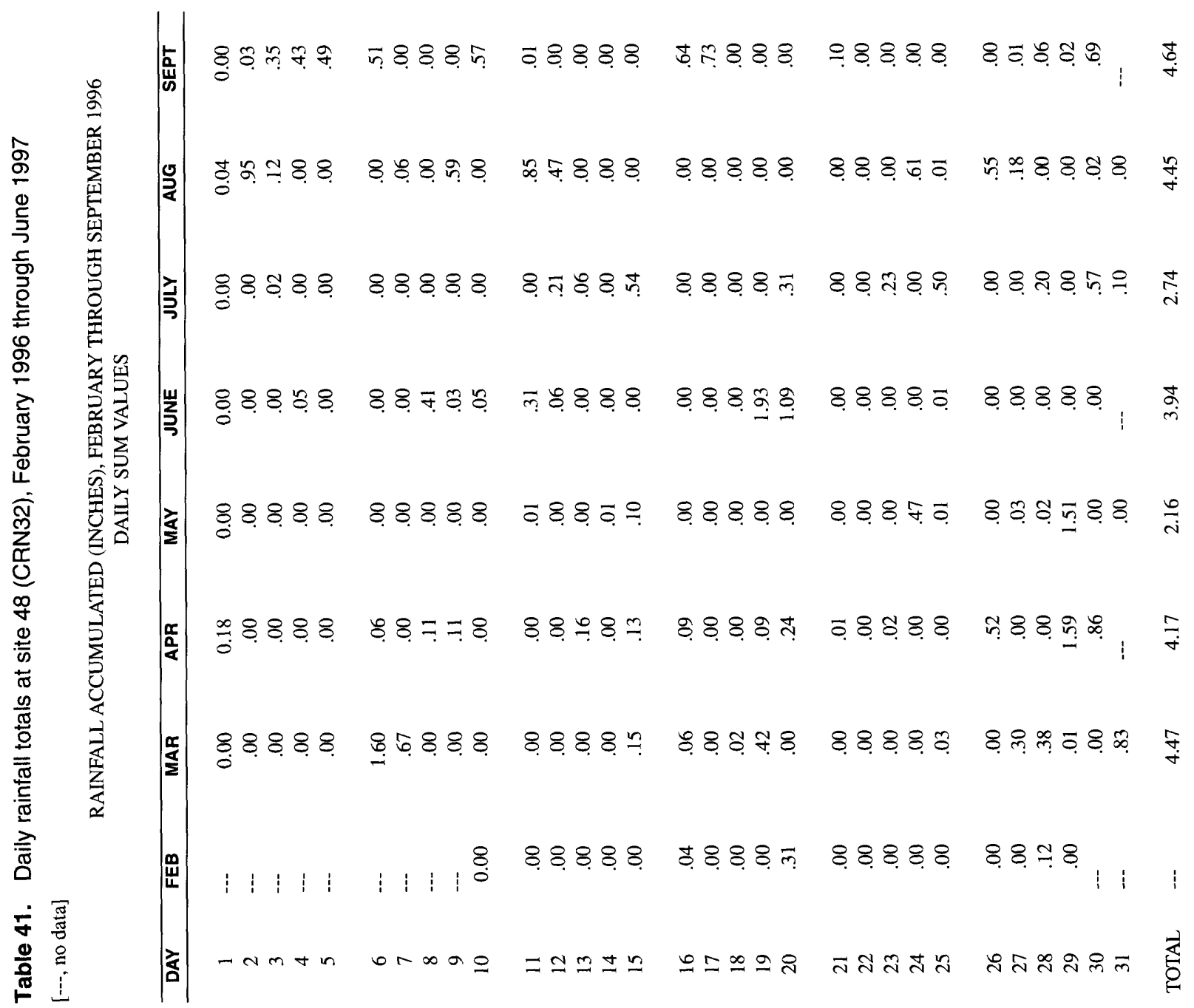




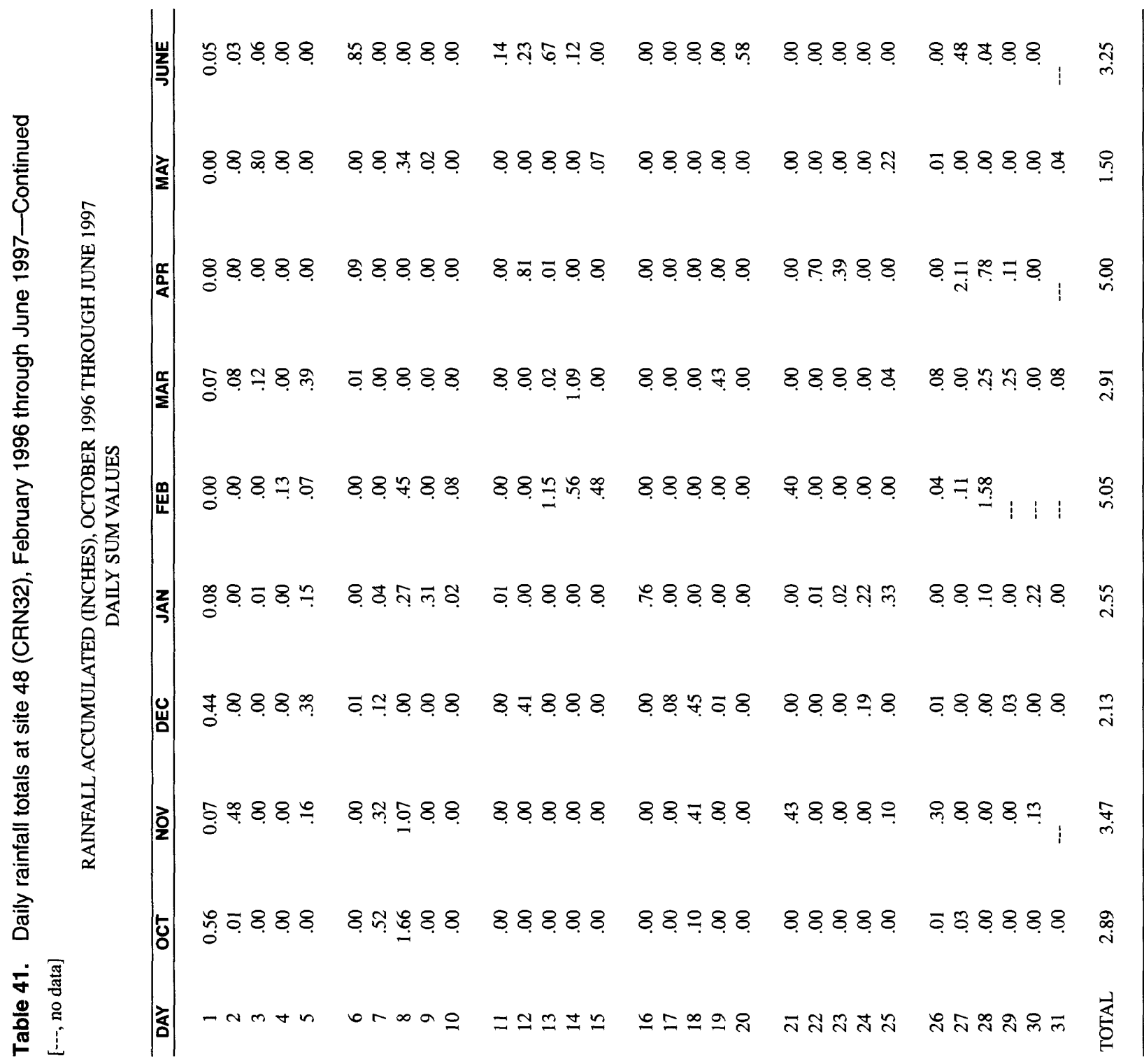




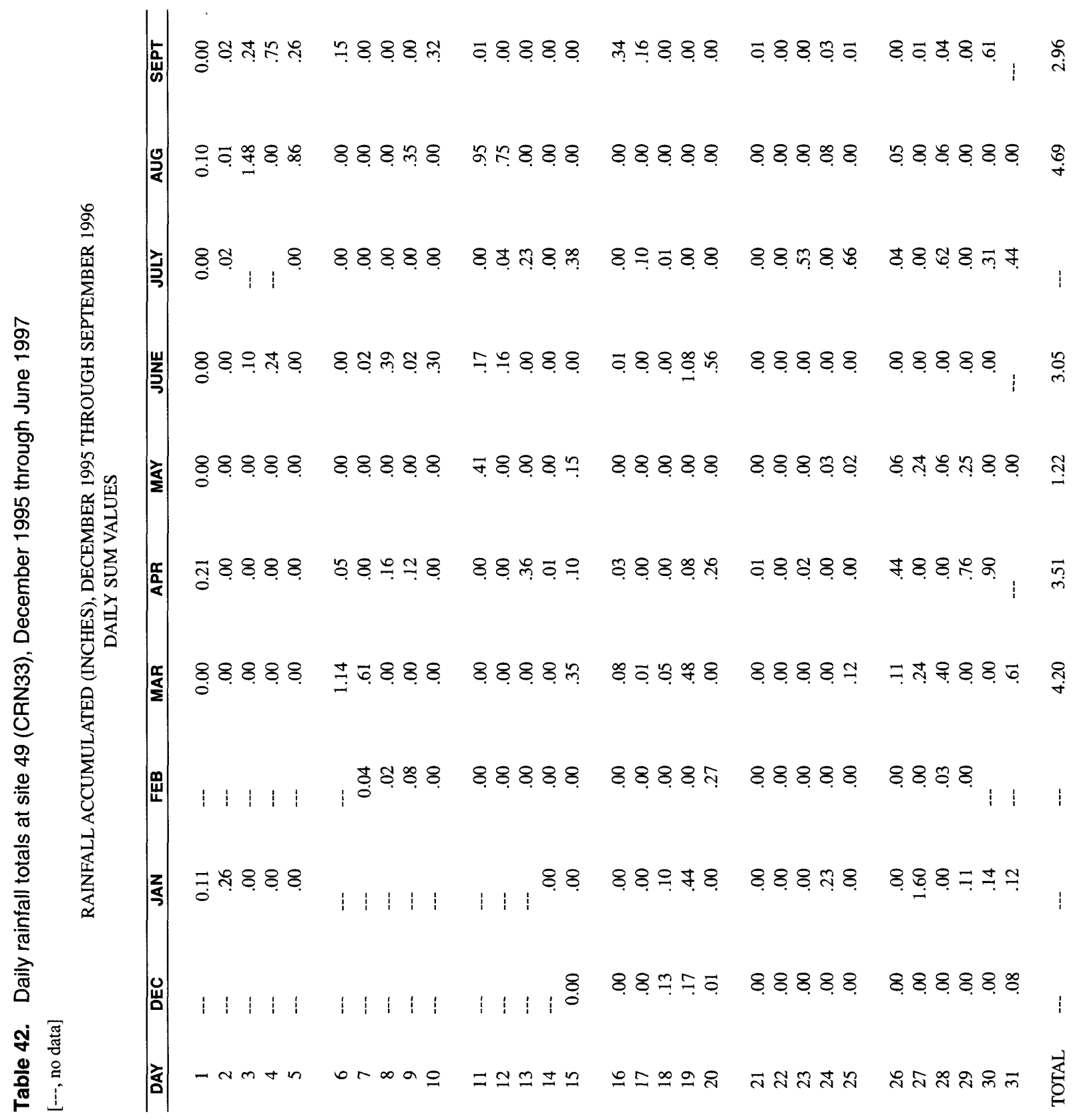




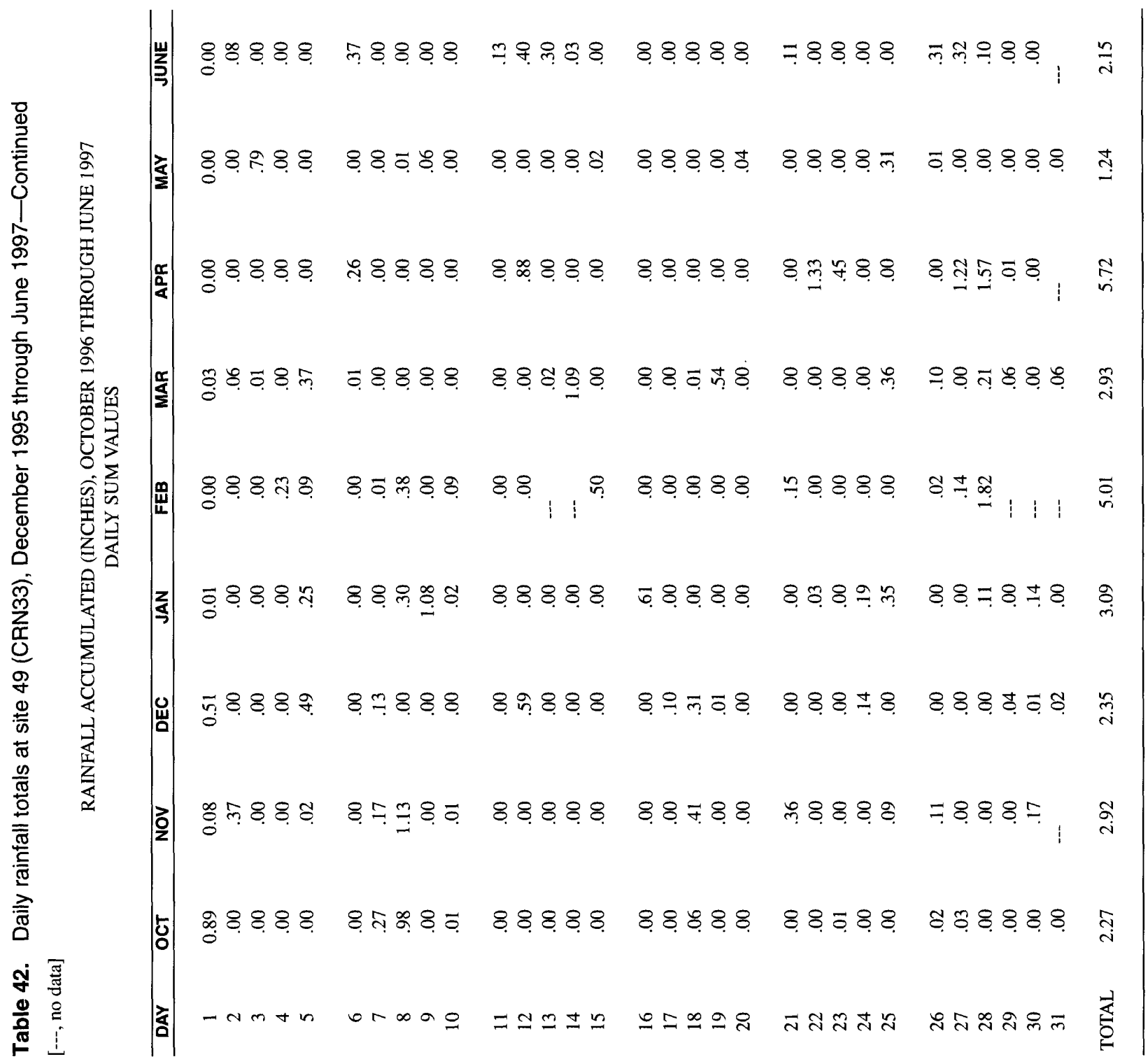




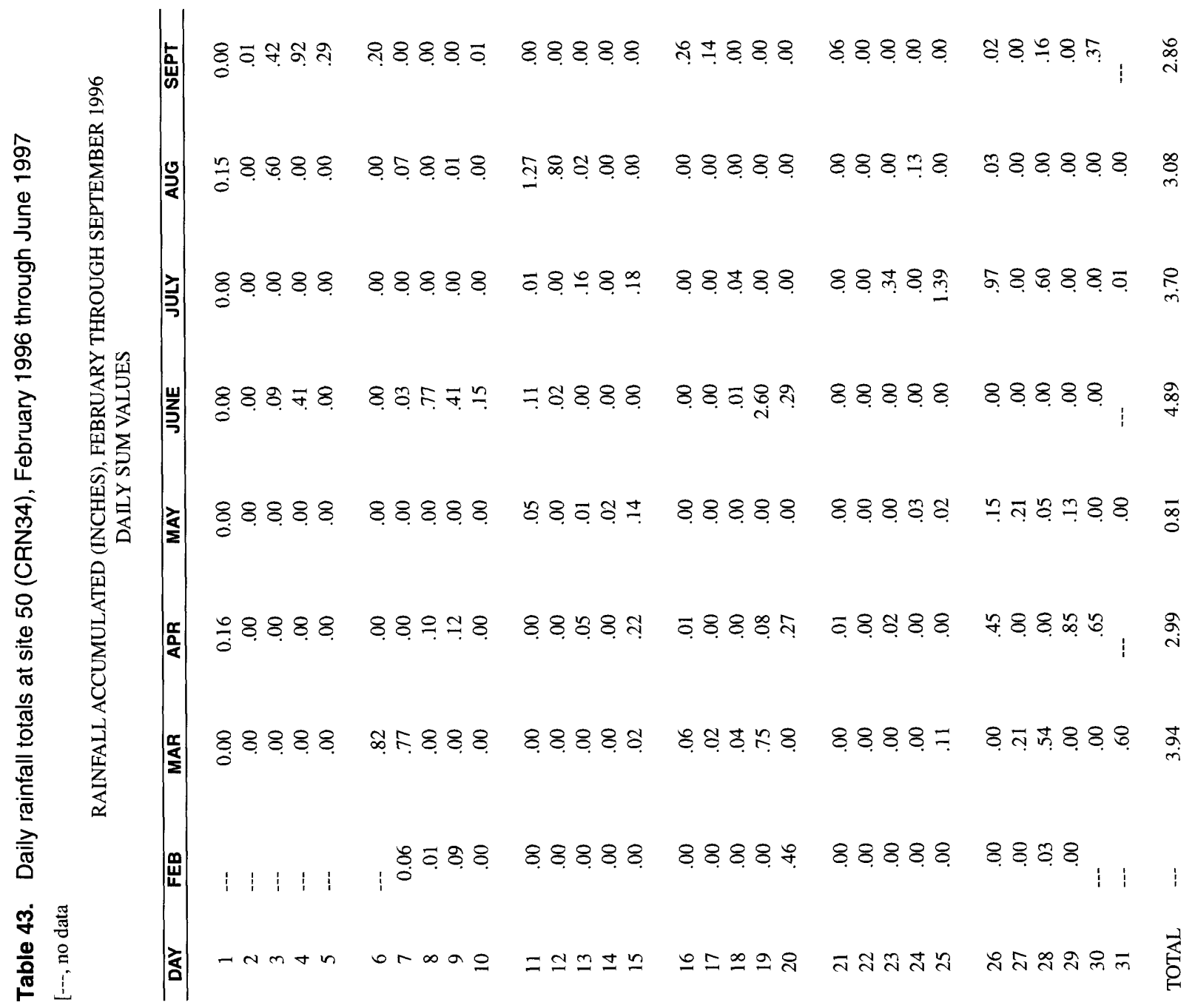




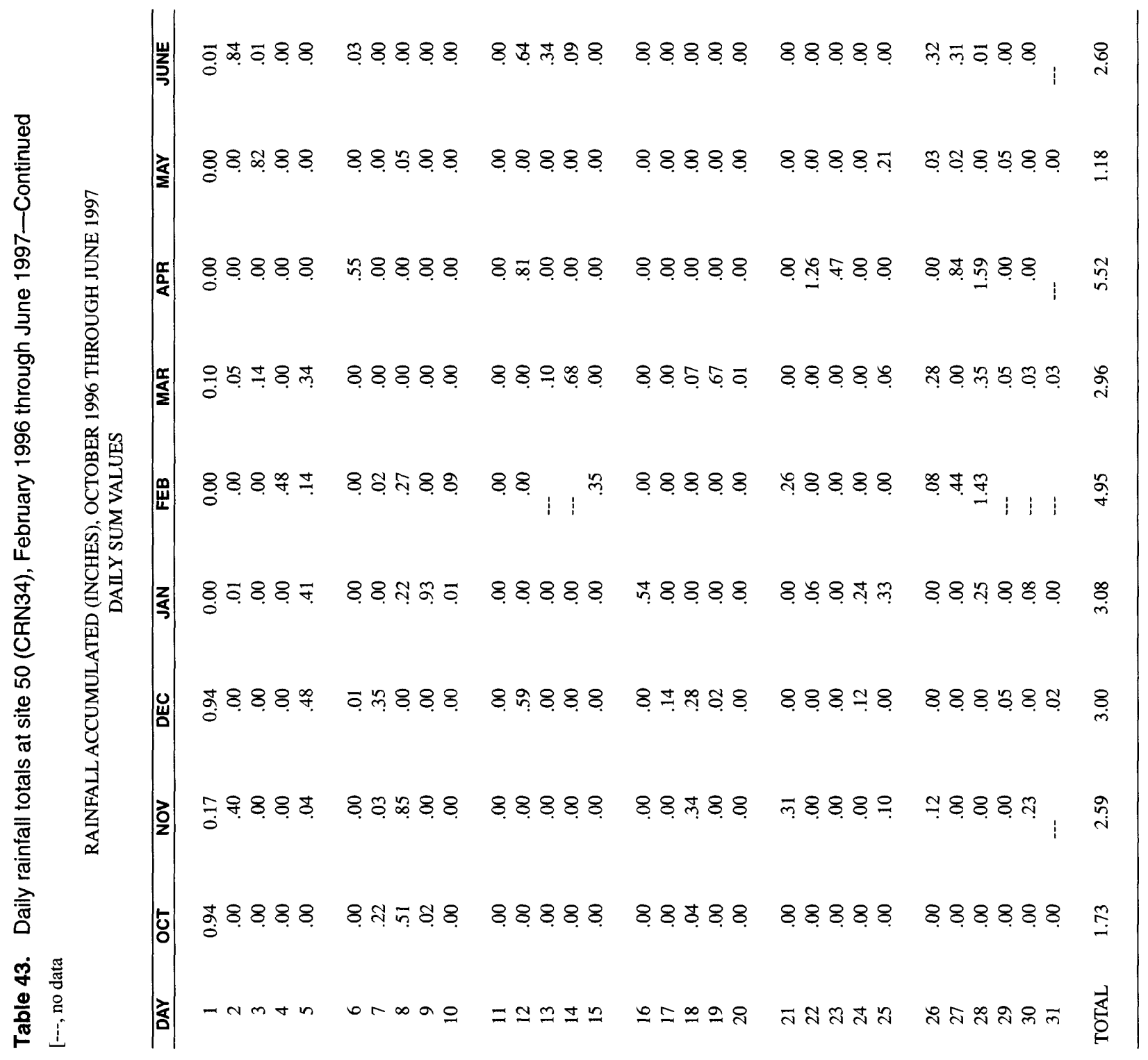




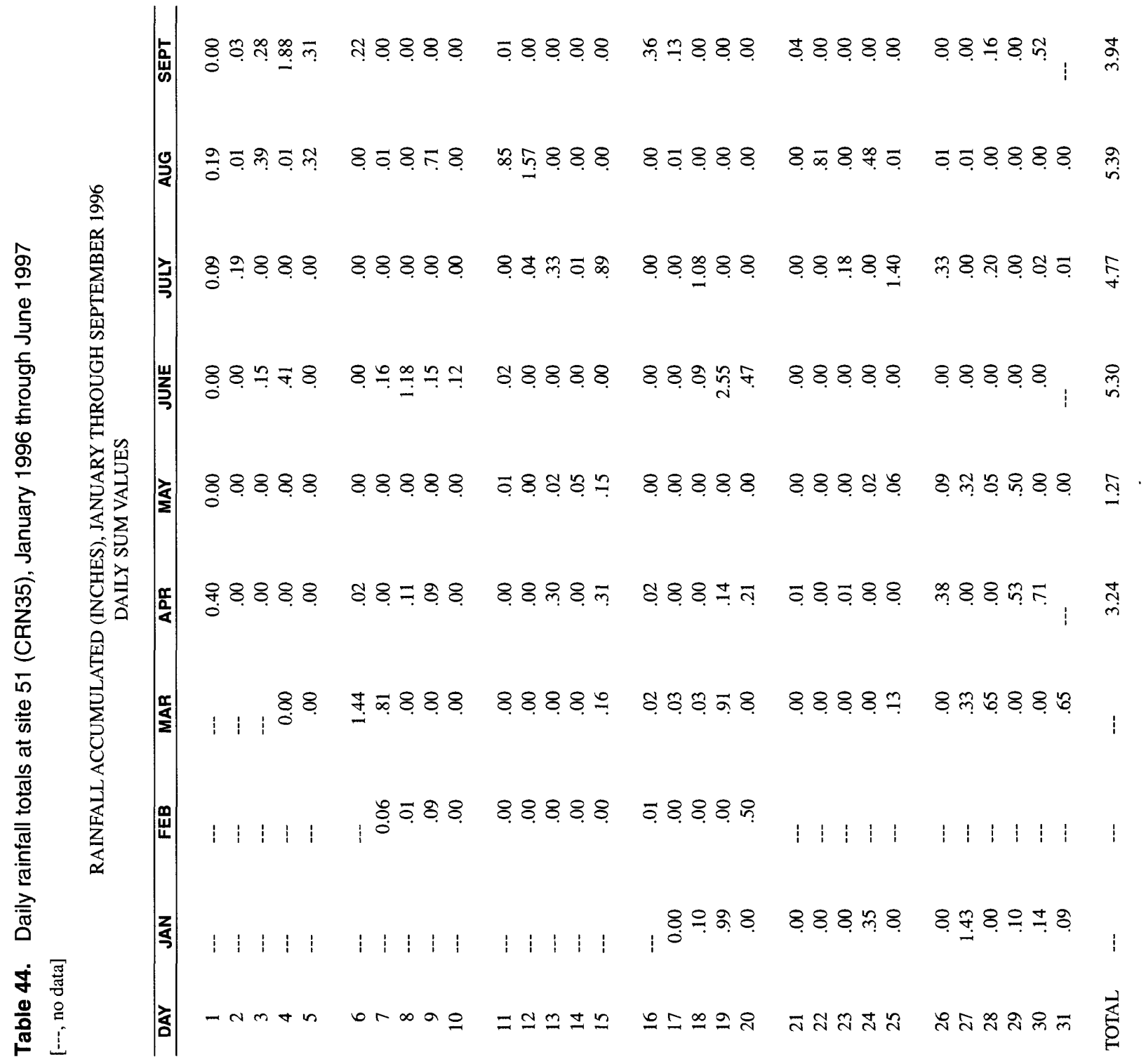




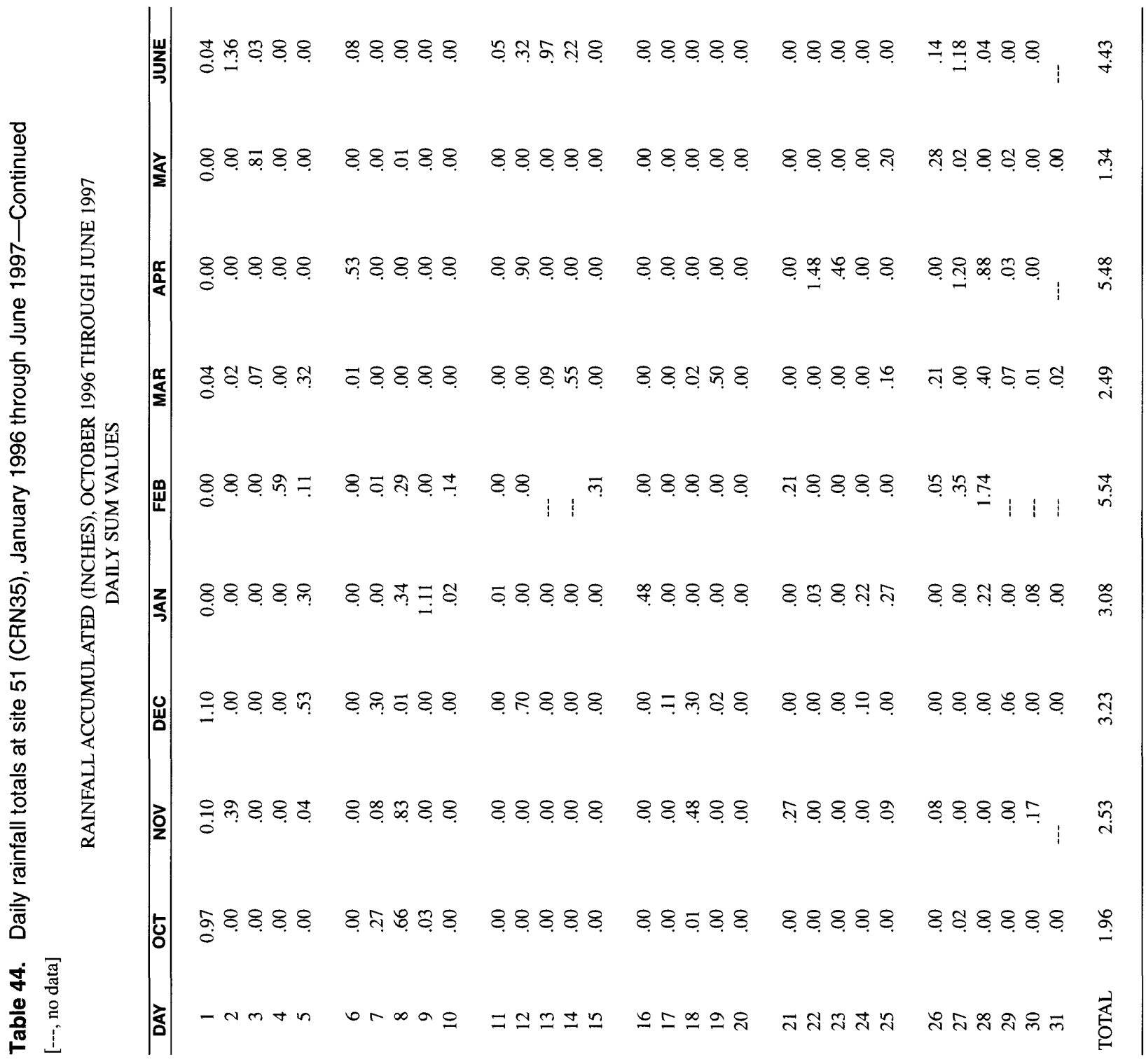




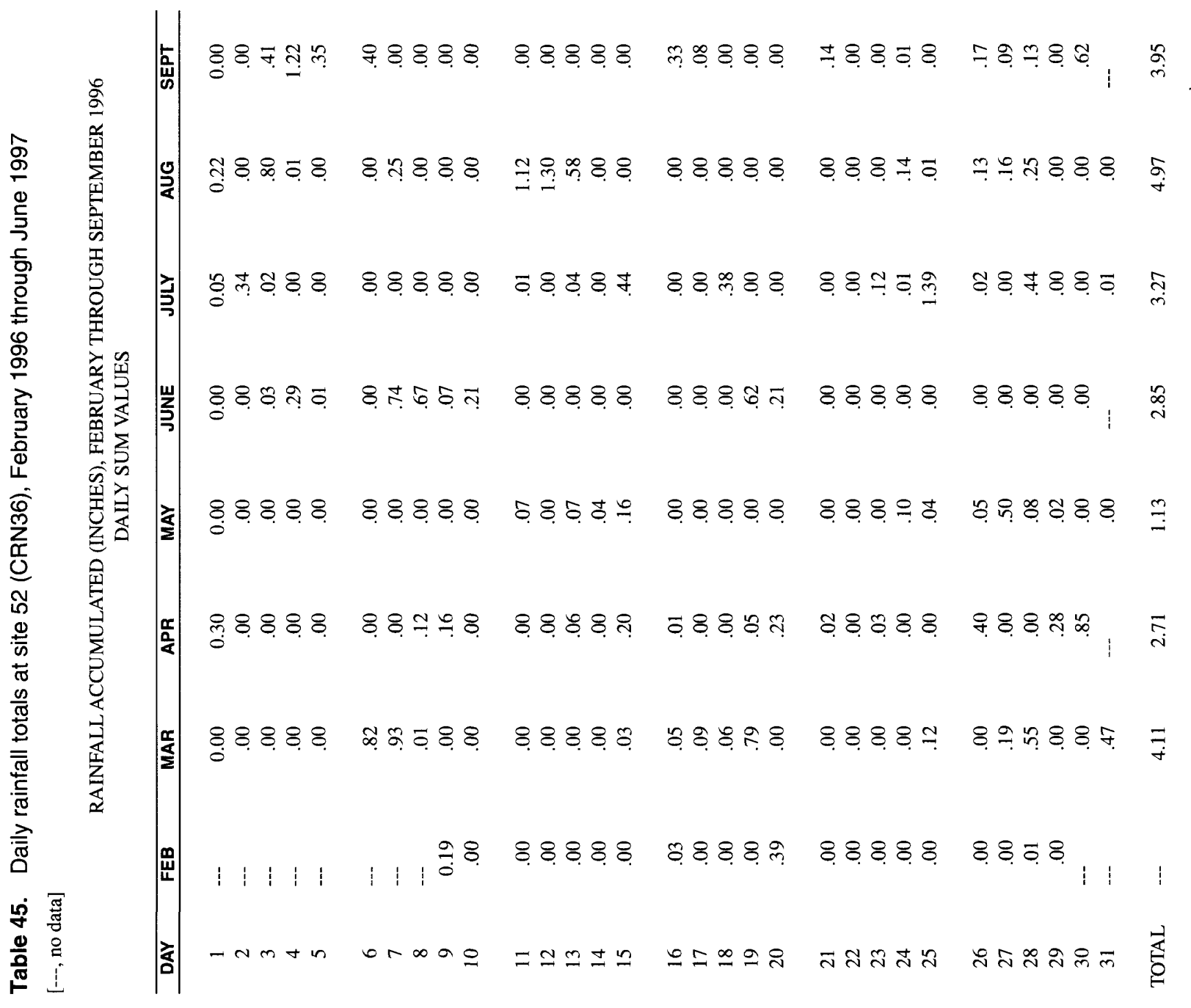




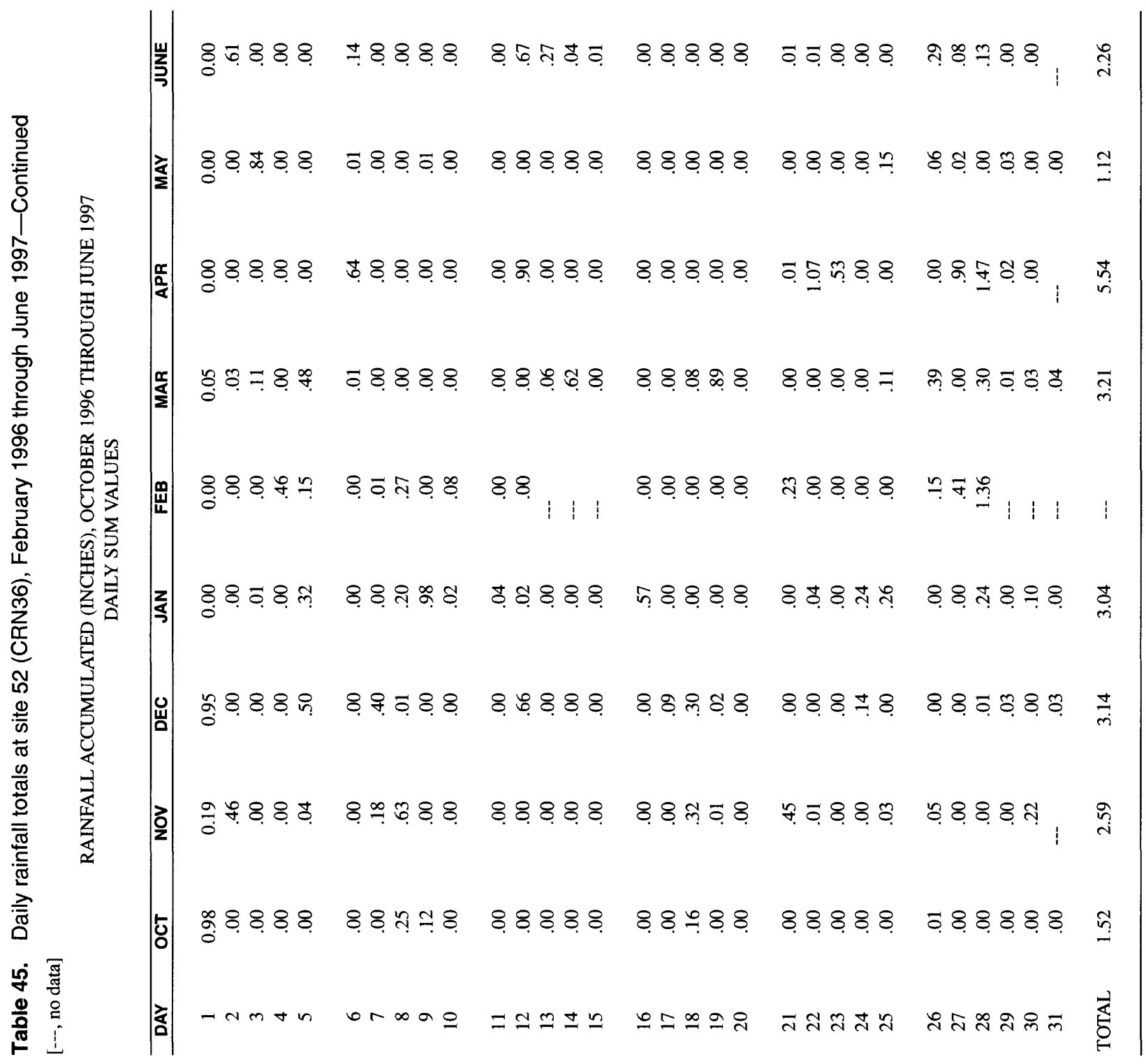




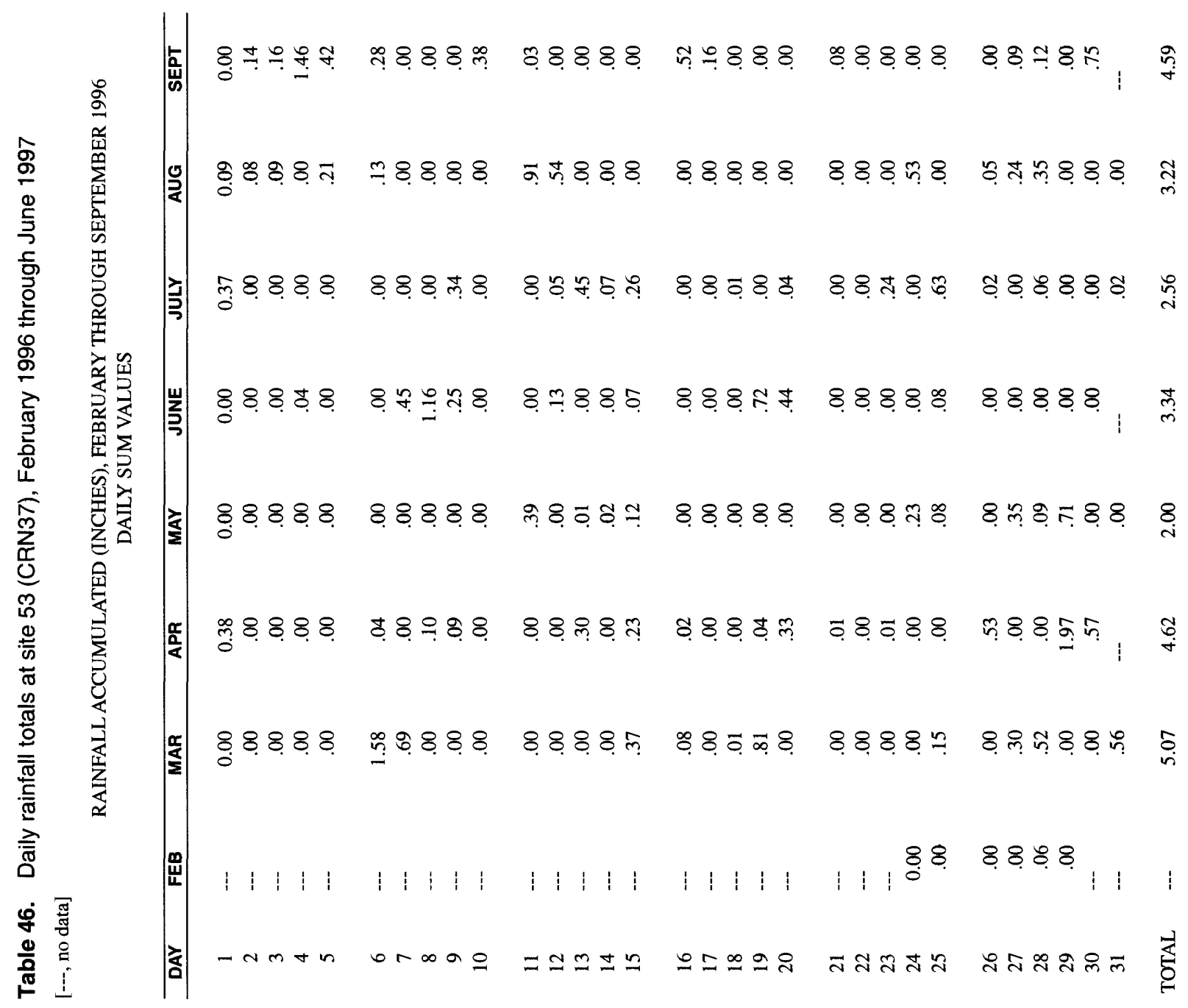




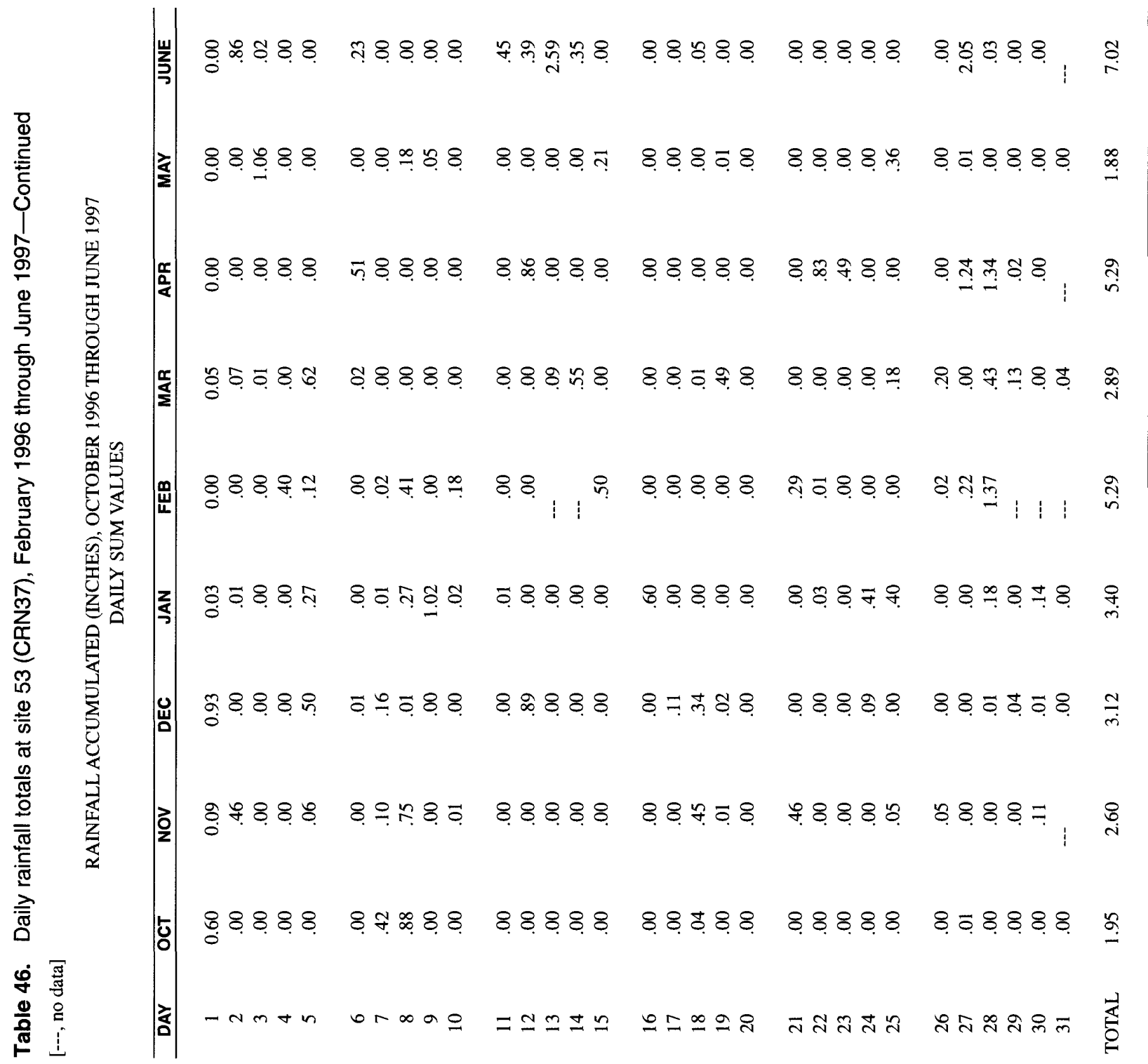




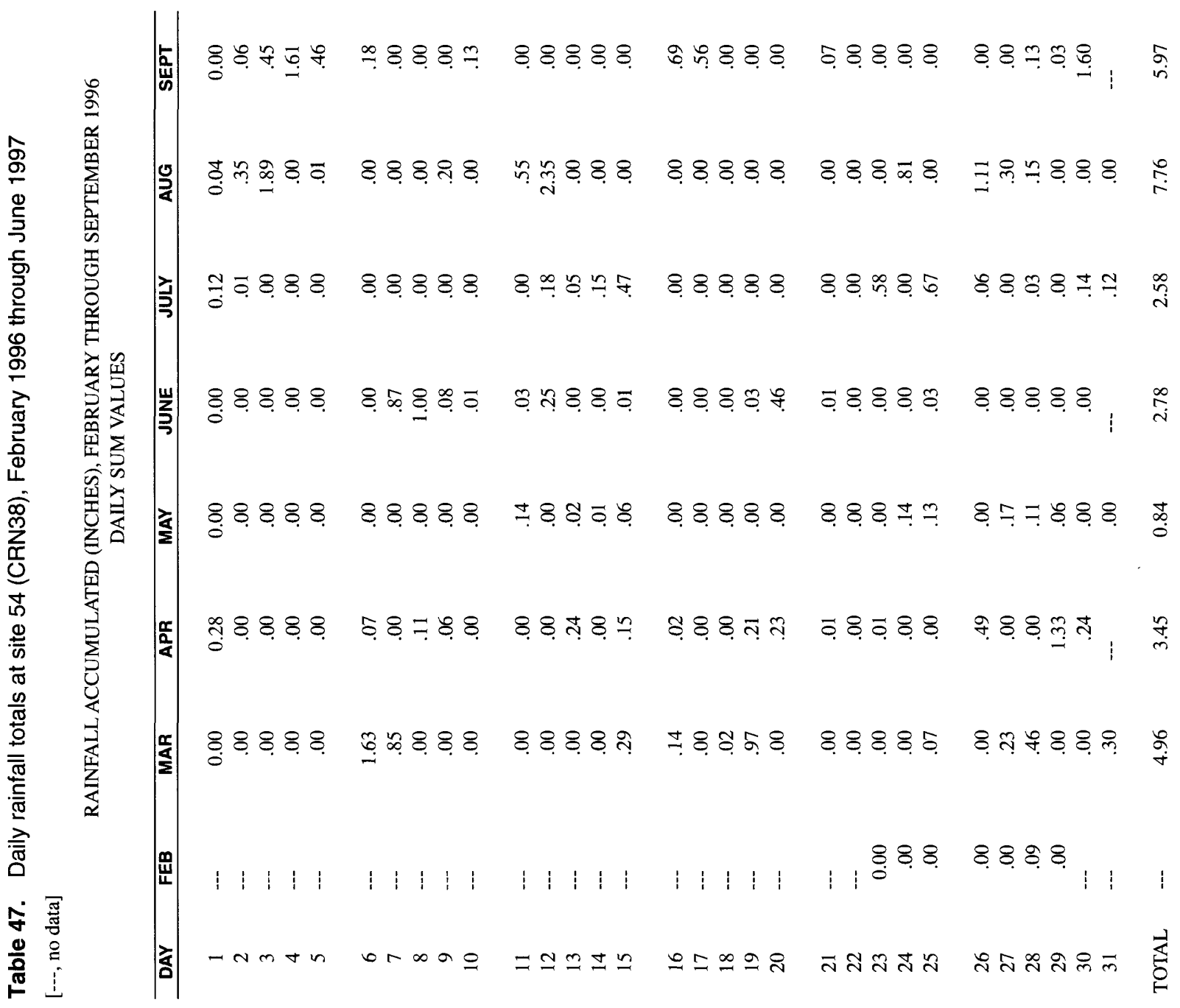




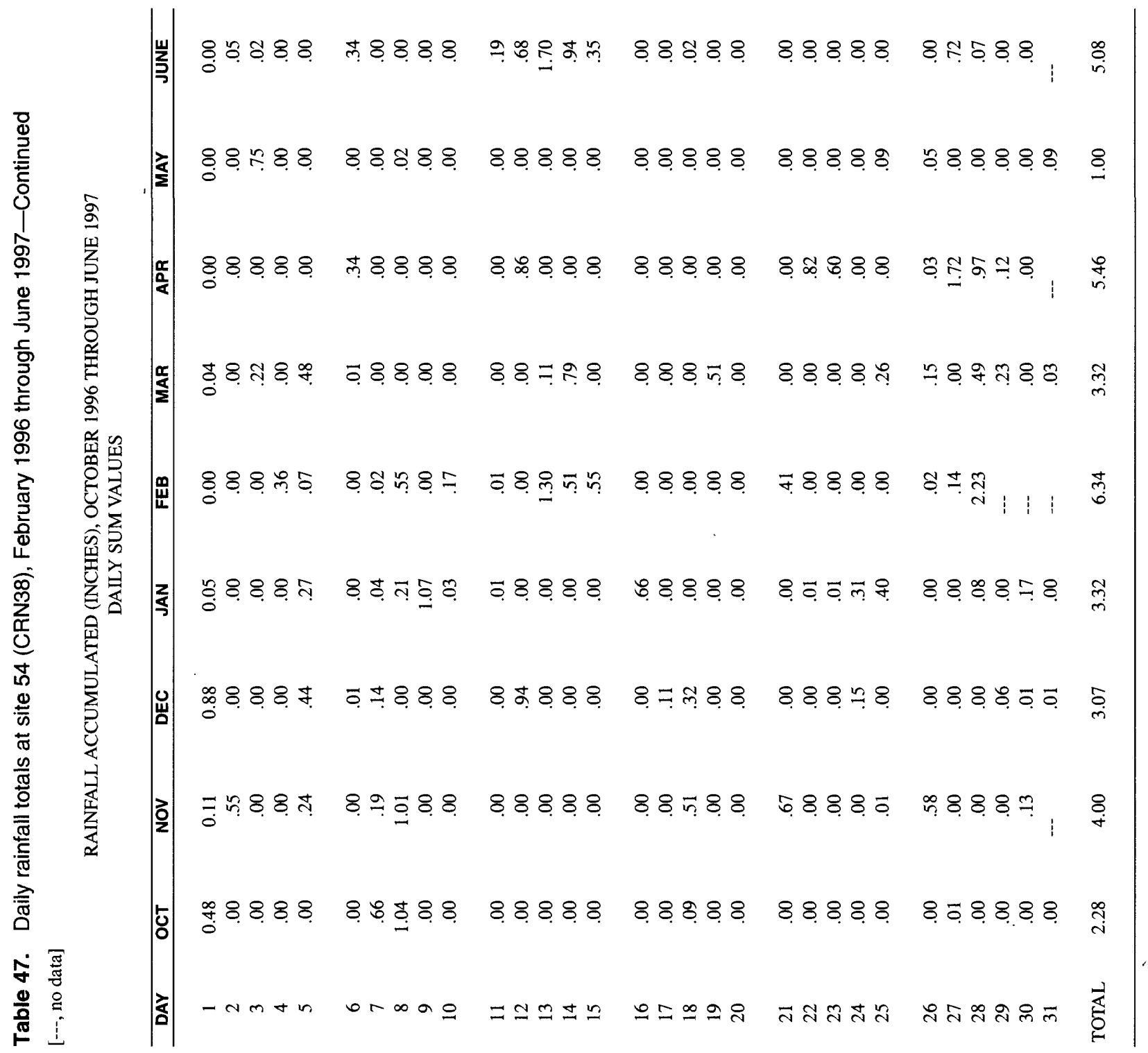




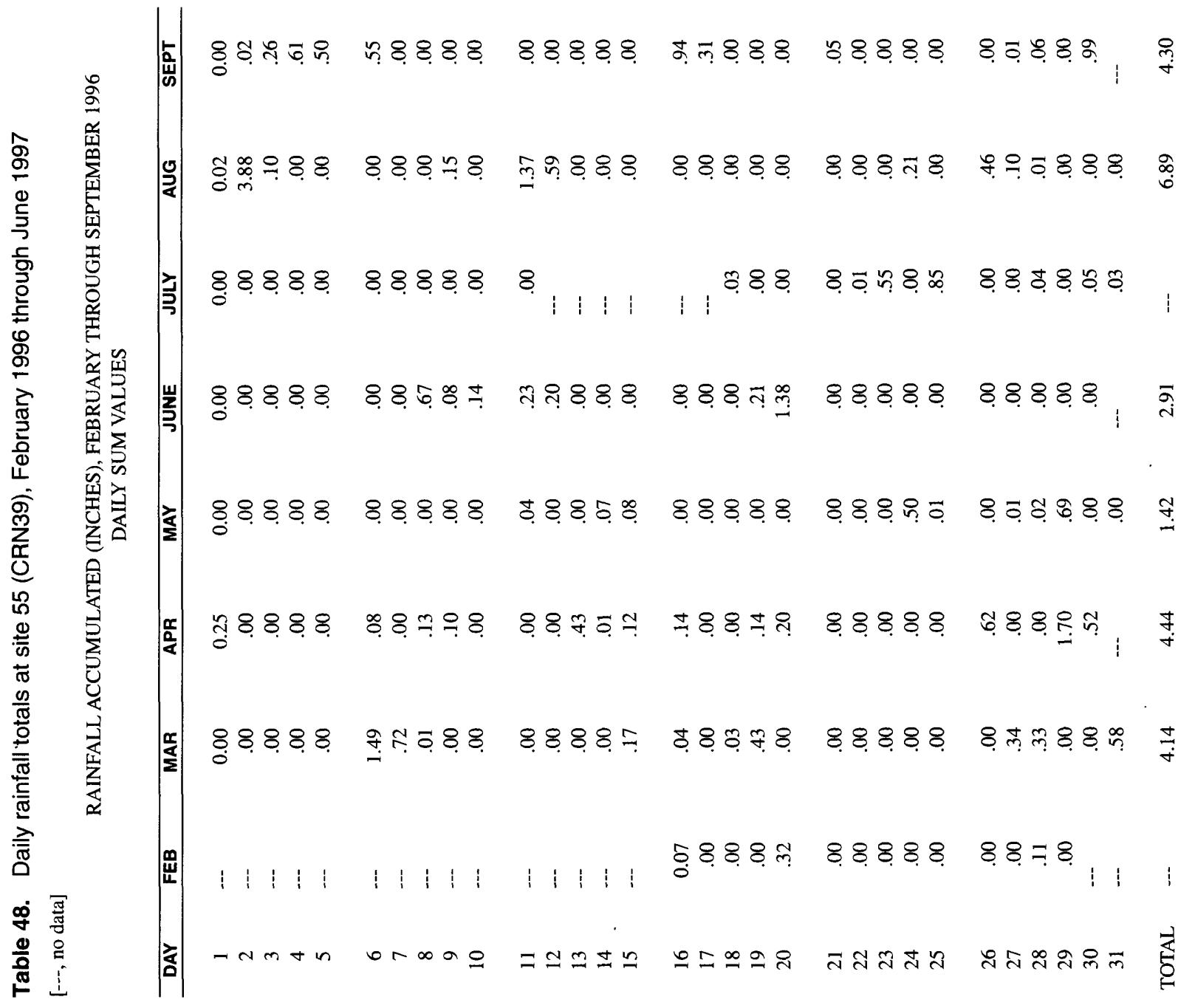




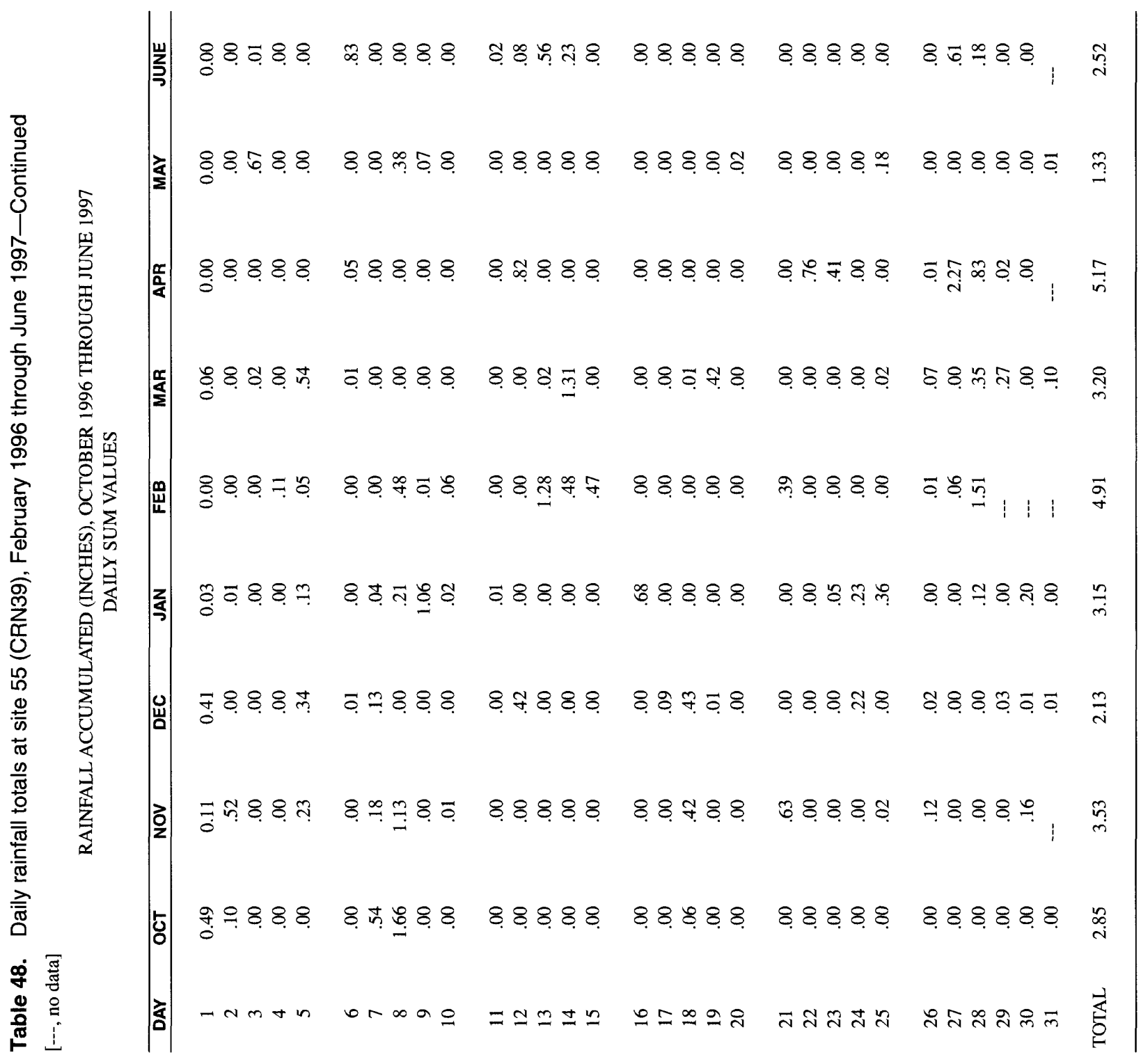




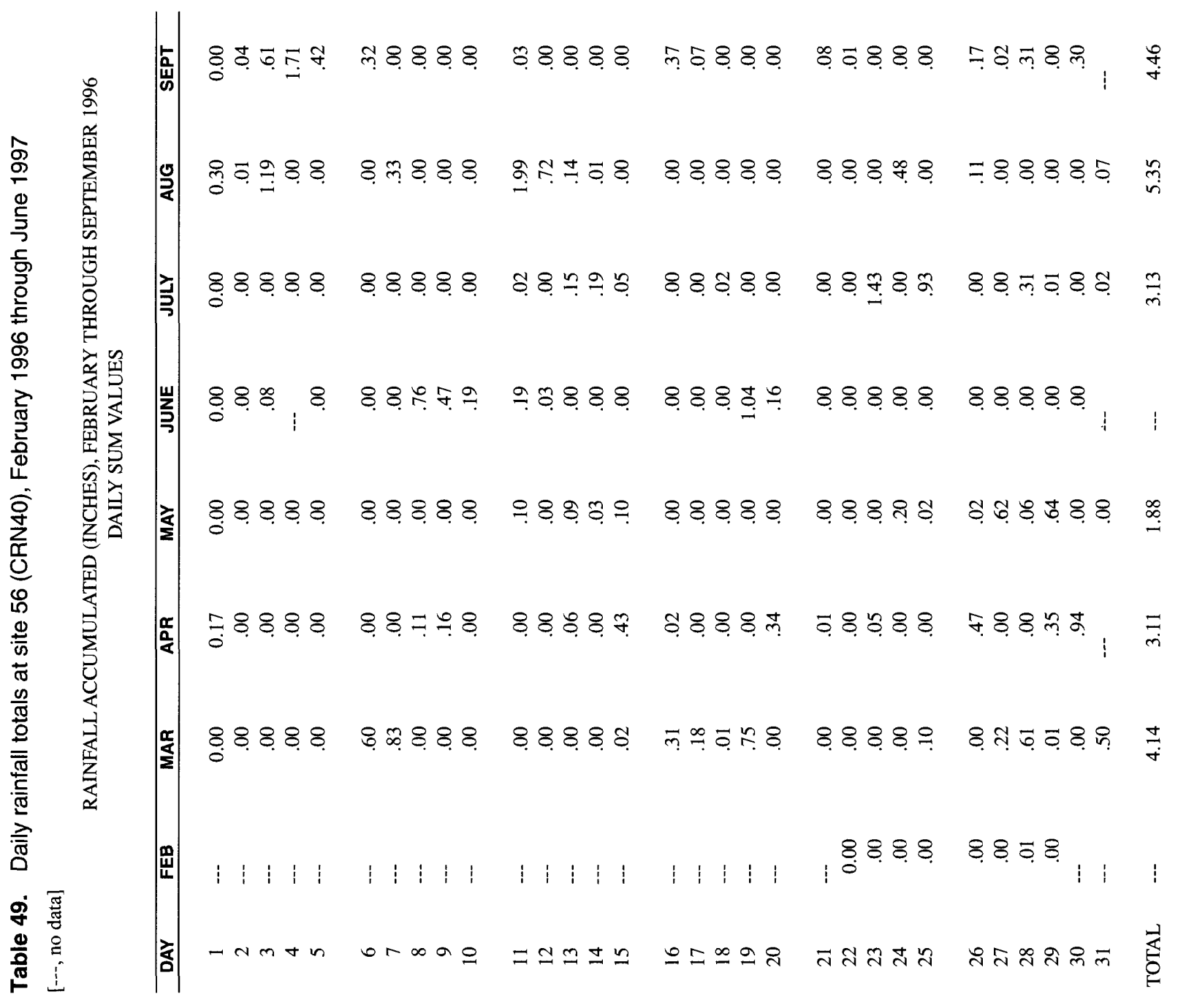




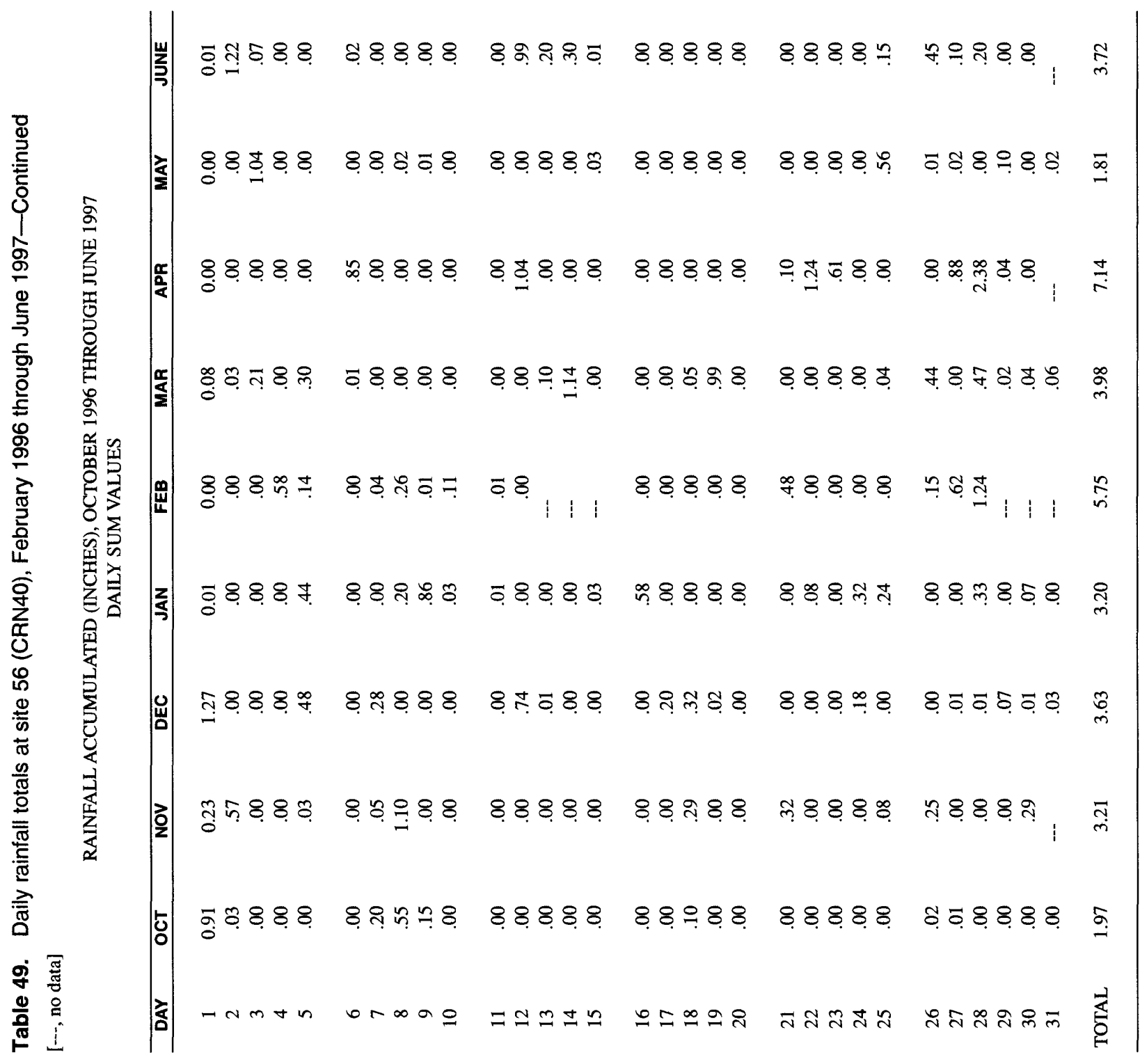




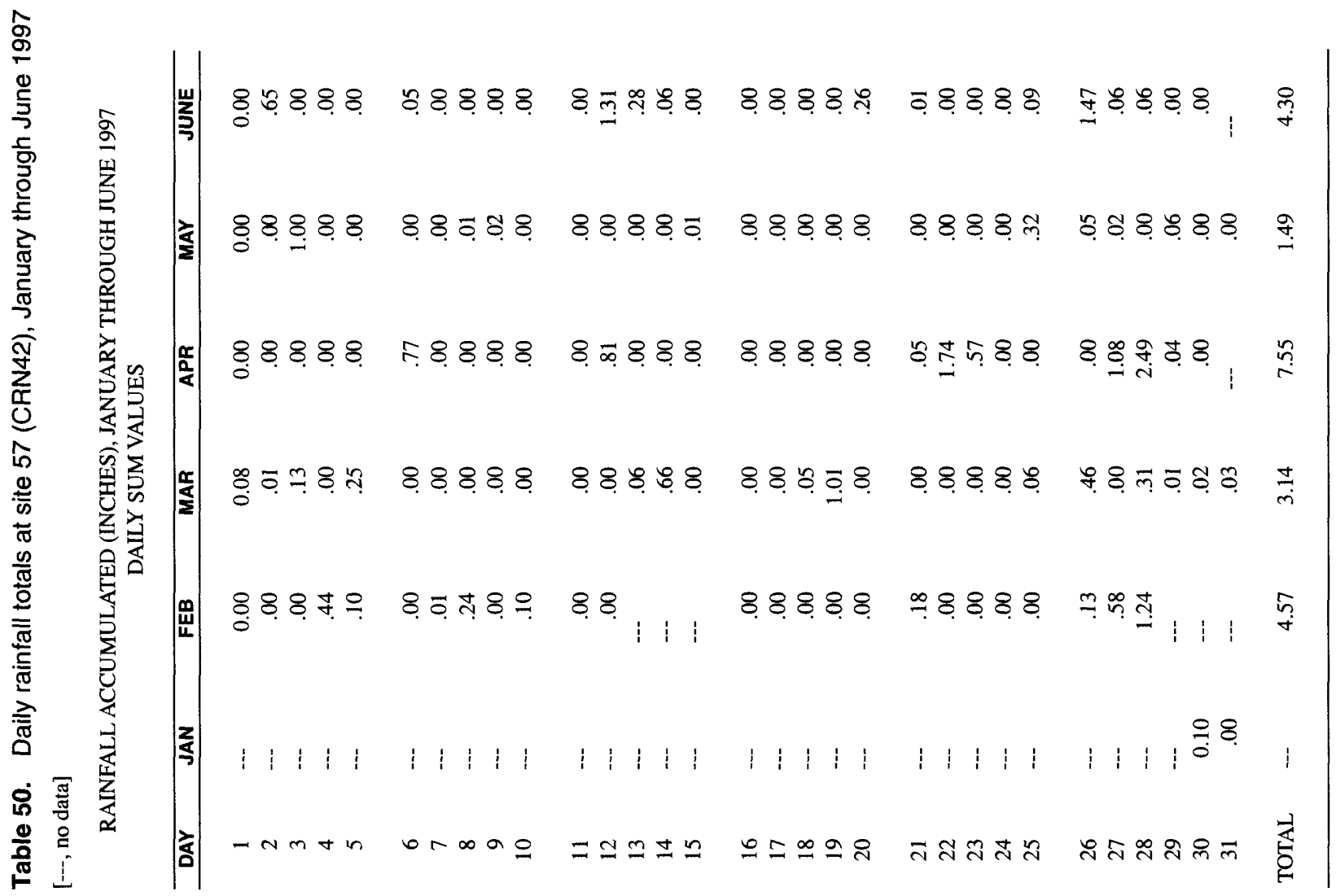




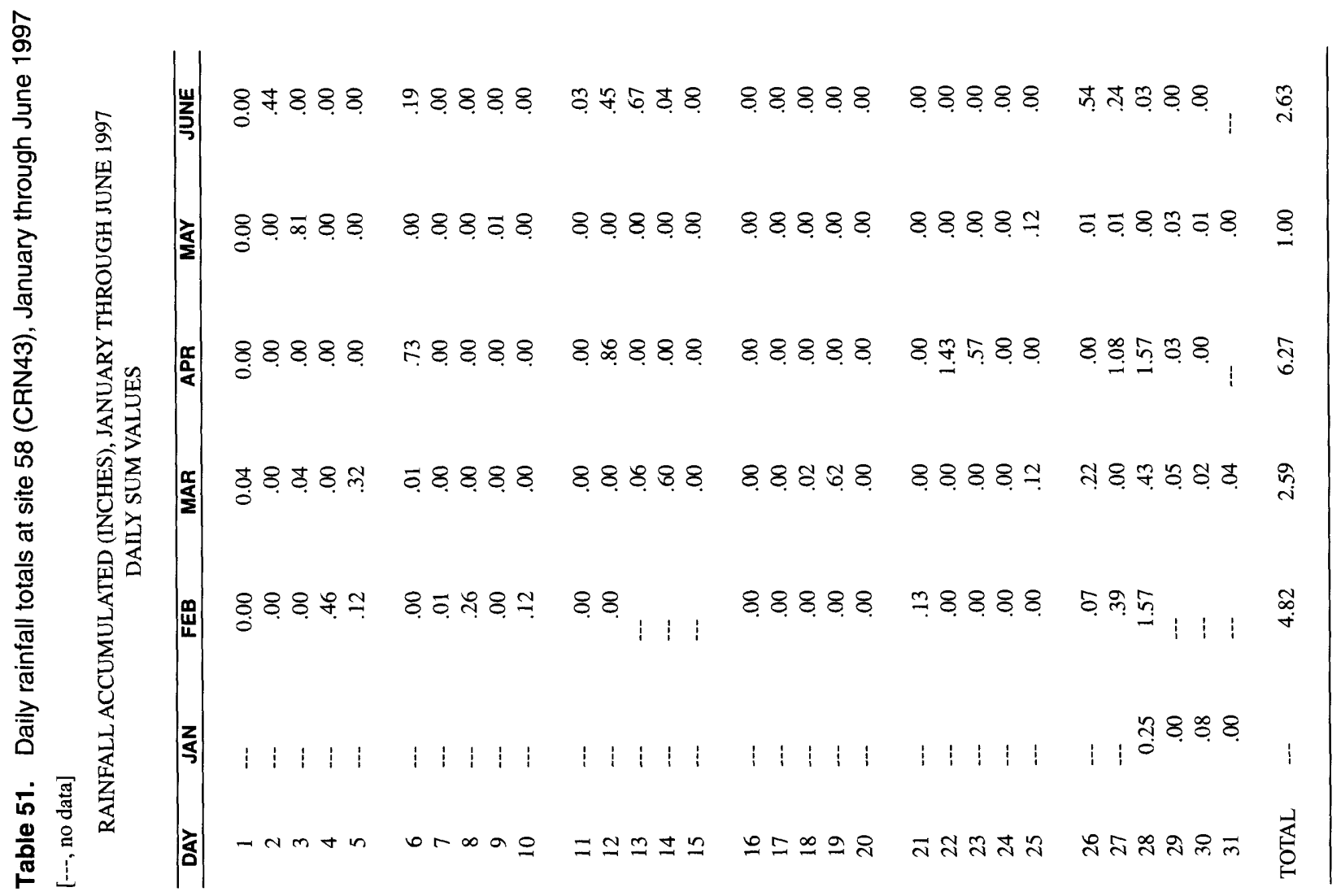




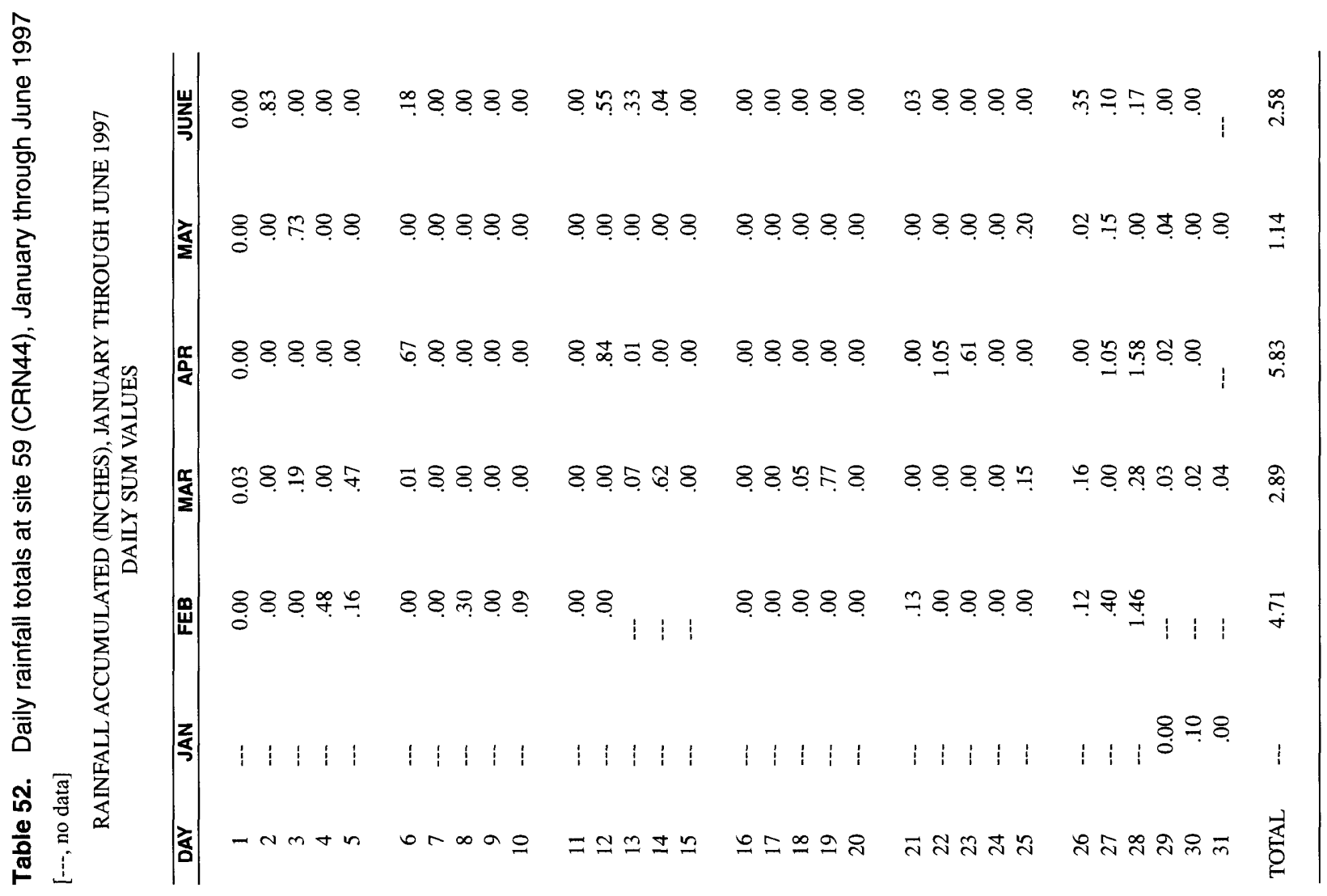




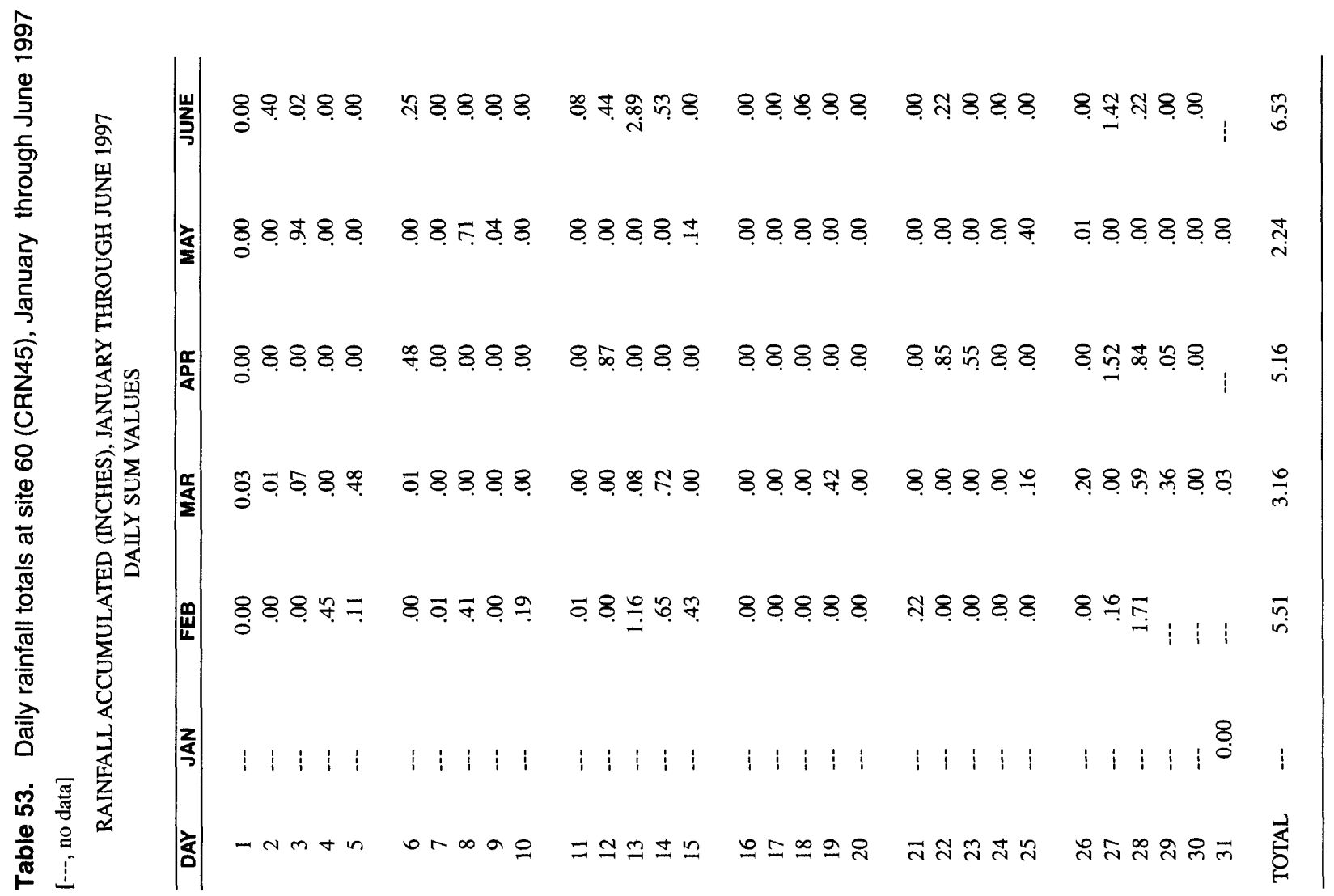




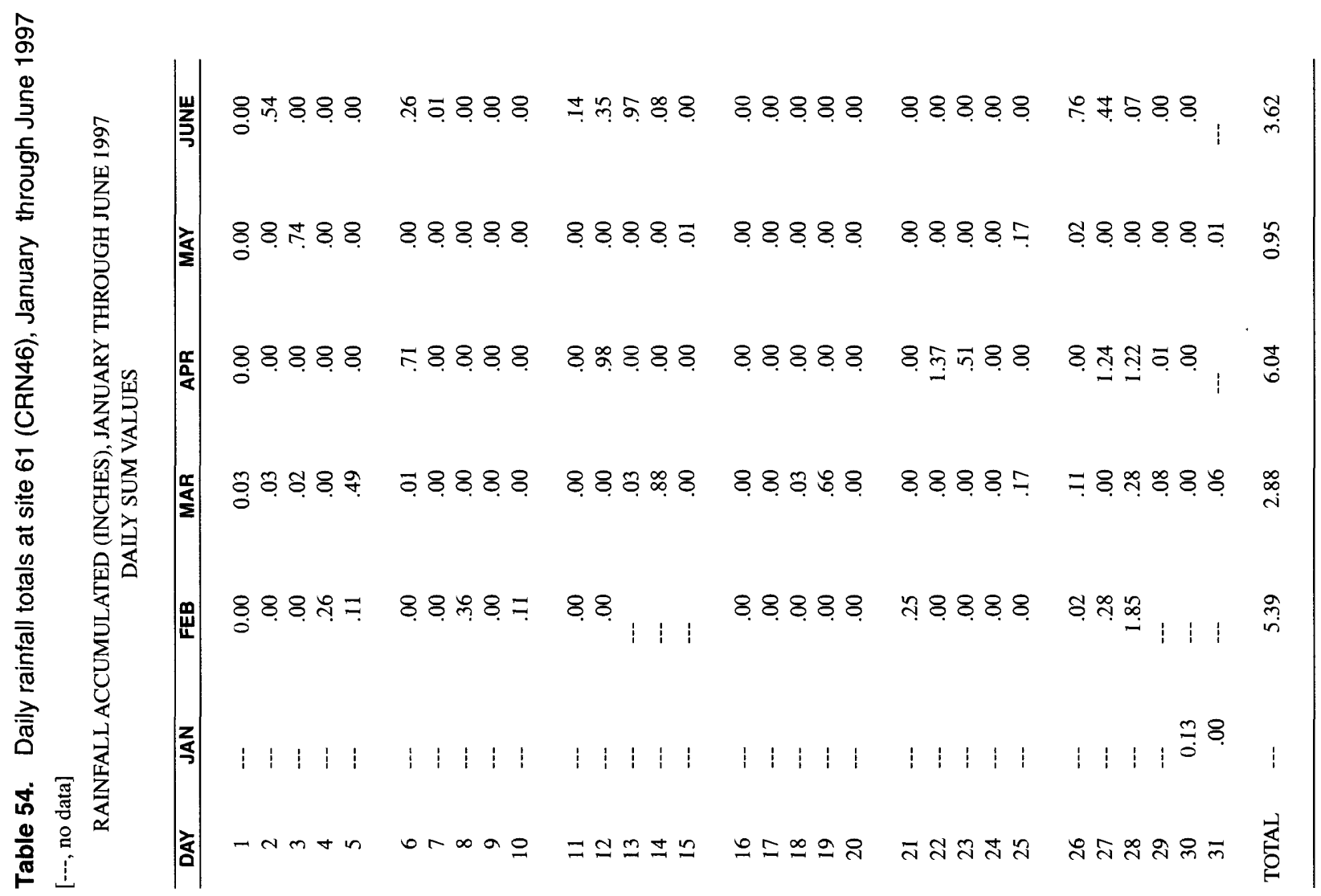


Table 55. Streamflow statistics at the streamflow and water-quality study sites, December 1993 through June 1997

\begin{tabular}{|c|c|c|c|c|}
\hline $\begin{array}{l}\text { Site no. } \\
\text { (fig. 1) }\end{array}$ & Period of record & $\begin{array}{l}\text { Daily mean discharge } \\
\text { for period of record } \\
\left(\mathrm{ft}^{3} / \mathrm{s}\right)\end{array}$ & $\begin{array}{l}\text { Maximum instantaneous } \\
\text { discharge recorded } \\
\left(\mathrm{ft}^{3} / \mathrm{s}\right)\end{array}$ & $\begin{array}{l}\text { Minimum instantaneous } \\
\text { discharge recorded } \\
\left(\mathrm{ft}^{3} / \mathrm{s}\right)\end{array}$ \\
\hline $\begin{array}{c}33^{\mathrm{a}} \\
{[\mathrm{CSW} 08]}\end{array}$ & 4/94-9/97 & 2.47 & $\begin{array}{c}493 \\
{[8 / 27 / 95]}\end{array}$ & $\begin{array}{c}0.11 \\
{[9 / 21 / 97]}\end{array}$ \\
\hline $\begin{array}{c}34^{\mathrm{a}} \\
{[\mathrm{CSW} 09]}\end{array}$ & 5/94-9/97 & 3.26 & $\begin{array}{c}652 \\
{[4 / 28 / 97]}\end{array}$ & $\begin{array}{c}\text { Not determined } \\
{[7 / 11 / 97]}\end{array}$ \\
\hline $\begin{array}{c}37 \\
{[\mathrm{CSW} 06]}\end{array}$ & $4 / 95-6 / 97$ & .081 & $\begin{array}{c}42 \\
{[6 / 19 / 95 ; 4 / 30 / 96]}\end{array}$ & $\begin{array}{c}0 \\
\text { [several days during the period of record] }\end{array}$ \\
\hline $\begin{array}{c}39 \\
{[\text { CSW05] }}\end{array}$ & $3 / 94-6 / 97$ & .036 & $\begin{array}{c}27 \\
{[5 / 29 / 96]}\end{array}$ & $\begin{array}{c}0 \\
\text { [several days during the period of record] }\end{array}$ \\
\hline $\begin{array}{c}40 \\
\text { [CSW03] }\end{array}$ & 7/94-6/97 & .009 & $\begin{array}{c}27 \\
{[8 / 27 / 95]}\end{array}$ & $\begin{array}{c}0 \\
\text { [several days during the period of record] }\end{array}$ \\
\hline $\begin{array}{c}41 \\
{[\mathrm{CSW} 02]}\end{array}$ & $12 / 93-6 / 97$ & .16 & $\begin{array}{c}334 \\
{[8 / 27 / 95]}\end{array}$ & $\begin{array}{c}0.010 \\
{[10 / 16 / 96 ; 3 / 5 / 97]}\end{array}$ \\
\hline $\begin{array}{c}42 \\
\text { [CSW04] }\end{array}$ & $12 / 93-6 / 97$ & .30 & $\begin{array}{c}294 \\
{[8 / 27 / 95]}\end{array}$ & $\begin{array}{c}0.001 \\
{[10 / 7-8 / 94]}\end{array}$ \\
\hline $\begin{array}{c}43 \\
{[\mathrm{CSW} 07]}\end{array}$ & $6 / 94-6 / 97$ & .42 & $\begin{array}{c}371 \\
{[7 / 3 / 95]}\end{array}$ & $\begin{array}{c}0 \\
\text { [several days during the period of record] }\end{array}$ \\
\hline $\begin{array}{c}44^{\mathrm{a}} \\
{[\mathrm{CSW} 10]}\end{array}$ & $11 / 96-9 / 97$ & 29 & $\begin{array}{c}990 \\
{[2 / 28 / 97]}\end{array}$ & $\begin{array}{c}2.1 \\
{[9 / 8-9 / 97]}\end{array}$ \\
\hline
\end{tabular}

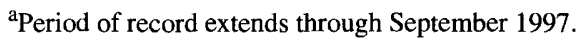




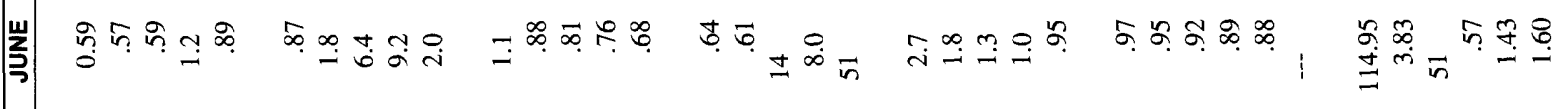

\

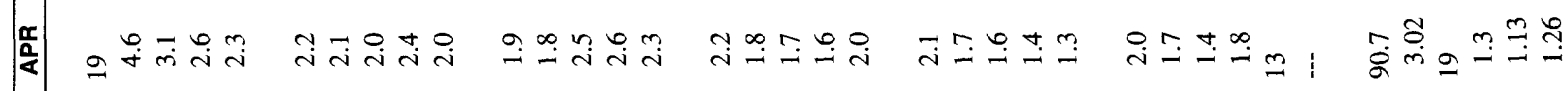

毣

逆

za

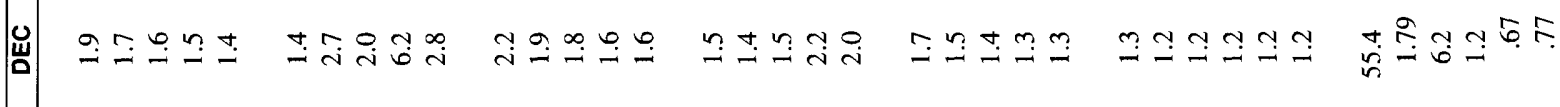

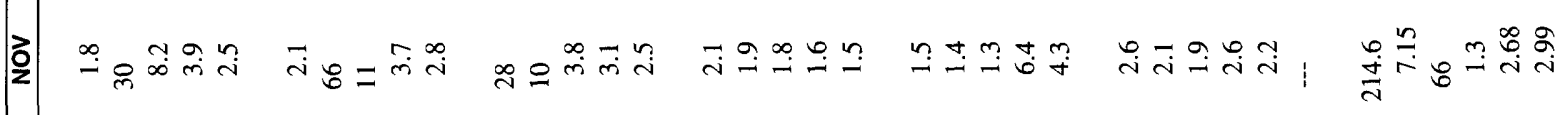

b 융

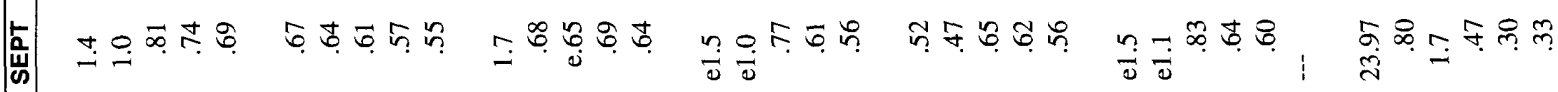

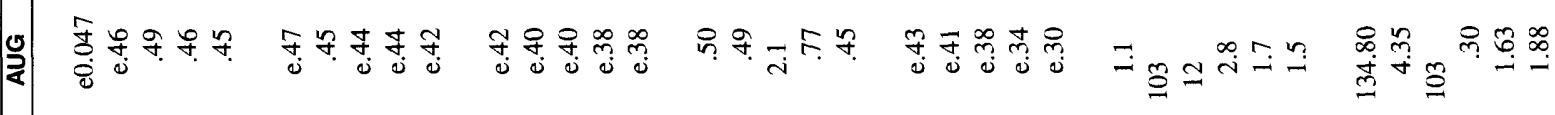

ᄀᄀ山

| 


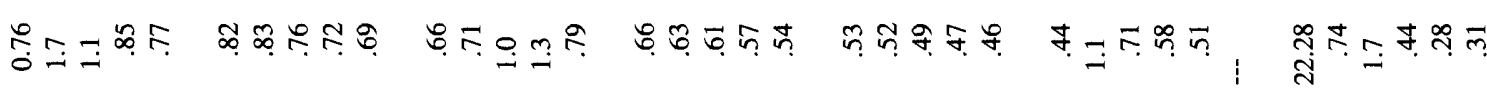

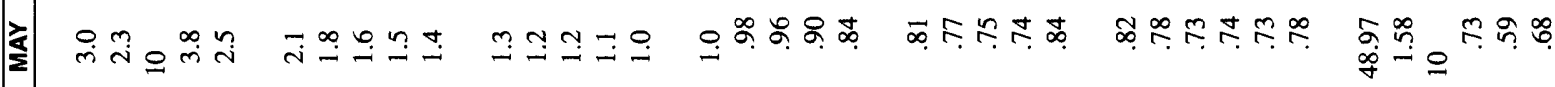

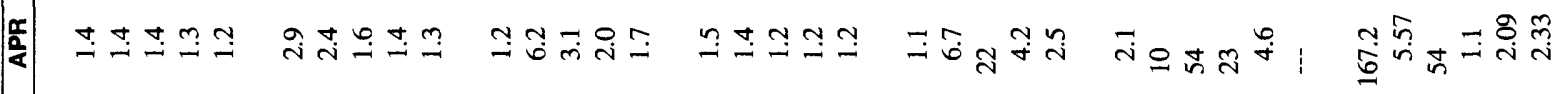

秥

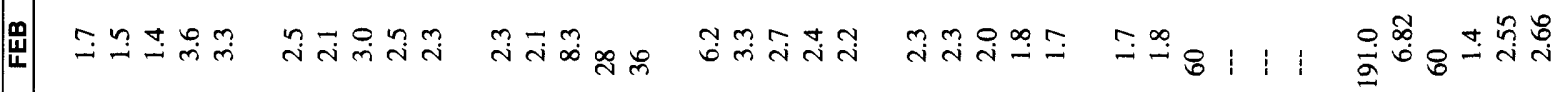

z

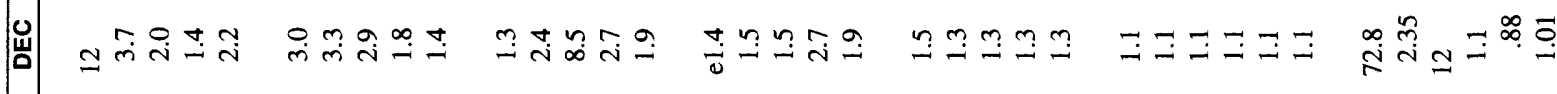

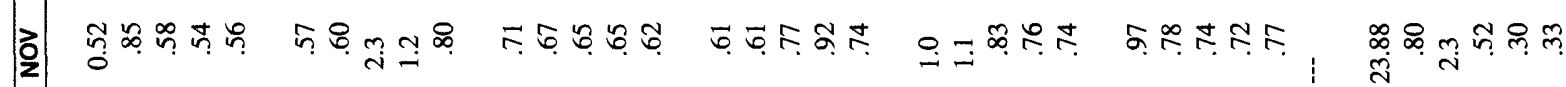

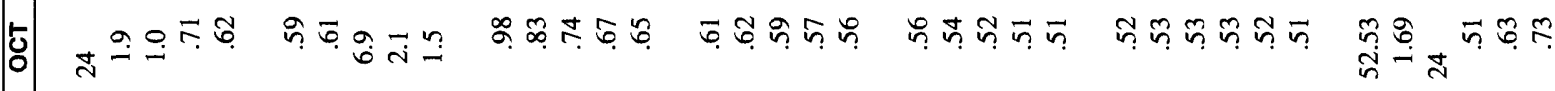

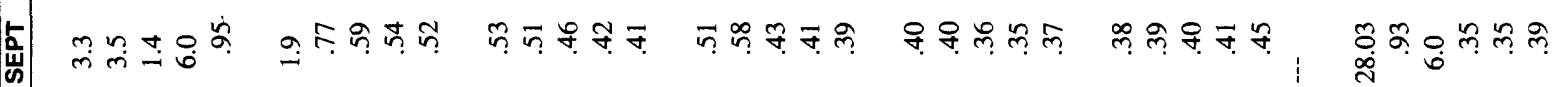

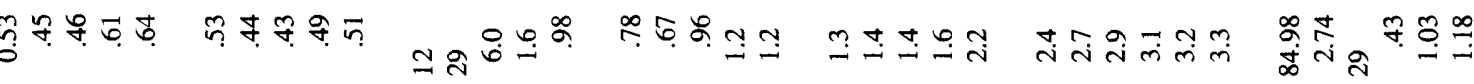

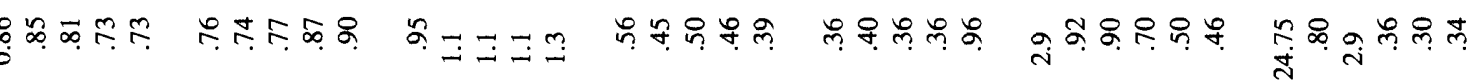

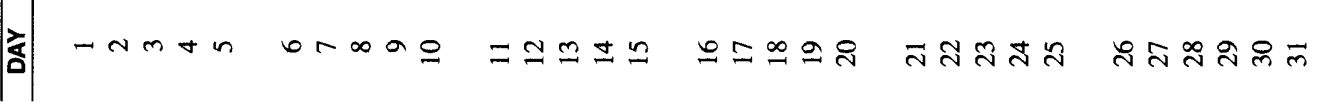

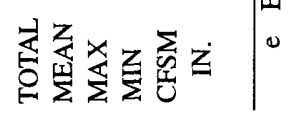




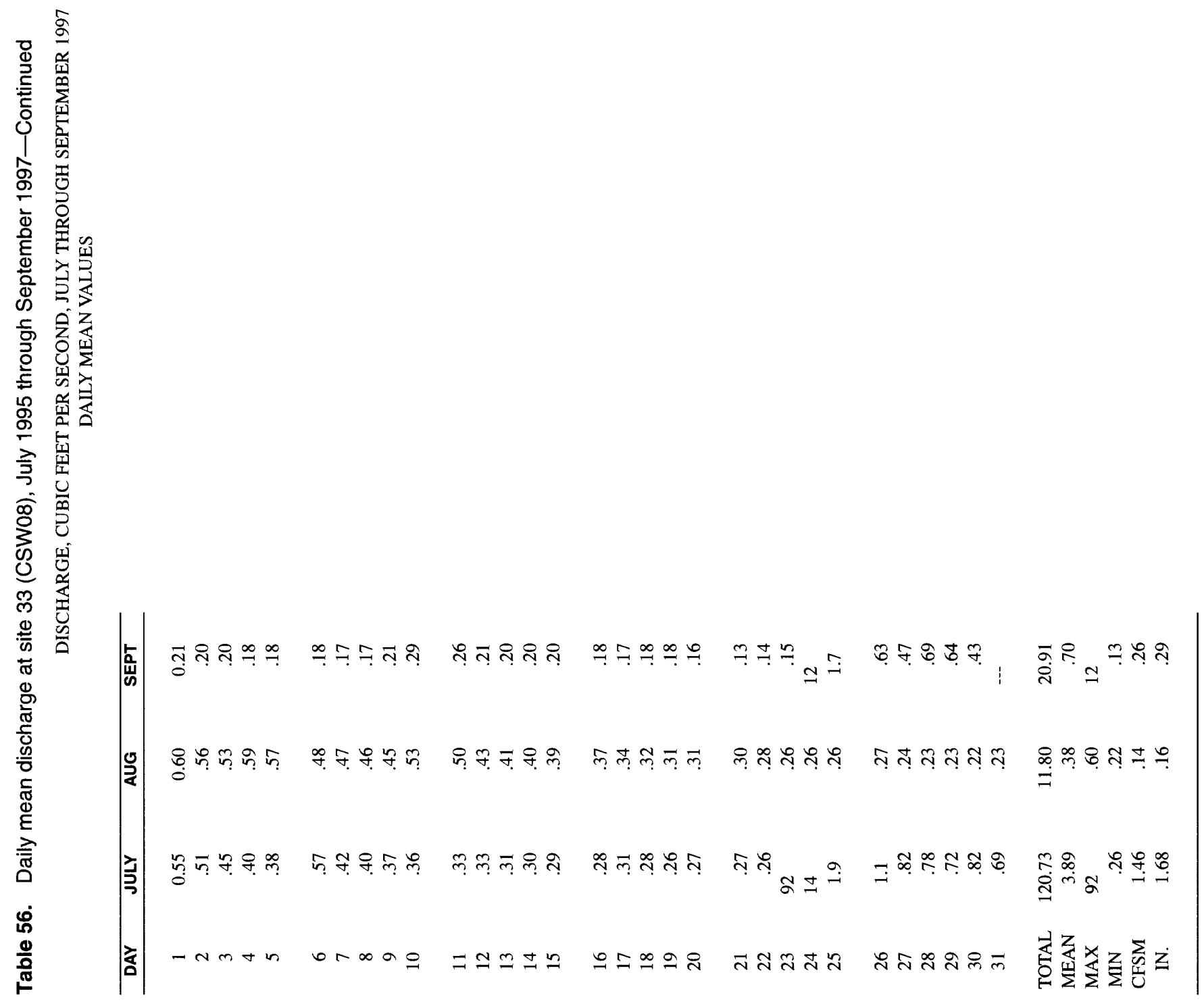


융유규

ฟ

䎹

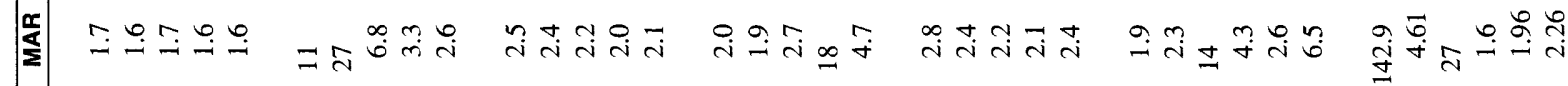

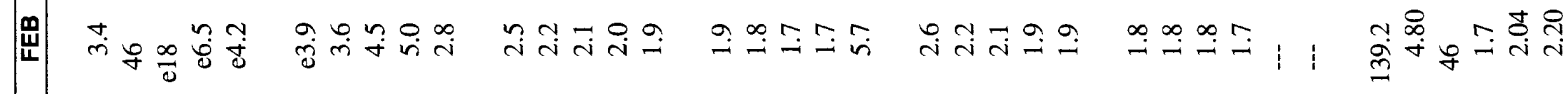

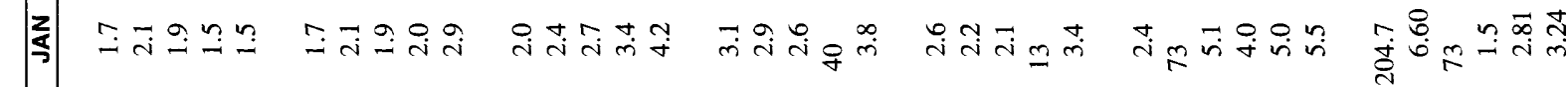

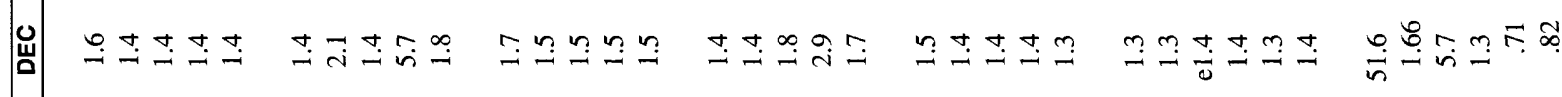

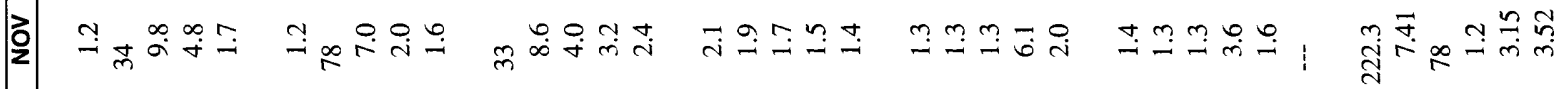

b

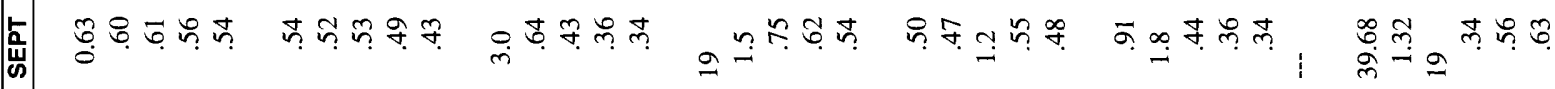

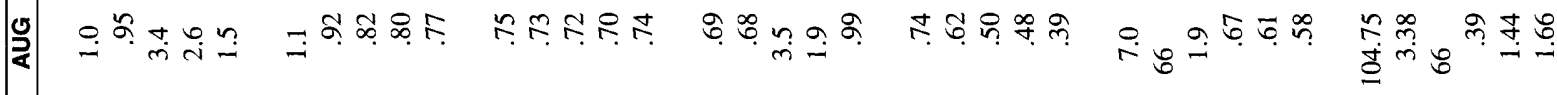

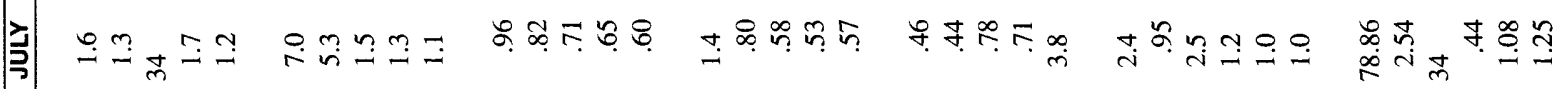

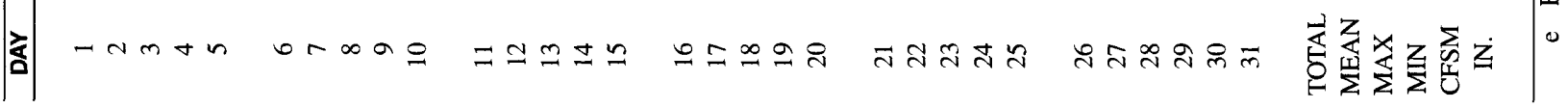


岁

爻

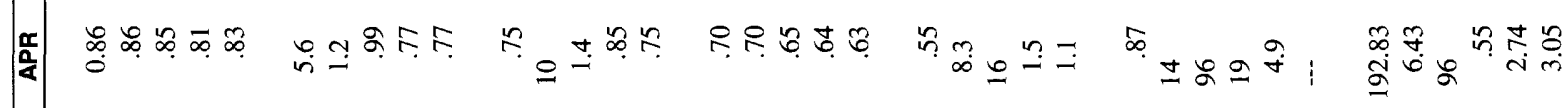

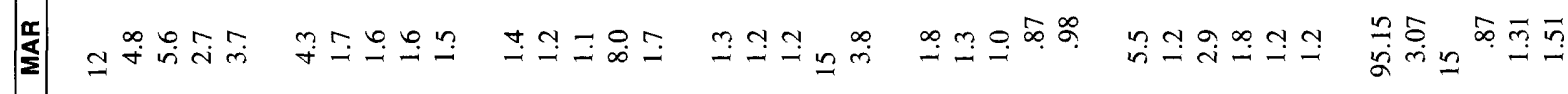

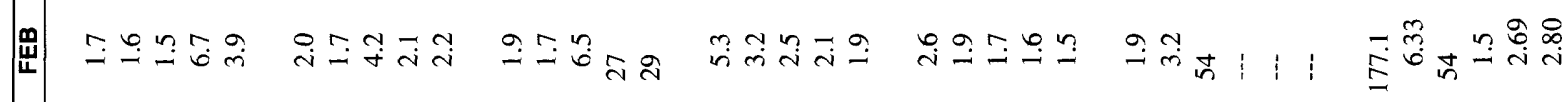

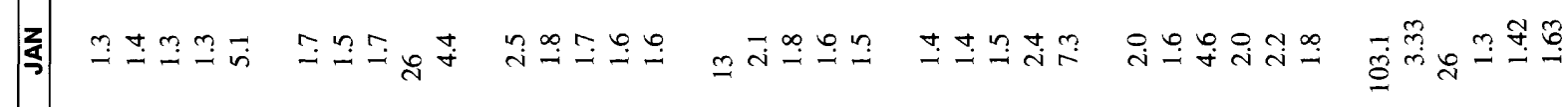

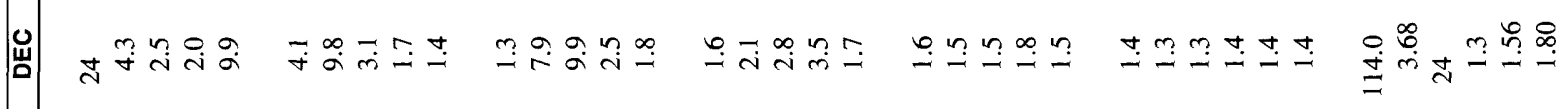

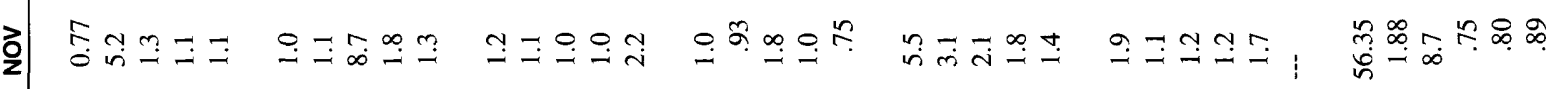

包

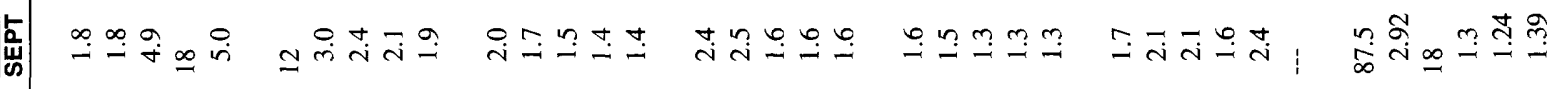

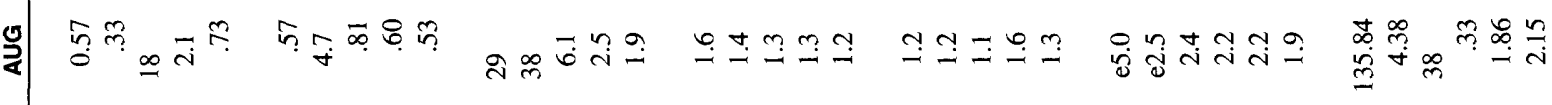

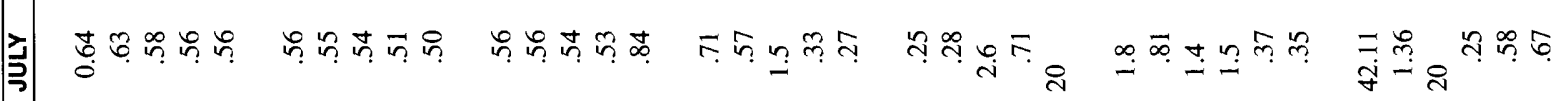

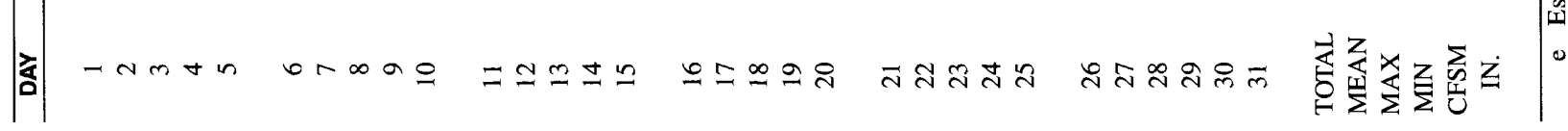




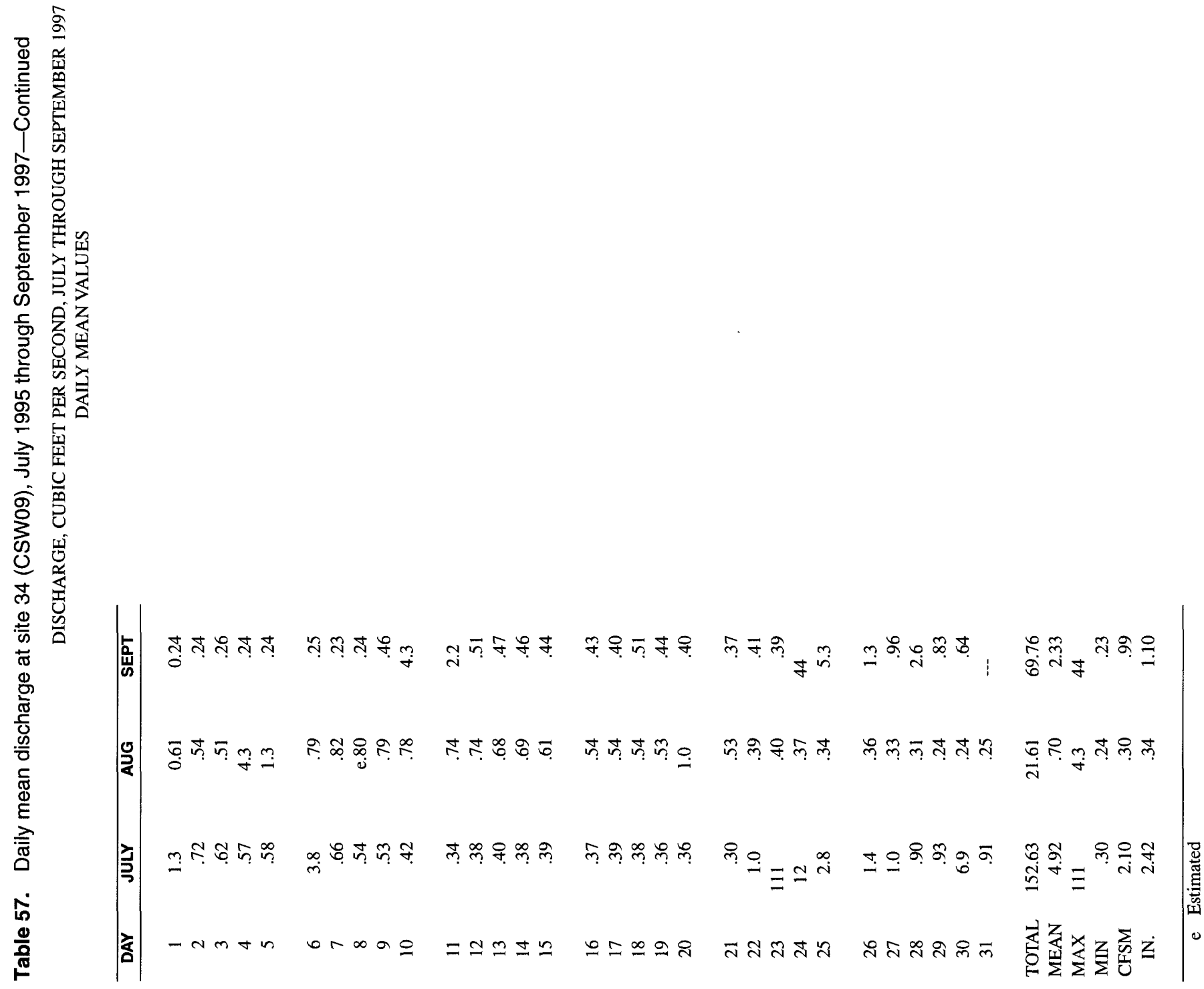




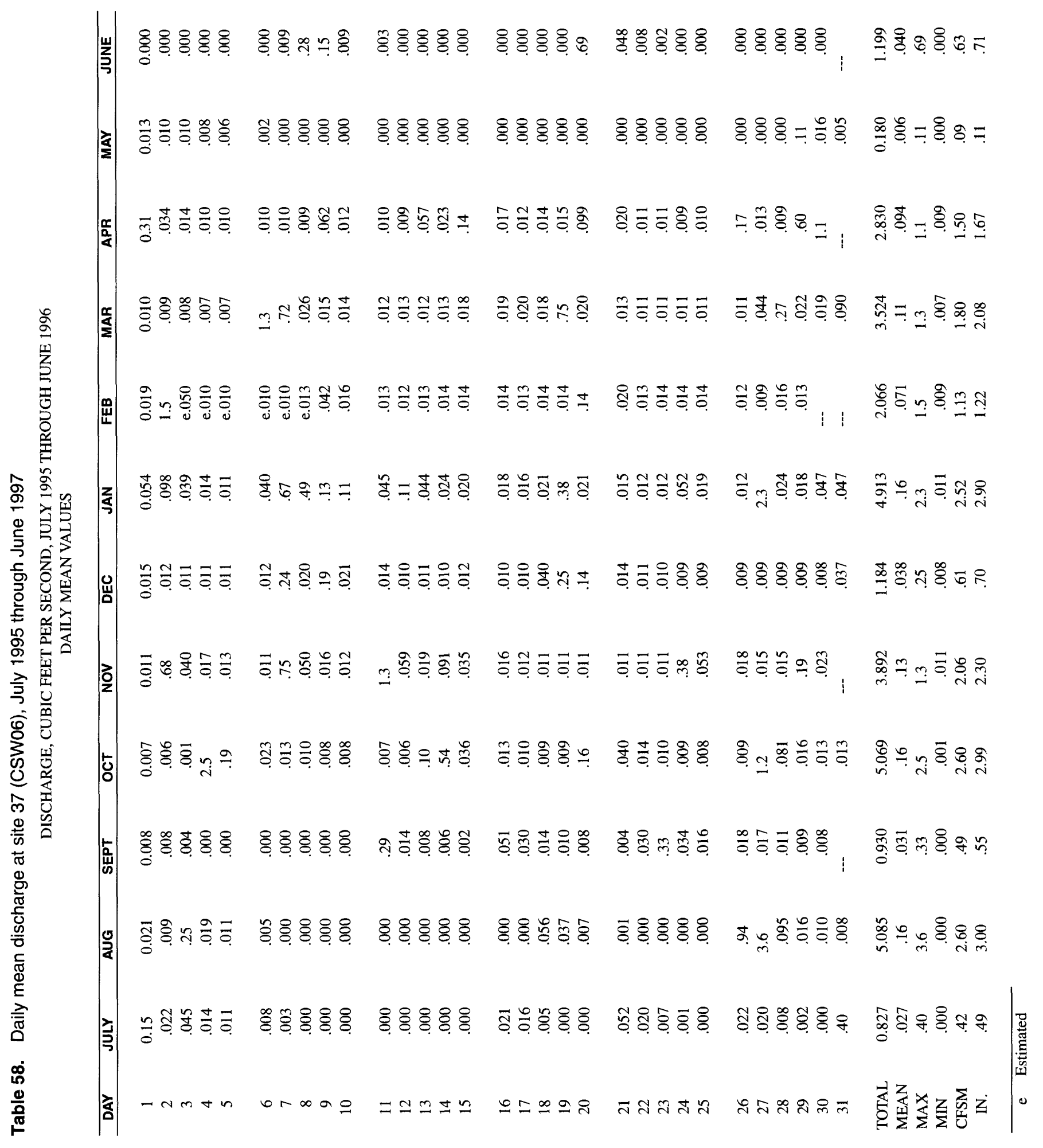




\begin{tabular}{|c|c|c|c|c|c|c|c|}
\hline$\frac{u}{5}$ & 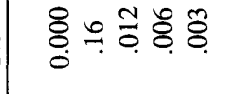 & 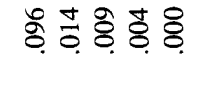 & จำ & ఫิ & 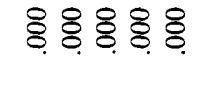 & 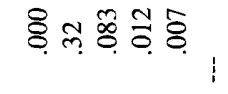 & 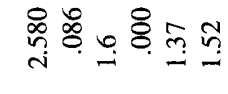 \\
\hline$\frac{2}{2}$ & 형 & 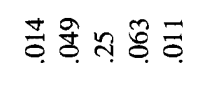 & 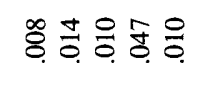 & 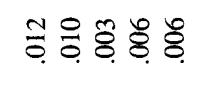 & 웅요 & 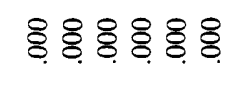 & 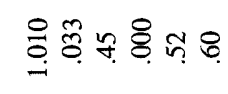 \\
\hline $\mid$ & 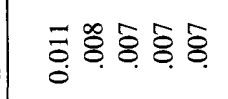 & 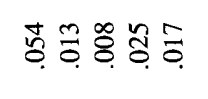 & ริำ & 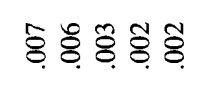 & 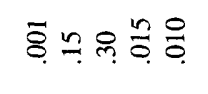 & 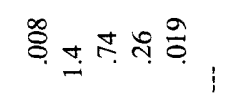 & 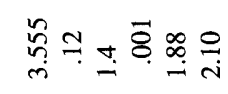 \\
\hline$\frac{\pi}{\frac{\pi}{2}}$ & 궁 & 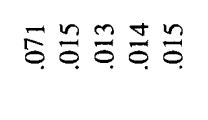 & 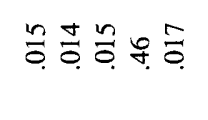 & ప் & 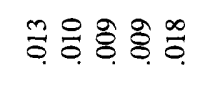 & 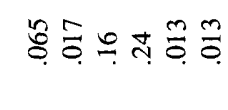 & 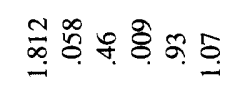 \\
\hline 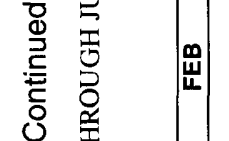 & 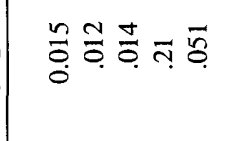 & 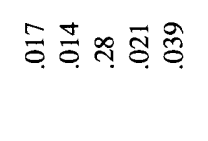 & 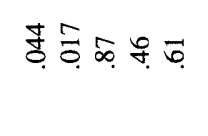 & 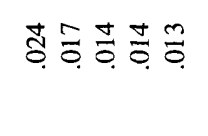 & 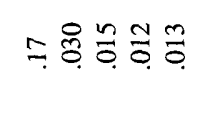 & 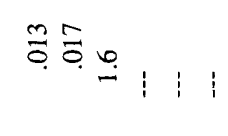 & 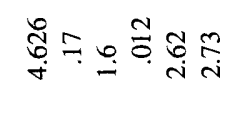 \\
\hline | & 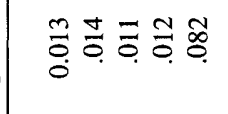 & 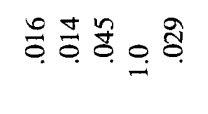 & 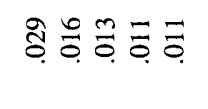 & 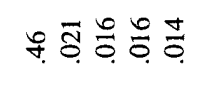 & 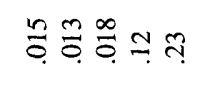 & 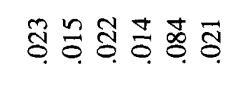 & 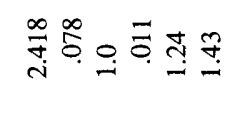 \\
\hline 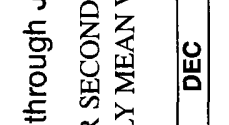 & 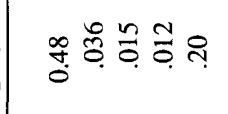 & 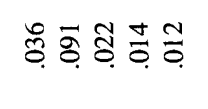 & 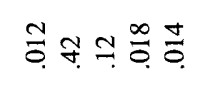 & 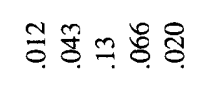 & 뭉 a & 훙 & 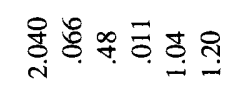 \\
\hline 定 & 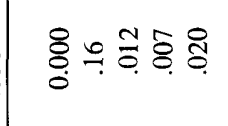 & 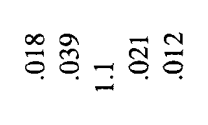 & 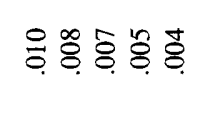 & $\overline{8}$ & 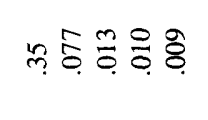 & 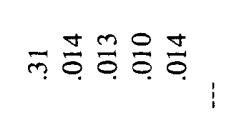 & 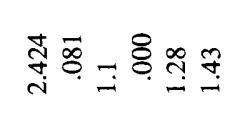 \\
\hline 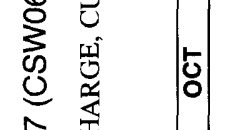 & $\bar{\infty}$ & 훙요 웅 & 范 & 훙ㅎㅇ용요 & \&: & $888 \%$ & 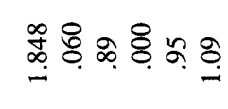 \\
\hline$\left|\begin{array}{|l}\mathbf{5} \\
\mathrm{w}\end{array}\right|$ & $8 \%$ & 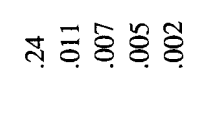 & 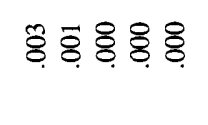 & 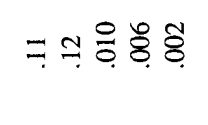 & $88 \% 8$ & కిำ & 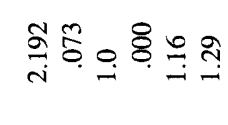 \\
\hline$\frac{5}{4}$ & 웡응 종 형 & 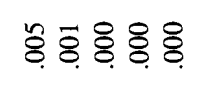 & 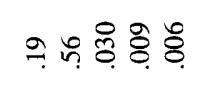 & $888 \%$ & 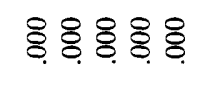 & 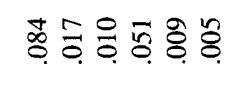 & 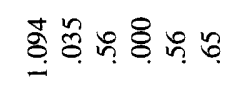 \\
\hline 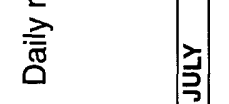 & $88 \% 8 \%$ & 웅 움웅용요 & 8 & 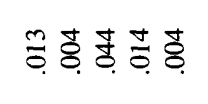 & 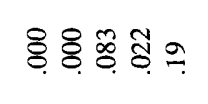 & 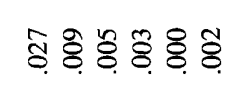 & 웅 \\
\hline $\mid$ & $-n m+n$ & orsag & $\Xi \simeq \cong \pm \cong$ & 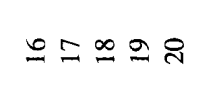 & สสฺส & 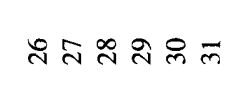 & 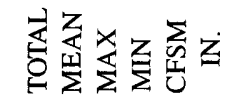 \\
\hline
\end{tabular}




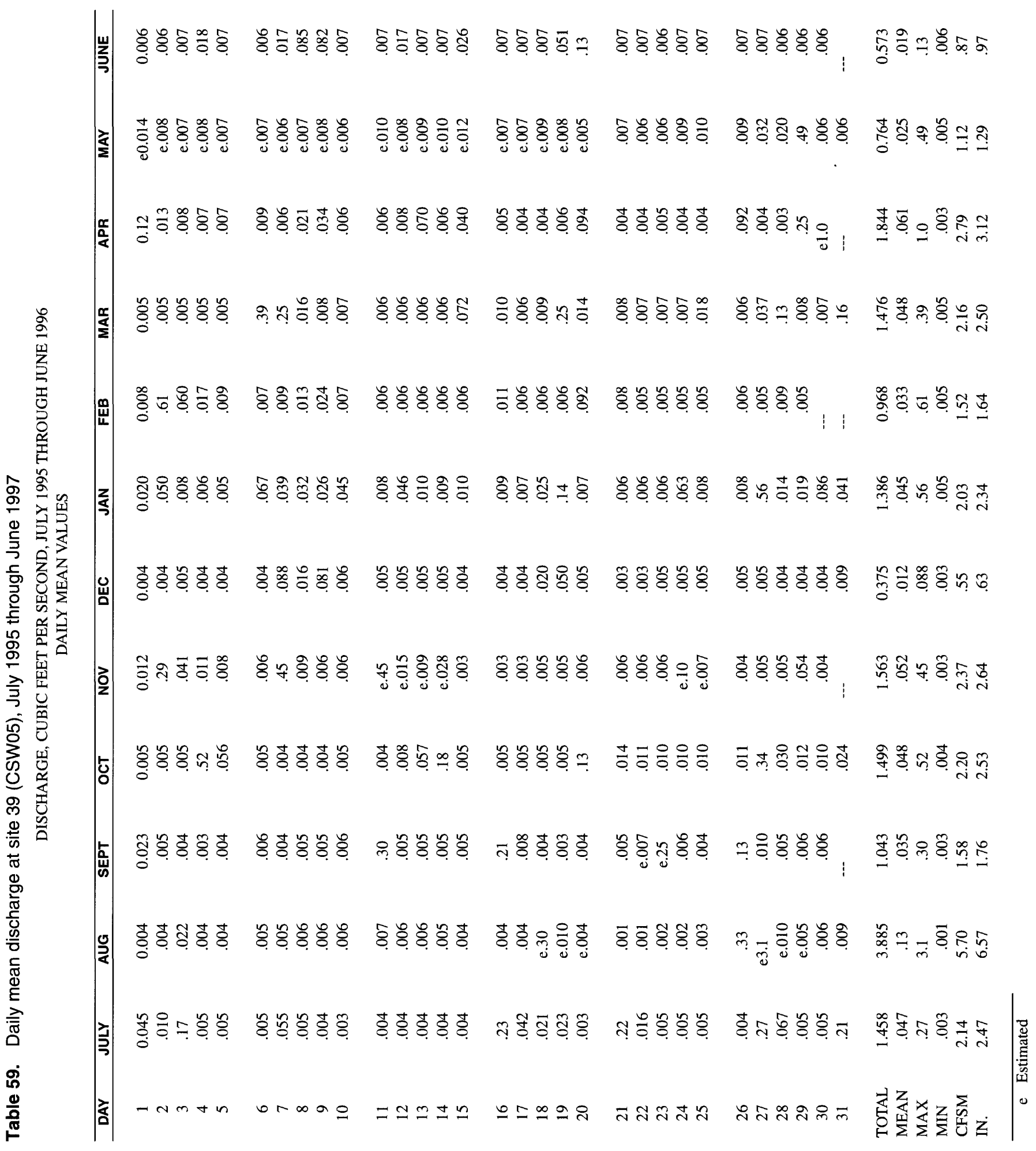




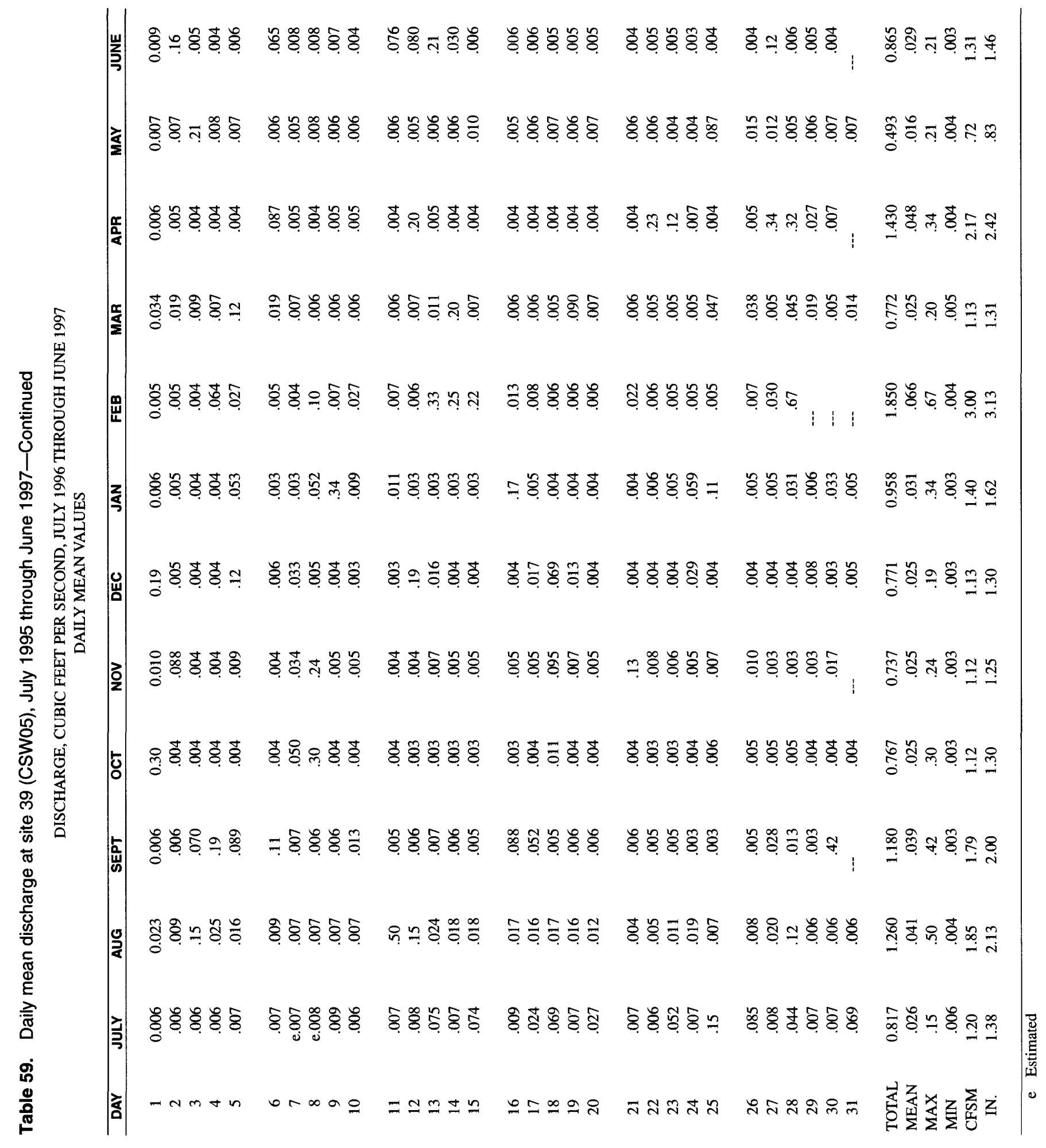




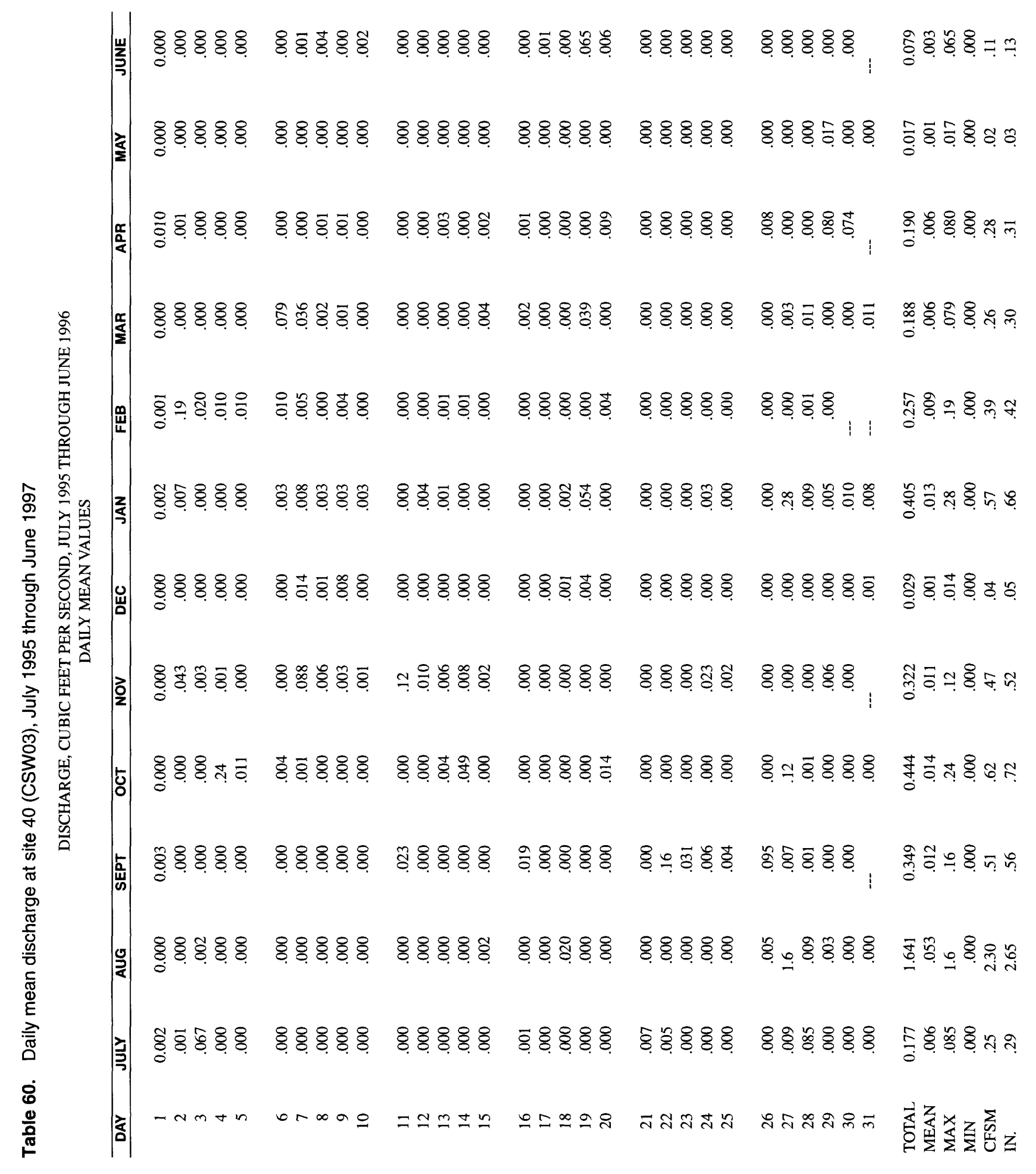




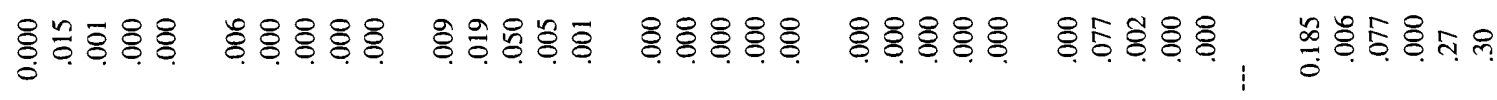

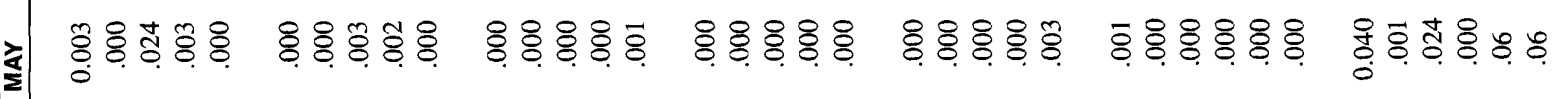

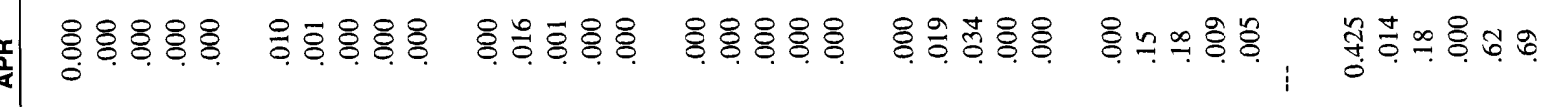

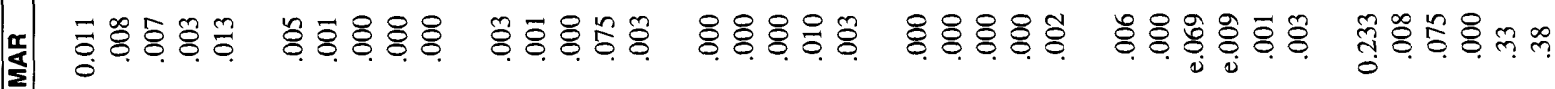

㞫

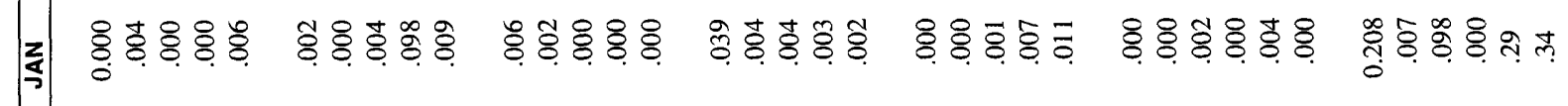

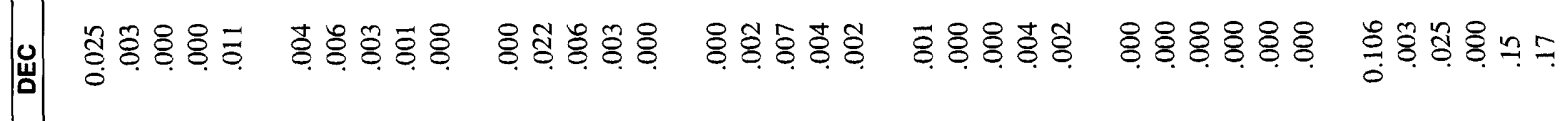

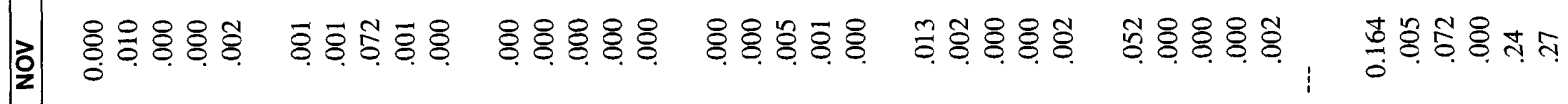

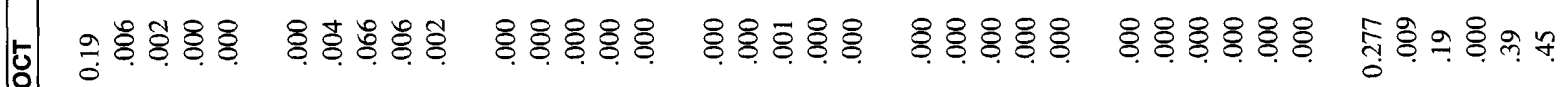

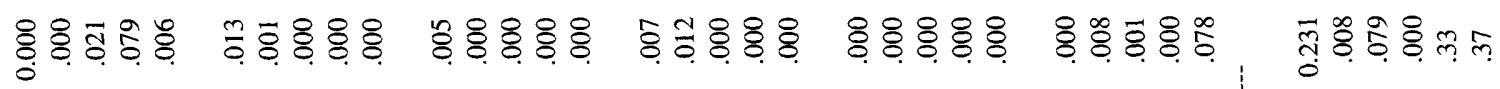

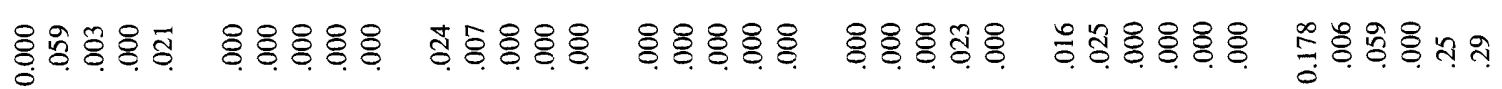

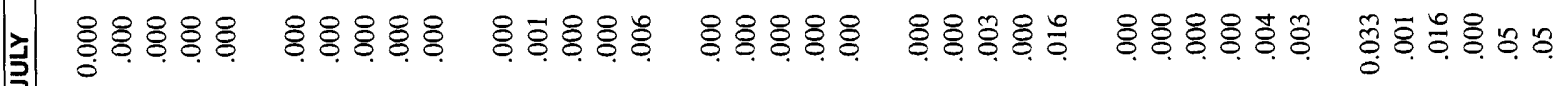




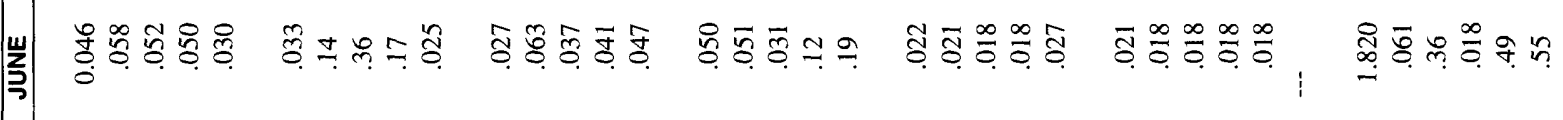

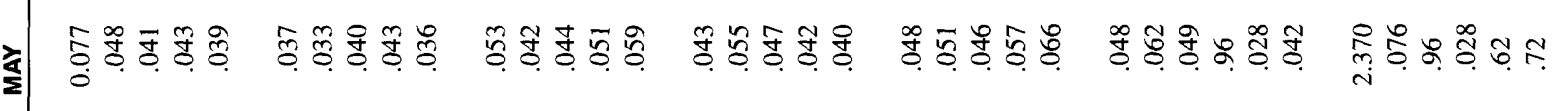

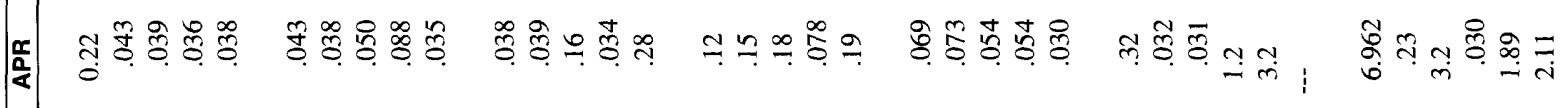

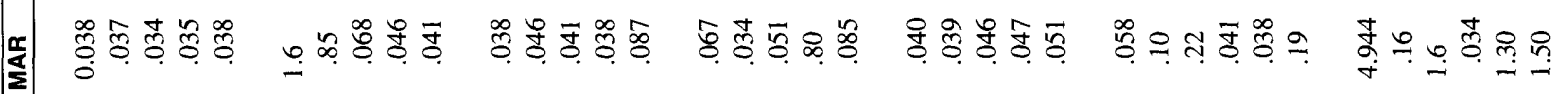

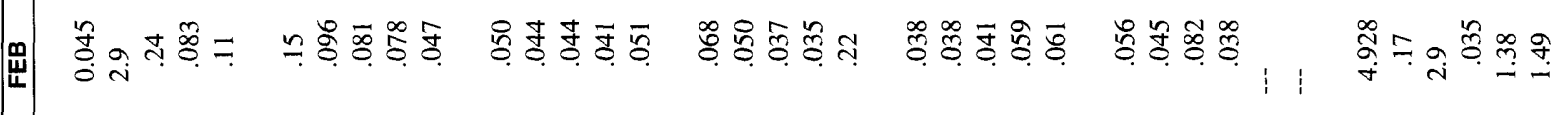

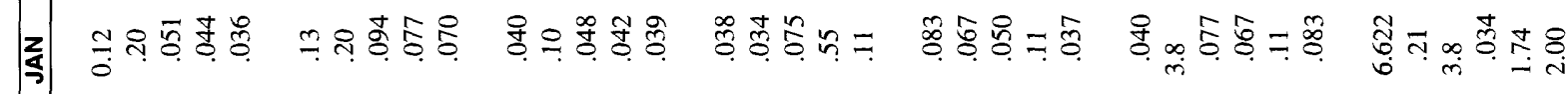

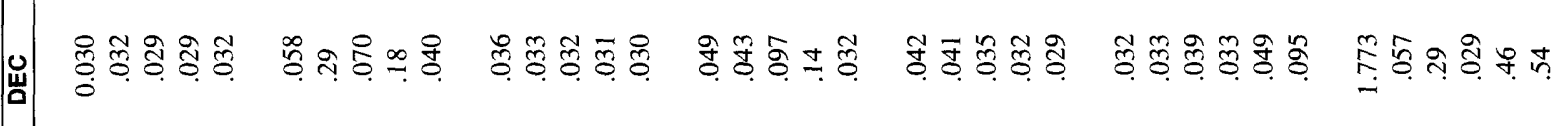

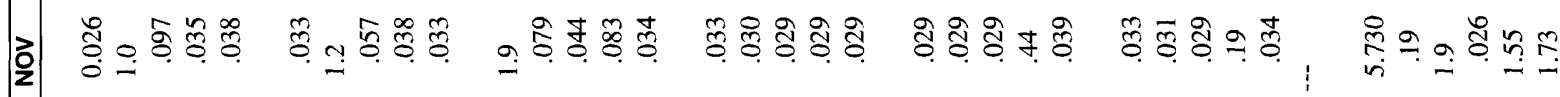

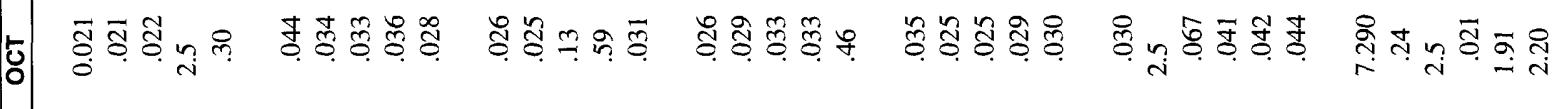

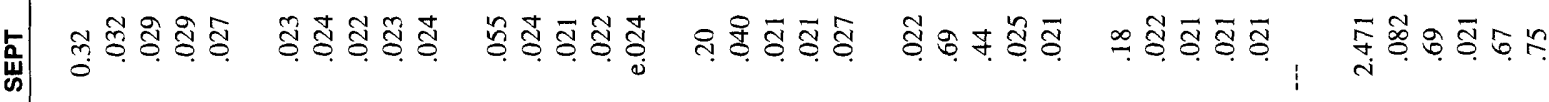

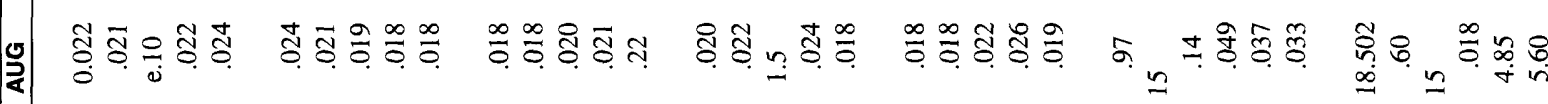

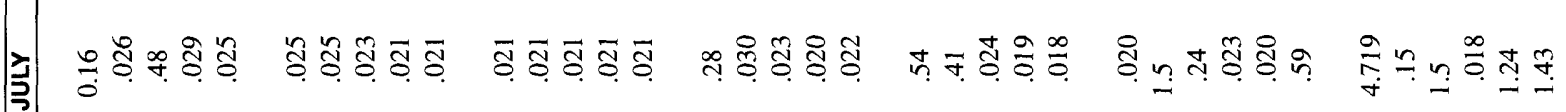




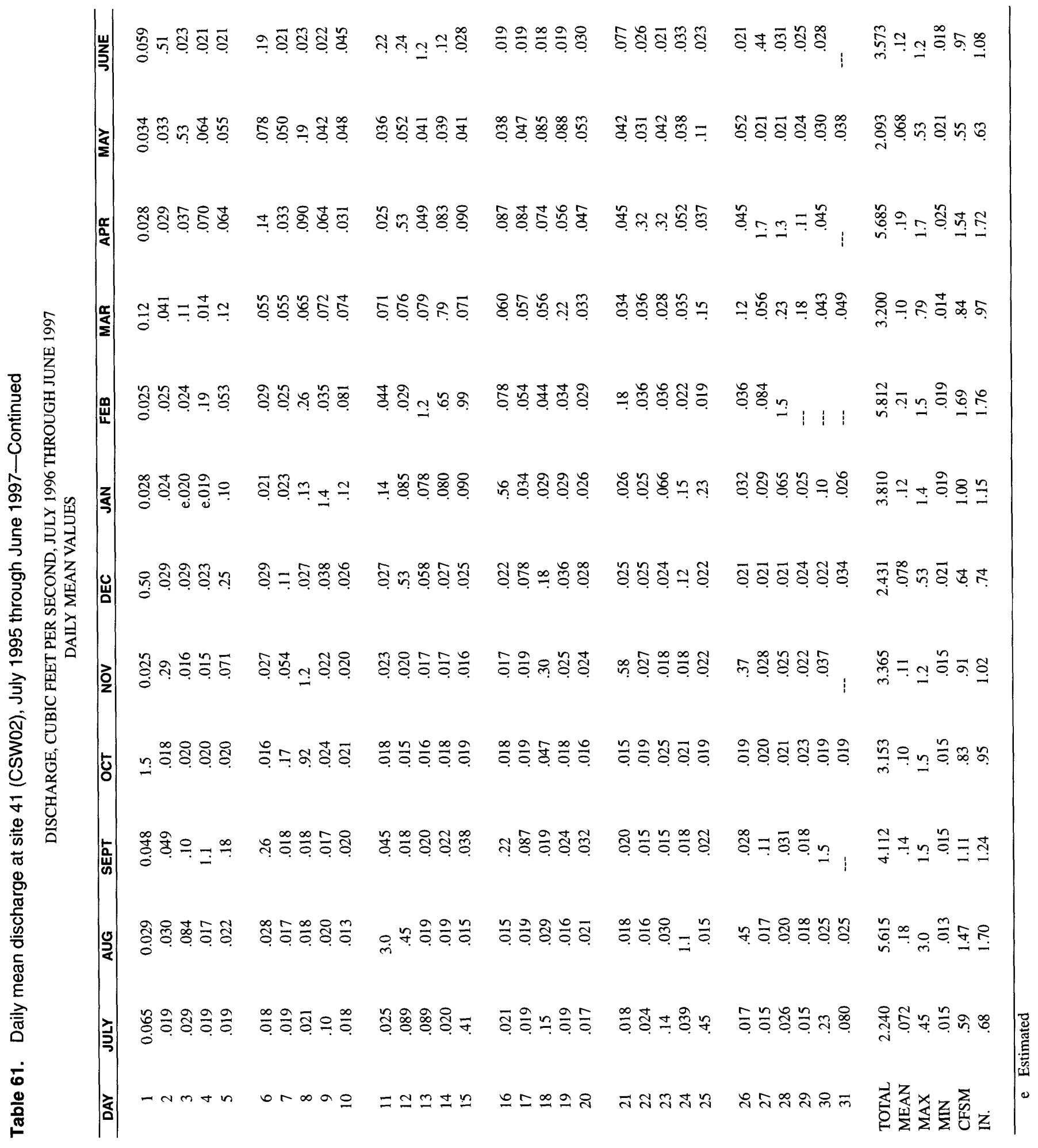




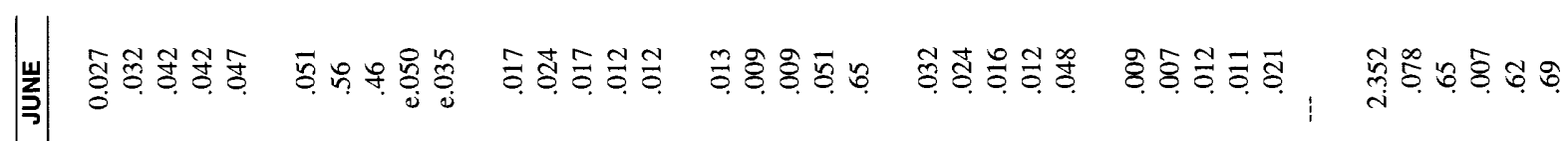

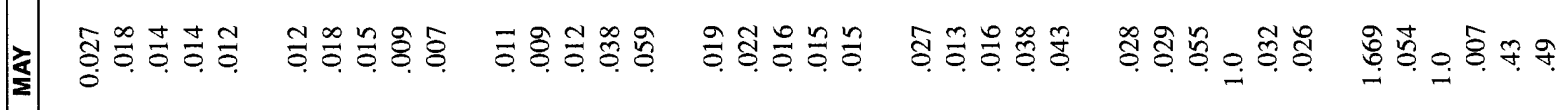

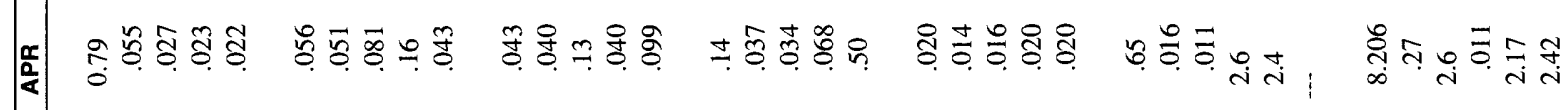

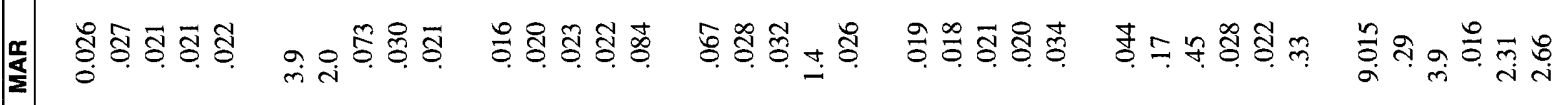

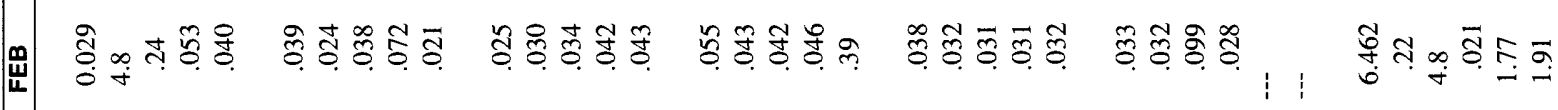

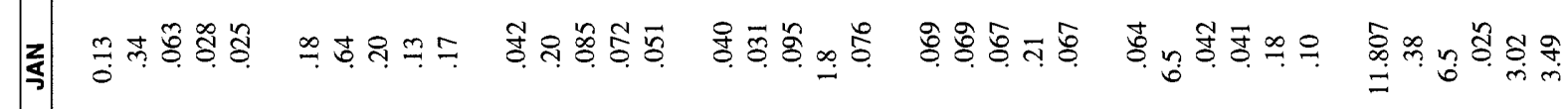

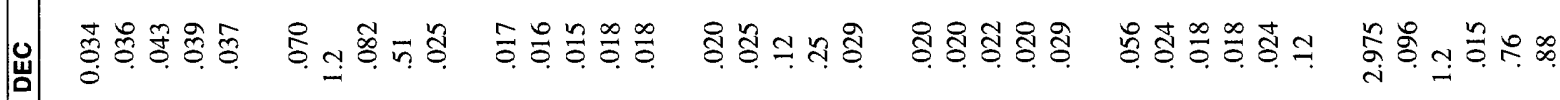

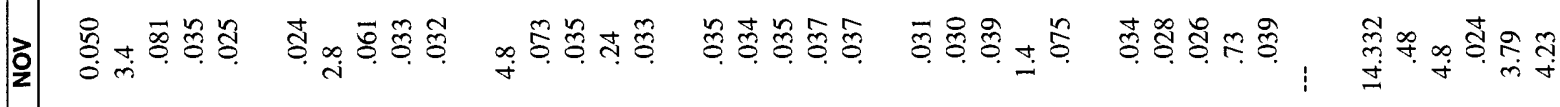

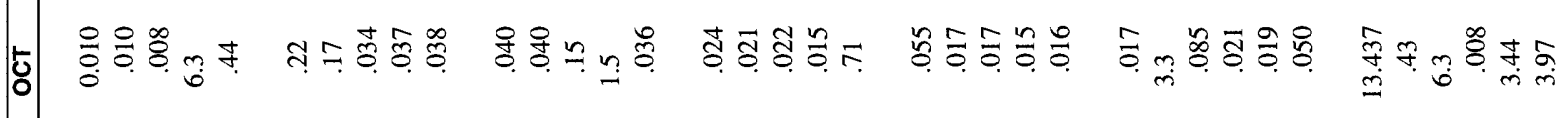

点 8

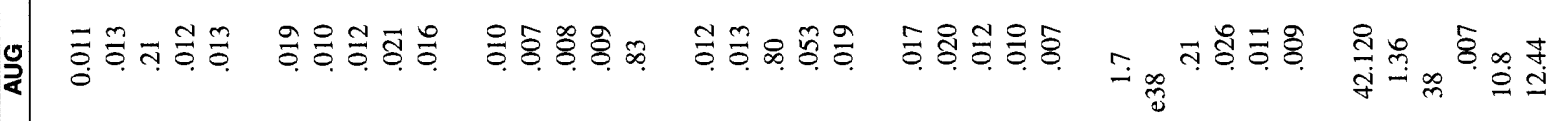

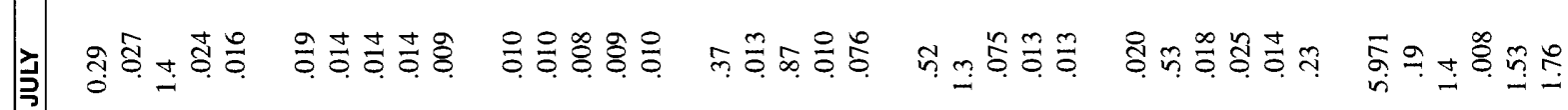

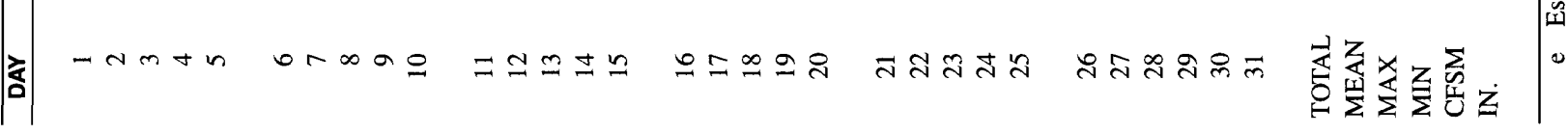




\begin{tabular}{|c|c|c|c|c|c|c|c|}
\hline & 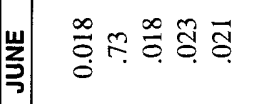 & 子 & 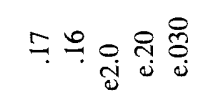 & 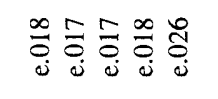 & 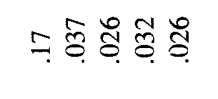 & 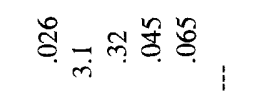 & 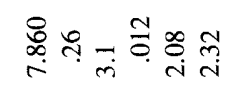 \\
\hline & $\frac{1}{2}$ & 중 & 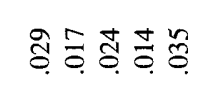 & 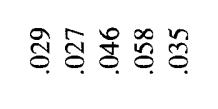 & 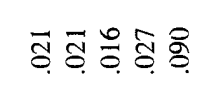 & 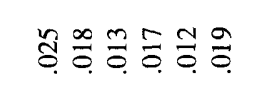 & 界 \\
\hline & $\frac{x}{4}$ & 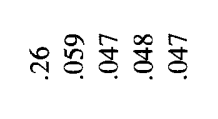 & 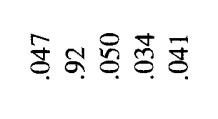 & 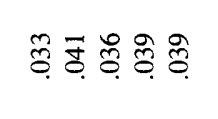 & 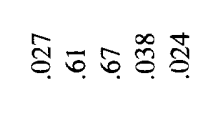 & $\underset{8}{8}$ & $\stackrel{\infty}{\stackrel{\infty}{g}}$ \\
\hline$\frac{\pi}{2}$ & $\frac{\alpha}{2}$ & $\simeq$ ฯ & חृق & 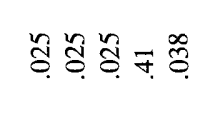 & ఫ్ర & 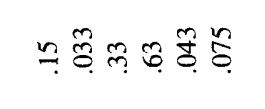 & 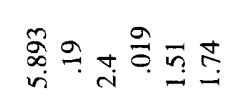 \\
\hline 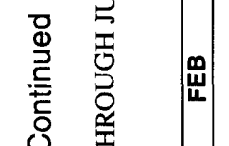 & 艛 & 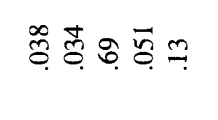 & 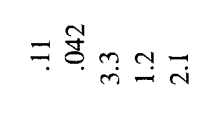 & $g$ & 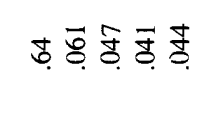 & 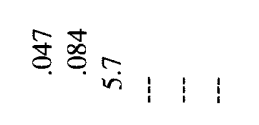 & 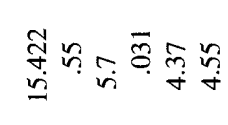 \\
\hline 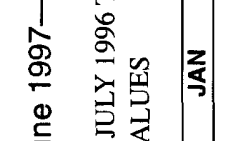 & 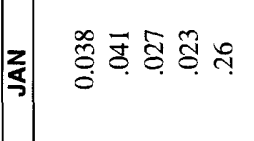 & 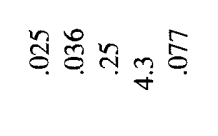 & 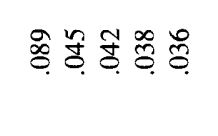 & 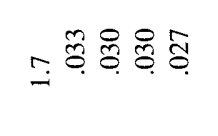 & 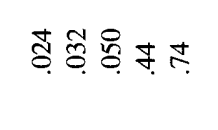 & 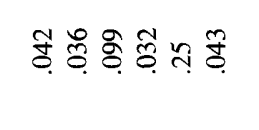 & 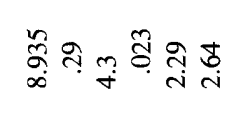 \\
\hline 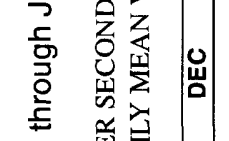 & هِ & 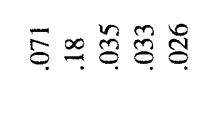 & $\check{\Xi}$ & 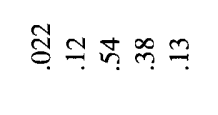 & $=$ & 중 중 & $\underset{\infty}{x}=$ \\
\hline 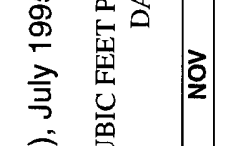 & 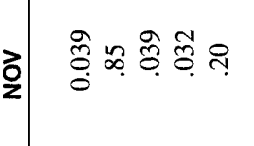 & 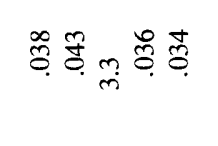 & 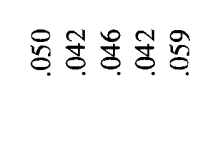 & 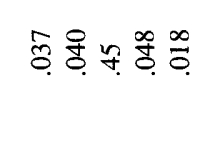 & 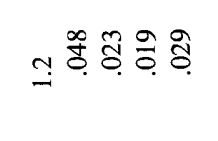 & 事 & वूले \\
\hline 告 & 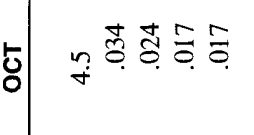 & 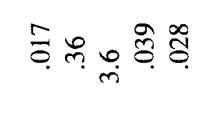 & 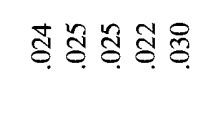 & 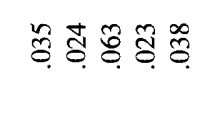 & 뭉 & 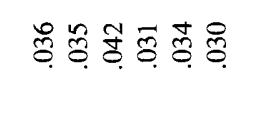 & कूष \\
\hline $\mid$ & 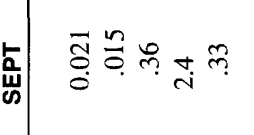 & 두ㅇㅝㅜ & جุ & 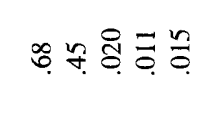 & 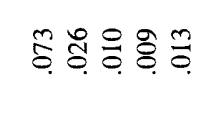 & 声 & $\stackrel{\infty}{\Delta=\infty}$ \\
\hline & 赵 & 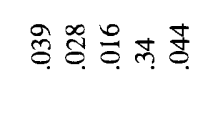 & 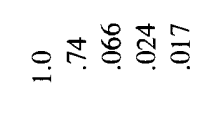 & 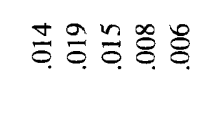 & 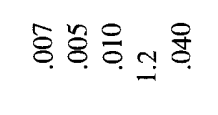 & 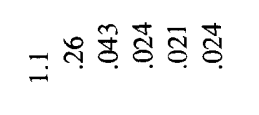 & 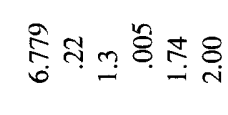 \\
\hline 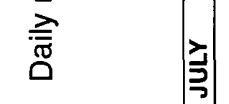 & 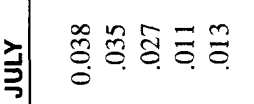 & 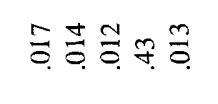 & 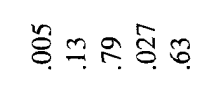 & 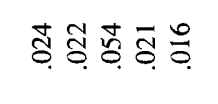 & 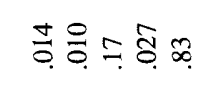 & 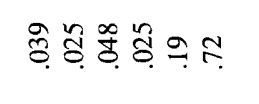 & $\underset{f}{q} \pm \infty$ \\
\hline 言 & 毠 & & $=3$ & $\therefore$ ते & 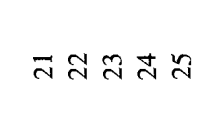 & 무 ה & \\
\hline
\end{tabular}




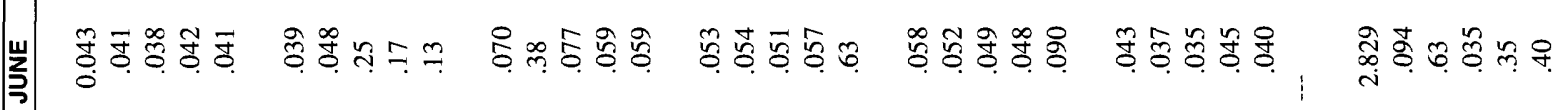

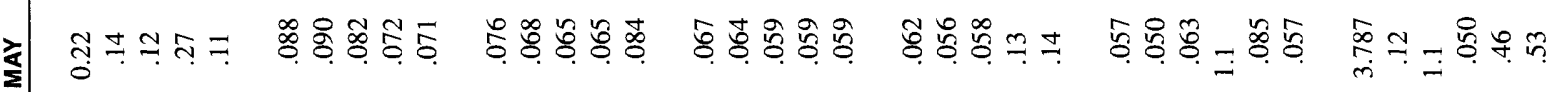

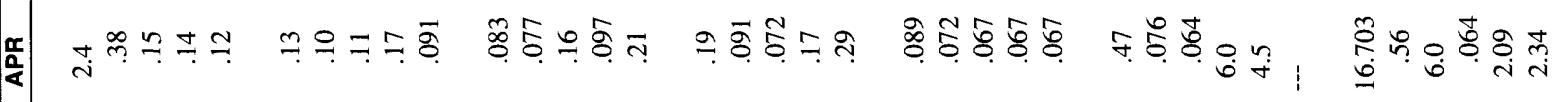

䇺

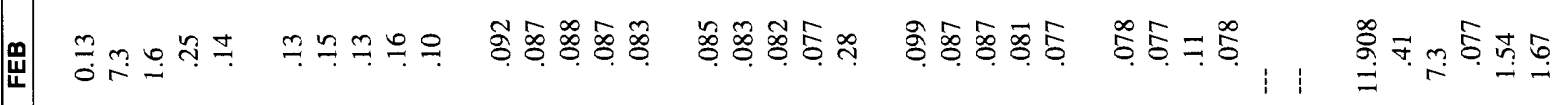

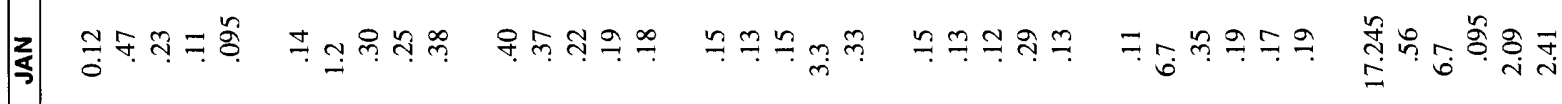

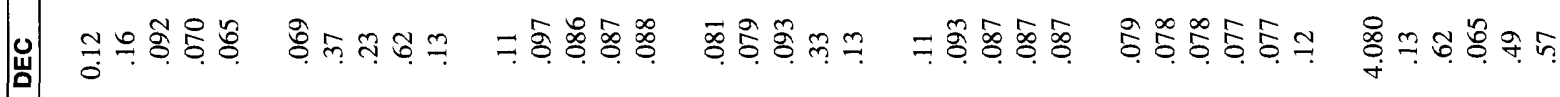

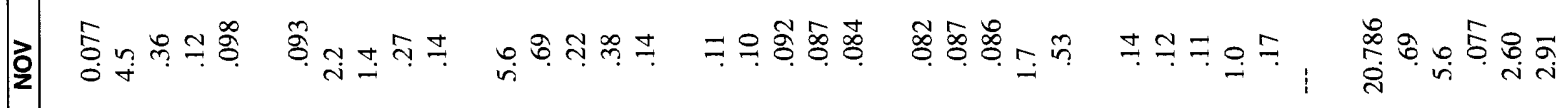

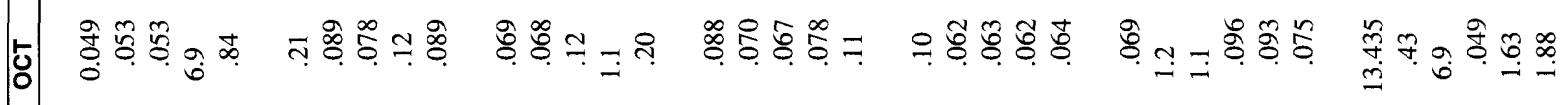

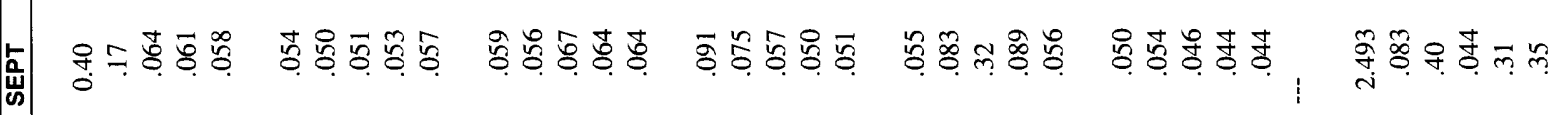

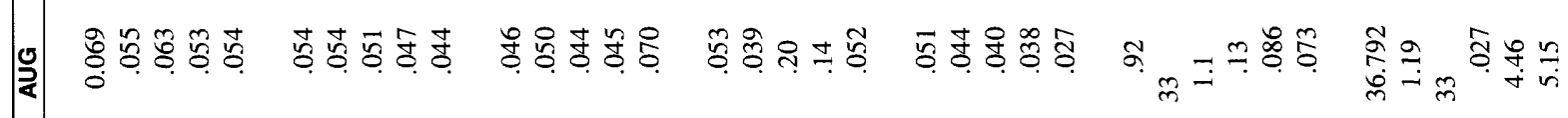

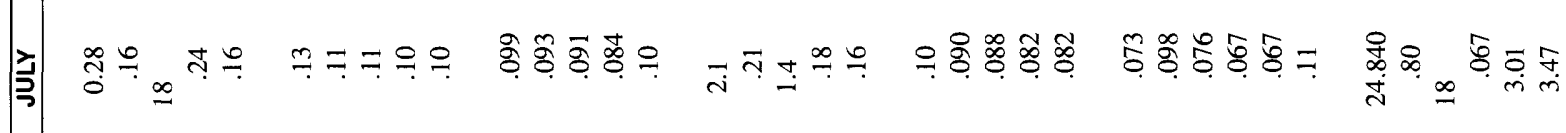

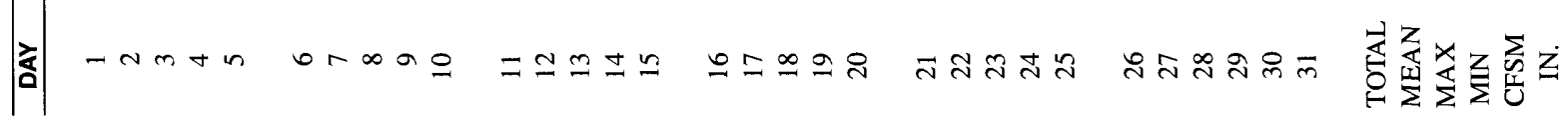




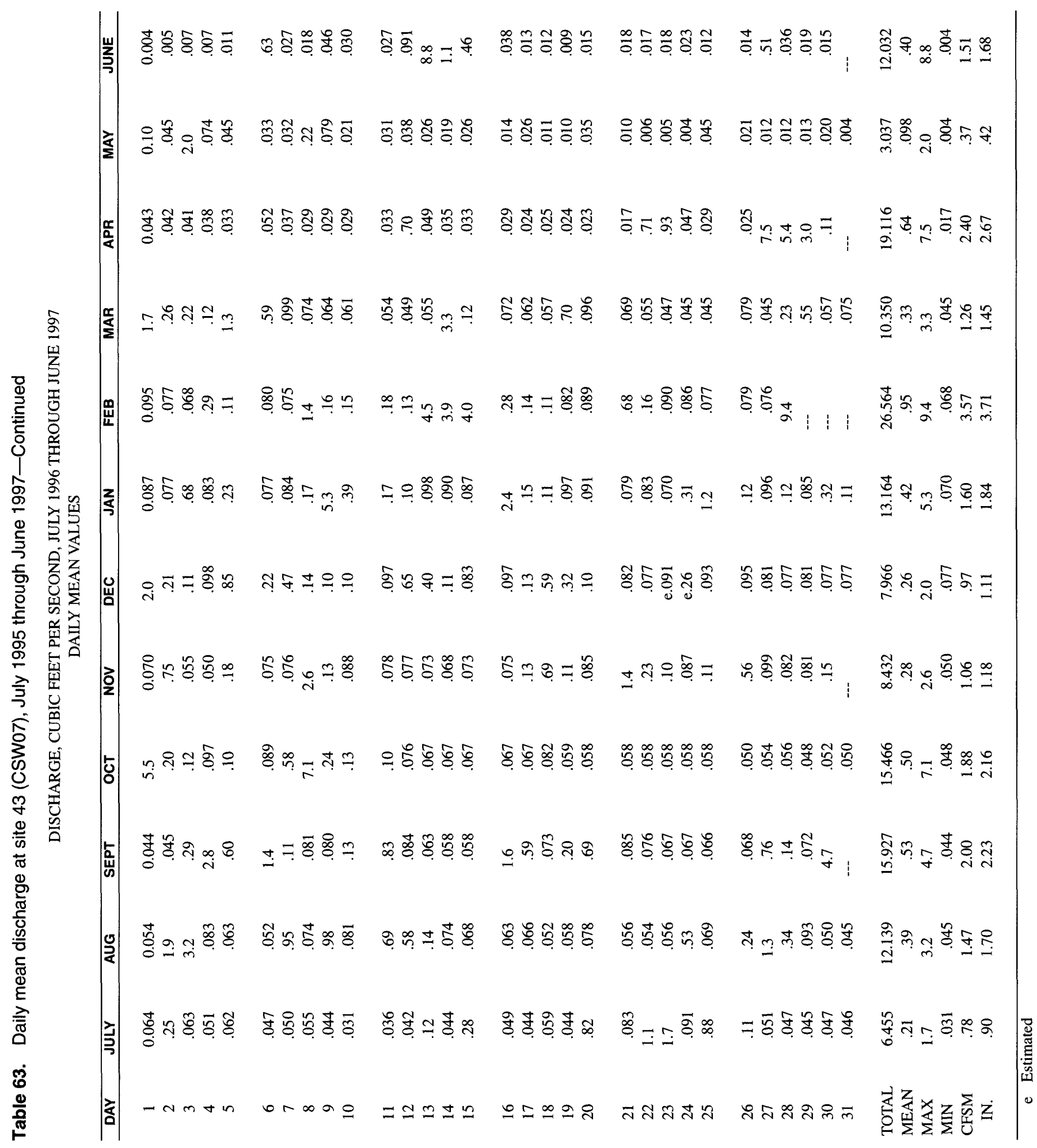




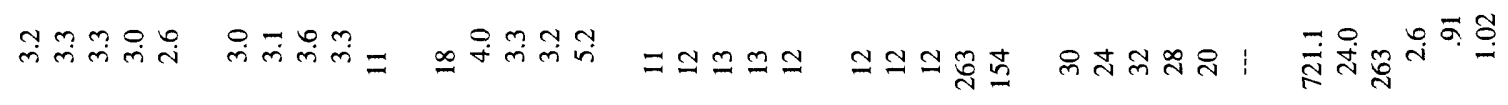

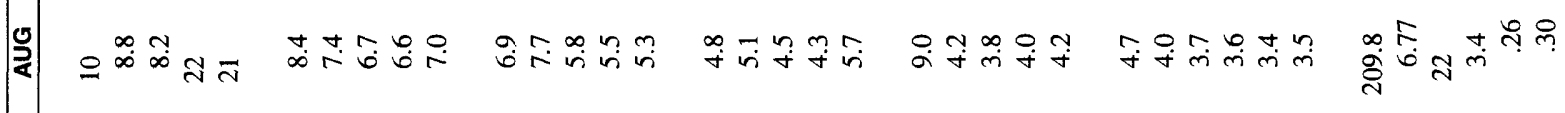

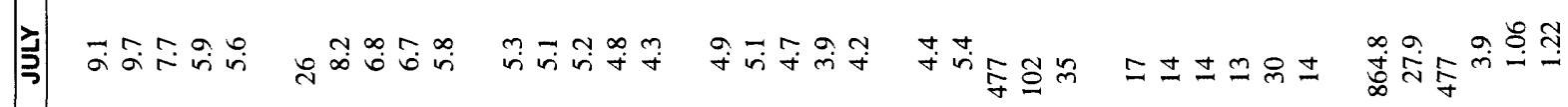

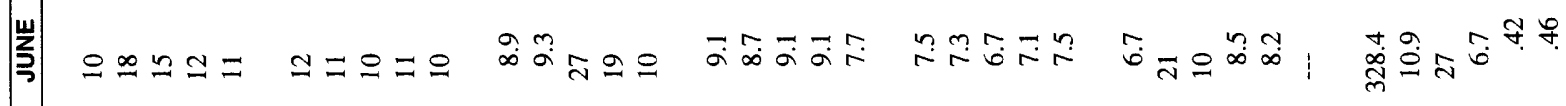

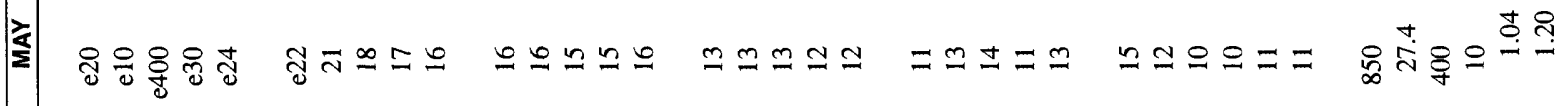
袮

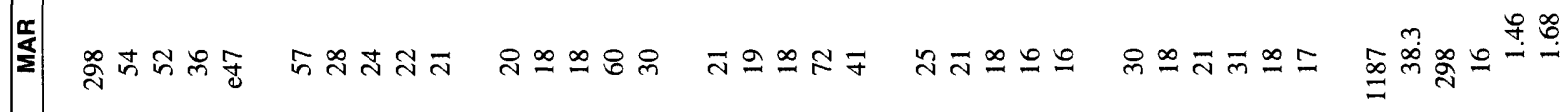

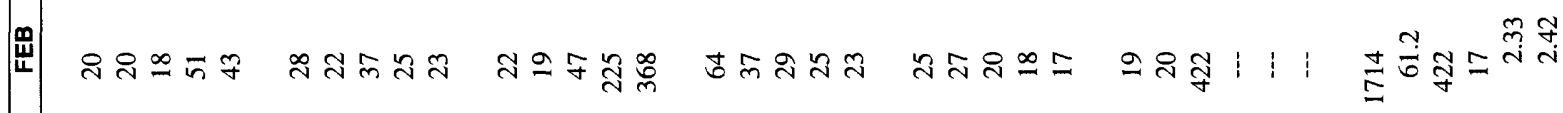

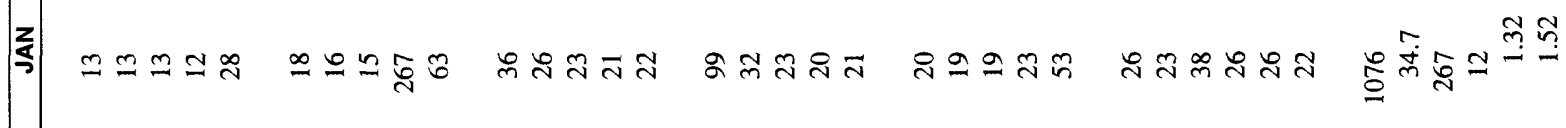

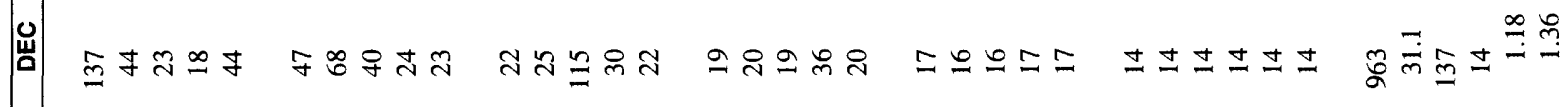

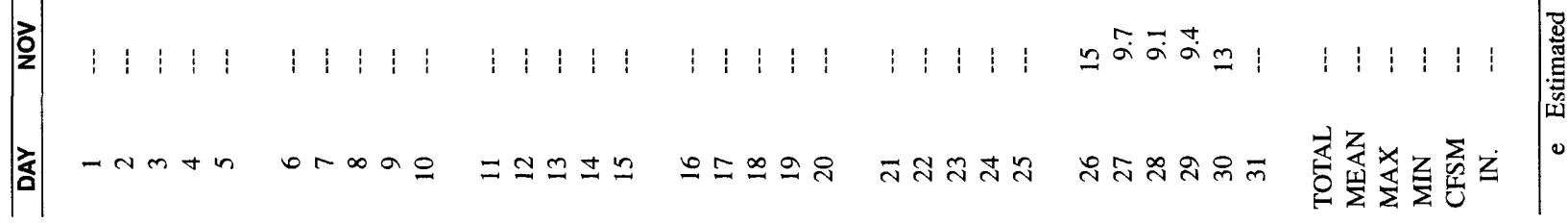


Table 65. Maximum, minimum, and median specific conductance and maximum and minimum water temperature recorded by monitors at the streamflow and water-quality study sites, October 1994 through September 1997

\begin{tabular}{|c|c|c|c|c|c|c|}
\hline \multirow{2}{*}{$\begin{array}{l}\text { Site no. } \\
\text { (fig. 1) }\end{array}$} & \multirow{2}{*}{ Period of record } & \multicolumn{3}{|c|}{$\begin{array}{l}\text { Specific conductance } \\
\left(\mu \mathrm{s} / \mathrm{cm} \text { at } 25^{\circ} \mathrm{C}\right)\end{array}$} & \multicolumn{2}{|c|}{$\begin{array}{l}\text { Water temperature } \\
\left({ }^{\circ} \mathrm{C}\right)\end{array}$} \\
\hline & & $\begin{array}{l}\text { Maximum } \\
\text { (Date) }\end{array}$ & $\begin{array}{c}\text { Minimum } \\
\text { (Date) }\end{array}$ & Median & $\begin{array}{c}\text { Maximum } \\
\text { (Date) }\end{array}$ & $\begin{array}{c}\text { Minimum } \\
\text { (Date) }\end{array}$ \\
\hline $\begin{array}{c}33^{\mathrm{a}} \\
{[\mathrm{CSW} 08]}\end{array}$ & $10 / 94-9 / 97$ & $\begin{array}{c}567 \\
(9 / 4 / 96)\end{array}$ & $\begin{array}{c}36 \\
(7 / 23 / 97)\end{array}$ & 162 & $\begin{array}{c}27.5 \\
(7 / 22 / 97)\end{array}$ & $\begin{array}{c}0.3 \\
(1 / 6 / 95)\end{array}$ \\
\hline $\begin{array}{c}34^{\mathrm{a}} \\
{[\mathrm{CSW} 09]}\end{array}$ & $10 / 94-9 / 97$ & $\begin{array}{c}441 \\
(1 / 9 / 96)\end{array}$ & $\begin{array}{c}17 \\
(7 / 17 / 96 ; 7 / 23 / 97)\end{array}$ & 113 & $\begin{array}{c}32.4 \\
(8 / 14 / 95)\end{array}$ & $\begin{array}{c}0.0 \\
(2 / 9 / 95 ; 1 / 7 / 96)\end{array}$ \\
\hline $\begin{array}{c}37 \\
\text { [CSW06] }\end{array}$ & $5 / 95-6 / 97$ & $\begin{array}{c}842 \\
(1 / 9 / 96)\end{array}$ & $\begin{array}{c}23 \\
(4 / 30 / 96 ; 4 / 27 / 97)\end{array}$ & 132 & $\begin{array}{c}38.1 \\
(6 / 23 / 96)\end{array}$ & $\begin{array}{c}0.3 \\
(1 / 7 / 96)\end{array}$ \\
\hline $\begin{array}{c}39 \\
\text { [CSW05] }\end{array}$ & $10 / 94-6 / 97$ & $\begin{array}{c}7,060 \\
(1 / 9 / 96)\end{array}$ & $\begin{array}{c}11 \\
(1 / 13 / 95)\end{array}$ & 356 & $\begin{array}{c}34.7 \\
(7 / 31 / 95)\end{array}$ & $\begin{array}{c}0.7 \\
(2 / 13 / 97)\end{array}$ \\
\hline $\begin{array}{c}40 \\
\text { [CSW03] }\end{array}$ & $10 / 94-6 / 97$ & $\begin{array}{c}3,000 \\
(1 / 12 / 96)\end{array}$ & $\begin{array}{c}10 \\
(10 / 10,14 / 94)\end{array}$ & 220 & $\begin{array}{c}31.0 \\
(8 / 15 / 95)\end{array}$ & $\begin{array}{c}0.4 \\
(2 / 5,6 / 96)\end{array}$ \\
\hline $\begin{array}{c}41 \\
{[C S W 02]}\end{array}$ & $10 / 94-6 / 97$ & $\begin{array}{c}4,480 \\
(2 / 4 / 96)\end{array}$ & $\begin{array}{c}12 \\
(6 / 19 / 95)\end{array}$ & 157 & $\begin{array}{c}31.6 \\
(8 / 15 / 95)\end{array}$ & $\begin{array}{c}2.0 \\
(2 / 13 / 97)\end{array}$ \\
\hline $\begin{array}{c}42 \\
\text { [CSW04] }\end{array}$ & $10 / 94-6 / 97$ & $\begin{array}{c}13,900 \\
(5 / 22 / 96)\end{array}$ & $\begin{array}{c}10 \\
(1 / 5 / 96)\end{array}$ & 286 & $\begin{array}{c}32.4 \\
(8 / 15 / 95)\end{array}$ & $\begin{array}{c}0.1 \\
(1 / 7 / 96)\end{array}$ \\
\hline $\begin{array}{c}43 \\
\text { [CSW07] }\end{array}$ & $10 / 94-6 / 97$ & $\begin{array}{c}933 \\
(2 / 10 / 95)\end{array}$ & $\begin{array}{c}27 \\
(1 / 7 / 96)\end{array}$ & 217 & $\begin{array}{c}33.0 \\
(6 / 24 / 97)\end{array}$ & $\begin{array}{c}0.0 \\
(1 / 6,2 / 7,9,12 / 25-30 / 95 \\
1 / 7,12,2 / 4-6 / 96 ; 1 / 18 / 97)\end{array}$ \\
\hline $\begin{array}{c}44^{\mathrm{b}} \\
{[\mathrm{CSW} 10]}\end{array}$ & $11 / 96-9 / 97$ & $\begin{array}{c}602 \\
(6 / 19 / 97)\end{array}$ & $\begin{array}{c}39 \\
(7 / 23 / 97)\end{array}$ & 146 & $\begin{array}{c}33.2 \\
(7 / 21 / 97)\end{array}$ & $\begin{array}{c}0.1 \\
(12 / 21 / 96)\end{array}$ \\
\hline
\end{tabular}

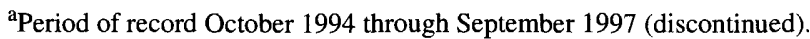

${ }^{b}$ Period of record November 1996 through September 1997 (discontinued). 
Table 66. Statistical summary of water-quality data at site 33 (CSW08), June 1994 through September 1997

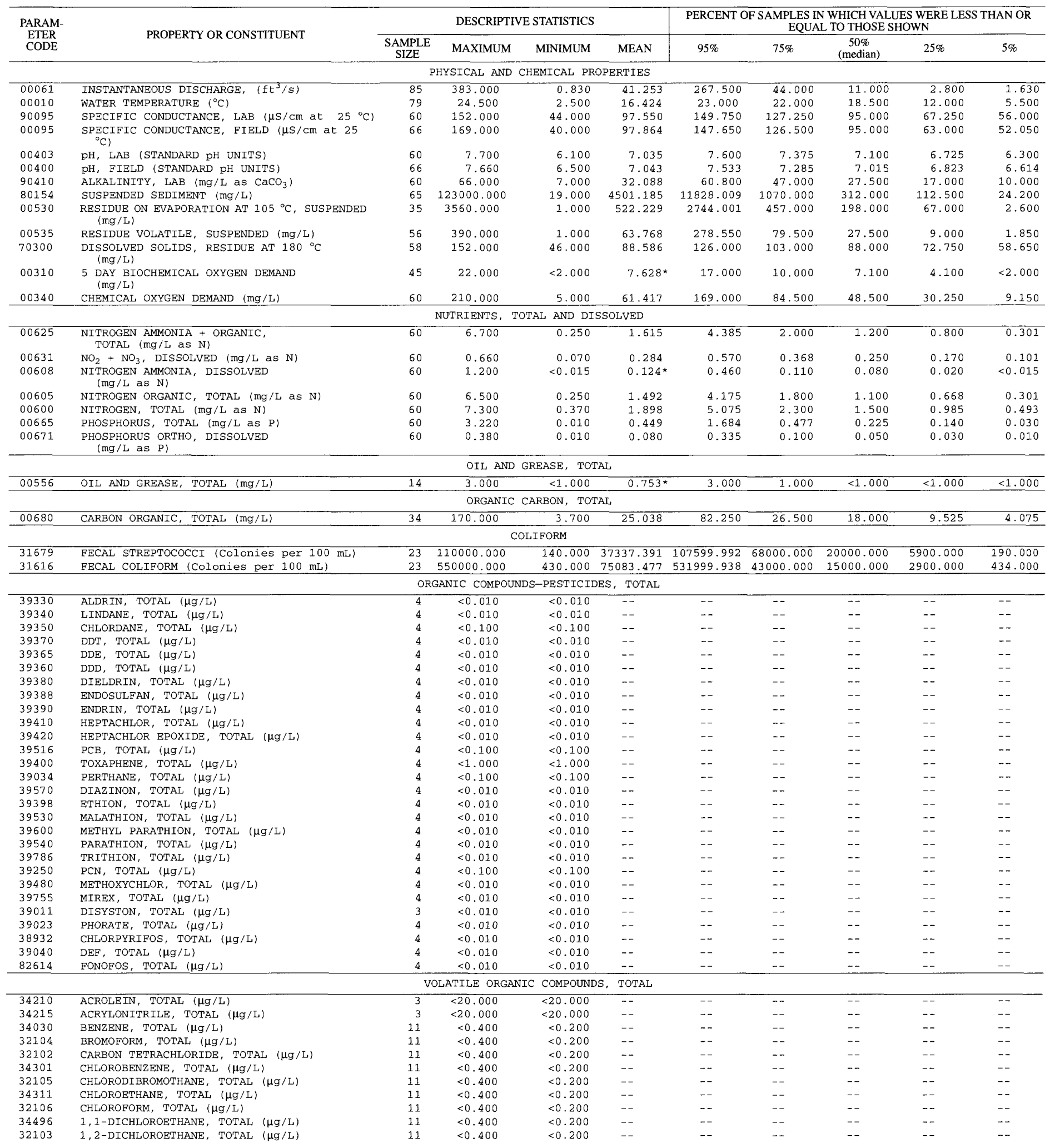

NOTE: Multiple detection limits during the period of record may result in different values flagged with a "<."

* Value is estimated by using a log-probability regression to predict the values of data below the detection limit 
Table 66. Statistical summary of water-quality data at site 33 (CSW08), June 1994 through September 1997—Continued

\begin{tabular}{|c|c|c|c|c|c|c|c|c|c|c|}
\hline \multirow{2}{*}{$\begin{array}{c}\text { PARAM- } \\
\text { ETER } \\
\text { CODE }\end{array}$} & \multirow{2}{*}{ PROPERTY OR CONSTITUENT } & \multicolumn{4}{|c|}{ DESCRIPTIVE STATISTICS } & \multicolumn{5}{|c|}{$\begin{array}{c}\text { PERCENT OF SAMPLES IN WHICH VALUES WERE LESS THAN OR } \\
\text { EQUAL TO THOSE SHOWN }\end{array}$} \\
\hline & & $\begin{array}{l}\text { SAMPLE } \\
\text { SIZE }\end{array}$ & MAXIMUM & MINIMUM & MEAN & $95 \%$ & $75 \%$ & $\begin{array}{c}50 \% \\
\text { (median) }\end{array}$ & $25 \%$ & $5 \%$ \\
\hline 34501 & 1.1-DICHLOROETHYLENE, TOTAL $(\mu \mathrm{g} / \mathrm{L})$ & 11 & $<0.400$ & $<0.200$ & -- & -- & -- & -- & -- & -- \\
\hline 34371 & ETHYLBENZENE， TOTAL $(\mu \mathrm{g} / \mathrm{L})$ & 11 & $<0.400$ & $<0.200$ & -. & -- & -. & -. & -- & -- \\
\hline 34413 & METHYL BROMIDE, TOTAL $(\mu \mathrm{g} / \mathrm{L})$ & 11 & $<0.400$ & $<0.200$ & -- & -- & -- & -. & -- & -- \\
\hline 34423 & METHYLENE CHLORIDE, TOTAL $(\mu \mathrm{g} / \mathrm{L})$ & 11 & $<0.400$ & $<0.200$ & -- & -- & -- & -- & -- & -- \\
\hline 34516 & $1,1,2,2$-TETRACHLOROETHANE，TOTAL $(\mu \mathrm{g} / \mathrm{L})$ & 11 & $<0.400$ & $<0.200$ & -- & -- & -- & -- & -- & -- \\
\hline 34475 & TETRACHLOROETHYLENE， TOTAL $(\mu \mathrm{g} / \mathrm{L})$ & 11 & $<0.400$ & $<0.200$ & -. & -. & -- & -- & -. & -- \\
\hline 34010 & TOLUENE, TOTAL $(\mu \mathrm{g} / \mathrm{L})$ & 11 & 0.400 & $<0.200$ & -- & 0.400 & $<0.400$ & $<0.200$ & $<0.200$ & $<0.200$ \\
\hline 34546 & $\begin{array}{l}\text { 1, 2-TRANSDICHLOROETHENE， TOTAL } \\
(\mu \mathrm{g} / \mathrm{L})\end{array}$ & 11 & $<0.400$ & $<0.200$ & -- & -- & -- & -- & -- & -- \\
\hline 34506 & $1,1,1$-TRICHLOROETHANE， TOTAL $(\mu \mathrm{g} / \mathrm{L})$ & 11 & $<0.400$ & $<0.200$ & -- & -- & -. & -- & - & -- \\
\hline 34511 & $1,1,2$-TRICHLOROETHANE， TOTAL $(\mu \mathrm{g} / \mathrm{L})$ & 11 & $<0.400$ & $<0.200$ & -- & -- & -- & -- & -- & -- \\
\hline 39180 & TRICHLOROETHYLENE, TOTAL $(\mu \mathrm{g} / \mathrm{L})$ & 11 & $<0.400$ & $<0.200$ & -- & -- & -- & -- & -- & -- \\
\hline 39175 & VINYL CHLORIDE, TOTAL $(\mu \mathrm{g} / \mathrm{L})$ & 11 & $<0.400$ & $<0.200$ & -- & -- & -- & -- & -- & -- \\
\hline 30217 & DIBROMOMETHANE, TOTAL $(\mu \mathrm{g} / \mathrm{L})$ & 11 & $<0.400$ & $<0.200$ & -- & -- & -- & -- & -- & -- \\
\hline 32101 & DICHLOROBROMOMETHANE, TOTAL $(\mu \mathrm{g} / \mathrm{L})$ & 11 & $<0.400$ & $<0.200$ & -- & -- & -- & -- & -- & -- \\
\hline 34668 & $\begin{array}{l}\text { DICHLORODIFLUOROMETHANE， TOTAL } \\
(\mu \mathrm{g} / \mathrm{L})\end{array}$ & 11 & $<0.400$ & $<0.200$ & -- & -- & -- & - & -- & -- \\
\hline 77651 & 1,2-DIBROMOETHANE, TOTAL $(\mu \mathrm{g} / \mathrm{L})$ & 11 & $<0.400$ & $<0.200$ & -- & -- & -- & -- & -- & -- \\
\hline 34418 & METHYLCHLORIDE, TOTAL $(\mu \mathrm{g} / \mathrm{L})$ & 11 & 0.200 & $<0.200$ & -- & 0.200 & $<0.400$ & $<0.200$ & $<0.200$ & $<0.200$ \\
\hline 34704 & $\begin{array}{l}\text { CIS } 1,3 \text {-DICHLOROPROPENE, TOTAL } \\
(\mu \mathrm{g} / \mathrm{L})\end{array}$ & 11 & $<0.400$ & $<0.200$ & -- & -- & -- & -- & -- & -- \\
\hline 34699 & TRANS 1,3 -DICHLOROPROPENE, TOTAL $(\mu \mathrm{g} / \mathrm{L})$ & 11 & $<0.400$ & $<0.200$ & -- & -- & -- & -- & -- & -- \\
\hline 77128 & STYRENE, TOTAL $(\mu \mathrm{g} / \mathrm{L})$ & 11 & $<0.400$ & $<0.200$ & -- & -- & -- & -- & -- & -- \\
\hline 81551 & XYLENE, TOTAL $(\mu \mathrm{g} / \mathrm{L})$ & 11 & $<0.400$ & $<0.200$ & -- & -- & -- & -- & -- & -- \\
\hline 82625 & DIBROMOCHLOROPROPANE， TOTAL $(\mu \mathrm{g} / \mathrm{L})$ & 11 & $<2.000$ & $<1.000$ & .. & -- & -- & -. & -- & -- \\
\hline 77168 & 1,1 -DICHLOROPROPENE， TOTAL $(\mu \mathrm{g} / \mathrm{L})$ & 11 & $<0.400$ & $<0.200$ & -- & -- & -- & -- & -- & -- \\
\hline 77170 & 2,2 -DICHLOROPROPANE，TOTAL $(\mu \mathrm{g} / \mathrm{L})$ & 11 & $<0.400$ & $<0.200$ & -- & -- & -- & -- & -- & -. \\
\hline 77173 & 1,3-DICHLOROPROPANE, TOTAL $(\mu \mathrm{g} / \mathrm{L})$ & 11 & $<0.400$ & $<0.200$ & -- & -- & -. & -- & -- & -- \\
\hline 77275 & 0-CHLOROTOLUENE, TOTAL $(\mu \mathrm{g} / \mathrm{L})$ & 11 & $<0.400$ & $<0.200$ & -- & -- & - & -- & -- & -- \\
\hline 77277 & P-CHLOROTOLUENE， TOTAL $(\mu \mathrm{g} / \mathrm{L})$ & 11 & $<0.400$ & $<0.200$ & -- & -- & -- & -- & -- & -- \\
\hline 77443 & 123-TRICHLOROPROPANE，TOTAL $(\mu \mathrm{g} / \mathrm{L})$ & 11 & $<0.400$ & $<0.200$ & -- & -- & -- & -- & -. & -- \\
\hline 77562 & 1112 -TETRACHLOROETHANE，TOTAL $(\mu \mathrm{g} / \mathrm{L})$ & 11 & $<0.400$ & $<0.200$ & -- & -. & -- & -- & -- & -- \\
\hline 78032 & TERTBUTYL METHYL ETHER, TOTAL $(\mu \mathrm{g} / \mathrm{L})$ & 11 & $<0.400$ & $<0.200$ & -- & -- & -- & -- & -- & -- \\
\hline 77297 & BROMOCHLORO METHANE, TOTAL $(\mu \mathrm{g} / \mathrm{L})$ & 11 & $<0.400$ & $<0.200$ & -- & -- & -- & -- & -- & -- \\
\hline 77093 & CIS-1,2-DICHLOROETHENE, TOTAL $(\mu \mathrm{g} / \mathrm{L})$ & 11 & $<0.400$ & $<0.200$ & -- & -- & -- & -- & -- & -- \\
\hline 34576 & 2-CHLOROETHYL VINYL ETHER, TOTAL $(\mu \mathrm{g} / \mathrm{L})$ & 8 & $<2.000$ & $<1.000$ & -- & -- & -- & -- & -- & -- \\
\hline 77223 & ISOPROPYL BENZENE, TOTAL $(\mu \mathrm{g} / \mathrm{L})$ & 11 & $<0.400$ & $<0.200$ & -- & -- & -- & -- & -- & -- \\
\hline 77224 & N-PROPYL BENZENE, TOTAL $(\mu \mathrm{g} / \mathrm{L})$ & 11 & $<0.400$ & $<0.200$ & -- & -- & $\ldots$ & -- & -- & -- \\
\hline 77350 & SEC-BUTYL BENZENE, TOTAL $(\mu \mathrm{g} / \mathrm{L})$ & 11 & $<0.400$ & $<0.200$ & -- & -- & -- & -- & -- & -- \\
\hline 77356 & P-ISOPROPYL TOLUENE, TOTAL $(\mu \mathrm{g} / \mathrm{L})$ & 11 & $<0.400$ & $<0.200$ & -- & -- & -- & -- & -- & -- \\
\hline 77342 & N-BUTYL BENZENE, TOTAL $(\mu \mathrm{g} / \mathrm{L}$ & 11 & $<0.400$ & $<0.200$ & -- & -- & -- & -- & -- & -- \\
\hline 77613 & $1,2,3$-TRICHLOROBENZENE，TOTAL $(\mu \mathrm{g} / \mathrm{L}$ & 11 & $<0.400$ & $<0.200$ & -. & -- & -- & -- & -- & -- \\
\hline 77652 & FREON-113， TOTAL $(\mu \mathrm{g} / \mathrm{L}$ & 11 & $<0.400$ & $<0.200$ & -- & -- & - & -- & -- & -- \\
\hline 77226 & MESITYLENE, TOTAL $(\mu \mathrm{g} / \mathrm{L}$ & 11 & $<0.400$ & $<0.200$ & -- & -- & -- & -- & -- & -- \\
\hline 81555 & BROMOBENZENE, TOTAL $(\mu \mathrm{g} / \mathrm{L}$ & 11 & $<0.400$ & $<0.200$ & -- & - & -- & -- & -- & $m$ \\
\hline & & MET & LS AND MINO & CONSTITUEN & S, TOTAL & & & & & \\
\hline 01097 & ANTIMONY, TOTAL $(\mu \mathrm{g} / \mathrm{L}$ as $\mathrm{Sb})$ & 41 & 18.000 & $<1.000$ & $2.510^{*}$ & 11.000 & 3.000 & $<1.000$ & $<1.000$ & $<1.000$ \\
\hline 01002 & ARSENIC, TOTAL $(\mu \mathrm{g} / \mathrm{L}$ as AS $)$ & 41 & 8.000 & $<1.000$ & $1.641 *$ & 6.000 & 2.000 & $<1.000$ & $<1.000$ & $<1.000$ \\
\hline 01012 & BERYLLIUM, TOTAL $(\mu \mathrm{g} / \mathrm{L}$ as Be) & 14 & 42.000 & $<10.000$ & -- & 42.000 & $<10.000$ & $<10.000$ & $<10.000$ & $<10.000$ \\
\hline 01027 & CADMIUM, TOTAL $(\mu \mathrm{g} / \mathrm{L}$ as $\mathrm{Cd})$ & 40 & 2.000 & $<1.000$ & -- & $<1.000$ & $<1.000$ & $<1.000$ & $<1.000$ & $<1.000$ \\
\hline 01034 & CHROMIUM, TOTAL $(\mu \mathrm{g} / \mathrm{L}$ as $\mathrm{Cr})$ & 41 & 100.000 & 1.000 & 20.512 & 79.200 & 22.500 & 15.000 & 3.000 & 1.000 \\
\hline 01042 & COPPER, TOTAL $(\mu \mathrm{g} / \mathrm{L}$ as $\mathrm{Cu})$ & 41 & 830.000 & 1.000 & 50.098 & 130.000 & 37.500 & 19.000 & 5.000 & 1.100 \\
\hline 01051 & LEAD, TOTAL $(\mu \mathrm{g} / \mathrm{L}$ as $\mathrm{Pb})$ & 41 & 36.000 & $<1.000$ & $9.718^{*}$ & 28.000 & 16.000 & 7.000 & 2.000 & $<1.000$ \\
\hline 71900 & MERCURY, TOTAL $(\mu \mathrm{g} / \mathrm{L}$ as $\mathrm{Hg})$ & 13 & 0.100 & $<0.100$ & -. & 0.100 & $<0.100$ & $<0.100$ & $<0.100$ & $<0.100$ \\
\hline 01067 & NICKEL, TOTAL $(\mu \mathrm{g} / \mathrm{L}$ as $\mathrm{Ni})$ & 41 & 28.000 & $<1.000$ & $7.457 *$ & 21.000 & 10.000 & 6.000 & 2.000 & $<1.000$ \\
\hline 01147 & SELENIUM, TOTAL $(\mu \mathrm{g} / \mathrm{L}$ as $\mathrm{Se})$ & 13 & 1.000 & $<1.000$ & -- & 1.000 & $<1.000$ & $<1.000$ & $<1.000$ & $<1.000$ \\
\hline 01077 & SILVER, TOTAL $(\mu \mathrm{g} / \mathrm{L}$ as $\mathrm{Ag})$ & 13 & 1.000 & $<1.000$ & -- & 1.000 & $<1.000$ & $<1.000$ & $<1.000$ & $<1.000$ \\
\hline 01092 & ZINC, TOTAL $(\mu \mathrm{g} / \mathrm{L}$ as $\mathrm{Zn})$ & 41 & 200.000 & $<1.000$ & $59.068 *$ & 140.000 & 70.000 & 50.000 & 30.000 & $<10.000$ \\
\hline 00720 & CYANIDE, TOTAL (mg/L as Cn) & 13 & $<0.010$ & $<0.010$ & -- & -- & -- & -- & -- & -- \\
\hline & & ORGAI & IC COMPOUND & ESTICIDES & DISSOLVED & & & & & \\
\hline 46342 & ALACHLOR, DISSOLVED $(\mu \mathrm{g} / \mathrm{L})$ & 3 & $<0.009$ & $<0.002$ & -- & -- & -- & -- & -- & $\cdots$ \\
\hline 04040 & DEETHYL ATRAZINE, DISSOLVED $(\mu \mathrm{g} / \mathrm{L})$ & 3 & 0.001 & $<0.002$ & -- & -- & -- & -- & -- & -- \\
\hline 39632 & ATRAZINE, DISSOLVED $(\mu \mathrm{g} / \mathrm{L})$ & 3 & 0.008 & 0.005 & -- & -- & -- & -- & -- & -- \\
\hline 82686 & METHYL AZINPHOS, DISSOLVED $(\mu \mathrm{g} / \mathrm{L})$ & 3 & $<0.040$ & $<0.001$ & -- & -- & -- & -- & -- & -- \\
\hline 82673 & BENFLURALIN， DISSOLVED $(\mu \mathrm{g} / \mathrm{L})$ & 3 & $<0.010$ & $<0.002$ & -- & -- & -- & -- & -- & -- \\
\hline 04028 & BUTYLATE, DISSOLVED $(\mu \mathrm{g} / \mathrm{L})$ & 3 & $<0.008$ & $<0.002$ & -. & $\ldots$ & -- & -- & $\ldots$ & -- \\
\hline 82680 & CARBARYL， DISSOLVED $(\mu \mathrm{g} / \mathrm{L})$ & 3 & 0.011 & $<0.050$ & -- & -- & -- & -- & -- & -- \\
\hline 82674 & CARBOFURAN， DISSOLVED $(\mu \mathrm{g} / \mathrm{L})$ & 3 & $<0.010$ & $<0.003$ & -- & -- & -- & -- & -- & -- \\
\hline 38933 & CHLORPYRIFOS, DISSOLVED $(\mu \mathrm{g} / \mathrm{L})$ & 3 & $<0.005$ & $<0.004$ & -- & -- & -- & -- & -- & -- \\
\hline 04041 & CYANAZINE, DISSOLVED $(\mu \mathrm{g} / \mathrm{L})$ & 3 & $<0.010$ & $<0.004$ & -- & -- & -- & -- & -- & - \\
\hline 82682 & DCPA， DISSOLVED $(\mu \mathrm{g} / \mathrm{L})$ & 3 & $<0.004$ & $<0.002$ & -- & -- & -- & -- & -- & -. \\
\hline 34653 & $\mathrm{P}, \mathrm{P}^{\prime}$ DDE, DISSOLVED $(\mu \mathrm{g} / \mathrm{L})$ & 3 & $<0.010$ & $<0.006$ & -- & -- & -- & -- & -- & -- \\
\hline 39572 & DIAZINON, DISSOLVED $(\mu \mathrm{g} / \mathrm{L})$ & 3 & $<0.008$ & $<0.002$ & -- & -- & -- & -- & -- & - \\
\hline 39381 & DIELDRIN, DISSOLVED $(\mu \mathrm{g} / \mathrm{L})$ & 3 & $<0.008$ & $<0.001$ & -- & -- & -- & -- & -- & -- \\
\hline 82660 & $\begin{array}{l}2,6 \text {-DIETHYL ANILINE, DISSOLVED } \\
(\mu \mathrm{g} / \mathrm{L})\end{array}$ & 3 & $<0.006$ & $<0.003$ & -- & -- & -- & -- & -- & -- \\
\hline 82662 & DIMETHOATE, DISSOLVED $(\mu \mathrm{g} / \mathrm{L})$ & 1 & $<0.020$ & -- & -- & -- & -- & -- & -- & -- \\
\hline
\end{tabular}


Table 66. Statistical summary of water-quality data at site 33 (CSW08), June 1994 through September 1997-Continued

\begin{tabular}{|c|c|c|c|c|c|c|c|c|c|c|}
\hline \multirow{2}{*}{$\begin{array}{c}\text { PARAM- } \\
\text { ETER } \\
\text { CODE }\end{array}$} & \multirow{2}{*}{ PROPERTY OR CONSTITUENT } & \multicolumn{4}{|c|}{ DESCRIPTIVE STATISTICS } & PERCEI & $\begin{array}{r}\text { AMPLF } \\
\text { EC }\end{array}$ & $\begin{array}{l}\text { NHICH VAI } \\
\text { IO THOSE }\end{array}$ & $\begin{array}{l}\text { WERE } \\
\text { /N }\end{array}$ & IAN OR \\
\hline & & $\begin{array}{l}\text { SAMPLE } \\
\text { SIZE }\end{array}$ & MAXIMUM & MINIMUM & MEAN & $95 \%$ & $75 \%$ & $\begin{array}{c}50 \% \\
\text { (median) }\end{array}$ & $25 \%$ & $5 \%$ \\
\hline 82677 & DISULFOTON, DISSOLVED $(\mu \mathrm{g} / \mathrm{L})$ & 3 & $<0.017$ & $<0.008$ & -- & -- & -- & -- & -- & -- \\
\hline 82668 & EPTC, DISSOLVED $(\mu \mathrm{g} / \mathrm{L})$ & 3 & $<0.005$ & $<0.002$ & -- & -- & -- & -- & - & -- \\
\hline 82663 & ETHALFLURALIN，DISSOLVED $(\mu \mathrm{g} / \mathrm{L})$ & 3 & $<0.010$ & $<0.004$ & $-\infty$ & -- & -- & -- & -- & -- \\
\hline 82672 & ETHOPROP， DISSOLVED $(\mu \mathrm{g} / \mathrm{L})$ & 3 & $<0.010$ & $<0.003$ & -- & -- & -. & -- & -- & -- \\
\hline 04095 & FONOFOS，DISSOLVED $(\mu \mathrm{g} / \mathrm{L})$ & 3 & $<0.008$ & $<0.003$ & -- & -- & -- & -- & -- & -- \\
\hline 34253 & ALPHA BHC, DISSOLVED $(\mu \mathrm{g} / \mathrm{L})$ & 3 & $<0.007$ & $<0.002$ & -- & -- & -- & -- & -- & -- \\
\hline 39341 & LINDANE， DISSOLVED $(\mu \mathrm{g} / \mathrm{L})$ & 3 & $<0.010$ & $<0.004$ & -- & -- & -- & -- & -- & -- \\
\hline 82666 & LINURON, DISSOLVED $(\mu \mathrm{g} / \mathrm{L})$ & 3 & $<0.040$ & $<0.002$ & -- & -- & -- & -- & -- & -- \\
\hline 39532 & MALATHION, DISSOLVED $(\mu \mathrm{g} / \mathrm{L})$ & 3 & $<0.010$ & $<0.005$ & -- & -- & -- & -- & -- & -- \\
\hline 82667 & METHYL PARATHION, DISSOLVED $(\mu \mathrm{g} / \mathrm{L})$ & 3 & $<0.040$ & $<0.006$ & -- & -- & -- & -- & -- & -- \\
\hline 39415 & METOLACHLOR, DISSOLVED $(\mu \mathrm{g} / \mathrm{L})$ & 3 & 0.006 & $<0.002$ & -- & -- & -- & -- & -- & -- \\
\hline 82630 & METRIBUZIN, DISSOLVED $(\mu \mathrm{g} / \mathrm{L})$ & 3 & $<0.010$ & $<0.004$ & -- & -- & -- & -- & -- & -- \\
\hline 82671 & MOLINATE， DISSOLVED $(\mu \mathrm{g} / \mathrm{L})$ & 3 & $<0.007$ & $<0.004$ & -- & -- & -- & -- & -- & -- \\
\hline 82684 & NAPROPAMIDE，DISSOLVED $(\mu \mathrm{g} / \mathrm{L})$ & 3 & $<0.010$ & $<0.003$ & -- & -- & -- & -- & - & -- \\
\hline 39542 & PARATHION，DISSOLVED $(\mu \mathrm{g} / \mathrm{L})$ & 3 & $<0.020$ & $<0.004$ & -- & -- & -- & -- & -- & -- \\
\hline 82669 & PEBULATE， DISSOLVED $(\mu \mathrm{g} / \mathrm{L})$ & 3 & $<0.009$ & $<0.004$ & -- & -- & -- & -- & -- & -- \\
\hline 82683 & PENDIMETHALIN， DISSOLVED $(\mu \mathrm{g} / \mathrm{L})$ & 3 & $<0.020$ & $<0.004$ & -- & -- & -- & -- & -- & -- \\
\hline 82687 & PERMETHRIN， DISSOLVED $(\mu \mathrm{g} / \mathrm{L})$ & 3 & $<0.020$ & $<0.005$ & -- & -- & -- & -- & -- & -- \\
\hline 82664 & PHORATE， DISSOLVED $(\mu \mathrm{g} / \mathrm{L})$ & 3 & $<0.010$ & $<0.002$ & -- & -- & -- & -- & -- & -- \\
\hline 82676 & PRONAMIDE， DISSOLVED $(\mu \mathrm{g} / \mathrm{L})$ & 3 & $<0.009$ & $<0.003$ & -- & -- & -- & -- & -- & -- \\
\hline 04037 & PROMETON，DISSOLVED $(\mu \mathrm{g} / \mathrm{L})$ & 3 & $<0.018$ & $<0.008$ & -- & -- & - & -- & -- & -- \\
\hline 04024 & PROPACHLOR，DISSOLVED $(\mu \mathrm{g} / \mathrm{L})$ & 3 & $<0.010$ & $<0.007$ & -- & -- & -- & -- & -- & -- \\
\hline 82679 & PROPANIL， DISSOLVED $(\mu \mathrm{g} / \mathrm{L})$ & 3 & $<0.020$ & $<0.004$ & -- & -- & -- & -- & -- & -- \\
\hline 82685 & PROPARGITE， DISSOLVED $(\mu \mathrm{g} / \mathrm{L})$ & 3 & $<0.013$ & $<0.006$ & -- & -- & -- & -- & -- & -- \\
\hline 04035 & SIMAZINE，DISSOLVED $(\mu \mathrm{g} / \mathrm{L})$ & 3 & $<0.008$ & $<0.005$ & -- & -- & -- & -- & -- & -- \\
\hline 82681 & THIOBENCARB， DISSOLVED $(\mu \mathrm{g} / \mathrm{L})$ & 3 & $<0.008$ & $<0.002$ & -- & -- & -- & -- & -- & -- \\
\hline 82670 & TEBUTHIURON， DISSOLVED $(\mu \mathrm{g} / \mathrm{L})$ & 3 & $<0.010$ & $<0.010$ & -- & -- & -- & -- & -- & -- \\
\hline 82665 & TERBACIL， DISSOLVED $(\mu \mathrm{g} / \mathrm{L})$ & 3 & $<0.030$ & $<0.007$ & -- & - & -- & -- & -- & -- \\
\hline 82675 & TERBUFOS， DISSOLVED $\langle\mu \mathrm{g} / \mathrm{L}\rangle$ & 3 & $<0.013$ & $<0.010$ & -- & -- & -- & -- & -- & -- \\
\hline 82678 & TRIALLATE， DISSOLVED $(\mu \mathrm{g} / \mathrm{L})$ & 3 & $<0.008$ & $<0.001$ & -- & -- & -- & -- & -- & -- \\
\hline 82661 & TRIFLURALIN, DISSOLVED $(\mu \mathrm{g} / \mathrm{L})$ & 3 & $<0.010$ & $<0.002$ & - & -- & -- & -- & -- & -- \\
\hline 39742 & $2,4,5-\mathrm{T}$, DISSOLVED $(\mu \mathrm{g} / \mathrm{L})$ & 3 & $<0.050$ & $<0.035$ & -- & -- & -- & -- & -- & -- \\
\hline 39732 & $2,4-D, \quad$ DISSOLVED $(\mu \mathrm{g} / \mathrm{L})$ & 3 & $<0.050$ & $<0.035$ & -- & -- & - & -- & -- & -- \\
\hline 38746 & $2,4-\mathrm{DB}$, DISSOLVED $(\mu \mathrm{g} / \mathrm{L})$ & 3 & $<0.050$ & $<0.035$ & -- & -- & -- & -- & -- & -- \\
\hline 49315 & ACIFLUORFEN, DISSOLVED $(\mu \mathrm{g} / \mathrm{L})$ & 3 & $<0.050$ & $<0.035$ & -- & -- & -- & -- & -- & -- \\
\hline 49312 & ALDICARB， DISSOLVED $(\mu \mathrm{g} / \mathrm{L})$ & 3 & $<0.050$ & $<0.016$ & -- & -- & -- & -- & -- & -- \\
\hline 49313 & ALDICARB SULFONE, DISSOLVED $(\mu \mathrm{g} / \mathrm{L})$ & 3 & $<0.050$ & $<0.016$ & -- & -- & -- & - & -- & -- \\
\hline 49314 & $\begin{array}{l}\text { ALDICARB SULFOXIDE, DISSOLVED } \\
(\mu \mathrm{g} / \mathrm{L})\end{array}$ & 3 & $<0.050$ & $<0.021$ & -- & -- & -- & -- & -- & -- \\
\hline 38711 & BENTAZON， DISSOLVED $(\mu \mathrm{g} / \mathrm{L})$ & 3 & $<0.050$ & $<0.014$ & -- & -- & -- & -- & -- & -- \\
\hline 04029 & BROMACIL, DISSOLVED $(\mu \mathrm{g} / \mathrm{L})$ & 3 & $<0.050$ & $<0.035$ & -- & -- & -- & -- & -- & -- \\
\hline 49311 & BROMOXYNIL， DISSOLVED $(\mu \mathrm{g} / \mathrm{L})$ & 3 & $<0.050$ & $<0.035$ & -- & -- & -- & -- & -- & -- \\
\hline 49310 & CARBARYL， DISSOLVED $(\mu \mathrm{g} / \mathrm{L})$ & 3 & $<0.050$ & $<0.008$ & - & -- & -- & -- & -- & -- \\
\hline 49309 & CARBOFURAN, DISSOLVED $(\mu \mathrm{g} / \mathrm{L})$ & 3 & $<0.050$ & $<0.028$ & -- & -- & -- & -- & -- & -- \\
\hline 49308 & 3-HYDROXY-CARBOFURAN $(\mu \mathrm{g} / \mathrm{L})$ & 3 & $<0.050$ & $<0.014$ & -- & -- & -- & -- & -- & -- \\
\hline 49307 & AMIBEN， DISSOLVED $(\mu \mathrm{g} / \mathrm{L})$ & 3 & $<0.050$ & $<0.011$ & -- & -- & -- & -- & -- & -- \\
\hline 49306 & CHLOROTHALONIL, DISSOLVED $(\mu \mathrm{g} / \mathrm{L})$ & 3 & $<0.050$ & $<0.035$ & -- & - & -- & -- & -- & -- \\
\hline 49305 & CLOPYRALID, DISSOLVED $(\mu \mathrm{g} / \mathrm{L})$ & 3 & $<0.050$ & $<0.050$ & -- & -- & -- & -- & -- & -- \\
\hline 49304 & DACTHALMONO-ACID, DISSOLVED $(\mu \mathrm{g} / \mathrm{L})$ & 3 & $<0.050$ & $<0.017$ & -- & -- & -- & -- & -- & -- \\
\hline 38442 & DICAMBA，DISSOLVED $(\mu \mathrm{g} / \mathrm{L})$ & 3 & $<0.050$ & $<0.035$ & -- & -- & -- & -- & -- & -- \\
\hline 49303 & DICHLOBENIL， DISSOLVED $(\mu \mathrm{g} / \mathrm{L})$ & 3 & $<0.050$ & $<0.020$ & -- & -- & -- & -- & -- & -- \\
\hline 49302 & DICHLORPROP, DISSOLVED $(\mu \mathrm{g} / \mathrm{L})$ & 3 & $<0.050$ & $<0.032$ & -- & -- & -- & -- & -- & -- \\
\hline 49301 & DINOSEB， DISSOLVED $(\mu \mathrm{g} / \mathrm{L})$ & 3 & $<0.050$ & $<0.035$ & -- & -- & -- & -- & -- & -- \\
\hline 49300 & DIURON， DISSOLVED $(\mu \mathrm{g} / \mathrm{L})$ & 3 & $<0.050$ & $<0.020$ & -- & -- & -- & -- & -- & -- \\
\hline 49299 & $\begin{array}{l}\text { 4, 6-DINITRO OCRESOL， DISSOLVED } \\
(\mu \mathrm{g} / \mathrm{L})\end{array}$ & 3 & $<0.050$ & $<0.035$ & -- & -- & -- & -- & -- & -- \\
\hline 49298 & ESFENVALERATE， DISSOLVED $(\mu \mathrm{g} / \mathrm{L})$ & 3 & $<0.050$ & $<0.019$ & -- & -- & -- & -- & -- & - \\
\hline 49297 & FENURON， DISSOLVED $(\mu \mathrm{g} / \mathrm{L})$ & 3 & $<0.050$ & $<0.013$ & -- & -- & -- & -- & -- & -- \\
\hline 38811 & FLUOMETURON， DISSOLVED ( $\mu \mathrm{g} / \mathrm{L})$ & 3 & $<0.050$ & $<0.035$ & -- & -- & -- & -- & -- & -- \\
\hline 38478 & LINURON， DISSOLVED $(\mu \mathrm{g} / \mathrm{L})$ & 3 & $<0.050$ & $<0.018$ & -- & -- & -- & -- & -- & -- \\
\hline 38482 & MCPA， DISSOLVED $(\mu \mathrm{g} / \mathrm{L})$ & 3 & $<0.050$ & $<0.050$ & -- & -- & -- & -- & -- & -- \\
\hline 38487 & MCPB， DISSOLVED $(\mu \mathrm{g} / \mathrm{L})$ & 3 & $<0.050$ & $<0.035$ & -- & -- & -- & -- & -- & -- \\
\hline 38501 & METHIOCARB， DISSOLVED $(\mu \mathrm{g} / \mathrm{L})$ & 3 & $<0.050$ & $<0.026$ & -- & -- & -- & -- & -- & -- \\
\hline 49296 & METHOMYL， DISSOLVED $(\mu \mathrm{g} / \mathrm{L})$ & 3 & $<0.050$ & $<0.017$ & -- & -- & -- & -- & -- & -- \\
\hline 49295 & 1-NAPHTHOL， DISSOLVED $(\mu \mathrm{g} / \mathrm{L})$ & 3 & $<0.050$ & $<0.007$ & -- & -- & -- & -- & -- & -- \\
\hline 49294 & NEBURON, DISSOLVED $(\mu \mathrm{g} / \mathrm{L})$ & 3 & $<0.050$ & $<0.015$ & -- & -- & -- & -- & -- & -- \\
\hline 49293 & NORFLURAZON， DISSOLVED $(\mu \mathrm{g} / \mathrm{L})$ & 3 & $<0.050$ & $<0.024$ & -- & -- & -- & -- & -- & -- \\
\hline 49292 & ORYZALIN， DISSOLVED $(\mu \mathrm{g} / \mathrm{L})$ & 3 & $<0.050$ & $<0.019$ & -- & - & -- & -- & -- & -- \\
\hline 38866 & OXAMYL，DISSOLVED $(\mu \mathrm{g} / \mathrm{L})$ & 3 & $<0.050$ & $<0.018$ & -- & -- & -- & -- & -- & -- \\
\hline 49291 & PICLORAM， DISSOLVED $(\mu \mathrm{g} / \mathrm{L})$ & 3 & $<0.050$ & $<0.050$ & -- & -- & -- & -- & -- & -- \\
\hline 49236 & PROPHAM, DISSOLVED $(\mu \mathrm{g} / \mathrm{L})$ & 3 & $<0.050$ & $<0.035$ & -- & -- & -- & -- & -- & -- \\
\hline 38538 & PROPOXUR, DISSOLVED $(\mu \mathrm{g} / \mathrm{L})$ & 3 & $<0.050$ & $<0.035$ & -- & -- & -- & -- & -- & -- \\
\hline 39762 & SILVEX，DISSOLVED $(\mu \mathrm{g} / \mathrm{L})$ & 3 & $<0.050$ & $<0.021$ & -- & -- & -- & -- & -- & -- \\
\hline 49235 & TRICLOPYR， DISSOLVED $(\mu \mathrm{g} / \mathrm{L})$ & 3 & $<0.050$ & $<0.050$ & -- & -- & -- & -- & -- & -- \\
\hline & & ORGAI & C COMPOUND & GANONITR & EN, TOT & & & & & \\
\hline 39057 & PROMETRYNE， TOTAL $(\mu \mathrm{g} / \mathrm{L})$ & 2 & $<0.1 .00$ & $<0.100$ & -- & -- & -- & -- & -- & -- \\
\hline 39056 & PROMETONE, TOTAL $(\mu \mathrm{g} / \mathrm{L})$ & 2 & $<0.200$ & $<0.200$ & -- & -- & -- & -- & -- & -- \\
\hline 39054 & SIMETRYNE, TOTAL $(\mu \mathrm{g} / \mathrm{L})$ & 2 & $<0.100$ & $<0.100$ & -- & -- & -- & -- & -- & -- \\
\hline 81757 & CYANAZINE, TOTAL $(\mu \mathrm{g} / \mathrm{L})$ & 2 & $<0.200$ & $<0.200$ & -- & -- & -- & -- & -- & -- \\
\hline 77825 & ALACHLOR, TOTAL $(\mu \mathrm{g} / \mathrm{L})$ & 2 & $<0.100$ & $<0.100$ & -- & -- & -- & -- & -- & -- \\
\hline 82611 & METRIBUZIN, TOTAL $(\mu \mathrm{g} / \mathrm{L})$ & 2 & $<0.100$ & $<0.100$ & -- & -- & -- & -- & -- & -- \\
\hline
\end{tabular}

NOTE: Multiple detection limits during the period of record may result in different values flagged with a "<."

* Value is estimated by using a log-probability regression to predict the values of data below the detection limit. 
Table 66. Statistical summary of water-quality data at site 33 (CSW08), June 1994 through September 1997-Continued

\begin{tabular}{|c|c|c|c|c|c|c|c|c|c|c|}
\hline \multirow{2}{*}{$\begin{array}{c}\text { PARAM- } \\
\text { ETER } \\
\text { CODE }\end{array}$} & \multirow{2}{*}{ PROPERTY OR CONSTITUENT } & \multicolumn{4}{|c|}{ DESCRIPTIVE STATISTICS } & \multicolumn{5}{|c|}{$\begin{array}{l}\text { PERCENT OF SAMPLES IN WHICH VALUES WERE LESS THAN OR } \\
\text { EQUAL TO THOSE SHOWN }\end{array}$} \\
\hline & & $\begin{array}{l}\text { SAMPLE } \\
\text { SIZE }\end{array}$ & MAXIMUM & MINIMUM & MEAN & $95 \%$ & $75 \%$ & $\begin{array}{c}50 \% \\
\text { (median) }\end{array}$ & $25 \%$ & $5 \%$ \\
\hline 30311 & TERBACIL， TOTAL $(\mu \mathrm{g} / \mathrm{L})$ & 2 & $<0.200$ & $<0.200$ & -- & -- & -- & -- & -- & -- \\
\hline 30245 & CARBOXIN, TOTAL $(\mu \mathrm{g} / \mathrm{L})$ & 2 & $<0.200$ & $<0.200$ & -- & -- & -- & -- & -- & -- \\
\hline 30264 & HEXAZINONE, TOTAL $(\mu \mathrm{g} / \mathrm{L})$ & 2 & $<0.200$ & $<0.200$ & -- & -- & -- & -- & -- & -- \\
\hline 30235 & BUTACHLOR, TOTAL $(\mu \mathrm{g} / \mathrm{L})$ & 2 & $<0.100$ & $<0.100$ & -- & -- & -- & -- & -- & -- \\
\hline 30236 & BUTYLATE, TOTAL $(\mu \mathrm{g} / \mathrm{L})$ & 2 & $<0.100$ & $<0.100$ & -- & -- & -- & -- & -- & -. \\
\hline 75981 & DE-ETHYLATRAZINE， TOTAL $(\mu \mathrm{g} / \mathrm{L})$ & 2 & $<0.200$ & $<0.200$ & -- & -- & -- & -- & -- & -- \\
\hline 39630 & ATRAZINE， TOTAL $(\mu \mathrm{g} / \mathrm{L})$ & 2 & $<0.100$ & $<0.100$ & -- & -- & -- & -- & -- & -- \\
\hline 39055 & SIMAZINE, TOTAL $(\mu \mathrm{g} / \mathrm{L})$ & 2 & $<0.100$ & $<0.100$ & -- & -- & -- & -- & -- & -- \\
\hline 39024 & PROPAZINE, TOTAL $\{\mu \mathrm{g} / \mathrm{L}\}$ & 2 & $<0.100$ & $<0.100$ & -- & -- & - - & -- & -. & -- \\
\hline 82184 & AMETRYNE, TOTAL $(\mu \mathrm{g} / \mathrm{L})$ & 2 & $<0.100$ & $<0.100$ & -. & -- & -- & -- & -- & -- \\
\hline 39030 & TRIFLURALIN, TOTAL $(\mu \mathrm{g} / \mathrm{L})$ & 2 & $<0.100$ & $<0.100$ & -- & -- & -- & -- & -- & -- \\
\hline 82612 & METOLACHLOR, TOTAL $(\mu \mathrm{g} / \mathrm{L})$ & 2 & $<0.200$ & $<0.200$ & - & -- & _- & -- & -- & -- \\
\hline 30234 & BROMACIL, TOTAL $(\mu \mathrm{g} / \mathrm{L})$ & 2 & $<0.200$ & $<0.200$ & -- & -- & -- & -- & -- & -- \\
\hline 30255 & DI PHENAMID, TOTAL $(\mu \mathrm{g} / \mathrm{L})$ & 2 & $<0.100$ & $<0.100$ & -- & -- & - - & -- & -- & -- \\
\hline 30324 & VERNOLATE, TOTAL $(\mu \mathrm{g} / \mathrm{L})$ & 2 & $<0.100$ & $<0.100$ & -- & -- & -- & -- & -- & -- \\
\hline 30254 & CYCLOATE, TOTAL $(\mu \mathrm{g} / \mathrm{L})$ & 2 & $<0.100$ & $<0.100$ & -- & -- & -- & -- & -- & -- \\
\hline 30295 & PROPACHLOR, TOTAL $(\mu \mathrm{g} / \mathrm{L})$ & 2 & $<0.100$ & $<0.100$ & -- & -- & -- & -- & -- & -- \\
\hline 75980 & DE-ISOPROPYLATRAZIN, TOTAL $(\mu \mathrm{g} / \mathrm{L})$ & 2 & $<0.200$ & $<0.200$ & -- & -- & -- & -- & -- & -- \\
\hline \multicolumn{11}{|c|}{ ORGANIC COMPOUNDS-HERBICIDES, TOTAL } \\
\hline 39730 & $2,4-\mathrm{D}$, TOTAL $(\mu \mathrm{g} / \mathrm{L})$ & 1. & $<0.010$ & -- & -- & -- & -- & -- & -- & -- \\
\hline 39760 & SILVEX, TOTAL $(\mu \mathrm{g} / \mathrm{L})$ & 1. & $<0.010$ & -- & -- & -- & -- & -- & -- & -. \\
\hline 39720 & PICLORAM, TOTAL $(\mu \mathrm{g} / \mathrm{L})$ & 1 & $<0.010$ & -- & - & -- & -- & -- & -- & -- \\
\hline 39740 & $2,4,5-\mathrm{T}$, TOTAL $(\mu \mathrm{g} / \mathrm{L})$ & 1. & $<0.010$ & -- & -- & -- & -- & -- & -- & -- \\
\hline 82183 & $2,4-\mathrm{DP}$, TOTAL $(\mu \mathrm{g} / \mathrm{L})$ & 1 & $<0.010$ & -- & -- & -- & -- & -- & -- & -- \\
\hline 82052 & DICAMBA, TOTAL $(\mu \mathrm{g} / \mathrm{L})$ & 1 & $<0.010$ & -- & -- & -- & -- & -- & -- & -- \\
\hline \multicolumn{11}{|c|}{ ORGANIC COMPOUNDS-CARBAMATE PESTICIDES, TOTAL } \\
\hline 39750 & SEVIN, TOTAL $(\mu \mathrm{g} / \mathrm{L})$ & 2 & $<0.500$ & $<0.500$ & -- & -- & -- & -- & -- & -- \\
\hline 39051 & METHOMYL, TOTAL $(\mu \mathrm{g} / \mathrm{L})$ & 2 & $<0.500$ & $<0.500$ & -- & -- & -- & -- & -- & -- \\
\hline 82619 & ALDICARD, TOTAL $(\mu \mathrm{g} / \mathrm{L})$ & 2 & $<0.500$ & $<0.500$ & -- & -- & -- & -. & -. & -- \\
\hline 30296 & PROPOXUR, TOTAL $(\mu \mathrm{g} / \mathrm{L})$ & 2 & $<0.500$ & $<0.500$ & -- & -- & -- & -- & -- & -- \\
\hline 39052 & PROPHAM, TOTAL $(\mu \mathrm{g} / \mathrm{L})$ & 2 & $<0.500$ & $<0.500$ & -- & -- & -- & -- & -- & -- \\
\hline 82615 & CARBOFURAN， TOTAL $(\mu \mathrm{g} / \mathrm{L})$ & 2 & $<0.500$ & $<0.500$ & -- & -- & -- & -- & -- & -- \\
\hline 77441 & 1 -NAPHTHOL， TOTAL $(\mu \mathrm{g} / \mathrm{L})$ & 2 & $<0.500$ & $<0.500$ & -- & -- & -- & -- & -- & -- \\
\hline 30282 & METHIOCARB, TOTAL $(\mu g / L)$ & 2 & $<0.500$ & $<0.500$ & -- & -- & -- & -- & -- & -- \\
\hline
\end{tabular}

NOTE: Multiple detection limits during the period of record may result in different values flagged with a "<."

* Value is estimated by using a log-probability regression to predict the values of data below the detection limit. 
Table 67. Statistical summary of water-quality data at site 34 (CSWO9), June 1994 through September 1997

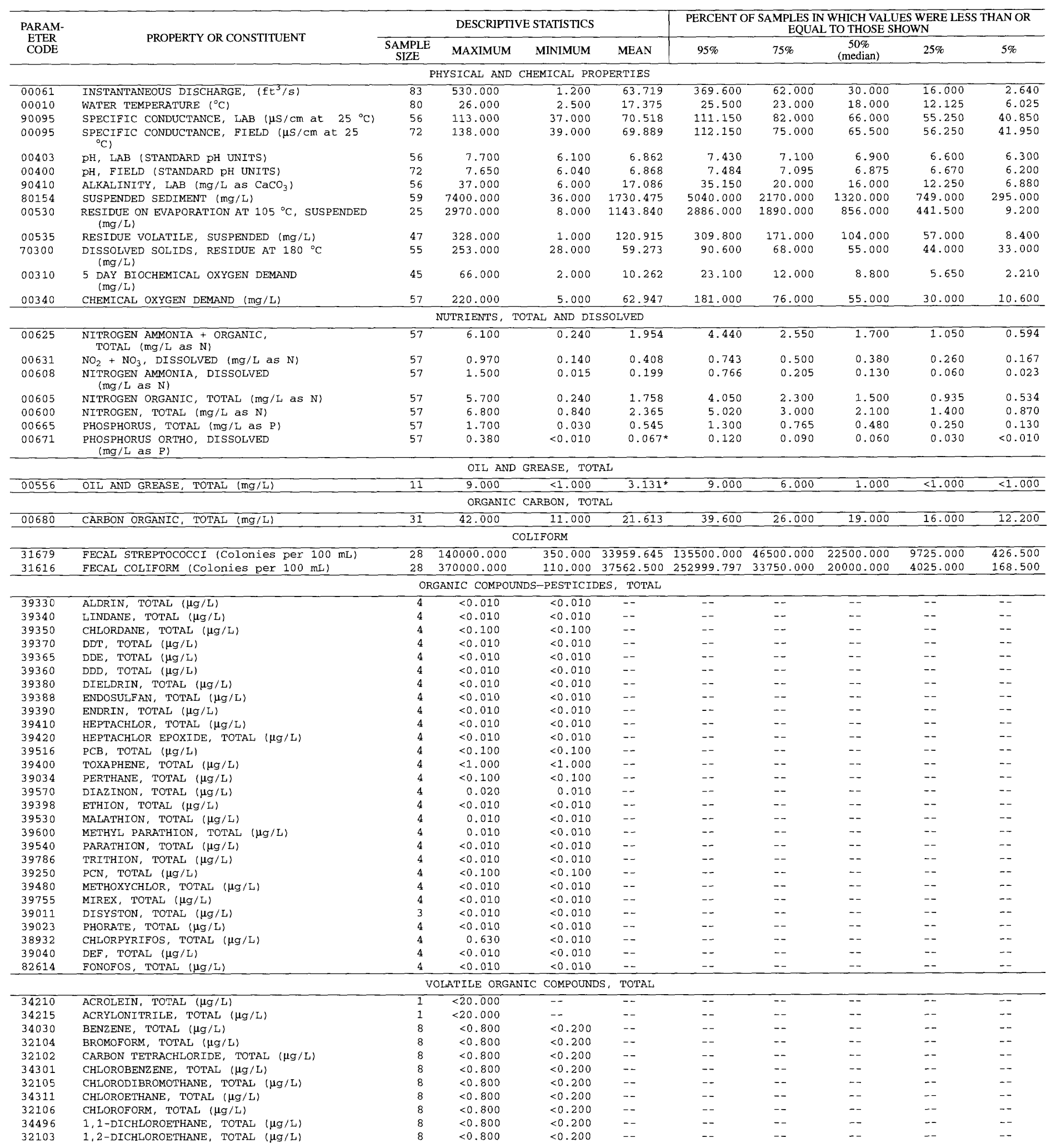

NOTE: Multiple detection limits during the period of record may result in different values flagged with a "<."

* Value is estimated by using a log-probability regression to predict the values of data below the detection limit. 
Table 67. Statistical summary of water-quality data at site 34 (CSW09), June 1994 through September 1997—Continued

\begin{tabular}{|c|c|c|c|c|c|c|c|c|c|c|}
\hline \multirow{2}{*}{$\begin{array}{c}\text { PARAM- } \\
\text { ETER } \\
\text { CODE }\end{array}$} & \multirow{2}{*}{ PROPERTY OR CONSTITUENT } & \multicolumn{4}{|c|}{ DESCRIPTIVE STATISTICS } & \multicolumn{5}{|c|}{$\begin{array}{l}\text { PERCENT OF SAMPLES IN WHICH VALUES WERE LESS THAN OR } \\
\text { EQUAL TO THOSE SHOWN }\end{array}$} \\
\hline & & $\begin{array}{l}\text { SAMPLE } \\
\text { SIZE }\end{array}$ & MAXIMUM & MINIMUM & MEAN & $95 \%$ & $75 \%$ & $\begin{array}{c}50 \% \\
\text { (median) }\end{array}$ & $25 \%$ & $5 \%$ \\
\hline 34501 & 1,1 -DICHLOROETHYLENE, TOTAL $(\mu \mathrm{g} / \mathrm{L})$ & 8 & $<0.800$ & $<0.200$ & -- & -- & -- & -- & -- & -- \\
\hline 34541 & 1,2 -DICHLOROPROPANE, TOTAL $(\mu \mathrm{g} / \mathrm{L})$ & 8 & $<0.800$ & $<0.200$ & -- & -- & -- & -- & -- & -- \\
\hline 34371 & ETHYLBENZENE， TOTAL $(\mu \mathrm{g} / \mathrm{L}$ ) & 8 & $<0.800$ & $<0.200$ & -. & -- & -. & -- & -- & -- \\
\hline 34413 & METHYL BROMIDE, TOTAL $(\mu \mathrm{g} / \mathrm{L})$ & 8 & $<0.800$ & $<0.200$ & -- & -- & -- & -- & -- & -- \\
\hline 34423 & METHYLENE CHLORIDE, TOTAL $(\mu \mathrm{g} / \mathrm{L})$ & 8 & $<0.800$ & $<0.200$ & -- & -- & -- & -- & -- & -- \\
\hline 34516 & $1,1,2,2$-TETRACHLOROETHANE, TOTAL $(\mu \mathrm{g} / \mathrm{L})$ & 8 & $<0.800$ & $<0.200$ & -- & -- & -- & -- & -- & -- \\
\hline 34475 & TETRACHLOROETHYLENE，TOTAL $(\mu \mathrm{g} / \mathrm{L})$ & 8 & $<0.800$ & $<0.200$ & -- & -- & -. & -- & -- & -- \\
\hline 34546 & $\begin{array}{l}1,2 \text {-TRANSDICHLOROETHENE, TOTAL } \\
(\mu \mathrm{g} / \mathrm{L})\end{array}$ & 8 & $<0.800$ & $<0.200$ & -- & -- & -- & -- & -- & -- \\
\hline 34506 & $1,1,1$-TRICHLOROETHANE, TOTAL $(\mu \mathrm{g} / \mathrm{L})$ & 8 & $<0.800$ & $<0.200$ & -- & -- & -- & - & -- & -- \\
\hline 34511 & $1,1,2$-TRICHLOROETHANE，TOTAL $(\mu \mathrm{g} / L)$ & 8 & $<0.800$ & $<0.200$ & -. & -- & -- & -- & -- & -- \\
\hline 39180 & TRICHLOROETHYLENE, TOTAL $(\mu \mathrm{g} / \mathrm{L})$ & 8 & $<0.800$ & $<0.200$ & -- & - & -- & -- & -- & -- \\
\hline 39175 & VINYL CHLORIDE, TOTAL $(\mu \mathrm{g} / \mathrm{L})$ & 8 & $<0.800$ & $<0.200$ & -- & -- & -- & -. & -- & -- \\
\hline 30217 & DIBROMOMETHANE， TOTAL $(\mu \mathrm{g} / \mathrm{L})$ & 8 & $<0.800$ & $<0.200$ & - & -- & -- & -- & -- & -- \\
\hline 32101 & DICHLOROBROMOMETHANE, TOTAL $(\mu \mathrm{g} / \mathrm{L})$ & 8 & $<0.800$ & $<0.200$ & -- & - & -- & -- & -- & -- \\
\hline 34668 & $\begin{array}{l}\text { DICHLORODIFLUOROMETHANE，TOTAL } \\
(\mu \mathrm{g} / \mathrm{L})\end{array}$ & 8 & $<0.800$ & $<0.200$ & -- & -- & -- & -- & -- & -- \\
\hline 77651 & 1,2 -DIBROMOETHANE, TOTAL $(\mu \mathrm{g} / \mathrm{L})$ & 8 & $<0.800$ & $<0.200$ & -- & -- & -- & -- & -- & - \\
\hline 34418 & METHYLCHLORIDE，TOTAL $(\mu \mathrm{g} / \mathrm{L})$ & 8 & $<0.800$ & $<0.200$ & -- & - & -- & -- & -- & -- \\
\hline 34704 & $\begin{array}{l}\text { CIS } 1,3 \text {-DICHLOROPROPENE， TOTAL } \\
(\mu \mathrm{g} / \mathrm{L})\end{array}$ & 8 & $<0.800$ & $<0.200$ & -- & -- & -- & -- & - & -- \\
\hline 34699 & TRANS 1,3 -DICHLOROPROPENE，TOTAL $(\mu \mathrm{g} / \mathrm{L})$ & 8 & $<0.800$ & $<0.200$ & -- & - & -- & -- & -- & -- \\
\hline 77128 & STYRENE, TOTAL $(\mu \mathrm{g} / \mathrm{L})$ & 8 & $<0.800$ & $<0.200$ & -- & -. & -- & -- & -. & -- \\
\hline 81551 & XYLENE, TOTAL $(\mu \mathrm{g} / \mathrm{L})$ & 8 & $<0.800$ & $<0.200$ & - & -- & -- & - & -- & -- \\
\hline 82625 & DIBROMOCHLOROPROPANE, TOTAL $(\mu \mathrm{g} / \mathrm{L})$ & 8 & $<4.000$ & $<1.000$ & -- & -- & -. & -- & -- & -. \\
\hline 771.68 & 1,1 -DICHLOROPROPENE, TOTAL $(\mu \mathrm{g} / \mathrm{L})$ & 8 & $<0.800$ & $<0.200$ & -- & -- & -. & -- & - & - \\
\hline 77170 & 2,2 -DICHLOROPROPANE, TOTAL $(\mu \mathrm{g} / \mathrm{L})$ & 8 & $<0.800$ & $<0.200$ & - & -- & -- & -- & -- & -- \\
\hline 77173 & 1,3-DICHLOROPROPANE, TOTAL $(\mu \mathrm{g} / \mathrm{L})$ & 8 & $<0.800$ & $<0.200$ & -. & -- & -. & -- & -- & -- \\
\hline 77275 & 0 -CHLOROTOLUENE, TOTAL $(\mu \mathrm{g} / \mathrm{L})$ & 8 & $<0.800$ & $<0.200$ & -. & -- & -- & -- & -. & -. \\
\hline 77277 & P-CHLOROTOLUENE, TOTAL $(\mu \mathrm{g} / \mathrm{L})$ & 8 & $<0.800$ & $<0.200$ & - & - & -- & -- & -- & -- \\
\hline 77443 & 123-TRICHLOROPROPANE, TOTAL $(\mu \mathrm{g} / \mathrm{L})$ & 8 & $<0,800$ & $<0.200$ & -- & -- & -- & -- & -- & -- \\
\hline 77562 & 1112-TETRACHLOROETHANE, TOTAL $(\mu \mathrm{g} / \mathrm{L})$ & 8 & $<0.800$ & $<0.200$ & - & -. & - & -- & -- & - \\
\hline 78032 & TERTBUTYL METHYL ETHER, TOTAL $(\mu \mathrm{g} / \mathrm{L})$ & 8 & $<0.800$ & $<0.200$ & -- & -- & -- & - & -- & - \\
\hline 77297 & BROMOCHLORO METHANE, TOTAL $(\mu \mathrm{g} / \mathrm{L})$ & 8 & $<0.800$ & $<0.200$ & -. & -- & -. & -. & - - & - - \\
\hline 77093 & CIS- 1,2 -DICHLOROETHENE, TOTAL $(\mu \mathrm{g} / \mathrm{L})$ & 8 & $<0.800$ & $<0.200$ & -. & -- & -- & -- & -- & -- \\
\hline 34576 & 2-CHLOROETHYL VINYL ETHER, TOTAL $(\mu \mathrm{g} / \mathrm{L})$ & 5 & $<4.000$ & $<1.000$ & -- & -- & -- & -- & -- & -- \\
\hline 77223 & ISOPROPYL BENZENE, TOTAL $(\mu \mathrm{g} / \mathrm{L})$ & 8 & $<0.800$ & $<0.200$ & -- & -- & -- & -- & - & -- \\
\hline 77224 & N-PROPYL BENZENE, TOTAL $(\mu \mathrm{g} / \mathrm{L})$ & 8 & $<0.800$ & $<0.200$ & - & - & -- & -- & -- & -- \\
\hline 77350 & SEC-BUTYL BENZENE, TOTAL $(\mu \mathrm{g} / \mathrm{L})$ & 8 & $<0.800$ & $<0.200$ & -- & -. & -- & -- & -- & -- \\
\hline 77356 & P-ISOPROPYL TOLUENE, TOTAL $(\mu \mathrm{g} / \mathrm{L})$ & 8 & $<0.800$ & $<0.200$ & -- & -- & -- & -- & -- & -- \\
\hline 77342 & N-BUTYL BENZENE, TOTAL $(\mu \mathrm{g} / \mathrm{L}$ & 8 & $<0.800$ & $<0.200$ & -- & - & - & -- & -- & -- \\
\hline 77613 & $1,2,3$-TRICHLOROBENZENE，TOTAL $(\mu \mathrm{g} / \mathrm{L}$ & 8 & $<0.800$ & $<0.200$ & -- & -- & -- & -- & -. & -- \\
\hline 77652 & FREON-113, TOTAL $(\mu \mathrm{g} / \mathrm{L}$ & 8 & $<0.800$ & $<0.200$ & - - & -. & -- & -- & - & - \\
\hline 77226 & MESITYLENE, TOTAL $(\mu \mathrm{g} / \mathrm{L}$ & 8 & $<0.800$ & $<0.200$ & -- & -- & -- & -- & -- & -- \\
\hline 81555 & BROMOBENZENE，TOTAL $(\mu \mathrm{g} / \mathrm{L}$ & 8 & $<0.800$ & $<0.200$ & - & $=$ & $z$ & -- & -- & -- \\
\hline & & MET & LS AND MINO & CONSTITUEN & S, TOTAL & & & & & \\
\hline 01097 & ANTIMONY, TOTAL $(\mu \mathrm{g} / \mathrm{L}$ as $\mathrm{Sb})$ & 43 & 25.000 & $<1.000$ & $2.392^{\star}$ & 14.000 & 1.000 & $<1.000$ & $<1.000$ & $<1.000$ \\
\hline 01002 & ARSENIC, TOTAL $(\mu \mathrm{g} / \mathrm{L}$ as As $)$ & 43 & 10.000 & $<1.000$ & $2.312 *$ & 7.000 & 3.000 & 1.000 & $<1.000$ & $<1.000$ \\
\hline 01012 & BERYLLIUM, TOTAL $(\mu \mathrm{g} / \mathrm{L}$ as $\mathrm{Be})$ & 13 & $<10.000$ & $<10.000$ & -- & -- & -- & -- & -- & -- \\
\hline 01027 & CADMIUM, TOTAL $(\mu \mathrm{g} / \mathrm{L}$ as $\mathrm{Cd})$ & 41 & 8.000 & $<1.000$ & -- & $<1.000$ & $<1.000$ & $<1.000$ & $<1.000$ & $<1.000$ \\
\hline 01034 & CHROMIUM, TOTAL $(\mu \mathrm{g} / \mathrm{L}$ as $\mathrm{Cr})$ & 41 & 96.000 & 2.000 & 28.927 & 73.700 & 44.500 & 23.000 & 11.500 & 3.400 \\
\hline 01042 & COPPER, TOTAL $(\mu \mathrm{g} / \mathrm{L}$ as $\mathrm{Cu})$ & 41 & 170.000 & 1.000 & 48.659 & 139.000 & 67.500 & 37.000 & 21.000 & 7.100 \\
\hline 01051 & LEAD, TOTAL $(\mu \mathrm{g} / \mathrm{t}$ as $\mathrm{Pb})$ & 41 & 49.000 & 1.000 & 23.195 & 48.800 & 29.000 & 20.000 & 16.000 & 1.800 \\
\hline 71900 & MERCURY, TOTAL $(\mu \mathrm{g} / \mathrm{L}$ as $\mathrm{Hg})$ & 15 & 0.110 & $<0.100$ & $0.094^{\star}$ & 0.110 & 0.100 & $<0.100$ & $<0.100$ & $<0.100$ \\
\hline 01067 & NICKEL, TOTAL $(\mu \mathrm{g} / L$ as Ni) & 41 & 30.000 & 1.000 & 13.098 & 27.900 & 19.000 & 12.000 & 8.500 & 1.200 \\
\hline 01147 & SELENIUM, TOTAL $(\mu \mathrm{g} / \mathrm{L}$ as $\mathrm{Se})$ & 15 & 2.000 & $<1.000$ & $\ldots$ & 2.000 & $<2.000$ & $<1.000$ & $<1.000$ & $<1.000$ \\
\hline 01077 & SILVER, TOTAL $(\mu \mathrm{g} / \mathrm{L}$ as Ag) & 13 & $<1.000$ & $<1.000$ & -- & -- & -- & -- & -- & -- \\
\hline 01092 & ZINC, TOTAL $(\mu \mathrm{g} / \mathrm{L}$ as $\mathrm{Zn})$ & 41 & 240.000 & 20.000 & 130.488 & 220.000 & 180.000 & 130.000 & 80.000 & 41.000 \\
\hline 00720 & CYANIDE, TOTAL (mg/L as Cn) & 15 & $<0.010$ & $<0.010$ & - & -- & -- & -- & -- & -- \\
\hline & & ORGAN & C COMPOUNDS & PESTICIDES & DISSOLVED & & & & & \\
\hline 46342 & ALACHLOR, DISSOLVED $(\mu \mathrm{g} / \mathrm{L})$ & 3 & 0.005 & $<0.002$ & -- & $=$ & -- & -- & -- & -- \\
\hline 04040 & DEETHYL ATRAZINE, DISSOLVED $(\mu \mathrm{g} / \mathrm{L})$ & 3 & 0.009 & $<0.010$ & -- & -- & -- & -- & -- & -- \\
\hline 39632 & ATRAZINE, DISSOLVED $(\mu \mathrm{g} / \mathrm{L})$ & 3 & 0.117 & 0.008 & -- & -- & -- & -- & -- & -- \\
\hline 82686 & METHYL AZINPHOS, DISSOLVED $(\mu \mathrm{g} / \mathrm{L})$ & 3 & $<0.070$ & $<0.001$ & -- & -- & -- & - & -- & -- \\
\hline 82673 & BENFLURALIN， DISSOLVED $(\mu \mathrm{g} / \mathrm{L})$ & 3 & 0.005 & $<0.020$ & -- & -- & -- & -- & -- & -- \\
\hline 04028 & BUTYLATE, DISSOLVED $(\mu \mathrm{g} / \mathrm{L})$ & 3 & $<0.010$ & $<0.002$ & -- & -- & -- & -. & -- & -- \\
\hline 82680 & CARBARYL, DISSOLVED $(\mu \mathrm{g} / \mathrm{L})$ & 3 & 0.190 & 0.040 & -- & -. & -- & -- & -- & -- \\
\hline 82674 & CARBOFURAN, DISSOLVED $(\mu \mathrm{g} / \mathrm{L})$ & 3 & $<0.020$ & $<0.003$ & -- & -- & -- & -- & -- & -- \\
\hline 38933 & CHLORPYRIFOS, DISSOLVED $(\mu \mathrm{g} / \mathrm{L})$ & 3 & 0.210 & $<0.004$ & -- & -- & - & -- & -- & -- \\
\hline 04041 & CYANAZINE， DISSOLVED $(\mu \mathrm{g} / \mathrm{L})$ & 3 & $<0.020$ & $<0.004$ & -- & -- & -- & -- & -- & -- \\
\hline 82682 & DCPA， DISSOLVED $(\mu \mathrm{g} / \mathrm{L})$ & 3 & $<0.008$ & $<0.002$ & -- & -- & -- & -- & -- & -- \\
\hline 34653 & P, $P^{\prime}$ DDE， DISSOLVED $(\mu \mathrm{g} / \mathrm{L})$ & 3 & $<0.020$ & $<0.006$ & -- & - & - & -- & -- & - \\
\hline 39572 & DIAZINON， DISSOLVED $(\mu \mathrm{g} / \mathrm{L})$ & 3 & 0.024 & $<0.002$ & - & -- & -- & -- & -- & -- \\
\hline 39381 & DIELDRIN，DISSOLVED $(\mu \mathrm{g} / \mathrm{L})$ & 3 & $<0.010$ & $<0.001$ & -- & -- & -. & -- & -- & -- \\
\hline 82660 & $\begin{array}{l}\text { 2,6-DIETHYL ANILINE, DISSOLVED } \\
(\mu \mathrm{g} / \mathrm{L})\end{array}$ & 3 & $<0.010$ & $<0.003$ & -- & -- & -- & -- & -- & -- \\
\hline 82662 & DIMETHOATE，DISSOLVED $(\mu \mathrm{g} / L)$ & 1 & $<0.050$ & -- & -- & -- & -- & -- & -- & -- \\
\hline
\end{tabular}

NOTE: Multiple detection limits during the period of record may result in different values flagged with a "<."

* Value is estimated by using a log-probability regression to predict the values of data below the detection limit. 
Table 67. Statistical summary of water-quality data at site 34 (CSW09), June 1994 through September 1997-Continued

\begin{tabular}{|c|c|c|c|c|c|c|c|c|c|c|}
\hline \multirow{2}{*}{$\begin{array}{l}\text { PARAM- } \\
\text { ETER } \\
\text { CODE }\end{array}$} & \multirow{2}{*}{ PROPERTY OR CONSTITUENT } & \multicolumn{4}{|c|}{ DESCRIPTIVE STATISTICS } & PERCEN & $\begin{array}{l}\text { AMPLE } \\
\text { EQ }\end{array}$ & $\begin{array}{l}\text { VHICH VA } \\
\text { rO THOSE }\end{array}$ & $\begin{array}{l}\text { WERE I } \\
\text { IN }\end{array}$ & IAN OR \\
\hline & & $\begin{array}{l}\text { SAMPLE } \\
\text { SIZE }\end{array}$ & MAXIMUM & MINIMUM & MEAN & $95 \%$ & $75 \%$ & $\begin{array}{c}50 \% \\
\text { (median) }\end{array}$ & $25 \%$ & $5 \%$ \\
\hline 82677 & DISULFOTON， DISSOLVED $(\mu \mathrm{g} / \mathrm{L})$ & 3 & $<0.017$ & $<0.010$ & -- & $\cdots$ & -- & -- & -- & -- \\
\hline 82668 & EPTC, DISSOLVED $(\mu \mathrm{g} / \mathrm{L})$ & 3 & $<0.010$ & $<0.002$ & $\ldots$ & $\ldots$ & -- & -- & -- & -- \\
\hline 82663 & ETHALFLURALIN，DISSOLVED $(\mu \mathrm{g} / \mathrm{L})$ & 3 & $<0.020$ & $<0.004$ & -- & -- & -- & -- & -. & -- \\
\hline 82672 & ETHOPROP， DISSOLVED $(\mu \mathrm{g} / \mathrm{L})$ & 3 & $<0.020$ & $<0.003$ & -- & -. & -- & -- & -- & -- \\
\hline 04095 & FONOFOS, DISSOLVED $(\mu \mathrm{g} / \mathrm{L})$ & 3 & $<0.010$ & $<0.003$ & -- & -- & -- & -- & -- & $\ldots$ \\
\hline 34253 & ALPHA BHC, DISSOLVED $(\mu \mathrm{g} / \mathrm{L})$ & 3 & $<0.010$ & $<0.002$ & -. & -- & -- & -- & -- & -- \\
\hline 39341 & LINDANE, DISSOLVED $(\mu \mathrm{g} / \mathrm{L})$ & 3 & $<0.020$ & $<0.004$ & -- & -- & -- & -- & -- & -- \\
\hline 39532 & MALATHION, DISSOLVED $(\mu \mathrm{g} / \mathrm{L})$ & 3 & $<0.020$ & $<0.005$ & -- & -- & -- & - & $\ldots$ & -- \\
\hline 82667 & METHYL PARATHION, DISSOLVED $(\mu \mathrm{g} / \mathrm{L})$ & 3 & $<0.070$ & $<0.006$ & -- & -- & - & -- & -- & -- \\
\hline 39415 & METOLACHLOR, DISSOLVED $(\mu \mathrm{g} / \mathrm{L})$ & 3 & 0.069 & 0.003 & $\ldots$ & $\ldots$ & -- & -- & -- & -- \\
\hline 82630 & METRIBUZIN， DISSOLVED $(\mu \mathrm{g} / \mathrm{L})$ & 3 & $<0.040$ & $<0.004$ & -- & -- & -- & -- & -- & -- \\
\hline 82671 & MOLINATE, DISSOLVED $(\mu \mathrm{g} / \mathrm{L})$ & 3 & $<0.010$ & $<0.004$ & -. & -. & -- & -- & -- & -- \\
\hline 82684 & NAPROPAMIDE, DISSOLVED $(\mu \mathrm{g} / \mathrm{L})$ & 3 & $<0.020$ & $<0.003$ & -- & -- & -. & -- & -- & -- \\
\hline 39542 & PARATHION， DISSOLVED $(\mu \mathrm{g} / \mathrm{L})$ & 3 & $<0.040$ & $<0.004$ & $\ldots$ & -- & -- & -- & -- & -- \\
\hline 82669 & PEBULATE, DISSOLVED $(\mu \mathrm{g} / \mathrm{L})$ & 3 & $<0.020$ & $<0.004$ & -- & -. & -- & -- & -- & -- \\
\hline 82683 & PENDIMETHALIN, DISSOLVED $(\mu \mathrm{g} / \mathrm{L})$ & 3 & 0.022 & $<0.020$ & -. & -- & -- & -- & -- & -- \\
\hline 82687 & PERMETHRIN， DISSOLVED $(\mu \mathrm{g} / \mathrm{L})$ & 3 & $<0.030$ & $<0.005$ & -- & -- & -- & -- & -- & -- \\
\hline 82676 & PRONAMIDE, DISSOLVED $(\mu \mathrm{g} / \mathrm{L})$ & 3 & $<0.020$ & $<0.003$ & -- & -- & -- & -- & -- & -- \\
\hline 04037 & PROMETON， DISSOLVED $(\mu \mathrm{g} / \mathrm{L})$ & 3 & 0.130 & $<0.010$ & -- & -- & -- & -- & -- & -- \\
\hline 04024 & PROPACHLOR, DISSOLVED $(\mu \mathrm{g} / \mathrm{L})$ & 3 & $<0.030$ & $<0.007$ & -- & -- & -- & -- & -- & -- \\
\hline 82679 & PROPANIL, DISSOLVED $(\mu \mathrm{g} / \mathrm{L})$ & 3 & $<0.030$ & $<0.004$ & -- & - & - & -- & - & -- \\
\hline 82685 & PROPARGITE， DISSOLVED $(\mu \mathrm{g} / \mathrm{L})$ & 3 & $<0.013$ & $<0.010$ & -- & -- & - & -- & -- & -- \\
\hline 04035 & SIMAZINE, DISSOLVED $(\mu \mathrm{g} / \mathrm{L})$ & 3 & $<0.010$ & $<0.005$ & - & - & -- & -- & -- & -- \\
\hline 82681 & THIOBENCARB, DISSOLVED $(\mu \mathrm{g} / \mathrm{L})$ & 3 & $<0.010$ & $<0.002$ & -- & -- & -- & -- & -- & -- \\
\hline 82670 & TEBUTHIURON, DISSOLVED $(\mu \mathrm{g} / \mathrm{L})$ & 3 & $<0.030$ & $<0.010$ & -- & -- & -- & -- & -- & - \\
\hline 82665 & TERBACIL， DISSOLVED $(\mu \mathrm{g} / \mathrm{L})$ & 3 & $<0.060$ & $<0.007$ & -- & -- & -- & -- & - & - \\
\hline 82675 & TERBUFOS, DISSOLVED $(\mu \mathrm{g} / \mathrm{L})$ & 3 & $<0.020$ & $<0.013$ & -- & -- & -- & -- & -- & -- \\
\hline 82678 & TRIALLATE， DISSOLVED $(\mu \mathrm{g} / \mathrm{L})$ & 3 & $<0.010$ & $<0.001$ & -- & -- & -- & -- & -- & -- \\
\hline 82661 & TRIFLURALIN, DISSOLVED $(\mu \mathrm{g} / \mathrm{L})$ & 3 & 0.004 & $<0.002$ & -- & -- & -- & -- & -- & -- \\
\hline 39742 & $2,4,5-\mathrm{T}$, DISSOLVED $(\mu \mathrm{g} / \mathrm{L})$ & 3 & $<0.050$ & $<0.035$ & -- & -. & -- & -- & -- & -- \\
\hline 39732 & $2,4-\mathrm{D}$, DISSOLVED $(\mu \mathrm{g} / \mathrm{L})$ & 3 & 1.800 & $<0.035$ & -- & -- & - & -- & + & -- \\
\hline 38746 & $2,4-\mathrm{DB}$, DISSOLVED $(\mu \mathrm{g} / \mathrm{L})$ & 3 & $<0.050$ & $<0.035$ & -- & -- & .. & -- & -- & -- \\
\hline 49315 & ACIFLUORFEN, DISSOLVED $(\mu \mathrm{g} / \mathrm{L})$ & 3 & $<0.050$ & $<0.035$ & -- & -- & $\ldots$ & -- & -- & -- \\
\hline 49312 & ALDICARB， DISSOLVED $(\mu \mathrm{g} / \mathrm{L})$ & 3 & $<0.050$ & $<0.016$ & -- & -- & -. & -. & -- & -- \\
\hline 49313 & ALDICARB SULFONE, DISSOLVED $(\mu \mathrm{g} / \mathrm{L})$ & 3 & $<0.050$ & $<0.016$ & -- & -- & - & +- & -- & -- \\
\hline 49314 & $\begin{array}{l}\text { ALDICARB SULFOXIDE, DISSOLVED } \\
(\mu \mathrm{g} / \mathrm{L})\end{array}$ & 3 & $<0.050$ & $<0.021$ & -- & -- & - & -- & + & -- \\
\hline 49310 & CARBARYL， DISSOLVED $(\mu \mathrm{g} / \mathrm{L})$ & 3 & 0.070 & $<0.008$ & $\ldots$ & -. & -- & -- & -- & -- \\
\hline 49309 & CARBOFURAN，DISSOLVED $(\mu \mathrm{g} / \mathrm{L})$ & 3 & $<0.050$ & $<0.028$ & -. & -- & -. & $\ldots$ & -- & -- \\
\hline 49308 & 3-HYDROXY-CARBOFURAN $(\mu \mathrm{g} / \mathrm{L})$ & 3 & $<0.050$ & $<0.014$ & -- & -- & $-\infty$ & $\ldots$ & -- & -- \\
\hline 49307 & AMIBEN, DISSOLVED $(\mu \mathrm{g} / \mathrm{L})$ & 3 & $<0.050$ & $<0.011$ & -- & -- & - & -- & -- & -- \\
\hline 49306 & CHLOROTHALONIL， DISSOLVED $(\mu \mathrm{g} / \mathrm{L})$ & 3 & $<0.050$ & $<0.035$ & -- & -- & -. & -- & -- & -- \\
\hline 49305 & CLOPYRALID, DISSOLVED $(\mu \mathrm{g} / \mathrm{L})$ & 3 & $<0.050$ & $<0.050$ & -- & -- & - & -- & -- & -- \\
\hline 49304 & DACTHALMONO-ACID， DISSOLVED $(\mu \mathrm{g} / \mathrm{L})$ & 3 & $<0.050$ & $<0.017$ & -. & .. & $-\infty$ & -- & -- & -- \\
\hline 38442 & DICAMBA， DISSOLVED $(\mu \mathrm{g} / \mathrm{L})$ & 3 & $<0.050$ & $<0.035$ & -- & -- & -. & -- & -- & -- \\
\hline 49303 & DICHLOBENIL, DISSOLVED $(\mu \mathrm{g} / \mathrm{L})$ & 3 & $<0.050$ & $<0.020$ & - & - & + & -- & -- & -- \\
\hline 49302 & DICHLORPROP， DISSOLVED $(\mu \mathrm{g} / \mathrm{L})$ & 3 & $<0.050$ & $<0.032$ & -- & -- & -- & -- & -- & -- \\
\hline 49301 & DINOSEB，DISSOLVED $(\mu \mathrm{g} / \mathrm{L})$ & 3 & $<0.050$ & $<0.035$ & -- & -- & -. & -- & -- & -. \\
\hline 49300 & DIURON， DISSOLVED $(\mu \mathrm{g} / \mathrm{L})$ & 3 & $<0.050$ & $<0.020$ & -- & -. & - & -- & -- & - \\
\hline 49299 & $\begin{array}{l}4,6 \text {-DINITRO OCRESOL， DISSOLVED } \\
(\mu \mathrm{g} / \mathrm{L})\end{array}$ & 3 & $<0.050$ & $<0.035$ & -- & -- & -- & - & - & -- \\
\hline 49298 & ESFENVALERATE， DISSOLVED $(\mu \mathrm{g} / \mathrm{L})$ & 3 & $<0.050$ & $<0.019$ & - & -- & $\ldots$ & -- & + & -- \\
\hline 49297 & FENURON, DISSOLVED $(\mu \mathrm{g} / \mathrm{L})$ & 3 & $<0.050$ & $<0.013$ & -- & -- & -- & -- & - & - \\
\hline 38811 & FLUOMETURON, DISSOLVED $(\mu \mathrm{g} / \mathrm{L})$ & 3 & $<0.050$ & $<0.035$ & -. & -- & $\ldots$ & -- & -- & -- \\
\hline 38478 & LINURON, DISSOLVED $(\mu \mathrm{g} / \mathrm{L})$ & 3 & $<0.050$ & $<0.018$ & -- & - & -- & -- & - & -- \\
\hline 38482 & MCPA, DISSOLVED $(\mu \mathrm{g} / \mathrm{L})$ & 3 & $<0.050$ & $<0.050$ & -- & -- & -. & -- & -- & -- \\
\hline 38487 & MCPB， DISSOLVED $(\mu \mathrm{g} / \mathrm{L})$ & 3 & $<0.050$ & $<0.035$ & $\ldots$ & -. & -- & -- & -- & -- \\
\hline 38501 & METHIOCARB, DISSOLVED $(\mu \mathrm{g} / \mathrm{L})$ & 3 & $<0.050$ & $<0.026$ & -- & -- & - & -- & -- & -- \\
\hline 49296 & METHOMYL， DISSOLVED $(\mu \mathrm{g} / \mathrm{L})$ & 3 & $<0.050$ & $<0.017$ & -- & -- & -- & -- & - & -. \\
\hline 49295 & 1-NAPHTHOL，DISSOLVED $(\mu \mathrm{g} / \mathrm{L})$ & 3 & $<0.050$ & $<0.007$ & -- & -- & - & - & -- & -- \\
\hline 49294 & NEBURON, DISSOLVED $(\mu \mathrm{g} / \mathrm{L})$ & 3 & $<0.050$ & $<0.015$ & -- & -- & -- & -- & -- & -- \\
\hline 49293 & NORFLURAZON, DISSOLVED $(\mu \mathrm{g} / \mathrm{L})$ & 3 & $<0.050$ & $<0.024$ & -- & -. & -- & -- & -- & + \\
\hline 49292 & ORYZALIN, DISSOLVED $(\mu \mathrm{g} / \mathrm{L})$ & 3 & $<0.050$ & $<0.019$ & -- & -- & -. & -- & -- & -- \\
\hline 38866 & OXAMYL, DISSOLVED $(\mu \mathrm{g} / \mathrm{L})$ & 3 & $<0.050$ & $<0.018$ & 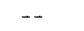 & - & -- & -- & -- & -- \\
\hline 49291 & PICLORAM, DISSOLVED $(\mu \mathrm{g} / \mathrm{L})$ & 3 & $<0.050$ & $<0.050$ & -- & -- & -- & -- & - & - \\
\hline 49236 & PROPHAM, DISSOLVED $(\mu \mathrm{g} / \mathrm{L})$ & 3 & $<0.050$ & $<0.035$ & -- & -- & -- & -- & - & - \\
\hline 38538 & PROPOXUR, DISSOLVED $(\mu \mathrm{g} / \mathrm{L})$ & 3 & $<0.050$ & $<0.035$ & -- & -- & - & - & -- & -- \\
\hline 39762 & SILVEX, DISSOLVED $(\mu \mathrm{g} / \mathrm{L})$ & 3 & $<0.050$ & $<0.021$ & -- & -- & -- & -- & -- & - \\
\hline 49235 & TRICLOPYR， DISSOLVED $(\mu \mathrm{g} / \mathrm{L})$ & 3 & 0.490 & $<0.050$ & -- & -- & -- & -- & -- & -- \\
\hline & & ORGA & COMPOUN & GANONITH & $\mathrm{NN}, \mathrm{T}$ & & & & & \\
\hline 39057 & PROMETRYNE, TOTAL $(\mu \mathrm{g} / \mathrm{L})$ & 1 & $<0.100$ & -- & $\ldots$ & - & -- & -- & -- & - \\
\hline 39056 & PROMETONE, TOTAL $(\mu \mathrm{g} / \mathrm{L})$ & 1 & $<0.200$ & - & -- & -- & -- & -- & - & -- \\
\hline 39054 & SIMETRYNE, TOTAL $(\mu \mathrm{g} / \mathrm{L})$ & 1 & $<0.100$ & -- & -- & -- & -- & -- & -- & -- \\
\hline 81757 & CYANAZINE, TOTAL $(\mu \mathrm{g} / \mathrm{L})$ & 1 & $<0.200$ & -- & -- & -- & -- & -- & -- & -- \\
\hline 77825 & ALACHLOR, TOTAL $(\mu \mathrm{g} / \mathrm{L})$ & 1 & $<0.100$ & -- & -- & -- & -- & -- & -- & -- \\
\hline 82611 & METRIBUZIN, TOTAL $(\mu \mathrm{g} / \mathrm{L})$ & 1 & $<0.100$ & -- & -- & -- & -- & -- & -- & -- \\
\hline
\end{tabular}

NOTE: Multiple detection limits during the period of record may result in different values flagged with a "<."

* Value is estimated by using a log-probability regression to predict the values of data below the detection limit. 
Table 67. Statistical summary of water-quality data at site 34 (CSW09), June 1994 through September 1997-Continued

\begin{tabular}{|c|c|c|c|c|c|c|c|c|c|c|}
\hline \multirow{2}{*}{$\begin{array}{l}\text { PARAM- } \\
\text { ETER } \\
\text { CODE }\end{array}$} & \multirow{2}{*}{ PROPERTY OR CONSTITUENT } & \multicolumn{4}{|c|}{ DESCRIPTIVE STATISTICS } & \multicolumn{5}{|c|}{$\begin{array}{c}\text { PERCENT OF SAMPLES IN WHICH VALUES WERE LESS THAN OR } \\
\text { EQUAL TO THOSE SHOWN }\end{array}$} \\
\hline & & $\begin{array}{l}\text { SAMPLE } \\
\text { SIZE }\end{array}$ & MAXIMUM & MINIMUM & MEAN & $95 \%$ & $75 \%$ & $\begin{array}{c}50 \% \\
\text { (median) }\end{array}$ & $25 \%$ & $5 \%$ \\
\hline 30311 & TERBACIL, TOTAL $(\mu \mathrm{g} / \mathrm{L})$ & 1 & $<0.200$ & $\overline{--}$ & - & -- & -- & -- & -- & -- \\
\hline 30264 & HEXAZINONE， TOTAL $(\mu \mathrm{g} / \mathrm{L})$ & 1 & $<0.200$ & -- & - & -- & -- & -- & -- & -- \\
\hline 30235 & BUTACHLOR, TOTAL $(\mu \mathrm{g} / \mathrm{L})$ & 1 & $<0.100$ & -- & -- & -- & -- & -- & -- & -- \\
\hline 30236 & BUTYLATE, TOTAL $(\mu \mathrm{g} / \mathrm{L})$ & 1 & $<0.100$ & -- & -- & -- & -- & - & -- & -- \\
\hline 75981 & DE-ETHYLATRAZINE，TOTAL $(\mu \mathrm{g} / \mathrm{L})$ & 1 & $<0.200$ & -- & - & -- & -- & -- & -- & -- \\
\hline 39630 & ATRAZINE, TOTAL $(\mu \mathrm{g} / \mathrm{L})$ & 1 & 0.100 & -- & -- & -- & -- & -- & -- & -- \\
\hline 39055 & SIMAZINE， TOTAL $(\mu \mathrm{g} / \mathrm{L})$ & 1 & $<0.100$ & -- & -- & -- & -- & -- & -- & -- \\
\hline 39024 & PROPAZINE, TOTAL $(\mu \mathrm{g} / \mathrm{L})$ & 1 & $<0.100$ & -- & -- & -- & -- & -- & -- & -- \\
\hline 82184 & AMETRYNE， TOTAL $(\mu \mathrm{g} / \mathrm{L})$ & 1 & $<0.100$ & -- & -- & -- & -- & -- & -- & -- \\
\hline 39030 & TRIFLURALIN, TOTAL $(\mu \mathrm{g} / \mathrm{L})$ & 1 & $<0.100$ & -- & -- & -- & -- & -- & -- & -- \\
\hline 82612 & METOLACHLOR, TOTAL $(\mu \mathrm{g} / L)$ & 1 & $<0.200$ & -- & - & -- & -- & -- & -- & -- \\
\hline 30234 & BROMACIL，TOTAL $(\mu \mathrm{g} / \mathrm{L})$ & 1 & $<0.200$ & -- & -- & -- & -- & -- & -- & -- \\
\hline 30255 & DIPHENAMID， TOTAL $(\mu \mathrm{g} / \mathrm{L})$ & 1 & $<0.100$ & -- & -- & -- & -- & -- & -- & -- \\
\hline 30324 & VERNOLATE, TOTAL $(\mu \mathrm{g} / \mathrm{L})$ & 1 & $<0.100$ & -- & -- & -- & -- & -- & -- & -- \\
\hline 30254 & CYCLOATE, TOTAL $(\mu \mathrm{g} / \mathrm{L})$ & 1 & $<0.100$ & -- & -- & -- & -- & -- & -- & -- \\
\hline 30295 & PROPACHLOR, TOTAL $(\mu \mathrm{g} / \mathrm{L})$ & 1 & $<0.100$ & -- & -- & -- & -- & -- & -- & -- \\
\hline 39730 & $2,4-D$, TOTAL $(\mu \mathrm{g} / \mathrm{L})$ & 1 & $<0.010$ & $\overline{--}$ & -- & -- & -- & -- & -- & -- \\
\hline 39760 & SILVEX, TOTAL $(\mu \mathrm{g} / \mathrm{L})$ & 1 & $<0.010$ & -- & - & - & -- & -- & -- & -- \\
\hline 39720 & PICLORAM, TOTAL $(\mu \mathrm{g} / \mathrm{L})$ & 1 & $<0.010$ & -- & -- & -- & -- & - & -- & -- \\
\hline 39740 & $2,4,5-\mathrm{T}$, TOTAL $(\mu \mathrm{g} / \mathrm{L})$ & 1 & $<0.010$ & -- & -- & -- & -- & -- & -- & -- \\
\hline 82183 & $2,4-\mathrm{DP}$, TOTAL $(\mu \mathrm{g} / \mathrm{L})$ & 1 & $<0.010$ & -- & -- & -- & -- & -- & -- & -- \\
\hline 82052 & DICAMBA，TOTAL $(\mu \mathrm{g} / \mathrm{L})$ & 1 & $<0.010$ & -- & -- & -- & -- & -- & -- & -- \\
\hline \multicolumn{11}{|c|}{ ORGANIC COMPOUNDS-CARBAMATE PESTICIDES, TOTAL } \\
\hline 39750 & SEVIN, TOTAL $(\mu \mathrm{g} / \mathrm{L})$ & 1. & $<0.500$ & -- & -- & -- & -- & -- & -- & -- \\
\hline 39051 & METHOMYL， TOTAL $(\mu \mathrm{g} / \mathrm{L})$ & 1 & $<0.500$ & -- & -- & -- & -- & -- & -- & -- \\
\hline 82619 & ALDICARD, TOTAL $(\mu \mathrm{g} / \mathrm{L})$ & 1 & $<0.500$ & -- & - & -- & -- & -- & -- & -- \\
\hline 30296 & PROPOXUR, TOTAL $(\mu \mathrm{g} / \mathrm{L})$ & 1 & $<0.500$ & -- & -- & -- & -- & -- & -- & -- \\
\hline 39052 & PROPHAM, TOTAL $(\mu \mathrm{g} / L)$ & 1 & $<0.500$ & -- & -- & -- & -- & -- & -- & -- \\
\hline 82615 & CARBOFURAN， TOTAL $(\mu \mathrm{g} / \mathrm{L})$ & 1 & $<0.500$ & -- & -- & -- & -- & -- & -- & -- \\
\hline 77441 & 1-NAPHTHOL，TOTAL $(\mu \mathrm{g} / \mathrm{L})$ & 1 & $<0.500$ & -- & -- & -- & -- & -- & -- & -- \\
\hline 30282 & METHIOCARB， TOTAL $(\mu \mathrm{g} / \mathrm{L})$ & 1 & $<0.500$ & - & -- & -- & -- & -- & -- & -- \\
\hline
\end{tabular}

NOTE: Multiple detection limits during the period of record may result in different values flagged with a "<."

* Value is estimated by using a log-probability regression to predict the values of data below the detection limit. 
Table 68. Statistical summary of water-quality data at site 37 (CSWO6), May 1995 through June 1997

\begin{tabular}{|c|c|c|c|c|c|c|c|c|c|c|}
\hline \multirow{2}{*}{$\begin{array}{l}\text { PARAM- } \\
\text { ETER } \\
\text { CODE }\end{array}$} & \multirow{2}{*}{ PROPERTY OR CONSTITUENT } & \multicolumn{4}{|c|}{ DESCRIPTIVE STATISTICS } & \multicolumn{5}{|c|}{$\begin{array}{c}\text { PERCENT OF SAMPLES IN WHICH VALUES WERE LESS THAN OR } \\
\text { EQUAL TO THOSE SHOWN }\end{array}$} \\
\hline & & $\begin{array}{l}\text { SAMPLE } \\
\text { SIZE }\end{array}$ & MAXIMUM & MINIMUM & MEAN & $95 \%$ & $75 \%$ & $\begin{array}{c}50 \% \\
\text { (median) }\end{array}$ & $25 \%$ & $5 \%$ \\
\hline \multicolumn{11}{|c|}{ PHYSICAL AND CHEMICAL PROPERTIES } \\
\hline 00061 & INSTANTANEOUS DISCHARGE, $\left(\mathrm{ft}^{3} / \mathrm{s}\right)$ & 60 & 40.000 & 0.010 & 3.519 & 23.210 & 3.275 & 1.035 & 0.507 & 0.082 \\
\hline 00010 & WATER TEMPERATURE $\left({ }^{\circ} \mathrm{C}\right)$ & 58 & 29.000 & 5.000 & 18.336 & 27.500 & 23.375 & 18.000 & 15.375 & 5.500 \\
\hline 90095 & SPECIFIC CONDUCTANCE, LAB $\left(\mu \mathrm{S} / \mathrm{cm}\right.$ at $\left.25^{\circ} \mathrm{C}\right)$ & 34 & 164.000 & 22.000 & 62.088 & 148.250 & 71.000 & 51.500 & 40.000 & 24.250 \\
\hline 00095 & $\begin{array}{l}\text { SPECIFIC CONDUCTANCE, FIELD ( } \mu \mathrm{S} / \mathrm{cm} \text { at } 25 \\
\left.{ }^{\circ} \mathrm{C}\right)\end{array}$ & 47 & 152.000 & 20.000 & 64.085 & 148.600 & 77.000 & 54.000 & 44.000 & 23.400 \\
\hline 00403 & pH, LAB (STANDARD pH UNITS) & 34 & 8.900 & 6.200 & 6.753 & 7.775 & 6.925 & 6.650 & 6.400 & 6.275 \\
\hline 00400 & pH， FIELD (STANDARD pH UNITS) & 47 & 7.950 & 6.000 & 6.634 & 7.452 & 6.750 & 6.600 & 6.500 & 6.140 \\
\hline 90410 & ALKALINITY, LAB (mg/L as $\mathrm{CaCO}_{3}$ ) & 34 & 52.000 & 5.600 & 12.241 & 32.500 & 14.000 & 8.900 & 7.000 & 5.825 \\
\hline 80154 & SUSPENDED SEDIMENT $(\mathrm{mg} / \mathrm{L})$ & 34 & 364.000 & 10.000 & 66.294 & 265.000 & 72.750 & 46.500 & 28.750 & 10.750 \\
\hline 00530 & $\begin{array}{l}\text { RESIDUE ON EVAPORATION AT } 105^{\circ} \mathrm{C} \text {, SUSPENDED } \\
(\mathrm{mg} / \mathrm{L})\end{array}$ & 19 & 111.000 & 4.000 & 29.000 & 111.000 & 32.000 & 20.000 & 8.000 & 4.000 \\
\hline 00535 & RESIDUE VOLATILE， SUSPENDED $(\mathrm{mg} / \mathrm{L})$ & 29 & 54.000 & $<1.000$ & $9.485^{*}$ & 50.000 & 10.000 & 5.000 & 2.000 & $<1.000$ \\
\hline 70300 & $\begin{array}{l}\text { DISSOLVED SOLIDS, RESIDUE AT } 180^{\circ} \mathrm{C} \\
(\mathrm{mg} / \mathrm{L})\end{array}$ & 34 & 190.000 & 19.000 & 51.559 & 133.000 & 54.000 & 41.000 & 35.500 & 21.250 \\
\hline 00310 & $\begin{array}{l}5 \text { DAY BIOCHEMICAL OXYGEN DEMAND } \\
(\mathrm{mg} / \mathrm{L})\end{array}$ & 34 & 20.000 & $<2.000$ & $6.352 *$ & 13.000 & 7.400 & 5.400 & 4.300 & $<2.000$ \\
\hline 00340 & CHEMICAL OXYGEN DEMAND (mg/L) & 34 & 190.000 & 5.000 & 31.735 & 90.250 & 34.250 & 27.500 & 18.000 & 8.750 \\
\hline \multicolumn{11}{|c|}{ NUTRIENTS, TOTAL AND DISSOLVED } \\
\hline 00625 & $\begin{array}{l}\text { NITROGEN AMMONIA + ORGANIC, } \\
\text { TOTAL (mg/L as N) }\end{array}$ & 34 & 2.600 & 0.360 & 0.835 & 2.000 & 0.962 & 0.720 & 0.575 & 0.360 \\
\hline 00631 & $\mathrm{NO}_{2}+\mathrm{NO}_{3}$, DISSOLVED $(\mathrm{mg} / \mathrm{L}$ as $\mathrm{N})$ & 34 & 0.850 & 0.050 & 0.322 & 0.760 & 0.450 & 0.320 & 0.190 & 0.080 \\
\hline 00608 & $\begin{array}{l}\text { NITROGEN AMMONIA, DISSOLVED } \\
(\mathrm{mg} / \mathrm{L} \text { as N) }\end{array}$ & 34 & 0.340 & $<0.015$ & $0.090^{\star}$ & 0.330 & 0.120 & 0.050 & 0.030 & $<0.015$ \\
\hline 00605 & NITROGEN ORGANIC, TOTAL (mg/L as N) & 34 & 2.600 & 0.310 & 0.746 & 1.925 & 0.805 & 0.665 & 0.530 & 0.340 \\
\hline 00600 & NITROGEN, TOTAL (mg/L as N) & 34 & 3.000 & 0.360 & 1.157 & 2.325 & 1.500 & 1.100 & 0.837 & 0.458 \\
\hline 00665 & PHOSPHORUS, TOTAL (mg/L as P) & 34 & 1.400 & 0.028 & 0.235 & 0.620 & 0.252 & 0.200 & 0.145 & 0.067 \\
\hline 00671 & $\begin{array}{l}\text { PHOSPHORUS ORTHO, DISSOLVED } \\
(\mathrm{mg} / \mathrm{L} \text { aS } \mathrm{P})\end{array}$ & 34 & 0.270 & 0.010 & 0.091 & 0.248 & 0.132 & 0.080 & 0.040 & 0.012 \\
\hline \multicolumn{11}{|c|}{ OIL AND GREASE, TOTAL } \\
\hline 00556 & OIL AND GREASE, TOTAL (mg/L) & 10 & 3.000 & $<1.000$ & $1.722^{*}$ & 3.000 & 3.000 & 1.000 & $<1.000$ & $<1.000$ \\
\hline \multicolumn{11}{|c|}{ ORGANIC CARBON, TOTAL } \\
\hline 00680 & CARBON ORGANIC, TOTAL $(\mathrm{mg} / \mathrm{L})$ & 19 & 20.000 & 5.700 & 11.400 & 20.000 & 14.000 & 10.000 & 8.700 & 5.700 \\
\hline & & & & SIFORM & & & & & & \\
\hline 31679 & FECAL STREPTOCOCCI (Colonies per $100 \mathrm{~mL}$ ) & 28 & 140000.000 & 450.000 & 45553.215 & 130999.984 & 70500.000 & 44500.000 & 16000.000 & 490.500 \\
\hline 31616 & FECAL COLIFORM (Colonies per $100 \mathrm{~mL}$ ) & 28 & 480000.000 & 90.000 & 77872.500 & 430499.906 & 71750.000 & 27500.000 & 7025.000 & 90.000 \\
\hline & & ORG & GANIC COMPO & S-PESTICID & DES, TOTAL & & & & & \\
\hline 39330 & ALDRIN， TOTAL $(\mu \mathrm{g} / \mathrm{L})$ & 3 & $<0.010$ & $<0.010$ & -- & -- & -- & -- & $\overline{--}$ & -- \\
\hline 39340 & LINDANE, TOTAL $(\mu \mathrm{g} / \mathrm{L})$ & 3 & $<0.010$ & $<0.010$ & -- & -- & -- & -- & -- & -- \\
\hline 39350 & CHLORDANE, TOTAL $(\mu \mathrm{g} / \mathrm{L})$ & 3 & $<0.100$ & $<0.100$ & -- & -- & -- & -- & -- & -- \\
\hline 39370 & DDT, TOTAL $(\mu \mathrm{g} / \mathrm{L})$ & 3 & $<0.010$ & $<0.010$ & -- & $-\cdots$ & -- & -- & -- & -- \\
\hline 39365 & DDE, TOTAL $(\mu \mathrm{g} / \mathrm{L})$ & 3 & $<0.010$ & $<0.010$ & -- & -- & -- & -- & -- & -- \\
\hline 39360 & DDD， TOTAL $(\mu \mathrm{g} / \mathrm{L})$ & 3 & $<0.010$ & $<0.010$ & -- & -. & -- & -- & -- & -- \\
\hline 39380 & DIELDRIN， TO'TAL $(\mu \mathrm{g} / \mathrm{L})$ & 3 & $<0.010$ & $<0.010$ & -- & -- & -- & -- & -- & -- \\
\hline 39388 & ENDOSULFAN, TOTAL $(\mu \mathrm{g} / \mathrm{L})$ & 3 & $<0.010$ & $<0.010$ & -- & -- & -- & -- & -- & -- \\
\hline 39390 & ENDRIN， TOTAL $(\mu \mathrm{g} / \mathrm{L})$ & 3 & $<0.010$ & $<0.010$ & -- & -- & -- & -- & -- & -- \\
\hline 39410 & HEPTACHLOR, TOTAL $(\mu \mathrm{g} / \mathrm{L})$ & 3 & $<0.010$ & $<0.010$ & -- & -- & -- & -- & -- & -- \\
\hline 39420 & HEPTACHLOR EPOXIDE, TOTAL $(\mu \mathrm{g} / \mathrm{L})$ & 3 & $<0.010$ & $<0.010$ & -- & -- & -- & -- & -- & -- \\
\hline 39516 & PCB， TOTAL $(\mu \mathrm{g} / \mathrm{L})$ & 3 & 0.100 & $<0.100$ & -- & -- & -- & -- & -- & -. \\
\hline 39400 & TOXAPHENE, TOTAL $(\mu \mathrm{g} / \mathrm{L})$ & 3 & $<1.000$ & $<1.000$ & -- & -- & -- & -- & -- & -- \\
\hline 39034 & PERTHANE，TOTAL $(\mu \mathrm{g} / \mathrm{L})$ & 3 & $<0.100$ & $<0.100$ & -- & -- & -- & -- & -- & -- \\
\hline 39570 & DIAZINON, TOTAL $(\mu \mathrm{g} / \mathrm{L})$ & 3 & 0.010 & $<0.010$ & -- & -- & -- & -- & -- & -- \\
\hline 39398 & ETHION, TOTAL $(\mu \mathrm{g} / \mathrm{L})$ & 3 & $<0.010$ & $<0.010$ & -- & -- & -- & -- & - & -- \\
\hline 39530 & MALATHION， TOTAL $(\mu \mathrm{g} / \mathrm{L})$ & 3 & 0.010 & $<0.010$ & -- & -- & -- & -- & -- & -- \\
\hline 39600 & METHYL PARATHION, TOTAL $(\mu \mathrm{g} / \mathrm{L})$ & 3 & 0.010 & $<0.010$ & $\rightarrow$ & -- & -- & -- & -- & -- \\
\hline 39540 & PARATHION, TOTAL $(\mu \mathrm{g} / \mathrm{L})$ & 3 & $<0.010$ & $<0.010$ & -- & -- & -- & -- & -- & -- \\
\hline 39786 & TRITHION， TOTAL $(\mu \mathrm{g} / \mathrm{L})$ & 3 & $<0.010$ & $<0.010$ & -- & -- & -- & -- & -- & -- \\
\hline 39250 & PCN, TOTAL $(\mu \mathrm{g} / \mathrm{L})$ & 3 & $<0.100$ & $<0.100$ & -- & -- & -- & -- & -- & -- \\
\hline 39480 & METHOXYCHLOR， TOTAL $(\mu \mathrm{g} / \mathrm{L})$ & 3 & $<0.010$ & $<0.010$ & -- & - & -- & -- & -- & -- \\
\hline 39755 & MIREX, TOTAL $(\mu \mathrm{g} / \mathrm{L})$ & 3 & $<0.010$ & $<0.010$ & -- & -- & -- & -- & -- & -- \\
\hline 39011 & DISYSTON, TOTAL $(\mu \mathrm{g} / \mathrm{L})$ & 3 & $<0.010$ & $<0.010$ & -- & -- & -- & -- & -- & -- \\
\hline 39023 & PHORATE, TOTAL $(\mu \mathrm{g} / \mathrm{L})$ & 3 & $<0.010$ & $<0.010$ & -- & -- & -- & -- & -- & -- \\
\hline 38932 & CHLORPYRIFOS, TOTAL $(\mu \mathrm{g} / \mathrm{L})$ & 3 & $<0.010$ & $<0.010$ & -- & -- & -- & -- & -- & -- \\
\hline 39040 & DEF， TOTAL $(\mu \mathrm{g} / \mathrm{L})$ & 3 & $<0.010$ & $<0.010$ & -- & -- & -- & -- & -- & -- \\
\hline 82614 & FONOFOS, TOTAL $(\mu \mathrm{g} / \mathrm{L})$ & 3 & $<0.010$ & $<0.010$ & - & -- & -- & -- & -- & -- \\
\hline & & & OLATILE ORG & COMPOUND & TOTAL & & & & & \\
\hline 34030 & BENZENE, TOTAL $(\mu \mathrm{g} / \mathrm{L})$ & 8 & $<2.000$ & $<0.200$ & -- & -- & -- & -- & -- & -- \\
\hline 32104 & BROMOFORM, TOTAL $(\mu \mathrm{g} / \mathrm{L})$ & 8 & $<2.000$ & $<0.200$ & -- & -- & -- & -- & -- & -- \\
\hline 32102 & CARBON TETRACHLORIDE, TOTAL $(\mu \mathrm{g} / \mathrm{L})$ & 8 & $<2.000$ & $<0.200$ & -- & -_ & -- & -- & -- & -- \\
\hline 34301 & CHLOROBENZENE, TOTAL $(\mu \mathrm{g} / \mathrm{L})$ & 8 & $<2.000$ & $<0.200$ & -- & -- & - & -- & -- & -- \\
\hline 32105 & CHLORODIBROMOTHANE, TOTAL $(\mu \mathrm{g} / \mathrm{L})$ & 8 & $<2.000$ & $<0.200$ & -- & -- & -- & -- & -- & -- \\
\hline 34311 & CHLOROETHANE, TOTAL $(\mu \mathrm{g} / \mathrm{L})$ & 8 & $<2.000$ & $<0.200$ & -- & -- & -- & -- & -- & -- \\
\hline 32106 & CHLOROFORM， TOTAL $(\mu \mathrm{g} / \mathrm{L})$ & 8 & $<2.000$ & $<0.200$ & -- & -- & -- & -- & -- & -- \\
\hline 34496 & 1,1 -DICHLOROETHANE, TOTAL $(\mu \mathrm{g} / \mathrm{L})$ & 8 & $<2.000$ & $<0.200$ & -- & -- & -- & -_ & -- & -- \\
\hline 32103 & 1,2-DICHLOROETHANE，TOTAL $(\mu \mathrm{g} / \mathrm{L})$ & 8 & $<2.000$ & $<0.200$ & -- & -- & -- & -- & -- & -- \\
\hline 34501 & 1,1 -DICHLOROETHYLENE, TOTAL $(\mu \mathrm{g} / \mathrm{L})$ & 8 & $<2.000$ & $<0.200$ & -- & -- & -- & -- & -- & -- \\
\hline 34541 & 1,2 -DICHLOROPROPANE, TOTAL $(\mu \mathrm{g} / \mathrm{L})$ & 8 & $<2.000$ & $<0.200$ & -- & -- & -- & -- & -- & -- \\
\hline
\end{tabular}

NOTE: Multiple detection limits during the period of record may result in different values flagged with a "<."

* Value is estimated by using a log-probability regression to predict the values of data below the detection limit. 
Table 68. Statistical summary of water-quality data at site 37 (CSW06), May 1995 through June 1997-Continued

\begin{tabular}{|c|c|c|c|c|c|c|c|c|c|c|}
\hline \multirow{2}{*}{$\begin{array}{l}\text { PARAM- } \\
\text { ETER } \\
\text { CODE }\end{array}$} & \multirow{2}{*}{ PROPERTY OR CONSTITUENT } & \multicolumn{4}{|c|}{ DESCRIPTIVE STATISTICS } & \multicolumn{5}{|c|}{$\begin{array}{c}\text { PERCENT OF SAMPLES IN WHICH VALUES WERE LESS THAN OR } \\
\text { EQUAL TO THOSE SHOWN }\end{array}$} \\
\hline & & $\begin{array}{l}\text { SAMPLE } \\
\text { SIZE }\end{array}$ & MAXIMUM & MINIMUM & MEAN & $95 \%$ & $75 \%$ & $\begin{array}{c}50 \% \\
\text { (median) }\end{array}$ & $25 \%$ & $5 \%$ \\
\hline 34371 & ETHYLBENZENE, TOTAL $(\mu \mathrm{g} / \mathrm{L})$ & 8 & $<2.000$ & $<0.200$ & $-\overline{-}$ & -- & -- & -- & -- & -- \\
\hline 34413 & METHYL BROMIDE, TOTAL $(\mu \mathrm{g} / \mathrm{L})$ & 8 & $<2.000$ & $<0.200$ & -- & -- & -- & -- & -- & - \\
\hline 34423 & METHYLENE CHLORIDE, TOTAL $(\mu \mathrm{g} / \mathrm{L})$ & 8 & $<2.000$ & $<0.200$ & -. & -. & -- & -- & -- & -- \\
\hline 34516 & $1,1,2,2$-TETRACHLOROETHANE，TOTAL $(\mu \mathrm{g} / \mathrm{L})$ & 8 & $<2.000$ & $<0.200$ & -- & -- & -- & -- & -- & -- \\
\hline 34475 & TETRACHLOROETHYLENE, TOTAL $(\mu \mathrm{g} / \mathrm{L})$ & 8 & $<2.000$ & $<0.200$ & -- & -- & -- & -- & -- & -- \\
\hline 34010 & TOLUENE, TOTAL $(\mu \mathrm{g} / \mathrm{L})$ & 8 & 0.300 & $<0.200$ & -. & 0.300 & $<0.800$ & $<0.800$ & $<0.400$ & $<0.400$ \\
\hline 34546 & $\begin{array}{l}\text { 1,2-TRANSDICHLOROETHENE， TOTAL } \\
(\mu \mathrm{g} / \mathrm{L})\end{array}$ & 8 & $<2.000$ & $<0.200$ & -- & -- & -- & -- & -- & -- \\
\hline 34506 & $1,1,1$-TRICHLOROETHANE，TOTAL $(\mu \mathrm{g} / \mathrm{L})$ & 8 & 1.300 & $<0.200$ & -. & 1.300 & $<0.800$ & $<0.400$ & $<0.400$ & $<0.400$ \\
\hline 34511 & $1,1,2$-TRICHLOROETHANE, TOTAL $(\mu \mathrm{g} / \mathrm{L})$ & 8 & $<2.000$ & $<0.200$ & -- & -- & -- & -- & -- & -- \\
\hline 39180 & TRICHLOROETHYLENE，TOTAL $(\mu \mathrm{g} / \mathrm{L})$ & 8 & $<2.000$ & $<0.200$ & -- & -- & -- & -- & -- & - - \\
\hline 39175 & VINYL CHLORIDE, TOTAL $\langle\mu \mathrm{g} / \mathrm{L}\rangle$ & 8 & $<2.000$ & $<0.200$ & -- & -- & -- & -- & -- & -- \\
\hline 30217 & DIBROMOMETHANE, TOTAL $(\mu \mathrm{g} / \mathrm{L})$ & 8 & $<2.000$ & $<0.200$ & -- & -- & -- & -- & -- & -- \\
\hline 32101 & DICHLOROBROMOMETHANE, TOTAL $(\mu \mathrm{g} / \mathrm{L})$ & 8 & $<2.000$ & $<0.200$ & -- & -- & -- & -- & -- & -- \\
\hline 34668 & $\begin{array}{l}\text { DICHLORODIFLUOROMETHANE, TOTAL } \\
(\mu \mathrm{g} / \mathrm{L})\end{array}$ & 8 & $<2.000$ & $<0.200$ & -- & -- & -- & -- & -- & -- \\
\hline 34488 & TRICHLOROFLUOROMETHANE, TOTAL $(\mu \mathrm{g} / \mathrm{L})$ & 8 & $<2.000$ & $<0.200$ & -- & -- & -- & -- & -- & -- \\
\hline 77651 & 1,2-DIBROMOETHANE, TOTAL $(\mu \mathrm{g} / \mathrm{L})$ & 8 & $<2.000$ & $<0.200$ & -- & -- & -- & -- & -- & -. \\
\hline 34704 & $\begin{array}{l}\text { CIS } 1,3 \text {-DICHLOROPROPENE, TOTAL } \\
(\mu \mathrm{g} / \mathrm{L})\end{array}$ & 8 & $<2.000$ & $<0.200$ & -- & -- & -- & -- & -- & -- \\
\hline 34699 & TRANS 1,3 -DICHLOROPROPENE, TOTAL $(\mu \mathrm{g} / \mathrm{L})$ & 8 & $<2.000$ & $<0.200$ & -- & -- & -- & -- & -- & -- \\
\hline 77128 & STYRENE, TOTAL $(\mu \mathrm{g} / \mathrm{L})$ & 8 & $<2.000$ & $<0.200$ & -- & -- & -- & -- & -- & -- \\
\hline 81551 & XYLENE, TOTAL $(\mu \mathrm{g} / \mathrm{L})$ & 8 & $<2.000$ & $<0.200$ & -. & -- & -- & -. & -- & -- \\
\hline 82625 & DIBROMOCHLOROPROPANE, TOTAL $(\mu \mathrm{g} / \mathrm{L})$ & 8 & $<10.000$ & $<1.000$ & -- & -- & -- & -- & -- & -- \\
\hline 77168 & 1,1 -DICHLOROPROPENE, TOTAL $(\mu \mathrm{g} / \mathrm{L})$ & 8 & $<2.000$ & $<0.200$ & -- & -- & -- & -- & -. & -- \\
\hline 77170 & 2,2 -DICHLOROPROPANE，TOTAL $(\mu \mathrm{g} / \mathrm{L})$ & 8 & $<2.000$ & $<0.200$ & -- & -- & -. & -- & -. & -- \\
\hline 77173 & 1,3 -DICHLOROPROPANE, TOTAL $(\mu \mathrm{g} / \mathrm{L})$ & 8 & $<2.000$ & $<0.200$ & -. & -- & -- & -- & -- & -. \\
\hline 77275 & 0 -CHLOROTOLUENE, TOTAL $(\mu \mathrm{g} / \mathrm{L})$ & 8 & $<2.000$ & $<0.200$ & -- & -- & -- & -- & -- & -- \\
\hline 77277 & P-CHLOROTOLUENE, TOTAL $(\mu \mathrm{g} / \mathrm{L})$ & 8 & $<2.000$ & $<0.200$ & -. & -- & -- & -- & -- & - \\
\hline 77443 & 123-TRICHLOROPROPANE, TOTAL $(\mu \mathrm{g} / \mathrm{L})$ & 8 & $<2.000$ & $<0.200$ & -. & -. & -- & .. & -- & -- \\
\hline 77562 & 1112 -TETRACHLOROETHANE, TOTAL $(\mu \mathrm{g} / \mathrm{L})$ & 8 & $<2.000$ & $<0.200$ & -- & -- & -- & -- & -- & - \\
\hline 78032 & TERTBUTYL METHYL ETHER, TOTAL $(\mu \mathrm{g} / \mathrm{L})$ & 8 & $<2.000$ & $<0.200$ & -- & -. & -. & .- & -- & -- \\
\hline 77297 & BROMOCHLORO METHANE, TOTAL $(\mu \mathrm{g} / \mathrm{L})$ & 8 & $<2.000$ & $<0.200$ & -- & -- & -- & -- & -- & -- \\
\hline 77093 & CIS-1,2-DICHLOROETHENE, TOTAL $(\mu \mathrm{g} / \mathrm{L})$ & 8 & $<2.000$ & $<0.200$ & -. & -- & _- & -. & -. & -. \\
\hline 34576 & 2-CHLOROETHYL VINYL ETHER, TOTAL $(\mu \mathrm{g} / \mathrm{L})$ & 5 & $<10.000$ & $<1.000$ & -- & -- & -. & -. & -. & -- \\
\hline 77223 & ISOPROPYL BENZENE, TOTAL $(\mu \mathrm{g} / \mathrm{L})$ & 8 & $<2.000$ & $<0.200$ & -- & - & -- & -- & -- & -- \\
\hline 77224 & N-PROPYL BENZENE, TOTAL $(\mu \mathrm{g} / \mathrm{L})$ & 8 & $<2.000$ & $<0.200$ & -- & -- & -- & -. & -. & -. \\
\hline 77353 & TERTBUTYL BENZENE, TOTAL $(\mu \mathrm{g} / \mathrm{L})$ & 8 & $<2.000$ & $<0.200$ & -- & -- & -- & -- & -- & -- \\
\hline 77222 & PSEUDOCUMENE, TOTAL $(\mu \mathrm{g} / \mathrm{L})$ & 8 & $<2.000$ & $<0.200$ & -- & -- & -. & -- & -- & -- \\
\hline 77613 & $1,2,3$-TRICHLOROBENZENE，TOTAL $(\mu \mathrm{g} / \mathrm{L}$ & 8 & $<2.000$ & $<0.200$ & -- & - - & -- & -- & -- & -- \\
\hline 77652 & FREON-113, TOTAL $(\mu \mathrm{g} / \mathrm{L}$ & 8 & $<2.000$ & $<0.200$ & -- & -- & -- & -- & - & -- \\
\hline 77226 & MESITYLENE, TOTAL $(\mu \mathrm{g} / \mathrm{L}$ & 8 & $<2.000$ & $<0.200$ & -- & -- & -- & -- & -- & -- \\
\hline 81555 & BROMOBENZENE, TOTAL $(\mu \mathrm{g} / \mathrm{L}$ & 8 & $<2.000$ & $<0.200$ & -- & -- & -- & -- & -- & -- \\
\hline & & MET & LS AND MINO & CONSTITUEN & CS, TOTAL & & & & & \\
\hline 01097 & ANTIMONY, TOTAL $(\mu \mathrm{g} / \mathrm{L}$ as $\mathrm{Sb})$ & 24 & 1.000 & $<1.000$ & -- & 1.000 & $<1.000$ & $<1.000$ & $<1.000$ & $<1.000$ \\
\hline 01002 & ARSENIC, TOTAL $(\mu \mathrm{g} / \mathrm{L}$ as As) & 24 & 1.000 & $<1.000$ & -- & $<1.000$ & $<1.000$ & $<1.000$ & $<1.000$ & $<1.000$ \\
\hline 01012 & BERYLLIUM, TOTAL $(\mu \mathrm{g} / \mathrm{L}$ as $\mathrm{Be})$ & 19 & $<10.000$ & $<10.000$ & -- & $-\infty$ & $-\infty$ & -- & -- & $\ldots$ \\
\hline 01027 & CADMIUM, TOTAL $(\mu \mathrm{g} / \mathrm{L}$ as $\mathrm{Cd})$ & 19 & $<1.000$ & $<1.000$ & -- & -- & -. & -. & -- & -- \\
\hline 01034 & CHROMIUM, TOTAL $(\mu \mathrm{g} / \mathrm{L}$ as $\mathrm{Cr})$ & 24 & 32.000 & $<1.000$ & $5.273^{*}$ & 15.000 & 5.000 & 3.000 & 2.000 & $<1.000$ \\
\hline 01042 & COPPER, TOTAL $(\mu \mathrm{g} / \mathrm{L}$ as $\mathrm{Cu})$ & 24 & 44.000 & 5.000 & 14.500 & 40.000 & 16.000 & 13.000 & 10.250 & 5.500 \\
\hline 01051 & LEAD, TOTAL $(\mu \mathrm{g} / \mathrm{L}$ as $\mathrm{Pb})$ & 24 & 41.000 & 1.000 & 5.333 & 33.750 & 5.750 & 2.500 & 2.000 & 1.000 \\
\hline 71900 & MERCURY, TOTAL $(\mu \mathrm{g} / \mathrm{L}$ as $\mathrm{Hg})$ & 24 & $<0.100$ & $<0.100$ & -- & -- & -- & -- & -- & -- \\
\hline 01067 & NICKEL, TOTAL $(\mu \mathrm{g} / \mathrm{L}$ as Ni) & 24 & 41.000 & 4.000 & 10.167 & 35.250 & 10.000 & 8.000 & 6.250 & 4.500 \\
\hline 01147 & SELENIUM, TOTAL $(\mu \mathrm{g} / \mathrm{L}$ as $\mathrm{Se})$ & 19 & $<1.000$ & $<1.000$ & -- & -. & -- & -- & -- & -- \\
\hline 01077 & SILVER, TOTAL $(\mu \mathrm{g} / \mathrm{L}$ as $\mathrm{Ag})$ & 19 & 2.000 & $<1.000$ & -- & 2.000 & $<1.000$ & $<1.000$ & $<1.000$ & $<1.000$ \\
\hline 01092 & ZINC, TOTAL $(\mu \mathrm{g} / \mathrm{L}$ as $\mathrm{zn})$ & 24 & 240.000 & 40.000 & 87.083 & 210.000 & 107.500 & 85.000 & 60.000 & 40.000 \\
\hline 00720 & CYANIDE, TOTAL (mg/L as Cn) & 15 & $<0.010$ & $<0.010$ & -- & -- & -- & -- & -- & - \\
\hline & & ORGAN & C COMPOUNDS & -PESTICIDES & DISSOLVED & & & & & \\
\hline 46342 & ALACHLOR, DISSOLVED $(\mu \mathrm{g} / \mathrm{L})$ & 2 & 0.010 & 0.009 & -- & -- & -- & -- & -- & -- \\
\hline 04040 & DEETHYL ATRAZINE, DISSOLVED $(\mu \mathrm{g} / \mathrm{L})$ & 2 & 0.008 & 0.007 & -- & -- & -- & -- & -. & -- \\
\hline 39632 & ATRAZINE，DISSOLVED $(\mu \mathrm{g} / \mathrm{L})$ & 2 & 0.084 & 0.028 & -- & -- & -- & -- & -- & -- \\
\hline 82686 & METHYL AZINPHOS, DISSOLVED $(\mu \mathrm{g} / \mathrm{L})$ & 2 & $<0.001$ & $<0.001$ & - & -- & -- & -- & -- & -- \\
\hline 82673 & BENFLURALIN， DISSOLVED $(\mu \mathrm{g} / \mathrm{L})$ & 2 & $<0.002$ & $<0.002$ & -- & -- & -- & -- & -- & -. \\
\hline 04028 & BUTYLATE, DISSOLVED $(\mu \mathrm{g} / \mathrm{L})$ & 2 & $<0.002$ & $<0.002$ & - & -- & -- & -- & -- & -- \\
\hline 82680 & CARBARYL, DISSOLVED $(\mu \mathrm{g} / \mathrm{L})$ & 2 & 0.027 & 0.014 & -- & -- & -- & -- & -- & -- \\
\hline 82674 & CARBOFURAN, DISSOLVED $(\mu \mathrm{g} / \mathrm{L})$ & 2 & 0.007 & $<0.003$ & -- & -- & -- & -- & -. & -- \\
\hline 38933 & CHLORPYRIFOS, DISSOLVED $(\mu \mathrm{g} / \mathrm{L})$ & 2 & $<0.004$ & $<0.004$ & -- & -- & -- & -- & -- & -- \\
\hline 04041 & CYANAZINE, DISSOLVED $(\mu \mathrm{g} / \mathrm{L})$ & 2 & 0.034 & $<0.004$ & -. & -. & -- & .. & -- & -- \\
\hline 82682 & DCPA, DISSOLVED $(\mu \mathrm{g} / \mathrm{L})$ & 2 & $<0.002$ & $<0.002$ & -- & -- & -- & -- & -- & -- \\
\hline 34653 & $\mathrm{P}, \mathrm{P}^{\prime}$ DDE, DISSOLVED $(\mu \mathrm{g} / \mathrm{L})$ & 2 & $<0.006$ & $<0.006$ & -- & -- & -- & -- & -- & -- \\
\hline 39572 & DIAZINON， DISSOLVED $(\mu \mathrm{g} / \mathrm{L})$ & 2 & $<0.002$ & $<0.002$ & -- & -- & -- & -- & -- & -- \\
\hline 39381 & DIELDRIN，DISSOLVED $(\mu \mathrm{g} / \mathrm{L})$ & 2 & $<0.001$ & $<0.001$ & -- & -. & .. & -. & -- & -- \\
\hline 82660 & $\begin{array}{l}2,6 \text {-DIETHYL ANILINE, DISSOLVED } \\
(\mu \mathrm{g} / \mathrm{L})\end{array}$ & 2 & $<0.003$ & $<0.003$ & -- & -- & -- & -- & -- & -- \\
\hline 82677 & DISULFOTON, DISSOLVED $(\mu \mathrm{g} / \mathrm{L})$ & 2 & $<0.017$ & $<0.017$ & -. & -- & -- & -- & -- & -- \\
\hline 82668 & EPTC, DISSOLVED $(\mu \mathrm{g} / \mathrm{L})$ & 2 & $<0.002$ & $<0.002$ & -- & -- & -- & -- & - & -- \\
\hline 82663 & ETHALFLURALIN, DISSOLVED $(\mu \mathrm{g} / \mathrm{L})$ & 2 & $<0.004$ & $<0.004$ & -- & -. & -- & - & -- & -- \\
\hline
\end{tabular}

NOTE: Multiple detection limits during the period of record may result in different values flagged with a "<."

* Value is estimated by using a log-probability regression to predict the values of data below the detection limit. 
Table 68. Statistical summary of water-quality data at site 37 (CSW06), May 1995 through June 1997-Continued

\begin{tabular}{|c|c|c|c|c|c|c|c|c|c|c|}
\hline \multirow{2}{*}{$\begin{array}{l}\text { PARAM- } \\
\text { ETER } \\
\text { CODE }\end{array}$} & \multirow{2}{*}{ PROPERTY OR CONSTITUENT } & \multicolumn{4}{|c|}{ DESCRIPTIVE STATISTICS } & PERCEN & $\begin{aligned} \text { AMPLE } \\
\text { EQ }\end{aligned}$ & $\begin{array}{l}\text { VHICH VAI } \\
\text { TO THOSE }\end{array}$ & $\begin{array}{ll}\text { WERE } \\
\text { VN }\end{array}$ & IAN OR \\
\hline & & $\begin{array}{l}\text { SAMPLE } \\
\text { SIZE }\end{array}$ & MAXIMUM & MINIMUM & MEAN & $95 \%$ & $75 \%$ & $\begin{array}{c}50 \% \\
\text { (median) }\end{array}$ & $25 \%$ & $5 \%$ \\
\hline$8 2 \longdiv { 6 7 2 }$ & ETHOPROP, DISSOLVED $(\mu \mathrm{g} / \mathrm{L})$ & 2 & $<0.003$ & $<0.003$ & -- & -- & -- & -- & $-\overline{-}$ & -- \\
\hline 04095 & FONOFOS, DISSOLVED $(\mu \mathrm{g} / \mathrm{L})$ & 2 & $<0.003$ & $<0.003$ & -- & -- & -- & -- & -- & -- \\
\hline 34253 & ALPHA BHC, DISSOLVED $(\mu \mathrm{g} / \mathrm{L})$ & 2 & $<0.002$ & $<0.002$ & -- & -- & -- & -- & -- & -- \\
\hline 39341 & LINDANE, DISSOLVED $(\mu \mathrm{g} / \mathrm{L})$ & 2 & $<0.004$ & $<0.004$ & -- & -- & -- & -- & -- & -- \\
\hline 82666 & LINURON， DISSOLVED $(\mu \mathrm{g} / \mathrm{L})$ & 2 & $<0.002$ & $<0.002$ & -- & -- & -- & -- & -- & -- \\
\hline 39532 & MALATHION, DISSOLVED $(\mu \mathrm{g} / \mathrm{L})$ & 2 & $<0.005$ & $<0.005$ & -. & -- & -- & -- & -- & -- \\
\hline 82667 & METHYL PARATHION, DISSOLVED $(\mu \mathrm{g} / \mathrm{L})$ & 2 & 0.031 & $<0.006$ & -- & -- & -- & -- & -- & -- \\
\hline 39415 & METOLACHLOR， DISSOLVED $(\mu \mathrm{g} / \mathrm{L})$ & 2 & 0.034 & 0.009 & -- & -- & -. & -- & -- & -- \\
\hline 82630 & METRIBUZIN, DISSOLVED $(\mu \mathrm{g} / \mathrm{L})$ & 2 & $<0.004$ & $<0.004$ & -- & -- & -- & -- & -- & -- \\
\hline 82671 & MOLINATE, DISSOLVED $(\mu \mathrm{g} / \mathrm{L})$ & 2 & $<0.004$ & $<0.004$ & -- & -- & -- & -- & -- & -- \\
\hline 82684 & NAPROPAMIDE, DISSOLVED $(\mu \mathrm{g} / \mathrm{L})$ & 2 & $<0.003$ & $<0.003$ & -- & -- & -- & - & -- & -- \\
\hline 39542 & PARATHION, DISSOLVED $(\mu \mathrm{g} / \mathrm{L})$ & 2 & $<0.004$ & $<0.004$ & -- & -- & -- & -- & -- & -- \\
\hline 82669 & PEBULATE， DISSOLVED $(\mu \mathrm{g} / \mathrm{L})$ & 2 & $<0.004$ & $<0.004$ & -- & -- & -- & -- & -- & -- \\
\hline 82683 & PENDIMETHALIN, DISSOLVED $(\mu \mathrm{g} / \mathrm{L})$ & 2 & $<0.004$ & $<0.004$ & -- & -- & -- & -- & -- & -- \\
\hline 82687 & PERMETHRIN, DISSOLVED $(\mu \mathrm{g} / \mathrm{L})$ & 2 & $<0.005$ & $<0.005$ & -- & -- & -- & -- & -- & -- \\
\hline 82664 & PHORATE, DISSOLVED $(\mu \mathrm{g} / \mathrm{L})$ & 2 & $<0.002$ & $<0.002$ & -- & -- & -- & -- & -- & -- \\
\hline 82676 & PRONAMIDE, DISSOLVED $(\mu \mathrm{g} / \mathrm{L})$ & 2 & 0.010 & $<0.003$ & -- & -- & -- & -- & -- & -- \\
\hline 04037 & PROMETON, DISSOLVED $(\mu \mathrm{g} / \mathrm{L})$ & 2 & 0.015 & $<0.018$ & -- & -- & -- & -- & -- & -- \\
\hline 04024 & PROPACHLOR, DISSOLVED $(\mu \mathrm{g} / \mathrm{L})$ & 2 & $<0.007$ & $<0.007$ & -- & -- & -- & -- & -- & -- \\
\hline 82679 & PROPANIL, DISSOLVED $(\mu \mathrm{g} / \mathrm{L})$ & 2 & 0.009 & $<0.004$ & -+ & -- & -- & -- & -- & -- \\
\hline 82685 & PROPARGITE, DISSOLVED $(\mu \mathrm{g} / \mathrm{L})$ & 2 & $<0.013$ & $<0.013$ & -- & -- & -- & -- & -- & -- \\
\hline 04035 & SIMAZINE, DISSOLVED $(\mu \mathrm{g} / \mathrm{L})$ & 2 & $<0.009$ & $<0.005$ & -- & -- & -- & -- & -- & -- \\
\hline 82681 & THIOBENCARB， DISSOLVED $(\mu \mathrm{g} / \mathrm{L})$ & 2 & $<0.002$ & $<0.002$ & -- & -- & -- & -- & -- & -- \\
\hline 82670 & TEBUTHIURON， DISSOLVED $(\mu \mathrm{g} / \mathrm{L})$ & 2 & 0.508 & 0.045 & -- & -- & -- & -- & -- & -- \\
\hline 82665 & TERBACIL, DISSOLVED $(\mu \mathrm{g} / \mathrm{L})$ & 2 & $<0.025$ & $<0.007$ & -- & -- & -- & -- & -- & -- \\
\hline 82675 & TERBUFOS， DISSOLVED $(\mu \mathrm{g} / \mathrm{L})$ & 2 & $<0.013$ & $<0.013$ & -- & -- & -- & -- & -- & -- \\
\hline 82678 & TRIALLATE, DISSOLVED $(\mu \mathrm{g} / \mathrm{L})$ & 2 & $<0.001$ & $<0.001$ & -- & -- & -- & -- & -- & -- \\
\hline 82661 & TRIFLURALIN， DISSOLVED $(\mu \mathrm{g} / \mathrm{L})$ & 2 & $<0.002$ & $<0.002$ & -- & -- & -- & -. & -. & -- \\
\hline 39742 & $2,4,5-\mathrm{T}$, DISSOLVED $(\mu \mathrm{g} / \mathrm{L})$ & 2 & $<0.035$ & $<0.035$ & -- & -- & -- & -. & -- & -- \\
\hline 39732 & $2,4-\mathrm{D}$, DISSOLVED $(\mu \mathrm{g} / \mathrm{L})$ & 2 & $<0.035$ & $<0.035$ & -- & -- & -- & -- & -- & -- \\
\hline 38746 & 2,4 -DB, DISSOLVED $(\mu \mathrm{g} / \mathrm{L})$ & 2 & $<0.035$ & $<0.035$ & -- & -- & -- & -- & -- & -- \\
\hline 4931.5 & ACIFLUORFEN， DISSOLVED $(\mu \mathrm{g} / \mathrm{L})$ & 2 & $<0.035$ & $<0.035$ & -- & -- & -- & -- & -- & -- \\
\hline 49312 & ALDICARB， DISSOLVED $(\mu \mathrm{g} / \mathrm{L})$ & 2 & $<0.016$ & $<0.016$ & -- & -- & -- & -- & -- & -- \\
\hline 49313 & ALDICARB SULFONE, DISSOLVED $(\mu \mathrm{g} / \mathrm{L})$ & 2 & $<0.016$ & $<0.016$ & -- & -- & -- & -- & -- & -- \\
\hline 49314 & $\begin{array}{l}\text { ALDICARB SULFOXIDE, DISSOLVED } \\
(\mu \mathrm{g} / \mathrm{L})\end{array}$ & 2 & $<0.021$ & $<0.021$ & -- & -- & -- & -- & -- & -- \\
\hline 38711 & BENTAZON, DISSOLVED $(\mu \mathrm{g} / \mathrm{L})$ & 2 & $<0.014$ & $<0.014$ & -- & -- & -- & -- & -- & -- \\
\hline 04029 & BROMACIL, DISSOLVED $(\mu \mathrm{g} / \mathrm{L})$ & 2 & $<0.035$ & $<0.035$ & -- & -- & -- & -- & -- & -- \\
\hline 49311 & BROMOXYNIL，DISSOLVED $(\mu \mathrm{g} / \mathrm{L})$ & 2 & $<0.035$ & $<0.035$ & -- & -- & -- & -- & -- & -- \\
\hline 49310 & CARBARYL，DISSOLVED $(\mu \mathrm{g} / \mathrm{L})$ & 2 & $<0.008$ & $<0.008$ & -- & -- & -- & -- & -- & -- \\
\hline 49309 & CARBOFURAN， DISSOLVED $(\mu \mathrm{g} / \mathrm{L})$ & 2 & $<0.028$ & $<0.028$ & -- & -- & -- & -- & -- & -- \\
\hline 49308 & 3-HYDROXY-CARBOFURAN $(\mu \mathrm{g} / \mathrm{L}\rangle$ & 2 & $<0.014$ & $<0.014$ & - & -- & -- & -- & -- & -- \\
\hline 49307 & AMIBEN， DISSOLVED $(\mu \mathrm{g} / \mathrm{L})$ & 2 & $<0.011$ & $<0.011$ & -- & -- & - & -- & -- & -- \\
\hline 49306 & CHLOROTHALONIL， DISSOLVED $\{\mu \mathrm{g} / \mathrm{L}\}$ & 2 & $<0.035$ & $<0.035$ & -- & -- & -. & -. & -- & -- \\
\hline 49305 & CLOPYRALID， DISSOLVED $(\mu \mathrm{g} / \mathrm{L})$ & 2 & $<0.050$ & $<0.050$ & -- & -- & -- & -- & -- & -- \\
\hline 49304 & DACTHALMONO-ACID， DISSOLVED $(\mu \mathrm{g} / \mathrm{L})$ & 2 & $<0.017$ & $<0.017$ & -- & -- & -- & -- & -- & -- \\
\hline 38442 & DICAMBA， DISSOLVED $(\mu \mathrm{g} / \mathrm{L})$ & 2 & $<0.035$ & $<0.035$ & -- & -- & -- & -- & -- & -- \\
\hline 49303 & DICHLOBENIL, DISSOLVED $(\mu \mathrm{g} / \mathrm{L})$ & 1 & $<0.020$ & - & -- & -- & -- & -- & -- & -- \\
\hline 49302 & DICHLORPROP， DISSOLVED $(\mu \mathrm{g} / \mathrm{L})$ & 2 & $<0.032$ & $<0.032$ & -- & -- & -- & -- & -- & -- \\
\hline 49301 & DINOSEB， DISSOLVED $(\mu \mathrm{g} / \mathrm{L})$ & 2 & $<0.035$ & $<0.035$ & -- & -- & -- & -- & -- & -- \\
\hline 49300 & DIURON, DISSOLVED $(\mu \mathrm{g} / \mathrm{L})$ & 2 & 5.700 & $<0.020$ & -- & -- & -- & -- & -. & -- \\
\hline 49299 & $\begin{array}{l}\text { 4. 6-DINITRO OCRESOL, DISSOLVED } \\
(\mu \mathrm{g} / \mathrm{L})\end{array}$ & 2 & $<0.035$ & $<0.035$ & -- & -- & -- & -- & -- & -- \\
\hline 49298 & ESFENVALERATE, DISSOLVED $(\mu \mathrm{g} / \mathrm{L})$ & 2 & $<0.019$ & $<0.019$ & -- & -- & -- & -- & -- & - \\
\hline 49297 & FENURON, DISSOLVED $(\mu \mathrm{g} / \mathrm{L})$ & 2 & $<0.013$ & $<0.013$ & -- & -- & -- & -- & -- & -- \\
\hline 38811 & FLUOMETURON, DISSOLVED $(\mu \mathrm{g} / \mathrm{L})$ & 2 & $<0.035$ & $<0.035$ & -- & -- & -- & -- & -- & -. \\
\hline 38478 & LINURON， DISSOLVED $(\mu \mathrm{g} / \mathrm{L})$ & 2 & $<0.018$ & $<0.018$ & -- & -- & -- & -- & -- & -- \\
\hline 38482 & MCPA, DISSOLVED $(\mu \mathrm{g} / \mathrm{L})$ & 2 & $<0.050$ & $<0.050$ & -- & -- & -- & -- & -- & -- \\
\hline 38487 & MCPB, DISSOLVED $(\mu \mathrm{g} / \mathrm{L})$ & 2 & $<0.035$ & $<0.035$ & -- & -- & -- & -- & -- & -- \\
\hline 38501 & METHIOCARB， DISSOLVED $(\mu \mathrm{g} / \mathrm{L})$ & 2 & $<0.026$ & $<0.026$ & -- & -- & -- & -- & -- & -- \\
\hline 49296 & METHOMYL， DISSOLVED $(\mu \mathrm{g} / \mathrm{L})$ & 2 & $<0.017$ & $<0.017$ & -- & -- & -- & -. & -- & -- \\
\hline 49295 & 1 -NAPHTHOL, DISSOLVED $(\mu \mathrm{g} / \mathrm{L})$ & 2 & $<0.007$ & $<0.007$ & -- & - & -- & -- & -- & -- \\
\hline 49294 & NEBURON， DISSOLVED $(\mu \mathrm{g} / \mathrm{L})$ & 2 & $<0.015$ & $<0.015$ & -- & -- & -- & -- & -- & -- \\
\hline 49293 & NORFLURAZON, DISSOLVED $(\mu \mathrm{g} / \mathrm{L})$ & 2 & $<0.024$ & $<0.024$ & -- & - & -- & -- & -- & -- \\
\hline 49292 & ORYZALIN, DISSOLVED $(\mu \mathrm{g} / \mathrm{L})$ & 2 & $<0.019$ & $<0.019$ & -- & -- & -- & -- & -- & -- \\
\hline 38866 & OXAMYL， DISSOLVED $(\mu \mathrm{g} / L)$ & 2 & $<0.018$ & $<0.018$ & -. & -- & -- & -- & -- & -- \\
\hline 49291 & PICLORAM, DISSOLVED $(\mu \mathrm{g} / \mathrm{L})$ & 2 & $<0.050$ & $<0.050$ & -- & -- & -- & -- & -- & -- \\
\hline 49236 & PROPHAM，DISSOLVED $(\mu \mathrm{g} / \mathrm{L})$ & 2 & $<0.035$ & $<0.035$ & -- & $\ldots$ & -- & -- & -- & -- \\
\hline 38538 & PROPOXUR, DISSOLVED $(\mu \mathrm{g} / \mathrm{L})$ & 2 & $<0.035$ & $<0.035$ & -- & -- & -- & -- & -- & -- \\
\hline 39762 & SILVEX， DISSOLVED $(\mu \mathrm{g} / \mathrm{L})$ & 2 & $<0.021$ & $<0.021$ & -. & -- & -- & -- & -- & -- \\
\hline 49235 & TRICLOPYR, DISSOLVED $(\mu \mathrm{g} / \mathrm{L})$ & 2 & $<0.050$ & $<0.050$ & -- & -- & -- & - & -- & -- \\
\hline & & ORGAN & C COMPOUNI & GANONITR & N, TO & & & & & \\
\hline $39 \overline{057}$ & PROMETRYNE， TOTAL $(\mu \mathrm{g} / \mathrm{L})$ & 1 & $<0.100$ & -- & -- & -- & -- & -- & $=-$ & $\overline{-r}$ \\
\hline 39056 & PROMETONE, TOTAL $(\mu \mathrm{g} / \mathrm{L})$ & 1 & $<0.200$ & -- & -- & -- & -- & -- & -- & -- \\
\hline 39054 & SIMETRYNE, TOTAL $(\mu \mathrm{g} / \mathrm{L})$ & 1 & $<0.100$ & -- & -- & -- & -- & -- & -- & -- \\
\hline 81.757 & CYANAZINE, TOTAL $(\mu \mathrm{g} / \mathrm{L})$ & 1 & $<0.200$ & -- & -- & -- & -- & -- & -- & -- \\
\hline 77825 & ALACHLOR, TOTAL $(\mu \mathrm{g} / \mathrm{L})$ & 1 & $<0.100$ & -- & - & - & -- & -- & -- & -- \\
\hline 82611 & METRIBUZIN, TOTAL $(\mu \mathrm{g} / \mathrm{L})$ & 1 & $<0.100$ & -- & -- & -. & -- & -- & -- & -- \\
\hline 30311 & TERBACIL, TOTAL $(\mu \mathrm{g} / \mathrm{L})$ & 1 & $<0.200$ & -- & -- & -- & -- & -- & -- & -- \\
\hline 30245 & CARBOXIN, TOTAL $(\mu \mathrm{g} / \mathrm{L})$ & 1 & $<0.200$ & -- & -- & -- & -- & -- & -- & -- \\
\hline 30264 & HEXAZINONE, TOTAL $(\mu \mathrm{g} / \mathrm{L})$ & 1 & $<0.200$ & -- & -- & -- & -- & -- & -- & -- \\
\hline
\end{tabular}

NOTE: Multiple detection limits during the period of record may result in different values flagged with a "<."

* Value is estimated by using a log-probability regression to predict the values of data below the detection limit. 
Table 68. Statistical summary of water-quality data at site 37 (CSW06), May 1995 through June 1997-Continued

\begin{tabular}{|c|c|c|c|c|c|c|c|c|c|c|}
\hline \multirow{2}{*}{$\begin{array}{l}\text { PARAM- } \\
\text { ETER } \\
\text { CODE }\end{array}$} & \multirow{2}{*}{ PROPERTY OR CONSTITUENT } & \multicolumn{4}{|c|}{ DESCRIPTIVE STATISTICS } & \multicolumn{5}{|c|}{$\begin{array}{c}\text { PERCENT OF SAMPLES IN WHICH VALUES WERE LESS THAN OR } \\
\text { EQUAL TO THOSE SHOWN }\end{array}$} \\
\hline & & $\begin{array}{l}\text { SAMPLE } \\
\text { SIZE }\end{array}$ & MAXIMUM & MINIMUM & MEAN & $95 \%$ & $75 \%$ & $\begin{array}{c}50 \% \\
\text { (median) }\end{array}$ & $25 \%$ & $5 \%$ \\
\hline 30235 & BUTACHLOR, TOTAL $(\mu \mathrm{g} / \mathrm{L})$ & 1 & $<0.100$ & -- & -- & -- & -- & -- & -- & -- \\
\hline 75981 & DE-ETHYLATRAZINE, TOTAL $(\mu \mathrm{g} / \mathrm{L})$ & 1 & $<0.200$ & -- & -- & -- & -- & -- & -- & -- \\
\hline 39630 & ATRAZINE, TOTAL $(\mu \mathrm{g} / \mathrm{L})$ & 1 & 0.100 & -- & -- & -- & -- & -- & -- & -- \\
\hline 39055 & SIMAZINE, TOTAL $(\mu \mathrm{g} / L)$ & 1 & $<0.100$ & -- & -- & -- & -- & -- & -- & -- \\
\hline 39024 & PROPAZINE, TOTAL $(\mu \mathrm{g} / \mathrm{L})$ & 1 & $<0.100$ & -- & -- & -- & -- & -- & -- & -- \\
\hline 82184 & AMETRYNE, TOTAL $(\mu \mathrm{g} / \mathrm{L})$ & 1 & $<0.100$ & -- & -- & -- & -- & -- & -- & -- \\
\hline 39030 & TRIFLURALIN，TOTAL $(\mu \mathrm{g} / L)$ & 1 & $<0.100$ & -- & -- & -- & -- & -- & -- & -- \\
\hline 82612 & METOLACHLOR, TOTAL $(\mu \mathrm{g} / \mathrm{L})$ & 1. & $<0.200$ & -- & -- & -- & -- & -- & -- & -- \\
\hline 30234 & BROMACIL, TOTAL $(\mu \mathrm{g} / \mathrm{L})$ & 1 & $<0.200$ & -- & -- & -- & -. & -- & -- & -- \\
\hline 30255 & DIPHENAMID, TOTAL $(\mu \mathrm{g} / \mathrm{L})$ & 1 & $<0.100$ & -- & -- & -- & -- & -- & -- & -- \\
\hline 30324 & VERNOLATE, TOTAL $(\mu \mathrm{g} / \mathrm{L})$ & 1 & $<0.100$ & -- & -- & -- & -- & -- & -- & -- \\
\hline 30254 & CYCLOATE, TOTAL $(\mu \mathrm{g} / \mathrm{L})$ & 1 & $<0.100$ & -- & -- & -- & -- & -- & -- & -- \\
\hline 30295 & PROPACHLOR, TOTAL $(\mu \mathrm{g} / \mathrm{L})$ & 1 & $<0.100$ & -- & -- & -- & -- & -- & -- & -- \\
\hline 75980 & DE-ISOPROPYLATRAZIN, TOTAL $(\mu \mathrm{g} / \mathrm{L})$ & 1 & $<0.200$ & -- & -- & -- & -- & -- & -- & -- \\
\hline \multicolumn{11}{|c|}{ ORGANIC COMPOUNDS-HERBICIDES, TOTAL } \\
\hline 39730 & $2,4-D$, TOTAL $(\mu \mathrm{g} / \mathrm{L})$ & 1 & $<0.010$ & -- & -- & -- & -- & -- & -- & -- \\
\hline 39720 & PICLORAM, TOTAL $(\mu \mathrm{g} / L)$ & 1 & $<0.010$ & -- & -- & -- & -- & -- & -- & -- \\
\hline 39740 & $2,4,5-\mathrm{T}$, TOTAL $(\mu \mathrm{g} / \mathrm{L})$ & 1 & $<0.010$ & -- & -- & - & -- & -- & -- & -- \\
\hline 82183 & $2,4-\mathrm{DP}$, TOTAL $(\mu \mathrm{g} / \mathrm{L})$ & 1 & $<0.010$ & -- & -- & -- & $-\infty$ & -- & -- & -- \\
\hline 82052 & DICAMBA， TOTAL $(\mu \mathrm{g} / \mathrm{L})$ & 1 & $<0.010$ & -- & -- & -- & -- & -- & -- & -- \\
\hline \multicolumn{11}{|c|}{ ORGANIC COMPOUNDS-CARBAMATE PESTICIDES, TOTAL } \\
\hline 39750 & SEVIN, TOTAL $(\mu \mathrm{g} / \mathrm{L})$ & 1 & $<0 . \overline{500}$ & -- & -- & -- & -- & $\overline{--}$ & -- & -- \\
\hline 39051 & METHOMYL, TOTAL $(\mu \mathrm{g} / \mathrm{L})$ & 1 & $<0.500$ & -- & -- & -- & -- & -- & -- & -- \\
\hline 82619 & ALDICARD, TOTAL $(\mu \mathrm{g} / \mathrm{L})$ & 1 & $<0.500$ & -- & -- & -- & -- & -- & -- & -- \\
\hline 30296 & PROPOXUR, TOTAL $(\mu \mathrm{g} / \mathrm{L})$ & 1 & $<0.500$ & -- & -- & -- & -- & -- & -- & -- \\
\hline 39052 & PROPHAM， TOTAL $(\mu \mathrm{g} / \mathrm{L})$ & 1 & $<0.500$ & - & -- & -- & -- & -- & -- & -- \\
\hline 82615 & CARBOFURAN, TOTAL $(\mu \mathrm{g} / \mathrm{L})$ & 1 & $<0.500$ & -- & - & -- & -- & -- & -- & -- \\
\hline 77441 & 1 -NAPHTHOL, TOTAL $(\mu \mathrm{g} / \mathrm{L})$ & 1 & $<0.500$ & -- & -- & -- & -- & -- & -- & -- \\
\hline 30282 & METHIOCARB， TOTAL $(\mu \mathrm{g} / \mathrm{L})$ & 1 & $<0.500$ & -- & -- & -- & -- & -- & -- & -- \\
\hline
\end{tabular}

NOTE: Multiple detection limits during the period of record may result in different values flagged with a "<."

* Value is estimated by using a log-probability regression to predict the values of data below the detection limit. 
Table 69. Statistical summary of water-quality data at site 39 (CSW05), June 1994 through June 1997

\begin{tabular}{|c|c|c|c|c|c|c|c|c|c|c|}
\hline \multirow{2}{*}{$\begin{array}{l}\text { PARAM- } \\
\text { ETER } \\
\text { CODE }\end{array}$} & \multirow{2}{*}{ PROPERTY OR CONSTITUENT } & \multicolumn{4}{|c|}{ DESCRIPTIVE STATISTICS } & \multicolumn{5}{|c|}{$\begin{array}{c}\text { PERCENT OF SAMPLES IN WHICH VALUES WERE LESS THAN OR } \\
\text { EQUAL TO THOSE SHOWN }\end{array}$} \\
\hline & & $\begin{array}{l}\text { SAMPLE } \\
\text { SIZE }\end{array}$ & MAXIMUM & MINIMUM & MEAN & $95 \%$ & $75 \%$ & $\begin{array}{c}50 \% \\
\text { (median) }\end{array}$ & $25 \%$ & $5 \%$ \\
\hline \multicolumn{11}{|c|}{ PHYSICAL AND CHEMICAL PROPERTIES } \\
\hline 00061 & INSTANTANEOUS DISCHARGE, $\left(\mathrm{ft}^{3} / \mathrm{s}\right)$ & 74 & 6.900 & $0 . \overline{002}$ & 1.131 & 4.850 & 1.610 & 0.460 & 0.156 & 0.010 \\
\hline 00010 & WATER TEMPERATURE $\left({ }^{\circ} \mathrm{C}\right)$ & 74 & 31.000 & 1.500 & 1.7 .453 & 28.000 & 23.500 & 18.250 & 13.250 & 4.000 \\
\hline 90095 & SPECIFIC CONDUCTANCE, LAB $\left(\mu \mathrm{S} / \mathrm{cm}\right.$ at $\left.25{ }^{\circ} \mathrm{C}\right)$ & 40 & 446.000 & 25.000 & 90.875 & 349.100 & 109.500 & 63.500 & 39.000 & 26.050 \\
\hline 00095 & $\begin{array}{l}\text { SPECIFIC CONDUCTANCE, FIELD }(\mu \mathrm{S} / \mathrm{cm} \text { at } 25 \\
\left.{ }^{\circ} \mathrm{C}\right)\end{array}$ & 56 & 426.000 & 11.000 & 77.804 & 21.5 .700 & 104.750 & 46.500 & 35.250 & 22.550 \\
\hline 00403 & pH， LAB (STANDARD pH UNITS) & 42 & 7.900 & 5.300 & 6.683 & 7.870 & 7.125 & 6.700 & 6.175 & 5.475 \\
\hline 00400 & pH, FIELD (STANDARD pH UNITS) & 54 & 7.870 & 5.200 & 6.753 & 7.290 & 6.935 & 6.800 & 6.600 & 6.032 \\
\hline 90410 & ALKALINITY, LAB (mg/L as $\mathrm{CaCO}_{3}$ ) & 43 & 147.000 & 2.000 & 18.891 & 101.800 & 21.000 & 9.000 & 6.200 & 2.600 \\
\hline 80154 & SUSPENDED SEDIMENT $(\mathrm{mg} / \mathrm{L})$ & 47 & 650.000 & 15.000 & 126.106 & 444.600 & 189.000 & 70.000 & 43.000 & 20.200 \\
\hline 00530 & $\begin{array}{l}\text { RESIDUE ON EVAPORATION AT } 105{ }^{\circ} \mathrm{C} \text {, SUSPENDED } \\
(\mathrm{mg} / \mathrm{L})\end{array}$ & 18 & 232.000 & $<1.000$ & $69.658 *$ & 232.000 & 138.000 & 28.000 & 21.000 & 6.000 \\
\hline 00535 & RESIDUE VOLATILE, SUSPENDED $(\mathrm{mg} / \mathrm{L})$ & 37 & 157.000 & $<1.000$ & $26.693^{*}$ & 126.000 & 45.000 & 10.000 & 5.000 & $<1.000$ \\
\hline 70300 & $\begin{array}{l}\text { DISSOLVED SOLIDS, RESIDUE AT } 180^{\circ} \mathrm{C} \\
(\mathrm{mg} / \mathrm{L})\end{array}$ & 43 & 297.000 & 1.000 & 69.535 & 223.400 & 87.000 & 48.000 & 27.000 & 3.600 \\
\hline 00310 & $\begin{array}{l}5 \text { DAY BIOCHEMICAL OXYGEN DEMAND } \\
(\mathrm{mg} / \mathrm{L})\end{array}$ & 30 & 33.000 & 2.000 & 12.080 & 32.450 & 15.000 & 9.150 & 4.375 & 2.055 \\
\hline 00340 & CHEMICAL OXYGEN DEMAND (mg/L) & 45 & 580.000 & 5.000 & 90.111 & 328.000 & 115.000 & 48.000 & 24.000 & 6.500 \\
\hline \multicolumn{11}{|c|}{ NUTRIENTS, TOTAL AND DISSOLVED } \\
\hline 00625 & $\begin{array}{l}\text { NITROGEN AMMONIA + ORGANIC, } \\
\text { TOTAL (mg/L as N) }\end{array}$ & 45 & 9.300 & 0.350 & 2.065 & 8.570 & 2.500 & 1.300 & 0.615 & 0.400 \\
\hline 00631 & $\mathrm{NO}_{2}+\mathrm{NO}_{3}$, DISSOLVED $(\mathrm{mg} / \mathrm{L}$ as $\mathrm{N})$ & 45 & 2.800 & 0.150 & 0.666 & 1.820 & 0.855 & 0.560 & 0.315 & 0.176 \\
\hline 00608 & $\begin{array}{l}\text { NITROGEN AMMONIA, DISSOLVED } \\
(\mathrm{mg} / \mathrm{L} \text { as } \mathrm{N})\end{array}$ & 45 & 2.300 & 0.015 & 0.380 & 1.650 & 0.525 & 0.190 & 0.080 & 0.020 \\
\hline 00605 & NITROGEN ORGANIC, TOTAL (mg/L as N) & 45 & 7.500 & 0.250 & 1.683 & 6.690 & 2.100 & 0.910 & 0.550 & 0.316 \\
\hline 00600 & NITROGEN, TOTAL (mg/L as N) & 45 & 11.000 & 0.660 & 2.732 & 10.220 & 3.250 & 1.600 & 1.100 & 0.714 \\
\hline 00665 & PHOSPHORUS, TOTAL (mg/L as P) & 45 & 1.500 & 0.020 & 0.450 & 1.410 & 0.680 & 0.260 & 0.160 & 0.069 \\
\hline 00671 & $\begin{array}{l}\text { PHOSPHORUS ORTHO, DISSOLVED } \\
(\mathrm{mg} / \mathrm{L} \text { aS } \mathrm{P})\end{array}$ & 45 & 1.000 & 0.010 & 0.241 & 0.982 & 0.280 & 0.140 & 0.085 & 0.015 \\
\hline \multicolumn{11}{|c|}{ OIL AND GREASE, TOTAL } \\
\hline 00556 & OIL AND GREASE, TOTAL (mg/L) & 14 & 19.000 & $<1.000$ & $5.791 *$ & 19.000 & 8.000 & 4.000 & 1.000 & $<1.000$ \\
\hline \multicolumn{11}{|c|}{ ORGANIC CARBON, TOTAL } \\
\hline 00680 & CARBON ORGANIC, TOTAL (mg/L) & 28 & 120.000 & 6.600 & 29.536 & 107.850 & 38.250 & 14.500 & 9.475 & 6.690 \\
\hline & & & & IFORM & & & & & & \\
\hline 31679 & FECAL STREPTOCOCCI (Colonies per $100 \mathrm{~mL}$ ) & 24 & 65000.000 & 72.000 & 12260.500 & 58250.000 & 19000.000 & 5700.000 & 2025.000 & 166.500 \\
\hline 31616 & FECAL COLIFORM (Colonies per $100 \mathrm{~mL}$ ) & 24 & 310000.000 & 60.000 & 39752.082 & 260000.000 & 45500.000 & 14550.000 & 720.000 & 70.000 \\
\hline & & & GANIC COMPO & S-PESTICID & ES, TOTAL & & & & & \\
\hline 39330 & ALDRIN, TOTAL $(\mu \mathrm{g} / \mathrm{L})$ & 4 & $<0.010$ & $<0.010$ & -- & -- & $\overline{--}$ & -- & -- & -- \\
\hline 39340 & LINDANE，TOTAL $(\mu \mathrm{g} / \mathrm{L})$ & 4 & $<0.010$ & $<0.010$ & -- & -- & -- & -- & -- & -- \\
\hline 39350 & CHLORDANE, TOTAL $(\mu \mathrm{g} / \mathrm{L})$ & 4 & $<0.100$ & $<0.100$ & -- & -- & -- & -- & -- & -- \\
\hline 39370 & DDT, TOTAL $(\mu \mathrm{g} / \mathrm{L})$ & 4 & $<0.010$ & $<0.010$ & -- & -- & -- & -- & -- & -- \\
\hline 39365 & DDE, TOTAL $(\mu \mathrm{g} / \mathrm{L})$ & 4 & $<0.010$ & $<0.010$ & -- & -- & -- & -- & -- & -- \\
\hline 39360 & DDD, TOTAL $(\mu \mathrm{g} / \mathrm{L})$ & 4 & $<0.010$ & $<0.010$ & -- & -- & -- & -- & -- & -- \\
\hline 39380 & DIELDRIN, TOTAL $(\mu \mathrm{g} / \mathrm{L})$ & 4 & $<0.010$ & $<0.010$ & -- & -- & -- & - & -- & -- \\
\hline 39388 & ENDOSULFAN, TOTAL $(\mu \mathrm{g} / \mathrm{L})$ & 4 & $<0.010$ & $<0.010$ & -- & -- & -- & -. & -- & -- \\
\hline 39390 & ENDRIN, TOTAL $(\mu \mathrm{g} / \mathrm{L})$ & 4 & $<0.010$ & $<0.010$ & -- & -- & -- & -- & -- & -- \\
\hline 39410 & HEPTACHLOR, TOTAL $(\mu \mathrm{g} / \mathrm{L})$ & 4 & $<0.010$ & $<0.010$ & -- & -- & -- & -- & -- & -- \\
\hline 39420 & HEPTACHLOR EPOXIDE， TOTAL $(\mu \mathrm{g} / \mathrm{L})$ & 4 & $<0.010$ & $<0.010$ & -. & -- & -. & -- & -- & -- \\
\hline 39516 & PCB, TOTAL $(\mu \mathrm{g} / \mathrm{L})$ & 4 & 0.100 & $<0.100$ & -- & -- & -- & -- & -- & -- \\
\hline 39400 & TOXAPHENE, TOTAL $(\mu \mathrm{g} / \mathrm{L})$ & 4 & $<1.000$ & $<1.000$ & -- & -- & -- & -- & -- & -- \\
\hline 39034 & PERTHANE, TOTAL $(\mu \mathrm{g} / \mathrm{L})$ & 4 & $<0.100$ & $<0.100$ & -- & -- & -- & -- & -- & -- \\
\hline 39570 & DIAZINON, TOTAL $(\mu \mathrm{g} / \mathrm{L})$ & 4 & 0.020 & $<0.010$ & -- & -- & -- & -- & -- & -- \\
\hline 39398 & ETHION, TOTAL $(\mu \mathrm{g} / \mathrm{L})$ & 4 & $<0.010$ & $<0.010$ & -- & -- & -- & -- & -- & -- \\
\hline 39530 & MALATHION, TOTAL $(\mu \mathrm{g} / \mathrm{L})$ & 4 & 0.010 & $<0.010$ & -- & -- & -- & -- & -- & -- \\
\hline 39600 & METHYL PARATHION, TOTAL $(\mu \mathrm{g} / \mathrm{L})$ & 4 & 0.010 & $<0.010$ & -- & -- & -- & -- & -- & -- \\
\hline 39540 & PARATHION, TOTAL $(\mu \mathrm{g} / \mathrm{L})$ & 4 & $<0.010$ & $<0.010$ & -- & -- & -- & -- & -- & -- \\
\hline 39786 & TRITHION, TOTAL $(\mu \mathrm{g} / \mathrm{L})$ & 4 & 0.020 & $<0.010$ & -- & -- & -- & -- & -- & -- \\
\hline 39250 & PCN, TOTAL $(\mu \mathrm{g} / \mathrm{L})$ & 4 & $<0.100$ & $<0.100$ & -- & -- & -- & -- & -- & -- \\
\hline 39480 & METHOXYCHLOR, TOTAL $(\mu \mathrm{g} / \mathrm{L})$ & 4 & $<0.010$ & $<0.010$ & -- & -- & -- & -- & -- & -- \\
\hline 39755 & MIREX, TOTAL $(\mu \mathrm{g} / \mathrm{L})$ & 4 & $<0.010$ & $<0.010$ & -- & -- & -- & -- & -- & -- \\
\hline 39011 & DISYSTON, TOTAL $(\mu \mathrm{g} / \mathrm{L})$ & 2 & $<0.010$ & $<0.010$ & -- & -- & -- & -- & -- & -- \\
\hline 39023 & PHORATE, TOTAL $(\mu \mathrm{g} / \mathrm{L})$ & 4 & $<0.010$ & $<0.010$ & -- & -. & -- & -- & -. & -- \\
\hline 38932 & CHLORPYRIFOS, TOTAL $(\mu \mathrm{g} / \mathrm{L})$ & 4 & 0.010 & $<0.010$ & -- & -- & -- & -- & -- & -- \\
\hline 39040 & DEF, TOTAL $(\mu \mathrm{g} / \mathrm{L})$ & 4 & $<0.010$ & $<0.010$ & -- & -- & -- & - & -- & -- \\
\hline 82614 & FONOFOS, TOTAL $(\mu \mathrm{g} / \mathrm{L})$ & 4 & $<0.010$ & $<0.010$ & -- & -- & -- & -- & -- & -- \\
\hline & & & OLATILE ORG & COMPOUND & S, TOTAL & & & & & \\
\hline 34210 & ACROLEIN, TOTAL $(\mu \mathrm{g} / \mathrm{L})$ & 1 & $<20.000$ & -- & -- & $\overline{--}$ & -- & -- & -- & -- \\
\hline 34215 & ACRYLONITRILE, TOTAL $(\mu \mathrm{g} / \mathrm{L})$ & 1 & $<20.000$ & -- & -- & -- & -- & - & -- & -- \\
\hline 34030 & BENZENE, TOTAL $(\mu \mathrm{g} / \mathrm{L})$ & 10 & $<2.000$ & $<0.200$ & -. & -- & -- & -- & -- & -- \\
\hline 32104 & BROMOFORM，TOTAL $(\mu \mathrm{g} / \mathrm{L})$ & 10 & $<2.000$ & $<0.200$ & -- & -- & -- & -- & -- & -- \\
\hline 32102 & CARBON TETRACHLORIDE, TOTAL $(\mu \mathrm{g} / \mathrm{L})$ & 10 & $<2.000$ & $<0.200$ & -- & -- & -- & -- & -- & -- \\
\hline 34301 & CHLOROBENZENE, TOTAL $(\mu \mathrm{g} / \mathrm{L})$ & 10 & $<2.000$ & $<0.200$ & -- & -- & -. & -- & -- & -- \\
\hline 32105 & CHLORODIBROMOTHANE，TOTAL $(\mu \mathrm{g} / \mathrm{L})$ & 10 & $<2.000$ & $<0.200$ & -- & -- & -- & -. & -- & -- \\
\hline 34311. & CHLOROETHANE，TOTAL $(\mu \mathrm{g} / \mathrm{L})$ & 10 & $<2.000$ & $<0.200$ & -- & -- & -- & -- & -- & -- \\
\hline 32106 & CHLOROFORM, TOTAL $(\mu \mathrm{g} / \mathrm{L})$ & 10 & $<2.000$ & $<0.200$ & -- & -- & -- & -- & -- & -- \\
\hline 34496 & 1,1 -DICHLOROETHANE, TOTAL $(\mu \mathrm{g} / \mathrm{L})$ & 10 & $<2.000$ & $<0.200$ & -- & -- & -- & -- & -- & -- \\
\hline 32103 & 1,2-DICHLOROETHANE，TOTAL $(\mu \mathrm{g} / \mathrm{L})$ & 10 & $<2.000$ & $<0.200$ & -- & -- & -- & -- & -- & -- \\
\hline
\end{tabular}

NOTE: Multiple detection limits during the period of record may result in different values flagged with a "<."

* Value is estimated by using a log-probability regression to predict the values of data below the detection limit. 
Table 69. Statistical summary of water-quality data at site 39 (CSW05), June 1994 through June 1997-Continued

\begin{tabular}{|c|c|c|c|c|c|c|c|c|c|c|}
\hline \multirow{2}{*}{$\begin{array}{l}\text { PARAM- } \\
\text { ETER } \\
\text { CODE }\end{array}$} & \multirow{2}{*}{ PROPERTY OR CONSTITUENT } & \multicolumn{4}{|c|}{ DESCRIPTIVE STATISTICS } & \multicolumn{5}{|c|}{$\begin{array}{c}\text { PERCENT OF SAMPLES IN WHICH VALUES WERE LESS THAN OR } \\
\text { EQUAL TO THOSE SHOWN }\end{array}$} \\
\hline & & $\begin{array}{l}\text { SAMPLE } \\
\text { SIZE }\end{array}$ & MAXIMUM & MINIMUM & MEAN & $95 \%$ & $75 \%$ & $\begin{array}{c}50 \% \\
\text { (median) }\end{array}$ & $25 \%$ & $5 \%$ \\
\hline 34501 & 1,1 -DICHLOROETHYLENE, TOTAL $(\mu \mathrm{g} / \mathrm{L})$ & 10 & $<2.000$ & $<0.200$ & -- & -- & -- & -- & -- & -- \\
\hline 34541 & 1,2 -DICHLOROPROPANE, TOTAL $(\mu \mathrm{g} / \mathrm{L})$ & 10 & $<2.000$ & $<0.200$ & - & -- & -- & -- & -- & -- \\
\hline 34371 & ETHYLBENZENE, TOTAL $(\mu \mathrm{g} / \mathrm{L})$ & 10 & $<2.000$ & $<0.200$ & -- & -- & -- & -- & -- & -- \\
\hline 34413 & METHYL BROMIDE, TOTAL $(\mu \mathrm{g} / \mathrm{L})$ & 10 & $<2.000$ & $<0.200$ & -- & -- & -- & -- & -- & -- \\
\hline 34423 & METHYLENE CHLORIDE, TOTAL $(\mu \mathrm{g} / \mathrm{L})$ & 10 & $<2.000$ & $<0.200$ & -- & -- & -- & -- & -- & -- \\
\hline 34516 & $1,1,2,2$-TETRACHLOROETHANE, TOTAL $(\mu \mathrm{g} / \mathrm{L})$ & 10 & $<2.000$ & $<0.200$ & -- & -- & -- & -- & -- & -- \\
\hline 34475 & TETRACHLOROETHYLENE, TOTAL $(\mu \mathrm{g} / \mathrm{L})$ & 10 & $<2.000$ & $<0.200$ & -- & -- & -- & -- & -- & -- \\
\hline 34010 & TOLUENE, TOTAL $(\mu \mathrm{g} / \mathrm{L})$ & 10 & $<2.000$ & $<0.200$ & -- & -- & -- & -- & -- & -- \\
\hline 34546 & $\begin{array}{l}1,2 \text {-TRANSDICHLOROETHENE, TOTAL } \\
(\mu \mathrm{g} / \mathrm{L})\end{array}$ & 10 & $<2.000$ & $<0.200$ & -- & -- & -- & -- & -- & -- \\
\hline 34506 & $1,1,1$-TRICHLOROETHANE, TOTAL $(\mu \mathrm{g} / \mathrm{L})$ & 10 & $<2.000$ & $<0.200$ & -- & -- & -- & -- & -- & -- \\
\hline 34511 & $1,1,2$-TRICHLOROETHANE, TOTAL $(\mu \mathrm{g} / \mathrm{L})$ & 10 & $<2.000$ & $<0.200$ & -- & -- & -- & -- & -- & -- \\
\hline 39180 & TRICHLOROETHYLENE, TOTAL $(\mu \mathrm{g} / \mathrm{L})$ & 10 & $<2.000$ & $<0.200$ & -- & -- & -- & -- & -- & -- \\
\hline 39175 & VINYL CHLORIDE, TOTAL $(\mu \mathrm{g} / \mathrm{L})$ & 10 & $<2.000$ & $<0.200$ & -- & -- & -- & -- & - & -- \\
\hline 30217 & DIBROMOMETHANE, TOTAL $(\mu \mathrm{g} / \mathrm{L})$ & 10 & $<2.000$ & $<0.200$ & -- & -- & -- & -- & -- & -- \\
\hline 32101 & DICHLOROBROMOMETHANE, TOTAL $(\mu \mathrm{g} / \mathrm{L})$ & 10 & $<2.000$ & $<0.200$ & -- & -- & -- & -- & -- & -- \\
\hline 34668 & $\begin{array}{l}\text { DICHLORODIFLUOROMETHANE， TOTAL } \\
(\mu \mathrm{g} / \mathrm{L})\end{array}$ & 10 & $<2.000$ & $<0.200$ & -- & -- & -- & -- & -- & -- \\
\hline 34488 & TRICHLOROFLUOROMETHANE，TOTAL $(\mu \mathrm{g} / \mathrm{L})$ & 10 & $<2.000$ & $<0.200$ & -- & -- & -- & -- & -- & -- \\
\hline 77651 & 1,2 -DIBROMOETHANE, TOTAL $(\mu \mathrm{g} / \mathrm{L})$ & 10 & $<2.000$ & $<0.200$ & -- & -- & -- & -- & -- & -- \\
\hline 34418 & METHYLCHLORIDE， TOTAL $(\mu \mathrm{g} / \mathrm{L})$ & 10 & $<2.000$ & $<0.200$ & -- & -- & -- & -- & -- & -- \\
\hline 34704 & $\begin{array}{l}\text { CIS } 1,3 \text {-DICHLOROPROPENE, TOTAL } \\
(\mu \mathrm{g} / \mathrm{L})\end{array}$ & 10 & $<2.000$ & $<0.200$ & -- & -- & -- & -- & - & -- \\
\hline 34699 & TRANS 1,3 -DICHLOROPROPENE, TOTAL $(\mu \mathrm{g} / \mathrm{L})$ & 10 & $<2.000$ & $<0.200$ & -- & -- & -- & -- & -- & -- \\
\hline 77128 & STYRENE, TOTAL $(\mu \mathrm{g} / \mathrm{L})$ & 10 & $<2.000$ & $<0.200$ & -- & -- & -- & -- & -- & -- \\
\hline 81551 & XYLENE, TOTAL $(\mu \mathrm{g} / \mathrm{L})$ & 10 & $<2.000$ & $<0.200$ & -- & -- & -- & -- & -- & -- \\
\hline 82625 & DIBROMOCHLOROPROPANE, TOTAL $(\mu \mathrm{g} / \mathrm{L})$ & 10 & $<10.000$ & $<1.000$ & -- & -- & -- & -- & -- & -- \\
\hline 77168 & 1,1 -DICHLOROPROPENE, TOTAL $(\mu \mathrm{g} / \mathrm{L})$ & 10 & $<2.000$ & $<0.200$ & -. & -- & -- & -- & -. & -- \\
\hline 77170 & 2,2 -DICHLOROPROPANE, TOTAL $(\mu \mathrm{g} / \mathrm{L})$ & 10 & $<2.000$ & $<0.200$ & -- & -- & -- & -- & -- & -- \\
\hline 77173 & 1,3-DICHLOROPROPANE, TOTAL $(\mu \mathrm{g} / \mathrm{L})$ & 10 & $<2.000$ & $<0.200$ & -- & -- & -- & -- & -- & $-\cdots$ \\
\hline 77275 & O-CHLOROTOLUENE, TOTAL $(\mu \mathrm{g} / \mathrm{L})$ & 10 & $<2.000$ & $<0.200$ & -. & -. & -- & -- & -- & -- \\
\hline 77277 & P-CHLOROTOLUENE, TOTAL $(\mu \mathrm{g} / \mathrm{L})$ & 10 & $<2.000$ & $<0.200$ & -- & -- & -- & -. & -- & -- \\
\hline 77443 & 123-TRICHLOROPROPANE, TOTAL $(\mu \mathrm{g} / \mathrm{L})$ & 10 & $<2.000$ & $<0.200$ & -- & -- & -- & -- & -- & -- \\
\hline 77562 & 1112-TETRACHLOROETHANE, TOTAL $(\mu \mathrm{g} / \mathrm{L})$ & 10 & $<2.000$ & $<0.200$ & -- & -- & -- & -- & -- & -- \\
\hline 78032 & TERTBUTYL METHYL ETHER, TOTAL $(\mu \mathrm{g} / \mathrm{L})$ & 10 & $<2.000$ & $<0.200$ & - & -- & -- & -- & -- & -- \\
\hline 77297 & BROMOCHLORO METHANE, TOTAL $(\mu \mathrm{g} / \mathrm{L})$ & 10 & $<2.000$ & $<0.200$ & -- & -- & -. & -- & -- & -- \\
\hline 77093 & CIS- 1,2 -DICHLOROETHENE, TOTAL $(\mu \mathrm{g} / \mathrm{L})$ & 10 & $<2.000$ & $<0.200$ & -- & -. & -- & -- & -- & -- \\
\hline 34576 & 2 -CHLOROETHYL VINYL ETHER, TOTAL $(\mu \mathrm{g} / \mathrm{L})$ & 5 & $<10.000$ & $<1.000$ & -- & -- & -- & -- & -- & -- \\
\hline 77223 & ISOPROPYL BENZENE, TOTAL $(\mu \mathrm{g} / \mathrm{L})$ & 10 & $<2.000$ & $<0.200$ & -- & -- & -- & - & -- & -- \\
\hline 77224 & N-PROPYL BENZENE, TOTAL $(\mu \mathrm{g} / \mathrm{L})$ & 10 & $<2.000$ & $<0.200$ & -- & -- & -- & -- & -- & -- \\
\hline 77353 & TERTBUTYL BENZENE, TOTAL $(\mu \mathrm{g} / \mathrm{L})$ & 10 & $<2.000$ & $<0.200$ & -- & -- & -- & -- & -- & -- \\
\hline 77222 & PSEUDOCUMENE, TOTAL $(\mu \mathrm{g} / \mathrm{L})$ & 10 & $<2.000$ & $<0.200$ & -- & -- & - & -- & -- & -- \\
\hline 77350 & SEC-BUTYL BENZENE, TOTAL $(\mu \mathrm{g} / \mathrm{L})$ & 10 & $<2.000$ & $<0.200$ & -- & -- & -- & -- & -- & -- \\
\hline 77356 & P-ISOPROPYL TOLUENE, TOTAL $(\mu \mathrm{g} / \mathrm{L})$ & 10 & $<2.000$ & $<0.200$ & -- & -- & -- & -- & -- & -- \\
\hline 77342 & N-BUTYL BENZENE， TOTAL $(\mu \mathrm{g} / \mathrm{L}$ & 10 & $<2.000$ & $<0.200$ & -- & -- & -- & -. & -. & -- \\
\hline 77613 & $1,2,3$-TRICHLOROBENZENE, TOTAL $(\mu \mathrm{g} / \mathrm{L}$ & 10 & $<2.000$ & $<0.200$ & -- & -- & -- & -- & -- & -- \\
\hline 77652 & FREON-113， TOTAL $(\mu \mathrm{g} / \mathrm{L}$ & 10 & $<2.000$ & $<0.200$ & -- & -- & -- & -- & -- & -- \\
\hline 77226 & MESITYLENE, TOTAL $(\mu \mathrm{g} / \mathrm{L}$ & 10 & $<2.000$ & $<0.200$ & -+ & -- & -- & -- & -- & -- \\
\hline 81555 & BROMOBENZENE, TOTAL $(\mu \mathrm{g} / \mathrm{L}$ & 10 & $<2.000$ & $<0.200$ & -- & -- & -- & -- & -- & -- \\
\hline & & MET & LS AND MIN & CONSTITUEN & IS, TOTAL & & & & & \\
\hline 01097 & ANTIMONY, TOTAL $(\mu \mathrm{g} / \mathrm{L}$ as $\mathrm{Sb})$ & 32 & 8.000 & $<1.000$ & $0.980^{\star}$ & 3.000 & 1.000 & $<1.000$ & $<1.000$ & $<1.000$ \\
\hline 01002 & ARSENIC, TOTAL $(\mu \mathrm{g} / \mathrm{L}$ as As) & 32 & 140.000 & $<1.000$ & -- & 29.000 & $<1.000$ & $<1.000$ & $<1.000$ & $<1.000$ \\
\hline 01012 & BERYLLIUM, TOTAL ( $\mu \mathrm{g} / \mathrm{L}$ as Be) & 12 & $<10.000$ & $<10.000$ & -- & -- & -- & -- & -- & -- \\
\hline 01027 & CADMIUM, TOTAL $(\mu \mathrm{g} / \mathrm{L}$ as $\mathrm{Cd})$ & 12 & 3.000 & $<1.000$ & -- & 3.000 & 1.000 & $<1.000$ & $<1.000$ & $<1.000$ \\
\hline 01034 & CHROMIUM, TOTAL $(\mu \mathrm{g} / \mathrm{L}$ as $\mathrm{Cr})$ & 32 & 25.000 & 1.000 & 9.969 & 23.700 & 14.000 & 8.000 & 4.250 & 1.000 \\
\hline 01042 & COPPER, TOTAL $(\mu \mathrm{g} / \mathrm{L}$ as $\mathrm{Cu})$ & 32 & 48.000 & 1.000 & 19.719 & 46.700 & 27.500 & 19.000 & 10.000 & 2.300 \\
\hline 01051 & LEAD, TOTAL $(\mu \mathrm{g} / \mathrm{L}$ as $\mathrm{Pb})$ & 32 & 66.000 & $<1.000$ & $22.617^{\star}$ & 60.000 & 38.000 & 18.000 & 8.000 & $<1.000$ \\
\hline 71900 & MERCURY，TOTAL ( $\mu \mathrm{g} / \mathrm{L}$ as $\mathrm{Hg})$ & 12 & 0.200 & $<0.100$ & -- & 0.200 & $<0.100$ & $<0.100$ & $<0.100$ & $<0.100$ \\
\hline 01067 & NICKEL, TOTAL $(\mu \mathrm{g} / \mathrm{L}$ as Ni) & 32 & 19.000 & 1.000 & 7.500 & 19.000 & 11.500 & 6.000 & 3.250 & 1.000 \\
\hline 01147 & SELENIUM, TOTAL $(\mu \mathrm{g} / \mathrm{L}$ as Se) & 12 & $<1.000$ & $<1.000$ & -- & -- & -- & -- & -- & -- \\
\hline 01077 & SILVER, TOTAL $(\mu \mathrm{g} / \mathrm{L}$ as $\mathrm{Ag})$ & 12 & $<1.000$ & $<1.000$ & -- & -- & - & -- & -- & -- \\
\hline 01092 & ZINC, TOTAL ( $\mu \mathrm{g} / \mathrm{L}$ as $\mathrm{Zn})$ & 32 & 700.000 & 40.000 & 207.500 & 550.500 & 272.500 & 175.000 & 82.500 & 46.500 \\
\hline 00720 & CYANIDE, TOTAL (mg/L as Cn) & 12 & $<0.010$ & $<0.010$ & -- & $\ldots$ & -- & -- & - & -- \\
\hline & & ORGAN & C COMPOUNI & PESTICIDES & DISSOLVED & & & & & \\
\hline 46342 & ALACHLOR， DISSOLVED $(\mu \mathrm{g} / \mathrm{L})$ & 3 & $<0.002$ & $<0.002$ & -- & -- & $\cdots$ & -- & -- & -- \\
\hline 04040 & DEETHYL ATRAZINE, DISSOLVED $(\mu \mathrm{g} / \mathrm{L})$ & 3 & 0.007 & $<0.002$ & -- & -- & -- & -- & -- & -- \\
\hline 39632 & ATRAZINE, DISSOLVED $(\mu \mathrm{g} / \mathrm{L})$ & 3 & 0.037 & $<0.001$ & -- & -- & -- & -- & -. & -- \\
\hline 82686 & METHYL AZINPHOS, DISSOLVED $(\mu \mathrm{g} / \mathrm{L})$ & 3 & $<0.001$ & $<0.001$ & -- & -- & -. & -- & -- & -- \\
\hline 82673 & BENFLURALIN， DISSOLVED $(\mu \mathrm{g} / \mathrm{L})$ & 3 & $<0.002$ & $<0.002$ & - & - & -- & -- & -- & -- \\
\hline 04028 & BUTYLATE, DISSOLVED $(\mu \mathrm{g} / \mathrm{L})$ & 3 & $<0.002$ & $<0.002$ & -- & -- & -- & -- & -- & -- \\
\hline 82680 & CARBARYL，DISSOLVED $(\mu \mathrm{g} / \mathrm{L})$ & 3 & 0.011 & $<0.003$ & -- & -- & -- & -- & -- & -- \\
\hline 82674 & CARBOFURAN, DISSOLVED $(\mu \mathrm{g} / \mathrm{L})$ & 3 & $<0.003$ & $<0.003$ & -- & -- & -- & -- & -- & -- \\
\hline 38933 & CHLORPYRIFOS, DISSOLVED $(\mu \mathrm{g} / \mathrm{L})$ & 3 & $<0.004$ & $<0.004$ & -- & -- & -- & -- & -- & -- \\
\hline 04041 & CYANAZINE, DISSOLVED $(\mu \mathrm{g} / \mathrm{L})$ & 3 & $<0.004$ & $<0.004$ &.- & -- & -- & -- & -- & -- \\
\hline 82682 & DCPA， DISSOLVED $(\mu \mathrm{g} / \mathrm{L})$ & 3 & 0.001 & $<0.002$ & -- & -- & -- & -- & -- & -- \\
\hline 34653 & P, P' DDE, DISSOLVED $(\mu \mathrm{g} / \mathrm{L})$ & 3 & $<0.006$ & $<0.006$ & -- & $\ldots$ & -- & -- & -- & -- \\
\hline 39572 & DIAZINON， DISSOLVED $(\mu \mathrm{g} / \mathrm{L})$ & 3 & $<0.002$ & $<0.002$ & -- & -- & -- & -- & - & -- \\
\hline 39381 & DIELDRIN， DISSOLVED $(\mu \mathrm{g} / \mathrm{L})$ & 3 & $<0.001$ & $<0.001$ & -- & -- & -- & -- & -- & -- \\
\hline 82660 & $\begin{array}{l}2,6 \text {-DIETHYL ANILINE, DISSOLVED } \\
(\mu \mathrm{g} / \mathrm{L})\end{array}$ & 3 & $<0.003$ & $<0.003$ & -- & -- & - & -- & -- & -- \\
\hline 82677 & DISULFOTON, DISSOLVED $(\mu \mathrm{g} / \mathrm{L})$ & 3 & $<0.017$ & $<0.017$ & -- & -- & -- & -- & -- & -- \\
\hline
\end{tabular}

NOTE: Multiple detection limits during the period of record may result in different values flagged with a "<."

* Value is estimated by using a log-probability regression to predict the values of data below the detection limit. 
Table 69. Statistical summary of water-quality data at site 39 (CSW05), June 1994 through June 1997—Continued

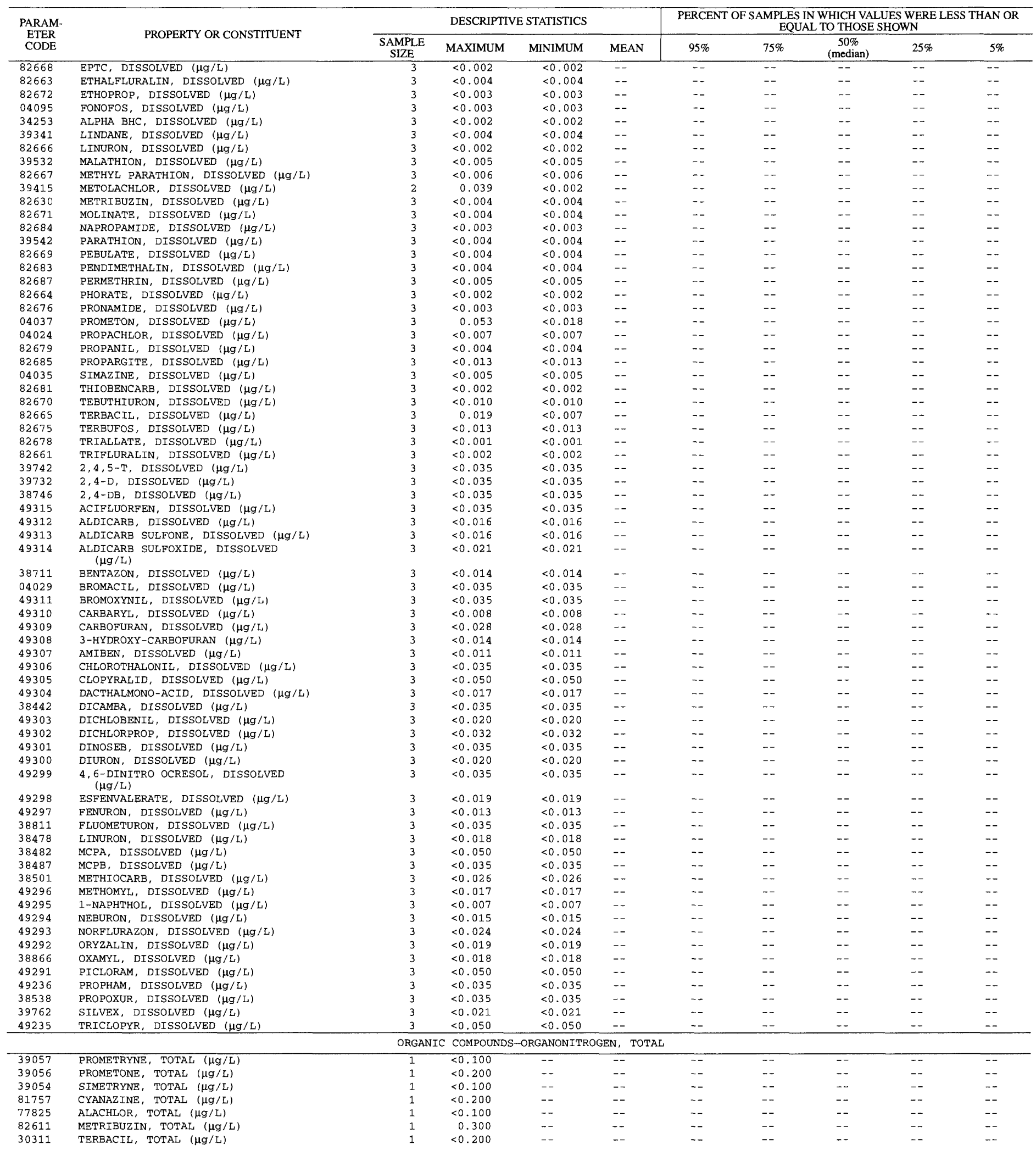

NOTE: Multiple detection limits during the period of record may result in different values flagged with a "<."

* Value is estimated by using a log-probability regression to predict the values of data below the detection limit. 
Table 69. Statistical summary of water-quality data at site 39 (CSW05), June 1994 through June 1997-Continued

\begin{tabular}{|c|c|c|c|c|c|c|c|c|c|c|}
\hline \multirow{2}{*}{$\begin{array}{l}\text { PARAM- } \\
\text { ETER } \\
\text { CODE }\end{array}$} & \multirow{2}{*}{ PROPERTY OR CONSTITUENT } & \multicolumn{4}{|c|}{ DESCRIPTIVE STATISTICS } & \multicolumn{5}{|c|}{$\begin{array}{c}\text { PERCENT OF SAMPLES IN WHICH VALUES WERE LESS THAN OR } \\
\text { EQUAL TO THOSE SHOWN }\end{array}$} \\
\hline & & $\begin{array}{l}\text { SAMPLE } \\
\text { SIZE }\end{array}$ & MAXIMUM & MINIMUM & MEAN & $95 \%$ & $75 \%$ & $\begin{array}{c}50 \% \\
\text { (median) }\end{array}$ & $25 \%$ & $5 \%$ \\
\hline 30245 & CARBOXIN, TOTAL $(\mu \mathrm{g} / \mathrm{L})$ & 1 & $<0.200$ & -- & -- & -- & -- & - & -- & -- \\
\hline 30235 & BUTACHLOR, TOTAL $(\mu \mathrm{g} / \mathrm{L})$ & 1 & $<0.100$ & -- & -- & -- & -- & -- & -- & - \\
\hline 30236 & BUTYLATE, TOTAL $(\mu \mathrm{g} / \mathrm{L})$ & 1 & $<0.100$ & -- & -- & -- & -- & -- & -- & -- \\
\hline 75981 & DE-ETHYLATRAZINE， TOTAL $(\mu \mathrm{g} / \mathrm{L})$ & 1 & $<0.200$ & -- & -- & -- & -- & -- & -- & -- \\
\hline 39630 & ATRAZINE, TOTAL $(\mu \mathrm{g} / \mathrm{L})$ & 1 & 0.200 & -- & -- & -- & -- & -- & -- & -- \\
\hline 39055 & SIMAZINE, TOTAL $(\mu \mathrm{g} / \mathrm{L})$ & 1 & $<0.100$ & -- & -- & -- & -- & -- & -- & -- \\
\hline 39024 & PROPAZINE, TOTAL $(\mu \mathrm{g} / \mathrm{L})$ & 1 & $<0.100$ & -- & -- & -- & -- & -- & -- & -- \\
\hline 82184 & AMETRYNE， TOTAL $(\mu \mathrm{g} / \mathrm{L})$ & 1 & $<0.100$ & -- & -- & -- & -- & -- & -- & -- \\
\hline 39030 & TRIFLURALIN， TOTAL $(\mu \mathrm{g} / \mathrm{L})$ & 1 & $<0.100$ & -- & -- & - & -- & -- & -- & -- \\
\hline 82612 & METOLACHLOR， TOTAL $(\mu \mathrm{g} / \mathrm{L})$ & 1 & $<0.200$ & -- & -- & -- & -- & -- & -- & -- \\
\hline 30234 & BROMACIL， TOTAL $(\mu \mathrm{g} / \mathrm{L})$ & 1 & $<0.200$ & -- & -- & -- & -- & -- & -- & -- \\
\hline 30255 & DIPHENAMID， TOTAL $(\mu \mathrm{g} / \mathrm{L})$ & 1 & $<0.100$ & -- & -- & -- & -. & -- & -- & -- \\
\hline 30324 & VERNOLATE, TOTAL $(\mu \mathrm{g} / \mathrm{L})$ & 1 & $<0.100$ & -- & -- & -- & -- & -- & -- & -- \\
\hline 30254 & CYCLOATE, TOTAL $(\mu \mathrm{g} / \mathrm{L})$ & 1 & $<0.100$ & -- & -- & -- & -. & -- & -- & -- \\
\hline 30295 & PROPACHLOR， TOTAL $(\mu \mathrm{g} / \mathrm{L})$ & 1 & $<0.100$ & -- & -- & -- & -- & -- & -. & -- \\
\hline 75980 & DE-ISOPROPYLATRAZIN， TOTAL $(\mu \mathrm{g} / \mathrm{L})$ & 1 & $<0.200$ & -- & -- & -- & -- & -- & -- & -- \\
\hline 39730 & $2,4-\mathrm{D}$, TOTAL $(\mu \mathrm{g} / \mathrm{L})$ & 1 & $<0.010$ & -- & -- & -- & -- & -- & -- & -- \\
\hline 39760 & SILVEX, TOTAL $(\mu \mathrm{g} / \mathrm{L})$ & 1 & $<0.010$ & -- & -- & -- & -- & -- & -- & -- \\
\hline 39720 & PICLORAM，TOTAL $(\mu \mathrm{g} / \mathrm{L})$ & 1 & $<0.010$ & -- & -- & -- & -- & -- & -- & -- \\
\hline 39740 & $2,4,5-\mathrm{T}$, TOTAL $(\mu \mathrm{g} / \mathrm{L})$ & 1 & $<0.010$ & -- & -- & -- & -- & -- & -- & -- \\
\hline 82183 & $2,4-\mathrm{DP}$, TOTAL $(\mu \mathrm{g} / \mathrm{L})$ & 1 & $<0.010$ & -- & -- & -- & -- & -- & -- & -- \\
\hline 82052 & DICAMBA, TOTAL $(\mu \mathrm{g} / \mathrm{L})$ & 1 & $<0.010$ & -- & -- & -- & -- & -- & -- & -- \\
\hline \multicolumn{11}{|c|}{ ORGANIC COMPOUNDS-CARBAMATE PESTICIDES, TOTAL } \\
\hline 39750 & SEVIN, TOTAL $(\mu g / L)$ & 1 & $<0.500$ & -- & -- & -- & -- & -- & -- & -- \\
\hline 39051 & METHOMYL， TOTAL $(\mu \mathrm{g} / \mathrm{L})$ & 1 & $<0.500$ & -- & -- & -- & -- & -- & -- & -- \\
\hline 82619 & ALDICARD, TOTAL $(\mu \mathrm{g} / \mathrm{L})$ & 1 & $<0.500$ & -- & -- & -. & -- & -- & -- & -- \\
\hline 30296 & PROPOXUR, TOTAL $(\mu \mathrm{g} / \mathrm{L})$ & 1 & $<0.500$ & -- & -- & -- & -- & -- & -- & -- \\
\hline 39052 & PROPHAM， TOTAL $(\mu \mathrm{g} / \mathrm{L})$ & 1 & $<0.500$ & -- & -- & -- & -- & -- & -- & -- \\
\hline 82615 & CARBOFURAN， TOTAL $(\mu \mathrm{g} / \mathrm{L})$ & 1 & $<0.500$ & -- & -- & -- & -- & -- & -- & -- \\
\hline 77441 & 1-NAPHTHOL， TOTAL $(\mu \mathrm{g} / \mathrm{L})$ & 1 & $<0.500$ & -- & -- & -- & -- & -- & $\cdots$ & -- \\
\hline 30282 & METHIOCARB， TOTAL $(\mu \mathrm{g} / \mathrm{L})$ & 1 & $<0.500$ & - & -- & -- & -- & - & -- & -- \\
\hline
\end{tabular}

NOTE: Multiple detection limits during the period of record may result in different values flagged with a "<."

* Value is estimated by using a log-probability regression to predict the values of data below the detection limit. 
Table 70. Statistical summary of water-quality data at site 40 (CSWO3), July 1994 through June 1997

\begin{tabular}{|c|c|c|c|c|c|c|c|c|c|c|}
\hline \multirow{2}{*}{$\begin{array}{l}\text { PARAM- } \\
\text { ETER } \\
\text { CODE }\end{array}$} & \multirow{2}{*}{ PROPERTY OR CONSTITUENT } & \multicolumn{4}{|c|}{ DESCRIPTIVE STATISTICS } & \multicolumn{5}{|c|}{$\begin{array}{c}\text { PERCENT OF SAMPLES IN WHICH VALUES WERE LESS THAN OR } \\
\text { EQUAL TO THOSE SHOWN }\end{array}$} \\
\hline & & $\begin{array}{l}\text { SAMPLE } \\
\text { SIZE }\end{array}$ & MAXIMUM & MINIMUM & MEAN & $95 \%$ & $75 \%$ & $\begin{array}{c}50 \% \\
\text { (median) }\end{array}$ & $25 \%$ & $5 \%$ \\
\hline \multicolumn{11}{|c|}{ PHYSICAL AND CHEMICAL PROPERTIES } \\
\hline$\overline{00061}$ & INSTANTANEOUS DISCHARGE, $\left(\mathrm{ft}^{3} / \mathrm{s}\right)$ & 62 & 21.000 & 0.001 & 0.676 & 2.405 & 0.303 & 0.105 & 0.020 & 0.010 \\
\hline 00010 & WATER TEMPERATURE $\left({ }^{\circ} \mathrm{C}\right)$ & 62 & 28.000 & 2.000 & 15.613 & 26.000 & 21.625 & 16.000 & 9.500 & 3.075 \\
\hline 90095 & SPECIFIC CONDUCTANCE, LAB ( $\mu \mathrm{S} / \mathrm{cm}$ at $25^{\circ} \mathrm{C}$ ) & 40 & 309.000 & 19.000 & 71.000 & 227.100 & 71.750 & 54.500 & 40.000 & 26.100 \\
\hline 00095 & $\begin{array}{l}\text { SPECIFIC CONDUCTANCE, FIELD }(\mu \mathrm{S} / \mathrm{cm} \text { at } 25 \\
\left.{ }^{\circ} \mathrm{C}\right)\end{array}$ & 50 & 317.000 & 13.000 & 69.140 & 273.300 & 65.000 & 52.000 & 38.000 & 19.950 \\
\hline 00403 & $\mathrm{pH}$, LAB (STANDARD $\mathrm{pH}$ UNITS) & 40 & 7.600 & 5.900 & 6.622 & 7.590 & 7.000 & 6.600 & 6.100 & 6.000 \\
\hline 00400 & $\mathrm{pH}$, FIELD (STANDARD pH UNITS) & 50 & 7.470 & 6.060 & 6.761 & 7.345 & 7.118 & 6.745 & 6.492 & 6.129 \\
\hline 90410 & ALKALINITY, LAB (mg/L as $\mathrm{CaCO}_{3}$ ) & 40 & 120.000 & 1.000 & 16.917 & 100.500 & 18.000 & 11.500 & 6.000 & 2.040 \\
\hline 80154 & SUSPENDED SEDIMENT (mg/L) & 41 & 1560.000 & 14.000 & 252.098 & 1417.000 & 287.000 & 87.000 & 48.500 & 19.000 \\
\hline 00530 & $\begin{array}{l}\text { RESIDUE ON EVAPORATION AT } 105^{\circ} \mathrm{C} \text {, SUSPENDED } \\
(\mathrm{mg} / \mathrm{L})\end{array}$ & 18 & 362.000 & 8.000 & 69.722 & 362.000 & 87.500 & 39.500 & 19.000 & 8.000 \\
\hline 00535 & RESIDUE VOLATILE, SUSPENDED (mg/L) & 37 & 586.000 & 1.000 & 54.324 & 296.200 & 67.500 & 21.000 & 7.500 & 2.800 \\
\hline 70300 & $\begin{array}{l}\text { DISSOLVED SOLIDS, RESIDUE AT } 180^{\circ} \mathrm{C} \\
(\mathrm{mg} / \mathrm{L})\end{array}$ & 40 & 192.000 & 14.000 & 56.450 & 148.700 & 62.000 & 47.000 & 32.250 & 14.400 \\
\hline 00310 & $\begin{array}{l}5 \text { DAY BIOCHEMICAL OXYGEN DEMAND } \\
(\mathrm{mg} / \mathrm{L})\end{array}$ & 30 & 43.000 & 1.400 & 11.050 & 39.700 & 11.750 & 7.600 & 5.825 & 2.005 \\
\hline 00340 & CHEMICAL OXYGEN DEMAND (mg/L) & 40 & 480.000 & 18.000 & 77.625 & 179.500 & 106.750 & 53.000 & 29.750 & 20.050 \\
\hline \multicolumn{11}{|c|}{ NUTRIENTS, TOTAL AND DISSOLVED } \\
\hline 00625 & $\begin{array}{l}\text { NITROGEN AMMONIA + ORGANIC, } \\
\text { TOTAL (mg/L as N) }\end{array}$ & 41 & 6.600 & 0.460 & 1.767 & 5.310 & 2.100 & 1.400 & 1.000 & 0.608 \\
\hline 00631 & $\mathrm{NO}_{2}+\mathrm{NO}_{3}$, DISSOLVED $(\mathrm{mg} / \mathrm{L}$ as $\mathrm{N})$ & 41 & 2.700 & 0.180 & 0.797 & 2.555 & 0.995 & 0.580 & 0.410 & 0.182 \\
\hline 00608 & $\begin{array}{l}\text { NITROGEN AMMONIA, DISSOLVED } \\
(\mathrm{mg} / \mathrm{L} \text { as N) }\end{array}$ & 41 & 4.500 & 0.015 & 0.487 & 3.450 & 0.480 & 0.270 & 0.075 & 0.016 \\
\hline 00605 & NITROGEN ORGANIC, TOTAL (mg/L as N) & 41 & 3.800 & 0.410 & 1.273 & 2.930 & 1.700 & 1.000 & 0.790 & 0.418 \\
\hline 00600 & NITROGEN, TOTAL (mg/L as $\mathrm{N})$ & 41 & 8.000 & 0.800 & 2.569 & 6.530 & 3.400 & 2.100 & 1.350 & 0.893 \\
\hline 00665 & PHOSPHORUS, TOTAL (mg/L AS P) & 41 & 1.300 & 0.060 & 0.381 & 0.968 & 0.525 & 0.290 & 0.175 & 0.121 \\
\hline 00671 & $\begin{array}{l}\text { PHOSPHORUS ORTHO, DISSOLVED } \\
(\mathrm{mg} / \mathrm{L} \text { aS } \mathrm{P})\end{array}$ & 41 & 0.790 & 0.022 & 0.178 & 0.530 & 0.205 & 0.120 & 0.060 & 0.034 \\
\hline \multicolumn{11}{|c|}{ OIL AND GREASE, TOTAL } \\
\hline 00556 & OIL AND GREASE, TOTAL (mg/L) & 6 & 6.000 & $<1.000$ & -- & 6.000 & 4.000 & $<1.000$ & $<1.000$ & $<1.000$ \\
\hline \multicolumn{11}{|c|}{ ORGANIC CARBON, TOTAL } \\
\hline 00680 & CARBON ORGANIC, TOTAL (mg/L) & 25 & 76.000 & 9.300 & 19.388 & 67.300 & 20.500 & 14.000 & 11.500 & 9.330 \\
\hline & & & & IFORM & & & & & & \\
\hline 31679 & FECAL STREPTOCOCCI (Colonies per $100 \mathrm{mI}$ ) & 21 & 640000.000 & 2100.000 & 92557.141 & 607999.875 & 74500.000 & 37000.000 & 10950.000 & 2260.000 \\
\hline 31616 & FECAL COLIFORM (Co:lonies per $100 \mathrm{~mL}$ ) & 21 & 590000.000 & 810.000 & 85667.141 & 562999.875 & 103500.000 & 29000.000 & 8850.000 & 949.000 \\
\hline & & & GANIC COMPOU & S-PESTICID & DES, TOTAL & & & & & \\
\hline 39330 & ALDRIN, TOTAL $(\mu \mathrm{g} / \mathrm{L})$ & 3 & $<0.010$ & $<0.010$ & -- & $\overline{--}$ & $\overline{--}$ & -- & -- & -- \\
\hline 39340 & LINDANE, TOTAL $(\mu \mathrm{g} / \mathrm{L})$ & 3 & 0.010 & $<0.010$ & -- & -- & -- & -- & -- & -- \\
\hline 39350 & CHLORDANE, TOTAL $(\mu \mathrm{g} / \mathrm{L})$ & 3 & 0.300 & $<0.100$ & -- & -- & -- & -- & -- & -- \\
\hline 39370 & DDT, TOTAL $(\mu \mathrm{g} / \mathrm{L})$ & 3 & $<0.010$ & $<0.010$ & -- & -- & -- & -- & -- & -- \\
\hline 39365 & DDE, TOTAL $(\mu \mathrm{g} / \mathrm{L})$ & 3 & $<0.010$ & $<0.010$ & -- & -- & -- & -- & -- & -- \\
\hline 39360 & DDD, TOTAL $(\mu \mathrm{g} / \mathrm{L})$ & 3 & $<0.010$ & $<0.010$ & -- & -- & -- & -- & -- & -- \\
\hline 39380 & DIELDRIN, TOTAL $(\mu \mathrm{g} / \mathrm{L})$ & 3 & 0.010 & $<0.010$ & -- & -- & -- & -- & -- & -- \\
\hline 39388 & ENDOSULFAN, TOTAL $(\mu \mathrm{g} / \mathrm{L})$ & 3 & $<0.010$ & $<0.010$ & -- & -- & -- & -- & -- & -- \\
\hline 39390 & ENDRIN, TOTAL $(\mu \mathrm{g} / \mathrm{L})$ & 3 & $<0.010$ & $<0.010$ & -- & -- & -- & -- & -- & -- \\
\hline 39410 & HEPTACHLOR, TOTAL $(\mu \mathrm{g} / \mathrm{L})$ & 3 & $<0.010$ & $<0.010$ & -- & -- & -- & -- & -- & -- \\
\hline 39420 & HEPTACHLOR EPOXIDE, TOTAL $(\mu \mathrm{g} / \mathrm{L})$ & 3 & 0.010 & $<0.010$ & -- & -- & -- & -- & -- & -- \\
\hline 39516 & PCB, TOTAL $(\mu \mathrm{g} / \mathrm{L})$ & 3 & 0.200 & $<0.100$ & -- & -- & -- & - & -- & -- \\
\hline 39400 & TOXAPHENE, TOTAL $(\mu \mathrm{g} / \mathrm{L})$ & 3 & $<1.000$ & $<1.000$ & -- & -- & -- & -- & - & -- \\
\hline 39034 & PERTHANE, TOTAL $(\mu \mathrm{g} / \mathrm{L})$ & 3 & $<0.100$ & $<0.100$ & -- & -- & -- & -- & -- & -- \\
\hline 39570 & DIAZINON, TOTAL $(\mu \mathrm{g} / \mathrm{L})$ & 3 & 0.010 & $<0.010$ & -- & -- & -- & -- & -- & -- \\
\hline 39398 & ETHION, TOTAL $(\mu \mathrm{g} / \mathrm{L})$ & 3 & $<0.010$ & $<0.010$ & -- & -- & -- & -- & -- & -- \\
\hline 39530 & MALATHION, TOTAL $(\mu \mathrm{g} / \mathrm{L})$ & 3 & 0.010 & $<0.010$ & -- & -- & -- & - & -- & -- \\
\hline 39600 & METHYL PARATHION, TOTAL $(\mu \mathrm{g} / \mathrm{L})$ & 3 & 0.010 & $<0.010$ & -- & -- & -- & -- & -- & -- \\
\hline 39540 & PARATHION, TOTAL $(\mu \mathrm{g} / \mathrm{L})$ & 3 & $<0.010$ & $<0.010$ & -- & -- & -- & -- & -- & -- \\
\hline 39786 & TRITHION, TOTAL $(\mu \mathrm{g} / \mathrm{L})$ & 3 & $<0.010$ & $<0.010$ & -- & -- & -- & -- & -- & -- \\
\hline 39250 & PCN, TOTAL $(\mu \mathrm{g} / \mathrm{L})$ & 3 & $<0.100$ & $<0.100$ & -- & -- & -- & -- & -- & -- \\
\hline 39480 & METHOXYCHLOR, TOTAL $(\mu \mathrm{g} / \mathrm{L})$ & 3 & $<0.010$ & $<0.010$ & -- & -- & -- & -- & -- & -- \\
\hline 39755 & MIREX, TOTAL $(\mu \mathrm{g} / \mathrm{L})$ & 3 & $<0.010$ & $<0.010$ & -- & -- & -- & -- & -- & -- \\
\hline 39011 & DISYSTON, TOTAL $(\mu \mathrm{g} / \mathrm{L})$ & 3 & $<0.010$ & $<0.010$ & -- & -- & -- & -- & -- & -- \\
\hline 39023 & PHORATE, TOTAL $(\mu \mathrm{g} / \mathrm{L})$ & 3 & $<0.010$ & $<0.010$ & -- & -- & - & -- & -- & -- \\
\hline 38932 & CHLORPYRIFOS, TOTAL $(\mu \mathrm{g} / \mathrm{L})$ & 3 & 0.060 & $<0.010$ & -- & -- & -- & -- & -- & -- \\
\hline 39040 & DEF, TOTAL $(\mu \mathrm{g} / \mathrm{L})$ & 3 & $<0.010$ & $<0.010$ & -- & -- & - & -- & -- & -- \\
\hline 82614 & FONOFOS, TOTAL $(\mu \mathrm{g} / \mathrm{L})$ & 3 & $<0.010$ & $<0.010$ & -- & -- & -- & -- & $\ldots$ & -- \\
\hline & & & OLATILE ORG & COMPOUND & DS, TOTAL & & & & & \\
\hline 34030 & BENZENE， TOTAL $(\mu \mathrm{g} / \mathrm{L})$ & 7 & $<1.000$ & $<0.200$ & -- & - & -- & -- & -- & -- \\
\hline 32104 & BROMOFORM, TOTAL $(\mu \mathrm{g} / \mathrm{L})$ & 7 & $<1.000$ & $<0.200$ & -- & -- & -- & -- & -- & -- \\
\hline 32102 & CARBON TETRACHLORIDE, TOTAL $(\mu \mathrm{g} / \mathrm{L})$ & 7 & $<1.000$ & $<0.200$ & -- & -- & -- & -- & -- & -- \\
\hline 34301 & CHLOROBENZENE， TOTAL $(\mu \mathrm{g} / \mathrm{L})$ & 7 & $<1.000$ & $<0.200$ & -- & -- & -- & -- & -- & -- \\
\hline 32105 & CHLORODIBROMOTHANE, TOTAL $(\mu \mathrm{g} / \mathrm{L})$ & 7 & $<1.000$ & $<0.200$ & -- & -- & -- & -- & -- & -- \\
\hline 34311 & CHLOROETHANE, TOTAL $(\mu \mathrm{g} / \mathrm{L})$ & 7 & $<1.000$ & $<0.200$ & -- & -- & -- & -- & -- & -- \\
\hline 32106 & CHLOROFORM, TOTAL $(\mu \mathrm{g} / \mathrm{L})$ & 7 & $<1.000$ & $<0.200$ & -- & -- & -- & -- & -- & -- \\
\hline 34496 & 1,1 -DICHLOROETHANE，TOTAL $(\mu \mathrm{g} / \mathrm{L})$ & 7 & $<1.000$ & $<0.200$ & -- & -- & -- & -- & -- & -- \\
\hline 32103 & 1,2 -DICHLOROETHANE, TOTAL $(\mu \mathrm{g} / \mathrm{L})$ & 7 & $<1.000$ & $<0.200$ & -- & - & -- & -- & -- & -- \\
\hline 34501 & 1,1 -DICHLOROETHYLENE, TOTAL $(\mu \mathrm{g} / \mathrm{L})$ & 7 & $<1.000$ & $<0.200$ & -- & -- & -- & -- & -- & -- \\
\hline 34541 & 1,2 -DICHLOROPROPANE, TOTAL $(\mu \mathrm{g} / \mathrm{L})$ & 7 & $<1.000$ & $<0.200$ & -- & -- & -- & -- & -- & - \\
\hline
\end{tabular}

NOTE: Multiple detection limits during the period of record may result in different values flagged with a "<."

* Value is estimated by using a log-probability regression to predict the values of data below the detection limit. 
Table 70. Statistical summary of water-quality data at site 40 (CSW03), July 1994 through June 1997-Continued

\begin{tabular}{|c|c|c|c|c|c|c|c|c|c|c|}
\hline \multirow{2}{*}{$\begin{array}{l}\text { PARAM- } \\
\text { ETER } \\
\text { CODE }\end{array}$} & \multirow{2}{*}{ PROPERTY OR CONSTITUENT } & \multicolumn{4}{|c|}{ DESCRIPTIVE STATISTICS } & \multicolumn{5}{|c|}{$\begin{array}{c}\text { PERCENT OF SAMPLES IN WHICH VALUES WERE LESS THAN OR } \\
\text { EQUAL TO THOSE SHOWN }\end{array}$} \\
\hline & & $\begin{array}{l}\text { SAMPLE } \\
\text { SIZE }\end{array}$ & MAXIMUM & MINIMUM & MEAN & $95 \%$ & $75 \%$ & $\begin{array}{c}50 \% \\
\text { (median) }\end{array}$ & $25 \%$ & $5 \%$ \\
\hline 34371 & ETHYLBENZENE， TOTAL $(\mu \mathrm{g} / \mathrm{L})$ & 7 & $<1.000$ & $<0.200$ & -- & -- & -- & -- & -- & -- \\
\hline 34423 & METHYLENE CHLORIDE，TOTAL $(\mu \mathrm{g} / \mathrm{L})$ & 7 & $<1.000$ & $<0.200$ & .- & -- & -- & -- & -- & -- \\
\hline 34516 & $1,1,2,2$-TETRACHLOROETHANE，TOTAL $(\mu \mathrm{g} / \mathrm{L})$ & 7 & $<1.000$ & $<0.200$ & -- & -- & -- & -- & -- & -- \\
\hline 34475 & TETRACHLOROETHYLENE, TOTAL $(\mu \mathrm{g} / L)$ & 7 & $<1.000$ & $<0.200$ & -- & -- & -- & -- & -- & -- \\
\hline 34010 & TOLUENE, TOTAL $(\mu \mathrm{g} / \mathrm{L})$ & 7 & 0.900 & $<0.200$ & - & 0.900 & $<1.000$ & $<0.800$ & $<0.400$ & $<0.400$ \\
\hline 34546 & $\begin{array}{l}\text { 1, 2-TRANSDICHLOROETHENE， TOTAL } \\
(\mu \mathrm{g} / \mathrm{L})\end{array}$ & 7 & $<1.000$ & $<0.200$ & -- & -- & -- & -- & -- & -- \\
\hline 34506 & $1,1,1$-TRICHLOROETHANE, TOTAL $(\mu \mathrm{g} / \mathrm{L})$ & 7 & $<1.000$ & $<0.200$ & -- & -- & -- & -- & -- & -- \\
\hline 34511 & $1,1,2$-TRICHLOROETHANE, TOTAL $(\mu \mathrm{g} / L)$ & 7 & $<1.000$ & $<0.200$ & -- & -- & -- & -- & -- & -- \\
\hline 39180 & TRICHLOROETHYLENE，TOTAL $(\mu \mathrm{g} / \mathrm{L})$ & 7 & $<1.000$ & $<0.200$ & -- & -- & -- & -- & -- & -- \\
\hline 39175 & VINYL CHLORIDE, TOTAL $(\mu \mathrm{g} / \mathrm{L})$ & 7 & $<1.000$ & $<0.200$ & -- & -- & -- & -- & -- & -- \\
\hline 30217 & DIBROMOMETHANE，TOTAL $(\mu \mathrm{g} / \mathrm{L})$ & 7 & $<1.000$ & $<0.200$ & -- & -- & -- & -- & -- & -- \\
\hline 32101 & DICHLOROBROMOMETHANE, TOTAL $(\mu \mathrm{g} / \mathrm{L})$ & 7 & $<1.000$ & $<0.200$ & -- & -- & -- & -- & -- & -- \\
\hline 34668 & $\begin{array}{l}\text { DICHLORODIFLUOROMETHANE, TOTAL } \\
(\mu \mathrm{g} / \mathrm{L})\end{array}$ & 7 & $<1.000$ & $<0.200$ & -- & -- & -- & -- & -- & -- \\
\hline 34488 & TRICHLOROFLUOROMETHANE, TOTAL $(\mu \mathrm{g} / \mathrm{L})$ & 7 & $<1.000$ & $<0.200$ & -- & -- & -- & -- & -- & -- \\
\hline 77651 & 1,2 -DIBROMOETHANE, TOTAL $(\mu \mathrm{g} / \mathrm{L})$ & 7 & $<1.000$ & $<0.200$ & -- & -- & -- & -- & -- & -- \\
\hline 34704 & $\begin{array}{l}\text { CIS } 1,3 \text {-DICHLOROPROPENE, TOTAL } \\
(\mu g / L)\end{array}$ & 7 & $<1.000$ & $<0.200$ & -- & -- & -- & -- & -- & -- \\
\hline 34699 & TRANS 1,3 -DICHLOROPROPENE, TOTAL $(\mu \mathrm{g} / \mathrm{L})$ & 7 & $<1.000$ & $<0.200$ & -- & -- & -- & -- & -- & -- \\
\hline 77128 & STYRENE, TOTAL $(\mu \mathrm{g} / \mathrm{L})$ & 7 & $<1.000$ & $<0.200$ & -- & -- & -- & -- & -- & -- \\
\hline 81551 & XYLENE，TOTAL $(\mu \mathrm{g} / L)$ & 7 & $<1.000$ & $<0.200$ & -- & -- & -- & -- & -- & -- \\
\hline 82625 & DIBROMOCHLOROPROPANE，TOTAL $(\mu \mathrm{g} / \mathrm{L})$ & 7 & $<5.000$ & $<1.000$ & -- & -- & -- & -- & -- & -- \\
\hline 77168 & 1,1-DICHLOROPROPENE, TOTAL $(\mu \mathrm{g} / \mathrm{L})$ & 7 & $<1.000$ & $<0.200$ & -- & -- & -- & -- & -- & -- \\
\hline 77170 & 2,2 -DICHLOROPROPANE, TOTAL $(\mu \mathrm{g} / \mathrm{L})$ & 7 & $<1.000$ & $<0.200$ & -- & -- & -- & -- & -- & -- \\
\hline 77173 & 1,3-DICHLOROPROPANE, TOTAL $(\mu \mathrm{g} / L)$ & 7 & $<1.000$ & $<0.200$ & -- & -- & -- & -- & -- & -- \\
\hline 77275 & 0-CHLOROTOLUENE，TOTAL $(\mu \mathrm{g} / \mathrm{L})$ & 7 & $<1.000$ & $<0.200$ & $-\rightarrow$ & -- & -- & -- & -- & -- \\
\hline 77277 & P-CHLOROTOLUENE, TOTAL $(\mu \mathrm{g} / \mathrm{L})$ & 7 & $<1.000$ & $<0.200$ & -- & -- & -- & -- & -- & -- \\
\hline 77443 & 123-TRICHLOROPROPANE，TOTAL $(\mu \mathrm{g} / \mathrm{L})$ & 7 & $<1.000$ & $<0.200$ & -- & -- & -- & -- & -- & -- \\
\hline 77562 & 1112-TETRACHLOROETHANE，TOTAL $(\mu \mathrm{g} / \mathrm{L})$ & 7 & $<1.000$ & $<0.200$ & -- & -- & -- & -- & -- & -- \\
\hline 78032 & TERTBUTYL METHYL ETHER, TOTAL $(\mu \mathrm{g} / L)$ & 7 & $<1.000$ & $<0.200$ & -- & -- & -- & -- & -- & -- \\
\hline 77297 & BROMOCHLORO METHANE， TOTAL $(\mu \mathrm{g} / \mathrm{L})$ & 7 & $<1.000$ & $<0.200$ & -- & -- & -- & -- & -. & -- \\
\hline 77093 & CIS- 1,2 -DICHLOROETHENE, TOTAL $(\mu \mathrm{g} / \mathrm{L})$ & 7 & $<1.000$ & $<0.200$ & -- & -- & -- & -- & -- & -- \\
\hline 34576 & 2-CHLOROETHYL VINYL ETHER, TOTAL $(\mu \mathrm{g} / L)$ & 3 & $<4.000$ & $<1.000$ & -- & -- & -- & -- & -- & -- \\
\hline 77223 & ISOPROPYL BENZENE, TOTAL $(\mu \mathrm{g} / \mathrm{L})$ & 7 & $<1.000$ & $<0.200$ & -- & -- & -- & -- & -- & -- \\
\hline 77224 & N-PROPYL BENZENE， TOTAL $(\mu \mathrm{g} / \mathrm{L})$ & 7 & $<1.000$ & $<0.200$ & - & -- & -- & -- & -- & -- \\
\hline 77353 & TERTBUTYL BENZENE, TOTAL $(\mu \mathrm{g} / \mathrm{L})$ & 7 & $<1.000$ & $<0.200$ & -- & -- & -- & -- & -- & -- \\
\hline 77222 & PSEUDOCUMENE, TOTAL $(\mu \mathrm{g} / \mathrm{L})$ & 7 & $<1.000$ & $<0.200$ & -- & -- & -- & -- & -- & -- \\
\hline 77342 & N-BUTYL BENZENE, TOTAL $(\mu \mathrm{g} / \mathrm{L}$ & 7 & $<1.000$ & $<0.200$ & -- & -- & -- & -- & -- & -- \\
\hline 77613 & $1,2,3$-TRICHLOROBENZENE, TOTAL $(\mu \mathrm{g} / \mathrm{L}$ & 7 & $<1.000$ & $<0.200$ & -- & -- & -- & -- & -- & -- \\
\hline 77652 & FREON-113, TOTAL $(\mu \mathrm{g} / \mathrm{L}$ & 7 & $<1.000$ & $<0.200$ & -- & -- & -- & -- & -- & -- \\
\hline 77226 & MESITYLENE, TOTAL $(\mu \mathrm{g} / \mathrm{L}$ & 7 & $<1.000$ & $<0.200$ & -- & -- & -- & -- & -- & -- \\
\hline 81555 & BROMOBENZENE，TOTAL $(\mu \mathrm{g} / \mathrm{L}$ & 7 & $<1.000$ & $<0.200$ & - & -- & -- & -- & -- & -- \\
\hline & & MET & LS AND MINO & CONSTITUEN & IS, TOTAL & & & & & \\
\hline 01097 & ANTIMONY, TOTAL $(\mu \mathrm{g} / L$ as Sb) & 29 & 7.000 & $<1.000$ & $0.836^{*}$ & 5.000 & $<1.000$ & $<1.000$ & $<1.000$ & $<1.000$ \\
\hline 01002 & ARSENIC, TOTAL $(\mu \mathrm{g} / L$ as AS $)$ & 29 & 3.000 & $<1.000$ & $1.165^{\star}$ & 2.000 & 2.000 & 1.000 & $<1.000$ & $<1.000$ \\
\hline 01012 & BERYLLIUM, TOTAL ( $\mu \mathrm{g} / \mathrm{L}$ as Be) & 9 & $<10.000$ & $<10.000$ & -- & -- & -- & -- & -- & -- \\
\hline 01027 & CADMIUM, TOTAL $(\mu \mathrm{g} / \mathrm{L}$ as $\mathrm{Cd})$ & 9 & 1.000 & $<1.000$ & - & 1.000 & $<1.000$ & $<1.000$ & $<1.000$ & $<1.000$ \\
\hline 01034 & CHROMIUM, TOTAL $(\mu \mathrm{g} / \mathrm{L}$ as $\mathrm{Cr})$ & 29 & 20.000 & 2.000 & 7.931 & 19.000 & 10.000 & 6.000 & 4.000 & 2.500 \\
\hline 01042 & COPPER, TOTAL ( $\mu \mathrm{g} / \mathrm{L}$ as $\mathrm{Cu})$ & 29 & 50.000 & 3.000 & 16.379 & 45.500 & 21.500 & 12.000 & 7.000 & 3.500 \\
\hline 01051 & LEAD, TOTAL $(\mu \mathrm{g} / L$ as $\mathrm{Pb})$ & 29 & 160.000 & 3.000 & 19.483 & 101.000 & 22.500 & 12.000 & 6.000 & 3.000 \\
\hline 71900 & MERCURY, TOTAL $(\mu \mathrm{g} / \mathrm{L}$ as $\mathrm{Hg})$ & 9 & 0.100 & $<0.100$ & -- & 0.100 & $<0.100$ & $<0.100$ & $<0.1 .00$ & $<0.100$ \\
\hline 01067 & NICKEL, TOTAL $(\mu \mathrm{g} / \mathrm{L}$ as Ni) & 29 & 23.000 & 1.000 & 6.552 & 18.500 & 8.500 & 6.000 & 4.000 & 1.500 \\
\hline 01147 & SELENIUM, TOTAL $(\mu \mathrm{g} / \mathrm{L}$ as $\mathrm{Se})$ & 9 & $<1.000$ & $<1.000$ & $-\cdots$ & -- & - & -- & -- & -- \\
\hline 01077 & SILVER, TOTAL $(\mu \mathrm{g} / \mathrm{L}$ as $\mathrm{Ag})$ & 9 & $<1.000$ & $<1.000$ & -- & -- & -- & -- & -- & -- \\
\hline 01092 & $\mathrm{ZINC}, \operatorname{TOTAL}(\mu \mathrm{g} / \mathrm{L}$ as $\mathrm{zn})$ & 28 & 490.000 & 30.000 & 96.786 & 359.500 & 107.500 & 75.000 & 60.000 & 34.500 \\
\hline 00720 & CYANIDE, TOTAL (mg/L as Cn) & 9 & 0.010 & $<0.010$ & -- & 0.010 & $<0.010$ & $<0.010$ & $<0.010$ & $<0.010$ \\
\hline & & ORGAN & COMPOUND & ESTICIDES & DISSOLVED & & & & & \\
\hline 46342 & ALACHLOR, DISSOLVED $(\mu \mathrm{g} / \mathrm{L})$ & 2 & 0.011 & $<0.002$ & -- & -- & -- & -- & -- & -- \\
\hline 04040 & DEETHYL ATRAZINE, DISSOLVED $(\mu \mathrm{g} / \mathrm{L})$ & 2 & 0.006 & $<0.002$ & -- & -- & -- & -- & -- & -- \\
\hline 39632 & ATRAZINE，DISSOLVED $(\mu \mathrm{g} / \mathrm{L})$ & 2 & 0.029 & $<0.001$ & -. & -- & -- & -- & -- & -- \\
\hline 82686 & METHYL AZINPHOS, DISSOLVED $(\mu \mathrm{g} / L)$ & 2 & $<0.001$ & $<0.001$ & -- & -- & -- & -- & -- & -- \\
\hline 82673 & BENFLURALIN, DISSOLVED $(\mu \mathrm{g} / \mathrm{L})$ & 2 & 0.037 & $<0.002$ & -- & - & -- & -- & -- & -- \\
\hline 04028 & BUTYLATE, DISSOLVED $(\mu \mathrm{g} / \mathrm{L})$ & 2 & $<0.002$ & $<0.002$ & -- & -- & -- & -- & -. & -- \\
\hline 82680 & CARBARYL， DISSOLVED $(\mu \mathrm{g} / \mathrm{L})$ & 2 & 0.787 & 0.370 & -- & -- & -- & -- & -- & -- \\
\hline 82674 & CARBOFURAN， DISSOLVED $(\mu \mathrm{g} / L)$ & 2 & $<0.003$ & $<0.003$ & - - & -- & -- & -- & -- & -- \\
\hline 38933 & CHLORPYRIFOS, DISSOLVED $(\mu \mathrm{g} / \mathrm{L})$ & 2 & 0.009 & $<0.004$ & -- & -- & -- & -- & -- & -- \\
\hline 04041 & CYANAZINE, DISSOLVED $(\mu \mathrm{g} / \mathrm{L})$ & 2 & $<0.004$ & $<0.004$ & -- & -- & -- & -- & -- & -- \\
\hline 82682 & DCPA， DISSOLVED $(\mu \mathrm{g} / \mathrm{L})$ & 2 & $<0.002$ & $<0.002$ & -- & -- & -- & -- & -- & -- \\
\hline 34653 & $P, P^{\prime}$ DDE, DISSOLVED $(\mu \mathrm{g} / L)$ & 2 & $<0.006$ & $<0.006$ & -- & -- & -- & -- & -- & -- \\
\hline 39572 & DIAZINON，DISSOLVED $(\mu \mathrm{g} / L)$ & 2 & 0.012 & $<0.002$ & -- & -- & -- & -- & -- & -- \\
\hline 39381 & DIELDRIN，DISSOLVED $(\mu \mathrm{g} / L)$ & 2 & $<0.001$ & $<0.001$ & -- & -- & -- & -- & -- & -- \\
\hline 82660 & $\begin{array}{l}2,6 \text {-DIETHYL ANILINE, DISSOLVED } \\
(\mu \mathrm{g} / L)\end{array}$ & 2 & $<0.003$ & $<0.003$ & -- & -- & -- & -- & -- & -- \\
\hline 82677 & DISULFOTON, DISSOLVED $(\mu \mathrm{g} / L)$ & 2 & $<0.017$ & $<0.017$ & -- & -- & -- & -- & -- & -- \\
\hline 82668 & EPTC， DISSOLVED $(\mu \mathrm{g} / \mathrm{L})$ & 2 & $<0.002$ & $<0.002$ & -- & -- & -- & -- & -- & -- \\
\hline 82663 & ETHALFLURALIN, DISSOLVED $(\mu \mathrm{g} / \mathrm{L})$ & 2 & $<0.004$ & $<0.004$ & -- & -- & -- & -- & -- & -- \\
\hline
\end{tabular}


Table 70. Statistical summary of water-quality data at site 40 (CSW03), July 1994 through June 1997-Continued

\begin{tabular}{|c|c|c|c|c|c|c|c|c|c|c|}
\hline \multirow{2}{*}{$\begin{array}{c}\text { PARAM- } \\
\text { ETER } \\
\text { CODE }\end{array}$} & \multirow{2}{*}{ PROPERTY OR CONSTITUENT } & \multicolumn{4}{|c|}{ DESCRIPTIVE STATISTICS } & PERCEN & $\begin{aligned} \text { AMPLE } \\
\text { EQ }\end{aligned}$ & $\begin{array}{l}\text { NHICH VAL } \\
\text { TO THOSE }\end{array}$ & NERE & IAN OR \\
\hline & & $\begin{array}{l}\text { SAMPLE } \\
\text { SIZE }\end{array}$ & MAXIMUM & MINIMUM & MEAN & $95 \%$ & $75 \%$ & $\begin{array}{c}50 \% \\
\text { (median) }\end{array}$ & $25 \%$ & $5 \%$ \\
\hline 82672 & ETHOPROP， DISSOLVED $(\mu \mathrm{g} / \mathrm{L})$ & 2 & $<0.003$ & $<0.003$ & -- & -- & - & -- & -- & -- \\
\hline 04095 & FONOFOS， DISSOLVED $(\mu \mathrm{g} / \mathrm{L})$ & 2 & $<0.003$ & $<0.003$ & -- & -- & -- & -- & -- & -- \\
\hline 34253 & ALPHA BHC, DISSOLVED $(\mu \mathrm{g} / \mathrm{L})$ & 2 & $<0.002$ & $<0.002$ & -- & -- & -- & -- & -- & -- \\
\hline 39341 & LINDANE，DISSOLVED $(\mu \mathrm{g} / \mathrm{L})$ & 2 & 0.006 & $<0.004$ & -- & -- & -- & -- & -- & -- \\
\hline 82666 & LINURON， DISSOLVED $(\mu \mathrm{g} / L)$ & 2 & $<0.002$ & $<0.002$ & -- & -- & -- & -- & -- & -- \\
\hline 39532 & MALATHION, DISSOLVED $(\mu \mathrm{g} / \mathrm{L})$ & 2 & 0.022 & $<0.005$ & -- & -- & -- & -- & -- & -- \\
\hline 82667 & METHYL PARATHION, DISSOLVED $(\mu \mathrm{g} / \mathrm{L})$ & 2 & 0.033 & $<0.006$ & -- & -- & -- & -- & -- & -- \\
\hline 3941.5 & METOLACHLOR， DISSOLVED $(\mu \mathrm{g} / \mathrm{L})$ & 2 & 0.038 & $<0.002$ & -- & -- & -- & -- & -- & -- \\
\hline 82630 & METRIBUZIN， DISSOLVED $(\mu \mathrm{g} / \mathrm{L})$ & 2 & $<0.004$ & $<0.004$ & -- & -- & -- & -- & -- & -- \\
\hline 82671 & MOLINATE, DISSOLVED $(\mu \mathrm{g} / \mathrm{L})$ & 2 & $<0.004$ & $<0.004$ & -- & -- & -- & -- & -- & -- \\
\hline 82684 & NAPROPAMIDE， DISSOLVED $(\mu \mathrm{g} / \mathrm{L})$ & 2 & $<0.003$ & $<0.003$ & -- & -- & -- & -- & -- & -- \\
\hline 39542 & PARATHION， DISSOLVED $(\mu \mathrm{g} / \mathrm{L})$ & 2 & $<0.004$ & $<0.004$ & -- & -- & -- & -- & -- & -- \\
\hline 82669 & PEBULATE， DISSOLVED $(\mu \mathrm{g} / \mathrm{L})$ & 2 & $<0.004$ & $<0.004$ & -- & -- & -- & -- & -- & -- \\
\hline 82683 & PENDIMETHALIN， DISSOLVED $(\mu \mathrm{g} / \mathrm{L})$ & 2 & $<0.004$ & $<0.004$ & -- & -- & -- & -- & -- & -- \\
\hline 82687 & PERMETHRIN， DISSOLVED $(\mu \mathrm{g} / \mathrm{L})$ & 2 & $<0.005$ & $<0.005$ & -- & - & -- & -- & -- & -- \\
\hline 82664 & PHORATE, DISSOLVED $(\mu \mathrm{g} / \mathrm{L})$ & 2 & $<0.002$ & $<0.002$ & -- & -- & -- & -- & -- & -- \\
\hline 82676 & PRONAMIDE， DISSOLVED $(\mu \mathrm{g} / L)$ & 2 & $<0.003$ & $<0.003$ & -- & -- & -- & -- & -- & -- \\
\hline 04037 & PROMETON, DISSOLVED $(\mu \mathrm{g} / \mathrm{L})$ & 2 & $<0.018$ & $<0.018$ & -- & -- & -- & -- & -- & -- \\
\hline 04024 & PROPACHLOR, DISSOLVED $(\mu \mathrm{g} / \mathrm{L})$ & 2 & $<0.007$ & $<0.007$ & -- & -- & -- & -- & -- & -- \\
\hline 82679 & PROPANIL, DISSOLVED $(\mu \mathrm{g} / \mathrm{L})$ & 2 & $<0.004$ & $<0.004$ & -- & -- & -- & -- & -- & -- \\
\hline 82685 & PROPARGITE, DISSOLVED $(\mu \mathrm{g} / \mathrm{L})$ & 2 & $<0.013$ & $<0.013$ & -- & -- & -- & -- & -- & -- \\
\hline 04035 & SIMAZINE， DISSOLVED $(\mu \mathrm{g} / \mathrm{L})$ & 2 & $<0.005$ & $<0.005$ & -- & -- & -- & -- & -- & -- \\
\hline 82681 & THIOBENCARB， DISSOLVED $(\mu \mathrm{g} / \mathrm{L})$ & 2 & $<0.002$ & $<0.002$ & -- & -- & -- & -- & -- & -- \\
\hline 82670 & TEBUTHIURON， DISSOLVED $(\mu \mathrm{g} / \mathrm{L})$ & 2 & 0.020 & $<0.010$ & -- & -- & -- & -- & -- & -- \\
\hline 82665 & TERBACIL， DISSOLVED $(\mu \mathrm{g} / \mathrm{L})$ & 2 & $<0.007$ & $<0.007$ & -- & -- & -- & -- & -- & -- \\
\hline 82675 & TERBUFOS， DISSOLVED $(\mu \mathrm{g} / \mathrm{L})$ & 2 & $<0.013$ & $<0.013$ & -- & -- & -- & -- & -- & -- \\
\hline 82678 & TRIALLATE, DISSOLVED $(\mu \mathrm{g} / \mathrm{L})$ & 2 & $<0.001$ & $<0.001$ & -- & -- & -- & -- & -- & -- \\
\hline 82661 & TRIFLURALIN， DISSOLVED $(\mu \mathrm{g} / \mathrm{L})$ & 2 & 0.023 & $<0.002$ & -- & -- & -- & -- & -- & -- \\
\hline 39742 & $2,4,5-\mathrm{T}$, DISSOLVED $(\mu \mathrm{g} / \mathrm{L})$ & 2 & $<0.035$ & $<0.035$ & - & -- & -- & -- & -- & -. \\
\hline 39732 & $2,4-\mathrm{D}$, DISSOLVED $(\mu \mathrm{g} / \mathrm{L})$ & 2 & $<0.035$ & $<0.035$ & -- & - & -- & -- & -- & -- \\
\hline 38746 & $2,4-\mathrm{DB}$, DISSOLVED $(\mu \mathrm{g} / \mathrm{L})$ & 2 & $<0.035$ & $<0.035$ & -- & -- & -- & -- & -- & - \\
\hline 49315 & ACIFLUORFEN， DISSOLVED $(\mu \mathrm{g} / \mathrm{L})$ & 2 & $<0.035$ & $<0.035$ & -- & -- & -- & -- & -- & -- \\
\hline 49312 & ALDICARB, DISSOLVED $(\mu \mathrm{g} / \mathrm{L})$ & 2 & $<0.016$ & $<0.016$ & -- & -- & -- & -- & -- & -- \\
\hline 49313 & ALDICARB SULFONE, DISSOLVED $(\mu \mathrm{g} / \mathrm{L})$ & 2 & $<0.016$ & $<0.016$ & -- & -- & -- & -- & -- & -- \\
\hline 49314 & $\begin{array}{l}\text { ALDICARB SULFOXIDE, DISSOLVED } \\
(\mu \mathrm{g} / \mathrm{L})\end{array}$ & 2 & $<0.021$ & $<0.021$ & -- & -- & -- & -- & -- & - \\
\hline 38711 & BENTAZON， DISSOLVED $(\mu \mathrm{g} / \mathrm{L})$ & 2 & $<0.014$ & $<0.014$ & -- & -- & -- & -- & -- & -- \\
\hline 04029 & BROMACIL, DISSOLVED $(\mu \mathrm{g} / \mathrm{L})$ & 2 & $<0.035$ & $<0.035$ & -- & - & -- & -- & -- & -- \\
\hline 49311 & BROMOXYNIL， DISSOLVED $(\mu \mathrm{g} / \mathrm{L})$ & 2 & $<0.035$ & $<0.035$ & -- & -- & -- & -- & -- & -- \\
\hline 49310 & CARBARYL， DISSOLVED $(\mu \mathrm{g} / \mathrm{L})$ & 2 & 0.540 & 0.090 & -- & -- & -- & -- & -- & -- \\
\hline 49309 & CARBOFURAN, DISSOLVED $(\mu \mathrm{g} / \mathrm{L})$ & 2 & $<0.028$ & $<0.028$ & -- & -- & -- & -- & -- & -- \\
\hline 49308 & 3 -HYDROXY-CARBOFURAN $(\mu \mathrm{g} / \mathrm{L})$ & 2 & $<0.014$ & $<0.014$ & -- & -- & -- & -- & -- & -- \\
\hline 49307 & AMIBEN， DISSOLVED $(\mu \mathrm{g} / \mathrm{L})$ & 2 & $<0.011$ & $<0.011$ & -- & -- & - & -- & -- & -- \\
\hline 49306 & CHLOROTHALONIL， DISSOLVED $(\mu \mathrm{g} / \mathrm{L})$ & 2 & $<0.035$ & $<0.035$ & -- & -- & -- & -- & -- & -- \\
\hline 49305 & CLOPYRALID， DISSOLVED $(\mu \mathrm{g} / \mathrm{L})$ & 2 & $<0.050$ & $<0.050$ & -- & -- & -- & -- & -- & -- \\
\hline 49304 & DACTHALMONO-ACID， DISSOLVED $(\mu \mathrm{g} / \mathrm{L})$ & 2 & $<0.017$ & $<0.017$ & -- & -- & -- & -- & -- & -- \\
\hline 38442 & DICAMBA， DISSOLVED $(\mu \mathrm{g} / \mathrm{L})$ & 2 & $<0.035$ & $<0.035$ & -- & -- & -- & -- & -- & -- \\
\hline 49303 & DICHLOBENIL, DISSOLVED $(\mu \mathrm{g} / \mathrm{L})$ & 1 & $<0.020$ & -- & -- & -- & -- & -- & -- & -- \\
\hline 49302 & DICHLORPROP, DISSOLVED $(\mu \mathrm{g} / \mathrm{L})$ & 2 & $<0.032$ & $<0.032$ & -- & -- & -- & -- & -- & -- \\
\hline 49301 & DINOSEB， DISSOLVED $(\mu \mathrm{g} / \mathrm{L})$ & 2 & $<0.035$ & $<0.035$ & $-\infty$ & -- & -- & -- & -- & -- \\
\hline 49300 & DIURON, DISSOLVED $(\mu \mathrm{g} / \mathrm{L})$ & 2 & $<0.020$ & $<0.020$ & -- & -- & -- & -- & -- & -- \\
\hline 49299 & $\begin{array}{l}\text { 4, 6-DINITRO OCRESOL， DISSOLVED } \\
(\mu \mathrm{g} / \mathrm{L})\end{array}$ & 2 & $<0.035$ & $<0.035$ & -- & -- & -- & -- & -- & -- \\
\hline 49298 & ESFENVALERATE，DISSOLVED $(\mu \mathrm{g} / \mathrm{L})$ & 2 & $<0.019$ & $<0.019$ & -- & -- & -- & -- & -- & -- \\
\hline 49297 & FENURON， DISSOLVED $(\mu \mathrm{g} / \mathrm{L})$ & 2 & $<0.013$ & $<0.013$ & -- & -- & -- & -- & -- & -- \\
\hline 38811 & FLUOMETURON， DISSOLVED $(\mu \mathrm{g} / \mathrm{L})$ & 2 & $<0.035$ & $<0.035$ & -- & -- & -- & -- & -- & -- \\
\hline 38478 & LINURON, DISSOLVED $(\mu \mathrm{g} / \mathrm{L})$ & 2 & $<0.018$ & $<0.018$ & -- & -- & -- & -- & -- & -- \\
\hline 38482 & MCPA， DISSOLVED $(\mu \mathrm{g} / \mathrm{L})$ & 2 & $<0.050$ & $<0.050$ & -- & -- & -- & -- & -- & -- \\
\hline 38487 & MCPB， DISSOLVED $(\mu \mathrm{g} / \mathrm{L})$ & 2 & $<0.035$ & $<0.035$ & -- & -- & -- & -- & -- & -- \\
\hline 38501 & METHIOCARB, DISSOLVED $(\mu \mathrm{g} / \mathrm{L})$ & 2 & $<0.026$ & $<0.026$ & -- & -- & -- & -- & -- & -- \\
\hline 49296 & METHOMYL， DISSOLVED $(\mu \mathrm{g} / \mathrm{L})$ & 2 & $<0.017$ & $<0.017$ & -- & -- & -- & -- & -- & -- \\
\hline 49295 & 1-NAPHTHOL， DISSOLVED $(\mu \mathrm{g} / \mathrm{L})$ & 2 & $<0.007$ & $<0.007$ & -- & -- & -- & -- & - & -- \\
\hline 49294 & NEBURON， DISSOLVED $(\mu \mathrm{g} / \mathrm{L})$ & 2 & $<0.015$ & $<0.015$ & -- & -- & -- & -- & -- & -- \\
\hline 49293 & NORFLURAZON, DISSOLVED $(\mu \mathrm{g} / \mathrm{L})$ & 2 & $<0.024$ & $<0.024$ & -- & -- & -- & -- & -- & - \\
\hline 49292 & ORYZALIN， DISSOLVED $(\mu \mathrm{g} / \mathrm{L})$ & 2 & $<0.019$ & $<0.019$ & -- & -- & -- & -- & -- & -- \\
\hline 38866 & OXAMYL， DISSOLVED $(\mu \mathrm{g} / \mathrm{L})$ & 2 & $<0.018$ & $<0.018$ & -- & -- & -- & -- & -- & -- \\
\hline 49291 & PICLORAM, DISSOLVED $(\mu \mathrm{g} / \mathrm{L})$ & 2 & $<0.050$ & $<0.050$ & -- & -- & -- & -- & -- & -- \\
\hline 49236 & PROPHAM, DISSOLVED $(\mu \mathrm{g} / \mathrm{L})$ & 2 & $<0.035$ & $<0.035$ & -- & -- & -- & -- & -- & -- \\
\hline 38538 & PROPOXUR， DISSOLVED $(\mu \mathrm{g} / \mathrm{L})$ & 2 & $<0.035$ & $<0.035$ & -- & -- & -- & -- & - & -- \\
\hline 39762 & SILVEX， DISSOLVED $(\mu \mathrm{g} / \mathrm{L})$ & 2 & $<0.021$ & $<0.021$ & -- & -- & -- & -- & -- & -- \\
\hline 49235 & TRICLOPYR， DISSOLVED $(\mu \mathrm{g} / \mathrm{L})$ & 2 & $<0.050$ & $<0.050$ & -- & -- & -- & -- & -- & -- \\
\hline & & ORGAN & COMPOUND & GANONITR & EN, TOT & & & & & \\
\hline 39057 & PROMETRYNE， TOTAL $(\mu \mathrm{g} / \mathrm{L})$ & 1 & 0.100 & -- & -- & -- & -- & -- & -- & -- \\
\hline 39056 & PROMETONE, TOTAL $(\mu \mathrm{g} / \mathrm{L})$ & 1 & $<0.200$ & -- & -- & -- & -- & - & -- & -- \\
\hline 39054 & SIMETRYNE, TOTAL $(\mu \mathrm{g} / \mathrm{L})$ & 1 & $<0.100$ & -- & -- & -- & -- & -- & -- & - \\
\hline 81757 & CYANAZINE, TOTAL $(\mu \mathrm{g} / \mathrm{L})$ & 1 & $<0.200$ & -- & -- & -- & -- & -- & $\rightarrow$ & -- \\
\hline 77825 & ALACHLOR, TOTAL $(\mu \mathrm{g} / \mathrm{L})$ & 1 & $<0.100$ & -- & -- & -- & -- & -- & -- & -- \\
\hline 82611 & METRIBUZIN, TOTAL $(\mu \mathrm{g} / \mathrm{L})$ & 1 & $<0.100$ & -- & -- & -- & -- & -- & -- & -- \\
\hline 30311 & TERBACIL, TOTAL $(\mu \mathrm{g} / \mathrm{L})$ & 1 & $<0.200$ & -- & -- & -- & -- & -- & -- & -- \\
\hline 30245 & CARBOXIN, TOTAL $(\mu \mathrm{g} / \mathrm{L})$ & 1 & $<0.200$ & -- & -- & -- & -- & -- & -- & -- \\
\hline 30264 & HEXAZINONE, TOTAL $(\mu \mathrm{g} / \mathrm{L})$ & 1 & $<0.200$ & -- & -- & -- & -- & -- & -- & -- \\
\hline
\end{tabular}

NOTE: Multiple detection limits during the period of record may result in different values flagged with a " $<$."

* Value is estimated by using a log-probability regression to predict the values of data below the detection limit. 
Table 70. Statistical summary of water-quality data at site 40 (CSW03), July 1994 through June 1997—Continued

\begin{tabular}{|c|c|c|c|c|c|c|c|c|c|c|}
\hline \multirow{2}{*}{$\begin{array}{c}\text { PARAM- } \\
\text { ETER } \\
\text { CODE }\end{array}$} & \multirow{2}{*}{ PROPERTY OR CONSTITUENT } & \multicolumn{4}{|c|}{ DESCRIPTIVE STATISTICS } & \multicolumn{5}{|c|}{$\begin{array}{c}\text { PERCENT OF SAMPLES IN WHICH VALUES WERE LESS THAN OR } \\
\text { EQUAL TO THOSE SHOWN }\end{array}$} \\
\hline & & $\begin{array}{l}\text { SAMPLE } \\
\text { SIZE }\end{array}$ & MAXIMUM & MINIMUM & MEAN & $95 \%$ & $75 \%$ & $\begin{array}{c}50 \% \\
\text { (median) }\end{array}$ & $25 \%$ & $5 \%$ \\
\hline 30235 & BUTACHLOR, TOTAL $(\mu \mathrm{g} / \mathrm{L})$ & 1 & $<0.100$ & -- & -- & -- & -- & -- & -- & -- \\
\hline 30236 & BUTYLATE， TOTAL $(\mu \mathrm{g} / \mathrm{L})$ & 1 & $<0.100$ & -- & -- & -- & -- & -- & -- & -- \\
\hline 75981 & DE-ETHYLATRAZINE，TOTAL $(\mu \mathrm{g} / \mathrm{L})$ & 1 & $<0.200$ & -- & -- & -- & -- & -- & -- & -- \\
\hline 39630 & ATRAZINE， TOTAL $(\mu \mathrm{g} / L)$ & 1 & $<0.100$ & -- & -- & -- & -- & -- & -- & -- \\
\hline 39055 & SIMAZINE，TOTAL $(\mu \mathrm{g} / L)$ & 1 & $<0.100$ & -- & -- & -- & -- & -- & -- & -- \\
\hline 39024 & PROPAZINE, TOTAL $(\mu \mathrm{g} / \mathrm{L})$ & 1 & $<0.100$ & -- & -- & -- & -- & -- & -- & -- \\
\hline 82184 & AMETRYNE，TOTAL $(\mu \mathrm{g} / \mathrm{L})$ & 1 & 0.100 & -- & -- & -- & -- & -- & -- & -- \\
\hline 39030 & TRIFLURALIN， TOTAL $(\mu \mathrm{g} / \mathrm{L})$ & 1 & $<0.100$ & -- & -- & -- & -- & -- & -- & -- \\
\hline 82612 & METOLACHLOR, TOTAL $(\mu \mathrm{g} / \mathrm{L})$ & 1 & $<0.200$ & -- & -- & -- & -- & -- & -- & -- \\
\hline 30234 & BROMACIL， TOTAL $(\mu \mathrm{g} / \mathrm{L})$ & 1 & $<0.200$ & -- & -- & -- & - & -- & -- & -- \\
\hline 30255 & DIPHENAMID，TOTAL $(\mu \mathrm{g} / \mathrm{L})$ & 1 & $<0.100$ & -- & -- & -- & -- & -- & -. & -- \\
\hline 30324 & VERNOLATE, TOTAL $(\mu \mathrm{g} / L)$ & 1 & $<0.100$ & -- & -- & -- & -- & -- & -- & -- \\
\hline 30254 & CYCLOATE, TOTAL $(\mu \mathrm{g} / L)$ & 1 & $<0.100$ & -- & -- & -- & -- & -- & -- & -- \\
\hline 30295 & PROPACHLOR, TOTAL $(\mu \mathrm{g} / \mathrm{L})$ & 1 & $<0.100$ & -- & -- & -- & -- & -- & -- & -- \\
\hline 75980 & DE-ISOPROPYLATRAZIN， TOTAL $(\mu \mathrm{g} / \mathrm{L})$ & 1 & $<0.200$ & - & $\cdots$ & -- & - & -- & -- & $\ldots$ \\
\hline \multicolumn{11}{|c|}{ ORGANIC COMPOUNDS-HERBICIDES, TOTAL } \\
\hline 39730 & $2,4-\mathrm{D}$, TOTAL $(\mu \mathrm{g} / \mathrm{L})$ & 1 & $<0.010$ & - & -- & -- & -- & -- & -- & -- \\
\hline 39760 & SILVEX， TOTAL $(\mu \mathrm{g} / \mathrm{L})$ & 1 & $<0.010$ & -- & -- & -- & -- & -- & -- & -- \\
\hline 39720 & PICLORAM, TOTAL $(\mu \mathrm{g} / \mathrm{L})$ & 1 & $<0.010$ & -- & -- & -- & - & -- & -- & -- \\
\hline 39740 & $2,4,5-\mathrm{T}$, TOTAL $(\mu \mathrm{g} / \mathrm{L})$ & 1 & $<0.010$ & -- & -- & -- & -- & -- & -- & -- \\
\hline 82183 & $2,4-\mathrm{DP}$, TOTAL $(\mu \mathrm{g} / \mathrm{L})$ & 1 & $<0.010$ & -- & -- & -- & -- & -- & -- & -- \\
\hline 82052 & DICAMBA, TOTAL $(\mu \mathrm{g} / \mathrm{L})$ & 1 & $<0.010$ & -- & -- & -- & -- & -- & -- & -- \\
\hline \multicolumn{11}{|c|}{ ORGANIC COMPOUNDS-CARBAMATE PESTICIDES, TOTAL } \\
\hline 39750 & SEVIN, TOTAL $(\mu \mathrm{g} / \mathrm{L})$ & 1 & $<0.500$ & -- & -- & -- & -- & $\overrightarrow{--}$ & -- & -- \\
\hline 39051 & METHOMYL, TOTAL $(\mu \mathrm{g} / \mathrm{L})$ & 1 & $<0.500$ & -- & -- & -- & -- & -- & -- & -- \\
\hline 82619 & ALDICARD, TOTAL $(\mu \mathrm{g} / \mathrm{L})$ & 1 & $<0.500$ & -- & -- & -- & -- & -- & -- & -- \\
\hline 30296 & PROPOXUR，TOTAL $(\mu \mathrm{g} / \mathrm{L})$ & 1 & $<0.500$ & -- & -. & -- & -- & -- & -- & -- \\
\hline 39052 & PROPHAM, TOTAL $(\mu \mathrm{g} / \mathrm{L})$ & 1 & $<0.500$ & -- & -- & -- & -- & -- & -- & -- \\
\hline 82615 & CARBOFURAN, TOTAL $(\mu \mathrm{g} / \mathrm{L})$ & 1 & $<0.500$ & -- & -- & -- & -- & -- & -- & -- \\
\hline 77441 & 1-NAPHTHOL，TOTAL $(\mu \mathrm{g} / \mathrm{L})$ & 1 & $<0.500$ & -- & -- & -- & -- & -- & -- & -- \\
\hline 30282 & METHIOCARB, TOTAL $(\mu \mathrm{g} / \mathrm{L})$ & 1 & $<0.500$ & -- & $\ldots$ & -- & -- & -- & -- & -- \\
\hline
\end{tabular}

NOTE: Multiple detection limits during the period of record may result in different values flagged with a "<."

* Value is estimated by using a log-probability regression to predict the values of data below the detection limit. 
Table 71. Statistical summary of water-quality data at site 41 (CSWO2), May 1994 through June 1997

\begin{tabular}{|c|c|c|c|c|c|c|c|c|c|c|}
\hline \multirow{2}{*}{$\begin{array}{l}\text { PARAM- } \\
\text { ETER } \\
\text { CODE }\end{array}$} & \multirow{2}{*}{ PROPERTY OR CONSTITUENT } & \multicolumn{4}{|c|}{ DESCRIPTIVE STATISTICS } & \multicolumn{5}{|c|}{$\begin{array}{l}\text { PERCENT OF SAMPLES IN WHICH VALUES WERE LESS THAN OR } \\
\text { EQUAL TO THOSE SHOWN }\end{array}$} \\
\hline & & $\begin{array}{l}\text { SAMPLE } \\
\text { SIZE }\end{array}$ & MAXIMUM & MINIMUM & MEAN & $95 \%$ & $75 \%$ & $\begin{array}{c}50 \% \\
\text { (median) }\end{array}$ & $25 \%$ & $5 \%$ \\
\hline \multicolumn{11}{|c|}{ PHYSICAL AND CHEMICAL PROPERTIES } \\
\hline 00061 & INSTANTANEOUS DISCHARGE, $\left(\mathrm{ft}^{3} / \mathrm{s}\right)$ & 73 & 153.000 & 0.030 & 9.053 & 61.400 & 4.900 & 1.400 & 0.410 & 0.181 \\
\hline 00010 & WATER TEMPERATURE $\left({ }^{\circ} \mathrm{C}\right)$ & 65 & 27.000 & 5.000 & 16.323 & 26.350 & 19.500 & 16.500 & 14.000 & 5.650 \\
\hline 90095 & SPECIFIC CONDUCTANCE, LAB $\left(\mu \mathrm{S} / \mathrm{cm}\right.$ at $\left.25^{\circ} \mathrm{C}\right)$ & 45 & 265.000 & 18.000 & 76.889 & 167.800 & 136.000 & 44.000 & 30.000 & 20.600 \\
\hline 00095 & $\begin{array}{l}\text { SPECIFIC CONDUCTANCE, FIELD ( } \mu \text { S/cm at } 25 \\
\left.{ }^{\circ} \mathrm{C}\right)\end{array}$ & 57 & 252.000 & 16.000 & 76.246 & 168.200 & 139.000 & 41.000 & 25.000 & 17.800 \\
\hline 00403 & pH， LAB (STANDARD pH UNITS) & 45 & 7.400 & 5.600 & 6.653 & 7.200 & 6.900 & 6.800 & 6.300 & 5.730 \\
\hline 00400 & pH，FIELD (STANDARD pH UNITS) & 53 & 7.220 & 5.940 & 6.709 & 7.051 & 6.885 & 6.730 & 6.575 & 6.300 \\
\hline 90410 & ALKALINITY, LAB (mg/L as $\mathrm{CaCO}_{3}$ ) & 45 & 61.000 & 2.000 & 21.038 & 55.700 & 45.000 & 10.000 & 6.500 & 3.300 \\
\hline 80154 & SUSPENDED SEDIMENT $(\mathrm{mg} / \mathrm{L})$ & 50 & 644.000 & 7.000 & 100.920 & 452.450 & 112.500 & 48.000 & 27.000 & 8.100 \\
\hline 00530 & $\begin{array}{l}\text { RESIDUE ON EVAPORATION AT } 105^{\circ} \mathrm{C} \text {, SUSPENDED } \\
(\mathrm{mg} / \mathrm{L})\end{array}$ & 21 & 608.000 & 4.000 & 79.667 & 576.800 & 75.000 & 18.000 & 13.500 & 4.300 \\
\hline 00535 & RESIDUE VOLATILE, SUSPENDED (mg/L) & 44 & 148.000 & $<1.000$ & $22.165^{\star}$ & 123.000 & 24.000 & 7.000 & 3.000 & $<1.000$ \\
\hline 70300 & $\begin{array}{l}\text { DISSOLVED SOLIDS, RESIDUE AT } 180^{\circ} \mathrm{C} \\
(\mathrm{mg} / \mathrm{L})\end{array}$ & 45 & 224.000 & 6.000 & 62.822 & 157.300 & 107.500 & 38.000 & 20.000 & 13.900 \\
\hline 00310 & $\begin{array}{l}5 \text { DAY BIOCHEMICAL OXYGEN DEMAND } \\
(\mathrm{mg} / \mathrm{L})\end{array}$ & 30 & 20.000 & $<2.000$ & $6.863 *$ & 15.000 & 9.000 & 5.800 & 3.600 & $<2.000$ \\
\hline 00340 & CHEMICAL OXYGEN DEMAND (mg/L) & 45 & 250.000 & $<5.000$ & $46.903 *$ & 170.000 & 51.000 & 29.000 & 20.000 & $<10.000$ \\
\hline \multicolumn{11}{|c|}{ NUTRIENTS, TOTAL AND DISSOLVED } \\
\hline 00625 & $\begin{array}{l}\text { NITROGEN AMMONIA + ORGANIC, } \\
\text { TOTAL (mg/L as N) }\end{array}$ & 47 & 9.800 & 0.300 & 1.368 & 5.300 & 1.500 & 0.830 & 0.600 & 0.304 \\
\hline 00631 & $\mathrm{NO}_{2}+\mathrm{NO}_{3}$, DISSOLVED $(\mathrm{mg} / \mathrm{L}$ as $\mathrm{N})$ & 47 & 1.800 & 0.100 & 0.615 & 1.566 & 1.100 & 0.390 & 0.230 & 0.122 \\
\hline 00608 & $\begin{array}{l}\text { NITROGEN AMMONIA， DISSOLVED } \\
(\mathrm{mg} / \mathrm{L} \text { as N) }\end{array}$ & 47 & 1.600 & 0.050 & 0.321 & 1.300 & 0.410 & 0.200 & 0.100 & 0.064 \\
\hline 00605 & NITROGEN ORGANIC, TOTAL (mg/L as N) & 47 & 9.000 & 0.160 & 1.049 & 3.820 & 1.200 & 0.650 & 0.390 & 0.176 \\
\hline 00600 & NITROGEN, TOTAL (mg/L as N) & 47 & 11.000 & 0.540 & 1.991 & 6.900 & 2.100 & 1.400 & 1.100 & 0.600 \\
\hline 00665 & PHOSPHORUS, TOTAL (mg/L as P) & 47 & 1.580 & 0.060 & 0.339 & 1.090 & 0.400 & 0.250 & 0.140 & 0.060 \\
\hline 00671 & $\begin{array}{l}\text { PHOSPHORUS ORTHO， DISSOLVED } \\
(\mathrm{mg} / \mathrm{L} \text { as P) }\end{array}$ & 47 & 0.980 & 0.010 & 0.185 & 0.784 & 0.230 & 0.120 & 0.050 & 0.024 \\
\hline \multicolumn{11}{|c|}{ OIL AND GREASE, TOTAL } \\
\hline 00556 & OIL AND GREASE， TOTAL (mg/L) & 16 & 5.000 & $<1.000$ & $1.257^{\star}$ & 5.000 & 2.000 & $<1.000$ & $<1.000$ & $<1.000$ \\
\hline \multicolumn{11}{|c|}{ ORGANIC CARBON, TOTAL } \\
\hline 00680 & CARBON ORGANIC, TOTAL (mg/L) & 29 & 82.000 & 2.000 & 18.066 & 77.000 & 18.500 & 11.000 & 7.000 & 2.450 \\
\hline & & & & IFORM & & & & & & \\
\hline 31679 & FECAL STREPTOCOCCI (COlonies per $100 \mathrm{~mL}$ ) & 24 & 105000.000 & 50.000 & 28364.584 & 101500.000 & 52000.000 & 16500.000 & 1725.000 & 165.000 \\
\hline 31616 & FECAL COLIFORM (Colonies per $100 \mathrm{~mL}$ ) & 24 & 420000.000 & 110.000 & 62146.668 & 390000.000 & 47000.000 & 26500.000 & 2450.000 & 150.000 \\
\hline & & ORC & GANIC COMPOUN & S-PESTICIDI & ES, TOTAL & & & & & \\
\hline 39330 & ALDRIN, TOTAL $(\mu \mathrm{g} / \mathrm{L})$ & 5 & $<0.010$ & $<0.010$ & -- & -- & -- & -- & -- & -- \\
\hline 39340 & LINDANE, TOTAL $(\mu \mathrm{g} / \mathrm{L})$ & 5 & 0.010 & $<0.010$ & -- & -- & -- & -- & -- & -- \\
\hline 39350 & CHLORDANE, TOTAL $(\mu \mathrm{g} / \mathrm{L})$ & 5 & 0.100 & $<0.100$ & -- & -- & -- & -- & -- & -- \\
\hline 39370 & DDT, TOTAL $(\mu \mathrm{g} / \mathrm{L})$ & 5 & 0.020 & $<0.010$ & -- & -- & -- & -. & -- & -- \\
\hline 39365 & DDE， TOTAL $(\mu \mathrm{g} / \mathrm{L})$ & 5 & $<0.010$ & $<0.010$ & -- & -- & -- & -- & -- & -- \\
\hline 39360 & DDD， TOTAL $(\mu \mathrm{g} / \mathrm{L})$ & 5 & $<0.010$ & $<0.010$ & -- & -- & -- & -- & -- & -- \\
\hline 39380 & DIELDRIN， TOTAL $(\mu \mathrm{g} / \mathrm{L})$ & 5 & 0.020 & $<0.010$ & -- & -- & -- & -- & -- & -- \\
\hline 39388 & ENDOSULFAN, TOTAL $(\mu \mathrm{g} / L)$ & 5 & $<0.010$ & $<0.010$ & -- & -- & -- & -- & -- & -- \\
\hline 39390 & ENDRIN， TOTAL $(\mu \mathrm{g} / \mathrm{L})$ & 5 & $<0.010$ & $<0.010$ & -- & -- & -- & -- & -- & - \\
\hline 39410 & HEPTACHLOR, TOTAL $(\mu \mathrm{g} / \mathrm{L})$ & 5 & $<0.010$ & $<0.010$ & -- & -- & -- & -- & -- & -- \\
\hline 39420 & HEPTACHLOR EPOXIDE, TOTAL $(\mu \mathrm{g} / \mathrm{L})$ & 5 & $<0.010$ & $<0.010$ & -- & -- & -- & -- & -- & -- \\
\hline 39516 & PCB， TOTAL $(\mu \mathrm{g} / \mathrm{L})$ & 5 & 0.100 & $<0.100$ & -- & -- & -- & -- & -- & -- \\
\hline 39400 & TOXAPHENE, TOTAL $(\mu \mathrm{g} / \mathrm{L})$ & 5 & $<1.000$ & $<1.000$ & -- & -- & -. & -- & -- & -- \\
\hline 39034 & PERTHANE， TOTAL $(\mu \mathrm{g} / \mathrm{L})$ & 5 & $<0.100$ & $<0.100$ & -- & -- & -- & -- & -- & -- \\
\hline 39570 & DIAZINON, TOTAL $(\mu \mathrm{g} / \mathrm{L})$ & 5 & 0.300 & $<0.010$ & -- & -- & -- & -- & -- & -- \\
\hline 39398 & ETHION, TOTAL $(\mu \mathrm{g} / \mathrm{L})$ & 5 & $<0.010$ & $<0.010$ & -- & -- & -- & .. & -- & -- \\
\hline 39530 & MALATHION, TOTAL $(\mu \mathrm{g} / L)$ & 5 & 0.100 & $<0.010$ & -- & -- & -- & -- & -- & -- \\
\hline 39600 & METHYL PARATHION, TOTAL $(\mu \mathrm{g} / \mathrm{L})$ & 5 & $<0.010$ & $<0.010$ & -- & -- & -- & -- & -- & -- \\
\hline 39540 & PARATHION, TOTAL $(\mu \mathrm{g} / \mathrm{L})$ & 5 & $<0.010$ & $<0.010$ & -- & -- & -- & -- & -- & -- \\
\hline 39786 & TRITHION, TOTAL $(\mu \mathrm{g} / L)$ & 5 & 0.010 & $<0.010$ & -- & -- & -- & -- & -- & -- \\
\hline 39250 & PCN, TOTAL $(\mu \mathrm{g} / \mathrm{L})$ & 5 & $<0.100$ & $<0.100$ & -- & -- & -- & -- & -- & -- \\
\hline 39480 & METHOXYCHLOR， TOTAL $(\mu \mathrm{g} / \mathrm{L})$ & 5 & $<0.010$ & $<0.010$ & -- & -- & -- & -- & -. & -- \\
\hline 39755 & MIREX, TOTAL $(\mu \mathrm{g} / \mathrm{L})$ & 5 & $<0.010$ & $<0.010$ & -- & -- & -- & -- & -- & -- \\
\hline 39011 & DISYSTON, TOTAL $(\mu \mathrm{g} / \mathrm{L})$ & 3 & $<0.010$ & $<0.010$ & -- & -- & -- & -- & -- & -- \\
\hline 39023 & PHORATE, TOTAL $(\mu \mathrm{g} / L)$ & 5 & $<0.010$ & $<0.010$ & -- & -- & -- & -- & -- & -- \\
\hline 38932 & CHLORPYRIFOS， TOTAL $(\mu \mathrm{g} / \mathrm{L})$ & 5 & 0.010 & $<0.010$ & -- & -- & -- & -- & -- & -- \\
\hline 39040 & DEF， TOTAL $(\mu \mathrm{g} / \mathrm{L})$ & 5 & $<0.010$ & $<0.010$ & -- & -- & -- & -- & -- & -- \\
\hline 82614 & FONOFOS, TOTAL $(\mu \mathrm{g} / \mathrm{L})$ & 5 & $<0.010$ & $<0.010$ & -- & -- & -- & -- & - & -- \\
\hline & & & OLATILE ORG & COMPOUND: & TOTAL & & & & & \\
\hline 34210 & ACROLEIN, TOTAL $(\mu \mathrm{g} / \mathrm{L})$ & 1 & $<20.000$ & -- & -- & -- & -- & -- & -- & -- \\
\hline 3421.5 & ACRYLONITRILE, TOTAL $(\mu \mathrm{g} / \mathrm{L})$ & 1 & $<20.000$ & -- & -- & -- & -- & -- & -- & -- \\
\hline 34030 & BENZENE, TOTAL $(\mu \mathrm{g} / L)$ & 12 & $<2.000$ & $<0.200$ & -- & -- & -- & -- & -- & -- \\
\hline 32104 & BROMOFORM, TOTAL $(\mu \mathrm{g} / \mathrm{L})$ & 12 & $<2.000$ & $<0.200$ & -- & -- & -- & -- & -- & -- \\
\hline 32102 & CARBON TETRACHLORIDE， TOTAL $(\mu \mathrm{g} / \mathrm{L})$ & 12 & $<2.000$ & $<0.200$ & -- & -- & -- & -- & -- & -- \\
\hline 34301 & CHLOROBENZENE， TOTAL $(\mu \mathrm{g} / \mathrm{L})$ & 12 & $<2.000$ & $<0.200$ & -- & -- & -- & -- & -- & -- \\
\hline 32105 & CHLORODIBROMOTHANE, TOTAL $(\mu \mathrm{g} / \mathrm{L})$ & 12 & $<2.000$ & $<0.200$ & -- & -- & -- & -- & -- & -- \\
\hline 34311 & CHLOROETHANE, TOTAL $(\mu \mathrm{g} / \mathrm{L})$ & 12 & $<2.000$ & $<0.200$ & -- & -- & -- & -- & -- & -- \\
\hline 32106 & CHLOROFORM, TOTAL $(\mu \mathrm{g} / \mathrm{L})$ & 12 & $<2.000$ & $<0.200$ & -- & -- & -- & -- & -- & - \\
\hline 34496 & 1,1 -DICHLOROETHANE, TOTAL $(\mu \mathrm{g} / \mathrm{L})$ & 12 & $<2.000$ & $<0.200$ & -- & -- & -- & -- & -- & -- \\
\hline 32103 & 1,2 -DICHLOROETHANE，TOTAL $(\mu \mathrm{g} / \mathrm{L})$ & 12 & $<2.000$ & $<0.200$ & -- & -- & -- & - & -- & -- \\
\hline
\end{tabular}

NOTE: Multiple detection limits during the period of record may result in different values flagged with a "<."

* Value is estimated by using a log-probability regression to predict the values of data below the detection limit. 
Table 71. Statistical summary of water-quality data at site 41 (CSW02), May 1994 through June 1997—Continued

\begin{tabular}{|c|c|c|c|c|c|c|c|c|c|c|}
\hline \multirow{2}{*}{$\begin{array}{c}\text { PARAM- } \\
\text { ETER } \\
\text { CODE }\end{array}$} & \multirow{2}{*}{ PROPERTY OR CONSTITUENT } & \multicolumn{4}{|c|}{ DESCRIPTIVE STATISTICS } & \multicolumn{5}{|c|}{$\begin{array}{l}\text { PERCENT OF SAMPLES IN WHICH VALUES WERE LESS THAN OR } \\
\text { EQUAL TO THOSE SHOWN }\end{array}$} \\
\hline & & $\begin{array}{l}\text { SAMPLE } \\
\text { SIZE }\end{array}$ & MAXIMUM & MINIMUM & MEAN & $95 \%$ & $75 \%$ & $\begin{array}{c}50 \% \\
\text { (median) }\end{array}$ & $25 \%$ & $5 \%$ \\
\hline 34501 & 1,1-DICHLOROETHYLENE，TOTAL $(\mu \mathrm{g} / \mathrm{L})$ & $\overline{12}$ & $<2.000$ & $<0.200$ & 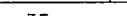 & -- & - & -- & $=$ & -- \\
\hline 34371 & ETHYLBENZENE, TOTAL $(\mu \mathrm{g} / \mathrm{L})$ & 12 & $<2.000$ & $<0.200$ & -- & - & -- & -- & -. & -- \\
\hline 34413 & METHYL BROMIDE, TOTAL $(\mu \mathrm{g} / \mathrm{L})$ & 12 & $<2.000$ & $<0.200$ & -- & -- & -. & -- & -- & -- \\
\hline 34423 & METHYLENE CHLORIDE, TOTAL $(\mu \mathrm{g} / \mathrm{L})$ & 12 & $<2.000$ & $<0.200$ & -- & -- & $\ldots$ & -- & $\ldots$ & -- \\
\hline 34516 & $1,1,2,2$-TETRACHLOROETHANE, TOTAL $(\mu \mathrm{g} / \mathrm{L})$ & 12 & $<2.000$ & $<0.200$ & -- & -- & -- & -. & -- & - \\
\hline 34475 & TETRACHLOROETHYLENE, TOTAL $(\mu \mathrm{g} / \mathrm{L})$ & 12 & 1.300 & $<0.400$ & $0.744^{*}$ & 1.300 & 0.900 & 0.600 & $<0.400$ & $<0.400$ \\
\hline 34546 & $\begin{array}{l}\text { 1, } 2 \text {-TRANSDICHLOROETHENE, TOTAL } \\
(\mu \mathrm{g} / \mathrm{L})\end{array}$ & 12 & $<2.000$ & $<0.200$ & -- & - & -- & -- & -- & -- \\
\hline 34506 & $1,1,1$-TRICHLOROETHANE，TOTAL $(\mu \mathrm{g} / \mathrm{L})$ & 12 & $<2.000$ & $<0.200$ & -- & -. & -- & -- & -- & -- \\
\hline 34511 & $1,1,2$-TRICHLOROETHANE, TOTAL $(\mu \mathrm{g} / \mathrm{L})$ & 12 & $<2.000$ & $<0.200$ & -. & -- & .. & .. & -. & -. \\
\hline 39180 & TRICHLOROETHYLENE，TOTAL $(\mu \mathrm{g} / \mathrm{L})$ & 12 & $<2.000$ & $<0.200$ & -- & - & -- & -- & - & -- \\
\hline 39175 & VINYL CHLORIDE, TOTAL $(\mu \mathrm{g} / \mathrm{L})$ & 12 & $<2.000$ & $<0.200$ & -_ & -- & _- & $\ldots$ & -. & .. \\
\hline 30217 & DIBROMOMETHANE, TOTAL $(\mu \mathrm{g} / \mathrm{L})$ & 12 & $<2.000$ & $<0.200$ & -- & -- & -- & -- & -- & -- \\
\hline 32101 & DICHLOROBROMOMETHANE, TOTAL $(\mu \mathrm{g} / \mathrm{L})$ & 12 & $<2.000$ & $<0.200$ & -- & -- & -. & .. & -. & -. \\
\hline 34668 & $\begin{array}{l}\text { DICHLORODIFLUOROMETHANE，TOTAL } \\
(\mu \mathrm{g} / \mathrm{L})\end{array}$ & 12 & $<2.000$ & $<0.200$ & -- & -- & -- & -- & -- & -- \\
\hline 77651 & 1,2-DIBROMOETHANE, TOTAL $(\mu \mathrm{g} / \mathrm{L})$ & 12 & $<2.000$ & $<0.200$ & -- & -- & -- & -- & -- & -- \\
\hline 34418 & METHYLCHLORIDE, TOTAL $(\mu \mathrm{g} / \mathrm{L})$ & 12 & $<2.000$ & $<0.200$ & -- & -- & - & -- & - & $\ldots$ \\
\hline 34704 & $\begin{array}{l}\text { CIS } 1,3 \text {-DICHLOROPROPENE, TOTAL } \\
(\mu \mathrm{g} / \mathrm{L})\end{array}$ & 12 & $<2.000$ & $<0.200$ & -- & - & - & -- & -- & -- \\
\hline 34699 & TRANS 1,3 -DICHLOROPROPENE, TOTAL $(\mu \mathrm{g} / \mathrm{L})$ & 12 & $<2.000$ & $<0.200$ & -- & -- & -- & -. & -- & -. \\
\hline 77128 & STYRENE, TOTAL $(\mu \mathrm{g} / \mathrm{L})$ & 12 & $<2.000$ & $<0.200$ & -- & -- & -- & -- & -- & -- \\
\hline 81551 & XYLENE, TOTAL $(\mu \mathrm{g} / \mathrm{L})$ & 12 & $<2.000$ & $<0.200$ & -- & -- & -- & -- & -- & - \\
\hline 82625 & DIBROMOCHLOROPROPANE，TOTAL $(\mu \mathrm{g} / \mathrm{L})$ & 12 & $<10.000$ & $<1.000$ & _- & -- & .. & _- & -. & -- \\
\hline 77168 & 1,1-DICHLOROPROPENE, TOTAL $(\mu \mathrm{g} / \mathrm{L})$ & 12 & $<2.000$ & $<0.200$ & -- & -. & .. & $\ldots$ & -- & .- \\
\hline 77170 & 2,2-DICHLOROPROPANE, TOTAL $(\mu \mathrm{g} / \mathrm{L})$ & 12 & $<2.000$ & $<0.200$ & -- & -- & -- & -- & -- & -- \\
\hline 77173 & 1,3-DICHLOROPROPANE, TOTAL $(\mu \mathrm{g} / \mathrm{L})$ & 12 & $<2.000$ & $<0.200$ & -- & -- & -. & -- & -- & -. \\
\hline 77275 & 0 -CHLOROTOLUENE, TOTAL $(\mu \mathrm{g} / \mathrm{L})$ & 12 & $<2.000$ & $<0.200$ & -. & -. & -- & -- & -. & -. \\
\hline 77277 & P-CHLOROTOLUENE, TOTAL $(\mu \mathrm{g} / \mathrm{L})$ & 12 & $<2.000$ & $<0.200$ & -- & -- & -. & -- & -. & -. \\
\hline 77443 & 123-TRICHLOROPROPANE, TOTAL $(\mu \mathrm{g} / \mathrm{L})$ & 12 & $<2.000$ & $<0.200$ & -- & -- & $-\ldots$ & -- & - & - \\
\hline 77562 & 1112 -TETRACHLOROETHANE, TOTAL $(\mu \mathrm{g} / \mathrm{L})$ & 12 & $<2.000$ & $<0.200$ & -. & -- & -. & -. & -. & -. \\
\hline 78032 & TERTBUTYL METHYL ETHER, TOTAL $(\mu \mathrm{g} / \mathrm{L})$ & 12 & $<2.000$ & $<0.200$ & -- & -- & .. & .. & -- & -- \\
\hline 77297 & BROMOCHLORO METHANE, TOTAL $(\mu \mathrm{g} / \mathrm{L})$ & 12 & $<2.000$ & $<0.200$ & -. & -. & -- & -- & -- & -- \\
\hline 77093 & CIS-1,2-DICHLOROETHENE, TOTAL $(\mu \mathrm{g} / \mathrm{L})$ & 12 & $<2.000$ & $<0.200$ & -- & -. & -- & -- & -. & -- \\
\hline 34576 & 2-CHLOROETHYL VINYL ETHER, TOTAL $(\mu \mathrm{g} / \mathrm{L})$ & 8 & $<10.000$ & $<1.000$ & -. & - & -- & -. & -- & $\ldots$ \\
\hline 77223 & ISOPROPYL BENZENE, TOTAL $(\mu \mathrm{g} / \mathrm{L})$ & 12 & $<2.000$ & $<0.200$ & .. & .. & -. & -. & -- & -. \\
\hline 77224 & N-PROPYL BENZENE, TOTAL $(\mu \mathrm{g} / \mathrm{L})$ & 12 & 0.600 & $<0.200$ & -- & 0.600 & $<0.800$ & $<0.400$ & $<0.200$ & $<0.200$ \\
\hline 77350 & SEC-BUTYL BENZENE, TOTAL $(\mu \mathrm{g} / \mathrm{L})$ & 12 & $<2.000$ & $<0.200$ & - & - & $\ldots$ & -- & - & $\ldots$ \\
\hline 77356 & P-ISOPROPYL TOLUENE, TOTAL $(\mu \mathrm{g} / \mathrm{L})$ & 12 & $<2.000$ & $<0.200$ & -- & -- & -- & -- & -- & -- \\
\hline 77342 & N-BUTYL BENZENE, TOTAL $(\mu \mathrm{g} / \mathrm{L}$ & 12 & $<2.000$ & $<0.200$ & -- & -- & - & -- & -. & -. \\
\hline 77613 & $1,2,3$-TRICHLOROBENZENE, TOTAL $(\mu \mathrm{g} / \mathrm{L}$ & 12 & $<2.000$ & $<0.200$ & -. & .. & -- & -- & -- & -- \\
\hline 77652 & FREON-113, TOTAL $(\mu \mathrm{g} / \mathrm{L}$ & 12 & $<2.000$ & $<0.200$ & -- & -- & -- & -- & -- & -- \\
\hline 77226 & MESITYLENE, TOTAL $(\mu \mathrm{g} / \mathrm{L}$ & 12 & 1.800 & $<0.200$ & _. & 1.800 & $<0.800$ & $<0.400$ & $<0.200$ & $<0.200$ \\
\hline 81555 & BROMOBENZENE，TOTAL $(\mu \mathrm{g} / \mathrm{L}$ & 12 & $<2.000$ & $<0.200$ & -- & -- & -- & -- & - & -- \\
\hline & & $\mathrm{ME}^{\prime}$ & LS AND MIN & ONSTITUED & S, TOTAL & & & & & \\
\hline 01097 & ANTIMONY, TOTAL $(\mu \mathrm{g} / \mathrm{L}$ as $\mathrm{Sb})$ & 31 & 5.000 & $<1.000$ & -+ & 1.000 & $<1.000$ & $<1.000$ & $<1.000$ & $<1.000$ \\
\hline 01002 & ARSENIC, TOTAL $(\mu \mathrm{g} / \mathrm{L}$ as AS) & 31 & 2.000 & $<1.000$ & -- & 2.000 & $<1.000$ & $<1.000$ & $<1.000$ & $<1.000$ \\
\hline 01012 & BERYLLIUM, TOTAL $(\mu \mathrm{g} / \mathrm{L}$ as $\mathrm{Be})$ & 12 & $<10.000$ & $<10.000$ & -- & -- & -- & -- & -- & -- \\
\hline 01027 & CADMIUM, TOTAL ( $\mu \mathrm{g} / \mathrm{L}$ as $\mathrm{Cd})$ & 12 & $<1.000$ & $<1.000$ & .. & -- & -- & -- & -- & -- \\
\hline 01034 & CHROMIUM, TOTAL $(\mu \mathrm{g} / \mathrm{L}$ as $\mathrm{Cr})$ & 31 & 26.000 & $<1.000$ & $5.356^{\star}$ & 15.000 & 7.000 & 4.000 & 2.000 & $<1.000$ \\
\hline 01042 & COPPER, TOTAL $(\mu \mathrm{g} / \mathrm{L}$ as $\mathrm{Cu})$ & 31 & 49.000 & $<1.000$ & 10.364 * & 25.000 & 13.000 & 8.000 & 4.000 & $<1.000$ \\
\hline 01051 & LEAD, TOTAL $(\mu \mathrm{g} / \mathrm{L}$ as $\mathrm{Pb})$ & 31 & 89.000 & 1.000 & 17.323 & 80.000 & 23.000 & 11.000 & 6.000 & 1.000 \\
\hline 71900 & MERCURY, TOTAL $(\mu \mathrm{g} / \mathrm{L}$ as $\mathrm{Hg})$ & 12 & 0.100 & $<0.100$ & -- & 0.100 & $<0.100$ & $<0.100$ & $<0.100$ & $<0.100$ \\
\hline 01067 & NICKEL, TOTAL $(\mu \mathrm{g} / \mathrm{L}$ as $\mathrm{Ni})$ & 31 & 14.000 & $<1.000$ & $3.055^{\star}$ & 8.000 & 3.000 & 2.000 & 1.000 & $<1.000$ \\
\hline 01147 & SELENIUM, TOTAL $(\mu \mathrm{g} / \mathrm{L}$ as $\mathrm{Se})$ & 12 & 1.000 & $<1.000$ & -- & 1.000 & $<1.000$ & $<1.000$ & $<1.000$ & $<1.000$ \\
\hline 01077 & SILVER, TOTAL $(\mu \mathrm{g} / \mathrm{L}$ as $\mathrm{Ag})$ & 12 & $<1.000$ & $<1.000$ & -- & -- & -- & -. & $\ldots$ & -. \\
\hline 01092 & $\mathrm{ZINC}, \operatorname{TOTAL}(\mu \mathrm{g} / \mathrm{L}$ as $\mathrm{zn})$ & 31 & 380.000 & 10.000 & 102.903 & 302.000 & 140.000 & 80.000 & 50.000 & 16.000 \\
\hline 00720 & CYANIDE, TOTAL (mg/L as $\mathrm{Cn}$ ) & 12 & $<0.010$ & $<0.010$ & -- & $\ldots$ & -- & -- & - & -- \\
\hline & & ORGAN & C COMPOUNDS & PESTICIDES, & DISSOLVED & & & & & \\
\hline 46342 & ALACHLOR， DISSOLVED $(\mu \mathrm{g} / \mathrm{L})$ & 4 & $<0.009$ & $<0.002$ & -- & - & -- & -- & -- & -- \\
\hline 04040 & DEETHYL ATRAZINE， DISSOLVED $(\mu \mathrm{g} / \mathrm{L})$ & 4 & $<0.005$ & $<0.002$ & -- & -- & -- & -- & - & $\ldots$ \\
\hline 39632 & ATRAZINE， DISSOLVED $(\mu \mathrm{g} / \mathrm{L})$ & 4 & 0.054 & $<0.020$ & -- & -- & $\ldots$ & -- & -- & -. \\
\hline 82686 & METHYL AZINPHOS, DISSOLVED $(\mu \mathrm{g} / \mathrm{L})$ & 4 & $<0.050$ & $<0.001$ & -- & -. & -- & -- & -. & -. \\
\hline 82673 & BENFLURALIN, DISSOLVED $(\mu \mathrm{g} / L)$ & 4 & 0.079 & $<0.002$ & -- & -. & -- & -- & -- & -- \\
\hline 04028 & BUTYLATE, DISSOLVED $(\mu \mathrm{g} / \mathrm{L})$ & 4 & $<0.008$ & $<0.002$ & -- & -- & - & -- & -- & -- \\
\hline 82680 & CARBARYL, DISSOLVED $(\mu \mathrm{g} / \mathrm{L})$ & 4 & 0.155 & $<0.003$ & -- & -- & -- & -- & -- & -- \\
\hline 82674 & CARBOFURAN， DISSOLVED $(\mu \mathrm{g} / \mathrm{L})$ & 4 & $<0.010$ & $<0.003$ & -- & -- & -- & -- & $\ldots$ & -. \\
\hline 38933 & CHLORPYRIFOS, DISSOLVED $(\mu \mathrm{g} / \mathrm{L})$ & 4 & $<0.008$ & $<0.004$ & -- & -- & -- & -- & - & -- \\
\hline 04041 & CYANAZINE, DISSOLVED $(\mu \mathrm{g} / \mathrm{L})$ & 4 & $<0.010$ & $<0.004$ & -. & -. & -- & -- & -- & -- \\
\hline 82682 & DCPA， DISSOLVED $(\mu \mathrm{g} / \mathrm{L})$ & 4 & 0.002 & $<0.002$ & - & -- & - & -- & -- & - \\
\hline 34653 & P, $P^{\prime}$ DDE， DISSOLVED $(\mu \mathrm{g} / \mathrm{L})$ & 4 & $<0.010$ & $<0.006$ & -- & -- & -- & -- & -- & -- \\
\hline 39572 & DIAZINON, DISSOLVED $(\mu \mathrm{g} / \mathrm{L})$ & 4 & 0.348 & $<0.002$ & -- & -- & -- & -- & -- & -- \\
\hline 39381 & DIELDRIN, DISSOLVED $(\mu \mathrm{g} / \mathrm{L})$ & 4 & 0.010 & $<0.001$ & -- & .- & -. & -- & -. & -- \\
\hline 82660 & $\begin{array}{l}\text { 2, 6-DIETHYL ANILINE, DISSOLVED } \\
(\mu \mathrm{g} / \mathrm{L})\end{array}$ & 4 & $<0.006$ & $<0.003$ & - & - & -- & -- & -- & -- \\
\hline 82662 & DIMETHOATE， DISSOLVED $(\mu \mathrm{g} / \mathrm{L})$ & 1 & $<0.020$ & -- & -. & _. & -- & -- & -- & -- \\
\hline
\end{tabular}

NOTE: Multiple detection limits during the period of record may result in different values flagged with a "<."

* Value is estimated by using a log-probability regression to predict the values of data below the detection limit. 
Table 71. Statistical summary of water-quality data at site 41 (CSW02), May 1994 through June 1997-Continued

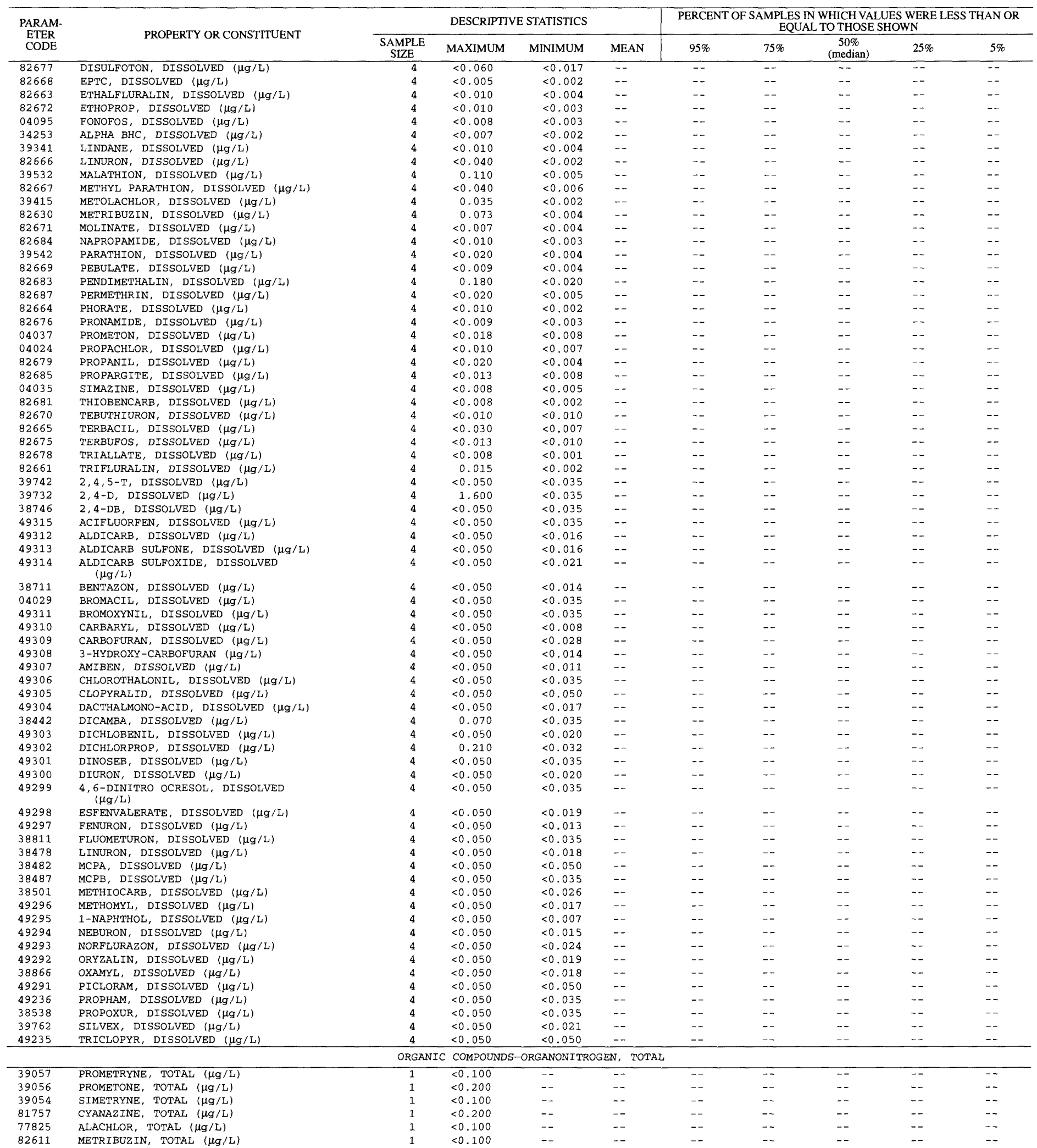

NOTE: Multiple detection limits during the period of record may result in different values flagged with a "<."

* Value is estimated by using a log-probability regression to predict the values of data below the detection limit. 
Table 71. Statistical summary of water-quality data at site 41 (CSW02), May 1994 through June 1997-Continued

\begin{tabular}{|c|c|c|c|c|c|c|c|c|c|c|}
\hline \multirow{2}{*}{$\begin{array}{l}\text { PARAM- } \\
\text { ETER } \\
\text { CODE }\end{array}$} & \multirow[b]{2}{*}{ PROPERTY OR CONSTITUENT } & \multicolumn{4}{|c|}{ DESCRIPTIVE STATISTICS } & \multicolumn{5}{|c|}{$\begin{array}{l}\text { PERCENT OF SAMPLES IN WHICH VALUES WERE LESS THAN OR } \\
\text { EQUAL TO THOSE SHOWN }\end{array}$} \\
\hline & & $\begin{array}{l}\text { SAMPLE } \\
\text { SIZE }\end{array}$ & MAXIMUM & MINIMUM & MEAN & $95 \%$ & $75 \%$ & $\begin{array}{c}50 \% \\
\text { (median) }\end{array}$ & $25 \%$ & $5 \%$ \\
\hline 30311 & TERBACIL， TOTAL $(\overline{\mu \mathrm{g} / L})$ & 1 & $<0.200$ & -- & -- & -- & -- & -- & -- & -- \\
\hline 30245 & CARBOXIN, TOTAL $(\mu \mathrm{g} / \mathrm{L})$ & 1 & $<0.200$ & -- & -- & -- & -- & -- & -- & -- \\
\hline 30264 & HEXAZINONE, TOTAL $(\mu \mathrm{g} / \mathrm{L})$ & 1 & $<0.200$ & - & -- & -- & -- & -- & -- & -- \\
\hline 30235 & BUTACHLOR, TOTAL $(\mu \mathrm{g} / \mathrm{L})$ & 1 & $<0.100$ & -. & -- & -- & -- & -- & -- & -- \\
\hline 30236 & BUTYLATE, TOTAL $(\mu \mathrm{g} / \mathrm{L})$ & 1 & $<0.100$ & -- & -- & -- & -- & -- & -- & -- \\
\hline 75981 & DE-ETHYLATRAZINE, TOTAL $(\mu \mathrm{g} / \mathrm{L})$ & 1 & $<0.200$ & -- & -- & -- & - & -- & -- & -- \\
\hline 39630 & ATRAZINE, TOTAL $(\mu \mathrm{g} / \mathrm{L})$ & 1 & $<0.100$ & -. & -- & -- & -- & -- & -- & -- \\
\hline 39055 & SIMAZINE, TOTAL $(\mu \mathrm{g} / \mathrm{L})$ & 1 & $<0.100$ & -- & -- & -- & -- & - & -- & -- \\
\hline 39024 & PROPAZINE, TOTAL $(\mu \mathrm{g} / \mathrm{L})$ & 1 & $<0.100$ & -. & -- & -- & -- & -. & -- & -- \\
\hline 82184 & AMETRYNE，TOTAL $(\mu \mathrm{g} / \mathrm{L})$ & 1 & $<0.100$ & -. & -- & -- & -- & -- & -- & -- \\
\hline 39030 & TRIFLURALIN, TOTAL $(\mu \mathrm{g} / \mathrm{L})$ & 1 & $<0.100$ & -- & -- & -- & -- & -- & -- & - \\
\hline 82612 & METOLACHLOR, TOTAL $(\mu \mathrm{g} / \mathrm{L})$ & 1 & $<0.200$ & -. & -- & -- & -- & -- & -- & -- \\
\hline 30234 & BROMACIL, TOTAL $(\mu \mathrm{g} / \mathrm{L})$ & 1 & 0.300 &.- & -- & -- & -- & -- & -- & -- \\
\hline 30255 & DIPHENAMID, TOTAL $(\mu \mathrm{g} / \mathrm{L})$ & 1 & $<0.100$ & .. & -- & -- & -- & -- & -- & -- \\
\hline 30324 & VERNOLATE, TOTAL $(\mu \mathrm{g} / \mathrm{L})$ & 1 & $<0.100$ & -- & -- & -- & -- & -- & -- & -- \\
\hline 30254 & CYCLOATE, TOTAL $(\mu \mathrm{g} / \mathrm{L})$ & 1 & $<0.100$ & - & -- & -- & -- & -- & -- & -- \\
\hline 30295 & PROPACHLOR, TOTAL $(\mu \mathrm{g} / \mathrm{L})$ & 1 & 0.100 & -. & -- & -- & -- & -. & -- & -. \\
\hline 75980 & DE-ISOPROPYLATRAZIN, TOTAL $(\mu \mathrm{g} / \mathrm{L})$ & 1 & $<0.200$ & -- & -- & -- & - & -- & -- & -- \\
\hline \multicolumn{11}{|c|}{ ORGANIC COMPOUNDS-HERBICIDES, TOTAL } \\
\hline 39730 & $2,4-\mathrm{D}$, TOTAL $(\mu \mathrm{g} / \mathrm{L})$ & 1 & 2.400 & $-\infty$ & -- & -- & -- & -- & -- & -- \\
\hline 39760 & SILVEX, TOTAL $(\mu \mathrm{g} / \mathrm{L})$ & 1 & $<0.010$ & -- & -. & -- & -- & -. & -- & -- \\
\hline 39720 & PICLORAM, TOTAL $(\mu \mathrm{g} / \mathrm{L})$ & 1 & $<0.010$ & -. & -- & -- & -- & -. & -- & -- \\
\hline 39740 & $2,4,5-\mathrm{T}$, TOTAL $(\mu \mathrm{g} / \mathrm{L})$ & 1 & $<0.010$ & -- & -- & -- & -- & -. & -- & -- \\
\hline 82183 & $2,4-\mathrm{DP}$, TOTAL $(\mu \mathrm{g} / \mathrm{L})$ & 1 & $<0.010$ & -_ & -. & -- & -- & -- & -- & -- \\
\hline 82052 & DICAMBA, TOTAL $(\mu \mathrm{g} / \mathrm{L})$ & 1 & 0.380 & -- & -- & -- & -- & -- & -- & -- \\
\hline \multicolumn{11}{|c|}{ ORGANIC COMPOUNDS-CARBAMATE PESTICIDES, TOTAL } \\
\hline 39750 & SEVIN, TOTAL $(\mu \mathrm{g} / \mathrm{L})$ & 1 & $<0.500$ & -- & -- & -- & -- & - & -- & -- \\
\hline 39051 & METHOMYL， TOTAL $(\mu \mathrm{g} / \mathrm{L})$ & 1 & $<0.500$ & -- & -- & -- & - - & - & -- & -- \\
\hline 82619 & ALDICARD, TOTAL $(\mu \mathrm{g} / \mathrm{L})$ & 1 & $<0.500$ & -. & -- & -- & -- & -- & -- & -- \\
\hline 30296 & PROPOXUR, TOTAL $(\mu \mathrm{g} / \mathrm{L})$ & 1 & $<0.500$ & -- & -- & -. & -- & -- & -- & -- \\
\hline 39052 & PROPHAM, TOTAL $(\mu \mathrm{g} / \mathrm{L})$ & 1 & $<0.500$ & -- & -- & -. & -- & -- & -- & -- \\
\hline 82615 & CARBOFURAN, TOTAL $(\mu \mathrm{g} / \mathrm{L})$ & 1 & $<0.500$ & -- & -- & -. & -- & -- & -- & -- \\
\hline 77441 & 1-NAPHTHOL, TOTAL $(\mu \mathrm{g} / \mathrm{L})$ & 1 & $<0.500$ & -- & -- & -- & -- & -- & -. & -- \\
\hline 30282 & METHIOCARB， TOTAL $(\mu \mathrm{g} / \mathrm{L})$ & 1 & $<0.500$ & -- & -- & -. & -- & -. & -- & -- \\
\hline
\end{tabular}

NOTE: Multiple detection limits during the period of record may result in different values flagged with a "<."

* Value is estimated by using a log-probability regression to predict the values of data below the detection limit 
Table 72. Statistical summary of water-quality data at site 42 (CSWO4), May 1994 through June 1997

\begin{tabular}{|c|c|c|c|c|c|c|c|c|c|c|}
\hline \multirow{2}{*}{$\begin{array}{l}\text { PARAM- } \\
\text { ETER } \\
\text { CODE }\end{array}$} & \multirow[b]{2}{*}{ PROPERTY OR CONSTITUENT } & \multicolumn{4}{|c|}{ DESCRIPTIVE STATISTICS } & \multicolumn{5}{|c|}{$\begin{array}{c}\text { PERCENT OF SAMPLES IN WHICH VALUES WERE LESS THAN OR } \\
\text { EQUAL TO THOSE SHOWN }\end{array}$} \\
\hline & & $\begin{array}{l}\text { SAMPLE } \\
\text { SIZE }\end{array}$ & MAXIMUM & MINIMUM & MEAN & $95 \%$ & $75 \%$ & $\begin{array}{c}50 \% \\
\text { (median) }\end{array}$ & $25 \%$ & $5 \%$ \\
\hline \multicolumn{11}{|c|}{ PHYSICAL AND CHEMICAL PROPERTIES } \\
\hline 00061 & INSTANTANEOUS DISCHARGE, $\left(\mathrm{ft}^{3} / \mathrm{s}\right)$ & 74 & 221.000 & 0.020 & 11.270 & 50.000 & 12.400 & 2.000 & 0.723 & 0.115 \\
\hline 00010 & WATER TEMPERATURE $\left({ }^{\circ} \mathrm{C}\right)$ & 66 & 25.000 & 2.500 & 15.205 & 24.150 & 20.000 & 15.750 & 10.750 & 3.500 \\
\hline 90095 & SPECIFIC CONDUCTANCE, LAB $\left(\mu \mathrm{S} / \mathrm{cm}\right.$ at $\left.25{ }^{\circ} \mathrm{C}\right)$ & 44 & 550.000 & 26.000 & 123.182 & 424.250 & 143.750 & 78.500 & 45.500 & 31.000 \\
\hline 00095 & $\begin{array}{l}\text { SPECIFIC CONDUCTANCE, FIELD ( } \mu \mathrm{S} / \mathrm{cm} \text { at } 25 \\
\left.{ }^{\circ} \mathrm{C}\right)\end{array}$ & 50 & 425.000 & 19.000 & 109.980 & 365.750 & 135.500 & 68.500 & 49.750 & 28.100 \\
\hline 00403 & pH, LAB (STANDARD pH UNITS) & 44 & 7.600 & 5.700 & 6.736 & 7.575 & 7.000 & 6.750 & 6.400 & 6.025 \\
\hline 00400 & pH，FIELD (STANDARD pH UNITS) & 50 & 7.710 & 6.210 & 6.917 & 7.359 & 7.043 & 6.885 & 6.795 & 6.51 .6 \\
\hline 90410 & ALKALINITY, LAB (mg/L as $\left.\mathrm{CaCO}_{3}\right)$ & 44 & 133.000 & 6.500 & 27.739 & 119.750 & 32.750 & 14.000 & 10.250 & 7.250 \\
\hline 80154 & SUSPENDED SEDIMENT $(\mathrm{mg} / \mathrm{L})$ & 47 & 1500.000 & 4.000 & 241.170 & 1158.799 & 235.000 & 103.000 & 62.000 & 25.800 \\
\hline 00530 & $\begin{array}{l}\text { RESIDUE ON EVAPORATION AT } 105^{\circ} \mathrm{C} \text {, SUSPENDED } \\
(\mathrm{mg} / \mathrm{L})\end{array}$ & 19 & 776.000 & 1.000 & 130.632 & 776.000 & 104.000 & 74.000 & 46.000 & 1.000 \\
\hline 00535 & RESIDUE VOLATILE, SUSPENDED (mg/L) & 42 & 172.000 & 1.000 & 25.905 & 117.850 & 26.500 & 13.000 & 8.000 & 1.150 \\
\hline 70300 & $\begin{array}{l}\text { DISSOLVED SOLIDS, RESIDUE AT } 180^{\circ} \mathrm{C} \\
(\mathrm{mg} / \mathrm{L})\end{array}$ & 44 & 340.000 & 1.000 & 86.000 & 254.000 & 103.750 & 67.500 & 38.500 & 16.000 \\
\hline 00310 & $\begin{array}{l}5 \text { DAY BIOCHEMICAL OXYGEN DEMAND } \\
(\mathrm{mg} / \mathrm{L})\end{array}$ & 29 & 31.000 & $<2.000$ & 10.457 * & 30.000 & 15.000 & 7.500 & 5.200 & $<2.000$ \\
\hline 00340 & CHEMICAL OXYGEN DEMAND (mg/L) & 44 & 220.000 & 5.000 & 59.773 & 177.500 & 81.750 & 42.000 & 30.000 & 15.500 \\
\hline \multicolumn{11}{|c|}{ NUTRIENTS, TOTAL AND DISSOLVED } \\
\hline 00625 & $\begin{array}{l}\text { NITROGEN AMMONIA + ORGANIC, } \\
\text { TOTAL }(\mathrm{mg} / \mathrm{L} \text { as N) }\end{array}$ & 45 & 11.000 & 0.400 & 2.202 & 7.740 & 2.000 & 1.400 & 1.000 & 0.617 \\
\hline 00631 & $\mathrm{NO}_{2}+\mathrm{NO}_{3}$, DISSOLVED (mg/L as $\left.\mathrm{N}\right)$ & 44 & 1.700 & 0.180 & 0.595 & 1.560 & 0.780 & 0.505 & 0.305 & 0.183 \\
\hline 00508 & $\begin{array}{l}\text { NITROGEN AMMONIA, DISSOLVED } \\
(\mathrm{mg} / \mathrm{L} \text { as N) }\end{array}$ & 45 & 5.200 & 0.015 & 0.499 & 2.510 & 0.460 & 0.250 & 0.140 & 0.029 \\
\hline 00605 & NITROGEN ORGANIC, TOTAL (mg/L as N) & 45 & 5.800 & 0.250 & 1.696 & 5.350 & 1.650 & 1.200 & 0.890 & 0.531 \\
\hline 00600 & NITROGEN, TOTAL (mg/L as N) & 45 & 12.000 & 0.930 & 2.776 & 9.340 & 2.600 & 2.100 & 1.400 & 1.000 \\
\hline 00665 & PHOSPHORUS, TOTAL (mg/L as P) & 45 & 4.600 & 0.060 & 0.550 & 1.430 & 0.575 & 0.390 & 0.235 & 0.066 \\
\hline 00671 & $\begin{array}{l}\text { PHOSPHORUS ORTHO, DISSOLVED } \\
(\mathrm{mg} / \mathrm{L} \text { aS } \mathrm{P})\end{array}$ & 45 & 4.800 & 0.010 & 0.361 & 1.276 & 0.380 & 0.150 & 0.080 & 0.020 \\
\hline \multicolumn{11}{|c|}{ OIL AND GREASE, TOTAI. } \\
\hline 00556 & OIL AND GREASE, TOTAL $(\mathrm{mg} / \mathrm{L})$ & 16 & 3.000 & $<1.000$ & $1.278^{\star}$ & 3.000 & 2.000 & 1.000 & $<1.000$ & $<1.000$ \\
\hline \multicolumn{11}{|c|}{ ORGANIC CARBON, TOTAL } \\
\hline 00680 & CARBON ORGANIC, TOTAL $(\mathrm{mg} / \mathrm{L})$ & 27 & 49.000 & 7.600 & 19.033 & 47.000 & 26.000 & 14.000 & 11.000 & 8.200 \\
\hline & & & & UIFORM & & & & & & \\
\hline 31679 & FECAL STREPTOCOCCI (Colonies per $100 \mathrm{~mL}$ ) & 29 & 540000.000 & 2400.000 & 66520.688 & 420000.000 & 55500.000 & 22000.000 & 5300.000 & 2450.000 \\
\hline 31616 & FECAL COLIFORM (Colonies per $100 \mathrm{~mL}$ ) & 29 & 700000.000 & 630.000 & 67343.445 & 505000.000 & 67500.000 & 15000.000 & 2400.000 & 675.000 \\
\hline & & ORC & GANIC COMPOUN & S-PESTICID & DES, TOTAL & & & & & \\
\hline 39330 & ALDRIN, TOTAL $(\mu \mathrm{g} / \mathrm{L})$ & 4 & 0.040 & $<0.010$ & $\overline{--}$ & -- & + & -- & -- & -- \\
\hline 39340 & LINDANE， TOTAL $(\mu \mathrm{g} / \mathrm{L})$ & 4 & 0.010 & $<0.010$ & -- & -- & -- & -- & -- & -- \\
\hline 39350 & CHLORDANE, TOTAL $(\mu \mathrm{g} / \mathrm{L})$ & 4 & $<0.100$ & $<0.100$ & -- & -- & -- & -- & -- & -- \\
\hline 39370 & DDT， TOTAL $(\mu \mathrm{g} / \mathrm{L})$ & 4 & $<0.010$ & $<0.010$ & -- & -- & -- & -- & -- & -- \\
\hline 39365 & DDE, TOTAL $(\mu \mathrm{g} / \mathrm{L})$ & 4 & $<0.010$ & $<0.010$ & -- & -- & -- & -- & -- & -- \\
\hline 39360 & DDD, TOTAL $(\mu \mathrm{g} / \mathrm{L})$ & 4 & $<0.010$ & $<0.010$ & -- & -- & -- & -- & -- & -- \\
\hline 39380 & DIELDRIN, TOTAL $(\mu \mathrm{g} / \mathrm{L})$ & 4 & $<0.010$ & $<0.010$ & -- & -- & -- & -- & -- & -- \\
\hline 39388 & ENDOSULFAN, TOTAL $(\mu \mathrm{g} / \mathrm{L})$ & 4 & $<0.010$ & $<0.010$ & -- & -- & -- & -- & -. & -- \\
\hline 39390 & ENDRIN, TOTAL $(\mu \mathrm{g} / \mathrm{L})$ & 4 & $<0.010$ & $<0.010$ & -- & -- & -- & -- & -- & -- \\
\hline 39410 & HEPTACHLOR, TOTAL $(\mu \mathrm{g} / \mathrm{L})$ & 4 & $<0.010$ & $<0.010$ & -- & -- & -- & -- & -- & -- \\
\hline 39420 & HEPTACHLOR EPOXIDE, TOTAL $(\mu \mathrm{g} / \mathrm{L})$ & 4 & $<0.010$ & $<0.010$ & -- & -- & -- & -- & -- & -- \\
\hline 39516 & PCB， TOTAL $(\mu \mathrm{g} / \mathrm{L})$ & 4 & $<0.100$ & $<0.100$ & -- & -- & -- & -- & -- & -- \\
\hline 39400 & TOXAPHENE, TOTAL $(\mu \mathrm{g} / \mathrm{L})$ & 4 & $<1.000$ & $<1.000$ & -- & -- & -- & -- & -- & -- \\
\hline 39034 & PERTHANE，TOTAL $(\mu \mathrm{g} / \mathrm{L})$ & 4 & $<0.100$ & $<0.100$ & -- & -- & -- & -- & -- & -- \\
\hline 39570 & DIAZINON, TOTAL $(\mu \mathrm{g} / \mathrm{L})$ & 4 & 0.060 & 0.010 & -- & -- & -- & -- & -- & -- \\
\hline 39398 & ETHION, TOTAL $(\mu \mathrm{g} / \mathrm{L})$ & 4 & $<0.010$ & $<0.010$ & -- & -- & -- & -- & -- & -- \\
\hline 39530 & MALATHION, TOTAL $(\mu \mathrm{g} / \mathrm{L})$ & 4 & 0.010 & $<0.010$ & -- & -- & -- & -- & -- & -- \\
\hline 39600 & METHYL PARATHION, TOTAL $(\mu \mathrm{g} / \mathrm{L})$ & 4 & 0.010 & $<0.010$ & -- & -- & -- & -- & -- & -- \\
\hline 39540 & PARATHION, TOTAL $(\mu \mathrm{g} / \mathrm{L})$ & 4 & $<0.010$ & $<0.010$ & -- & -- & -- & -- & -- & -- \\
\hline 39786 & TRITHION, TOTAL $(\mu \mathrm{g} / \mathrm{L})$ & 4 & $<0.010$ & $<0.010$ & -- & -- & -- & -- & -- & -- \\
\hline 39250 & PCN, TOTAL $(\mu \mathrm{g} / \mathrm{L})$ & 4 & $<0.100$ & $<0.100$ & -- & -- & -- & -- & -- & -- \\
\hline 39480 & METHOXYCHLOR, TOTAL $(\mu \mathrm{g} / \mathrm{L})$ & 4 & 0.010 & $<0.010$ & -- & -- & -- & -- & -- & -- \\
\hline 39755 & MIREX， TOTAL $(\mu \mathrm{g} / \mathrm{L})$ & 4 & $<0.010$ & $<0.010$ & -- & -- & -- & -- & -- & -- \\
\hline 39011 & DISYSTON, TOTAL $\{\mu \mathrm{g} / \mathrm{L}\rangle$ & 4 & $<0.010$ & $<0.010$ & -- & -- & -- & -- & -- & -- \\
\hline 39023 & PHORATE, TOTAL $(\mu \mathrm{g} / \mathrm{L})$ & 4 & $<0.010$ & $<0.010$ & -- & -- & -- & -- & -- & -- \\
\hline 38932 & CHLORPYRIFOS, TOTAL $(\mu \mathrm{g} / \mathrm{L})$ & 4 & 0.020 & $<0.010$ & -- & -- & -- & -- & -- & -- \\
\hline 39040 & DEF, TOTAL $(\mu \mathrm{g} / \mathrm{L})$ & 4 & $<0.010$ & $<0.010$ & - & -- & -- & -- & - & -- \\
\hline 82614 & FONOFOS, TOTAL $(\mu \mathrm{g} / \mathrm{L})$ & 4 & $<0.010$ & $<0.010$ & -- & -- & -- & -- & -- & -- \\
\hline & & & OLATILE ORGAN & C COMPOUND & DS, TOTAL & & & & & \\
\hline 34210 & ACROLEIN, TOTAL $(\mu \mathrm{g} / \mathrm{L})$ & 2 & $<20.000$ & $<20.000$ & -- & - & -- & - & -- & -- \\
\hline 34215 & ACRYLONITRILE, TOTAL $(\mu \mathrm{g} / \mathrm{L})$ & 2 & $<20.000$ & $<20.000$ & -- & -- & -- & -- & -- & - \\
\hline 34030 & BENZENE, TOTAL $(\mu \mathrm{g} / \mathrm{L})$ & 14 & $<8.000$ & $<0.200$ & -- & -- & -- & -- & -- & -- \\
\hline 32104 & BROMOFORM, TOTAL $(\mu \mathrm{g} / \mathrm{L})$ & 14 & $<8.000$ & $<0.200$ & -. & -- & -- & -- & -- & -- \\
\hline 32102 & CARBON TETRACHLORIDE, TOTAL $(\mu \mathrm{g} / \mathrm{L})$ & 14 & $<8.000$ & $<0.200$ & -- & -- & -- & -- & -- & -- \\
\hline 34301 & CHLOROBENZENE, TOTAL $(\mu \mathrm{g} / \mathrm{L})$ & 14 & $<8.000$ & $<0.200$ & -- & -- & -- & -- & -- & -. \\
\hline 32105 & CHLORODIBROMOTHANE, TOTAL $(\mu \mathrm{g} / \mathrm{L})$ & 14 & $<8.000$ & $<0.200$ & -- & -- & -- & -- & -- & -- \\
\hline 34311 & CHLOROETHANE, TOTAL $(\mu \mathrm{g} / \mathrm{L})$ & 14 & $<8.000$ & $<0.200$ & -. & -- & -- & -- & -- & -- \\
\hline 32106 & CHLOROFORM， TOTAL $(\mu \mathrm{g} / \mathrm{L})$ & 1.4 & $<8.000$ & $<0.200$ & -- & -- & -- & -. & -- & -- \\
\hline 34496 & 1,1 -DICHLOROETHANE, TOTAL $(\mu \mathrm{g} / \mathrm{L})$ & 1.4 & $<8.000$ & $<0.200$ & -- & -- & -- & -- & -- & -- \\
\hline 32103 & 1,2 -DICHLOROETHANE, TOTAL $(\mu \mathrm{g} / \mathrm{L})$ & 14 & $<8.000$ & $<0.200$ & -- & -- & -- & -- & -- & -- \\
\hline
\end{tabular}

NOTE: Multiple detection limits during the period of record may result in different values flagged with a "<."

* Value is estimated by using a log-probability regression to predict the values of data below the detection limit. 
Table 72. Statistical summary of water-quality data at site 42 (CSW04), May 1994 through June 1997-Continued

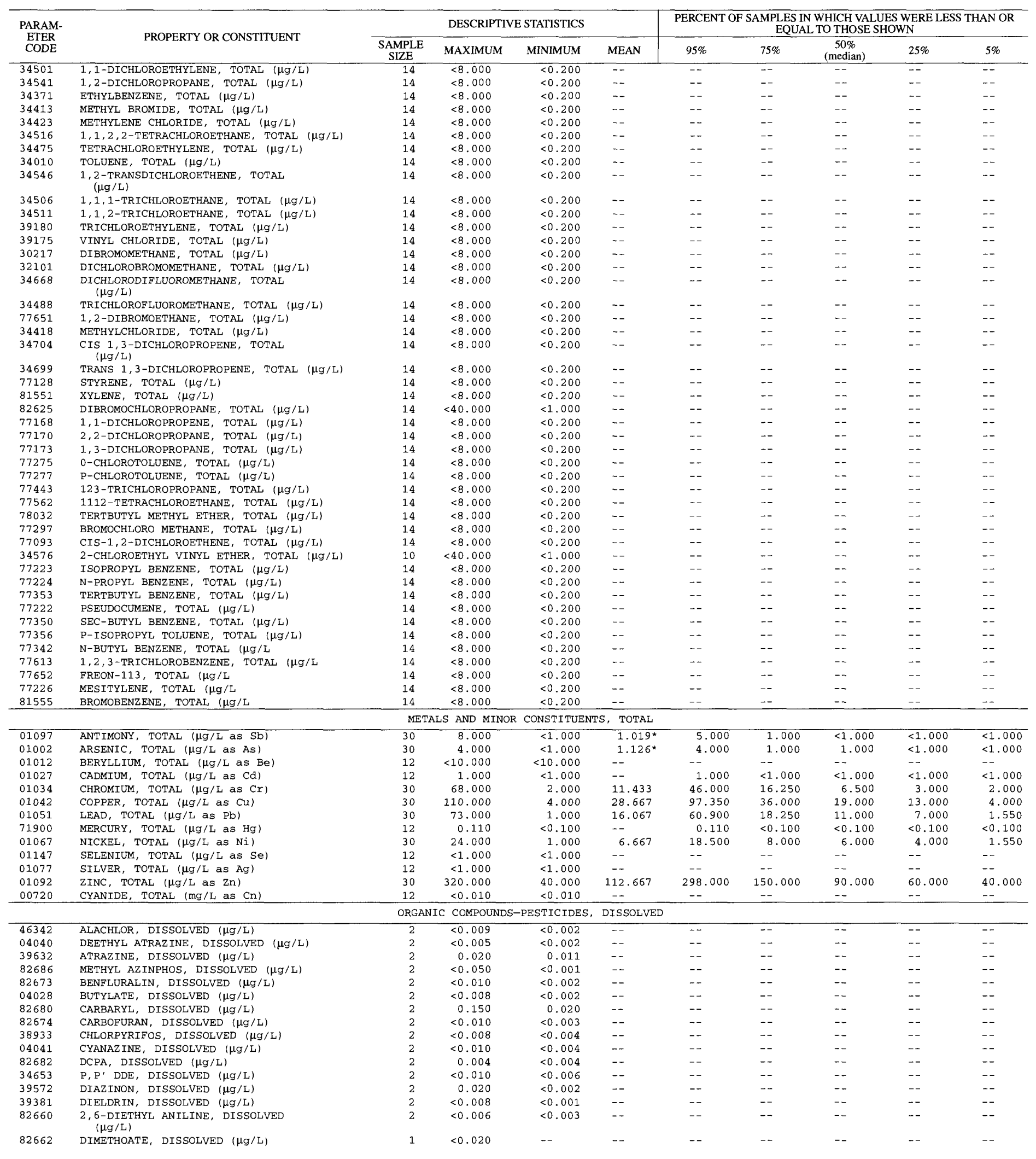

NOTE: Multiple detection limits during the period of record may result in different values flagged with a "<."

* Value is estimated by using a log-probability regression to predict the values of data below the detection limit. 
Table 72. Statistical summary of water-quality data at site 42 (CSW04), May 1994 through June 1997-Continued

\begin{tabular}{|c|c|c|c|c|c|c|c|c|c|c|}
\hline \multirow{2}{*}{$\begin{array}{l}\text { PARAM- } \\
\text { ETER } \\
\text { CODE }\end{array}$} & \multirow{2}{*}{ PROPERTY OR CONSTITUENT } & \multicolumn{4}{|c|}{ DESCRIPTIVE STATISTICS } & PERCEN & $\begin{array}{l}\text { AMPLE } \\
\text { EQ }\end{array}$ & $\begin{array}{l}\text { VHICH VAI } \\
\text { IO THOSE }\end{array}$ & WERE & IAN OR \\
\hline & & $\begin{array}{l}\text { SAMPLE } \\
\text { SIZE }\end{array}$ & MAXIMUM & MINIMUM & MEAN & $95 \%$ & $75 \%$ & $\begin{array}{c}50 \% \\
\text { (median) }\end{array}$ & $25 \%$ & $5 \%$ \\
\hline 82677 & DISULFOTON， DISSOLVED $(\mu \mathrm{g} / \mathrm{L})$ & 2 & $<0.060$ & $<0.017$ & -- & -- & -- & -- & -- & -- \\
\hline 82668 & EPTC, DISSOLVED $(\mu \mathrm{g} / \mathrm{L})$ & 2 & $<0.005$ & $<0.002$ & -- & -- & -- & -- & -- & -. \\
\hline 82663 & ETHALFLURALIN， DISSOLVED $(\mu \mathrm{g} / \mathrm{L})$ & 2 & $<0.010$ & $<0.004$ & -- & -- & -- & -- & -- & -- \\
\hline 82672 & ETHOPROP， DISSOLVED $(\mu \mathrm{g} / \mathrm{L})$ & 2 & $<0.010$ & $<0.003$ & -- & -- & -- & -- & -- & -- \\
\hline 04095 & FONOFOS, DISSOLVED $(\mu \mathrm{g} / \mathrm{L})$ & 2 & $<0.008$ & $<0.003$ & - & -- & -- & -- & -- & -- \\
\hline 34253 & ALPHA BHC, DISSOLVED $(\mu \mathrm{g} / \mathrm{L})$ & 2 & $<0.007$ & $<0.002$ & -- & -- & -- & -- & -- & -- \\
\hline 39341 & LINDANE， DISSOLVED $(\mu \mathrm{g} / \mathrm{L})$ & 2 & $<0.010$ & $<0.004$ & -- & -- & -- & -- & -- & -- \\
\hline 82666 & LINURON, DISSOLVED $(\mu \mathrm{g} / \mathrm{L})$ & 2 & $<0.040$ & $<0.002$ & -- & -- & -- & -- & -- & -- \\
\hline 39532 & MALATHION, DISSOLVED $(\mu \mathrm{g} / \mathrm{L})$ & 2 & 0.026 & $<0.010$ & -- & -- & -- & -- & -- & -- \\
\hline 82667 & METHYL PARATHION, DISSOLVED $(\mu \mathrm{g} / \mathrm{L})$ & 2 & $<0.040$ & $<0.006$ & -- & -- & -- & -- & -- & -- \\
\hline 39415 & METOLACHLOR，DISSOLVED $(\mu \mathrm{g} / \mathrm{L})$ & 2 & 0.010 & $<0.002$ & -- & -- & -- & -- & -. & -- \\
\hline 82630 & METRIBUZIN，DISSOLVED $(\mu \mathrm{g} / \mathrm{L})$ & 2 & $<0.010$ & $<0.004$ & -- & -- & -- & -- & -- & -- \\
\hline 82671 & MOLINATE, DISSOLVED $(\mu \mathrm{g} / \mathrm{L})$ & 2 & $<0.007$ & $<0.004$ & -- & -- & -- & -- & -- & -- \\
\hline 82684 & NAPROPAMIDE，DISSOLVED $(\mu \mathrm{g} / \mathrm{L})$ & 2 & $<0.010$ & $<0.003$ & -- & -- & -- & -. & -- & -- \\
\hline 39542 & PARATHION， DISSOLVED $(\mu \mathrm{g} / \mathrm{L})$ & 2 & $<0.020$ & $<0.004$ & -- & -- & -- & -- & -- & -- \\
\hline 82669 & PEBULATE，DISSOLVED $(\mu \mathrm{g} / \mathrm{L})$ & 2 & $<0.009$ & $<0.004$ & -- & -- & -- & -- & -- & -. \\
\hline 82683 & PENDIMETHALIN， DISSOLVED $(\mu \mathrm{g} / \mathrm{L})$ & 2 & 0.210 & $<0.020$ & -- & -- & -- & -- & -- & -- \\
\hline 82687 & PERMETHRIN， DISSOLVED $(\mu \mathrm{g} / \mathrm{L})$ & 2 & $<0.020$ & $<0.005$ & -- & -- & -- & -- & -- & -- \\
\hline 82664 & PHORATE, DISSOLVED $(\mu \mathrm{g} / \mathrm{L})$ & 2 & $<0.010$ & $<0.002$ & -- & -- & -- & -- & -- & $\ldots$ \\
\hline 82676 & PRONAMIDE, DISSOLVED $(\mu \mathrm{g} / \mathrm{L})$ & 2 & $<0.009$ & $<0.003$ & -- & -- & -- & -- & -- & -- \\
\hline 04037 & PROMETON， DISSOLVED $(\mu \mathrm{g} / \mathrm{L})$ & 2 & 0.030 & $<0.018$ & -. & -- & -- & -- & -- & -- \\
\hline 04024 & PROPACHLOR, DISSOLVED $(\mu \mathrm{g} / \mathrm{L})$ & 2 & $<0.010$ & $<0.007$ & -- & -- & -- & -- & -- & -- \\
\hline 82679 & PROPANIL，DISSOLVED $(\mu \mathrm{g} / \mathrm{L})$ & 2 & $<0.020$ & $<0.004$ & -- & -- & -- & -- & -- & -- \\
\hline 82685 & PROPARGITE, DISSOLVED $(\mu \mathrm{g} / \mathrm{L})$ & 2 & $<0.013$ & $<0.008$ & -- & -- & -- & -- & -- & -- \\
\hline 04035 & SIMAZINE, DISSOLVED $(\mu \mathrm{g} / \mathrm{L})$ & 2 & $<0.008$ & $<0.005$ & -- & -- & -- & - & -- & -- \\
\hline 82681 & THIOBENCARB， DISSOLVED $(\mu \mathrm{g} / \mathrm{L})$ & 2 & $<0.008$ & $<0.002$ & -- & -- & -- & -- & -- & -- \\
\hline 82670 & TEBUTHIURON, DISSOLVED $(\mu \mathrm{g} / \mathrm{L})$ & 2 & 0.050 & $<0.010$ & -- & -- & -- & -. & -- & -- \\
\hline 82665 & TERBACIL， DISSOLVED $(\mu \mathrm{g} / \mathrm{L})$ & 2 & $<0.030$ & $<0.007$ & -- & -- & -- & -- & -- & -- \\
\hline 82675 & TERBUFOS, DISSOLVED $(\mu \mathrm{g} / \mathrm{L})$ & 2 & $<0.013$ & $<0.010$ & -- & -- & -- & -- & -- & -- \\
\hline 82678 & TRIALLATE， DISSOLVED $(\mu \mathrm{g} / \mathrm{L})$ & 2 & $<0.008$ & $<0.001$ & -- & -- & -- & -- & -- & -- \\
\hline 82661 & TRIFLURALIN， DISSOLVED $(\mu \mathrm{g} / \mathrm{L})$ & 2 & $<0.010$ & $<0.002$ & -. & -- & -- & -- & -- & -- \\
\hline 39742 & $2,4,5-T$, DISSOLVED $(\mu \mathrm{g} / \mathrm{L})$ & 3 & $<0.050$ & $<0.035$ & -- & -- & -- & -- & -- & -- \\
\hline 39732 & $2,4-D$, DISSOLVED $(\mu \mathrm{g} / \mathrm{L})$ & 3 & 1.200 & $<0.035$ & -- & -- & -- & -- & -- & -- \\
\hline 38746 & $2,4-D B$, DISSOLVED $(\mu \mathrm{g} / \mathrm{L})$ & 3 & $<0.050$ & $<0.035$ & -- & -- & -- & -- & -- & -- \\
\hline 49315 & ACIFLUORFEN，DISSOLVED $(\mu \mathrm{g} / \mathrm{L})$ & 3 & $<0.050$ & $<0.035$ & -- & -- & -- & -- & -- & -- \\
\hline 49312 & ALDICARB， DISSOLVED $(\mu \mathrm{g} / \mathrm{L})$ & 3 & $<0.050$ & $<0.016$ & -- & -- & -- & -- & -- & -- \\
\hline 49313 & ALDICARB SULFONE, DISSOLVED $(\mu \mathrm{g} / L)$ & 3 & $<0.050$ & $<0.016$ & -- & -- & -- & -- & -- & -- \\
\hline 49314 & $\begin{array}{l}\text { ALDICARB SULFOXIDE, DISSOLVED } \\
(\mu \mathrm{g} / \mathrm{L})\end{array}$ & 3 & $<0.050$ & $<0.021$ & -- & -- & -- & -- & -- & -- \\
\hline 38711 & BENTAZON, DISSOLVED $(\mu \mathrm{g} / \mathrm{L})$ & 3 & $<0.050$ & $<0.014$ & -- & -- & -- & -- & -- & -- \\
\hline 04029 & BROMACIL, DISSOLVED $(\mu \mathrm{g} / L)$ & 3 & $<0.050$ & $<0.035$ & -- & -- & -- & -- & -- & -- \\
\hline 49311 & BROMOXYNIL, DISSOLVED $(\mu \mathrm{g} / \mathrm{L})$ & 3 & $<0.050$ & $<0.035$ & -- & -- & -- & -- & -- & -- \\
\hline 49310 & CARBARYL， DISSOLVED $(\mu \mathrm{g} / \mathrm{L})$ & 3 & $<0.050$ & $<0.008$ & -- & -- & -- & -- & -- & -- \\
\hline 49309 & CARBOFURAN, DISSOLVED $(\mu \mathrm{g} / \mathrm{L})$ & 3 & $<0.050$ & $<0.028$ & -- & -- & - & -- & -- & -- \\
\hline 49308 & 3-HYDROXY-CARBOFURAN $(\mu \mathrm{g} / \mathrm{L})$ & 3 & $<0.050$ & $<0.014$ & -- & -- & -- & -- & -- & -- \\
\hline 49307 & AMIBEN， DISSOLVED $(\mu \mathrm{g} / \mathrm{L})$ & 3 & $<0.050$ & $<0.011$ & -- & -- & -- & -- & -- & -- \\
\hline 49306 & CHLOROTHALONIL, DISSOLVED $(\mu \mathrm{g} / \mathrm{L})$ & 3 & $<0.050$ & $<0.035$ & -- & -- & -- & -- & -- & -- \\
\hline 49305 & CLOPYRALID，DISSOLVED $(\mu \mathrm{g} / \mathrm{L})$ & 3 & $<0.050$ & $<0.050$ & -- & -- & -- & -- & -- & -- \\
\hline 49304 & DACTHALMONO-ACID, DISSOLVED $(\mu \mathrm{g} / \mathrm{L})$ & 3 & $<0.050$ & $<0.017$ & -- & -- & -- & -- & -- & -- \\
\hline 38442 & DICAMBA，DISSOLVED $(\mu \mathrm{g} / \mathrm{L})$ & 3 & $<0.050$ & $<0.035$ & -- & -- & -- & -. & -- & -- \\
\hline 49303 & DICHLOBENIL, DISSOLVED $(\mu \mathrm{g} / \mathrm{L})$ & 3 & $<0.050$ & $<0.020$ & -- & -- & -- & -- & -- & -- \\
\hline 49302 & DICHLORPROP， DISSOLVED $(\mu \mathrm{g} / \mathrm{L})$ & 3 & $<0.050$ & $<0.032$ & -- & -- & -- & -- & -- & -- \\
\hline 49301 & DINOSEB，DISSOLVED $(\mu \mathrm{g} / \mathrm{L})$ & 3 & $<0.050$ & $<0.035$ & -- & -- & - & -- & -- & -- \\
\hline 49300 & DIURON, DISSOLVED $(\mu \mathrm{g} / \mathrm{L})$ & 3 & $<0.050$ & $<0.020$ & -- & -- & -- & -- & -- & -- \\
\hline 49299 & $\begin{array}{l}\text { 4, } 6 \text {-DINITRO OCRESOL, DISSOLVED } \\
(\mu \mathrm{g} / \mathrm{L})\end{array}$ & 3 & $<0.050$ & $<0.035$ & -- & -- & -- & -- & -- & -- \\
\hline 49298 & ESFENVALERATE，DISSOLVED $(\mu \mathrm{g} / \mathrm{L})$ & 3 & $<0.050$ & $<0.019$ & -- & -- & -- & -- & -- & -- \\
\hline 49297 & FENURON，DISSOLVED $(\mu \mathrm{g} / \mathrm{L})$ & 3 & $<0.050$ & $<0.013$ & -- & -- & $\ldots$ & -- & -- & -- \\
\hline 38811 & FLUOMETURON，DISSOLVED $(\mu \mathrm{g} / L)$ & 3 & $<0.050$ & $<0.035$ & -- & -- & -- & -- & -- & -- \\
\hline 38478 & LINURON， DISSOLVED $(\mu \mathrm{g} / \mathrm{L})$ & 3 & $<0.050$ & $<0.018$ & -- & -- & -. & -- & -- & -- \\
\hline 38482 & MCPA， DISSOLVED $(\mu \mathrm{g} / \mathrm{L})$ & 3 & $<0.050$ & $<0.050$ & -- & -- & -- & -- & -- & -- \\
\hline 38487 & MCPB， DISSOLVED $(\mu \mathrm{g} / \mathrm{L})$ & 3 & $<0.050$ & $<0.035$ & -- & -- & -- & -- & -- & -- \\
\hline 38501 & METHIOCARB， DISSOLVED $(\mu \mathrm{g} / \mathrm{L})$ & 3 & $<0.050$ & $<0.026$ & -- & -- & -- & -- & -- & -- \\
\hline 49296 & METHOMYL， DISSOLVED $(\mu \mathrm{g} / \mathrm{L})$ & 3 & $<0.050$ & $<0.017$ & -- & -- & -- & -- & -- & -- \\
\hline 49295 & 1-NAPHTHOL， DISSOLVED $(\mu \mathrm{g} / \mathrm{L})$ & 3 & $<0.050$ & $<0.007$ & -- & -- & -- & -- & -. & -- \\
\hline 49294 & NEBURON， DISSOLVED $(\mu \mathrm{g} / \mathrm{L})$ & 3 & $<0.050$ & $<0.015$ & -- & -- & -- & -- & -- & -- \\
\hline 49293 & NORFLURAZON, DISSOLVED $(\mu \mathrm{g} / \mathrm{L})$ & 3 & $<0.050$ & $<0.024$ & -- & -- & -- & -- & -- & -- \\
\hline 49292 & ORYZALIN, DISSOLVED $(\mu \mathrm{g} / \mathrm{L})$ & 3 & $<0.050$ & $<0.019$ & -- & -- & -- & -- & -- & -- \\
\hline 38866 & OXAMYL， DISSOLVED $(\mu \mathrm{g} / \mathrm{L})$ & 3 & $<0.050$ & $<0.018$ & -- & -- & -- & -- & -- & -- \\
\hline 49291 & PICLORAM， DISSOLVED $(\mu \mathrm{g} / \mathrm{L})$ & 3 & $<0.050$ & $<0.050$ & -- & -- & -- & -- & -- & -- \\
\hline 49236 & PROPHAM， DISSOLVED $(\mu \mathrm{g} / \mathrm{L})$ & 3 & $<0.050$ & $<0.035$ & -- & -- & -- & -- & -- & -- \\
\hline 38538 & PROPOXUR，DISSOLVED $(\mu \mathrm{g} / \mathrm{L})$ & 3 & $<0.050$ & $<0.035$ & -- & -- & -- & -- & -- & -- \\
\hline 39762 & SILVEX, DISSOLVED $(\mu \mathrm{g} / \mathrm{L})$ & 3 & $<0.050$ & $<0.021$ & -- & -- & -- & -- & -- & -- \\
\hline 49235 & TRICLOPYR, DISSOLVED $(\mu \mathrm{g} / \mathrm{L})$ & 3 & $<0.050$ & $<0.050$ & -- & -- & -- & -- & -- & -- \\
\hline & & ORGAN & COMPOUN & GANONITRC & $\mathrm{NN}, \mathrm{TC}$ & & & & & \\
\hline 39057 & PROMETRYNE, TOTAL $(\mu \mathrm{g} / \mathrm{L})$ & 1 & $<0.100$ & -- & -- & -- & -- & -- & $\overline{--}$ & -- \\
\hline 39056 & PROMETONE，TOTAL $(\mu \mathrm{g} / \mathrm{L})$ & 1 & $<0.200$ & -- & -- & -- & - & -- & -- & -- \\
\hline 39054 & SIMETRYNE，TOTAL $(\mu \mathrm{g} / L)$ & 1 & $<0.100$ & -- & -- & -- & -- & -- & -- & -- \\
\hline 81757 & CYANAZINE，TOTAL $(\mu \mathrm{g} / \mathrm{L})$ & 1 & $<0.200$ & -- & -- & -- & -- & -- & -- & -- \\
\hline 77825 & ALACHLOR， TOTAL $(\mu \mathrm{g} / \mathrm{L})$ & 1 & $<0.100$ & -- & -- & -- & -- & - & -- & -- \\
\hline 82611 & METRIBUZIN, TOTAL $(\mu \mathrm{g} / \mathrm{L})$ & 1 & $<0.100$ & -- & -- & -- & -- & -- & -- & -- \\
\hline
\end{tabular}

NOTE: Multiple detection limits during the period of record may result in different values flagged with a "<."

* Value is estimated by using a log-probability regression to predict the values of data below the detection limit. 
Table 72. Statistical summary of water-quality data at site 42 (CSW04), May 1994 through June 1997-Continued

\begin{tabular}{|c|c|c|c|c|c|c|c|c|c|c|}
\hline \multirow{2}{*}{$\begin{array}{l}\text { PARAM- } \\
\text { ETER } \\
\text { CODE }\end{array}$} & \multirow{2}{*}{ PROPERTY OR CONSTITUENT } & \multicolumn{4}{|c|}{ DESCRIPTIVE STATISTICS } & \multicolumn{5}{|c|}{$\begin{array}{c}\text { PERCENT OF SAMPLES IN WHICH VALUES WERE LESS THAN OR } \\
\text { EQUAL TO THOSE SHOWN }\end{array}$} \\
\hline & & $\begin{array}{l}\text { SAMPLE } \\
\text { SIZE }\end{array}$ & MAXIMUM & MINIMUM & MEAN & $95 \%$ & $75 \%$ & $\begin{array}{c}50 \% \\
\text { (median) }\end{array}$ & $25 \%$ & $5 \%$ \\
\hline 30311 & TERBACIL, TOTAL $(\mu \mathrm{g} / \mathrm{L})$ & 1 & 0.300 & -- & -- & -- & -- & -- & -- & -- \\
\hline 30264 & HEXAZ INONE, TOTAL $(\mu \mathrm{g} / L)$ & 1 & $<0.200$ & - & -- & -- & -- & -- & -- & -- \\
\hline 30235 & BUTACHLOR, TOTAL $(\mu \mathrm{g} / \mathrm{L})$ & 1 & $<0.100$ & -- & -- & -- & -- & -- & -- & -- \\
\hline 30236 & BUTYLATE, TOTAL $(\mu \mathrm{g} / \mathrm{L})$ & 1 & $<0.100$ & -- & -- & -- & -- & -- & -- & -- \\
\hline 75981 & DE-ETHYLATRAZINE, TOTAL $(\mu \mathrm{g} / \mathrm{L})$ & 1 & $<0.200$ & -- & -- & -- & -- & -- & -- & -- \\
\hline 39630 & ATRAZINE, TOTAL $(\mu \mathrm{g} / L)$ & 1 & $<0.100$ & -- & -- & -- & -- & -- & -- & -- \\
\hline 39055 & SIMAZINE, TOTAL $(\mu \mathrm{g} / L)$ & 1 & $<0.100$ & -- & -- & -- & - & - & -- & -- \\
\hline 39024 & PROPAZINE, TOTAL $(\mu \mathrm{g} / \mathrm{L})$ & 1 & $<0.100$ & -- & -- & -- & -- & -- & -- & -- \\
\hline 82184 & AMETRYNE, TOTAL $(\mu \mathrm{g} / \mathrm{L})$ & 1 & $<0.100$ & -- & -- & -- & -- & -- & -- & -- \\
\hline 39030 & TRIFLURALIN, TOTAL $(\mu \mathrm{g} / \mathrm{L})$ & 1 & $<0.100$ & -- & -- & -- & -- & -- & +- & -- \\
\hline 82612 & METOLACHLOR, TOTAL $(\mu \mathrm{g} / \mathrm{L})$ & 1 & $<0.200$ & -- & -- & -- & -- & -- & -- & -- \\
\hline 30234 & BROMACIL, TOTAL $(\mu \mathrm{g} / \mathrm{L})$ & 1 & $<0.200$ & - & -- & -- & -- & -- & -- & -- \\
\hline 30255 & DIPHENAMID, TOTAL $(\mu \mathrm{g} / \mathrm{L})$ & 1 & $<0.100$ & -- & -- & -- & -- & -- & -- & -- \\
\hline 30324 & VERNOLATE, TOTAL $(\mu \mathrm{g} / \mathrm{L})$ & 1 & $<0.100$ & -- & -- & -- & -- & -- & -- & -- \\
\hline 30254 & CYCLOATE, TOTAL $\langle\mu \mathrm{g} / L\rangle$ & 1 & $<0.100$ & -- & -- & -- & -- & -- & -- & -- \\
\hline 30295 & PROPACHLOR, TOTAL $(\mu \mathrm{g} / \mathrm{L})$ & 1 & $<0.100$ & -- & -- & -- & -- & -- & -- & -- \\
\hline 39730 & $2,4-\mathrm{D}$, TOTAL $(\mu \mathrm{g} / \mathrm{L})$ & 1 & 3.700 & -- & -- & -- & -- & $\overline{--}$ & -- & -- \\
\hline 39760 & SILVEX, TOTAL $(\mu \mathrm{g} / \mathrm{L})$ & 1 & $<0.010$ & -- & -- & -- & -- & -- & -- & -- \\
\hline 39720 & PICLORAM, TOTAL $(\mu \mathrm{g} / \mathrm{L})$ & 1 & $<0.010$ & -- & -- & -- & -- & -- & -- & -- \\
\hline 39740 & $2,4,5-\mathrm{T}$, TOTAL $(\mu \mathrm{g} / \mathrm{L})$ & 1 & $<0.010$ & -- & -- & -- & $-\cdots$ & -- & -- & -- \\
\hline 82183 & 2,4 -DP, TOTAL $(\mu \mathrm{g} / \mathrm{L})$ & 1 & $<0.010$ & -- & -- & -- & -- & -- & -- & -- \\
\hline 82052 & DICAMBA， TOTAL $(\mu \mathrm{g} / \mathrm{L})$ & 1 & 0.650 & -- & -- & $\ldots$ & -- & -- & -- & -- \\
\hline \multicolumn{11}{|c|}{ ORGANIC COMPOUNDS-CARBAMATE PESTICIDES, TOTAL } \\
\hline 39750 & SEVIN, TOTAL $(\mu \mathrm{g} / \overline{\mathrm{L}})$ & 1 & $<0.500$ & $\overline{--}$ & -- & -- & -- & -- & -- & -- \\
\hline 39051 & METHOMYL, TOTAL $(\mu \mathrm{g} / \mathrm{L})$ & 1 & $<0.500$ & -- & -- & -- & -- & -- & -- & -- \\
\hline 82619 & ALDICARD, TOTAL $(\mu \mathrm{g} / \mathrm{L})$ & 1 & $<0.500$ & -- & -- & -- & -- & -- & -- & -- \\
\hline 30296 & PROPOXUR, TOTAL $(\mu \mathrm{g} / \mathrm{L})$ & 1. & $<0.500$ & -- & -- & -- & -- & -- & -- & -- \\
\hline 39052 & PROPHAM, TOTAL $(\mu \mathrm{g} / \mathrm{L})$ & 1 & $<0.500$ & -- & -- & -- & -- & -- & -- & -- \\
\hline 82615 & CARBOFURAN, TOTAL $(\mu \mathrm{g} / \mathrm{L})$ & 1 & $<0.500$ & -- & -- & -- & -- & -- & -- & -- \\
\hline 77441 & 1-NAPHTHOL, TOTAL $(\mu \mathrm{g} / \mathrm{L})$ & 1. & $<0.500$ & -- & -- & -- & -- & -- & -- & -- \\
\hline 30282 & METHIOCARB, TOTAL $(\mu \mathrm{g} / \mathrm{L})$ & 1 & $<0.500$ & -- & -- & -- & -- & -- & -- & -- \\
\hline
\end{tabular}

NOTE: Multiple detection limits during the period of record may result in different values flagged with a "<."

* Value is estimated by using a log-probability regression to predict the values of data below the detection limit. 
Table 73. Statistical summary of water-quality data at site 43 (CSW07), June 1994 through June 1997

\begin{tabular}{|c|c|c|c|c|c|c|c|c|c|c|}
\hline \multirow{2}{*}{$\begin{array}{c}\text { PARAM- } \\
\text { ETER } \\
\text { CODE }\end{array}$} & \multirow{2}{*}{ PROPERTY OR CONSTITUENT } & \multicolumn{4}{|c|}{ DESCRIPTIVE STATISTICS } & \multicolumn{5}{|c|}{$\begin{array}{c}\text { PERCENT OF SAMPLES IN WHICH VALUES WERE LESS THAN OR } \\
\text { EQUAL TO THOSE SHOWN }\end{array}$} \\
\hline & & $\begin{array}{l}\text { SAMPLE } \\
\text { SIZE }\end{array}$ & MAXIMUM & MINIMUM & MEAN & $95 \%$ & $75 \%$ & $\begin{array}{c}50 \% \\
\text { (median) }\end{array}$ & $25 \%$ & $5 \%$ \\
\hline \multicolumn{11}{|c|}{ PHYSICAL AND CHEMICAL PROPERTIES } \\
\hline 00061 & INSTANTANEOUS DISCHARGE, $\left(\mathrm{ft}^{3} / \mathrm{s}\right)$ & 67 & 344.000 & 0.020 & 12.036 & 34.800 & 10.000 & 2.700 & 0.870 & 0.194 \\
\hline 00010 & WATER TEMPERATURE $\left({ }^{\circ} \mathrm{C}\right)$ & 66 & 29.000 & 5.000 & 17.311 & 27.000 & 23.000 & 18.500 & 12.875 & 6.675 \\
\hline 90095 & SPECIFIC CONDUCTANCE, LAB $\left(\mu \mathrm{S} / \mathrm{cm}\right.$ at $\left.25^{\circ} \mathrm{C}\right)$ & 47 & 316.000 & 33.000 & 105.511 & 246.400 & 128.000 & 94.000 & 70.000 & 37.000 \\
\hline 00095 & $\begin{array}{l}\text { SPECIFIC CONDUCTANCE, FIELD ( } \mu \text { S/CM at } 25 \\
\left.{ }^{\circ} \mathrm{C}\right)\end{array}$ & 61 & 304.000 & 26.000 & 105.148 & 228.800 & 136.500 & 97.000 & 65.000 & 33.000 \\
\hline 00403 & $\mathrm{pH}$, LAB (STANDARD pH UNITS) & 47 & 7.700 & 6.200 & 6.915 & 7.660 & 7.100 & 6.900 & 6.600 & 6.300 \\
\hline 00400 & pH, FIELD (STANDARD PH UNITS) & 58 & 7.950 & 6.600 & 7.039 & 7.481 & 7.200 & 7.000 & 6.898 & 6.648 \\
\hline 90410 & ALKALINITY, LAB (mg/L as $\left(\mathrm{CaCO}_{3}\right.$ ) & 47 & 145.000 & 8.000 & 29.936 & 88.200 & 36.000 & 22.000 & 16.000 & 11.200 \\
\hline 80154 & SUSPENDED SEDIMENT (mg/L) & 49 & 19400.000 & 44.000 & 2079.735 & 12000.000 & 1980.000 & 678.000 & 251.000 & 89.500 \\
\hline 00530 & $\begin{array}{l}\text { RESIDUE ON EVAPORATION AT } 105^{\circ} \mathrm{C} \text {, SUSPENDED } \\
(\mathrm{mg} / \mathrm{L})\end{array}$ & 19 & 23900.000 & 17.000 & 2071.790 & 23900.000 & 1140.000 & 368.000 & 90.000 & 17.000 \\
\hline 00535 & RESIDUE VOLATILE， SUSPENDED (mg/L) & 40 & 2200.000 & 1.000 & 174.875 & 1179.500 & 131.000 & 47.500 & 21.000 & 3.150 \\
\hline 70300 & $\begin{array}{l}\text { DISSOLVED SOLIDS, RESIDUE AT } 180^{\circ} \mathrm{C} \\
(\mathrm{mg} / \mathrm{L})\end{array}$ & 46 & 326.000 & 22.000 & 82.413 & 223.700 & 85.250 & 64.500 & 49.500 & 26.700 \\
\hline 00310 & $\begin{array}{l}5 \text { DAY BIOCHEMICAL OXYGEN DEMAND } \\
(\mathrm{mg} / \mathrm{L})\end{array}$ & 31 & 27.000 & $<2.000$ & $7.492 \star$ & 20.000 & 8.800 & 5.700 & 3.800 & $<2.000$ \\
\hline 00340 & CHEMICAL OXYGEN DEMAND (mg/L) & 47 & 650.000 & 7.000 & 72.830 & 298.000 & 72.000 & 48.000 & 25.000 & 11.000 \\
\hline \multicolumn{11}{|c|}{ NUTRIENTS, TOTAL AND DISSOLVED } \\
\hline 00625 & $\begin{array}{l}\text { NITROGEN AMMONIA + ORGANIC, } \\
\text { TOTAL (mg/L as N) }\end{array}$ & 48 & 7.800 & 0.330 & 1.867 & 6.665 & 1.975 & 1.200 & 0.892 & 0.418 \\
\hline 00631 & $\mathrm{NO}_{2}+\mathrm{NO}_{3}$, DISSOLVED $(\mathrm{mg} / \mathrm{L}$ as $\mathrm{N})$ & 47 & 1.310 & 0.050 & 0.281 & 0.926 & 0.380 & 0.200 & 0.110 & 0.050 \\
\hline 00608 & $\begin{array}{l}\text { NITROGEN AMMONIA, DISSOLVED } \\
(\mathrm{mg} / \mathrm{L} \text { as } \mathrm{N})\end{array}$ & 47 & 1.100 & 0.015 & 0.171 & 0.566 & 0.220 & 0.090 & 0.050 & 0.019 \\
\hline 00605 & NITROGEN ORGANIC, TOTAL (mg/L as N) & 48 & 7.700 & 0.240 & 1.698 & 6.610 & 1.700 & 0.970 & 0.777 & 0.321 \\
\hline 00600 & NITROGEN, TOTAL (mg/L as N) & 48 & 8.300 & 0.450 & 2.141 & 6.775 & 2.525 & 1.500 & 1.000 & 0.587 \\
\hline 00665 & PHOSPHORUS, TOTAL (mg/L as P) & 48 & 33.500 & 0.110 & 3.035 & 17.275 & 2.768 & 0.920 & 0.512 & 0.185 \\
\hline 00671. & $\begin{array}{l}\text { PHOSPHORUS ORTHO, DISSOLVED } \\
(\mathrm{mg} / \mathrm{L} \text { aS } \mathrm{P})\end{array}$ & 47 & 1.230 & 0.010 & 0.141 & 0.688 & 0.090 & 0.060 & 0.030 & 0.014 \\
\hline \multicolumn{11}{|c|}{ OIL AND GREASE, TOTAL } \\
\hline 00556 & OIL AND GREASE, TOTAL (mg/L) & 12 & 4.000 & $<1.000$ & $1.156^{*}$ & 4.000 & 1.000 & $<1.000$ & $<1.000$ & $<1.000$ \\
\hline \multicolumn{11}{|c|}{ ORGANIC CARBON, TOTAL } \\
\hline 00680 & CARBON ORGANIC, TOTAL (mg/L) & 26 & 56.000 & 4.400 & 18.023 & 46.550 & 22.250 & 16.000 & 12.000 & 5.450 \\
\hline & & & & OLIFORM & & & & & & \\
\hline 31679 & FECAL STREPTOCOCCI (Colonies per $100 \mathrm{~mL}$ ) & 19 & 70000.000 & 80.000 & 21698.947 & 70000.000 & 29000.000 & 12000.000 & 6900.000 & 80.000 \\
\hline 31616 & FECAL COLIFORM (Colonies per $100 \mathrm{~mL}$ ) & 19 & 92000.000 & $<1000.000$ & $19280.203^{*}$ & 92000.000 & 28000.000 & 13000.000 & 1200.000 & 160.000 \\
\hline & & & RGANIC COMPC & NDS-PESTICI & DES, TOTAL & & & & & \\
\hline 39330 & ALDRIN, TOTAL $(\mu \mathrm{g} / \mathrm{L})$ & 3 & $<0.010$ & $<0.010$ & -- & -- & -- & -- & $\overline{--}$ & $\overline{--}$ \\
\hline 39340 & LINDANE, TOTAL $(\mu \mathrm{g} / \mathrm{L})$ & 3 & $<0.010$ & $<0.010$ & -- & -- & -- & -- & -- & -- \\
\hline 39350 & CHLORDANE, TOTAL $(\mu \mathrm{g} / \mathrm{L})$ & 3 & $<0.100$ & $<0.100$ & -- & -- & $\cdots$ & -- & -- & -- \\
\hline 39370 & DDT, TOTAL $(\mu \mathrm{g} / \mathrm{L})$ & 3 & $<0.010$ & $<0.010$ & -- & -. & - & -- & -- & -- \\
\hline 39365 & DDE, TOTAL $(\mu \mathrm{g} / \mathrm{L})$ & 3 & $<0.010$ & $<0.010$ & -- & -- & - & -- & -- & -- \\
\hline 39360 & DDD， TOTAL $(\mu \mathrm{g} / \mathrm{L})$ & 3 & $<0.010$ & $<0.010$ & -- & -- & -- & -- & -- & -- \\
\hline 39380 & DIELDRIN， TOTAL $(\mu \mathrm{g} / \mathrm{L})$ & 3 & $<0.020$ & $<0.010$ & -- & -- & -- & -- & -- & -- \\
\hline 39388 & ENDOSULFAN, TOTAL $(\mu \mathrm{g} / \mathrm{L})$ & 3 & $<0.010$ & $<0.010$ & -- & -- & -- & -- & -- & -- \\
\hline 39390 & ENDRIN，TOTAL $(\mu \mathrm{g} / \mathrm{L})$ & 3 & $<0.010$ & $<0.010$ & -- & -- & -- & -- & -- & -- \\
\hline 39410 & HEPTACHLOR, TOTAL $(\mu \mathrm{g} / \mathrm{L})$ & 3 & $<0.010$ & $<0.010$ & -- & -- & -- & -- & -- & -- \\
\hline 39420 & HEPTACHLOR EPOXIDE, TOTAL $(\mu \mathrm{g} / \mathrm{L})$ & 3 & $<0.010$ & $<0.010$ & -- & -- & -- & -- & -- & -- \\
\hline 39516 & PCB， TOTAL $(\mu \mathrm{g} / \mathrm{L})$ & 3 & $<0.100$ & $<0.100$ & -- & -. & - & -- & -- & -- \\
\hline 39400 & TOXAPHENE, TOTAL $(\mu \mathrm{g} / \mathrm{L})$ & 3 & $<1.000$ & $<1.000$ & -- & -- & -- & -- & -- & -- \\
\hline 39034 & PERTHANE， TOTAL $(\mu \mathrm{g} / \mathrm{L})$ & 3 & $<0.100$ & $<0.100$ & -- & -- & -- & -- & -- & -- \\
\hline 39570 & DIAZINON, TOTAL $(\mu \mathrm{g} / \mathrm{L})$ & 3 & 0.080 & 0.010 & -- & -- & - & -- & -- & -- \\
\hline 39398 & ETHION， TOTAL $(\mu \mathrm{g} / \mathrm{L})$ & 3 & $<0.010$ & $<0.010$ & -. & -- & -- & -- & -- & -- \\
\hline 39530 & MALATHION, TOTAL $(\mu \mathrm{g} / \mathrm{L})$ & 3 & 0.020 & $<0.010$ & -- & -- & - & -- & -- & -- \\
\hline 39600 & METHYL PARATHION, TOTAL $(\mu \mathrm{g} / \mathrm{L})$ & 3 & 0.010 & $<0.010$ & -- & -- & -- & -- & -- & -- \\
\hline 39540 & PARATHION, TOTAL $(\mu \mathrm{g} / \mathrm{L})$ & 3 & $<0.010$ & $<0.010$ & -- & -- & -- & -- & -- & -- \\
\hline 39786 & TRITHION, TOTAL $(\mu \mathrm{g} / \mathrm{L})$ & 3 & $<0.010$ & $<0.010$ & -- & -- & -- & -- & -- & -- \\
\hline 39250 & PCN， TOTAL $(\mu \mathrm{g} / \mathrm{L})$ & 3 & $<0.100$ & $<0.100$ & -- & -- & -- & -- & -- & -- \\
\hline 39480 & METHOXYCHLOR, TOTAL $(\mu \mathrm{g} / \mathrm{L})$ & 3 & $<0.010$ & $<0.010$ & -- & -- & -- & -- & -- & -- \\
\hline 39755 & MIREX, TOTAL $(\mu \mathrm{g} / \mathrm{L})$ & 3 & $<0.010$ & $<0.010$ & -- & -- & -- & -- & -- & -- \\
\hline 39011 & DISYSTON， TOTAL $(\mu \mathrm{g} / \mathrm{L})$ & 3 & $<0.010$ & $<0.010$ & -- & -- & -- & -- & -- & -- \\
\hline 39023 & PHORATE, TOTAL $(\mu \mathrm{g} / \mathrm{L})$ & 3 & $<0.010$ & $<0.010$ & -- & -- & -- & -- & -- & -- \\
\hline 38932 & CHLORPYRIFOS, TOTAL $(\mu \mathrm{g} / \mathrm{L})$ & 3 & 0.050 & $<0.010$ & -- & -- & -- & -- & -- & -- \\
\hline 39040 & DEF， TOTAL $(\mu \mathrm{g} / \mathrm{L})$ & 3 & $<0.010$ & $<0.010$ & -- & -- & -- & -- & -- & -- \\
\hline 82614 & FONOFOS, TOTAL $(\mu \mathrm{g} / \mathrm{L})$ & 3 & $<0.010$ & $<0.010$ & -- & -- & - & - & -- & -- \\
\hline & & & VOLATILE ORGP & IIC COMPOUN & DS, TOTAL & & & & & \\
\hline 34210 & ACROLEIN, TOTAL $(\mu \mathrm{g} / \mathrm{L})$ & 1 & $<20.000$ & -- & -- & -- & -- & -- & -- & -- \\
\hline 34215 & ACRYLONITRILE, TOTAL $(\mu \mathrm{g} / L)$ & 1 & $<20.000$ & -- & -- & -- & -- & -- & -- & -- \\
\hline 34030 & BENZENE, TOTAL $(\mu \mathrm{g} / \mathrm{L})$ & 9 & $<2.000$ & $<0.200$ & -- & -- & - & -- & -- & -- \\
\hline 32104 & BROMOFORM，TOTAL $(\mu \mathrm{g} / \mathrm{L})$ & 9 & $<2.000$ & $<0.200$ & -- & -- & -- & -- & -- & -- \\
\hline 32102 & CARBON TETRACHLORIDE, TOTAL $(\mu \mathrm{g} / \mathrm{L})$ & 9 & $<2.000$ & $<0.200$ & -- & -- & -- & -- & -- & -- \\
\hline 34301 & CHLOROBENZENE， TOTAL $(\mu \mathrm{g} / \mathrm{L})$ & 9 & $<2.000$ & $<0.200$ & -- & -- & -- & -- & -- & -- \\
\hline 32105 & CHLORODIBROMOTHANE, TOTAL $(\mu \mathrm{g} / \mathrm{L})$ & 9 & $<2.000$ & $<0.200$ & -- & -- & - & -- & -- & -- \\
\hline 34311 & CHLOROETHANE, TOTAL $(\mu \mathrm{g} / \mathrm{L})$ & 9 & $<2.000$ & $<0.200$ & -- & -- & -- & -- & -- & -- \\
\hline 32106 & CHLOROFORM， TOTAL $(\mu \mathrm{g} / \mathrm{L})$ & 9 & $<2.000$ & $<0.200$ & -- & -- & -- & $\ldots$ & -- & -- \\
\hline 34496 & 1,1 -DICHLOROETHANE, TOTAL $(\mu \mathrm{g} / \mathrm{L})$ & 9 & $<2.000$ & $<0.200$ & -- & - & -- & -- & -- & -- \\
\hline 32103 & 1,2-DICHLOROETHANE, TOTAL $(\mu \mathrm{g} / \mathrm{L})$ & 9 & $<2.000$ & $<0.200$ & -- & -- & -- & -- & -- & -- \\
\hline
\end{tabular}

NOTE: Multiple detection limits during the period of record may result in different values flagged with a "<."

* Value is estimated by using a log-probability regression to predict the values of data below the detection limit. 
Table 73. Statistical summary of water-quality data at site 43 (CSW07), June 1994 through June 1997-Continued

\begin{tabular}{|c|c|c|c|c|c|c|c|c|c|c|}
\hline \multirow{2}{*}{$\begin{array}{l}\text { PARAM- } \\
\text { ETER } \\
\text { CODE }\end{array}$} & \multirow{2}{*}{ PROPERTY OR CONSTITUENT } & \multicolumn{4}{|c|}{ DESCRIPTIVE STATISTICS } & \multicolumn{5}{|c|}{$\begin{array}{c}\text { PERCENT OF SAMPLES IN WHICH VALUES WERE LESS THAN OR } \\
\text { EQUAL TO THOSE SHOWN }\end{array}$} \\
\hline & & $\begin{array}{l}\text { SAMPLE } \\
\text { SIZE }\end{array}$ & MAXIMUM & MINIMUM & MEAN & $95 \%$ & $75 \%$ & $\begin{array}{c}50 \% \\
\text { (median) }\end{array}$ & $25 \%$ & $5 \%$ \\
\hline 34501 & 1,1-DICHLOROETHYLENE, TOTAL $(\mu \mathrm{g} / \mathrm{L})$ & 9 & $<2.000$ & $<0.200$ & -- & -- & -- & -- & -- & -- \\
\hline 34371 & ETHYLBENZENE, TOTAL $(\mu \mathrm{g} / \mathrm{L})$ & 9 & $<2.000$ & $<0.200$ & -- & -- & -- & -- & -- & -- \\
\hline 34413 & METHYL BROMIDE, TOTAL $(\mu \mathrm{g} / \mathrm{L})$ & 9 & $<2.000$ & $<0.200$ & -- & -- & -- & -- & -- & -- \\
\hline 34423 & METHYLENE CHLORIDE, TOTAL $(\mu \mathrm{g} / \mathrm{L})$ & 9 & $<2.000$ & $<0.200$ & -- & -- & -- & -- & -- & -- \\
\hline 34516 & $1,1,2,2$-TETRACHLOROETHANE，TOTAL $(\mu \mathrm{g} / \mathrm{L})$ & 9 & $<2.000$ & $<0.200$ & -- & -- & -- & -- & -- & -- \\
\hline 34475 & TETRACHLOROETHYLENE，TOTAL $(\mu \mathrm{g} / \mathrm{L})$ & 9 & $<2.000$ & $<0.200$ & -- & -- & -- & -- & -- & -- \\
\hline 34546 & $\begin{array}{l}\text { 1, 2-TRANSDICHLOROETHENE, TOTAL } \\
(\mu \mathrm{g} / \mathrm{L})\end{array}$ & 9 & $<2.000$ & $<0.200$ & -- & -- & - & -- & -- & -- \\
\hline 34506 & $1,1,1$-TRICHLOROETHANE, TOTAL $(\mu \mathrm{g} / \mathrm{L})$ & 9 & $<2.000$ & $<0.200$ & -- & -- & -- & -- & -- & -- \\
\hline 34511 & $1,1,2$-TRICHLOROETHANE，TOTAL $(\mu \mathrm{g} / \mathrm{L})$ & 9 & $<2.000$ & $<0.200$ & -- & -- & -- & -- & -. & -- \\
\hline 39180 & TRICHLOROETHYLENE, TOTAL $(\mu \mathrm{g} / \mathrm{L})$ & 9 & $<2.000$ & $<0.200$ & -- & -- & -- & -- & -- & -- \\
\hline 39175 & VINYL CHLORIDE, TOTAL $(\mu \mathrm{g} / \mathrm{L})$ & 9 & $<2.000$ & $<0.200$ & -- & -- & - & -- & -- & -- \\
\hline 30217 & DIBROMOMETHANE, TOTAL $(\mu \mathrm{g} / \mathrm{L})$ & 9 & $<2.000$ & $<0.200$ & -- & -- & -- & -- & -- & -- \\
\hline 32101 & DICHLOROBROMOMETHANE， TOTAL $(\mu \mathrm{g} / \mathrm{L})$ & 9 & $<2.000$ & $<0.200$ & -- & -- & -- & -- & -- & -- \\
\hline 34668 & $\begin{array}{l}\text { DICHLORODIFLUOROMETHANE， TOTAL } \\
(\mu \mathrm{g} / \mathrm{L})\end{array}$ & 9 & $<2.000$ & $<0.200$ & -- & -- & -- & -- & -- & -- \\
\hline 77651 & 1,2 -DIBROMOETHANE, TOTAL $(\mu \mathrm{g} / \mathrm{L})$ & 9 & $<2.000$ & $<0.200$ & -- & -- & -- & -. & -. & -- \\
\hline 34418 & METHYLCHLORIDE，TOTAL $(\mu \mathrm{g} / \mathrm{L})$ & 9 & $<2.000$ & $<0.200$ & -- & -- & -- & -- & -- & -- \\
\hline 34704 & $\begin{array}{l}\text { CIS } 1,3 \text {-DICHLOROPROPENE, TOTAL } \\
(\mu \mathrm{g} / \mathrm{L})\end{array}$ & 9 & $<2.000$ & $<0.200$ & -- & -- & -- & -- & -- & -- \\
\hline 34699 & TRANS 1,3 -DICHLOROPROPENE, TOTAL $(\mu \mathrm{g} / \mathrm{L})$ & 9 & $<2.000$ & $<0.200$ & -- & -- & -- & -- & -- & -- \\
\hline 77128 & STYRENE, TOTAL $(\mu \mathrm{g} / \mathrm{L})$ & 9 & $<2.000$ & $<0.200$ & -- & -- & -- & -- & -- & -- \\
\hline 81551 & XYLENE, TOTAL $(\mu \mathrm{g} / \mathrm{L})$ & 9 & $<2.000$ & $<0.200$ & -- & -- & -- & -- & -- & -- \\
\hline 82625 & DIBROMOCHLOROPROPANE， TOTAL $(\mu \mathrm{g} / \mathrm{L})$ & 9 & $<10.000$ & $<1.000$ & -- & -- & -- & -- & -- & -- \\
\hline 77168 & 1, 1 -DICHLOROPROPENE，TOTAL $(\mu \mathrm{g} / \mathrm{L})$ & 9 & $<2.000$ & $<0.200$ & -- & -- & -. & -- & -- & -- \\
\hline 77170 & 2,2 -DICHLOROPROPANE, TOTAL $(\mu \mathrm{g} / \mathrm{L})$ & 9 & $<2.000$ & $<0.200$ & -- & -. & -- & -- & -- & -- \\
\hline 77173 & 1,3-DICHLOROPROPANE, TOTAL $(\mu \mathrm{g} / \mathrm{L})$ & 9 & $<2.000$ & $<0.200$ & -- & -- & -- & -- & -- & -- \\
\hline 77275 & 0-CHLOROTOLUENE， TOTAL $(\mu \mathrm{g} / \mathrm{L})$ & 9 & $<2.000$ & $<0.200$ & -- & -- & -- & -- & -- & -- \\
\hline 77277 & P-CHLOROTOLUENE, TOTAL $(\mu \mathrm{g} / \mathrm{L})$ & 9 & $<2.000$ & $<0.200$ & -- & -- & -- & -- & -. & -- \\
\hline 77443 & 123-TRICHLOROPROPANE, TOTAL $(\mu \mathrm{g} / \mathrm{L})$ & 9 & $<2.000$ & $<0.200$ & -- & -- & -- & -- & -- & -- \\
\hline 77562 & 1112 -TETRACHLOROETHANE, TOTAL $(\mu \mathrm{g} / \mathrm{L})$ & 9 & $<2.000$ & $<0.200$ & -- & -- & -- & -- & -- & -- \\
\hline 78032 & TERTBUTYL METHYL ETHER, TOTAL $(\mu \mathrm{g} / \mathrm{L})$ & 9 & $<2.000$ & $<0.200$ & -- & -- & -- & -- & -- & -- \\
\hline 77297 & BROMOCHLORO METHANE, TOTAL $(\mu \mathrm{g} / \mathrm{L})$ & 9 & $<2.000$ & $<0.200$ & -- & -- & -- & -- & -- & -- \\
\hline 77093 & CIS-1,2-DICHLOROETHENE, TOTAL $(\mu \mathrm{g} / \mathrm{L})$ & 9 & $<2.000$ & $<0.200$ & -- & -- & -- & -- & -- & -- \\
\hline 34576 & 2 -CHLOROETHYL VINYL ETHER, TOTAL $(\mu \mathrm{g} / \mathrm{L})$ & 6 & $<2.000$ & $<1.000$ & -- & -- & -- & -- & -- & -. \\
\hline 77223 & ISOPROPYL BENZENE, TOTAL $(\mu \mathrm{g} / \mathrm{L})$ & 9 & $<2.000$ & $<0.200$ & -- & -- & -- & -- & -- & -- \\
\hline 77224 & N-PROPYL BENZENE，TOTAL $(\mu \mathrm{g} / \mathrm{L})$ & 9 & $<2.000$ & $<0.200$ & -- & -- & -- & -- & -- & -- \\
\hline 77350 & SEC-BUTYL BENZENE, TOTAL $(\mu \mathrm{g} / \mathrm{L})$ & 9 & $<2.000$ & $<0.200$ & -- & - & -- & -- & -- & -- \\
\hline 77356 & P-ISOPROPYL TOLUENE, TOTAL $(\mu \mathrm{g} / \mathrm{L})$ & 9 & $<2.000$ & $<0.200$ & -- & -- & -- & -- & -- & -- \\
\hline 77342 & N-BUTYL BENZENE, TOTAL $(\mu \mathrm{g} / \mathrm{L}$ & 9 & $<2.000$ & $<0.200$ & -- & -- & $\ldots$ & -- & -- & -- \\
\hline 77613 & $1,2,3$-TRICHLOROBENZENE, TOTAL $(\mu \mathrm{g} / \mathrm{L}$ & 9 & $<2.000$ & $<0.200$ & -- & -- & -- & -- & -- & -- \\
\hline 77652 & FREON-1.13, TOTAL $(\mu \mathrm{g} / \mathrm{L}$ & 9 & $<2.000$ & $<0.200$ & -- & -- & -- & -- & -- & -- \\
\hline 77226 & MESITYLENE， TOTAL $(\mu \mathrm{g} / \mathrm{L}$ & 9 & $<2.000$ & $<0.200$ & -- & -. & -- & -- & -- & -- \\
\hline 81555 & BROMOBENZENE，TOTAL $(\mu \mathrm{g} / \mathrm{L}$ & 9 & $<2.000$ & $<0.200$ & -- & -- & -- & -- & -- & -- \\
\hline & & MET & TALS AND MI & CONSTITU & TS, TOTAL & & & & & \\
\hline 01097 & ANTIMONY, TOTAL $(\mu \mathrm{g} / \mathrm{L}$ as $\mathrm{Sb})$ & 33 & 20.000 & $<1.000$ & $2.714^{\star}$ & 19.000 & $<5.000$ & $<1.000$ & $<1.000$ & $<1.000$ \\
\hline 01002 & ARSENIC, TOTAL $(\mu \mathrm{g} / \mathrm{L}$ as As) & 33 & 59.000 & $<1.000$ & $9.719 *$ & 41.000 & 10.000 & 2.000 & $<1.000$ & $<1.000$ \\
\hline 01012 & BERYLLIUM, TOTAL ( $\mu \mathrm{g} / \mathrm{L}$ as Be) & 14 & 710.000 & $<10.000$ & -- & 710.000 & $<10.000$ & $<10.000$ & $<10.000$ & $<10.000$ \\
\hline 01027 & CADMIUM, TOTAL $(\mu \mathrm{g} / \mathrm{L}$ as $\mathrm{Cd})$ & 13 & $<1.000$ & $<1.000$ & -. & -- & -- & -- & -. & -- \\
\hline 01034 & CHROMIUM, TOTAL $(\mu \mathrm{g} / \mathrm{L}$ as $\mathrm{Cr})$ & 33 & 1100.000 & 6.000 & 173.000 & 862.000 & 155.000 & 49.000 & 18.500 & 6.700 \\
\hline 01042 & COPPER, TOTAL $(\mu \mathrm{g} / \mathrm{L}$ as $\mathrm{Cu})$ & 33 & 1200.000 & 3.000 & 154.606 & 969.000 & 175.000 & 36.000 & 11.500 & 3.000 \\
\hline 01051 & LEAD, TOTAL $(\mu \mathrm{g} / \mathrm{L}$ as $\mathrm{Pb})$ & 33 & 130.000 & 1.000 & 28.333 & 130.000 & 30.000 & 10.000 & 5.000 & 1.700 \\
\hline 71900 & MERCURY, TOTAL $(\mu \mathrm{g} / \mathrm{L}$ as $\mathrm{Hg})$ & 32 & 0.700 & $<0.100$ & $0.146^{\star}$ & 0.700 & 0.200 & $<0.100$ & $<0.100$ & $<0.100$ \\
\hline 01067 & NICKEL, TOTAL $(\mu \mathrm{g} / \mathrm{L}$ as Ni) & 33 & 1200.000 & 4.000 & 154.939 & 983.000 & 160.000 & 44.000 & 18.500 & 6.100 \\
\hline 01147 & SELENIUM, TOTAL $(\mu \mathrm{g} / \mathrm{L}$ as $\mathrm{Se})$ & 13 & 2.000 & $<1.000$ & -- & 2.000 & $<5.000$ & $<1.000$ & $<1.000$ & $<1.000$ \\
\hline 01077 & SILVER, TOTAL $(\mu \mathrm{g} / \mathrm{L}$ as Ag) & 13 & $<1.000$ & $<1.000$ & -- & -- & -- & -- & -- & -- \\
\hline 01092 & ZINC, TOTAL $(\mu \mathrm{g} / \mathrm{L}$ as $\mathrm{Zn})$ & 32 & 1800.000 & 20.000 & 227.813 & 1130.500 & 247.500 & 90.000 & 42.500 & 26.500 \\
\hline 00720 & CYANIDE, TOTAL (mg/L as Cn) & 13 & $<0.010$ & $<0.010$ & -- & -- & -- & -- & -- & -- \\
\hline & & ORGA & NIC COMPOU & PESTICIDE & DISSOLVEI & & & & & \\
\hline 46342 & ALACHLOR, DISSOLVED $(\mu \mathrm{g} / \mathrm{L})$ & 2 & 0.027 & $<0.002$ & -- & -- & -- & $\cdots$ & -- & -- \\
\hline 04040 & DEETHYL ATRAZINE，DISSOLVED $(\mu \mathrm{g} / \mathrm{L})$ & 2 & $<0.002$ & $<0.002$ & -- & -- & -- & -- & -- & -- \\
\hline 39632 & ATRAZINE，DISSOLVED $(\mu \mathrm{g} / \mathrm{L})$ & 2 & 0.010 & $<0.001$ & -- & -. & -- & $\ldots$ & -- & -- \\
\hline 82686 & METHYL AZINPHOS, DISSOLVED $(\mu \mathrm{g} / \mathrm{L})$ & 2 & $<0.001$ & $<0.001$ & -- & -- & -- & -- & -- & -- \\
\hline 82673 & BENFLURALIN, DISSOLVED $(\mu \mathrm{g} / \mathrm{L})$ & 2 & 0.011 & 0.006 & -. & -- & -- & -- & -- & -- \\
\hline 04028 & BUTYLATE, DISSOLVED $(\mu \mathrm{g} / \mathrm{L})$ & 2 & $<0.002$ & $<0.002$ & -- & -- & -- & -- & -- & - \\
\hline 82680 & CARBARYL， DISSOLVED $(\mu \mathrm{g} / \mathrm{L})$ & 2 & 0.310 & 0.045 & -- & -- & -- & -- & -- & -- \\
\hline 82674 & CARBOFURAN， DISSOLVED $(\mu \mathrm{g} / \mathrm{L})$ & 2 & $<0.003$ & $<0.003$ & -- & -- & -- & -- & -- & -- \\
\hline 38933 & CHLORPYRIFOS, DISSOLVED $(\mu \mathrm{g} / \mathrm{L})$ & 2 & 0.032 & $<0.004$ & -- & -- & -- & -- & -- & -- \\
\hline 04041 & CYANAZINE, DISSOLVED $(\mu \mathrm{g} / \mathrm{L})$ & 2 & $<0.004$ & $<0.004$ & -- & -- & -- & -- & -- & -- \\
\hline 82682 & DCPA， DISSOLVED $(\mu \mathrm{g} / \mathrm{L})$ & 2 & 0.013 & $<0.002$ & -- & $-\infty$ & -- & -- & -- & -- \\
\hline 34653 & P, $P^{\prime}$ DDE, DISSOLVED $(\mu \mathrm{g} / L)$ & 2 & $<0.006$ & $<0.006$ & -- & -- & -- & -- & -- & -- \\
\hline 39572 & DIAZINON， DISSOLVED $(\mu \mathrm{g} / \mathrm{L})$ & 2 & 0.110 & $<0.002$ & -- & -- & -- & -- & -- & -- \\
\hline 39381 & DIELDRIN，DISSOLVED $(\mu \mathrm{g} / \mathrm{L})$ & 2 & $<0.001$ & $<0.001$ & -- & -- & -- & -- & -- & -- \\
\hline 82660 & $\begin{array}{l}\text { 2, 6-DIETHYL ANILINE, DISSOLVED } \\
(\mu \mathrm{g} / \mathrm{L})\end{array}$ & 2 & $<0.003$ & $<0.003$ & -- & -- & -- & -- & -- & -- \\
\hline 82677 & DISULFOTON， DISSOLVED $(\mu \mathrm{g} / \mathrm{L})$ & 2 & $<0.017$ & $<0.017$ & -- & -- & -. & -- & -- & -- \\
\hline
\end{tabular}

NOTE: Multiple detection limits during the period of record may result in different values flagged with a "<."

* Value is estimated by using a log-probability regression to predict the values of data below the detection limit. 
Table 73. Statistical summary of water-quality data at site 43 (CSW07), June 1994 through June 1997—Continued

\begin{tabular}{|c|c|c|c|c|c|c|c|c|c|c|}
\hline \multirow{2}{*}{$\begin{array}{c}\text { PARAM- } \\
\text { ETER } \\
\text { CODE }\end{array}$} & \multirow{2}{*}{ PROPERTY OR CONSTITUENT } & \multicolumn{4}{|c|}{ DESCRIPTIVE STATISTICS } & PERCEN & $\begin{array}{r}\text { AMPLE } \\
\text { EQ }\end{array}$ & $\begin{array}{l}\text { VHICH VAI } \\
\text { TO THOSE }\end{array}$ & WERE & AN OR \\
\hline & & $\begin{array}{l}\text { SAMPLE } \\
\text { SIZE }\end{array}$ & MAXIMUM & MINIMUM & MEAN & $95 \%$ & $75 \%$ & $\begin{array}{c}50 \% \\
\text { (median) }\end{array}$ & $25 \%$ & $5 \%$ \\
\hline 82668 & EPTC, DISSOLVED $(\mu \mathrm{g} / \mathrm{L})$ & 2 & $<0.002$ & $<0.002$ & -- & -- & -- & -- & -- & -- \\
\hline 82663 & ETHALFLURALIN, DISSOLVED $(\mu \mathrm{g} / \mathrm{L})$ & 2 & $<0.004$ & $<0.004$ & -- & -- & -- & -- & -- & -- \\
\hline 82672 & ETHOPROP， DISSOLVED $(\mu \mathrm{g} / \mathrm{L})$ & 2 & $<0.003$ & $<0.003$ & -- & -- & -- & -- & -- & -- \\
\hline 04095 & FONOFOS, DISSOLVED $(\mu \mathrm{g} / \mathrm{L})$ & 2 & $<0.003$ & $<0.003$ & -- & -- & -- & -- & -- & -- \\
\hline 34253 & ALPHA BHC, DISSOLVED $(\mu \mathrm{g} / \mathrm{L})$ & 2 & $<0.002$ & $<0.002$ & -- & -- & -- & -- & -- & -- \\
\hline 39341 & LINDANE, DISSOLVED $(\mu \mathrm{g} / \mathrm{L})$ & 2 & $<0.004$ & $<0.004$ & -- & -- & -- & -- & -- & -- \\
\hline 82666 & LINURON, DISSOLVED $(\mu \mathrm{g} / \mathrm{L})$ & 2 & $<0.002$ & $<0.002$ & +- & -- & -- & -- & -- & -- \\
\hline 39532 & MALATHION, DISSOLVED $(\mu \mathrm{g} / \mathrm{L})$ & 2 & $<0.005$ & $<0.005$ & -- & -- & -- & -- & -- & -- \\
\hline 82667 & METHYL PARATHION, DISSOLVED $(\mu \mathrm{g} / \mathrm{L})$ & 2 & $<0.006$ & $<0.006$ & -- & -- & -- & -- & -- & -- \\
\hline 39415 & METOLACHLOR, DISSOLVED $(\mu \mathrm{g} / \mathrm{L})$ & 2 & 0.038 & 0.021 & -- & -- & -- & -- & -- & -- \\
\hline 82630 & METRIBUZIN，DISSOLVED $(\mu \mathrm{g} / \mathrm{L})$ & 2 & $<0.030$ & $<0.004$ & -- & -- & -- & -- & -- & -- \\
\hline 82671 & MOLINATE, DISSOLVED $(\mu \mathrm{g} / \mathrm{L})$ & 2 & $<0.004$ & $<0.004$ & -- & -- & -- & -- & -- & -- \\
\hline 82684 & NAPROPAMIDE， DISSOLVED $(\mu \mathrm{g} / \mathrm{L})$ & 2 & $<0.003$ & $<0.003$ & -- & -- & -- & -- & -- & -- \\
\hline 39542 & PARATHION， DISSOLVED $(\mu \mathrm{g} / \mathrm{L})$ & 2 & $<0.004$ & $<0.004$ & -- & -- & -- & -- & -- & -- \\
\hline 82669 & PEBULATE， DISSOLVED $(\mu \mathrm{g} / \mathrm{L})$ & 2 & $<0.004$ & $<0.004$ & -- & -- & -- & -- & -- & -- \\
\hline 82683 & PENDIMETHALIN, DISSOLVED $(\mu \mathrm{g} / \mathrm{L})$ & 2 & 0.094 & $<0.004$ & -+ & -- & -- & -- & -- & -- \\
\hline 82687 & PERMETHRIN， DISSOLVED $(\mu \mathrm{g} / \mathrm{L})$ & 2 & $<0.005$ & $<0.005$ & -- & -- & -- & -- & -- & -- \\
\hline 82664 & PHORATE， DISSOLVED $(\mu \mathrm{g} / \mathrm{L})$ & 2 & $<0.002$ & $<0.002$ & -- & -- & -- & -- & -- & -- \\
\hline 82676 & PRONAMIDE， DISSOLVED $(\mu \mathrm{g} / \mathrm{L})$ & 2 & $<0.060$ & $<0.003$ & -- & -- & -- & -- & -- & -- \\
\hline 04037 & PROMETON, DISSOLVED $(\mu \mathrm{g} / \mathrm{L})$ & 2 & $<0.018$ & $<0.018$ & -- & -- & -- & -- & -- & -- \\
\hline 04024 & PROPACHLOR， DISSOLVED $(\mu \mathrm{g} / \mathrm{L})$ & 2 & $<0.007$ & $<0.007$ & -- & -- & -- & -- & -- & -- \\
\hline 82679 & PROPANIL，DISSOLVED $(\mu \mathrm{g} / \mathrm{L})$ & 2 & $<0.004$ & $<0.004$ & -- & -- & -- & -- & -- & -- \\
\hline 82685 & PROPARGITE, DISSOLVED $(\mu \mathrm{g} / \mathrm{L})$ & 2 & $<0.013$ & $<0.013$ & -- & -- & -- & -- & -- & -- \\
\hline 04035 & SIMAZINE, DISSOLVED $(\mu \mathrm{g} / \mathrm{L})$ & 2 & $<0.005$ & $<0.005$ & -- & -- & -- & -- & -- & -- \\
\hline 82681 & THIOBENCARB，DISSOLVED $(\mu \mathrm{g} / \mathrm{L})$ & 2 & $<0.002$ & $<0.002$ & -- & -- & -- & -- & -- & -- \\
\hline 82670 & TEBUTHIURON， DISSOLVED $(\mu \mathrm{g} / \mathrm{L})$ & 2 & $<0.010$ & $<0.010$ & -- & -- & -- & -- & -- & -- \\
\hline 82665 & TERBACIL， DISSOLVED $(\mu \mathrm{g} / \mathrm{L})$ & 2 & $<0.007$ & $<0.007$ & -- & -- & -- & -- & -- & -- \\
\hline 82675 & TERBUFOS, DISSOLVED $(\mu \mathrm{g} / \mathrm{L})$ & 2 & $<0.013$ & $<0.013$ & -- & -- & -- & -- & -- & -- \\
\hline 82678 & TRIALLATE, DISSOLVED $(\mu \mathrm{g} / \mathrm{L})$ & 2 & $<0.001$ & $<0.001$ & -- & -- & -- & -- & -- & -- \\
\hline 82661 & TRIFLURALIN， DISSOLVED $(\mu \mathrm{g} / \mathrm{L})$ & 2 & 0.008 & 0.005 & -- & -- & -- & -- & -- & -- \\
\hline 39742 & $2,4,5-T$, DISSOLVED $(\mu \mathrm{g} / \mathrm{L})$ & 2 & $<0.035$ & $<0.035$ & -- & -- & -- & -- & -- & -- \\
\hline 39732 & $2,4-D$, DISSOLVED $(\mu \mathrm{g} / \mathrm{L})$ & 2 & $<0.035$ & $<0.035$ & -- & -- & -- & -- & -- & -- \\
\hline 38746 & $2,4-\mathrm{DB}$, DISSOLVED $(\mu \mathrm{g} / \mathrm{L})$ & 2 & $<0.035$ & $<0.035$ & -- & -- & -- & -- & -- & - \\
\hline 49315 & ACIFLUORFEN, DISSOLVED $(\mu \mathrm{g} / \mathrm{L})$ & 2 & $<0.035$ & $<0.035$ & -- & -- & -- & -- & -- & -- \\
\hline 49312 & ALDICARB， DISSOLVED $(\mu \mathrm{g} / \mathrm{L})$ & 2 & $<0.016$ & $<0.016$ & -- & -- & -- & -- & -- & -- \\
\hline 49313 & ALDICARB SULFONE, DISSOLVED $(\mu \mathrm{g} / \mathrm{L})$ & 2 & $<0.016$ & $<0.016$ & -- & -- & -- & -- & -- & -- \\
\hline 49314 & $\begin{array}{l}\text { ALDICARB SULFOXIDE, DISSOLVED } \\
(\mu \mathrm{g} / \mathrm{L})\end{array}$ & 2 & $<0.021$ & $<0.021$ & -- & -- & - & - & -- & - \\
\hline 38711 & BENTAZON, DISSOLVED $(\mu \mathrm{g} / \mathrm{L})$ & 2 & $<0.014$ & $<0.014$ & -- & -- & -- & - & -- & -- \\
\hline 04029 & BROMACIL， DISSOLVED $(\mu \mathrm{g} / \mathrm{L})$ & 2 & $<0.035$ & $<0.035$ & -- & -- & -- & -- & -- & -- \\
\hline 49311 & BROMOXYNIL， DISSOLVED $(\mu \mathrm{g} / \mathrm{L})$ & 2 & $<0.035$ & $<0.035$ & -- & - & -- & -- & -- & -- \\
\hline 49310 & CARBARYL， DISSOLVED $(\mu \mathrm{g} / \mathrm{L})$ & 2 & 0.150 & $<0.008$ & -- & -- & -- & -- & -- & -- \\
\hline 49309 & CARBOFURAN, DISSOLVED $(\mu \mathrm{g} / \mathrm{L})$ & 2 & $<0.028$ & $<0.028$ & -- & -- & -- & -- & -- & -- \\
\hline 49308 & 3-HYDROXY-CARBOFURAN $(\mu \mathrm{g} / \mathrm{L})$ & 2 & $<0.014$ & $<0.014$ & -- & -- & -- & -- & -- & -- \\
\hline 49307 & AMIBEN, DISSOLVED $(\mu \mathrm{g} / \mathrm{L})$ & 2 & $<0.011$ & $<0.011$ & -- & -- & - & -- & -- & -- \\
\hline 49306 & CHLOROTHALONIL， DISSOLVED $(\mu \mathrm{g} / \mathrm{L})$ & 2 & $<0.035$ & $<0.035$ & -- & -- & -- & -- & -- & -- \\
\hline 49305 & CLOPYRALID， DISSOLVED $(\mu \mathrm{g} / \mathrm{L})$ & 2 & $<0.050$ & $<0.050$ & -- & -- & -- & -- & -- & -- \\
\hline 49304 & DACTHALMONO-ACID，DISSOLVED $(\mu \mathrm{g} / \mathrm{L})$ & 2 & $<0.017$ & $<0.017$ & -- & -- & -- & -- & -- & -- \\
\hline 38442 & DICAMBA， DISSOLVED $(\mu \mathrm{g} / \mathrm{L})$ & 2 & $<0.035$ & $<0.035$ & -- & -- & -- & -- & -- & -- \\
\hline 49303 & DICHLOBENIL， DISSOLVED $(\mu \mathrm{g} / \mathrm{L})$ & 2 & $<0.020$ & $<0.020$ & -- & -- & -- & -- & -- & - \\
\hline 49302 & DICHLORPROP， DISSOLVED $(\mu \mathrm{g} / \mathrm{L})$ & 2 & $<0.032$ & $<0.032$ & -- & -- & -- & -- & -- & -- \\
\hline 49301 & DINOSEB， DISSOLVED $(\mu \mathrm{g} / \mathrm{L})$ & 2 & $<0.035$ & $<0.035$ & -- & -- & -- & -- & -- & -- \\
\hline 49300 & DIURON， DISSOLVED $(\mu \mathrm{g} / \mathrm{L})$ & 2 & $<0.020$ & $<0.020$ & -- & -- & -- & -- & -- & -- \\
\hline 49299 & $\begin{array}{l}\text { 4, 6-DINITRO OCRESOL, DISSOLVED } \\
(\mu \mathrm{g} / \mathrm{L})\end{array}$ & 2 & $<0.035$ & $<0.035$ & - & -- & -- & - & -- & -- \\
\hline 49298 & ESFENVALERATE， DISSOLVED $(\mu \mathrm{g} / \mathrm{L})$ & 2 & $<0.019$ & $<0.019$ & -- & -- & -- & -- & -- & -- \\
\hline 49297 & FENURON， DISSOLVED $(\mu \mathrm{g} / \mathrm{L})$ & 2 & $<0.013$ & $<0.013$ & -- & -- & -- & -- & -- & -- \\
\hline 38811 & FLUOMETURON， DISSOLVED $(\mu \mathrm{g} / \mathrm{L})$ & 2 & $<0.035$ & $<0.035$ & -- & -- & -- & -- & -- & -- \\
\hline 38478 & LINURON, DISSOLVED $(\mu \mathrm{g} / \mathrm{L})$ & 2 & $<0.018$ & $<0.018$ & -- & -- & -- & -- & -- & -- \\
\hline 38482 & MCPA， DISSOLVED $(\mu \mathrm{g} / \mathrm{L})$ & 2 & $<0.050$ & $<0.050$ & -- & -- & -- & -- & -- & -- \\
\hline 38487 & MCPB， DISSOLVED $(\mu \mathrm{g} / \mathrm{L})$ & 2 & $<0.035$ & $<0.035$ & -- & -- & -- & - & -- & -- \\
\hline 38501 & METHIOCARB， DISSOLVED $(\mu \mathrm{g} / \mathrm{L})$ & 2 & $<0.026$ & $<0.026$ & -- & -- & -- & -- & -- & -- \\
\hline 49296 & METHOMYL， DISSOLVED $(\mu \mathrm{g} / \mathrm{L})$ & 2 & $<0.017$ & $<0.017$ & -- & -- & -- & -- & -- & -- \\
\hline 49295 & 1-NAPHTHOL， DISSOLVED $(\mu \mathrm{g} / \mathrm{L})$ & 2 & $<0.007$ & $<0.007$ & -- & -- & -- & - & - & -- \\
\hline 49294 & NEBURON， DISSOLVED $(\mu \mathrm{g} / \mathrm{L})$ & 2 & $<0.015$ & $<0.015$ & -- & -- & -- & -- & -- & -- \\
\hline 49293 & NORFLURAZON， DISSOLVED $(\mu \mathrm{g} / \mathrm{L})$ & 2 & $<0.024$ & $<0.024$ & -- & -- & - & -- & -- & -- \\
\hline 49292 & ORYZALIN， DISSOLVED $(\mu \mathrm{g} / \mathrm{L})$ & 2 & $<0.019$ & $<0.019$ & -- & -- & -- & -- & -- & -- \\
\hline 38866 & OXAMYL，DISSOLVED $(\mu \mathrm{g} / \mathrm{L})$ & 2 & $<0.018$ & $<0.018$ & -- & -- & -+ & - & -- & -- \\
\hline 49291 & PICLORAM， DISSOLVED $(\mu \mathrm{g} / \mathrm{L})$ & 2 & $<0.050$ & $<0.050$ & -- & -- & -- & -- & -- & -- \\
\hline 49236 & PROPHAM， DISSOLVED $(\mu \mathrm{g} / \mathrm{L})$ & 2 & $<0.035$ & $<0.035$ & -- & -- & -- & -- & -- & -- \\
\hline 38538 & PROPOXUR， DISSOLVED $(\mu \mathrm{g} / \mathrm{L})$ & 2 & $<0.035$ & $<0.035$ & -- & -- & -- & -- & -- & -- \\
\hline 39762 & SILVEX， DISSOLVED $(\mu \mathrm{g} / \mathrm{L})$ & 2 & $<0.021$ & $<0.021$ & -- & -- & -- & -- & -- & -- \\
\hline 49235 & TRICLOPYR, DISSOLVED $(\mu \mathrm{g} / \mathrm{L})$ & 2 & $<0.050$ & $<0.050$ & -- & -- & -- & -- & -- & -- \\
\hline & & ORGAI & IC COMPOUN & RGANONIT & GEN, TO & & & & & \\
\hline 39057 & PROMETRYNE, TOTAL $(\mu \mathrm{g} / \mathrm{L})$ & 1 & $<0.100$ & -- & -- & -- & -- & -- & -- & -- \\
\hline 39056 & PROMETONE, TOTAL $(\mu \mathrm{g} / \mathrm{L})$ & 1 & $<0.200$ & -- & -- & -- & -- & -- & -- & -- \\
\hline 39054 & SIMETRYNE，TOTAL $(\mu \mathrm{g} / \mathrm{L})$ & 1 & $<0.100$ & -- & -- & -- & -- & -- & -- & -- \\
\hline 81757 & CYANAZINE, TOTAL $(\mu \mathrm{g} / \mathrm{L})$ & 1 & $<0.200$ & -- & -- & -- & -- & -- & -- & -- \\
\hline 77825 & ALACHLOR，TOTAL $(\mu \mathrm{g} / \mathrm{L})$ & 1. & $<0.100$ & -- & -- & -- & -- & -- & -- & -- \\
\hline 82611 & METRIBUZIN, TOTAL $(\mu \mathrm{g} / \mathrm{L})$ & 1 & $<0.100$ & -- & -- & -- & -- & -- & -- & -- \\
\hline 30311 & TERBACIL, TOTAL $(\mu \mathrm{g} / \mathrm{L})$ & 1 & $<0.200$ & -- & -- & -- & -- & -- & -- & -- \\
\hline
\end{tabular}

NOTE: Multiple detection limits during the period of record may result in different values flagged with a "<."

* Value is estimated by using a log-probability regression to predict the values of data below the detection limit. 
Table 73. Statistical summary of water-quality data at site 43 (CSW07), June 1994 through June 1997-Continued

\begin{tabular}{|c|c|c|c|c|c|c|c|c|c|c|}
\hline \multirow{2}{*}{$\begin{array}{l}\text { PARAM- } \\
\text { ETER } \\
\text { CODE }\end{array}$} & \multirow{2}{*}{ PROPERTY OR CONSTITUENT } & \multicolumn{4}{|c|}{ DESCRIPTIVE STATISTICS } & \multicolumn{5}{|c|}{$\begin{array}{c}\text { PERCENT OF SAMPLES IN WHICH VALUES WERE LESS THAN OR } \\
\text { EQUAL TO THOSE SHOWN }\end{array}$} \\
\hline & & $\begin{array}{l}\text { SAMPLE } \\
\text { SIZE }\end{array}$ & MAXIMUM & MINIMUM & MEAN & $95 \%$ & $75 \%$ & $\begin{array}{c}50 \% \\
\text { (median) }\end{array}$ & $25 \%$ & $5 \%$ \\
\hline 30245 & CARBOXIN, TOTAL $(\mu \mathrm{g} / \mathrm{L})$ & 1 & $<0.200$ & -- & - & -- & -- & -- & -- & -- \\
\hline 30264 & HEXAZINONE, TOTAL $(\mu \mathrm{g} / \mathrm{L})$ & 1 & $<0.200$ & -- & -- & -- & -- & -- & -- & -- \\
\hline 30235 & BUTACHLOR， TOTAL $\langle\mu \mathrm{g} / \mathrm{L})$ & 1 & $<0.100$ & -- & -- & -- & -- & -- & -- & -- \\
\hline 30236 & BUTYLATE, TOTAL $(\mu \mathrm{g} / \mathrm{L})$ & 1 & 0.200 & -- & -- & -- & -- & -- & -- & -- \\
\hline 75981 & DE-ETHYLATRAZINE，TOTAL $(\mu \mathrm{g} / \mathrm{L})$ & 1 & $<0.200$ & -- & -- & -- & -- & -- & -- & -- \\
\hline 39630 & ATRAZINE, TOTAL $(\mu \mathrm{g} / \mathrm{L})$ & 1 & 0.200 & -- & -- & -- & -- & -- & -- & -- \\
\hline 39055 & SIMAZINE, TOTAL $(\mu \mathrm{g} / \mathrm{L})$ & 1 & 0.200 & -- & -- & -- & -- & -- & -- & -- \\
\hline 39024 & PROPAZINE, TOTAL $(\mu \mathrm{g} / \mathrm{L})$ & 1 & $<0.100$ & -- & -. & -- & -- & -- & -- & -- \\
\hline 82184 & AMETRYNE， TOTAL $(\mu \mathrm{g} / \mathrm{L})$ & 1 & $<0.100$ & -- & -- & -- & -- & -- & -- & -- \\
\hline 39030 & TRIFLURALIN， TOTAL $(\mu \mathrm{g} / \mathrm{L})$ & 1 & $<0.100$ & -- & -- & -- & -- & -- & -- & -- \\
\hline 82612 & METOLACHLOR, TOTAL $(\mu \mathrm{g} / \mathrm{L})$ & 1 & $<0.200$ & -- & -- & -- & -- & -- & -- & -- \\
\hline 30234 & BROMACIL, TOTAL $(\mu \mathrm{g} / \mathrm{L})$ & 1 & 0.300 & -- & -- & -- & -- & -- & -- & -- \\
\hline 30255 & DIPHENAMID, TOTAL $(\mu \mathrm{g} / \mathrm{L})$ & 1 & $<0.100$ & -- & -- & -- & -. & -- & -- & -- \\
\hline 30324 & VERNOLATE, TOTAL $(\mu \mathrm{g} / \mathrm{L})$ & 1 & $<0.100$ & -- & -- & -- & -- & -- & -- & -- \\
\hline 30254 & CYCLOATE, TOTAL $(\mu \mathrm{g} / \mathrm{L})$ & 1 & $<0.100$ & -- & -- & -- & -- & -- & -- & -- \\
\hline 30295 & PROPACHLOR, TOTAL $(\mu \mathrm{g} / \mathrm{L})$ & 1 & $<0.100$ & -- & -- & -- & -- & -- & -- & -- \\
\hline 75980 & DE-ISOPROPYLATRAZIN，TOTAL $(\mu \mathrm{g} / \mathrm{L})$ & 1. & $<0.200$ & -- & -- & -- & -- & -- & -- & -- \\
\hline \multicolumn{11}{|c|}{ ORGANIC COMPOUNDS-HERBICIDES, TOTAL } \\
\hline 39730 & $2,4-\mathrm{D}$, TOTAL $(\mu \mathrm{g} / \mathrm{L})$ & 1 & 0.740 & -- & -- & -- & -- & -- & -- & -- \\
\hline 39760 & SILVEX，TOTAL $(\mu \mathrm{g} / \mathrm{L})$ & 1 & $<0.010$ & -- & -- & -- & -- & -- & -- & -- \\
\hline 39720 & PICLORAM, TOTAL $(\mu \mathrm{g} / \mathrm{L})$ & 1 & $<0.010$ & -- & -- & -- & -- & -- & -- & -- \\
\hline 39740 & $2,4,5-\mathrm{T}$, TOTAL $(\mu \mathrm{g} / \mathrm{L})$ & 1 & $<0.010$ & -- & -- & -- & -- & -- & -- & -- \\
\hline 82183 & $2,4-\mathrm{DP}$, TOTAL $(\mu \mathrm{g} / \mathrm{L})$ & 1 & $<0.010$ & -- & -- & -- & -- & -- & -- & -- \\
\hline 82052 & DICAMBA， TOTAL $(\mu \mathrm{g} / \mathrm{L})$ & 1 & 0.170 & -- & -- & -- & -- & -- & -- & -- \\
\hline \multicolumn{11}{|c|}{ ORGANIC COMPOUNDS-CARBAMATE PESTICIDES, TOTAL } \\
\hline 39750 & SEVIN, TOTAL $(\mu \mathrm{g} / \mathrm{L})$ & 1 & $<0.500$ & -- & -- & -- & -- & -- & -- & -- \\
\hline 39051 & METHOMYL, TOTAL $(\mu \mathrm{g} / \mathrm{L})$ & 1 & $<0.500$ & -- & -- & -- & -- & -- & -- & -- \\
\hline 82619 & ALDICARD, TOTAL $(\mu \mathrm{g} / \mathrm{L})$ & 1 & $<0.500$ & -- & -- & -- & -- & -- & -- & -- \\
\hline 30296 & PROPOXUR， TOTAL $(\mu \mathrm{g} / \mathrm{L})$ & 1 & $<0.500$ & -- & -- & -- & -- & -- & -- & -- \\
\hline 39052 & PROPHAM， TOTAL $(\mu \mathrm{g} / \mathrm{L})$ & 1 & $<0.500$ & -- & -- & -- & -- & -- & -- & -- \\
\hline 82615 & CARBOFURAN， TOTAL $(\mu \mathrm{g} / \mathrm{L})$ & 1 & $<0.500$ & -- & -- & -- & -- & -- & -- & -- \\
\hline 77441 & 1-NAPHTHOL， TOTAL $(\mu \mathrm{g} / \mathrm{L})$ & 1 & $<0.500$ & -- & -- & -- & -- & -- & -- & -- \\
\hline 30282 & METHIOCARB， TOTAL $(\mu \mathrm{g} / \mathrm{L})$ & 1 & $<0.500$ & -- & -- & -- & -- & -- & -- & -- \\
\hline
\end{tabular}

NOTE: Multiple detection limits during the period of record may result in different values flagged with a "<."

* Value is estimated by using a log-probability regression to predict the values of data below the detection limit. 
Table 74. Statistical summary of water-quality data at site 44 (CSW10), November 1996 through September 1997

\begin{tabular}{|c|c|c|c|c|c|c|c|c|c|c|}
\hline \multirow{2}{*}{$\begin{array}{c}\text { PARAM- } \\
\text { ETER } \\
\text { CODE }\end{array}$} & \multirow{2}{*}{ PROPERTY OR CONSTITUENT } & \multicolumn{4}{|c|}{ DESCRIPTIVE STATISTICS } & \multicolumn{5}{|c|}{$\begin{array}{c}\text { PERCENT OF SAMPLES IN WHICH VALUES WERE LESS THAN OR } \\
\text { EQUAL TO THOSE SHOWN }\end{array}$} \\
\hline & & $\begin{array}{l}\text { SAMPLE } \\
\text { SIZE }\end{array}$ & MAXIMUM & MINIMUM & MEAN & $95 \%$ & $75 \%$ & $\begin{array}{c}50 \% \\
\text { (median) }\end{array}$ & $25 \%$ & $5 \%$ \\
\hline \multicolumn{11}{|c|}{ PHYSICAL AND CHEMICAL PROPERTIES } \\
\hline 00061 & INSTANTANEOUS DISCHARGE， $\left(\mathrm{ft}^{3} / \mathrm{s}\right)$ & 28 & 864.000 & 18.000 & 200.893 & 715.950 & 296.000 & 134.500 & 54.500 & 18.450 \\
\hline 00010 & WATER TEMPERATURE $\left({ }^{\circ} \mathrm{C}\right)$ & 28 & 25.500 & 3.000 & 11.250 & 25.275 & 13.000 & 11.500 & 5.375 & 3.225 \\
\hline 90095 & SPECIFIC CONDUCTANCE, LAB ( $\mu \mathrm{S} / \mathrm{cm}$ at $\left.25^{\circ} \mathrm{C}\right)$ & 21 & 142.000 & 59.000 & 99.000 & 141.800 & 115.500 & 99.000 & 76.500 & 59.000 \\
\hline 00095 & $\begin{array}{l}\text { SPECIFIC CONDUCTANCE, FIELD ( } \mu \text { S/ } \mathrm{cm} \text { at } 25 \\
{ }^{\circ} \mathrm{C} \text { ) }\end{array}$ & 27 & 143.000 & 22.000 & 100.037 & 138.600 & 120.000 & 104.000 & 85.000 & 35.600 \\
\hline 00403 & pH， LAB (STANDARD pH UNITS) & 21 & 7.600 & 6.700 & 7.138 & 7.590 & 7.400 & 7.100 & 6.950 & 6.700 \\
\hline 00400 & pH，FIELD (STANDARD pH UNITS) & 27 & 7.610 & 6.650 & 7.181 & 7.590 & 7.330 & 7.150 & 7.060 & 6.724 \\
\hline 90410 & ALKALINITY, LAB (mg/L as $\mathrm{CaCO}_{3}$ ) & 23 & 52.000 & 11.000 & 28.087 & 51.000 & 36.000 & 27.000 & 21.000 & 11.000 \\
\hline 80154 & SUSPENDED SEDIMENT $\langle\mathrm{mg} / \mathrm{L}\rangle$ & 24 & 2490.000 & 31.000 & 650.167 & 2372.500 & 922.000 & 425.500 & 176.250 & 46.250 \\
\hline 00530 & $\begin{array}{l}\text { RESIDUE ON EVAPORATION AT } 105^{\circ} \mathrm{C} \text {, SUSPENDED } \\
(\mathrm{mg} / \mathrm{L})\end{array}$ & 23 & 1790.000 & 17.000 & 492.217 & 1730.000 & 748.000 & 333.000 & 128.000 & 23.600 \\
\hline 00535 & RESIDUE VOLATILE, SUSPENDED $(\mathrm{mg} / \mathrm{L})$ & 23 & 230.000 & 1.000 & 62.826 & 226.000 & 102.000 & 44.000 & 16.000 & 2.400 \\
\hline 70300 & $\begin{array}{l}\text { DISSOLVED SOLIDS, RESIDUE AT } 180^{\circ} \mathrm{C} \\
(\mathrm{mg} / \mathrm{L})\end{array}$ & 23 & 103.000 & 44.000 & 73.261 & 101.400 & 86.000 & 74.000 & 62.000 & 44.800 \\
\hline 00310 & $\begin{array}{l}5 \text { DAY BIOCHEMICAL OXYGEN DEMAND } \\
(\mathrm{mg} / \mathrm{L})\end{array}$ & 23 & 15.000 & 2.000 & 6.935 & 14.600 & 9.600 & 5.900 & 4.100 & 2.140 \\
\hline 00340 & CHEMICAL OXYGEN DEMAND $(\mathrm{mg} / \mathrm{L})$ & 23 & 120.000 & 5.000 & 25.870 & 103.400 & 28.000 & 22.000 & 17.000 & 5.200 \\
\hline \multicolumn{11}{|c|}{ NUTRIENTS, TOTAL AND DISSOLVED } \\
\hline 00625 & $\begin{array}{l}\text { NITROGEN AMMONIA + ORGANIC, } \\
\text { TOTAL (mg/L as N) }\end{array}$ & 23 & 3.000 & 0.250 & 1.526 & 2.980 & 2.000 & 1.400 & 0.840 & 0.296 \\
\hline 00631 & $\mathrm{NO}_{2}+\mathrm{NO}_{3}$, DISSOLVED $(\mathrm{mg} / \mathrm{L}$ as $\mathrm{N})$ & 23 & 0.890 & 0.380 & 0.584 & 0.858 & 0.660 & 0.570 & 0.510 & 0.384 \\
\hline 00508 & $\begin{array}{l}\text { NITROGEN AMMONIA, DISSOLVED } \\
(\mathrm{mg} / \mathrm{L} \text { as N) }\end{array}$ & 23 & 0.470 & $<0.015$ & $0.163^{*}$ & 0.430 & 0.230 & 0.120 & 0.050 & $<0.015$ \\
\hline 00605 & NITROGEN ORGANIC, TOTAL (mg/L as N) & 23 & 2.800 & 0.250 & 1.363 & 2.760 & 1.800 & 1.200 & 0.780 & 0.288 \\
\hline 00600 & NITROGEN，TOTAL (mg/L as N) & 23 & 3.700 & 0.770 & 2.103 & 3.660 & 2.700 & 1.900 & 1.400 & 0.836 \\
\hline 00655 & PHOSPHORUS, TOTAL (mg/L as P) & 23 & 1.180 & 0.050 & 0.466 & 1.162 & 0.750 & 0.380 & 0.180 & 0.052 \\
\hline 00671 & $\begin{array}{l}\text { PHOSPHORUS ORTHO, DISSOLVED } \\
(\mathrm{mg} / \mathrm{L} \text { as } \mathrm{P})\end{array}$ & 23 & 0.110 & $<0.010$ & $0.039 *$ & 0.080 & 0.060 & 0.034 & 0.014 & $<0.010$ \\
\hline \multicolumn{11}{|c|}{ OIL AND GREASE, TOTAL } \\
\hline 00556 & OIL AND GREASE, TOTAL (mg/L) & 2 & 2.000 & $<1.000$ & -- & -- & -- & -- & -- & -- \\
\hline \multicolumn{11}{|c|}{ ORGANIC CARBON, TOTAL } \\
\hline 00580 & CARBON ORGANIC, TOTAL (mg/L) & 16 & 57.000 & 3.500 & 18.150 & 57.000 & 23.750 & 16.500 & 10.250 & 3.600 \\
\hline & & & & LIFORM & & & & & & \\
\hline 31679 & FECAL STREPTOCOCCI (Colonies per $100 \mathrm{~mL}$ ) & 10 & 420000.000 & 560.0001 & 51855.000 & 420000.000 & 372500.000 & 68000.000 & 9247.500 & 560.000 \\
\hline 31616 & FECAL COLIFORM (Colonies per $100 \mathrm{~mL}$ ) & 10 & 51000.000 & 180.000 & 21634.000 & 51000.000 & 38750.000 & 24500.000 & 495.000 & 180.000 \\
\hline & & ORC & GANIC COMPOUN & S-PESTICID & ES, TOTAL & & & & & \\
\hline 39330 & ALDRIN, TOTAL $(\mu \mathrm{g} / \mathrm{L})$ & 1 & $<0.010$ & -- & -- & -- & -- & -- & -- & -- \\
\hline 39340 & LINDANE, TOTAL $(\mu \mathrm{g} / \mathrm{L})$ & 1 & $<0.010$ & -- & -- & -- & -- & -- & -- & -- \\
\hline 39350 & CHLORDANE, TOTAL $(\mu \mathrm{g} / \mathrm{L})$ & 1 & $<0.100$ & -- & -- & -- & -- & -- & -- & -- \\
\hline 39370 & DDT, TOTAL $(\mu \mathrm{g} / \mathrm{L})$ & 1 & $<0.010$ & -- & -- & -- & -- & -- & -. & -- \\
\hline 39365 & $\mathrm{DDE}$, TOTAL $(\mu \mathrm{g} / \mathrm{L})$ & 1 & $<0.010$ & -- & -- & -- & -- & -- & -- & -- \\
\hline 39360 & DDD, TOTAL $(\mu \mathrm{g} / \mathrm{L})$ & 1 & $<0.010$ & -- & -- & -- & -- & -- & -- & -- \\
\hline 39380 & DIELDRIN, TOTAL $(\mu \mathrm{g} / \mathrm{L})$ & 1 & $<0.010$ & -- & -- & -- & -- & -- & -- & -- \\
\hline 39388 & ENDOSULFAN, TOTAL $\{\mu \mathrm{g} / \mathrm{L}\rangle$ & 1 & $<0.010$ & -. & -- & -- & -- & -- & -- & -- \\
\hline 39390 & ENDRIN, TOTAL $(\mu \mathrm{g} / \mathrm{L})$ & 1 & $<0.010$ & -- & -- & -- & -- & -- & - & -- \\
\hline 39410 & HEPTACHLOR, TOTAL $(\mu \mathrm{g} / \mathrm{L})$ & 1 & $<0.010$ & -- & -- & -- & -- & -- & -- & -- \\
\hline 39420 & HEPTACHLOR EPOXIDE, TOTAL $(\mu \mathrm{g} / \mathrm{L})$ & 1 & $<0.010$ & -- & -- & -- & -- & -- & -- & -- \\
\hline 39516 & PCB，TOTAL $(\mu \mathrm{g} / \mathrm{L})$ & 1 & $<0.100$ & -- & -- & -- & -- & -- & -- & -- \\
\hline 39400 & TOXAPHENE, TOTAL $(\mu \mathrm{g} / \mathrm{L})$ & 1 & $<1.000$ & -- & -- & -- & -- & -- & -. & -- \\
\hline 39034 & PERTHANE, TOTAL $(\mu \mathrm{g} / \mathrm{L})$ & 1 & $<0.100$ & -- & -- & -- & -- & -- & -- & -- \\
\hline 39570 & DIAZINON, TOTAL $(\mu \mathrm{g} / \mathrm{L})$ & 1 & 0.110 & -- & -- & -- & -- & -- & -- & -- \\
\hline 39398 & ETHION, TOTAL $(\mu \mathrm{g} / \mathrm{L})$ & 1 & $<0.010$ & -- & -- & -- & -- & -- & -- & -- \\
\hline 39530 & MALATHION, TOTAL $(\mu \mathrm{g} / \mathrm{L})$ & 1 & $<0.010$ & -- & -- & -- & -- & -- & -- & -- \\
\hline 39600 & METHYL PARATHION，TOTAL $(\mu \mathrm{g} / \mathrm{L})$ & 1 & $<0.010$ & -. & -- & -- & -- & -- & -- & -- \\
\hline 39540 & PARATHION, TOTAL $(\mu \mathrm{g} / \mathrm{L})$ & 1 & $<0.010$ & -- & -- & -- & -- & -- & -- & -- \\
\hline 39786 & TRITHION, TOTAL $(\mu \mathrm{g} / \mathrm{L})$ & 1 & $<0.010$ & -. & -- & -- & -- & -- & $\ldots$ & - \\
\hline 39250 & PCN, TOTAL $(\mu \mathrm{g} / \mathrm{L})$ & 1 & $<0.100$ & -- & -- & -- & -- & -- & -- & -- \\
\hline 39480 & METHOXYCHLOR， TOTAL $(\mu \mathrm{g} / \mathrm{L})$ & 1 & $<0.010$ & -- & -- & -- & -- & -- & -- & -- \\
\hline 39755 & MIREX，TOTAL $(\mu \mathrm{g} / \mathrm{L})$ & 1 & $<0.010$ & -. & -- & .. & -. & -. & -- & -- \\
\hline 39011 & DISYSTON, TOTAL $(\mu \mathrm{g} / \mathrm{L})$ & 1 & $<0.010$ & -- & -- & -- & -- & $\rightarrow-$ & -- & -- \\
\hline 39023 & PHORATE, TOTAL $(\mu \mathrm{g} / \mathrm{L})$ & 1 & $<0.010$ & -- & -- & -- & -- & -- & -- & -- \\
\hline 38932 & CHLORPYRIFOS, TOTAL $(\mu \mathrm{g} / \mathrm{L})$ & 1 & 0.010 & -- & -- & -- & -- & -- & -- & -- \\
\hline 39040 & DEF, TOTAL $(\mu \mathrm{g} / \mathrm{L})$ & 1 & $<0.010$ & -- & -- & -- & -- & -. & -- & -- \\
\hline 82614 & FONOFOS, TOTAL $(\mu \mathrm{g} / \mathrm{L})$ & 1 & $<0.010$ & -- & -- & -- & -- & -- & -- & -- \\
\hline & & & DLATILE ORG & COMPOU & TOTAL & & & & & \\
\hline 34030 & BENZENE, TOTAL $(\mu \mathrm{g} / \mathrm{L})$ & 1 & $<0.200$ & -- & -- & -- & -- & -- & -- & -- \\
\hline 32104 & BROMOFORM, TOTAL $(\mu \mathrm{g} / \mathrm{L})$ & 1 & $<0.200$ & -- & -- & -- & -- & -- & -- & -- \\
\hline 32102 & CARBON TETRACHLORIDE, TOTAL $(\mu \mathrm{g} / \mathrm{L})$ & 1 & $<0.200$ & -- & -- & -- & -. & -- & -- & -- \\
\hline 34301 & CHLOROBENZENE，TOTAL $(\mu \mathrm{g} / \mathrm{L})$ & 1 & $<0.200$ & -- & -- & -- & -- & -- & -- & -- \\
\hline 32105 & CHLORODIBROMOTHANE，TOTAL $(\mu \mathrm{g} / L)$ & 1 & $<0.200$ & -- & -- & -. & -- & -. & -- & -- \\
\hline 34311 & CHLOROETHANE, TOTAL $(\mu \mathrm{g} / \mathrm{L})$ & 1 & $<0.200$ & -- & -- & -- & -- & -- & -- & -- \\
\hline 32106 & CHLOROFORM，TOTAL $(\mu \mathrm{g} / \mathrm{L})$ & 1 & $<0.200$ & -- & -- & - & -- & -- & -- & -- \\
\hline 34496 & 1,1 -DICHLOROETHANE，TOTAL $(\mu \mathrm{g} / \mathrm{L})$ & 1 & $<0.200$ & -- & -. & -- & -- & $\ldots$ & -- & -- \\
\hline 32103 & 1,2 -DICHLOROETHANE, TOTAL $(\mu \mathrm{g} / \mathrm{L})$ & 1 & $<0.200$ & -- & -- & -- & -- & -- & -- & -- \\
\hline 34501 & 1,1-DICHLOROETHYLENE, TOTAL $(\mu \mathrm{g} / \mathrm{L})$ & 1 & $<0.200$ & -- & -- & -- & -- & -- & -- & -- \\
\hline 34541 & 1,2 -DICHLOROPROPANE, TOTAL $(\mu \mathrm{g} / \mathrm{L})$ & 1 & $<0.200$ & -- & -- & -- & -- & -- & -- & -- \\
\hline
\end{tabular}

NOTE: Multiple detection limits during the period of record may result in different values flagged with a "<."

* Value is estimated by using a log-probability regression to predict the values of data below the detection limit. 
Table 74. Statistical summary of water-quality data at site 44 (CSW10), November 1996 through September 1997-Continued

\begin{tabular}{|c|c|c|c|c|c|c|c|c|c|c|}
\hline \multirow{2}{*}{$\begin{array}{l}\text { PARAM- } \\
\text { ETER } \\
\text { CODE }\end{array}$} & \multirow{2}{*}{ PROPERTY OR CONSTITUENT } & \multicolumn{4}{|c|}{ DESCRIPTIVE STATISTICS } & \multicolumn{5}{|c|}{$\begin{array}{l}\text { PERCENT OF SAMPLES IN WHICH VALUES WERE LESS THAN OR } \\
\text { EQUAL TO THOSE SHOWN }\end{array}$} \\
\hline & & $\begin{array}{l}\text { SAMPLE } \\
\text { SIZE }\end{array}$ & MAXIMUM & MINIMUM & MEAN & $95 \%$ & $75 \%$ & $\begin{array}{c}50 \% \\
\text { (median) }\end{array}$ & $25 \%$ & $5 \%$ \\
\hline 34371 & ETHYLBENZENE, TOTAL $(\mu \mathrm{g} / \mathrm{L})$ & 1 & $<0.200$ & -- & -- & -- & -- & -- & -- & -- \\
\hline 34413 & METHYL BROMIDE, TOTAL $(\mu \mathrm{g} / \mathrm{L})$ & 1 & $<0.200$ & -- & -- & -- & -- & -- & -- & -- \\
\hline 34423 & METHYLENE CHLORIDE, TOTAL $(\mu \mathrm{g} / \mathrm{L})$ & 1 & $<0.200$ & -- & -- & -- & -- & -- & -- & -. \\
\hline 34516 & $1,1,2,2$-TETRACHLOROETHANE, TOTAL $(\mu \mathrm{g} / \mathrm{L})$ & 1 & $<0.200$ & -- & -- & -. & -- & -- & -- & -- \\
\hline 34475 & TETRACHLOROETHYLENE, TOTAL $(\mu \mathrm{g} / \mathrm{L})$ & 1 & $<0.200$ & - & -- & -- & -- & -- & -- & -- \\
\hline 34010 & TOLUENE, TOTAL $(\mu \mathrm{g} / \mathrm{L})$ & 1 & $<0.200$ & -- & -- & -- & -- & -- & -- & -- \\
\hline 34546 & $\begin{array}{l}1,2 \text {-TRANSDICHLOROETHENE， TOTAL } \\
(\mu \mathrm{g} / \mathrm{L})\end{array}$ & 1 & $<0.200$ & -- & - & -- & -- & -- & -- & -- \\
\hline 34506 & $1,1,1$-TRICHLOROETHANE, TOTAL $(\mu \mathrm{g} / \mathrm{L})$ & 1 & $<0.200$ & -- & -- & -- & -- & - & -- & -- \\
\hline 34511 & $1,1,2$-TRICHLOROETHANE, TOTAL $\langle\mu \mathrm{g} / \mathrm{L}\rangle$ & 1 & $<0.200$ & -- & -- & -- & -- & -- & -- & -- \\
\hline 39180 & TRICHLOROETHYLENE，TOTAL $\langle\mu \mathrm{g} / \mathrm{L}\rangle$ & 1 & $<0.200$ & -- & -- & -- & -- & -- & -. & -- \\
\hline 39175 & VINYL CHLORIDE, TOTAL $(\mu \mathrm{g} / \mathrm{L})$ & 1 & $<0.200$ & -- & -- & -- & -- & -- & -- & -- \\
\hline 30217 & DIBROMOMETHANE, TOTAL $(\mu \mathrm{g} / \mathrm{L})$ & 1 & $<0.200$ & -- & -- & -- & -- & -- & -- & -- \\
\hline 32101 & DICHLOROBROMOMETHANE, TOTAL $(\mu \mathrm{g} / \mathrm{L})$ & 1 & $<0.200$ & -- & -- & -- & -- & - & -- & -- \\
\hline 34668 & $\begin{array}{l}\text { DICHLORODIFLUOROMETHANE, TOTAL } \\
(\mu \mathrm{g} / \mathrm{L})\end{array}$ & 1 & $<0.200$ & -- & -- & -- & -- & -- & -- & -- \\
\hline 34488 & TRICHLOROFLUOROMETHANE，TOTAL $(\mu \mathrm{g} / \mathrm{L})$ & 1 & $<0.200$ & -- & -- & -- & -- & -- & -- & -. \\
\hline 77651 & 1,2-DIBROMOETHANE, TOTAL $(\mu \mathrm{g} / \mathrm{L})$ & 1 & $<0.200$ & -- & -- & -- & -- & -- & -- & -- \\
\hline 34418 & METHYLCHLORIDE, TOTAL $(\mu \mathrm{g} / \mathrm{L})$ & 1 & $<0.200$ & -- & - & -- & -- & -- & -- & -- \\
\hline 34704 & $\begin{array}{l}\text { CIS } 1,3-D I C H L O R O P R O P E N E, \text { TOTAL } \\
(\mu \mathrm{g} / \mathrm{L})\end{array}$ & 1 & $<0.200$ & -- & -- & -- & -- & -- & -- & -- \\
\hline 34699 & TRANS 1,3 -DICHLOROPROPENE, TOTAL $(\mu \mathrm{g} / \mathrm{L})$ & 1 & $<0.200$ & -- & $\ldots$ & -- & - & -- & -- & -- \\
\hline 77128 & STYRENE, TOTAL $(\mu \mathrm{g} / \mathrm{L})$ & 1 & $<0.200$ & -- & -- & -- & -- & -- & -- & -- \\
\hline 81551 & XYLENE, TOTAL $(\mu \mathrm{g} / L)$ & 1 & $<0.200$ & -- & -- & -- & -- & -- & -- & -- \\
\hline 82625 & DIBROMOCHLOROPROPANE, TOTAL $(\mu \mathrm{g} / \mathrm{L})$ & 1 & $<1.000$ & -- & -- & -- & -- & -- & -- & -- \\
\hline 77168 & 1,1 -DICHLOROPROPENE, TOTAL $(\mu \mathrm{g} / \mathrm{L})$ & 1 & $<0.200$ & -- & -. & -- & .. & -- & -- & -- \\
\hline 77170 & 2,2 -DICHLOROPROPANE, TOTAL $(\mu \mathrm{g} / \mathrm{L})$ & 1 & $<0.200$ & -- & -- & -- & -- & -- & -- & -- \\
\hline 77173 & 1,3 -DICHLOROPROPANE, TOTAL $(\mu \mathrm{g} / \mathrm{L})$ & 1 & $<0.200$ & -- & -- & -- & -- & -. & -- & -- \\
\hline 77275 & 0 -CHLOROTOLUENE, TOTAL $(\mu \mathrm{g} / \mathrm{L})$ & 1 & $<0.200$ & -- & -- & -- & -- & -- & -- & -- \\
\hline 77277 & P-CHLOROTOLUENE, TOTAL $(\mu \mathrm{g} / \mathrm{L})$ & 1 & $<0.200$ & -- & -- & -- & -- & -- & -- & - \\
\hline 77443 & 123-TRICHLOROPROPANE，TOTAL $(\mu \mathrm{g} / \mathrm{L})$ & 1 & $<0.200$ & -- & -- & -- & -- & -- & -- & -- \\
\hline 77562 & 1112 -TETRACHLOROETHANE, TOTAL $(\mu \mathrm{g} / \mathrm{L})$ & 1 & $<0.200$ & -- & $\ldots$ & -- & $\ldots$ & -- & -- & -- \\
\hline 78032 & TERTBUTYL METHYL ETHER, TOTAL $(\mu \mathrm{g} / \mathrm{L})$ & 1 & $<0.200$ & -- & -. & -- & -- & -- & -- & -- \\
\hline 77297 & BROMOCHLORO METHANE, TOTAL $(\mu \mathrm{g} / \mathrm{L})$ & 1 & $<0.200$ & -- & -- & -- & -- & -- & -- & -- \\
\hline 77093 & CIS-1,2-DICHLOROETHENE, TOTAL $\{\mu \mathrm{g} / \mathrm{L}\}$ & 1 & $<0.200$ & -- & -- & -- & -- & -- & -- & -- \\
\hline 77223 & ISOPROPYL BENZENE, TOTAL $(\mu \mathrm{g} / \mathrm{L})$ & 1 & $<0.200$ & -- & -- & -- & -- & -. & -- & -- \\
\hline 77224 & N-PROPYL BENZENE, TOTAL $(\mu \mathrm{g} / \mathrm{L})$ & 1 & $<0.200$ & -- & -- & -- & -- & -- & -- & -- \\
\hline 77353 & TERTBUTYL BENZENE, TOTAL $(\mu \mathrm{g} / \mathrm{L})$ & 1 & $<0.200$ & -- & -- & -- & -- & -- & -- & -- \\
\hline 77222 & PSEUDOCUMENE, TOTAL $(\mu \mathrm{g} / \mathrm{L})$ & 1 & $<0.200$ & $\ldots$ & -- & -- & -- & -- & -- & -- \\
\hline 77350 & SEC-BUTYL BENZENE, TOTAL $(\mu \mathrm{g} / \mathrm{L})$ & 1. & $<0.200$ & -- & -- & -- & -- & -- & -- & -- \\
\hline 77356 & P-ISOPROPYL TOLUENE, TOTAL $(\mu \mathrm{g} / \mathrm{L})$ & 1 & $<0.200$ & -. & -. & -- & -- & -- & -- & -- \\
\hline 77342 & N-BUTYL BENZENE, TOTAL $(\mu \mathrm{g} / \mathrm{L}$ & 1 & $<0.200$ & -- & $\therefore$ & -- & -. & -- & -- & -- \\
\hline 77613 & $1,2,3$-TRICHLOROBENZENE, TOTAL $(\mu \mathrm{g} / \mathrm{L}$ & 1. & $<0.200$ & .- & -- & -. & -. & -- & -- & -- \\
\hline 77652 & FREON-113, TOTAL $(\mu \mathrm{g} / \mathrm{L}$ & 1 & $<0.200$ & -- & -- & - & -- & -. & -- & -- \\
\hline 77226 & MESITYLENE, TOTAL $(\mu \mathrm{g} / \mathrm{L}$ & 1. & $<0.200$ & -- & -- & -- & -- & -- & -- & -- \\
\hline 81555 & BROMOBENZENE, TOTAL $(\mu \mathrm{g} / \mathrm{L}$ & 1 & $<0.200$ & -- & $\cdots$ & -- & -- & -- & -- & -- \\
\hline & & MET & ES AND MINO & CONSTITUEI & S, TOTAL & & & & & \\
\hline 01097 & ANTIMONY, TOTAL $(\mu \mathrm{g} / \mathrm{L}$ as $\mathrm{Sb})$ & 19 & 3.000 & $<1.000$ & -- & 3.000 & $<1.000$ & $<1.000$ & $<1.000$ & $<1.000$ \\
\hline 01002 & ARSENIC, TOTAL $(\mu \mathrm{g} / \mathrm{L}$ as As) & 19 & 12.000 & $<1.000$ & $3.002 *$ & 12.000 & 5.000 & 2.000 & $<1.000$ & $<1.000$ \\
\hline 01012 & BERYLLIUM, TOTAL $(\mu \mathrm{g} / \mathrm{L}$ as $\mathrm{Be})$ & 19 & $<10.000$ & $<10.000$ & -- & -- & -- & -- & -- & -- \\
\hline 01027 & CADMIUM, TOTAL $(\mu \mathrm{g} / \mathrm{L}$ as Cd $)$ & 19 & 1.000 & $<1.000$ & -- & 1.000 & $<1.000$ & $<1.000$ & $<1.000$ & $<1.000$ \\
\hline 01034 & CHROMIUM, TOTAL $(\mu \mathrm{g} / \mathrm{L}$ as $\mathrm{Cr})$ & 19 & 69.000 & 1.000 & 24.158 & 69.000 & 34.000 & 20.000 & 9.000 & 1.000 \\
\hline 01042 & COPPER, TOTAL $(\mu \mathrm{g} / \mathrm{L}$ as $\mathrm{Cu})$ & 19 & 110.000 & 3.000 & 52.158 & 110.000 & 84.000 & 49.000 & 20.000 & 3.000 \\
\hline 01051 & LEAD, TOTAL $(\mu \mathrm{g} / \mathrm{L}$ as $\mathrm{Pb})$ & 19 & 25.000 & 1.000 & 9.684 & 25.000 & 14.000 & 9.000 & 4.000 & 1.000 \\
\hline 71900 & MERCURY, TOTAL $(\mu \mathrm{g} / \mathrm{L}$ as $\mathrm{Hg})$ & 19 & 0.400 & $<0.100$ & $0.073 *$ & 0.400 & 0.100 & $<0.100$ & $<0.100$ & $<0.100$ \\
\hline 01067 & NICKEL, TOTAL $(\mu \mathrm{g} / \mathrm{L}$ as $\mathrm{Ni})$ & 19 & 25.000 & 2.000 & 8.947 & 25.000 & 9.000 & 8.000 & 5.000 & 2.000 \\
\hline 01147 & SELENIUM, TOTAL $(\mu \mathrm{g} / \mathrm{L}$ as Se) & 19 & $<1.000$ & $<1.000$ & -- & -- & -- & -- & -- & -- \\
\hline 01077 & SILVER, TOTAL $(\mu \mathrm{g} / \mathrm{L}$ as Ag) & 19 & 8.000 & $<1.000$ & -- & -- & -- & -- & -- & -- \\
\hline 01092 & $\mathrm{ZINC}$, TOTAL $(\mu \mathrm{g} / \mathrm{L}$ as $\mathrm{Zn})$ & 19 & 200.000 & 30.000 & 79.474 & 200.000 & 90.000 & 70.000 & 50.000 & 30.000 \\
\hline 00720 & CYANIDE, TOTAL (mg/L as Cn) & 17 & $<0.010$ & $<0.010$ & -- & -. & -- & -- & -- & -- \\
\hline & & ORGAN & C COMPOUNI & EESTICIDES & DISSOLVEL & & & & & \\
\hline 45342 & ALACHLOR, DISSOLVED $(\mu \mathrm{g} / \mathrm{L})$ & 1 & $<0.002$ & -- & -- & -- & -- & -- & -- & -- \\
\hline 04040 & DEETHYL ATRAZINE， DISSOLVED $(\mu \mathrm{g} / \mathrm{L})$ & 1 & 0.064 & -- & -- & -- & -- & -- & -- & $-\infty$ \\
\hline 39632 & ATRAZINE, DISSOLVED $(\mu \mathrm{g} / \mathrm{L})$ & 1 & 0.662 & -- & -- & -- & -- & -- & -- & -- \\
\hline 82686 & METHYL AZINPHOS, DISSOLVED $(\mu \mathrm{g} / \mathrm{L})$ & 1 & $<0.001$ & -- & -- & -- & -- & -- & -- & -- \\
\hline 82673 & BENFLURALIN, DISSOLVED $(\mu \mathrm{g} / \mathrm{L})$ & 1 & 0.009 & -- & -- & -- & -- & -- & -- & -- \\
\hline 04028 & BUTYLATE, DISSOLVED $(\mu \mathrm{g} / \mathrm{L})$ & 1 & $<0.002$ & -- & .. & -- & -- & -- & -- & -- \\
\hline 82680 & CARBARYL， DISSOLVED $\{\mu \mathrm{g} / \mathrm{L}\}$ & 1 & $<0.010$ & -- & -- & -- & -- & -- & -- & -- \\
\hline 82674 & CARBOFURAN, DISSOLVED $(\mu \mathrm{g} / \mathrm{L})$ & 1 & $<0.003$ & - & -- & -- & -- & -- & -- & -- \\
\hline 38933 & CHLORPYRIFOS，DISSOLVED $(\mu \mathrm{g} / \mathrm{L})$ & 1 & $<0.004$ & -- & -- & -- & -- & -- & -- & -. \\
\hline 04041 & CYANAZINE, DISSOLVED $(\mu \mathrm{g} / \mathrm{L})$ & 1 & $<0.004$ & -- & -- & -- & -- & -- & -- & -- \\
\hline 82682 & DCPA, DISSOLVED $(\mu \mathrm{g} / \mathrm{L})$ & 1 & 0.001 & -- & -- & -- & -- & -- & -- & -- \\
\hline 34653 & P, P' DDE, DISSOLVED $(\mu \mathrm{g} / \mathrm{L})$ & 1 & $<0.005$ & -- & -- & - & -- & - & -- & -- \\
\hline 39572 & DIAZINON, DISSOLVED $(\mu \mathrm{g} / \mathrm{L})$ & 1 & 0.119 & -- & -- & -- & -- & -- & -- & -- \\
\hline 39381 & DIELDRIN，DISSOLVED $(\mu \mathrm{g} / \mathrm{L})$ & 1 & $<0.001$ & -. & -- & - - & - & -- & -. & -- \\
\hline 82660 & $\begin{array}{l}2,6 \text {-DIETHYL ANILINE, DISSOLVED } \\
(\mu \mathrm{g} / \mathrm{L})\end{array}$ & 1 & $<0.003$ & -- & -- & -- & -- & -- & -- & -- \\
\hline 82677 & DISULFOTON， DISSOLVED $(\mu \mathrm{g} / \mathrm{L})$ & 1 & $<0.017$ & -- & -- & -- & -- & -- & -- & -- \\
\hline 82668 & EPTC, DISSOLVED $(\mu \mathrm{g} / \mathrm{L})$ & 1 & $<0.002$ & -- & -- & -- & -- & -- & -- & -- \\
\hline 82663 & ETHALFLURALIN, DISSOLVED $(\mu \mathrm{g} / \mathrm{L})$ & 1 & $<0.004$ & -- & -- & -. & -- & -- & -. & -- \\
\hline 82672 & ETHOPROP， DISSOLVED $(\mu \mathrm{g} / \mathrm{L})$ & 1 & $<0.003$ & -- & -- & -- & -- & -- & -- & -- \\
\hline
\end{tabular}

NOTE: Multiple detection limits during the period of record may result in different values flagged with a "<."

* Value is estimated by using a log-probability regression to predict the values of data below the detection limit. 
Table 74. Statistical summary of water-quality data at site 44 (CSW10), November 1996 through September 1997-Continued

\begin{tabular}{|c|c|c|c|c|c|c|c|c|c|c|}
\hline \multirow{2}{*}{$\begin{array}{c}\text { PARAM- } \\
\text { ETER } \\
\text { CODE }\end{array}$} & \multirow{2}{*}{ PROPERTY OR CONSTITUENT } & \multicolumn{4}{|c|}{ DESCRIPTIVE STATISTICS } & \multicolumn{5}{|c|}{$\begin{array}{c}\text { PERCENT OF SAMPLES IN WHICH VALUES WERE LESS THAN OR } \\
\text { EQUAL TO THOSE SHOWN }\end{array}$} \\
\hline & & $\begin{array}{l}\text { SAMPLE } \\
\text { SIZE }\end{array}$ & MAXIMUM & MINIMUM & MEAN & $95 \%$ & $75 \%$ & $\begin{array}{c}50 \% \\
\text { (median) }\end{array}$ & $25 \%$ & $5 \%$ \\
\hline 04095 & FONOFOS, DISSOLVED $(\mu \mathrm{g} / \mathrm{L})$ & 1 & $<0.003$ & -- & -- & -- & -- & -- & -- & -- \\
\hline 34253 & ALPHA BHC, DISSOLVED $(\mu \mathrm{g} / \mathrm{L})$ & 1 & $<0.002$ & -- & -- & -- & -- & -- & -- & -- \\
\hline 39341 & LINDANE，DISSOLVED $(\mu \mathrm{g} / \mathrm{L})$ & 1 & $<0.004$ & -- & -- & -- & -- & -- & -- & -- \\
\hline 82666 & LINURON，DISSOLVED $(\mu \mathrm{g} / \mathrm{L})$ & 1 & $<0.002$ & -- & -- & -- & -- & -. & -- & -- \\
\hline 39532 & MALATHION, DISSOLVED $(\mu \mathrm{g} / \mathrm{L})$ & 1 & $<0.005$ & -- & -- & -- & -- & -- & -- & -- \\
\hline 82667 & METHYL PARATHION, DISSOLVED $(\mu \mathrm{g} / \mathrm{L})$ & 1 & $<0.006$ & -- & -- & -- & -- & -- & -- & -- \\
\hline 3941.5 & METOLACHLOR, DISSOLVED $(\mu \mathrm{g} / \mathrm{L})$ & 1 & 0.118 & -- & -- & -- & -- & -- & -- & -- \\
\hline 82630 & METRIBUZIN， DISSOLVED $(\mu \mathrm{g} / \mathrm{L})$ & 1 & $<0.004$ & -- & -- & -- & -- & -- & -- & -- \\
\hline 82671 & MOLINATE， DISSOLVED $(\mu \mathrm{g} / \mathrm{L})$ & 1 & $<0.004$ & -- & -- & -- & -- & -- & -- & -- \\
\hline 82684 & NAPROPAMIDE， DISSOLVED $(\mu \mathrm{g} / \mathrm{L})$ & 1 & $<0.003$ & -- & -- & -- & -- & -- & -- & -- \\
\hline 39542 & PARATHION, DISSOLVED $(\mu \mathrm{g} / \mathrm{L})$ & 1 & $<0.004$ & -- & -- & -- & -- & -- & -- & -- \\
\hline 82669 & PEBULATE, DISSOLVED $(\mu \mathrm{g} / \mathrm{L})$ & 1 & $<0.004$ & -- & -- & -- & -- & -- & -- & -- \\
\hline 82683 & PENDIMETHALIN, DISSOLVED $(\mu \mathrm{g} / \mathrm{L})$ & 1 & 0.032 & -- & -- & -- & -- & -- & -- & -- \\
\hline 82687 & PERMETHRIN， DISSOLVED $(\mu \mathrm{g} / \mathrm{L})$ & 1 & $<0.005$ & -- & -- & -- & -- & -- & -- & -- \\
\hline 82664 & PHORATE， DISSOLVED $(\mu \mathrm{g} / \mathrm{L})$ & 1 & $<0.002$ & -- & -- & -- & -- & -- & -- & -- \\
\hline 82676 & PRONAMIDE， DISSOLVED $(\mu \mathrm{g} / \mathrm{L})$ & 1 & $<0.003$ & -- & -- & -- & -- & -- & - & -- \\
\hline 04037 & PROMETON， DISSOLVED $(\mu \mathrm{g} / \mathrm{L})$ & 1 & 0.011 & -- & -- & -- & -- & -- & -- & -- \\
\hline 04024 & PROPACHLOR, DISSOLVED $(\mu \mathrm{g} / \mathrm{L})$ & 1 & $<0.007$ & -- & -- & -- & - & -- & -- & -- \\
\hline 82679 & PROPANIL，DISSOLVED $(\mu \mathrm{g} / \mathrm{L})$ & 1 & $<0.004$ & -- & -- & -- & -- & -- & -- & -- \\
\hline 82685 & PROPARGITE，DISSOLVED $(\mu \mathrm{g} / \mathrm{L})$ & 1 & $<0.013$ & -- & -- & -- & -- & -- & - & -- \\
\hline 04035 & SIMAZINE, DISSOLVED $(\mu \mathrm{g} / \mathrm{L})$ & 1 & 0.041 & -- & -- & -- & -- & -- & -- & -- \\
\hline 82681 & THIOBENCARB， DISSOLVED $(\mu \mathrm{g} / \mathrm{L})$ & 1 & $<0.002$ & -- & -- & -- & - & -- & - & -- \\
\hline 82670 & TEBUTHIURON，DISSOLVED $(\mu \mathrm{g} / \mathrm{L})$ & 1 & 0.063 & -- & -- & -- & -- & -- & -- & -- \\
\hline 82665 & TERBACIL， DISSOLVED $(\mu \mathrm{g} / \mathrm{L})$ & 1 & $<0.007$ & -- & -- & -- & -- & -- & -- & -- \\
\hline 82675 & TERBUFOS, DISSOLVED $(\mu \mathrm{g} / \mathrm{L})$ & 1 & $<0.013$ & -- & -- & -- & -- & -- & -- & -- \\
\hline 82678 & TRIALLATE, DISSOLVED $(\mu \mathrm{g} / \mathrm{L})$ & 1 & $<0.001$ & -- & -- & -- & -- & -- & -- & -- \\
\hline 82661 & TRIFLURALIN, DISSOLVED $(\mu \mathrm{g} / \mathrm{L})$ & 1 & 0.010 & -- & -- & -- & -- & -- & -- & -- \\
\hline 39742 & $2,4,5-T, \quad$ DISSOLVED $(\mu \mathrm{g} / \mathrm{L})$ & 1 & $<0.035$ & -- & -- & -- & -- & -- & -- & -- \\
\hline 39732 & $2,4-\mathrm{D}$, DISSOLVED $(\mu \mathrm{g} / \mathrm{L})$ & 1 & 2.800 & -- & -- & -- & -- & -- & -- & -- \\
\hline 38746 & $2,4-D B ，$ DISSOLVED $(\mu \mathrm{g} / \mathrm{L})$ & 1 & $<0.035$ & -- & -- & -- & -- & -- & -- & -- \\
\hline 49315 & ACIFLUORFEN，DISSOLVED $(\mu \mathrm{g} / \mathrm{L})$ & 1 & $<0.035$ & -- & -- & -- & -- & -- & -- & -- \\
\hline 49312 & ALDICARB, DISSOLVED $(\mu \mathrm{g} / \mathrm{L})$ & 1 & $<0.016$ & -- & -- & -- & -- & -- & -- & - \\
\hline 49313 & ALDICARB SULFONE, DISSOLVED $(\mu \mathrm{g} / \mathrm{L})$ & 1 & $<0.016$ & -- & -- & -- & -- & -- & - & -- \\
\hline 49314 & $\begin{array}{l}\text { ALDICARB SULFOXIDE， DISSOLVED } \\
(\mu \mathrm{g} / \mathrm{L})\end{array}$ & 1 & $<0.021$ & -- & - & -- & -- & -- & -- & - \\
\hline 38711 & BENTAZON， DISSOLVED $(\mu \mathrm{g} / \mathrm{L})$ & 1 & $<0.014$ & -- & -- & -- & -- & -- & -- & -- \\
\hline 04029 & BROMACIL， DISSOLVED $(\mu \mathrm{g} / \mathrm{L})$ & 1 & $<0.035$ & -- & -- & -- & -- & -- & -- & -- \\
\hline 49311 & BROMOXYNIL， DISSOLVED $(\mu \mathrm{g} / \mathrm{L})$ & 1 & $<0.035$ & -- & -- & -- & -- & -- & -- & -- \\
\hline 49310 & CARBARYL, DISSOLVED $(\mu \mathrm{g} / \mathrm{L})$ & 1 & $<0.008$ & -- & -- & -- & -- & -- & -- & -- \\
\hline 49309 & CARBOFURAN, DISSOLVED $(\mu \mathrm{g} / \mathrm{L})$ & 1 & $<0.028$ & -- & -- & -- & -- & -- & -- & - \\
\hline 49308 & 3-HYDROXY-CARBOFURAN $(\mu \mathrm{g} / \mathrm{L})$ & 1 & $<0.014$ & -- & -- & -- & -- & -- & -- & - \\
\hline 49307 & AMIBEN， DISSOLVED $(\mu \mathrm{g} / \mathrm{L})$ & 1 & $<0.011$ & -- & -- & -- & -- & -- & -- & -- \\
\hline 49306 & CHLOROTHALONIL， DISSOLVED $(\mu \mathrm{g} / \mathrm{L})$ & 1. & $<0.035$ & -- & -- & -- & -- & -- & -- & -- \\
\hline 49305 & CLOPYRALID， DISSOLVED $(\mu \mathrm{g} / \mathrm{L})$ & 1. & $<0.050$ & -- & -- & -- & -- & -- & -- & -- \\
\hline 49304 & DACTHALMONO-ACID, DISSOLVED $(\mu \mathrm{g} / \mathrm{L})$ & 1 & $<0.017$ & -- & -- & -- & -- & -- & -- & -- \\
\hline 38442 & DICAMBA， DISSOLVED $(\mu \mathrm{g} / \mathrm{L})$ & 1 & $<0.035$ & -- & -- & -- & -- & -- & -- & -- \\
\hline 49302 & DICHLORPROP， DISSOLVED $(\mu \mathrm{g} / \mathrm{L})$ & 1. & $<0.032$ & -- & -- & -- & -- & -- & -- & -- \\
\hline 49301 & DINOSEB， DISSOLVED $(\mu \mathrm{g} / \mathrm{L})$ & 1 & $<0.035$ & -- & -- & -- & -- & -- & -- & -- \\
\hline 49300 & DIURON， DISSOLVED $(\mu \mathrm{g} / \mathrm{L})$ & 1. & $<0.020$ & -- & -- & -- & -- & -- & -- & -- \\
\hline 49299 & $\begin{array}{l}\text { 4, 6-DINITRO OCRESOL, DISSOLVED } \\
(\mu \mathrm{g} / \mathrm{L})\end{array}$ & 1. & $<0.035$ & -- & -- & -- & -- & -- & -- & -- \\
\hline 49298 & ESFENVALERATE， DISSOLVED $(\mu \mathrm{g} / \mathrm{L})$ & 1 & $<0.019$ & -- & - & -- & -- & -- & -- & -- \\
\hline 49297 & FENURON, DISSOLVED $(\mu \mathrm{g} / \mathrm{L})$ & 1. & $<0.013$ & -- & -- & -- & -- & -- & - & -- \\
\hline 38811 & FLUOMETURON， DISSOLVED $(\mu \mathrm{g} / \mathrm{L})$ & 1 & $<0.035$ & $-\infty$ & -- & -- & -- & -- & -- & -- \\
\hline 38478 & LINURON, DISSOLVED $(\mu \mathrm{g} / \mathrm{L})$ & 1 & $<0.018$ & -- & -- & -- & -- & -- & -- & -- \\
\hline 38482 & MCPA, DISSOLVED $(\mu \mathrm{g} / \mathrm{L})$ & 1 & 0.780 & -- & -- & -- & -- & -- & -- & -- \\
\hline 38487 & MCPB, DISSOLVED $(\mu \mathrm{g} / \mathrm{L})$ & 1 & $<0.035$ & -- & -- & -- & -- & -- & -- & -- \\
\hline 38501 & METHIOCARB， DISSOLVED $(\mu \mathrm{g} / \mathrm{L})$ & 1 & $<0.026$ & -- & -- & -- & -- & -- & -- & -- \\
\hline 49296 & METHOMYL， DISSOLVED $(\mu \mathrm{g} / \mathrm{L})$ & 1 & $<0.017$ & -- & -- & -- & -- & -- & -- & - \\
\hline 49295 & 1-NAPHTHOL， DISSOLVED $(\mu \mathrm{g} / \mathrm{L})$ & 1 & $<0.007$ & -- & -- & -- & -- & -- & -- & -- \\
\hline 49294 & NEBURON, DISSOLVED $(\mu \mathrm{g} / \mathrm{L})$ & 1 & $<0.015$ & -- & -- & -- & - & -- & -- & -- \\
\hline 49293 & NORFLURAZON， DISSOLVED $(\mu \mathrm{g} / \mathrm{L})$ & 1 & $<0.024$ & -- & -- & -- & -- & -- & -- & -- \\
\hline 49292 & ORYZALIN， DISSOLVED $(\mu \mathrm{g} / \mathrm{L})$ & 1 & $<0.019$ & - & -- & -- & -- & -- & -- & -- \\
\hline 38866 & OXAMYL， DISSOLVED $(\mu \mathrm{g} / \mathrm{L})$ & 1. & $<0.018$ & -- & -- & -- & -- & -- & -- & -- \\
\hline 49291 & PICLORAM， DISSOLVED $(\mu \mathrm{g} / \mathrm{L})$ & 1 & $<0.050$ & -- & -- & -- & -- & -- & -- & -- \\
\hline 49236 & PROPHAM， DISSOLVED $(\mu \mathrm{g} / \mathrm{L})$ & 1 & $<0.035$ & -- & -- & -- & -- & -- & -- & -- \\
\hline 38538 & PROPOXUR, DISSOLVED $(\mu \mathrm{g} / \mathrm{L})$ & 1. & $<0.035$ & -- & -- & -- & -- & -- & -- & -- \\
\hline 39762 & SILVEX，DISSOLVED $(\mu \mathrm{g} / \mathrm{L})$ & 1 & $<0.021$ & -- & -- & -- & -- & -- & -. & -- \\
\hline 49235 & TRICLOPYR, DISSOLVED $(\mu \mathrm{g} / \mathrm{L})$ & 1 & $<0.050$ & -- & -- & -- & -- & -- & -- & -- \\
\hline
\end{tabular}

NOTE: Multiple detection limits during the period of record may result in different values flagged with a "<."

* Value is estimated by using a log-probability regression to predict the values of data below the detection limit. 
Table 75. Water-quality data at site 33 (CSW08), July 1995 through September 1997

\begin{tabular}{|c|c|c|c|c|c|c|c|c|c|c|}
\hline DATE & TIME & $\begin{array}{c}\text { RAIN- } \\
\text { FALL } \\
\text { ACCUM } \\
(I N) \\
(00045)\end{array}$ & $\begin{array}{c}\text { DIS- } \\
\text { CHARGE, } \\
\text { INST. } \\
\text { CUBIC } \\
\text { FEET PER } \\
\text { SECOND } \\
(00061) \\
\end{array}$ & $\begin{array}{c}\text { TEMPERA- } \\
\text { TURE } \\
\text { WATER } \\
\text { DEG. C) } \\
(00010) \\
\end{array}$ & $\begin{array}{c}\text { SPE- } \\
\text { CIFIC } \\
\text { CONDUC- } \\
\text { TANCE } \\
\text { LAB } \\
\text { (US/CM) } \\
(90095) \\
\end{array}$ & $\begin{array}{c}\text { SPE- } \\
\text { CIFIC } \\
\text { CONDUC- } \\
\text { TANCE } \\
\text { (US/CM) } \\
(00095\end{array}$ & $\begin{array}{c}\text { PH } \\
\text { WATER } \\
\text { RAW LAB } \\
\text { (STAN- } \\
\text { DARD } \\
\text { UNITS) } \\
(00403)\end{array}$ & $\begin{array}{c}\text { PH } \\
\text { WATER } \\
\text { RAW } \\
\text { FIELD } \\
\text { (STAN- } \\
\text { DARD } \\
\text { UNITS) } \\
(00400) \\
\end{array}$ & $\begin{array}{c}\text { ALKA- } \\
\text { LINITY } \\
\text { LAB } \\
\text { (MG/L } \\
\text { AS } \\
\text { CACO3) } \\
(90410) \\
\end{array}$ & $\begin{array}{c}\text { SEDI- } \\
\text { MENT, } \\
\text { SUS- } \\
\text { PENDED } \\
(\text { MG /L) } \\
(80154)\end{array}$ \\
\hline \multicolumn{11}{|l|}{ JUL 1995} \\
\hline $07 \ldots$ & 0138 & 1.03 & 4.3 & 21.5 & 109 & 102 & 6.9 & 7.00 & 42 & 153 \\
\hline $07 \ldots$ & 0238 & 1.03 & 21 & 22.0 & 84 & 78 & 7.0 & 6.90 & 29 & 1320 \\
\hline $07 \ldots$ & 0343 & 1.03 & 11 & 21.5 & 81 & 75 & 6.7 & 6.80 & 23 & 577 \\
\hline $16 \ldots$ & 1647 & 1.43 & 250 & 22.5 & -- & -- & -- & -- & -- & 1680 \\
\hline $16 \ldots$ & 1709 & 1.43 & 288 & 22.0 & -- & -- & -- & - & -- & -- \\
\hline $16 \ldots$ & 1812 & 1.43 & 116 & 22.5 & -- & -- & -- & -- & -- & -- \\
\hline \multicolumn{11}{|l|}{ OCT } \\
\hline $20 \ldots$ & 2025 & 1.21 & 4.5 & 16.5 & 109 & 100 & 6.6 & 7.16 & 31 & 402 \\
\hline $20 \ldots$ & 2136 & 1.21 & 117 & 16.0 & 70 & 63 & 6.4 & 7.25 & 22 & 3960 \\
\hline $20 \ldots$ & 2249 & 1.21 & 63 & 16.0 & 63 & 57 & 6.5 & 6.99 & 16 & 1070 \\
\hline $20 \ldots$ & 2327 & 1.21 & 44 & 16.0 & 62 & 55 & 6.4 & 6.83 & 16 & 607 \\
\hline $27 \ldots$ & 2330 & 1.92 & 96 & 16.0 & -- & -- & -- & -- & -- & -- \\
\hline \multicolumn{11}{|l|}{ NOV } \\
\hline $\begin{array}{c}11 \ldots \\
\text { FEB } 1996\end{array}$ & 1238 & 1.02 & 14 & 12.5 & -- & 104 & -- & 6.65 & -- & -- \\
\hline $20 \ldots$ & 0859 & 0.42 & 3.3 & 8.5 & 136 & 124 & 7.7 & 6.84 & 50 & 45 \\
\hline $20 \ldots$ & 0940 & 0.42 & 3.8 & 8.5 & -- & 122 & -- & 6.87 & -- & -- \\
\hline \multicolumn{11}{|l|}{ MAR } \\
\hline $19 \ldots$ & 0706 & 0.63 & 17 & 10.0 & 110 & 101 & 7.4 & 7.10 & 40 & 213 \\
\hline $19 \ldots$ & 0720 & 0.63 & 35 & 9.5 & 95 & 85 & 7.4 & 7.00 & 32 & 1390 \\
\hline $19 \ldots$ & 0906 & 0.63 & 57 & 9.5 & 71 & 62 & 7.1 & 6.92 & 19 & 1740 \\
\hline $19 \ldots$ & 1122 & 0.63 & 33 & 10.0 & 71 & 63 & 7.1 & 6.72 & 16 & 463 \\
\hline \multicolumn{11}{|l|}{ APR } \\
\hline$\underset{\text { JUN }}{03} \cdots$ & \multicolumn{9}{|c|}{ JUN } & 23 \\
\hline $07 \ldots$ & 1925 & 0.87 & 2.8 & 20.5 & -- & 139 & -- & 6.66 & -- & -- \\
\hline $07 \ldots$ & 1930 & 0.87 & 2.8 & 20.0 & 145 & 138 & 7.6 & 6.97 & 61 & 517 \\
\hline $07 \ldots$ & 2135 & 0.87 & 8.4 & 20.0 & 105 & 102 & 7.5 & 7.40 & 39 & 840 \\
\hline $08 \ldots$ & 0450 & 0.87 & 1.6 & 18.5 & 129 & 121 & 7.6 & 7.28 & 50 & 175 \\
\hline $08 \ldots$ & 2030 & 1.17 & 48 & 20.5 & 68 & 63 & 7.0 & 7.03 & 22 & 2670 \\
\hline $08 \ldots$ & 2211 & 1.17 & 25 & 20.0 & 67 & 62 & 7.1 & 7.00 & 17 & 721 \\
\hline \multicolumn{11}{|l|}{ JUL } \\
\hline $15 \ldots$ & 1435 & 0.68 & 2.3 & 22.5 & -- & 115 & -- & 7.66 & -- & -- \\
\hline $15 \ldots$ & 1632 & 0.68 & 2.9 & 22.0 & -- & 147 & -- & 7.55 & -- & -- \\
\hline $25 \ldots$ & 1935 & 0.94 & 6.1 & 23.0 & 144 & 138 & 7.5 & 7.37 & 57 & 228 \\
\hline $25 \ldots$ & 1955 & 0.94 & 5.0 & 23.0 & -- & -- & -- & -- & -- & -- \\
\hline $25 \ldots$ & 2216 & 0.94 & 2.5 & 22.5 & 121 & 113 & 7.3 & 7.44 & 42 & 263 \\
\hline \multicolumn{11}{|l|}{ AUG } \\
\hline $11 \ldots$ & 2058 & 3.61 & 1.8 & 21.5 & 141 & 134 & 7.3 & 7.35 & 57 & 179 \\
\hline $11 \ldots$ & 2348 & 3.61 & 289 & 22.0 & 50 & 42 & 7.0 & 6.68 & 10 & 123000 \\
\hline $12 \ldots$ & 0210 & 3.61 & 56 & 22.0 & 56 & 55 & 7.0 & 6.66 & 12 & 1460 \\
\hline $12 \ldots$ & 0703 & 3.61 & 14 & 22.0 & 76 & 73 & 7.1 & 6.87 & 22 & 190 \\
\hline \multicolumn{11}{|l|}{ OCT } \\
\hline $08 \ldots$ & 0724 & 1.08 & 19 & 14.5 & 97 & 90 & 7.0 & 7.07 & 26 & 504 \\
\hline $08 \ldots$ & 0832 & 1.08 & 17 & 15.0 & -- & 79 & -- & 7.12 & -- & 621 \\
\hline $08 \ldots$ & 1048 & 1.08 & 10 & 15.5 & 85 & 79 & 7.0 & 7.12 & 25 & 183 \\
\hline NOV & & & & & & & & & & \\
\hline $08 \ldots$ & 1045 & 0.57 & 1.0 & 17.0 & -- & 169 & -- & 7.09 & -- & -- \\
\hline $\mathrm{DEC}$ & & & & & & & & & & \\
\hline $01 \ldots$ & 0926 & 0.85 & 2.5 & 11.0 & 152 & 140 & 7.5 & 7.30 & 55 & 58 \\
\hline $01 \ldots$ & 1324 & 0.85 & 41 & 12.0 & -- & 92 & $\cdots$ & 7.05 & -- & -- \\
\hline $01 \ldots$ & 1334 & 0.85 & 39 & -- & 95 & 94 & 7.4 & 7.30 & 25 & 2570 \\
\hline $01 \ldots$ & 1642 & 0.85 & 19 & -- & 92 & 89 & 7.3 & 7.04 & 23 & 247 \\
\hline FEB 1997 & & & & & & & & & & \\
\hline $04 \ldots$ & 0641 & 0.45 & 3.5 & 10.5 & 137 & 130 & 7.3 & 7.34 & 51 & 66 \\
\hline $13 \ldots$ & 1624 & -- & 3.3 & 4.5 & 129 & 148 & 7.5 & 6.98 & 41 & 19 \\
\hline $13 \ldots$ & 1900 & -- & 16 & 2.5 & 103 & 113 & 7.3 & 7.16 & 30 & 494 \\
\hline $13 \ldots$ & 2329 & -- & 37 & 2.5 & 84 & 92 & 7.2 & 7.10 & 20 & 763 \\
\hline $14 \ldots$ & 0530 & -- & 22 & 6.5 & 82 & 91 & 7.1 & 6.90 & 20 & 435 \\
\hline $14 \ldots$ & 1007 & -- & 14 & 6.5 & -- & 93 & -- & 6.94 & -- & -- \\
\hline MAY & & & & & & & & & & \\
\hline $07 \ldots$ & 0740 & 0.00 & 1.9 & 12.5 & 144 & 134 & 7.5 & 6.80 & 57 & 23 \\
\hline $\begin{array}{l}\text { JUN } \\
\qquad 02 \ldots\end{array}$ & 1422 & 0.62 & 7.9 & 18.5 & -- & 129 & -- & 7.37 & -- & -- \\
\hline $02 \ldots$ & 1426 & 0.62 & 7.4 & 18.5 & 135 & 126 & 7.6 & 7.65 & 50 & 410 \\
\hline JUL & & & & & & & & & & \\
\hline $23 \ldots$ & 0047 & 5.45 & 33 & 24.5 & 106 & 96 & 7.0 & 6.64 & 36 & 2210 \\
\hline $23 \ldots$ & 0318 & 5.45 & 75 & 23.5 & 61 & 54 & 6.8 & 7.06 & 11 & 1650 \\
\hline $23 \ldots$ & 0753 & 5.45 & 383 & 23.5 & 44 & 40 & 6.7 & 6.99 & 10 & 116000 \\
\hline
\end{tabular}


Table 75. Water-quality data at site 33 (CSW08), July 1995 through September 1997-Continued

\begin{tabular}{|c|c|c|c|c|c|c|c|c|c|c|}
\hline DATE & $\begin{array}{c}\text { RESIDUE } \\
\text { TOTAL } \\
\text { AT } 105 \\
\text { DEG. C, } \\
\text { SUS- } \\
\text { PENDED } \\
\text { (MG /L) } \\
(00530) \\
\end{array}$ & $\begin{array}{l}\text { RESIDUE } \\
\text { VOLA- } \\
\text { TILE, } \\
\text { SUS- } \\
\text { PENDED } \\
\text { (MG/L) } \\
\text { (00535) }\end{array}$ & $\begin{array}{c}\text { SOLIDS, } \\
\text { RESIDUE } \\
\text { AT } 180 \\
\text { DEG. C } \\
\text { DIS- } \\
\text { SOLVED } \\
(M G / L) \\
(70300) \\
\end{array}$ & $\begin{array}{c}\text { OXYGEN } \\
\text { DEMAND, } \\
\text { BIO- } \\
\text { CHEMICAL } \\
5 \text { DAY } \\
(\text { MG /L) } \\
(00310) \\
\end{array}$ & $\begin{array}{c}\text { OXYGEN } \\
\text { DEMAND, } \\
\text { CHEM- } \\
\text { ICAL } \\
\text { (HIGH } \\
\text { LEVEL) } \\
(\text { MG } / L) \\
(00340) \\
\end{array}$ & $\begin{array}{c}\text { NITRO- } \\
\text { GEN, AM- } \\
\text { MONIA + } \\
\text { ORGANIC } \\
\text { TOTAL } \\
\text { (MG/L } \\
\text { AS N) } \\
(00625) \\
\end{array}$ & $\begin{array}{c}\text { NITRO- } \\
\text { GEN, } \\
\text { NO2 +NO3 } \\
\text { DIS- } \\
\text { SOLVED } \\
\text { (MG/L } \\
\text { AS N) } \\
(00631) \\
\end{array}$ & $\begin{array}{c}\text { NITRO- } \\
\text { GEN, } \\
\text { AMMONIA } \\
\text { DIS- } \\
\text { SOLVED } \\
\text { (MG / L } \\
\text { AS N) } \\
(00608) \\
\end{array}$ & $\begin{array}{l}\text { NITRO- } \\
\text { GEN, } \\
\text { ORGANIC } \\
\text { TOTAL } \\
\text { (MG / L } \\
\text { AS N) } \\
(00605) \\
\end{array}$ & $\begin{array}{c}\text { NITRO- } \\
\text { GEN, } \\
\text { TOTAL } \\
\text { (MG/L } \\
\text { A.S N) } \\
(00600)\end{array}$ \\
\hline \multicolumn{11}{|l|}{ JUL 1995} \\
\hline $07 \ldots$ & -- & 23 & -- & 7.4 & 47 & 0.80 & 0.400 & 0.080 & 0.72 & 1.2 \\
\hline $07 \ldots$ & -- & 164 & -- & 12 & 100 & 1.0 & 0.430 & 0.100 & 0.90 & 1.4 \\
\hline $07 \ldots$ & -- & 82 & 86 & 11 & 100 & 1.7 & 0.370 & 0.220 & 1.5 & 2.1 \\
\hline $16 \ldots$ & -- & 197 & -- & -- & - & -- & -- & -- & -- & -- \\
\hline $16 \ldots$ & -- & -- & -- & -- & -- & -- & -- & -- & -- & -- \\
\hline $16 \ldots$ & - & - & -- & -- & -- & -- & -- & -- & -- & -- \\
\hline \multicolumn{11}{|l|}{$\mathrm{OCT}$} \\
\hline $20 \ldots$ & -- & $-\infty$ & 84 & 4.1 & 37 & 1.3 & 0.200 & 0.100 & 1.2 & 1.5 \\
\hline $20 \ldots$ & -- & -- & 64 & 11 & 120 & 4.4 & 0.660 & 0.220 & 4.2 & 5.1 \\
\hline $20 \ldots$ & -- & -- & 69 & 6.6 & 69 & 2.0 & 0.320 & 0.110 & 1.9 & 2.3 \\
\hline $20 \ldots$ & -- & -- & 75 & 5.6 & 59 & 1.5 & 0.280 & 0.090 & 1.4 & 1.8 \\
\hline $27 \ldots$ & -- & -- & -- & -- & -- & -- & -- & -- & -- & -- \\
\hline \multicolumn{11}{|l|}{ NOV } \\
\hline $\begin{array}{c}11 \ldots \\
\text { FEB } 1996\end{array}$ & -- & -- & -- & -- & -- & -- & -- & -- & -- & -- \\
\hline $20 \ldots$ & 6 & 3 & 126 & 2.4 & 15 & 0.38 & 0.310 & $<0.040$ & 0.38 & 0.69 \\
\hline $20 \ldots$ & -- & -- & -- & -- & -- & -- & -- & -- & -- & -- \\
\hline \multicolumn{11}{|l|}{ MAR } \\
\hline $19 \ldots$ & 170 & 27 & 75 & 3.1 & 32 & 0.83 & 0.200 & $<0.015$ & 0.83 & 1.0 \\
\hline $19 \ldots$ & 1010 & 134 & 66 & 7.1 & 68 & 3.3 & 0.270 & 0.040 & 3.3 & 3.6 \\
\hline $19 \ldots$ & 494 & 72 & 59 & 6.8 & 75 & 3.0 & 0.480 & 0.180 & 2.8 & 3.5 \\
\hline $19 \ldots$ & 135 & 15 & 73 & 4.0 & 48 & 1.2 & 0.550 & 0.120 & 1.1 & 1.7 \\
\hline \multicolumn{11}{|l|}{ APR } \\
\hline $03 \ldots$ & 3 & 1 & 77 & $<2.0$ & 12 & 0.38 & 0.160 & $<0.015$ & 0.38 & 0.54 \\
\hline \multicolumn{11}{|l|}{ JUN } \\
\hline $07 \ldots$ & -- & -- & -- & -- & -- & -- & -- & - & -- & -- \\
\hline $07 \ldots$ & 408 & 56 & 109 & 11 & 35 & 1.9 & 0.420 & 0.110 & 1.8 & 2.3 \\
\hline $07 \ldots$ & 664 & 100 & 85 & 17 & 65 & 3.2 & 0.560 & 0.130 & 3.1 & 3.8 \\
\hline $08 \ldots$ & 60 & 8 & 100 & 7.2 & 15 & 0.67 & 0.310 & 0.030 & 0.64 & 0.98 \\
\hline $08 \ldots$ & 1770 & 273 & 62 & 20 & 32 & 6.7 & 0.420 & 0.160 & 6.5 & 7.1 \\
\hline $08 \ldots$ & 455 & 70 & 59 & 10 & 48 & 2.2 & 0.240 & 0.060 & 2.1 & 2.4 \\
\hline \multicolumn{11}{|l|}{ JUL } \\
\hline $15 \ldots$ & -- & -- & -- & -- & -- & -- & -- & -- & -- & -- \\
\hline $15 \ldots$ & -- & -- & -- & -- & -- & -- & -- & -- & -- & -- \\
\hline $25 \ldots$ & 198 & 38 & 113 & 8.8 & 29 & 1.4 & 0.330 & $<0.015$ & 1.4 & 1.7 \\
\hline $25 \ldots$ & -- & -- & -- & -- & -- & -- & -- & -- & -- & -- \\
\hline $25 \ldots$ & 174 & 26 & 152 & 10 & 34 & 1.6 & 0.600 & 0.100 & 1.5 & 2.2 \\
\hline \multicolumn{11}{|l|}{ AUG } \\
\hline $11 \ldots$ & 84 & 14 & 103 & 8.0 & 14 & 1.1 & 0.280 & 0.020 & 1.1 & 1.4 \\
\hline $11 \ldots$ & 2540 & 310 & 46 & 14 & 53 & 2.2 & 0.170 & 0.040 & 2.2 & 2.4 \\
\hline $12 \ldots$ & 294 & 42 & 59 & 7.4 & 57 & 1.4 & 0.140 & 0.030 & 1.4 & 1.5 \\
\hline $12 \ldots$ & 77 & 14 & 70 & 5.1 & 31 & 0.93 & 0.120 & $<0.015$ & 0.93 & 1.0 \\
\hline \multicolumn{11}{|l|}{$\mathrm{OCT}$} \\
\hline $08 \ldots$ & 222 & 22 & 86 & 6.8 & 30 & 1.7 & 0.360 & 0.050 & 1.6 & 2.1 \\
\hline $08 \ldots$ & -- & -- & -- & -- & -- & -- & -- & -- & -- & -- \\
\hline $08 \ldots$ & 67 & 7 & 74 & 8.0 & 36 & 1.3 & 0.170 & 0.050 & 1.2 & 1.5 \\
\hline NOV & & & & & & & & & & \\
\hline $08 \ldots$ & -- & -- & -- & -- & -- & - & -- & -- & -- & -- \\
\hline $\mathrm{DEC}$ & & & & & & & & & & \\
\hline $01 \ldots$ & 17 & 4 & 107 & 3.8 & 17 & 0.37 & 0.230 & $<0.015$ & 0.37 & 0.60 \\
\hline $01 \ldots$ & -- & -- & -- & -- & -- & -- & -- & -- & -- & -- \\
\hline $01 \ldots$ & 393 & 56 & 93 & 12 & 63 & 1.9 & 0.260 & 0.100 & 1.8 & 2.2 \\
\hline $01 \ldots$ & 78 & 13 & 92 & 6.3 & 48 & 1.2 & 0.240 & 0.080 & 1.1 & 1.4 \\
\hline FEB 1997 & & & & & & & & & & \\
\hline $04 \ldots$ & 44 & 7 & 98 & $<2.0$ & 9 & 0.31 & 0.310 & $<0.015$ & 0.31 & 0.62 \\
\hline $13 \ldots$ & 13 & 2 & 91 & $<2.0$ & 5 & 0.41 & 0.190 & $<0.015$ & 0.41 & 0.60 \\
\hline $13 \ldots$ & 457 & 65 & 77 & 7.8 & 43 & 2.6 & 0.300 & 0.100 & 2.5 & 2.9 \\
\hline $13 \ldots$ & 452 & 58 & 69 & 6.8 & 51 & 2.5 & 0.330 & 0.110 & 2.4 & 2.8 \\
\hline $14 \ldots$ & 74 & 9 & 72 & 2.8 & 29 & 0.98 & 0.290 & 0.080 & 0.90 & 1.3 \\
\hline $14 \ldots$ & $\ldots$ & - & - & -- & -- & -- & -- & $\ldots$ & -- & -- \\
\hline MAY & & & & & & & & & & \\
\hline $07 \ldots$ & 1 & $<1$ & 106 & $<2.0$ & $<5$ & 0.25 & 0.240 & $<0.015$ & 0.25 & 0.49 \\
\hline JUN & & & & & & & & & & \\
\hline $02 \ldots$ & -- & - & -- & -- & -- & -- & -- & -- & -- & -- \\
\hline $02 \ldots$ & 370 & 58 & 103 & 9.2 & 19 & 1.8 & 0.450 & 0.050 & 1.7 & 2.2 \\
\hline JUL & & & & & & & & & & \\
\hline $23 \ldots$ & 2300 & 390 & 90 & 22 & 210 & 6.7 & 0.570 & 0.480 & 6.2 & 7.3 \\
\hline $23 \ldots$ & 1200 & 180 & 64 & 15 & 120 & 4.1 & 0.480 & 0.420 & 3.7 & 4.6 \\
\hline $23 \ldots$ & 3560 & 230 & 52 & 8.0 & 120 & 2.8 & 0.230 & 0.460 & 2.3 & 3.0 \\
\hline
\end{tabular}


Table 75. Water-quality data at site 33 (CSW08), July 1995 through September 1997-Continued

\begin{tabular}{|c|c|c|c|c|c|c|c|c|c|c|}
\hline DATE & $\begin{array}{l}\text { PHOS- } \\
\text { PHORUS } \\
\text { TOTAL } \\
\text { (MG / L } \\
\text { AS P) } \\
(00665) \\
\end{array}$ & $\begin{array}{c}\text { PHOS- } \\
\text { PHORUS } \\
\text { ORTHO, } \\
\text { DIS- } \\
\text { SOLVED } \\
\text { (MG /L } \\
\text { AS P) } \\
(00671) \\
\end{array}$ & $\begin{array}{c}\text { OIL AND } \\
\text { GREASE, } \\
\text { TOTAL } \\
\text { RECOV. } \\
\text { GRAVI- } \\
\text { METRIC } \\
\text { (MG/L) } \\
(00556) \\
\end{array}$ & $\begin{array}{c}\text { CARBON, } \\
\text { ORGANIC } \\
\text { TOTAL } \\
\text { (MG / L } \\
\text { AS C) } \\
(00680) \\
\end{array}$ & $\begin{array}{c}\text { STREP- } \\
\text { TOCOCCI } \\
\text { FECAL, } \\
\text { (COLS. } \\
\text { PER } \\
100 \mathrm{ML} \text { ) } \\
(31679) \\
\end{array}$ & $\begin{array}{c}\text { COLI- } \\
\text { FORM, } \\
\text { FECAL, } \\
0.45 \\
\text { UM-MF } \\
\text { (COLS. ' } \\
100 \mathrm{ML} \text { ) } \\
\text { (31616) }\end{array}$ & $\begin{array}{c}\text { ANTI- } \\
\text { MONY, } \\
\text { TOTAL } \\
\text { (UG/L } \\
\text { AS SB) } \\
(01097)\end{array}$ & $\begin{array}{c}\text { ARSENIC } \\
\text { TOTAL } \\
\text { (UG/L } \\
\text { AS AS) } \\
(01002) \\
\end{array}$ & $\begin{array}{c}\text { BERYL- } \\
\text { LIUM, } \\
\text { TOTAL } \\
\text { RECOVER- } \\
\text { ABLE } \\
\text { (UG/L } \\
\text { AS BE) } \\
(01012) \\
\end{array}$ & $\begin{array}{c}\text { CADMIUM } \\
\text { WATER } \\
\text { UNFLTRD } \\
\text { TOTAL } \\
\text { (UG/L } \\
\text { AS CD) } \\
(01027) \\
\end{array}$ \\
\hline \multicolumn{11}{|l|}{ JUL 1995} \\
\hline $07 \ldots$ & 0.140 & 0.100 & -- & 15 & -- & $\ldots$ & $<1$ & $<1$ & -- & $<1$ \\
\hline $07 \ldots$ & 0.150 & 0.050 & -- & 23 & -- & -- & $<1$ & $<1$ & -- & $<1$ \\
\hline $07 \ldots$ & 0.360 & 0.100 & -- & -- & -- & - & -- & -- & -- & -- \\
\hline $16 \ldots$ & -- & -- & $<1$ & -- & 110000 & 460000 & -- & - & -- & -- \\
\hline $16 \ldots$ & -- & -- & -- & -- & 98000 & 550000 & -. & -- & -- & -- \\
\hline $16 \ldots$ & -- & -- & -- & -- & 65000 & 340000 & -- & -- & -- & -- \\
\hline \multicolumn{11}{|l|}{ OCT } \\
\hline $20 \ldots$ & 0.440 & 0.170 & -- & 17 & -- & -- & $<1$ & 2 & -- & $<1$ \\
\hline $20 \ldots$ & 1.42 & 0.140 & -- & 44 & -- & -- & 4 & 6 & -- & $<1$ \\
\hline $20 \ldots$ & 0.480 & 0.110 & -- & -- & -- & -- & -- & -- & -- & -- \\
\hline $20 \ldots$ & 0.340 & 0.090 & -- & -- & -- & -- & -- & -- & -- & -- \\
\hline $27 \ldots$ & -- & -- & -- & -- & 38000 & 43000 & -- & -- & -- & -- \\
\hline \multicolumn{11}{|l|}{ NOV } \\
\hline \multicolumn{11}{|l|}{ FEB 1996} \\
\hline $20 \ldots$ & 0.010 & 0.010 & -- & 4.2 & -- & -- & 6 & $<1$ & -- & $<1$ \\
\hline $20 \ldots$ & -- & -- & $<1$ & -- & K630 & $\mathrm{K} 1200$ & -- & + & -- & -- \\
\hline \multicolumn{11}{|l|}{ MAR } \\
\hline $19 \ldots$ & 0.700 & 0.040 & -- & 28 & -- & -- & 13 & 4 & -- & $<1$ \\
\hline $19 \ldots$ & 0.430 & 0.060 & -- & 6.1 & -- & -- & $<1$ & 2 & -- & $<1$ \\
\hline $19 \ldots$ & 0.190 & 0.030 & -- & -- & $\mathrm{K} 18000$ & $\mathrm{~K} 15000$ & - & -- & -- & -- \\
\hline \multicolumn{11}{|l|}{ APR } \\
\hline $03 \ldots$ & 0.030 & $<0.010$ & $<1$ & -- & 140 & 940 & 3 & $<1$ & $<10$ & $<1$ \\
\hline \multicolumn{11}{|l|}{ JUN } \\
\hline $07 \ldots$ & -- & -- & -- & -- & -- & -- & -- & -- & -- & -- \\
\hline $07 \ldots$ & 0.480 & 0.090 & -- & -- & -- & -- & 3 & 2 & -- & $<1$ \\
\hline $07 \ldots$ & 0.830 & 0.050 & -- & -- & -- & -- & 7 & 2 & -- & $<1$ \\
\hline $08 \ldots$ & 0.080 & 0.020 & - & -- & - & -- & -- & -- & -- & -- \\
\hline $08 \ldots$ & 1.56 & 0.050 & -- & -- & -- & -- & 18 & 2 & -- & $<1$ \\
\hline $08 \ldots$ & 0.440 & 0.020 & -- & -- & -- & -- & 5 & $<1$ & -- & $<1$ \\
\hline \multicolumn{11}{|l|}{ JUL } \\
\hline $15 \ldots$ & $\sim$ & -- & $<1$ & -- & 24000 & 35000 & $\ldots$ & -- & -- & -- \\
\hline $15 \ldots$ & -- & -- & -- & -- & 20000 & 4300 & -- & -- & -- & -- \\
\hline $25 \ldots$ & 0.290 & 0.030 & -- & 18 & -- & - & 8 & 2 & -- & $<1$ \\
\hline $25 \ldots$ & -- & -- & -- & -- & 68000 & 50000 & -- & -- & -- & $\ldots$ \\
\hline $25 \ldots$ & 0.330 & 0.080 & -- & 21 & -- & -- & -- & -- & -- & -- \\
\hline \multicolumn{11}{|l|}{ AUG } \\
\hline $11 \ldots$ & 0.190 & 0.060 & - - & 11 & -- & -. & $<1$ & $<1$ & 42 & - \\
\hline $11 \ldots$ & 3.22 & 0.040 & -- & 53 & -- & -- & 3 & 4 & -- & $<1$ \\
\hline $12 \ldots$ & 0.330 & 0.020 & -- & -- & -- & -- & -- & -- & -- & -. \\
\hline $12 \ldots$ & 0.150 & 0.010 & -- & -- & -. & -. & -- & -- & -- & -- \\
\hline \multicolumn{11}{|l|}{ OCT } \\
\hline $08 \ldots$ & 0.440 & 0.100 & - - & 22 & -- & -- & $<1$ & 2 & -- & $<1$ \\
\hline $08 \ldots$ & - & -. & -- & -- & 90000 & 34000 & -- & -- & -- & - \\
\hline $08 \ldots$ & 0.200 & 0.035 & -- & -- & -- & -- & -- & -- & -- & -- \\
\hline NOV & & & & & & & & & & \\
\hline $\begin{array}{l}08 \ldots \\
\text { DEC }\end{array}$ & -- & -- & 3 & -- & 5900 & K7100 & -- & -- & -- & -- \\
\hline $01 \ldots$ & 0.060 & 0.014 & -- & 7.0 & -- & -- & 4 & 1 & -- & $<1$ \\
\hline $01 \ldots$ & -- & -- & -- & -- & 98000 & 33000 & -- & -- & -- & -- \\
\hline $01 \ldots$ & 0.660 & 0.100 & -- & 34 & -- & -- & $<1$ & 2 & -- & $<1$ \\
\hline $01 \ldots$ & 0.210 & 0.041 & -- & -- & -- & -- & -- & $\ldots$ & -- & - \\
\hline FEB 1997 & & & & & & & & & & \\
\hline $04 \ldots$ & 0.060 & 0.013 & 1 & 4.8 & 4600 & K1200 & $<1$ & $<1$ & -- & $<1$ \\
\hline $13 \ldots$ & 0.047 & 0.012 & -- & 3.7 & -- & -- & $<1$ & $<1$ & -- & $<1$ \\
\hline $13 \ldots$ & 0.970 & 0.340 & -- & 22 & -- & -- & $<1$ & 2 & -- & $<1$ \\
\hline $13 \ldots$ & 0.810 & 0.370 & -- & 25 & -- & -- & $<1$ & 2 & -- & $<1$ \\
\hline $14 \ldots$ & 0.260 & 0.140 & -- & -- & -- & -- & $<1$ & $<1$ & -- & 2 \\
\hline $14 \ldots$ & -- & -- & -- & -- & 2900 & $\mathrm{~K} 450$ & -- & -- & -- & - \\
\hline MAY & & & & & & & & & & \\
\hline $07 \ldots$ & 0.028 & $<0.010$ & 2 & -- & 390 & 430 & $<1$ & $<1$ & -- & $<1$ \\
\hline JUN & & & & & & & & & & \\
\hline $02 \ldots$ & -- & -- & -- & -- & -- & -- & -- & -- & -- & -- \\
\hline $02 \ldots$ & 0.560 & 0.014 & 1 & 18 & 24000 & 35000 & $<1$ & $<1$ & -- & $<1$ \\
\hline JUL & & & & & & & & & & \\
\hline $23 \ldots$ & 2.17 & 0.030 & -- & 170 & -- & -- & $<1$ & 7 & -- & $<1$ \\
\hline $23 \ldots$ & 1.28 & 0.180 & -- & 42 & -- & -- & $<1$ & 4 & -- & $<1$ \\
\hline $23 \ldots$ & 1.69 & 0.110 & -- & 25 & -- & -- & $<1$ & 8 & -- & $<1$ \\
\hline
\end{tabular}

K Results based on colony count outside the acceptable range (nonideal colony count). 
Table 75. Water-quality data at site 33 (CSW08), July 1995 through September 1997—Continued

\begin{tabular}{|c|c|c|c|c|c|c|c|c|c|}
\hline DATE & $\begin{array}{c}\text { CHRO- } \\
\text { MIUM, } \\
\text { TOTAL } \\
\text { RECOV- } \\
\text { ERABLE } \\
\text { (UG/L } \\
\text { AS CR) } \\
(01034) \\
\end{array}$ & $\begin{array}{c}\text { COPPER, } \\
\text { TOTAL } \\
\text { RECOV- } \\
\text { ERABLE } \\
\text { (UG/L } \\
\text { AS CU) } \\
(01042 \text { ) } \\
\end{array}$ & $\begin{array}{c}\text { LEAD, } \\
\text { TOTAL } \\
\text { RECOV- } \\
\text { ERABLE } \\
\text { (UG/L } \\
\text { AS PB) } \\
(01051) \\
\end{array}$ & $\begin{array}{c}\text { MERCURY } \\
\text { TOTAL } \\
\text { RECOV- } \\
\text { ERABLE } \\
\text { (UG/L } \\
\text { AS HG) } \\
(71900) \\
\end{array}$ & $\begin{array}{l}\text { NICKEL, } \\
\text { TOTAL } \\
\text { RECOV- } \\
\text { ERABLE } \\
\text { (UG/L } \\
\text { AS NI) } \\
\text { (01067) } \\
\end{array}$ & $\begin{array}{c}\text { SELE- } \\
\text { NIUM, } \\
\text { TOTAL } \\
\text { (UG/L } \\
\text { AS SE) } \\
(01147 \text { ) } \\
\end{array}$ & $\begin{array}{c}\text { SILVER, } \\
\text { TOTAL } \\
\text { RECOV- } \\
\text { ERABLE } \\
\text { (UG/L } \\
\text { AS AG) } \\
(01077) \\
\end{array}$ & $\begin{array}{l}\text { ZINC, } \\
\text { TOTAL } \\
\text { RECOV- } \\
\text { ERABLE } \\
\text { (UG/L } \\
\text { AS ZN) } \\
(01092) \\
\end{array}$ & $\begin{array}{c}\text { CYANIDE } \\
\text { TOTAL } \\
\text { (MG/L } \\
\text { AS CN) } \\
(00720) \\
\end{array}$ \\
\hline \multicolumn{10}{|l|}{ JUL 1995} \\
\hline $07 \ldots$ & 4 & 5 & 2 & -- & 2 & -- & -- & 10 & -- \\
\hline $07 \ldots$ & 22 & 36 & 16 & -- & 12 & -- & -- & 50 & -- \\
\hline $07 \ldots$ & -- & -- & -- & -- & -- & -- & -- & -- & - \\
\hline $16 \ldots$ & -- & -- & -- & -- & -- & -- & -- & -- & -- \\
\hline $16 \ldots$ & -- & - & - & -- & -- & -- & -- & -- & - \\
\hline $\begin{array}{l}16 \ldots \\
\text { OCT }\end{array}$ & -- & -- & -- & -- & -- & - & -- & -- & - \\
\hline $20 \ldots$ & 16 & 36 & 7 & -. & 5 & $\ldots$ & -- & 40 & - - \\
\hline $20 \ldots$ & 80 & 130 & 28 & -- & 28 & -- & -- & 140 & -- \\
\hline $20 \ldots$ & -- & -- & -- & -- & -- & -- & -- & -- & - \\
\hline $20 \ldots$ & - & -- & -- & -- & -- & -- & -- & - & -- \\
\hline $27 \ldots$ & -- & -- & -- & -- & -- & -- & -- & -- & -- \\
\hline \multicolumn{10}{|l|}{ NOV } \\
\hline $\begin{array}{c}11 \ldots \\
\mathrm{FEB} \\
1996\end{array}$ & -- & -- & - & -- & -- & -- & -- & -- & - \\
\hline $20 \ldots$ & 3 & 4 & $<1$ & -- & $<1$ & -. & -- & 30 & -. \\
\hline $20 \ldots$ & -- & -- & -- & -- & - & -- & -. & - & -- \\
\hline \multicolumn{10}{|l|}{ MAR } \\
\hline $19 \ldots$ & 7 & 61 & 4 & -- & 1 & - & -- & 50 & - \\
\hline $19 \ldots$ & 71 & 60 & 18 & -- & 18 & - & -- & 100 & -- \\
\hline $19 \ldots$ & 33 & 33 & 14 & -- & 10 & - & -- & 110 & -- \\
\hline $19 \ldots$ & -- & - & - & -- & -- & -- & -- & -- & -- \\
\hline \multicolumn{10}{|l|}{ APR } \\
\hline $03 \ldots$ & 3 & $<1$ & 2 & 0.10 & $<1$ & $<1$ & 1 & 30 & $<0.010$ \\
\hline \multicolumn{10}{|l|}{ JUN } \\
\hline $07 \ldots$ & -- & -- & -- & -- & -- & -- & -- & -- & - - \\
\hline $07 \ldots$ & 16 & 38 & 6 & -- & 6 & - & -- & 50 & -- \\
\hline $07 \ldots$ & 21 & 830 & 13 & -- & 13 & -- & -- & 70 & -- \\
\hline $08 \ldots$ & - & -- & -- & -- & - & -- & -- & -- & -- \\
\hline $08 \ldots$ & 100 & 110 & 29 & -- & 28 & -. & - & 140 & -- \\
\hline $08 \ldots$ & 18 & 32 & 9 & -- & 13 & - & -- & 60 & -- \\
\hline \multicolumn{10}{|l|}{ JUL } \\
\hline $15 \ldots$ & -- & -- & -- & -- & -- & - & -- & -- & -- \\
\hline $15 \ldots$ & -- & -- & -- & -- & -- & -- & -- & -- & -- \\
\hline $25 \ldots$ & 15 & 18 & 4 & -- & 6 & -. & -- & 70 & -- \\
\hline $25 \ldots$ & -- & -- & -- & -- & -- & -- & -- & -- & -- \\
\hline $25 \ldots$ & -- & -- & -- & -- & -- & -- & -- & -- & -- \\
\hline \multicolumn{10}{|l|}{ AUG } \\
\hline $11 \ldots$ & 8 & 13 & 2 & -- & 8 & -. & -- & $<1$ & -- \\
\hline $11 \ldots$ & 72 & 130 & 28 & -- & 21 & -- & -- & 190 & -- \\
\hline $12 \ldots$ & -- & -- & -- & -- & -- & - & -- & -- & -- \\
\hline $12 \ldots$ & -- & -- & -- & -- & -- & -- & -- & -+ & -- \\
\hline \multicolumn{10}{|l|}{ OCT } \\
\hline $08 \ldots$ & 20 & 8 & 6 & -- & 7 & - & -- & 80 & -- \\
\hline $08 \ldots$ & -- & -- & -- & -- & -- & -- & -- & -- & -- \\
\hline $08 \ldots$ & -- & -- & -- & -- & $\ldots$ & -. & -- & -- & -- \\
\hline \multicolumn{10}{|l|}{ NOV } \\
\hline${ }_{D E C}^{08 \ldots}$ & -- & -- & -- & -- & -- & -. & -- & -- & -- \\
\hline $01 \ldots$ & 3 & 6 & 1 & -. & $<1$ & -. & -- & 30 & -- \\
\hline $01 \ldots$ & -- & -- & -- & -- & -- & -- & -- & - & -- \\
\hline $01 \ldots$ & 27 & 35 & 7 & -- & 4 & -- & -- & 50 & -- \\
\hline $01 \ldots$ & -- & -- & -- & -- & -- & -- & -- & -- & -- \\
\hline FEB 1997 & & & & & & & & & \\
\hline $04 \ldots$ & 5 & $<1$ & 3 & -- & $<1$ & -- & -- & 60 & -- \\
\hline $13 \ldots$ & 10 & 12 & $<1$ & -- & $<1$ & -- & -- & 30 & -- \\
\hline $13 \ldots$ & 11 & 11 & 8 & -- & 7 & -- & -- & 70 & -- \\
\hline $13 \ldots$ & 4 & 37 & 8 & -- & 9 & -- & -- & 70 & -- \\
\hline $14 \ldots$ & 23 & 10 & 2 & -- & 3 & -- & -- & 50 & -- \\
\hline $14 \ldots$ & -- & -- & -- & -- & -- & -- & -- & -- & -- \\
\hline MAY & & & & & & & & & \\
\hline $07 \ldots$ & 2 & 2 & $<1$ & -- & 2 & -- & -- & 30 & -- \\
\hline $\begin{array}{l}\text { JUN } \\
\quad 02 \ldots\end{array}$ & -- & -. & -- & -- & -- & -- & -- & -- & -- \\
\hline $02 \ldots$ & 16 & 20 & 7 & -- & 6 & -- & -- & 70 & -- \\
\hline JUL & & & & & & & & & \\
\hline $23 \ldots$ & 3 & 19 & 8 & -- & 3 & - & -- & 100 & -- \\
\hline $23 \ldots$ & 55 & 41 & 18 & -- & 18 & -- & -- & 130 & -- \\
\hline $23 \ldots$ & 61 & 68 & 36 & -- & 20 & -- & -- & 200 & -- \\
\hline
\end{tabular}


Table 76. Water-quality data at site 34 (CSW09), July 1995 through September 1997

\begin{tabular}{|c|c|c|c|c|c|c|c|c|c|c|}
\hline DATE & TIME & $\begin{array}{c}\text { RAIN- } \\
\text { FALL } \\
\text { ACCUM } \\
\text { (IN) } \\
(00045)\end{array}$ & $\begin{array}{c}\text { DIS- } \\
\text { CHARGE, } \\
\text { INST. } \\
\text { CUBIC } \\
\text { FEET PER } \\
\text { SECOND } \\
(00061) \\
\end{array}$ & $\begin{array}{c}\text { TEMPER- } \\
\text { ATURE } \\
\text { WATER } \\
\text { DEG. C) } \\
(00010)\end{array}$ & $\begin{array}{c}\text { SPE- } \\
\text { CIFIC } \\
\text { CONDUC- } \\
\text { TANCE } \\
\text { LAB } \\
\text { (US/CM) } \\
(90095)\end{array}$ & $\begin{array}{c}\text { SPE- } \\
\text { CIFIC } \\
\text { CONDUC- } \\
\text { TANCE } \\
\text { (US /CM) } \\
(00095\end{array}$ & $\begin{array}{c}\text { PH } \\
\text { WATER } \\
\text { RAW LAB } \\
\text { (STAN- } \\
\text { DARD } \\
\text { UNITS) } \\
\text { (00403) }\end{array}$ & $\begin{array}{c}\text { PH } \\
\text { WATER } \\
\text { RAW } \\
\text { FIELD } \\
\text { (STAN- } \\
\text { DARD } \\
\text { UNITS) } \\
(00400) \\
\end{array}$ & $\begin{array}{c}\text { ALKA- } \\
\text { LINITY } \\
\text { LAB } \\
\text { (MG/L AS } \\
\text { CACO3) } \\
(90410)\end{array}$ & $\begin{array}{c}\text { SEDI- } \\
\text { MENT, } \\
\text { SUS- } \\
\text { PENDED } \\
\text { (MG/L) } \\
(80154) \\
\end{array}$ \\
\hline \multicolumn{11}{|l|}{ JUL 1995} \\
\hline $06 \ldots$ & 2200 & 1.40 & 21 & 21.0 & 61 & 58 & 6.5 & 6.20 & 14 & 1320 \\
\hline $06 \ldots$ & 2303 & 1.40 & 107 & 24.0 & 65 & 60 & 6.6 & 6.60 & 13 & 1810 \\
\hline $07 \ldots$ & 0110 & 1.40 & 23 & 24.0 & 69 & 65 & 6.9 & 6.60 & 15 & 482 \\
\hline $28 \ldots$ & 1150 & 0.25 & 11 & 23.5 & -- & 65 & -- & 6.16 & -- & -- \\
\hline \multicolumn{11}{|l|}{ AUG } \\
\hline $03 \ldots$ & 1446 & 0.52 & 27 & 25.0 & 71 & 67 & 6.9 & 6.80 & 19 & 1980 \\
\hline $03 \ldots$ & 1506 & 0.52 & 37 & 26.0 & 62 & 59 & 6.8 & 6.60 & 16 & 1550 \\
\hline $03 \ldots$ & 1542 & 0.52 & 17 & 26.0 & 74 & 70 & 7.2 & 6.50 & 18 & 771 \\
\hline $03 \ldots$ & 1601 & 0.52 & 13 & 26.0 & -- & 71 & -- & 7.11 & -- & -- \\
\hline $03 \ldots$ & 1653 & 0.52 & 6.9 & 25.5 & -- & 73 & -- & 7.08 & -- & -- \\
\hline \multicolumn{11}{|l|}{ OCT } \\
\hline $20 \ldots$ & 2049 & 0.63 & 30 & 17.5 & 113 & 102 & 6.7 & 6.88 & 18 & 1290 \\
\hline $20 \ldots$ & 2138 & 0.63 & 62 & 17.5 & 81 & 74 & 6.6 & 6.91 & 14 & 1420 \\
\hline $20 \ldots$ & 2216 & 0.63 & 26 & 17.0 & 82 & 74 & 6.6 & 6.87 & 17 & 852 \\
\hline \multicolumn{11}{|l|}{ NOV } \\
\hline $11 \ldots$ & 1143 & 1.20 & 29 & 12.5 & -- & 75 & -- & 6.81 & -- & -- \\
\hline $11 \ldots$ & 1155 & 1.20 & 30 & 13.0 & $\ldots$ & 68 & -- & 6.85 & -- & -- \\
\hline $11 \ldots$ & 1425 & 1.20 & 83 & 13.5 & -- & 54 & -- & 6.87 & -- & -- \\
\hline \multicolumn{11}{|l|}{ JAN 1996} \\
\hline $24 \ldots$ & 0912 & 0.50 & 27 & 9.0 & 104 & 96 & 7.4 & 6.92 & 21 & 1510 \\
\hline $24 \ldots$ & 0955 & 0.50 & 91 & 9.0 & 77 & 69 & 7.7 & 6.75 & 13 & 1590 \\
\hline $24 \ldots$ & 1127 & 0.50 & 34 & 8.0 & 93 & 69 & 7.6 & 6.83 & 14 & 577 \\
\hline \multicolumn{11}{|l|}{ MAR } \\
\hline $19 \ldots$ & 0644 & 0.81 & 38 & 9.5 & 75 & 70 & 7.3 & 6.88 & 20 & 1320 \\
\hline $19 \ldots$ & 0704 & 0.81 & 90 & 9.5 & 66 & 60 & 7.0 & 6.72 & 14 & 2930 \\
\hline $19 \ldots$ & 0841 & 0.81 & 49 & 10.0 & 80 & 73 & 7.2 & 6.83 & 16 & 659 \\
\hline $19 \ldots$ & 0914 & 0.81 & 36 & 10.5 & -- & 72 & -- & 6.81 & -- & 492 \\
\hline \multicolumn{11}{|l|}{ APR } \\
\hline $03 \ldots$ & 1255 & 0.00 & 2.6 & 16.0 & 112 & 108 & 7.4 & 6.88 & 35 & 36 \\
\hline JUN & & & & & & & & & & \\
\hline $08 \ldots$ & 1904 & 1.43 & 29 & 21.5 & -- & 63 & -- & 7.43 & -- & -- \\
\hline $08 \ldots$ & 1910 & 1.43 & 49 & 21.5 & 55 & 51 & 6.9 & 7.23 & 13 & 3690 \\
\hline $08 \ldots$ & 2013 & 1.43 & 156 & 22.0 & 53 & 49 & 6.9 & 7.04 & 12 & 3680 \\
\hline $08 \ldots$ & 2133 & 1.43 & 39 & 22.0 & 66 & 62 & 7.1 & 6.96 & 16 & 1020 \\
\hline JUL & & & & & & & & & & \\
\hline $15 \ldots$ & 1500 & 0.24 & 1.2 & 18.0 & -- & 138 & -- & 7.65 & -- & -- \\
\hline $15 \ldots$ & 1545 & 0.24 & 1.7 & 22.5 & -- & 136 & -- & 7.53 & -- & -- \\
\hline $25 \ldots$ & 1927 & 1.96 & 140 & 25.0 & -- & 54 & -- & 7.56 & -- & -- \\
\hline $25 \ldots$ & 1928 & 1.96 & 136 & 25.0 & -- & -- & -- & -- & -- & -- \\
\hline AUG & & & & & & & & & & \\
\hline $07 \ldots$ & 1530 & 0.40 & 57 & 25.5 & -- & -- & -- & -- & -- & 3620 \\
\hline $07 \ldots$ & 1541 & 0.40 & 73 & 25.0 & 55 & 47 & 6.5 & 6.04 & 6.0 & 3140 \\
\hline $07 \ldots$ & 1643 & 0.40 & 22 & 25.0 & 62 & 60 & 6.9 & 6.30 & 12 & 1080 \\
\hline $07 \ldots$ & 1740 & 0.40 & 12 & 25.5 & -- & 57 & -- & 7.29 & -- & -- \\
\hline $11 \ldots$ & 2046 & 3.37 & 114 & 23.0 & 41 & 40 & 7.1 & 6.37 & 7.0 & 3180 \\
\hline $11 \ldots$ & 2352 & 3.37 & 395 & 23.5 & 40 & 40 & 6.9 & 6.40 & 8.0 & 996 \\
\hline $12 \ldots$ & 0212 & 3.37 & 58 & 22.0 & 54 & 52 & 6.8 & 6.43 & 9.0 & -- \\
\hline $\mathrm{OCT}$ & & & & & & & & & & \\
\hline $08 \ldots$ & 0242 & 0.87 & 16 & 14.0 & 66 & 63 & 7.0 & 6.80 & 20 & 614 \\
\hline $08 \ldots$ & 0522 & 0.87 & 35 & 14.5 & 56 & 52 & 6.8 & 7.06 & 12 & 522 \\
\hline $08 \ldots$ & 0748 & 0.87 & 20 & 14.5 & 62 & 59 & 6.8 & 7.00 & 14 & 295 \\
\hline NOV & & & & & & & & & & \\
\hline $08 \ldots$ & 1013 & 0.73 & 3.7 & 18.0 & -- & 118 & -- & 7.16 & -. & -- \\
\hline $08 \ldots$ & 1038 & 0.73 & 24 & 18.0 & 94 & 87 & 7.2 & 7.23 & 30 & 1070 \\
\hline $08 \ldots$ & 1133 & 0.73 & 74 & 18.0 & 59 & 53 & 7.1 & 7.03 & 14 & 1680 \\
\hline $08 \ldots$ & 1242 & 0.73 & 32 & 17.0 & 72 & 69 & 7.1 & 6.97 & 18 & 579 \\
\hline FEB 1997 & & & & & & & & & & \\
\hline $04 \ldots$ & 0531 & 0.45 & 22 & 10.5 & 93 & 86 & 6.9 & 6.86 & 17 & 516 \\
\hline $04 \ldots$ & 0717 & 0.45 & 16 & 10.0 & -- & 82 & -- & 6.89 & -- & -- \\
\hline $13 \ldots$ & 1704 & -- & 20 & 2.5 & -- & 91 & -- & 6.93 & 36 & 905 \\
\hline $14 \ldots$ & 0925 & -- & 10 & 3.5 & -- & 108 & -- & 7.08 & -- & -- \\
\hline MAY & & & & & & & & & & \\
\hline $07 \ldots$ & 0630 & 0.00 & 1.7 & 12.5 & 111 & 108 & 7.4 & 6.66 & 37 & 41 \\
\hline JUN & & & & & & & & & & \\
\hline $02 \ldots$ & 1334 & 0.41 & 20 & 20.0 & 96 & 89 & 7.4 & 7.31 & 27 & 1600 \\
\hline $02 \ldots$ & 1342 & 0.41 & 28 & 20.0 & -- & 66 & -- & 7.46 & -- & -- \\
\hline $02 \ldots$ & 1407 & 0.41 & 49 & 20.5 & 46 & 43 & 7.0 & 7.14 & -- & 2170 \\
\hline $02 \ldots$ & 1508 & 0.41 & 11 & 20.0 & -- & 65 & -- & 6.82 & -- & -- \\
\hline JUL 1997 & & & & & & & & & & \\
\hline $22 \ldots$ & 2350 & 4.31 & 36 & 24.0 & 80 & 75 & 6.9 & 6.70 & 22 & 2580 \\
\hline $23 \ldots$ & 0024 & 4.31 & 142 & 25.5 & 54 & 52 & 6.6 & 6.73 & 10 & 2570 \\
\hline $23 \ldots$ & 0615 & 4.31 & 530 & 24.0 & 42 & 39 & 6.7 & 6.50 & 9.0 & 7400 \\
\hline
\end{tabular}


Table 76. Water-quality data at site 34 (CSW09), July 1995 through September 1997-Continued

\begin{tabular}{|c|c|c|c|c|c|c|c|c|c|c|}
\hline DATE & $\begin{array}{c}\text { RESIDUE } \\
\text { TOTAL } \\
\text { AT } 105 \\
\text { DEG. C, } \\
\text { SUS- } \\
\text { PENDED } \\
(\text { MG } / \text { L) } \\
(00530) \\
\end{array}$ & $\begin{array}{l}\text { RESIDUE } \\
\text { VOLA- } \\
\text { TILE, } \\
\text { SUS- } \\
\text { PENDED } \\
\text { (MG/L) } \\
(00535) \\
\end{array}$ & $\begin{array}{l}\text { SOLIDS, } \\
\text { RESIDUE } \\
\text { AT } 180 \\
\text { DEG. C } \\
\text { DIS- } \\
\text { SOLVED } \\
(\mathrm{MG} / \mathrm{L}) \\
(70300) \\
\end{array}$ & $\begin{array}{c}\text { OXYGEN } \\
\text { DEMAND, } \\
\text { BIO- } \\
\text { CHEMI- } \\
\text { CAL } \\
5 \mathrm{DAY} \\
(\mathrm{MG} / \mathrm{L}) \\
(00310) \\
\end{array}$ & $\begin{array}{c}\text { OXYGEN } \\
\text { DEMAND, } \\
\text { CHEM- } \\
\text { ICAL } \\
\text { (HIGH } \\
\text { LEVEL) } \\
\text { (MG/L) } \\
(00340) \\
\end{array}$ & $\begin{array}{l}\text { NITRO- } \\
\text { GEN, AM- } \\
\text { MONIA + } \\
\text { ORGANIC } \\
\text { TOTAL } \\
\text { (MG / L } \\
\text { AS N) } \\
(00625) \\
\end{array}$ & $\begin{array}{c}\text { NITRO- } \\
\text { GEN, } \\
\text { NO2+NO3 } \\
\text { DIS- } \\
\text { SOLVED } \\
\text { (MG/L } \\
\text { AS N) } \\
(00631) \\
\end{array}$ & $\begin{array}{l}\text { NITRO- } \\
\text { GEN, } \\
\text { AMMONIA } \\
\text { DIS- } \\
\text { SOLVED } \\
\text { (MG/L } \\
\text { AS N) } \\
(00608) \\
\end{array}$ & $\begin{array}{l}\text { NITRO- } \\
\text { GEN, } \\
\text { ORGANIC } \\
\text { TOTAL } \\
\text { (MG/L AS } \\
\text { N) } \\
(00605) \\
\end{array}$ & $\begin{array}{l}\text { NITRO- } \\
\text { GEN, } \\
\text { TOTAL } \\
\text { (MG/L } \\
\text { AS N) } \\
(00600) \\
\end{array}$ \\
\hline \multicolumn{11}{|l|}{ JUL 1995} \\
\hline $06 \ldots$ & -- & 107 & -- & 11 & 87 & 0.90 & 0.490 & 0.140 & 0.76 & 1.4 \\
\hline $06 \ldots$ & -- & 127 & -- & 10 & 87 & 1.2 & 0.500 & 0.300 & 0.90 & 1.7 \\
\hline $07 \ldots$ & -- & 45 & 64 & 7.1 & 59 & 1.1 & 0.410 & 0.150 & 0.95 & 1.5 \\
\hline $28 \ldots$ & -- & -- & -- & -- & $\ldots$ & -- & -- & -- & -- & -- \\
\hline \multicolumn{11}{|l|}{ AUG } \\
\hline $03 \ldots$ & -- & 174 & 57 & 12 & 100 & 2.5 & 0.340 & 0.190 & 2.3 & 2.8 \\
\hline $03 \ldots$ & - & -- & 49 & 10 & 83 & 2.0 & 0.320 & 0.180 & 1.8 & 2.3 \\
\hline $03 \ldots$ & -- & 60 & 57 & 8.5 & 65 & 1.6 & 0.400 & 0.150 & 1.5 & 2.0 \\
\hline $03 \ldots$ & -- & -- & -- & -- & -- & -- & -- & -- & -- & -- \\
\hline $03 \ldots$ & -- & -- & -- & -- & -- & -- & -- & -- & -- & -- \\
\hline \multicolumn{11}{|l|}{ OCT } \\
\hline $20 \ldots$ & -- & -- & 75 & 6.3 & 48 & 3.5 & 0.660 & 1.50 & 2.0 & 4.2 \\
\hline $20 \ldots$ & -- & -- & 61 & 6.8 & 51 & 3.2 & 0.550 & 1.00 & 2.2 & 3.7 \\
\hline $20 \ldots$ & -- & -- & 253 & 5.2 & 42 & 2.2 & 0.480 & 0.740 & 1.5 & 2.7 \\
\hline \multicolumn{11}{|l|}{ NOV } \\
\hline $11 \ldots$ & -- & -- & -- & -- & -- & -- & -- & -- & -- & -- \\
\hline $11 \ldots$ & -- & -- & -- & -- & -- & -- & -- & -- & -- & -- \\
\hline $11 \ldots$ & -- & -- & -- & -- & -- & -- & -- & -- & -- & -- \\
\hline \multicolumn{11}{|l|}{ JAN 1996} \\
\hline $24 \ldots$ & -- & -- & 79 & 9.9 & 62 & 1.8 & 0.670 & 0.100 & 1.7 & 2.5 \\
\hline $24 \ldots$ & -- & -- & 63 & 9.1 & 52 & 1.8 & 0.380 & 0.130 & 1.7 & 2.2 \\
\hline $24 \ldots$ & -- & -- & 75 & 4.5 & 41 & 1.0 & 0.390 & 0.090 & 0.91 & 1.4 \\
\hline \multicolumn{11}{|l|}{ MAR } \\
\hline $19 \ldots$ & 1110 & 124 & 46 & 6.1 & 59 & 2.8 & 0.450 & 0.160 & 2.6 & 3.2 \\
\hline $19 \ldots$ & 2140 & 238 & 37 & 7.5 & 82 & 4.8 & 0.360 & 0.320 & 4.5 & 5.2 \\
\hline $19 \ldots$ & 443 & 50 & 52 & 4.4 & 42 & 1.9 & 0.360 & 0.200 & 1.7 & 2.3 \\
\hline $19 \ldots$ & -- & -- & -- & -- & -- & -- & -- & -- & -- & -- \\
\hline \multicolumn{11}{|l|}{ APR } \\
\hline $03 \ldots$ & 12 & 4 & 74 & $<2.0$ & 7 & 0.54 & 0.440 & $<0.015$ & 0.54 & 0.98 \\
\hline JUN & & & & & & & & & & \\
\hline $08 \ldots$ & -- & -- & -- & -- & -- & -- & -- & -- & -- & -- \\
\hline $08 \ldots$ & 2690 & 305 & 45 & 9.2 & 75 & 3.0 & 0.470 & 0.110 & 2.9 & 3.5 \\
\hline $08 \ldots$ & 2180 & 247 & 39 & 9.7 & 61 & 3.5 & 0.350 & 0.100 & 3.4 & 3.8 \\
\hline $08 \ldots$ & 565 & 65 & 51 & 6.9 & 16 & 1.7 & 0.440 & 0.060 & 1.6 & 2.1 \\
\hline JUL & & & & & & & & & & \\
\hline $15 \ldots$ & -- & -- & -- & -- & -- & -- & -- & -- & -- & -- \\
\hline $15 \ldots$ & -- & -- & -- & -- & -- & -- & -- & -- & -- & -- \\
\hline $25 \ldots$ & -- & -- & -- & -- & -- & -- & -- & -- & -- & -- \\
\hline $25 \ldots$ & -- & -- & -- & -- & -- & -- & -- & -- & -- & -- \\
\hline AUG & & & & & & & & & & \\
\hline $07 \ldots$ & -- & -- & -- & -- & -- & -- & -- & -- & -- & -- \\
\hline $07 \ldots$ & 2400 & 270 & 34 & 8.8 & 11 & 1.8 & 0.680 & 0.130 & 1.7 & 2.5 \\
\hline $07 \ldots$ & 1010 & 110 & 47 & 7.3 & 15 & 1.6 & 0.450 & 0.060 & 1.5 & 2.0 \\
\hline $07 \ldots$ & -- & -- & -- & -- & -- & -- & -- & -- & -- & -- \\
\hline $11 \ldots$ & 2590 & 290 & 28 & 10 & 19 & 2.5 & 0.280 & 0.060 & 2.4 & 2.8 \\
\hline $11 \ldots$ & 600 & 72 & 36 & 3.9 & 29 & 1.4 & 0.280 & 0.050 & 1.3 & 1.7 \\
\hline $12 \ldots$ & -- & -- & 50 & 3.9 & 40 & 1.2 & 0.380 & 0.027 & 1.2 & 1.6 \\
\hline $\mathrm{OCT}$ & & & & & & & & & & \\
\hline $08 \ldots$ & 440 & 44 & 41 & 66 & 25 & 1.4 & 0.260 & 0.060 & 1.3 & 1.7 \\
\hline $08 \ldots$ & 342 & 32 & 36 & 6.5 & 25 & 1.2 & 0.200 & 0.080 & 1.1 & 1.4 \\
\hline $08 \ldots$ & 187 & 15 & 43 & 6.3 & 25 & 1.0 & 0.220 & 0.050 & 0.95 & 1.2 \\
\hline NOV & & & & & & & & & & \\
\hline $08 \ldots$ & -- & -- & -- & -- & -- & -- & -- & -- & -- & -- \\
\hline $08 \ldots$ & 856 & 104 & 66 & 12 & 30 & 2.0 & 0.200 & 0.024 & 2.0 & 2.2 \\
\hline $08 \ldots$ & 1140 & 120 & 41 & 12 & 27 & 2.0 & 0.180 & 0.040 & 2.0 & 2.2 \\
\hline $08 \ldots$ & 406 & 50 & 45 & 11 & 29 & 1.5 & 0.220 & 0.050 & 1.5 & 1.7 \\
\hline FEB 1997 & & & & & & & & & & \\
\hline $04 \ldots$ & 477 & 71 & 61 & 11 & 30 & 1.3 & 0.970 & 0.230 & 1.1 & 2.3 \\
\hline $04 \ldots$ & - & - & -- & -- & -- & -- & -- & -- & -- & -- \\
\hline $13 \ldots$ & 800 & 112 & 55 & 13 & 40 & 3.3 & 0.260 & 0.180 & 3.1 & 3.6 \\
\hline $14 \ldots$ & $\ldots$ & -- & - & -- & -- & -- & - & -- & -- & -- \\
\hline MAY & & & & & & & & & & \\
\hline $07 \ldots$ & 8 & $<1$ & 93 & $<2.0$ & $<5$ & 0.24 & 0.630 & $<0.015$ & 0.24 & 0.87 \\
\hline JUN & & & & & & & & & & \\
\hline $02 \ldots$ & 1240 & 160 & 74 & 17 & 72 & 2.7 & 0.660 & 0.150 & 2.5 & 3.4 \\
\hline $02 \ldots$ & -- & -- & -- & -- & -- & -- & -- & -- & -- & -- \\
\hline $02 \ldots$ & 1640 & 204 & 29 & 12 & 55 & 2.5 & 0.520 & 0.170 & 2.3 & 3.0 \\
\hline $02 \ldots$ & - & -- & -- & -- & -- & -- & -- & -- & -- & -- \\
\hline JUL 1997 & & & & & & & & & & \\
\hline $22 \ldots$ & 2970 & 260 & 65 & 17 & 85 & 3.3 & 0.500 & 0.440 & 2.9 & 3.8 \\
\hline $23 \ldots$ & 1630 & 192 & 45 & 13 & 32 & 2.5 & 0.430 & 0.400 & 2.1 & 2.9 \\
\hline $23 \ldots$ & 720 & 88 & 34 & 6.3 & 28 & 1.5 & 0.290 & 0.340 & 1.2 & 1.8 \\
\hline
\end{tabular}


Table 76. Water-quality data at site 34 (CSW09), July 1995 through September 1997-Continued

\begin{tabular}{|c|c|c|c|c|c|c|c|c|c|c|}
\hline DATE & $\begin{array}{l}\text { PHOS- } \\
\text { PHORUS } \\
\text { TOTAL } \\
\text { (MG / L } \\
\text { AS P) } \\
(00665)\end{array}$ & $\begin{array}{c}\text { PHOS- } \\
\text { PHORUS } \\
\text { ORTHO, } \\
\text { DIS- } \\
\text { SOLVED } \\
\text { (MG/L AS } \\
\text { P) } \\
(00671) \\
\end{array}$ & $\begin{array}{l}\text { OIL AND } \\
\text { GREASE, } \\
\text { TOTAL } \\
\text { RECOV. } \\
\text { GRAVI- } \\
\text { METRIC } \\
\text { (MG / L) } \\
(00556)\end{array}$ & $\begin{array}{c}\text { CARBON, } \\
\text { ORGANIC } \\
\text { TOTAL } \\
\text { (MG/L AS } \\
\text { C) } \\
(00680) \\
\end{array}$ & $\begin{array}{c}\text { STREPTO- } \\
\text { COCCI } \\
\text { FECAL, } \\
\text { (COLS. } \\
\text { PER } \\
100 \mathrm{ML} \text { ) } \\
(31679) \\
\end{array}$ & $\begin{array}{c}\text { COLI- } \\
\text { FORM, } \\
\text { FECAL, } \\
0.45 \\
\text { UM-MF } \\
\text { (COLS.) } \\
100 \mathrm{ML}) \\
(31616)\end{array}$ & $\begin{array}{l}\text { ANTI- } \\
\text { MONY, } \\
\text { TOTAL } \\
\text { (UG / L } \\
\text { AS SB) } \\
(01097 \text { ) }\end{array}$ & $\begin{array}{c}\text { ARSENIC } \\
\text { TOTAL } \\
\text { (UG/L } \\
\text { AS AS) } \\
(01002) \\
\end{array}$ & $\begin{array}{c}\text { BERYL- } \\
\text { LIUM, } \\
\text { TOTAL } \\
\text { RECOV- } \\
\text { ERABLE } \\
\text { (UG / L AS } \\
\text { BE) } \\
(01012) \\
\end{array}$ & $\begin{array}{l}\text { CADMIUM } \\
\text { WATER } \\
\text { UNFLTRD } \\
\text { TOTAL } \\
\text { (UG/L } \\
\text { AS CD) } \\
(01027 \text { ) }\end{array}$ \\
\hline \multicolumn{11}{|l|}{ JUL 1995} \\
\hline $06 \ldots$ & 0.160 & 0.110 & -- & 16 & -- & -- & $<1$ & 1 & -- & $<1$ \\
\hline $06 \ldots$ & 0.220 & 0.120 & -- & 14 & -- & -- & $<1$ & 1 & -- & $<1$ \\
\hline $07 \ldots$ & 0.170 & 0.090 & -- & -- & -- & -- & -- & -- & -- & -- \\
\hline $28 \ldots$ & -- & -- & $<1$ & -- & K140000 & 370000 & -- & -- & -- & -- \\
\hline \multicolumn{11}{|l|}{ AUG } \\
\hline $03 \ldots$ & 0.590 & 0.060 & -- & 34 & -- & -- & $<1$ & $<1$ & -- & $<1$ \\
\hline $03 \ldots$ & 0.590 & 0.070 & -- & 27 & -- & -- & $<1$ & $<1$ & -- & $<1$ \\
\hline $03 \ldots$ & 0.440 & 0.090 & -- & -- & -- & -- & -- & -- & -- & -- \\
\hline $03 \ldots$ & - & -- & -- & -- & 79000 & $\mathrm{~K} 100000$ & -- & -- & -- & -- \\
\hline $03 \ldots$ & -- & -- & -- & -- & 51000 & 63000 & -- & -- & -- & -- \\
\hline \multicolumn{11}{|l|}{ OCT } \\
\hline $20 \ldots$ & 0.520 & 0.110 & -- & 21 & -- & -- & 2 & 3 & -- & $<1$ \\
\hline $20 \ldots$ & 0.790 & 0.380 & -- & 19 & -- & -- & $<1$ & 2 & -- & $<1$ \\
\hline $20 \ldots$ & 0.510 & 0.240 & -- & -- & -- & -- & -- & -- & -- & -- \\
\hline \multicolumn{11}{|l|}{ NOV } \\
\hline $11 \ldots$ & -- & -- & -- & -- & 5000 & K72 00 & -- & -- & -- & -- \\
\hline $11 \ldots$ & -- & -- & -- & -- & 22000 & K7600 & -- & -- & -- & -- \\
\hline $11 \ldots$ & -- & -- & -- & -- & 8800 & 20000 & -- & -- & -- & -- \\
\hline \multicolumn{11}{|l|}{ JAN 1996} \\
\hline $24 \ldots$ & 0.590 & 0.050 & -- & 24 & -- & -- & $<1$ & 4 & -- & $<1$ \\
\hline $24 \ldots$ & 0.680 & 0.050 & -- & 23 & 28000 & 3800 & 1 & 3 & -- & $<1$ \\
\hline $24 \ldots$ & 0.310 & 0.060 & -- & -- & $\mathrm{K} 12000$ & 2200 & -- & -- & -- & -- \\
\hline \multicolumn{11}{|l|}{ MAR } \\
\hline $19 \ldots$ & 0.870 & 0.090 & -- & 26 & -- & -- & 6 & 4 & -- & $<1$ \\
\hline $19 \ldots$ & 1.14 & 0.080 & -- & 42 & -- & -- & 7 & 8 & -- & 8 \\
\hline $19 \ldots$ & 0.360 & 0.090 & -- & -- & 22000 & 4700 & -- & -- & -- & -- \\
\hline $19 \ldots$ & -- & -- & -- & -- & -- & -- & -- & -- & -- & -- \\
\hline \multicolumn{11}{|l|}{ APR } \\
\hline $03 \ldots$ & 0.030 & $<0.010$ & 6 & -- & 350 & 110 & $<1$ & $<1$ & $<10$ & $<1$ \\
\hline JUN & & & & & & & & & & \\
\hline $08 \ldots$ & -- & -- & -- & -- & -- & -- & -- & $\ldots$ & -- & -- \\
\hline $08 \ldots$ & 0.900 & 0.010 & 9 & -- & -- & -- & 25 & 6 & -- & $<1$ \\
\hline $08 \ldots$ & 1.05 & $<0.010$ & -- & -- & -- & -- & 24 & 4 & -- & $<1$ \\
\hline $08 \ldots$ & 0.390 & 0.020 & 9 & -- & -- & -- & 9 & $<1$ & -- & $<1$ \\
\hline JUL & & & & & & & & & & \\
\hline $15 \ldots$ & -- & -- & $<1$ & -- & 30000 & K79000 & $+\infty$ & -- & -- & -- \\
\hline $15 \ldots$ & -- & -- & -- & -- & 23000 & 20000 & -- & -- & -- & -- \\
\hline $25 \ldots$ & -- & -- & -- & -- & 33000 & 28000 & -- & -- & -- & -- \\
\hline $25 \ldots$ & -- & -- & -- & -- & 42000 & $\mathrm{~K} 110000$ & -- & -- & - & -- \\
\hline AUG & & & & & & & & & & \\
\hline $07 \ldots$ & -- & -- & -- & -- & -- & -- & -- & -- & -- & -- \\
\hline $07 \ldots$ & 0.800 & $<0.010$ & -- & 31 & -- & -- & 2 & 6 & -- & $<1$ \\
\hline $07 \ldots$ & 0.470 & 0.020 & -- & -- & 61000 & 38000 & -- & -- & -- & -- \\
\hline $07 \ldots$ & -- & -- & -- & -- & 35000 & 34000 & -- & -- & -- & -- \\
\hline $11 \ldots$ & 0.720 & 0.010 & -- & -- & -- & $\cdots$ & $<1$ & 10 & -- & $<1$ \\
\hline $11 \ldots$ & 0.490 & 0.060 & -- & 18 & -- & -- & $<1$ & 3 & -- & $<1$ \\
\hline $12 \ldots$ & 0.180 & 0.040 & -- & -- & -- & -- & $\rightarrow$ & -- & -- & -- \\
\hline OCT & & & & & & & & & & \\
\hline $08 \ldots$ & 0.370 & 0.018 & -- & 17 & -- & -- & $<1$ & 1 & -- & $<1$ \\
\hline $08 \ldots$ & 0.370 & 0.060 & -- & 15 & -- & -- & $<1$ & 2 & -- & $<1$ \\
\hline $08 \ldots$ & 0.240 & 0.037 & -- & -- & 29000 & 21000 & -- & -- & -- & -- \\
\hline NOV & & & & & & & & & & \\
\hline $08 \ldots$ & -- & -- & 3 & -- & K11000 & K21000 & -- & -- & -- & -- \\
\hline $08 \ldots$ & 0.650 & 0.040 & -- & 14 & -- & -- & 14 & 3 & $\rightarrow$ & $<1$ \\
\hline $08 \ldots$ & 0.770 & 0.070 & -- & 16 & K19000 & 24000 & 2 & 3 & -- & $<1$ \\
\hline $08 \ldots$ & 0.390 & 0.070 & -- & -- & $\mathrm{K} 12000$ & $\mathrm{~K} 11000$ & -- & -- & -- & -- \\
\hline FEB 1997 & & & & & & & & & & \\
\hline $04 \ldots$ & 0.420 & 0.049 & $\cdots$ & 16 & -- & -- & $<1$ & 7 & -- & $<1$ \\
\hline $04 \ldots$ & -- & -- & 1 & -- & 21000 & $\mathrm{~K} 10000$ & -- & -- & -- & -- \\
\hline $13 \ldots$ & 0.760 & 0.050 & -- & -- & -- & -- & $<1$ & 3 & -- & 1 \\
\hline $14 \ldots$ & -- & -- & -- & -- & 5400 & 2700 & -- & -- & -- & -- \\
\hline MAY & & & & & & & & & & \\
\hline $07 \ldots$ & 0.041 & $<0.010$ & 1 & -- & 520 & 240 & $<1$ & $<1$ & -- & $<1$ \\
\hline JUN & & & & & & & & & & \\
\hline $02 \ldots$ & 0.680 & 0.012 & -- & -. & -- & -- & $<1$ & $<1$ & -- & $<1$ \\
\hline $02 \ldots$ & -- & -- & -- & -- & -- & -- & -- & -- & -- & -- \\
\hline $02 \ldots$ & 0.860 & $<0.010$ & -- & -- & -- & -- & $<1$ & 2 & -- & $<1$ \\
\hline $02 \ldots$ & -- & -- & -- & -- & 48000 & $\mathrm{~K} 2200$ & -- & -- & -- & -- \\
\hline JUL 1997 & & & & & & & & & & \\
\hline $22 \ldots$ & 0.970 & 0.046 & -- & 18 & -- & -- & $<1$ & 5 & -- & $<1$ \\
\hline $23 \ldots$ & 0.790 & 0.020 & -- & 17 & - & -- & 4 & 3 & -- & $<1$ \\
\hline $23 \ldots$ & 0.550 & 0.032 & -- & 14 & - & -- & $<1$ & 3 & -- & $<1$ \\
\hline
\end{tabular}

K Results based on colony count outside the acceptable range (nonideal colony count). 
Table 76. Water-quality data at site 34 (CSW09), July 1995 through September 1997-Continued

\begin{tabular}{|c|c|c|c|c|c|c|c|c|c|}
\hline DATE & $\begin{array}{c}\text { CHRO- } \\
\text { MIUM, } \\
\text { TOTAL } \\
\text { RECOV- } \\
\text { ERABLE } \\
\text { (UG/L } \\
\text { AS CR) } \\
(01034) \\
\end{array}$ & $\begin{array}{c}\text { COPPER, } \\
\text { TOTAL } \\
\text { RECOV- } \\
\text { ERABLE } \\
\text { (UG / L AS } \\
\text { CU) } \\
(01042) \\
\end{array}$ & $\begin{array}{l}\text { LEAD, } \\
\text { TOTAL } \\
\text { RECOV- } \\
\text { ERABLE } \\
\text { (UG/L } \\
\text { AS PB) } \\
(01051) \\
\end{array}$ & $\begin{array}{c}\text { MERCURY } \\
\text { TOTAL } \\
\text { RECOV- } \\
\text { ERABLE } \\
\text { (UG/L AS } \\
\text { HG) } \\
(71900) \\
\end{array}$ & $\begin{array}{c}\text { NICKEL, } \\
\text { TOTAL } \\
\text { RECOVER- } \\
\text { ABLE } \\
\text { (UG/L } \\
\text { AS NI) } \\
\text { (01067) } \\
\end{array}$ & $\begin{array}{c}\text { SELE- } \\
\text { NIUM, } \\
\text { TOTAL } \\
\text { (UG/L } \\
\text { AS SE) } \\
(01147) \\
\end{array}$ & $\begin{array}{c}\text { SILVER, } \\
\text { TOTAL } \\
\text { RECOV- } \\
\text { ERABLE } \\
\text { (UG/L } \\
\text { AS AG) } \\
\text { (01077) } \\
\end{array}$ & $\begin{array}{c}\text { ZINC, } \\
\text { TOTAL } \\
\text { RECOV- } \\
\text { ERABLE } \\
\text { (UG/L } \\
\text { AS ZN) } \\
(01092) \\
\end{array}$ & $\begin{array}{c}\text { CYANIDE } \\
\text { TOTAL } \\
\text { (MG/L AS } \\
\text { CN) } \\
(00720) \\
\end{array}$ \\
\hline \multicolumn{10}{|l|}{ JUL 1995} \\
\hline $06 \ldots$ & 19 & 31 & 19 & -- & 13 & -- & -- & 80 & -- \\
\hline $06 \ldots$ & 11 & 30 & 23 & -- & 7 & -- & -- & 80 & -- \\
\hline $07 \ldots$ & -- & -- & -- & -- & -- & -- & -- & -- & -- \\
\hline $28 \ldots$ & -- & -- & -- & -- & -- & -- & -- & -- & -- \\
\hline \multicolumn{10}{|l|}{$A \cup G$} \\
\hline $03 \ldots$ & 14 & 35 & 24 & -- & 9 & -- & -- & 110 & -- \\
\hline $03 \ldots$ & 12 & 24 & 22 & -- & 6 & -- & -- & 100 & -- \\
\hline $03 \ldots$ & -- & -- & -- & -- & -- & -- & -- & -- & -- \\
\hline $03 \ldots$ & -- & -- & -- & -- & -- & -- & -- & -- & -- \\
\hline $03 \ldots$ & -- & -- & -- & -- & -- & -- & -- & -- & -- \\
\hline \multicolumn{10}{|l|}{$\mathrm{OCT}$} \\
\hline $\begin{array}{l}20 \ldots \\
20\end{array}$ & $\begin{array}{l}28 \\
27\end{array}$ & $\begin{array}{l}45 \\
55\end{array}$ & $\begin{array}{l}18 \\
21\end{array}$ & -- & $\begin{array}{r}11 \\
9\end{array}$ & -- & -- & 130 & -- \\
\hline $\begin{array}{l}20 \ldots \\
20 \ldots\end{array}$ & $\begin{array}{l}21 \\
--\end{array}$ & -- & $-\ldots$ & -- & $\begin{array}{r}9 \\
--\end{array}$ & -- & -- & 110 & -- \\
\hline \multicolumn{10}{|l|}{ NOV $20 . \cdots$} \\
\hline $11 \ldots$ & -- & - & -- & -- & -. & -- & -- & -- & -- \\
\hline $11 \ldots$ & -- & -- & -- & -- & -- & -- & -- & -- & -- \\
\hline \multirow{2}{*}{\multicolumn{10}{|c|}{ JAN 1996}} \\
\hline & & & & & & & & & \\
\hline $24 \ldots$ & 45 & 66 & 26 & -- & 20 & -- & -- & 150 & -- \\
\hline $24 \ldots$ & 38 & 53 & 25 & -- & 14 & -- & -- & 120 & -- \\
\hline $24 \ldots$ & -- & -- & - & -- & -- & -- & -- & $\ldots$ & -- \\
\hline \multicolumn{10}{|l|}{ MAR } \\
\hline $19 \ldots$ & 57 & 77 & 30 & -- & 19 & -- & -- & 200 & -- \\
\hline $19 \ldots$ & 74 & 90 & 47 & -- & 27 & -- & -- & 220 & -- \\
\hline $19 \ldots$ & -- & -- & -- & -- & -- & -- & -- & -- & -- \\
\hline $19 \ldots$ & -- & - & -- & -- & -- & -- & -- & -- & -- \\
\hline \multicolumn{10}{|l|}{ APR } \\
\hline \multicolumn{10}{|l|}{ JUN } \\
\hline $08 \ldots$ & -- & -- & -. & -- & -- & -- & -- & -. & -- \\
\hline $08 \ldots$ & 71 & 130 & 49 & -- & 28 & -- & -- & 210 & -- \\
\hline $08 \ldots$ & 69 & 91 & 43 & -- & 27 & -- & -- & 180 & -- \\
\hline $08 \ldots$ & 20 & 22 & 16 & -- & 9 & -- & -- & 80 & -- \\
\hline \multicolumn{10}{|l|}{ JUL } \\
\hline $15 \ldots$ & -- & -- & -- & -- & -- & -- & -- & -- & -- \\
\hline $15 \ldots$ & -- & -- & - & -- & -- & -- & -- & -- & $-\cdot$ \\
\hline $25 \ldots$ & -- & -- & - & -- & -- & -- & -- & -- & -- \\
\hline $25 \ldots$ & -- & -- & -- & -- & -- & -- & -- & -- & -- \\
\hline AUG & & & & & & & & & \\
\hline $07 \ldots$ & -- & -- & -- & -- & -- & -- & -- & -- & -- \\
\hline $07 \ldots$ & 55 & 140 & 49 & -- & 30 & -- & -- & 220 & -- \\
\hline $07 \ldots$ & -- & -- & -- & -- & -- & -- & -- & -- & -- \\
\hline $07 \ldots$ & -- & -- & -- & -- & - & -- & -. & -- & -- \\
\hline $11 \ldots$ & 96 & 170 & 41 & -- & 21 & -- & -- & 200 & -- \\
\hline $11 \ldots$ & 25 & 46 & 13 & -- & 21 & -- & -- & 80 & -- \\
\hline $12 \ldots$ & -- & -- & -- & -- & -- & -- & -- & -- & -- \\
\hline OCT & & & & & & & & & \\
\hline $08 \ldots$ & 26 & 58 & 12 & -- & 11 & -- & -- & 130 & -- \\
\hline $08 \ldots$ & 20 & 89 & 9 & -- & 7 & -- & -- & 100 & -- \\
\hline $08 \ldots$ & -- & -- & -- & -- & -- & -- & -- & -- & -- \\
\hline NoV & & & & & & & & & \\
\hline $08 \ldots$ & -- & -- & -- & -- & -- & -- & -- & -- & -- \\
\hline $08 \ldots$ & 45 & 58 & 15 & -- & 23 & -- & -- & 180 & -- \\
\hline $08 \ldots$ & 44 & 70 & 22 & -- & 20 & -- & -- & 170 & -- \\
\hline $08 \ldots$ & -- & -- & -- & -- & -- & -- & - & -- & -- \\
\hline FEB 1997 & & & & & & & & & \\
\hline $04 \ldots$ & 23 & 7 & 17 & -- & $<1$ & -- & -- & 130 & -- \\
\hline $04 \ldots$ & -- & -- & -- & -- & -- & -- & -- & -- & -- \\
\hline $13 \ldots$ & 10 & 19 & 19 & -- & 12 & -- & -- & 140 & -- \\
\hline $14 \ldots$ & -- & -- & -- & -- & -- & -- & $\cdots$ & -- & -. \\
\hline MAY & & & & & & & & & \\
\hline$\underset{\text { JUN }}{07 \ldots}$ & 2 & 8 & $<1$ & -- & 3 & -- & -- & 40 & -- \\
\hline $02 \ldots$ & 31 & 21 & 25 & -- & 14 & -- & -- & 200 & -- \\
\hline $02 \ldots$ & -- & -- & -- & -- & -- & -- & -- & - & -- \\
\hline $02 \ldots$ & 28 & 80 & 32 & -- & 15 & -- & -- & 180 & -- \\
\hline $02 \ldots$ & -- & -- & -- & -- & -- & -- & -- & -- & -- \\
\hline JUL 1997 & & & & & & & & & \\
\hline $22 \ldots$ & 45 & 68 & 35 & -- & 12 & -- & -- & 240 & -- \\
\hline $23 \ldots$ & 60 & 67 & 38 & -- & 19 & -- & -- & 210 & -- \\
\hline $23 \ldots$ & 29 & 39 & 18 & -- & 11 & -- & -- & 150 & -- \\
\hline
\end{tabular}


Table 77. Water-quality data at site 37 (CSWO6), July 1995 through June 1997

\begin{tabular}{|c|c|c|c|c|c|c|c|c|c|c|}
\hline DATE & TIME & $\begin{array}{c}\text { RAIN- } \\
\text { FALL } \\
\text { ACCUM } \\
(\text { IN }) \\
(00045)\end{array}$ & $\begin{array}{c}\text { DIS- } \\
\text { CHARGE, } \\
\text { INST. } \\
\text { CUBIC } \\
\text { FEET PER } \\
\text { SECOND } \\
(00061)\end{array}$ & $\begin{array}{c}\text { TEMPER- } \\
\text { ATURE } \\
\text { WATER } \\
\text { DEG. C) } \\
(00010)\end{array}$ & $\begin{array}{c}\text { SPE- } \\
\text { CIFIC } \\
\text { CONDUC- } \\
\text { TANCE } \\
\text { LAB } \\
\text { (US/CM) } \\
(90095)\end{array}$ & $\begin{array}{c}\text { SPE- } \\
\text { CIFIC } \\
\text { CONDUC- } \\
\text { TANCE } \\
\text { (US/CM) } \\
(00095\end{array}$ & $\begin{array}{c}\text { PH } \\
\text { WATER } \\
\text { RAW LAB } \\
\text { (STAN- } \\
\text { DARD } \\
\text { UNITS) } \\
(00403)\end{array}$ & $\begin{array}{c}\text { PH } \\
\text { WATER } \\
\text { RAW } \\
\text { FIELD } \\
\text { (STAN- } \\
\text { DARD } \\
\text { UNITS) } \\
(00400)\end{array}$ & $\begin{array}{c}\text { ALKA- } \\
\text { LINITY } \\
\text { LAB } \\
\text { (MG/L AS } \\
\text { CACO3) } \\
(90410)\end{array}$ & $\begin{array}{l}\text { SEDI- } \\
\text { MENT, } \\
\text { SUS- } \\
\text { PENDED } \\
(\text { MG / L) } \\
(80154)\end{array}$ \\
\hline \multicolumn{11}{|l|}{ JUL 1995} \\
\hline $21 \ldots$ & 1909 & 0.33 & 0.08 & -- & -- & -- & -- & -- & -- & -- \\
\hline $21 \ldots$ & 1943 & 0.33 & 0.77 & -- & -- & -- & -- & -- & -- & -- \\
\hline $21 \ldots$ & 2040 & 0.33 & 0.39 & 27.0 & $\ldots$ & $\ldots$ & -- & -- & -- & -- \\
\hline $31 \ldots$ & 1437 & 0.99 & 1.5 & 29.0 & 60 & 53 & 6.3 & 6.10 & 24 & 364 \\
\hline $31 \ldots$ & 1456 & 0.99 & 7.0 & 27.5 & 48 & 45 & 6.2 & 6.30 & 7.2 & 68 \\
\hline $31 \ldots$ & 1500 & 0.99 & 6.4 & 27.5 & -- & - & -- & -- & - & -. \\
\hline $31 \ldots$ & 1522 & 0.99 & 3.2 & 27.0 & -- & -- & -- & -- & -- & -- \\
\hline $31 \ldots$ & 1545 & 0.99 & 1.4 & 27.0 & 57 & 53 & 6.4 & 6.30 & 8.9 & 29 \\
\hline $31 \ldots$ & 1804 & 0.99 & 2.2 & 26.0 & 51 & 48 & 6.3 & 6.00 & 8.9 & 20 \\
\hline $31 \ldots$ & 1846 & 0.99 & 1.1 & 25.5 & 51 & 50 & 7.4 & 6.20 & 9.2 & 15 \\
\hline \multicolumn{11}{|l|}{ OCT } \\
\hline $04 \ldots$ & 0511 & 2.86 & 0.44 & 20.5 & -- & 69 & -- & 6.61 & -- & -- \\
\hline $04 \ldots$ & 0535 & 2.86 & 6.1 & 20.5 & 37 & 29 & 6.3 & 6.57 & 8.0 & 62 \\
\hline $04 \ldots$ & 0608 & 2.86 & 2.4 & 20.0 & 40 & 31 & 6.5 & 6.58 & 7.0 & 25 \\
\hline \multicolumn{11}{|l|}{ JAN 1996} \\
\hline $24 \ldots$ & 1115 & 0.13 & 0.25 & 9.5 & -- & 145 & -- & 6.72 & -- & -- \\
\hline \multicolumn{11}{|l|}{$\mathrm{FEB}$} \\
\hline $20 \ldots$ & 0624 & 0.17 & 0.39 & 9.0 & 138 & 125 & 7.1 & 6.74 & 23 & 36 \\
\hline $20 \ldots$ & 0801 & 0.17 & 0.69 & 9.0 & 100 & 88 & 7.0 & 6.68 & 13 & 38 \\
\hline $20 \ldots$ & 0832 & 0.17 & 0.59 & 9.0 & - & 80 & -- & 7.23 & -- & -- \\
\hline $20 \ldots$ & 1105 & 0.17 & 0.21 & 10.0 & 93 & 81 & 7.0 & 6.75 & 14 & 28 \\
\hline \multicolumn{11}{|l|}{ APR } \\
\hline $29 \ldots$ & 1332 & 0.89 & 0.53 & 20.5 & 69 & 55 & 6.9 & 6.50 & 20 & 59 \\
\hline $29 \ldots$ & 1336 & 0.89 & 0.89 & 20.5 & - & 63 & -- & 6.52 & -- & -- \\
\hline $29 \ldots$ & 1347 & 0.89 & 8.2 & 20.5 & -- & 44 & -- & 6.56 & -- & -- \\
\hline $29 \ldots$ & 1423 & 0.89 & 5.8 & 20.5 & 37 & 29 & 6.9 & 6.87 & 7.0 & 143 \\
\hline $29 \ldots$ & 1708 & 0.89 & 0.81 & 20.0 & 46 & 47 & 7.0 & 6.67 & 10 & 34 \\
\hline \multicolumn{11}{|l|}{ AUG } \\
\hline $11 \ldots$ & 2116 & 0.77 & 0.27 & 25.0 & 83 & 79 & 6.7 & 6.35 & 8.0 & 55 \\
\hline $11 \ldots$ & 2324 & 0.77 & 1.2 & 24.5 & 50 & 50 & 6.8 & 6.64 & 7.0 & 57 \\
\hline \multicolumn{11}{|l|}{ OCT } \\
\hline $07 \ldots$ & 2248 & 1.46 & 0.21 & 15.5 & 77 & 72 & 6.5 & 6.76 & 14 & 45 \\
\hline $08 \ldots$ & 0056 & 1.46 & 1.9 & 15.0 & 40 & 34 & 6.5 & 6.74 & 7.0 & 39 \\
\hline $08 \ldots$ & 0214 & 1.46 & 3.2 & 15.0 & 33 & 27 & 6.4 & 6.61 & 6.0 & 59 \\
\hline $08 \ldots$ & 0650 & 1.46 & 0.97 & 15.0 & 40 & 37 & 6.4 & 6.67 & 8.0 & 75 \\
\hline \multicolumn{11}{|l|}{ NOV } \\
\hline $\begin{array}{r}18 \ldots \\
\text { JAN } 1997\end{array}$ & 1615 & 0.45 & 0.02 & 11.5 & -- & 132 & -- & 7.95 & -- & -- \\
\hline $24 \ldots$ & 1230 & 0.23 & 0.21 & 6.5 & 143 & 138 & 6.9 & 6.81 & 26 & 40 \\
\hline $24 \ldots$ & 1253 & 0.23 & 0.34 & 6.5 & - & 151 & -- & 6.67 & -- & - \\
\hline $25 \ldots$ & 0312 & 0.31 & 1.5 & 5.0 & 62 & 58 & 6.9 & 6.73 & 12 & 87 \\
\hline $25 \ldots$ & 0447 & 0.31 & 0.69 & 5.5 & 52 & 47 & 6.9 & 6.59 & 9.0 & 48 \\
\hline $25 \ldots$ & 0905 & 0.31 & 0.12 & 5.5 & -- & -- & -- & -- & -- & - \\
\hline \multicolumn{11}{|l|}{ MAY } \\
\hline $03 \ldots$ & 0602 & 0.80 & 0.50 & 17.5 & 92 & 85 & 7.1 & 6.81 & 25 & 72 \\
\hline $03 \ldots$ & 0607 & 0.80 & 0.73 & 18.0 & - & 77 & -- & 6.51 & -- & -- \\
\hline $03 \ldots$ & 0623 & 0.80 & 2.0 & 18.0 & 60 & 57 & 7.0 & 6.89 & 14 & 67 \\
\hline $03 \ldots$ & 1030 & 0.80 & 1.2 & 18.0 & 52 & 49 & 6.9 & 6.88 & 10 & 41 \\
\hline $07 \ldots$ & 1445 & 0.00 & 0.01 & 27.5 & 164 & 152 & 8.9 & 7.60 & 52 & 27 \\
\hline \multicolumn{11}{|l|}{ JUN } \\
\hline $02 \ldots$ & 1446 & 0.66 & 1.2 & 23.0 & -- & 65 & -- & 6.76 & -- & -- \\
\hline $02 \ldots$ & 1456 & 0.66 & 5.1 & 22.5 & -- & 54 & -. & 6.79 & $\ldots$ & $\ldots$ \\
\hline $06 \ldots$ & 1212 & 0.36 & 0.39 & 17.0 & -- & 66 & -- & 6.26 & -- & -- \\
\hline
\end{tabular}


Table 77. Water-quality data at site 37 (CSW06), July 1995 through June 1997—Continued

\begin{tabular}{|c|c|c|c|c|c|c|c|c|c|c|}
\hline DATE & $\begin{array}{c}\text { RESIDUE } \\
\text { TOTAL } \\
\text { AT } 105 \\
\text { DEG. C, } \\
\text { SUS- } \\
\text { PENDED } \\
\text { (MG / L) } \\
(00530) \\
\end{array}$ & $\begin{array}{l}\text { RESIDUE } \\
\text { VOLA- } \\
\text { TILE, } \\
\text { SUS- } \\
\text { PENDED } \\
(\text { MG / L) } \\
(00535) \\
\end{array}$ & $\begin{array}{c}\text { SOLIDS, } \\
\text { RESIDUE } \\
\text { AT } 180 \\
\text { DEG. C } \\
\text { DIS- } \\
\text { SOLVED } \\
(\text { MG } / \mathrm{L}) \\
(70300) \\
\end{array}$ & $\begin{array}{c}\text { OXYGEN } \\
\text { DEMAND, } \\
\text { BIO- } \\
\text { CHEMI- } \\
\text { CAL } \\
5 \mathrm{DAY} \\
(\mathrm{MG} / \mathrm{L}) \\
(00310) \\
\end{array}$ & $\begin{array}{c}\text { OXYGEN } \\
\text { DEMAND, } \\
\text { CHEM- } \\
\text { ICAL } \\
\text { (HIGH } \\
\text { LEVEL) } \\
\text { (MG / L) } \\
(00340) \\
\end{array}$ & $\begin{array}{c}\text { NITRO- } \\
\text { GEN, AM- } \\
\text { MONIA + } \\
\text { ORGANIC } \\
\text { TOTAL } \\
\text { (MG/L } \\
\text { AS N) } \\
(00625) \\
\end{array}$ & $\begin{array}{c}\text { NITRO- } \\
\text { GEN, } \\
\text { NO2+NO3 } \\
\text { DIS- } \\
\text { SOLVED } \\
\text { (MG/L } \\
\text { AS N) } \\
(00631) \\
\end{array}$ & $\begin{array}{c}\text { NITRO- } \\
\text { GEN, } \\
\text { AMMONIA } \\
\text { DIS- } \\
\text { SOLVED } \\
\text { (MG / L } \\
\text { AS N) } \\
(00608) \\
\end{array}$ & $\begin{array}{c}\text { NITRO- } \\
\text { GEN, } \\
\text { ORGANIC } \\
\text { TOTAL } \\
\text { (MG/L AS } \\
\text { N) } \\
(00605) \\
\end{array}$ & $\begin{array}{c}\text { NITRO- } \\
\text { GEN, } \\
\text { TOTAL } \\
\text { (MG/L } \\
\text { AS N) } \\
(00600)\end{array}$ \\
\hline \multicolumn{11}{|l|}{ JUL 1995} \\
\hline $21 \ldots$ & -- & -- & -- & -- & -- & -- & -- & -- & -- & -- \\
\hline $21 \ldots$ & -- & -- & -- & -- & -- & - & -- & -- & -- & -- \\
\hline $21 \ldots$ & -- & -- & -- & -- & -- & -- & -. & -- & -- & -- \\
\hline $31 \ldots$ & -- & 50 & 46 & $>24$ & 190 & 2.6 & 0.450 & 0.040 & 2.6 & 3.0 \\
\hline $31 \ldots$ & -- & -- & 36 & 6.5 & 34 & 0.90 & 0.360 & 0.180 & 0.72 & 1.3 \\
\hline $31 \ldots$ & -- & -- & -- & -- & -- & -- & -- & -- & -- & -- \\
\hline $31 \ldots$ & -- & -- & -- & -- & -- & -- & -- & -- & -- & -- \\
\hline $31 \ldots$ & -- & -- & 50 & 7.2 & 43 & 1.0 & 0.450 & 0.120 & 0.88 & 1.5 \\
\hline $31 \ldots$ & -- & -- & 50 & 6.2 & 35 & 0.80 & 0.350 & 0.050 & 0.75 & 1.1 \\
\hline $31 \ldots$ & -- & 11 & 44 & 4.3 & 31 & 0.70 & 0.370 & 0.040 & 0.66 & 1.1 \\
\hline \multicolumn{11}{|l|}{ OCT } \\
\hline $04 \ldots$ & -- & -- & -- & -- & -- & -- & -- & -- & -- & -- \\
\hline $04 \ldots$ & -- & -- & 36 & 5.4 & 22 & 0.42 & 0.190 & 0.070 & 0.35 & 0.61 \\
\hline $04 \ldots$ & -- & -- & 38 & 4.7 & 25 & 0.36 & 0.250 & 0.050 & 0.31 & 0.61 \\
\hline \multicolumn{11}{|l|}{ JAN 1996} \\
\hline $24 \ldots$ & -- & -- & -- & -- & -- & -- & -- & -- & -. & -- \\
\hline \multicolumn{11}{|l|}{$\mathrm{FEB}$} \\
\hline $20 \ldots$ & 9 & 3 & 114 & 9.8 & 31 & 0.70 & 0.730 & 0.120 & 0.58 & 1.4 \\
\hline $20 \ldots$ & 8 & 3 & 88 & 7.4 & 27 & 0.66 & 0.850 & 0.120 & 0.54 & 1.5 \\
\hline $20 \ldots$ & -- & -- & -- & -- & -- & -- & -- & -- & -- & -- \\
\hline $20 \ldots$ & 4 & 2 & 90 & 4.8 & 29 & 0.62 & 0.540 & 0.070 & 0.55 & 1.2 \\
\hline \multicolumn{11}{|l|}{$\mathrm{APR}$} \\
\hline $29 \ldots$ & 32 & 8 & 54 & 11 & 45 & 1.3 & 0.210 & 0.070 & 1.2 & 1.5 \\
\hline $29 \ldots$ & -- & -- & -- & -- & - & -- & -- & -- & -- & -- \\
\hline $29 \ldots$ & -- & -- & -- & -- & -- & -- & -- & -- & -- & -- \\
\hline $29 \ldots$ & 111 & 13 & 24 & 5.4 & 24 & 0.84 & 0.190 & 0.060 & 0.78 & 1.0 \\
\hline $29 \ldots$ & 9 & $<1$ & 37 & 8.0 & 25 & 0.85 & 0.190 & $<0.015$ & 0.85 & 1.0 \\
\hline \multicolumn{11}{|l|}{ AUG } \\
\hline $11 \ldots$ & 7 & 2 & 75 & 11 & 40 & 1.3 & 0.470 & 0.080 & 1.2 & 1.8 \\
\hline $11 \ldots$ & 6 & 2 & 40 & 8.1 & 25 & 0.59 & 0.340 & 0.027 & 0.56 & 0.93 \\
\hline \multicolumn{11}{|l|}{ OCT } \\
\hline $07 \ldots$ & 6 & 2 & 47 & 4.2 & 18 & 0.52 & 0.100 & 0.020 & 0.50 & 0.62 \\
\hline $08 \ldots$ & 20 & $<1$ & 22 & 3.5 & 12 & 0.71 & 0.200 & 0.040 & 0.67 & 0.91 \\
\hline $08 \ldots$ & 26 & 2 & 19 & 2.7 & 5 & 0.76 & 0.140 & 0.026 & 0.73 & 0.90 \\
\hline $08 \ldots$ & 32 & 2 & 25 & 2.7 & 10 & 0.92 & 0.190 & $<0.015$ & 0.92 & 1.1 \\
\hline \multicolumn{11}{|l|}{ NOV } \\
\hline $18 \ldots$ & -- & -- & -- & -- & -- & -- & -- & -- & -- & -- \\
\hline \multicolumn{11}{|l|}{ JAN 1997} \\
\hline $24 \ldots$ & 15 & 5 & 86 & 3.0 & 11 & 0.53 & 0.360 & 0.080 & 0.45 & 0.89 \\
\hline $24 \ldots$ & -- & -- & - & -- & -- & -- & -- & -- & -- & -- \\
\hline $25 \ldots$ & 53 & 10 & 39 & 4.8 & 18 & 0.73 & 0.340 & 0.040 & 0.69 & 1.1 \\
\hline $25 \ldots$ & 20 & 5 & 32 & 3.2 & 12 & 0.59 & 0.240 & 0.025 & 0.57 & 0.83 \\
\hline $25 \ldots$ & -- & -- & -- & -- & -- & $\sim$ & -- & -- & -- & -- \\
\hline \multicolumn{11}{|l|}{ MAY } \\
\hline $03 \ldots$ & 94 & 34 & 74 & 20 & 57 & 1.8 & 0.260 & 0.120 & 1.7 & 2.1 \\
\hline $03 \ldots$ & -- & -- & -- & -- & -- & -- & -- & -- & -- & -- \\
\hline $03 \ldots$ & 60 & 11 & 51 & 13 & 31 & 0.95 & 0.370 & 0.130 & 0.82 & 1.3 \\
\hline $03 \ldots$ & 31 & 4 & 48 & 6.9 & 20 & 0.67 & 0.170 & $<0.015$ & 0.67 & 0.84 \\
\hline $07 \ldots$ & 8 & 1 & 190 & 4.4 & 12 & 0.36 & $<0.050$ & $<0.015$ & 0.36 & 0.36 \\
\hline \multicolumn{11}{|l|}{ JUN } \\
\hline $02 \ldots$ & -- & -- & -- & -- & -- & -- & -- & -- & -- & -- \\
\hline $02 \ldots$ & -- & -- & -- & -- & -- & -- & -- & -- & -- & -- \\
\hline $06 \ldots$ & -- & -- & -- & -- & -- & -- & -- & -- & -- & -- \\
\hline
\end{tabular}


Table 77. Water-quality data at site 37 (CSW06), July 1995 through June 1997—Continued

\begin{tabular}{|c|c|c|c|c|c|c|c|c|c|c|}
\hline DATE & $\begin{array}{l}\text { PHOS- } \\
\text { PHORUS } \\
\text { TOTAL } \\
\text { (MG / L } \\
\text { AS P) } \\
(00665) \\
\end{array}$ & $\begin{array}{c}\text { PHOS- } \\
\text { PHORUS } \\
\text { ORTHO, } \\
\text { DIS- } \\
\text { SOLVED } \\
\text { (MG/L AS } \\
\text { P) } \\
(00671) \\
\end{array}$ & $\begin{array}{c}\text { OIL AND } \\
\text { GREASE, } \\
\text { TOTAL } \\
\text { RECOV. } \\
\text { GRAVI- } \\
\text { METRIC } \\
\text { (MG/L) } \\
\text { (00556) }\end{array}$ & $\begin{array}{c}\text { CARBON, } \\
\text { ORGANIC } \\
\text { TOTAL } \\
\text { (MG/L AS } \\
\text { C) } \\
(00680)\end{array}$ & $\begin{array}{c}\text { STREPTO- } \\
\text { COCCI } \\
\text { FECAL, } \\
\text { (COLS. } \\
\text { PER } \\
100 \mathrm{ML}) \\
(31679) \\
\end{array}$ & $\begin{array}{c}\text { COLI- } \\
\text { FORM, } \\
\text { FECAL, } \\
0.45 \\
\text { UM-MF } \\
\text { (COLS. / } \\
\text { (00 ML) } \\
(31616) \\
\end{array}$ & $\begin{array}{c}\text { ANTI- } \\
\text { MONY, } \\
\text { TOTAL } \\
\text { (UG/L } \\
\text { AS SB) } \\
(01097) \\
\end{array}$ & $\begin{array}{c}\text { ARSENIC } \\
\text { TOTAL } \\
\text { (UG } / L \\
\text { AS AS) } \\
(01002 \text { ) }\end{array}$ & $\begin{array}{c}\text { BERYL- } \\
\text { LIUM, } \\
\text { TOTAL } \\
\text { RECOV- } \\
\text { ERABLE } \\
\text { (UG/L AS } \\
\text { BE) } \\
(01012) \\
\end{array}$ & $\begin{array}{c}\text { CADMIUM } \\
\text { WATER } \\
\text { UNFLTRD } \\
\text { TOTAL } \\
\text { (UG /L } \\
\text { AS CD) } \\
\text { (01027) }\end{array}$ \\
\hline \multicolumn{11}{|l|}{ JUL 1995} \\
\hline $21 \ldots$ & -- & -- & $<1$ & -- & 75000 & 370000 & -- & -- & -- & -- \\
\hline $21 \ldots$ & -. & -- & -- & -- & K120000 & 480000 & -- & -- & -- & -- \\
\hline $21 \ldots$ & -- & -- & -- & -- & K110000 & 24000 & -- & -- & -- & -- \\
\hline $31 \ldots$ & 1.40 & 0.240 & -- & -- & $\ldots$ & -- & $<1$ & $<1$ & $<10$ & $<1$ \\
\hline $31 \ldots$ & 0.220 & 0.180 & -- & -- & $\mathrm{K} 45000$ & K110000 & $<1$ & $<1$ & $<10$ & $<1$ \\
\hline $31 \ldots$ & -- & -- & -- & -- & -- & -- & -- & -- & -- & -- \\
\hline $31 \ldots$ & -- & -- & -- & +- & 44000 & 290000 & -- & -- & - & -- \\
\hline $31 \ldots$ & 0.330 & 0.270 & -- & -- & 53000 & 320000 & -- & -- & -- & -- \\
\hline $31 \ldots$ & 0.230 & 0.160 & -- & -- & -- & - & $<1$ & $<1$ & $<10$ & $<1$ \\
\hline $31 \ldots$ & 0.170 & 0.150 & -- & -- & -- & -- & - & -- & -- & -- \\
\hline \multicolumn{11}{|l|}{$\mathrm{OCT}$} \\
\hline $04 \ldots$ & -- & -- & $<1$ & -- & K140000 & K74000 & -- & -- & -- & -- \\
\hline $04 \ldots$ & 0.250 & 0.150 & -- & 8.7 & K63000 & K63000 & $<1$ & $<1$ & $<10$ & $<1$ \\
\hline $04 \ldots$ & 0.230 & 0.190 & -- & -- & -- & -- & -- & -- & -- & -- \\
\hline \multicolumn{11}{|l|}{ JAN 1996} \\
\hline $24 \ldots$ & -- & -- & 1 & -- & K540 & K90 & -- & -- & -- & -- \\
\hline \multicolumn{11}{|l|}{ FEB } \\
\hline $20 \ldots$ & 0.150 & 0.040 & -- & 10 & -- & -- & 1 & $<1$ & $<10$ & $<1$ \\
\hline $20 \ldots$ & 0.110 & 0.060 & -- & 9.8 & -- & -- & 1 & $<1$ & $<10$ & $<1$ \\
\hline $20 \ldots$ & -- & -- & -- & -- & 2000 & K90 & -- & -- & -- & -- \\
\hline $20 \ldots$ & 0.100 & 0.050 & -- & -- & -- & -- & -- & -- & -- & -- \\
\hline \multicolumn{11}{|l|}{ APR } \\
\hline $29 \ldots$ & 0.200 & 0.050 & -- & -- & -- & -- & $<1$ & $<1$ & $<10$ & $<1$ \\
\hline $29 \ldots$ & -- & -- & -- & -- & -- & -- & -- & -- & - & -- \\
\hline $29 \ldots$ & -- & -- & 2 & 19 & 46000 & K7700 & -- & -- & -- & -- \\
\hline $29 \ldots$ & 0.350 & 0.040 & -- & 9.4 & 53000 & K6800 & $<1$ & $<1$ & $<10$ & $<1$ \\
\hline $29 \ldots$ & 0.160 & 0.040 & -- & -- & -- & -- & -- & -- & -- & -- \\
\hline \multicolumn{11}{|l|}{ AUG } \\
\hline $11 \ldots$ & 0.190 & 0.100 & -- & 18 & -- & -- & $<1$ & $<1$ & $<10$ & $<1$ \\
\hline $11 \ldots$ & 0.180 & 0.140 & -- & 11 & -- & -- & $<1$ & $<1$ & $<10$ & $<1$ \\
\hline \multicolumn{11}{|l|}{ OCT } \\
\hline $07 \ldots$ & 0.028 & 0.050 & -- & 9.6 & -- & -- & $<1$ & $<1$ & $<10$ & $<1$ \\
\hline $08 \ldots$ & 0.130 & 0.120 & -- & 7.4 & -- & -- & $<1$ & $<1$ & $<10$ & $<1$ \\
\hline $08 \ldots$ & 0.230 & 0.080 & -- & 6.8 & -- & -- & $<1$ & $<1$ & $<10$ & $<1$ \\
\hline $08 \ldots$ & 0.270 & 0.080 & -- & -- & -- & -- & -- & -- & -- & -- \\
\hline \multicolumn{11}{|l|}{ NOV } \\
\hline $\begin{array}{c}18 \ldots \\
\text { JAN } 1997\end{array}$ & -- & -- & \multicolumn{2}{|c|}{ JAN 1997} & 3000 & K200 & -- & -- & -- & -- \\
\hline $24 \ldots$ & 0.080 & 0.012 & -- & 6.6 & -- & -- & $<1$ & 1 & -- & -- \\
\hline $24 \ldots$ & -- & -- & 3 & -- & 2900 & $<100$ & -- & -- & -- & -- \\
\hline $25 \ldots$ & 0.190 & 0.020 & -- & 10 & -- & -- & $<1$ & $<1$ & -- & -- \\
\hline $25 \ldots$ & 0.100 & 0.021 & -- & -- & -- & -- & -- & -- & -- & -- \\
\hline $25 \ldots$ & -- & -- & $\cdots$ & -- & K1600 & K360 & -- & -- & -- & -- \\
\hline \multicolumn{11}{|l|}{ MAY } \\
\hline $03 \ldots$ & 0.360 & 0.050 & -- & -- & -- & -- & $<1$ & $<1$ & -- & -- \\
\hline $03 \ldots$ & -- & -- & -- & -- & -- & -- & -- & -- & -- & -- \\
\hline $03 \ldots$ & 0.210 & 0.030 & -- & -- & -- & -- & $<1$ & $<1$ & -- & -- \\
\hline $03 \ldots$ & 0.150 & 0.019 & $-\overline{-}$ & -- & - & -- & -- & - & -- & -- \\
\hline $07 \ldots$ & 0.090 & $<0.010$ & 2 & -- & K450 & K90 & $<1$ & $<1$ & -- & -- \\
\hline \multicolumn{11}{|l|}{ JUN } \\
\hline $02 \ldots$ & -- & -- & 3 & 14 & 73000 & 35000 & -- & -- & -- & -- \\
\hline $02 \ldots$ & -- & -- & -- & 20 & 80000 & 58000 & -- & -- & -- & -- \\
\hline $06 \ldots$ & -- & -- & -- & -- & K16000 & K14000 & -- & -- & -- & -- \\
\hline
\end{tabular}

K Results based on colony count outside the acceptable range (nonideal colony count). 
Table 77. Water-quality data at site 37 (CSW06), July 1995 through June 1997-Continued

\begin{tabular}{|c|c|c|c|c|c|c|c|c|c|}
\hline DATE & $\begin{array}{c}\text { CHRO- } \\
\text { MIUM, } \\
\text { TOTAL } \\
\text { RECOV- } \\
\text { ERABLE } \\
\text { (UG/L } \\
\text { AS CR) } \\
(01034) \\
\end{array}$ & $\begin{array}{c}\text { COPPER, } \\
\text { TOTAL } \\
\text { RECOV- } \\
\text { ERABLE } \\
\text { (UG/L AS } \\
\text { CU) } \\
(01042) \\
\end{array}$ & $\begin{array}{c}\text { LEAD, } \\
\text { TOTAL } \\
\text { RECOV- } \\
\text { ERABLE } \\
\text { (UG/L } \\
\text { AS PB) } \\
(01051) \\
\end{array}$ & $\begin{array}{c}\text { MERCURY } \\
\text { TOTAL } \\
\text { RECOV- } \\
\text { ERABLE } \\
\text { (UG/L AS } \\
\text { HG) } \\
(71900) \\
\end{array}$ & $\begin{array}{c}\text { NICKEL, } \\
\text { TOTAL } \\
\text { RECOVER- } \\
\text { ABLE } \\
\text { (UG/L } \\
\text { AS NI) } \\
(01067) \\
\end{array}$ & $\begin{array}{l}\text { SELE- } \\
\text { NIUM, } \\
\text { TOTAL } \\
\text { (UG /L } \\
\text { AS SE) } \\
(01147) \\
\end{array}$ & $\begin{array}{c}\text { SILVER, } \\
\text { TOTAL, } \\
\text { RECOV- } \\
\text { ERABLE } \\
\text { (UG/L } \\
\text { AS AG) } \\
(01077) \\
\end{array}$ & $\begin{array}{c}\text { ZINC, } \\
\text { TOTAL } \\
\text { RECOV- } \\
\text { ERABLE } \\
\text { (UG/L } \\
\text { AS ZN) } \\
(01092) \\
\end{array}$ & $\begin{array}{c}\text { CYANIDE } \\
\text { TOTAL } \\
\text { (MG/L AS } \\
\text { CN) } \\
(00720) \\
\end{array}$ \\
\hline \multicolumn{10}{|l|}{ JUL 1995} \\
\hline $21 \ldots$ & - & -- & -- & -- & -- & -- & -- & -- & -- \\
\hline $21 \ldots$ & -- & -- & -- & -- & -- & -- & -- & -- & -- \\
\hline $21 \ldots$ & -- & -- & -- & -- & -- & -- & -- & -- & -- \\
\hline $31 \ldots$ & 12 & 44 & 11 & $<0.10$ & 41 & $<1$ & $<1$ & 240 & $<0.010$ \\
\hline $31 \ldots$ & 2 & 16 & 3 & $<0.10$ & 8 & $<1$ & $<1$ & 70 & $<0.010$ \\
\hline $31 \ldots$ & -- & -- & -- & -- & -- & -- & -- & $\ldots$ & -- \\
\hline $31 \ldots$ & -- & -- & -- & -- & -- & -- & -- & -- & -- \\
\hline $31 \ldots$ & - & -- & -- & -- & -- & -- & -- & -- & -- \\
\hline $31 \ldots$ & 2 & 12 & 2 & $<0.10$ & 7 & $<1$ & $<1$ & 70 & $<0.010$ \\
\hline $31 \ldots$ & - & - & - & -- & -. & -- & - & -- & -- \\
\hline \multicolumn{10}{|l|}{ Ост } \\
\hline $04 \ldots$ & -- & -- & -- & -- & -- & -- & -- & -- & -- \\
\hline $04 \ldots$ & 4 & 14 & 5 & $<0.10$ & 8 & $<1$ & $<1$ & 110 & $<0.010$ \\
\hline $04 \ldots$ & -- & -- & -- & -- & -- & -- & -- & -- & -- \\
\hline \multicolumn{10}{|l|}{ JAN 1996} \\
\hline $24 \ldots$ & -- & -- & -- & -- & -- & -- & - & -- & -- \\
\hline \multicolumn{10}{|l|}{$\mathrm{FEB}$} \\
\hline $20 \ldots$ & 3 & 7 & 2 & $<0.10$ & 10 & $<1$ & 2 & 120 & -- \\
\hline $20 \ldots$ & 3 & 7 & 2 & $<0.10$ & 9 & $<1$ & 2 & 90 & -- \\
\hline $20 \ldots$ & -- & -- & -. & -- & -- & - & -- & -- & -- \\
\hline $20 \ldots$ & -- & $\ldots$ & -- & -- & -- & -- & -- & -- & -- \\
\hline \multicolumn{10}{|l|}{$A P R$} \\
\hline $29 \ldots$ & 15 & 14 & $<1$ & $<0.10$ & 18 & $<1$ & $<1$ & 40 & $<0.010$ \\
\hline $29 \ldots$ & -. & -- & -- & -- & -- & -- & -- & -- & -- \\
\hline $29 \ldots$ & - & -- & -- & -- & -- & -- & -- & -- & -- \\
\hline $29 \ldots$ & 32 & 21 & 41 & $<0.10$ & 18 & $<1$ & $<1$ & 90 & $<0.010$ \\
\hline $29 \ldots$ & -- & - & -- & -- & -- & -- & -- & -- & -- \\
\hline \multicolumn{10}{|l|}{ AUG } \\
\hline $11 \ldots$ & 2 & 13 & 2 & $<0.10$ & 10 & $<1$ & $<1$ & 120 & -- \\
\hline $11 \ldots$ & 2 & 10 & 1 & $<0.10$ & 7 & $<1$ & $<1$ & 70 & -- \\
\hline \multicolumn{10}{|l|}{ OCT } \\
\hline $07 \ldots$ & 3 & 19 & 2 & $<0.10$ & 9 & $<1$ & $<1$ & 80 & $<0.010$ \\
\hline $08 \ldots$ & 3 & 11 & 2 & $<0.10$ & 6 & $<1$ & $<1$ & 60 & $<0.010$ \\
\hline $08 \ldots$ & 4 & 11 & 2 & $<0.10$ & 6 & $<1$ & $<1$ & 50 & $<0.010$ \\
\hline $08 \ldots$ & - & $\ldots$ & -- & -- & -. & - & - & -- & -- \\
\hline \multicolumn{10}{|l|}{ Nov } \\
\hline $18 \ldots$ & -- & - & -- & -- & -- & -- & -- & -- & -- \\
\hline $24 \ldots$ & 4 & 8 & 7 & $<0.10$ & 6 & -- & -- & 120 & -- \\
\hline $24 \ldots$ & - & -. & -. & -- & -- & -- & -- & - & -- \\
\hline $25 \ldots$ & 7 & 14 & 12 & $<0.10$ & 6 & -- & -- & 100 & -- \\
\hline $25 \ldots$ & - & - & - & -- & -- & -- & -- & -. & -- \\
\hline $25 \ldots$ & -- & -- & -- & -- & -- & -- & -- & -- & -- \\
\hline \multicolumn{10}{|l|}{ MAY } \\
\hline $03 \ldots$ & 7 & 17 & 8 & $<0.10$ & 14 & -- & -- & 110 & -- \\
\hline $03 \ldots$ & -- & -- & -- & -- & -- & -- & -- & -- & -- \\
\hline $03 \ldots$ & 5 & 16 & 6 & $<0.10$ & 10 & -- & -- & 90 & -- \\
\hline $03 \ldots$ & -- & - & -- & -- & $\ldots$ & -- & -- & - & -. \\
\hline $07 \ldots$ & 3 & 5 & 3 & $<0.10$ & 7 & -- & -- & 40 & -- \\
\hline \multicolumn{10}{|l|}{ JUN } \\
\hline $02 \ldots$ & -- & -- & -- & -- & -- & -- & -- & -- & -- \\
\hline $02 \ldots$ & -- & -- & -- & -- & -- & -- & -- & -- & -- \\
\hline $06 \ldots$ & - & -- & -- & -- & -- & -- & -- & -- & -- \\
\hline
\end{tabular}


Table 78. Water-quality data at site 39 (CSW05), July 1995 through June 1997

\begin{tabular}{|c|c|c|c|c|c|c|c|c|c|c|}
\hline DATE & TIME & $\begin{array}{c}\text { RAIN- } \\
\text { FALL } \\
\text { ACCUM } \\
(\text { IN }) \\
(00045)\end{array}$ & $\begin{array}{c}\text { DIS- } \\
\text { CHARGE, } \\
\text { INST. } \\
\text { CUBIC } \\
\text { FEET PER } \\
\text { SECOND } \\
(00061) \\
\end{array}$ & $\begin{array}{c}\text { TEMPER- } \\
\text { ATURE } \\
\text { WATER } \\
\text { DEG. C) } \\
(00010)\end{array}$ & $\begin{array}{c}\text { SPE- } \\
\text { CIFIC } \\
\text { CONDUC- } \\
\text { TANCE } \\
\text { LAB } \\
\text { (US/CM) } \\
(90095)\end{array}$ & $\begin{array}{c}\text { SPE- } \\
\text { CIFIC } \\
\text { CONDUC- } \\
\text { TANCE } \\
(\text { US /CM }) \\
(00095 \\
\end{array}$ & $\begin{array}{c}\text { PH } \\
\text { WATER } \\
\text { RAW LAB } \\
\text { (STAN- } \\
\text { DARD } \\
\text { UNITS) } \\
(00403 \text { ) }\end{array}$ & $\begin{array}{c}\text { PH } \\
\text { WATER } \\
\text { RAW } \\
\text { FIELD } \\
\text { (STAN- } \\
\text { DARD } \\
\text { UNITS) } \\
(00400) \\
\end{array}$ & $\begin{array}{c}\text { ALKA- } \\
\text { LINITY } \\
\text { LAB } \\
\text { (MG/LAS } \\
\text { CACO3) } \\
(90410)\end{array}$ & $\begin{array}{c}\text { SEDI- } \\
\text { MENT, } \\
\text { SUS- } \\
\text { PENDED } \\
\text { (MG / L) } \\
(80154)\end{array}$ \\
\hline \multicolumn{11}{|l|}{ JUL 1995} \\
\hline $07 \ldots$ & 0156 & 0.39 & 2.3 & 24.5 & -- & -- & -- & - & -- & -- \\
\hline $21 \ldots$ & 1719 & 1.51 & 0.044 & 29.0 & -- & -- & -- & -- & -- & -- \\
\hline $27 \ldots$ & 1528 & 1.16 & 0.002 & 23.5 & -- & -- & -- & -- & -- & -- \\
\hline $27 \ldots$ & 1610 & 1.16 & 0.24 & 23.5 & -- & -- & -- & -- & -- & -- \\
\hline $31 \ldots$ & 1504 & 0.14 & 0.11 & 24.5 & -- & -- & -- & -- & -- & -- \\
\hline $31 \ldots$ & 1508 & 0.14 & 6.9 & 24.5 & -- & -- & -- & -- & -- & -- \\
\hline $31 \ldots$ & 1528 & 0.14 & 3.3 & 24.5 & -- & -- & -- & -- & -- & -- \\
\hline $31 \ldots$ & 1545 & 0.14 & 0.73 & 24.0 & -- & -- & -- & -- & -- & -- \\
\hline \multicolumn{11}{|l|}{ OCT } \\
\hline $04 \ldots$ & 0439 & 1.97 & 0.08 & 21.0 & -- & 28 & -- & 7.23 & -- & 64 \\
\hline $04 \ldots$ & 0509 & 1.97 & 0.85 & 20.5 & -- & 20 & -- & 6.69 & -- & 59 \\
\hline $04 \ldots$ & 0603 & 1.97 & 0.36 & 20.0 & 56 & 46 & 6.9 & 6.75 & 12 & 26 \\
\hline $04 \ldots$ & 0900 & 1.97 & 2.7 & 18.5 & -- & -- & -- & -- & -- & -- \\
\hline $04 \ldots$ & 0912 & 1.97 & 3.6 & 18.5 & 32 & 24 & 6.7 & 6.61 & 8.0 & 74 \\
\hline \multicolumn{11}{|l|}{ NOV } \\
\hline $11 \ldots$ & 1258 & 1.13 & 2.1 & 15.0 & -- & 33 & -- & 6.99 & -- & -- \\
\hline \multicolumn{11}{|l|}{ JAN 1996} \\
\hline $24 \ldots$ & 0710 & 0.24 & 0.11 & 10.5 & 166 & 152 & 7.5 & 7.06 & 24 & 114 \\
\hline $24 \ldots$ & 0723 & 0.24 & 0.09 & 11.0 & -- & 155 & -- & 7.14 & - & -- \\
\hline $24 \ldots$ & 0845 & 0.24 & 0.95 & 11.0 & 50 & 45 & 7.5 & 6.64 & 25 & 213 \\
\hline $24 \ldots$ & 1025 & 0.24 & 0.22 & 10.5 & 63 & 56 & 7.3 & 6.83 & 11 & 50 \\
\hline \multicolumn{11}{|l|}{ MAR } \\
\hline $06 \ldots$ & 1118 & 1.43 & 0.44 & 14.0 & -- & 109 & -- & 6.61 & -- & -- \\
\hline \multicolumn{11}{|l|}{ APR } \\
\hline $03 \ldots$ & 1015 & 0.00 & 0.01 & 12.0 & 358 & 350 & 7.9 & 7.87 & 114 & 41 \\
\hline $26 \ldots$ & 0906 & 0.45 & 1.1 & 18.5 & 64 & 74 & 7.2 & 6.80 & 11 & 189 \\
\hline $26 \ldots$ & 0914 & 0.45 & 0.99 & 18.5 & -- & 44 & -- & 6.83 & -- & -- \\
\hline $26 \ldots$ & 1002 & 0.45 & 0.76 & 18.0 & 37 & 37 & 7.3 & 6.70 & 8.0 & 64 \\
\hline $26 \ldots$ & 1008 & 0.45 & 0.73 & 17.5 & -- & 35 & -- & 6.84 & -- & 48 \\
\hline $26 \ldots$ & 1029 & 0.45 & 0.92 & 17.5 & -- & 38 & -- & 6.78 & -- & -- \\
\hline $26 \ldots$ & 1034 & 0.45 & 0.88 & 17.5 & -- & 38 & -- & 6.81 & 6.0 & 44 \\
\hline $26 \ldots$ & 1138 & 0.45 & 0.46 & 16.5 & 33 & 37 & 7.2 & 6.92 & 7.0 & 24 \\
\hline \multicolumn{11}{|l|}{ JUL } \\
\hline $31 \ldots$ & 2206 & 0.29 & 6.4 & 24.0 & 71 & 66 & 6.0 & 5.83 & 2.0 & 423 \\
\hline $31 \ldots$ & 2217 & 0.29 & 2.1 & 24.0 & 82 & 78 & 6.6 & 6.36 & 5.0 & 70 \\
\hline $31 \ldots$ & 2306 & 0.29 & 0.16 & 24.0 & 158 & 149 & 7.1 & 6.40 & 16 & 65 \\
\hline \multicolumn{11}{|l|}{$\mathrm{OCT}$} \\
\hline $07 \ldots$ & 1550 & 1.31 & 0.01 & 18.5 & -- & 192 & -- & 7.25 & -- & -- \\
\hline $07 \ldots$ & 1725 & 1.31 & 0.04 & 18.0 & -- & 106 & -- & 7.27 & -- & -- \\
\hline $07 \ldots$ & 1742 & 1.31 & 0.02 & 18.0 & 114 & 105 & 7.1 & 7.35 & 21 & 93 \\
\hline $07 \ldots$ & 2211 & 1.31 & 0.25 & 16.0 & - & 40 & 6.9 & 6.99 & 8.0 & 89 \\
\hline $08 \ldots$ & 0130 & 1.31 & 0.82 & 15.5 & 44 & 38 & 6.9 & 6.98 & 9.0 & 41 \\
\hline $08 \ldots$ & 0638 & 1.31 & 0.24 & 15.5 & 85 & 78 & 7.2 & 7.01 & 16 & 60 \\
\hline \multicolumn{11}{|l|}{ JAN 1997} \\
\hline $16 \ldots$ & 0102 & 0.61 & 0.53 & 9.5 & 74 & 65 & 6.8 & 6.89 & 6.0 & 190 \\
\hline $16 \ldots$ & 0156 & 0.61 & 3.4 & 8.0 & 25 & 11 & 6.7 & 6.34 & 3.0 & 309 \\
\hline $16 \ldots$ & 0405 & 0.61 & 0.32 & 6.5 & 52 & 45 & 6.9 & 6.72 & 9.0 & 32 \\
\hline $24 \ldots$ & 1119 & 0.28 & 0.61 & 7.0 & -- & 47 & -- & 6.83 & -- & -- \\
\hline \multicolumn{11}{|l|}{ MAY } \\
\hline $07 \ldots$ & 0525 & 0.00 & 0.01 & 13.5 & 446 & 426 & 7.9 & 6.57 & 147 & 22 \\
\hline $25 \ldots$ & 1449 & 0.41 & 5.6 & 26.0 & 37 & 36 & 6.0 & 6.56 & 3.0 & 459 \\
\hline $25 \ldots$ & 1454 & 0.41 & 3.2 & 25.5 & -- & 35 & -- & 6.71 & -- & -- \\
\hline $25 \ldots$ & 1540 & 0.41 & 0.11 & 25.0 & 95 & 88 & 6.6 & 6.84 & 12 & 26 \\
\hline \multicolumn{11}{|l|}{ JUN } \\
\hline $06 \ldots$ & 1102 & 0.36 & 0.14 & 18.0 & -- & 42 & -- & 6.78 & -- & -- \\
\hline
\end{tabular}


Table 78. Water-quality data at site 39 (CSW05), July 1995 through June 1997—Continued

\begin{tabular}{|c|c|c|c|c|c|c|c|c|c|c|}
\hline DATE & $\begin{array}{l}\text { RESIDUE } \\
\text { TOTAL } \\
\text { AT } 105 \\
\text { DEG. C, } \\
\text { SUS- } \\
\text { PENDED } \\
\text { (MG/L) } \\
(00530) \\
\end{array}$ & $\begin{array}{l}\text { RESIDUE } \\
\text { VOLA- } \\
\text { TILE, } \\
\text { SUS- } \\
\text { PENDED } \\
(\text { MG /L) } \\
(00535) \\
\end{array}$ & $\begin{array}{l}\text { SOLIDS, } \\
\text { RESIDUE } \\
\text { AT } 180 \\
\text { DEG. C } \\
\text { DIS- } \\
\text { SOLVED } \\
(M G / L) \\
(70300) \\
\end{array}$ & $\begin{array}{c}\text { OXYGEN } \\
\text { DEMAND, } \\
\text { BIO- } \\
\text { CHEMI- } \\
\text { CAL } \\
5 \mathrm{DAY} \\
(\mathrm{MG} / \mathrm{L}) \\
(00310)\end{array}$ & $\begin{array}{c}\text { OXYGEN } \\
\text { DEMAND, } \\
\text { CHEM- } \\
\text { ICAL } \\
\text { (HIGH } \\
\text { LEVEL) } \\
\text { (MG/L) } \\
(00340)\end{array}$ & $\begin{array}{c}\text { NITRO- } \\
\text { GEN, AM- } \\
\text { MONIA + } \\
\text { ORGANIC } \\
\text { TOTAL } \\
\text { (MG/L } \\
\text { AS N) } \\
(00625) \\
\end{array}$ & $\begin{array}{c}\text { NITRO- } \\
\text { GEN, } \\
\text { NO2 +NO3 } \\
\text { DIS- } \\
\text { SOLVED } \\
\text { (MG/L } \\
\text { AS N) } \\
(00631) \\
\end{array}$ & $\begin{array}{c}\text { NITRO- } \\
\text { GEN, } \\
\text { AMMONIA } \\
\text { DIS- } \\
\text { SOLVED } \\
\text { (MG/L } \\
\text { AS N) } \\
(00608) \\
\end{array}$ & $\begin{array}{l}\text { NITRO- } \\
\text { GEN, } \\
\text { ORGANIC } \\
\text { TOTAL } \\
\text { (MG / L AS } \\
\text { N) } \\
(00605) \\
\end{array}$ & $\begin{array}{c}\text { NITRO- } \\
\text { GEN, } \\
\text { TOTAL } \\
\text { (MG/L } \\
\text { AS N) } \\
(00600) \\
\end{array}$ \\
\hline \multicolumn{11}{|l|}{ JUL 1995} \\
\hline $07 \ldots$ & -- & -- & -- & -- & -- & -- & -- & -- & -- & -- \\
\hline $21 \ldots$ & $\ldots$ & -- & -- & -- & -- & $\ldots$ & - & - & -- & - \\
\hline $27 \ldots$ & -- & -- & -- & -- & -- & -- & -- & -- & -- & -- \\
\hline $27 \ldots$ & -- & -- & -- & -- & -- & -- & $\ldots$ & -- & -- & -- \\
\hline $31 \ldots$ & -- & -- & -- & -- & -- & -- & -- & -- & -- & -- \\
\hline $31 \ldots$ & -- & -- & -- & -- & -- & -- & -- & -- & -- & -- \\
\hline $31 \ldots$ & -- & -- & -- & - & -- & -- & -- & -- & -- & -- \\
\hline $31 \ldots$ & -- & -- & -- & -- & -- & -- & -- & -- & -- & $\ldots$ \\
\hline \multicolumn{11}{|l|}{ OCT } \\
\hline $04 \ldots$ & -- & -- & -- & -- & 18 & 0.76 & 0.550 & 0.060 & 0.70 & 1.3 \\
\hline $04 \ldots$ & -- & -- & -- & -- & 18 & 0.61 & 0.170 & 0.120 & 0.49 & 0.78 \\
\hline $04 \ldots$ & -- & -- & 42 & 5.2 & 24 & 0.67 & 0.570 & 0.080 & 0.59 & 1.2 \\
\hline $04 \ldots$ & -- & -- & -- & -- & - & -- & -- & -- & - & - \\
\hline $04 \ldots$ & -- & -- & 27 & 2.9 & 22 & 0.62 & 0.530 & 0.060 & 0.56 & 1.1 \\
\hline \multicolumn{11}{|l|}{ NOV } \\
\hline \multicolumn{11}{|l|}{ JAN 1996} \\
\hline $24 \ldots$ & -- & -- & 130 & 25 & 100 & 7.1 & 1.30 & 0.890 & 6.2 & 8.4 \\
\hline $24 \ldots$ & -- & -- & -- & - & - & $\ldots$ & -- & -- & $\ldots$ & $\ldots$ \\
\hline $24 \ldots$ & -- & -- & 42 & 11 & 89 & 1.3 & 0.480 & 0.350 & 0.95 & 1.8 \\
\hline $24 \ldots$ & -- & - & 54 & 3.4 & 25 & 0.55 & 0.220 & 0.080 & 0.47 & 0.77 \\
\hline \multicolumn{11}{|l|}{ MAR } \\
\hline $06 \ldots$ & -- & -- & -- & -- & -- & -- & -- & -- & -- & -- \\
\hline \multicolumn{11}{|l|}{ APR } \\
\hline $03 \ldots$ & $<1$ & $<1$ & 205 & $<2.0$ & $<5$ & 0.35 & 0.600 & 0.020 & 0.33 & 0.95 \\
\hline $26 \ldots$ & 156 & 62 & 74 & $>33$ & 120 & 5.1 & 1.13 & 0.690 & 4.4 & 6.2 \\
\hline $26 \ldots$ & -- & -- & - & -- & -- & -- & -- & -- & -- & -- \\
\hline $26 \ldots$ & 41 & 10 & 32 & 14 & 41 & 1.3 & 0.320 & 0.190 & 1.1 & 1.6 \\
\hline $26 \ldots$ & -- & - & -- & -- & -- & -- & -- & -- & -- & -- \\
\hline $26 \ldots$ & -- & -- & -- & $\ldots$ & +- & -- & -- & -- & $\ldots$ & -- \\
\hline $26 \ldots$ & 24 & 4 & 31 & 15 & 25 & 1.0 & 0.260 & 0.090 & 0.91 & 1.3 \\
\hline $26 \ldots$ & 14 & 1 & 30 & 6.0 & 22 & 0.78 & 0.290 & 0.060 & 0.72 & 1.1 \\
\hline \multicolumn{11}{|l|}{ JUL } \\
\hline $31 \ldots$ & 207 & 45 & 48 & 12 & 49 & 2.8 & 0.600 & 0.780 & 2.0 & 3.4 \\
\hline $31 \ldots$ & 41 & 10 & 62 & 9.6 & 24 & 1.5 & 0.600 & 0.430 & 1.1 & 2.1 \\
\hline $31 \ldots$ & 28 & 7 & 120 & 7.1 & 29 & 1.6 & 0.880 & 0.290 & 1.3 & 2.5 \\
\hline \multicolumn{11}{|l|}{$\mathrm{OCT}$} \\
\hline $07 \ldots$ & -- & -- & -- & -- & -- & -- & $\ldots$ & -- & -- & -- \\
\hline $07 \ldots$ & -- & -- & -- & -- & -- & -- & -- & -- & -- & -- \\
\hline $07 \ldots$ & 28 & 4 & 87 & 12 & 75 & 2.2 & 1.02 & 0.340 & 1.9 & 3.2 \\
\hline $07 \ldots$ & 32 & 9 & 23 & 6.6 & 25 & 0.88 & 0.320 & 0.100 & 0.78 & 1.2 \\
\hline $08 \ldots$ & 21 & 4 & 12 & 3.7 & 10 & 0.58 & 0.230 & 0.040 & 0.54 & 0.81 \\
\hline $08 \ldots$ & 21 & $<1$ & 51 & 3.4 & 25 & 0.91 & 0.790 & 0.050 & 0.86 & 1.7 \\
\hline \multicolumn{11}{|l|}{ JAN 1997} \\
\hline $16 \ldots$ & 138 & 38 & 49 & 14 & 87 & 2.0 & 0.800 & 0.390 & 1.6 & 2.8 \\
\hline $16 \ldots$ & 226 & 50 & 10 & 7.3 & 85 & 1.4 & 0.150 & 0.080 & 1.3 & 1.5 \\
\hline $16 \ldots$ & 13 & 4 & 38 & 4.6 & 15 & 0.72 & 0.360 & 0.020 & 0.70 & 1.1 \\
\hline $24 \ldots$ & -- & -- & -- & -- & -- & -- & -- & -- & -- & -- \\
\hline \multicolumn{11}{|l|}{ MAY } \\
\hline $07 \ldots$ & 6 & 2 & 297 & 3.1 & $<5$ & 0.40 & 1.12 & $<0.015$ & 0.40 & 1.5 \\
\hline $25 \ldots$ & 232 & 64 & 58 & $>32$ & 120 & 2.9 & 0.580 & 0.240 & 2.7 & 3.5 \\
\hline $25 \ldots$ & -- & -- & -- & -- & -- & -- & -- & -- & -- & -- \\
\hline $25 \ldots$ & 23 & 7 & 95 & 15 & 55 & 2.5 & 0.850 & 0.250 & 2.2 & 3.3 \\
\hline \multicolumn{11}{|l|}{ JUN } \\
\hline $06 \ldots$ & -- & -- & -- & -- & -- & -- & -- & -- & -- & -- \\
\hline
\end{tabular}


Table 78. Water-quality data at site 39 (CSW05), July 1995 through June 1997-Continued

\begin{tabular}{|c|c|c|c|c|c|c|c|c|c|c|}
\hline DATE & $\begin{array}{c}\text { PHOS- } \\
\text { PHORUS } \\
\text { TOTAL } \\
\text { (MG/L } \\
\text { AS P) } \\
(00665)\end{array}$ & $\begin{array}{c}\text { PHOS- } \\
\text { PHORUS } \\
\text { ORTHO, } \\
\text { DIS- } \\
\text { SOLVED } \\
\text { (MG/L AS } \\
\text { P) } \\
(00671) \\
\end{array}$ & $\begin{array}{c}\text { OIL AND } \\
\text { GREASE, } \\
\text { TOTAL } \\
\text { RECOV. } \\
\text { GRAVI- } \\
\text { METRIC } \\
(\text { MG /L) } \\
(00556) \\
\end{array}$ & $\begin{array}{c}\text { CARBON, } \\
\text { ORGANIC } \\
\text { TOTAL } \\
\text { (MG/L AS } \\
\text { C) } \\
(00680) \\
\end{array}$ & $\begin{array}{c}\text { STREPTO- } \\
\text { COCCI } \\
\text { FECAL, } \\
\text { (COLS. } \\
\text { PER } \\
100 \mathrm{ML}) \\
(31679) \\
\end{array}$ & $\begin{array}{c}\text { COLI- } \\
\text { FORM, } \\
\text { FECAL, } \\
0.45 \\
\text { UM-MF } \\
\text { (COLS. , } \\
100 \mathrm{ML} \text { ) } \\
(31616) \\
\end{array}$ & $\begin{array}{c}\text { ANTI- } \\
\text { MONY, } \\
\text { TOTAL } \\
\text { (UG / L } \\
\text { AS SB) } \\
(01097) \\
\end{array}$ & $\begin{array}{c}\text { ARSENIC } \\
\text { TOTAL } \\
\text { (UG/L } \\
\text { AS AS) } \\
(01002)\end{array}$ & $\begin{array}{c}\text { BERYL- } \\
\text { LIUM, } \\
\text { TOTAL } \\
\text { RECOV- } \\
\text { ERABLE } \\
\text { (UG/L AS } \\
\text { BE) } \\
(01012) \\
\end{array}$ & $\begin{array}{l}\text { CADMIUM } \\
\text { WATER } \\
\text { UNFLTRD } \\
\text { TOTAL } \\
\text { (UG/L } \\
\text { AS CD) } \\
(01027)\end{array}$ \\
\hline \multicolumn{11}{|l|}{ JUL 1995} \\
\hline $07 \ldots$ & -- & -- & $<1$ & -- & -- & -- & -- & -- & -- & -- \\
\hline $21 \ldots$ & -- & -- & 3 & -- & -- & -- & -- & -- & -- & -- \\
\hline $27 \ldots$ & -- & -- & -- & -- & 21000 & 96000 & -- & -- & -- & -- \\
\hline $27 \ldots$ & -- & -- & -- & -- & 65000 & 38000 & -- & -- & -- & -- \\
\hline $31 \ldots$ & -- & -- & -- & -- & 7400 & $\mathrm{~K} 110000$ & -- & -- & -- & -- \\
\hline $31 \ldots$ & -- & -- & -- & -- & 4700 & 37000 & -- & -- & -- & -- \\
\hline $31 \ldots$ & -- & - & -- & -- & -- & -- & -- & -- & -- & -- \\
\hline $31 \ldots$ & -- & -- & -- & -- & 38000 & K90000 & -- & -- & -- & -- \\
\hline \multicolumn{11}{|l|}{$\mathrm{OCT}$} \\
\hline $04 \ldots$ & 0.250 & 0.150 & -- & 8.1 & -- & -- & 1 & $<1$ & -- & -- \\
\hline $04 \ldots$ & 0.140 & 0.070 & -- & 7.2 & -- & -- & $<1$ & $<1$ & -- & -- \\
\hline $04 \ldots$ & 0.190 & 0.130 & -- & -- & -- & -- & -- & -- & -- & -- \\
\hline $04 \ldots$ & -- & -- & -- & -- & 29000 & 37000 & -- & -- & -- & -- \\
\hline $04 \ldots$ & 0.240 & 0.150 & -- & 6.9 & -- & -- & 1 & $<1$ & -- & -- \\
\hline \multicolumn{11}{|l|}{ NOV } \\
\hline $\begin{array}{c}11 \ldots \\
\text { JAN } 1996\end{array}$ & -- & -- & -- & -- & 5800 & 2000 & -- & -- & -- & -- \\
\hline $24 \ldots$ & 0.210 & 0.100 & -- & 40 & -- & -- & 3 & 1 & -- & -- \\
\hline $24 \ldots$ & -- & -- & 6 & -- & K1600 & K180 & -- & -- & -- & -- \\
\hline $24 \ldots$ & 0.240 & 0.070 & -- & 22 & -- & -- & $<1$ & 1 & -- & -- \\
\hline $24 \ldots$ & 0.090 & 0.050 & -- & -- & -- & -- & -- & -- & -- & -- \\
\hline \multicolumn{11}{|l|}{ MAR } \\
\hline \multicolumn{10}{|l|}{ APR } & -- \\
\hline $03 \ldots$ & 0.020 & $<0.010$ & $<1$ & -- & 630 & 60 & 3 & $<1$ & $<10$ & $<1$ \\
\hline $26 \ldots$ & 0.820 & 0.200 & 19 & 66 & 5600 & 310000 & 1 & $<1$ & -- & -- \\
\hline $26 \ldots$ & -- & -- & -- & -- & -- & -- & -- & -- & -- & -- \\
\hline $26 \ldots$ & 0.260 & 0.140 & 4 & 12 & $\mathrm{~K} 13000$ & 48000 & $<1$ & $<1$ & -- & -- \\
\hline $26 \ldots$ & -- & -- & -- & -- & -- & -- & -- & -- & -- & -- \\
\hline $26 \ldots$ & -- & -- & -- & -- & -- & -- & -- & -- & $\cdots$ & -- \\
\hline $26 \ldots$ & 0.180 & 0.100 & -- & 9.3 & 8200 & K20000 & $<1$ & $<1$ & -- & -- \\
\hline $26 \ldots$ & 0.140 & 0.070 & -- & -- & K5500 & 28000 & -- & -- & -- & -- \\
\hline \multicolumn{11}{|l|}{ JUL } \\
\hline $31 \ldots$ & 1.50 & 1.00 & -- & 14 & -- & -- & $<1$ & $<1$ & -- & -- \\
\hline $31 \ldots$ & 1.02 & 0.890 & -- & 11 & -- & -- & $<1$ & $<1$ & -- & -- \\
\hline $31 \ldots$ & 1.18 & 0.940 & -- & -- & -- & -- & -- & -- & -- & - \\
\hline \multicolumn{11}{|l|}{ OCT } \\
\hline $\begin{array}{l}07 \ldots \\
07 \ldots\end{array}$ & $\begin{array}{l}-- \\
--\end{array}$ & -- & $\begin{array}{l}6 \\
4\end{array}$ & $\begin{array}{l}-- \\
--\end{array}$ & $\begin{array}{r}31000 \\
9300\end{array}$ & $\begin{array}{r}20000 \\
3600\end{array}$ & $\begin{array}{l}-- \\
--\end{array}$ & -- & $\begin{array}{l}-- \\
--\end{array}$ & -- \\
\hline $07 \ldots$ & 0.640 & 0.380 & -- & 27 & - & -- & $<1$ & 1 & -- & -- \\
\hline $07 \ldots$ & 0.360 & 0.210 & -- & 10 & -- & -- & $<1$ & $<1$ & -- & -- \\
\hline $08 \ldots$ & 0.260 & 0.140 & -- & 6.6 & -- & -- & $<1$ & $<1$ & -- & -- \\
\hline $08 \ldots$ & 0.520 & 0.440 & -- & -- & -- & -- & -- & -- & -- & -- \\
\hline \multicolumn{11}{|l|}{ JAN 1997} \\
\hline $16 \ldots$ & 0.200 & 0.060 & -- & 14 & -- & -- & $<1$ & $<1$ & -- & -- \\
\hline $16 \ldots$ & 0.280 & 0.026 & -- & 10 & -- & -- & $<1$ & $<1$ & -- & -- \\
\hline $16 \ldots$ & 0.140 & 0.150 & -- & -- & -- & -- & -- & -- & -- & -- \\
\hline $24 \ldots$ & -- & -- & 8 & -- & 2100 & K630 & -- & -- & -- & -- \\
\hline \multicolumn{11}{|l|}{ MAY } \\
\hline $07 \ldots$ & 0.060 & 0.011 & 1 & -- & $\mathrm{K} 72$ & 310 & $<1$ & $<1$ & -- & -- \\
\hline $25 \ldots$ & 0.740 & 0.070 & -- & -- & -- & -- & $<1$ & $<1$ & -- & -- \\
\hline $25 \ldots$ & -- & -- & -- & -- & -- & -- & -- & -- & -- & -- \\
\hline $25 \ldots$ & 0.560 & 0.280 & -- & -- & -- & -- & $<1$ & $<1$ & -- & -- \\
\hline \multicolumn{11}{|l|}{ JUN } \\
\hline $06 \ldots$ & -- & -- & -- & 12 & K8000 & K9100 & -- & -- & -- &.- \\
\hline
\end{tabular}

K Results based on colony count outside the acceptable range (nonideal colony count). 
Table 78. Water-quality data at site 39 (CSW05), July 1995 through June 1997—Continued

\begin{tabular}{|c|c|c|c|c|c|c|c|c|c|}
\hline DATE & $\begin{array}{c}\text { CHRO- } \\
\text { MIUM, } \\
\text { TOTAL } \\
\text { RECOV- } \\
\text { ERABLE } \\
\text { (UG / L } \\
\text { AS CR) } \\
(01034) \\
\end{array}$ & $\begin{array}{c}\text { COPPER, } \\
\text { TOTAL } \\
\text { RECOV- } \\
\text { ERABLE } \\
\text { (UG/L AS } \\
\text { CU) } \\
(01042) \\
\end{array}$ & $\begin{array}{c}\text { LEAD, } \\
\text { TOTAL } \\
\text { RECOV- } \\
\text { ERABLE } \\
\text { (UG/L } / \\
\text { AS PB) } \\
(01051) \\
\end{array}$ & $\begin{array}{c}\text { MERCURY } \\
\text { TOTAL } \\
\text { RECOV- } \\
\text { ERABLE } \\
\text { (UG/L AS } \\
\text { HG) } \\
(71900) \\
\end{array}$ & $\begin{array}{c}\text { NICKEL, } \\
\text { TOTAL } \\
\text { RECOVER- } \\
\text { ABLE } \\
\text { (UG / } \\
\text { AS NI) } \\
(01067) \\
\end{array}$ & $\begin{array}{c}\text { SELE- } \\
\text { NIUM, } \\
\text { TOMAL } \\
\text { (UG / L } \\
\text { AS SE) } \\
(01147 \text { ) }\end{array}$ & $\begin{array}{c}\text { SILVER, } \\
\text { TOTAL } \\
\text { RECOV- } \\
\text { ERABLE } \\
\text { (UG/L } \\
\text { AS AG) } \\
(01077 \text { ) } \\
\end{array}$ & $\begin{array}{c}\text { ZINC, } \\
\text { TOTAL } \\
\text { RECOV- } \\
\text { ERABLE } \\
\text { (UG/L } \\
\text { AS ZN) } \\
(01092) \\
\end{array}$ & $\begin{array}{c}\text { CYANIDE } \\
\text { TOTAL } \\
\text { (MG/L AS } \\
\text { CN) } \\
(00720) \\
\end{array}$ \\
\hline \multicolumn{10}{|l|}{ JUL 1995} \\
\hline $07 \ldots$ & -- & -- & -- & -- & -- & -- & -- & -- & -- \\
\hline $21 \ldots$ & -- & -- & -- & -- & -- & -- & -- & -- & -- \\
\hline $27 \ldots$ & -- & -- & -- & -- & -- & -- & -- & -- & -- \\
\hline $27 \ldots$ & -- & -- & -- & -- & -- & -- & -- & -- & -- \\
\hline $31 \ldots$ & -- & -- & -- & -- & -- & -- & -- & -- & -- \\
\hline $31 \ldots$ & -- & -- & -- & -- & -- & -- & -- & -- & -- \\
\hline $31 \ldots$ & -- & -- & -- & -- & -- & -- & -- & -- & -- \\
\hline $31 \ldots$ & -- & -- & -- & -- & -- & -- & -- & -- & -- \\
\hline \multicolumn{10}{|l|}{ ОСТ } \\
\hline $04 \ldots$ & 6 & 10 & 8 & -- & 4 & -- & -- & 160 & -- \\
\hline $04 \ldots$ & 8 & 10 & 9 & -- & 1 & -- & -- & 80 & -- \\
\hline $04 \ldots$ & -- & -- & -- & -- & -- & -- & -- & -- & -- \\
\hline $04 \ldots$ & -- & -- & -- & -- & -- & -- & -- & -- & -- \\
\hline $04 \ldots$ & 8 & 10 & 8 & -- & 6 & -- & -- & 120 & -- \\
\hline \multicolumn{10}{|l|}{ Nov } \\
\hline \multicolumn{10}{|l|}{ JAN 1996} \\
\hline $24 \ldots$ & 23 & 22 & 20 & -- & 13 & -- & -- & 120 & -- \\
\hline $24 \ldots$ & -- & -- & -- & -- & -- & -- & -- & -- & -- \\
\hline $24 \ldots$ & 25 & 26 & 39 & -- & 9 & -- & -- & 190 & -- \\
\hline $24 \ldots$ & -- & -- & -- & -- & -- & -- & -- & -- & -- \\
\hline \multicolumn{10}{|l|}{ MAR } \\
\hline $06 \ldots$ & -- & -- & -- & -- & -- & -- & -- & -- & -- \\
\hline \multicolumn{10}{|l|}{ APR } \\
\hline $03 \ldots$ & 2 & $<1$ & $<1$ & 0.11 & 2 & $<1$ & $<1$ & 50 & $<0.010$ \\
\hline $26 \ldots$ & $<1$ & 21 & $<1$ & -- & 6 & -- & -- & 230 & -- \\
\hline $26 \ldots$ & -- & -- & -- & -- & -- & -- & -- & -- & -- \\
\hline $26 \ldots$ & 6 & 13 & $<1$ & -- & 5 & -- & -- & 80 & -- \\
\hline $26 \ldots$ & -- & -- & -- & -- & -- & -- & -- & -- & -- \\
\hline $26 \ldots$ & -- & -- & -- & -- & -- & -- & -- & -- & -- \\
\hline $26 \ldots$ & 5 & 7 & $<1$ & -- & 1 & -- & -- & 60 & -- \\
\hline \multirow{2}{*}{\multicolumn{10}{|c|}{ JUL }} \\
\hline & & & & & & & & & \\
\hline $31 \ldots$ & 14 & 24 & 24 & -- & 19 & -- & -- & 240 & -- \\
\hline $31 \ldots$ & 8 & 9 & 4 & -- & 2 & -- & -- & 80 & -- \\
\hline \multirow{2}{*}{\multicolumn{10}{|c|}{ OCT }} \\
\hline & & & & & & & & & \\
\hline $07 \ldots$ & -- & -- & -- & -- & -- & -- & -- & -- & -- \\
\hline $07 \ldots$ & -- & - & -- & -- & -- & -- & -- & -- & -- \\
\hline $07 \ldots$ & 10 & 8 & 18 & -- & 8 & -- & -- & 170 & -- \\
\hline $07 \ldots$ & 8 & 39 & 11 & -- & 4 & -- & -- & 130 & -- \\
\hline $08 \ldots$ & 6 & 18 & 5 & -- & 3 & -- & -- & 90 & -- \\
\hline $08 \ldots$ & -- & -- & -- & -- & -- & -- & -- & -- & -- \\
\hline \multicolumn{10}{|l|}{ JAN 1997} \\
\hline $16 \ldots$ & 16 & 22 & 42 & -- & 6 & -- & -- & 250 & -- \\
\hline $16 \ldots$ & 23 & 28 & 37 & -- & 2 & -- & -- & 180 & -- \\
\hline $16 \ldots$ & -- & -. & -- & -- & -- & -- & -- & -- & -- \\
\hline $24 \ldots$ & -- & -- & -- & -- & -- & -- & -- & -- & -- \\
\hline \multicolumn{10}{|l|}{ MAY } \\
\hline $07 \ldots$ & 2 & 3 & 1 & -. & 4 & -- & -- & 90 & -- \\
\hline $25 \ldots$ & 4 & 26 & 47 & -- & 10 & -- & -- & 280 & -- \\
\hline $25 \ldots$ & -- & -- & -- & -- & -- & -- & -- & -- & -- \\
\hline \multirow{2}{*}{\multicolumn{10}{|c|}{ JUN }} \\
\hline & & & & & & & & & \\
\hline $06 \ldots$ & -- & -- & -- & -- & -- & -- & -- & -- & -- \\
\hline
\end{tabular}


Table 79. Water-quality data at site 40 (CSWO3), July 1995 through June 1997

\begin{tabular}{|c|c|c|c|c|c|c|c|c|c|c|}
\hline DATE & TIME & $\begin{array}{c}\text { RAIN- } \\
\text { FALL } \\
\text { ACCUM } \\
(\text { IN }) \\
(00045) \\
\end{array}$ & $\begin{array}{c}\text { DIS- } \\
\text { CHARGE, } \\
\text { INST. } \\
\text { CUBIC } \\
\text { FEET PER } \\
\text { SECOND } \\
(00061) \\
\end{array}$ & $\begin{array}{c}\text { TEMPER- } \\
\text { ATURE } \\
\text { WATER } \\
\text { DEG. C) } \\
(00010) \\
\end{array}$ & $\begin{array}{c}\text { SPE- } \\
\text { CIFIC } \\
\text { CONDUC- } \\
\text { TANCE } \\
\text { LAB } \\
\text { (US/CM) } \\
\text { (90095) }\end{array}$ & $\begin{array}{c}\text { SPE- } \\
\text { CIFIC } \\
\text { CONDUC- } \\
\text { TANCE } \\
\text { (US/CM) } \\
(00095 \\
\end{array}$ & $\begin{array}{c}\text { PH } \\
\text { WATER } \\
\text { RAW LAB } \\
\text { (STAN- } \\
\text { DARD } \\
\text { UNITS) } \\
(00403 \text { ) }\end{array}$ & $\begin{array}{c}\text { PH } \\
\text { WATER } \\
\text { RAW } \\
\text { FIELD } \\
\text { (STAN- } \\
\text { DARD } \\
\text { UNITS) } \\
(00400) \\
\end{array}$ & $\begin{array}{c}\text { ALKA- } \\
\text { LINITY } \\
\text { LAB } \\
\text { (MG/L AS } \\
\text { CACO3) } \\
(90410)\end{array}$ & $\begin{array}{c}\text { SEDI- } \\
\text { MENT, } \\
\text { SUS- } \\
\text { PENDED } \\
\text { (MG/L) } \\
(80154) \\
\end{array}$ \\
\hline \multicolumn{11}{|l|}{ AUG 1995} \\
\hline $27 \ldots$ & 0426 & 9.37 & 21 & 22.0 & 40 & 29 & 6.6 & 6.80 & 8.0 & 527 \\
\hline \multicolumn{11}{|l|}{$\mathrm{OCT}$} \\
\hline $04 \ldots$ & 0453 & 2.28 & 0.05 & 21.0 & 31 & 25 & 6.2 & 6.50 & 6.0 & 53 \\
\hline $04 \ldots$ & 0513 & 2.28 & 0.18 & 20.5 & 38 & 39 & 6.3 & 6.28 & 9.0 & 228 \\
\hline $04 \ldots$ & 0545 & 2.28 & 0.21 & 20.0 & -- & -- & -- & -- & -- & -- \\
\hline $04 \ldots$ & 0622 & 2.28 & 0.05 & 20.5 & 46 & 39 & 6.7 & 6.67 & 12 & 58 \\
\hline $04 \ldots$ & 0856 & 2.28 & 1.2 & 19.0 & 39 & 31 & 6.8 & 6.75 & 15 & 1440 \\
\hline \multicolumn{11}{|l|}{ NOV } \\
\hline $\begin{array}{r}11 \ldots \\
\text { MAR } 1996\end{array}$ & \multicolumn{9}{|c|}{ MAR 1996} & -- \\
\hline $06 \ldots$ & 1054 & 1.23 & 0.11 & 12.5 & -- & 59 & -- & 6.66 & -- & -- \\
\hline $27 \ldots$ & 1845 & 0.55 & 0.02 & 10.5 & 63 & 58 & 6.9 & 7.10 & 6.0 & 63 \\
\hline $28 \ldots$ & 0722 & 0.55 & 0.01 & 7.0 & 66 & 61 & 7.6 & 7.11 & 20 & 28 \\
\hline $28 \ldots$ & 1004 & 0.55 & 0.01 & 8.0 & -- & 114 & -- & 7.23 & -- & -- \\
\hline \multicolumn{11}{|l|}{ JUN } \\
\hline $07 \ldots$ & 1931 & 0.37 & 0.15 & 24.5 & -- & 45 & -- & 6.58 & -- & -- \\
\hline $07 \ldots$ & 1934 & 0.37 & 0.06 & 24.5 & 49 & 51 & 6.0 & 6.08 & 6.0 & 56 \\
\hline \multicolumn{11}{|l|}{ JUL } \\
\hline $23 \ldots$ & 1735 & 0.23 & 0.01 & 28.0 & -- & 96 & -- & 7.20 & -- & -- \\
\hline $30 \ldots$ & 1245 & 0.89 & 0.18 & 26.5 & 40 & 32 & 6.0 & 6.23 & 2.0 & 96 \\
\hline $30 \ldots$ & 1310 & 0.89 & 0.01 & 26.0 & -- & 54 & -- & 6.17 & -- & -- \\
\hline \multicolumn{11}{|l|}{ AUG } \\
\hline $11 \ldots$ & 1900 & 0.70 & 0.10 & 25.5 & 41 & 40 & 6.6 & 6.17 & 3.0 & 45 \\
\hline $11 \ldots$ & 1920 & 0.70 & 0.34 & 25.0 & 33 & 30 & 6.8 & 6.46 & 5.0 & 233 \\
\hline \multicolumn{11}{|l|}{ SEP } \\
\hline $11 \ldots$ & 1307 & 0.05 & 0.29 & 25.5 & -- & 53 & -- & 6.80 & -- & -- \\
\hline $11 \ldots$ & 1317 & 0.05 & 0.15 & 26.0 & -- & 56 & -- & 6.8 & -- & -- \\
\hline \multicolumn{11}{|l|}{ OCT } \\
\hline $07 \ldots$ & 2210 & 1.60 & 0.03 & 15.5 & 46 & 39 & 7.0 & 7.14 & 10 & 44 \\
\hline $08 \ldots$ & 0208 & 1.60 & 0.16 & 15.0 & 41 & 32 & 7.1 & 7.15 & 12 & 63 \\
\hline $08 \ldots$ & 0518 & 1.60 & 0.42 & 15.0 & 55 & 47 & 7.0 & 7.18 & 18 & 87 \\
\hline $08 \ldots$ & 0640 & 1.60 & 0.10 & 15.5 & 97 & 47 & 7.2 & 7.18 & 34 & 42 \\
\hline \multicolumn{11}{|l|}{ NOV } \\
\hline $18 \ldots$ & 1616 & 0.37 & 0.01 & 12.0 & -- & 51 & -- & 7.03 & -- & -- \\
\hline \multicolumn{11}{|l|}{ FEB 1997} \\
\hline $04 \ldots$ & 0403 & 0.28 & 0.02 & 11.5 & 68 & 63 & 6.6 & 6.73 & 5.0 & 61 \\
\hline $04 \ldots$ & 1039 & 0.28 & 0.001 & 9.5 & -- & 118 & -- & 6.74 & - & -- \\
\hline $13 \ldots$ & 1243 & 1.17 & 0.03 & 5.5 & 41 & 38 & 6.9 & 6.06 & $<1.0$ & 163 \\
\hline $13 \ldots$ & 1311 & 1.17 & 0.02 & 5.5 & -- & 40 & -- & 7.25 & -- & -- \\
\hline $13 \ldots$ & 1724 & 1.17 & 0.25 & 2.0 & 38 & 33 & 7.0 & 6.47 & 12 & 200 \\
\hline $14 \ldots$ & 0141 & 1.17 & 0.10 & 3.0 & 79 & 75 & 7.2 & 6.94 & 27 & 98 \\
\hline $14 \ldots$ & 0902 & 1.17 & 0.01 & 5.5 & -- & 258 & -- & 7.18 & -- & -- \\
\hline \multicolumn{11}{|l|}{ MAY } \\
\hline $03 \ldots$ & 0552 & 0.77 & 0.76 & 16.0 & -- & 40 & -- & 6.91 & -- & -- \\
\hline $03 \ldots$ & 0554 & 0.77 & 0.51 & 17.0 & 59 & 49 & 7.0 & 6.94 & 14 & 437 \\
\hline $03 \ldots$ & 0652 & 0.77 & 0.03 & 17.0 & 69 & 80 & 7.2 & 7.15 & 20 & 34 \\
\hline $03 \ldots$ & 1034 & 0.77 & 0.08 & 17.5 & 54 & 63 & 7.2 & 7.30 & 20 & 52 \\
\hline $03 \ldots$ & 1322 & 0.77 & 0.01 & 16.5 & 231 & 292 & 7.6 & 7.47 & 104 & 29 \\
\hline \multicolumn{11}{|l|}{ JUN } \\
\hline $06 \ldots$ & 1023 & 0.47 & 0.01 & 16.0 & -- & 56 & -- & 6.67 & -- & -- \\
\hline
\end{tabular}


Table 79. Water-quality data at site 40 (CSW03), July 1995 through June 1997-Continued

\begin{tabular}{|c|c|c|c|c|c|c|c|c|c|c|}
\hline DATE & $\begin{array}{c}\text { RESIDUE } \\
\text { TOTAL } \\
\text { AT } 105 \\
\text { DEG. C, } \\
\text { SUS- } \\
\text { PENDED } \\
\text { (MG/L) } \\
(00530) \\
\end{array}$ & $\begin{array}{c}\text { RESIDUE } \\
\text { VOLA- } \\
\text { TILE, } \\
\text { SUS- } \\
\text { PENDED } \\
\text { (MG/L) } \\
(00535)\end{array}$ & $\begin{array}{c}\text { SOLIDS, } \\
\text { RESIDUE } \\
\text { AT } 180 \\
\text { DEG. C } \\
\text { DIS- } \\
\text { SOLVED } \\
\text { (MG/L) } \\
(70300) \\
\end{array}$ & $\begin{array}{c}\text { OXYGEN } \\
\text { DEMAND, } \\
\text { BIO- } \\
\text { CHEMI- } \\
\text { CAL } \\
5 \mathrm{DAY} \\
(\mathrm{MG} / \mathrm{L}) \\
(00310) \\
\end{array}$ & $\begin{array}{c}\text { OXYGEN } \\
\text { DEMAND, } \\
\text { CHEM- } \\
\text { ICAL } \\
\text { (HIGH } \\
\text { LEVEL) } \\
\text { (MG/L) } \\
(00340)\end{array}$ & $\begin{array}{c}\text { NITRO- } \\
\text { GEN, AM- } \\
\text { MONIA + } \\
\text { ORGANIC } \\
\text { TOTAL } \\
\text { (MG/L } \\
\text { AS N) } \\
(00625) \\
\end{array}$ & $\begin{array}{c}\text { NITRO- } \\
\text { GEN, } \\
\text { NO2+NO3 } \\
\text { DIS- } \\
\text { SOLVED } \\
\text { (MG/L } \\
\text { AS N) } \\
(00631) \\
\end{array}$ & $\begin{array}{c}\text { NITRO- } \\
\text { GEN, } \\
\text { AMMONIA } \\
\text { DIS- } \\
\text { SOLVED } \\
\text { (MG/L } \\
\text { AS N) } \\
(00608) \\
\end{array}$ & $\begin{array}{l}\text { NI TRO- } \\
\text { GEN, } \\
\text { ORGANIC } \\
\text { TOTAL } \\
\text { (MG/L AS } \\
\text { N) } \\
(00605) \\
\end{array}$ & $\begin{array}{c}\text { NITRO- } \\
\text { GEN, } \\
\text { TOTAL } \\
\text { (MG/L } \\
\text { AS N) } \\
(00600)\end{array}$ \\
\hline \multicolumn{11}{|l|}{ AUG 1995} \\
\hline${ }_{\text {OCT }}^{27 \ldots}$ & -- & 77 & 22 & 1.4 & 42 & 0.93 & 0.610 & 0.050 & 0.88 & 1.5 \\
\hline $04 \ldots$ & -- & -- & 24 & 8.9 & 59 & 1.2 & 0.480 & 0.290 & 0.91 & 1.7 \\
\hline $04 \ldots$ & -- & -- & 39 & 11 & 54 & 2.4 & 0.450 & 0.200 & 2.2 & 2.8 \\
\hline $04 \ldots$ & -- & -- & -- & -- & -- & -- & -- & -- & -- & -- \\
\hline $04 \ldots$ & -- & -- & 41 & 4.5 & 24 & 0.78 & 0.570 & 0.120 & 0.66 & 1.3 \\
\hline $04 \ldots$ & -- & -- & 44 & 6.1 & 51 & 1.5 & 0.260 & 0.070 & 1.4 & 1.8 \\
\hline \multicolumn{11}{|l|}{ NoV } \\
\hline $\begin{array}{r}11 \ldots \\
\operatorname{MAR} 1996\end{array}$ & -- & -- & -- & -- & -- & -- & -- & -- & -- & -- \\
\hline $06 \ldots$ & -- & -- & $\ldots$ & -- & - & -- & $\ldots$ & -- & -- & $\ldots$ \\
\hline $27 \ldots$ & 48 & 22 & 48 & 6.8 & 54 & 1.5 & 1.11 & 0.430 & 1.1 & 2.6 \\
\hline $28 \ldots$ & 9 & 3 & 49 & 4.9 & 25 & 0.69 & 0.530 & 0.090 & 0.60 & 1.2 \\
\hline $28 \ldots$ & -- & -- & -- & $-\rightarrow$ & -- & -- & -- & -- & -- & -- \\
\hline \multicolumn{11}{|l|}{ JUN } \\
\hline $07 \ldots$ & -- & -- & -- & -- & -- & -- & -- & -- & -- & -- \\
\hline $07 \ldots$ & 29 & 21 & 103 & $>31$ & 110 & 2.5 & 1.36 & 0.450 & 2.0 & 3.9 \\
\hline \multicolumn{11}{|l|}{ JUL } \\
\hline $23 \ldots$ & -- & -- & -- & -- & -- & -- & -- & -- & -- & -- \\
\hline $30 \ldots$ & 74 & 16 & 35 & 5.9 & 27 & 1.1 & 0.840 & 0.180 & 0.92 & 1.9 \\
\hline $30 \ldots$ & -- & -- & -- & -- & $\rightarrow-$ & -- & -- & -- & -- & $\cdots$ \\
\hline \multicolumn{11}{|l|}{ AUG } \\
\hline $11 \ldots$ & 19 & 7 & 37 & 10 & 34 & 1.4 & 0.800 & 0.350 & 1.0 & 2.2 \\
\hline $11 \ldots$ & 172 & 34 & 25 & 8.9 & 21 & 1.1 & 0.490 & 0.160 & 0.94 & 1.6 \\
\hline \multicolumn{11}{|l|}{ SEP } \\
\hline $11 \ldots$ & -- & -- & -+ & - & -- & -- & -- & -- & -- & -- \\
\hline $11 \ldots$ & -- & -- & -- & -- & -- & -- & -- & -- & -- & -- \\
\hline $\begin{array}{l}\text { OCT } \\
\quad 07 \ldots\end{array}$ & 31 & 13 & 38 & 15 & 40 & 0.68 & 0.410 & 0.070 & 0.61 & 1.1 \\
\hline $08 \ldots$ & 39 & 8 & 25 & 6.4 & 18 & 0.68 & 0.240 & 0.017 & 0.66 & 0.92 \\
\hline $08 \ldots$ & 40 & 6 & 37 & 5.0 & 28 & 1.0 & 0.360 & 0.019 & $0 . \dot{98}$ & 1.4 \\
\hline $08 \ldots$ & 8 & $<1$ & 66 & 6.1 & 25 & 1.0 & 0.760 & 0.016 & 0.98 & 1.8 \\
\hline \multicolumn{11}{|l|}{ NOV } \\
\hline \multicolumn{10}{|l|}{ FEB 1997} & -- \\
\hline $04 \ldots$ & 45 & 20 & 47 & 7.1 & 52 & 1.8 & 2.15 & 0.460 & 1.3 & 4.0 \\
\hline $04 \ldots$ & - & -- & -- & -- & -- & -- & -- & -- & -- & -- \\
\hline $13 \ldots$ & 148 & 58 & 32 & 8.1 & 78 & 2.4 & 0.700 & 0.120 & 2.3 & 3.1 \\
\hline $13 \ldots$ & - & -- & -- & -- & -- & -- & -- & -- & -- & -- \\
\hline $13 \ldots$ & 128 & 28 & 33 & 4.0 & 25 & 1.4 & 0.180 & 0.050 & 1.3 & 1.6 \\
\hline $14 \ldots$ & 19 & 3 & 56 & 2.5 & 20 & 0.46 & 0.430 & 0.050 & 0.41 & 0.89 \\
\hline $14 \ldots$ & -- & -- & -- & -- & $-\infty$ & -- & -- & -- & -- & -- \\
\hline \multicolumn{11}{|l|}{ MAY } \\
\hline $03 \ldots$ & -- & -- & -- & -- & -- & -- & -- & -- & -- & -- \\
\hline $03 \ldots$ & 362 & 82 & 51 & 22 & 120 & 3.3 & 0.590 & 0.300 & 3.0 & 3.9 \\
\hline $03 \ldots$ & 23 & 7 & 57 & 10 & 32 & 1.2 & 0.680 & 0.150 & 1.0 & 1.9 \\
\hline $03 \ldots$ & 42 & 11 & 47 & 8.4 & 29 & 0.83 & 0.230 & 0.050 & 0.78 & 1.1 \\
\hline $03 \ldots$ & 19 & 4 & 150 & 5.6 & 34 & 1.1 & 0.220 & $<0.015$ & 1.1 & 1.3 \\
\hline \multicolumn{11}{|l|}{ JUN } \\
\hline $06 \ldots$ & -- & -- & -- & -- & - & -- & -- & -- & -- & -- \\
\hline
\end{tabular}


Table 79. Water-quality data at site 40 (CSW03), July 1995 through June 1997-Continued

\begin{tabular}{|c|c|c|c|c|c|c|c|c|c|c|}
\hline DATE & $\begin{array}{l}\text { PHOS- } \\
\text { PHORUS } \\
\text { TOTAL } \\
\text { (MG / L } \\
\text { AS P) } \\
(00665)\end{array}$ & $\begin{array}{c}\text { PHOS- } \\
\text { PHORUS } \\
\text { ORTHO, } \\
\text { DIS- } \\
\text { SOLVED } \\
\text { (MG/L AS } \\
\text { P) } \\
(00671) \\
\end{array}$ & $\begin{array}{l}\text { OIL AND } \\
\text { GREASE, } \\
\text { TOTAL } \\
\text { RECOV. } \\
\text { GRAVI- } \\
\text { METRIC } \\
\text { (MG/L) } \\
\text { (00556) } \\
\end{array}$ & $\begin{array}{c}\text { CARBON, } \\
\text { ORGANIC } \\
\text { TOTAL } \\
\text { (MG / L AS } \\
\text { C) } \\
(00680)\end{array}$ & $\begin{array}{c}\text { STREPTO- } \\
\text { COCCI } \\
\text { FECAL, } \\
\text { (COLS. } \\
\text { PER } \\
100 \mathrm{ML}) \\
(31679) \\
\end{array}$ & $\begin{array}{c}\text { COLI- } \\
\text { FORM, } \\
\text { FECAL, } \\
0.45 \\
\text { UM-MF } \\
\text { (COLS. , } \\
100 \mathrm{ML} \text { ) } \\
(31616) \\
\end{array}$ & $\begin{array}{c}\text { ANTI- } \\
\text { MONY, } \\
\text { TOTAL } \\
\text { (UG/L } \\
\text { AS SB) } \\
(01097)\end{array}$ & $\begin{array}{c}\text { ARSENIC } \\
\text { TOTAL } \\
\text { (UG/L } \\
\text { AS AS) } \\
(01002) \\
\end{array}$ & $\begin{array}{c}\text { BERYL- } \\
\text { LIUM, } \\
\text { TOTAL } \\
\text { RECOV- } \\
\text { ERABLE } \\
\text { (UG/L AS } \\
\text { BE) } \\
(01012) \\
\end{array}$ & $\begin{array}{l}\text { CADMIUM } \\
\text { WATER } \\
\text { UNFLTRD } \\
\text { TOTAL } \\
\text { (UG/L } \\
\text { AS CD) } \\
(01027) \\
\end{array}$ \\
\hline \multicolumn{11}{|l|}{ AUG 1995} \\
\hline${ }_{\mathrm{OCT}}^{27 \ldots}$ & 0.680 & 0.170 & -- & 10 & -- & -- & 7 & $<1$ & -- & -- \\
\hline $04 \ldots$ & 0.250 & 0.160 & -- & 14 & -- & -- & $<1$ & $<1$ & -- & -- \\
\hline $04 \ldots$ & 0.510 & 0.160 & - & 13 & -- & -- & $<1$ & $<1$ & -- & -- \\
\hline $04 \ldots$ & -- & -- & $<1$ & -- & 60000 & $\mathrm{~K} 65000$ & -- & -- & $\cdots$ & -- \\
\hline $04 \ldots$ & 0.240 & 0.150 & - & -- & -- & -- & -- & -- & -- & -- \\
\hline $04 \ldots$ & 0.860 & 0.100 & -- & 12 & K67000 & 53000 & 1 & $<1$ & -- & -- \\
\hline \multicolumn{11}{|l|}{ NOV } \\
\hline $\begin{array}{c}11 \ldots \\
\text { MAR } 1996\end{array}$ & -- & -- & -- & -- & 27000 & 21000 & -- & -- & -- & -- \\
\hline $06 \ldots$ & -- & -- & -- & -- & K82000 & $\mathrm{K} 12000$ & -- & -- & -- & -- \\
\hline $27 \ldots$ & 0.150 & 0.060 & -- & 17 & - & -- & 3 & 1 & $\rightarrow$ & -- \\
\hline $28 \ldots$ & 0.120 & 0.050 & -- & 9.4 & -- & -- & $<1$ & $<1$ & -- & $\ldots$ \\
\hline $28 \ldots$ & -- & -- & -. & -- & 41000 & 3400 & -- & -- & -- & -- \\
\hline \multicolumn{11}{|l|}{ JUN } \\
\hline $07 \ldots$ & -- & -- & -- & -- & -- & -- & -- & -- & -- & -- \\
\hline $07 \ldots$ & 0.340 & 0.260 & -- & -- & -- & -- & 3 & 2 & -- & -- \\
\hline \multicolumn{11}{|l|}{ JUL } \\
\hline $23 \ldots$ & -- & -- & 6 & -- & 37000 & 29000 & -- & -- & -- & - \\
\hline $30 \ldots$ & 0.220 & 0.140 & -- & 11 & - & -- & 5 & 1 & -- & -- \\
\hline $30 \ldots$ & -- & -- & -- & -- & $\mathrm{K} 66000$ & $\mathrm{~K} 87000$ & -- & - & -- & -- \\
\hline \multicolumn{11}{|l|}{ AUG } \\
\hline $11 \ldots$ & 0.200 & 0.130 & -- & 13 & -- & -- & $<1$ & 2 & -- & -- \\
\hline $11 \ldots$ & 0.420 & 0.100 & -- & 13 & -- & -- & $<1$ & 2 & -- & -- \\
\hline \multicolumn{11}{|l|}{ SEP } \\
\hline $11 \ldots$ & -- & -- & -- & -- & 320000 & K190000 & -- & -- & -- & -- \\
\hline $11 \ldots$ & -- & -- & -- & -- & 240000 & K1 80000 & -- & -- & -- & -- \\
\hline \multicolumn{11}{|l|}{ OCT } \\
\hline $07 \ldots$ & 0.170 & 0.070 & -- & 14 & -- & -- & $<1$ & $<1$ & -- & -- \\
\hline $08 \ldots$ & 0.180 & 0.060 & -- & 9.3 & -- & -- & $<1$ & 1 & -- & -- \\
\hline $08 \ldots$ & 0.280 & 0.100 & $\rightarrow$ & 11 & -- & -- & $<1$ & 2 & $\ldots$ & -- \\
\hline $08 \ldots$ & 0.270 & 0.120 & -- & -- & -- & -- & -- & -- & -- & -- \\
\hline \multicolumn{11}{|l|}{ NOV } \\
\hline $\begin{array}{c}18 \ldots \\
\text { FEB } 1997\end{array}$ & - & -- & 4 & -- & K14000 & $\mathrm{K} 7700$ & -- & -- & -- & -- \\
\hline $04 \ldots$ & 0.130 & 0.060 & -- & 13 & -- & -- & $<1$ & 1 & -- & -- \\
\hline $04 \ldots$ & -- & -- & -- & -- & 7900 & 26000 & -- & -- & -- & -- \\
\hline $13 \ldots$ & 0.290 & 0.039 & -- & 21 & -- & -- & $<1$ & 2 & -- & -- \\
\hline $13 \ldots$ & -- & -- & $<1$ & -- & 2100 & 4900 & -- & -- & -- & -- \\
\hline $13 \ldots$ & 0.310 & 0.060 & -- & 14 & -- & -- & $<1$ & $<1$ & -- & -- \\
\hline $14 \ldots$ & 0.140 & 0.120 & -- & -- & -- & -- & -- & -- & -- & -- \\
\hline $14 \ldots$ & -- & -- & -- & -- & 7300 & 2200 & -- & -- & -- & -- \\
\hline \multicolumn{11}{|l|}{ MAY } \\
\hline $03 \ldots$ & -- & -- & -- & -- & -- & -- & -- & -- & -- & -- \\
\hline $03 \ldots$ & 0.700 & 0.060 & -- & -- & -- & -- & $<1$ & $<1$ & -- & -- \\
\hline $03 \ldots$ & 0.160 & 0.044 & -- & -- & -- & -- & -- & -- & -- & -- \\
\hline $03 \ldots$ & 0.150 & 0.033 & -- & -- & -- & -- & $<1$ & 1 & -- & -- \\
\hline $03 \ldots$ & 0.130 & 0.022 & -- & -- & -- & -- & -- & -- & -- & -- \\
\hline \multicolumn{11}{|l|}{ JUN } \\
\hline $06 \ldots$ & -- & -- & -- & 14 & $\mathrm{~K} 14000$ & $\mathrm{~K} 120000$ & -- & -- & -- & -- \\
\hline
\end{tabular}

$\mathrm{K}$ Results based on colony count outside the acceptable range (nonideal colony count). 
Table 79. Water-quality data at site 40 (CSWO3), July 1995 through June 1997-Continued

\begin{tabular}{|c|c|c|c|c|c|c|c|c|c|}
\hline DATE & $\begin{array}{c}\text { CHRO- } \\
\text { MIUM, } \\
\text { TOTAL } \\
\text { RECOV- } \\
\text { ERABLE } \\
\text { (UG/L } \\
\text { AS CR) } \\
(01034) \\
\end{array}$ & $\begin{array}{c}\text { COPPER, } \\
\text { TOTAL } \\
\text { RECOV- } \\
\text { ERABLE } \\
\text { (UG/L AS } \\
\text { CU) } \\
(01042) \\
\end{array}$ & $\begin{array}{c}\text { LEAD, } \\
\text { TOTAL } \\
\text { RECOV- } \\
\text { ERABLE } \\
\text { (UG/L } \\
\text { AS PB) } \\
(01051) \\
\end{array}$ & $\begin{array}{c}\text { MERCURY } \\
\text { TOTAL } \\
\text { RECOV- } \\
\text { ERABLE } \\
\text { (UG/L AS } \\
\text { HG) } \\
(71900) \\
\end{array}$ & $\begin{array}{c}\text { NICKEL, } \\
\text { TOTAL } \\
\text { RECOVER- } \\
\text { ABLE } \\
\text { (UG/L } \\
\text { AS NI) } \\
(01067) \\
\end{array}$ & $\begin{array}{c}\text { SELE- } \\
\text { NIUM, } \\
\text { TOTAL } \\
\text { (UG/L } \\
\text { AS SE) } \\
(01147) \\
\end{array}$ & $\begin{array}{l}\text { SILVER, } \\
\text { TOTAL } \\
\text { RECOV- } \\
\text { ERABLE } \\
\text { (UG/L } \\
\text { AS AG) } \\
(01077 \text { ) } \\
\end{array}$ & $\begin{array}{c}\text { ZINC, } \\
\text { TOTAL } \\
\text { RECOV- } \\
\text { ERABLE } \\
\text { (UG/L } \\
\text { AS ZN) } \\
(01092) \\
\end{array}$ & $\begin{array}{c}\text { CYANIDE } \\
\text { TOTAL } \\
\text { (MG/L AS } \\
\text { CN) } \\
(00720) \\
\end{array}$ \\
\hline \multicolumn{10}{|l|}{ AUG 1995} \\
\hline${ }_{\text {OCT }}^{27 \ldots}$ & 12 & 18 & 42 & -- & 4 & -- & -- & 80 & -- \\
\hline $04 \ldots$ & 4 & 9 & 5 & -- & 2 & -- & - & 80 & -. \\
\hline $04 \ldots$ & 10 & 22 & 12 & -. & 6 & -- & - & 110 & -- \\
\hline $04 \ldots$ & $\cdots$ & $\ldots$ & -- & - & -- & - & -- & -- & -- \\
\hline $04 \ldots$ & - & -- & -- & -- & -- & -- & -- & -- & -- \\
\hline $04 \ldots$ & 18 & 30 & 36 & -- & 10 & -- & -- & 110 & -- \\
\hline \multicolumn{10}{|l|}{ NOV } \\
\hline $\begin{array}{r}11 \ldots \\
\text { MAR } 1996\end{array}$ & -- & -- & - & -- & -- & -- & -- & -- & -- \\
\hline $06 \ldots$ & -. & -- & -- & -- & -. & -- & -- & -- & -- \\
\hline $27 \ldots$ & 4 & 6 & 7 & -- & 2 & -- & - & 110 & -- \\
\hline $28 \ldots$ & 4 & 4 & 3 & -- & $<1$ & -- & -- & 50 & -- \\
\hline $28 \ldots$ & -. & -- & $\cdots$ & -- & -- & -- & - & $\ldots$ & -- \\
\hline \multicolumn{10}{|l|}{ JUN } \\
\hline $07 \ldots$ & -- & -- & -- & -- & -- & -- & -- & -- & -- \\
\hline $07 \ldots$ & 7 & 12 & 5 & -- & 7 & - & -- & 70 & $-\infty$ \\
\hline \multicolumn{10}{|l|}{ JUL } \\
\hline $23 \ldots$ & -- & -- & -- & -- & -- & -- & -- & -- & -- \\
\hline $30 \ldots$ & 8 & 11 & 8 & -- & 8 & -- & -- & 70 & -- \\
\hline $30 \ldots$ & - & -- & - & -- & - & -- & -- & -- & -- \\
\hline \multicolumn{10}{|l|}{ AUG } \\
\hline $11 \ldots$ & 3 & 5 & 3 & -- & 4 & -- & -- & 60 & -- \\
\hline $11 \ldots$ & 10 & 17 & 14 & -. & 6 & -- & -- & 60 & -- \\
\hline \multicolumn{10}{|l|}{$S E P$} \\
\hline $11 \ldots$ & -- & -- & -- & -- & -- & -- & -- & -- & -- \\
\hline$\underset{\text { OCT }}{11 \ldots}$ & -- & -- & -- & -- & -- & -- & -- & -- & -- \\
\hline $07 \ldots$ & 3 & 8 & 4 & -- & 2 & -- & -- & 80 & -- \\
\hline $08 \ldots$ & 5 & 15 & 5 & - & 2 & -- & -- & 70 & -- \\
\hline $08 \ldots$ & 5 & 16 & 6 & -- & 4 & -- & -- & -- & -- \\
\hline \multicolumn{7}{|l|}{ NoV } & -- & -- & -- \\
\hline $\begin{array}{r}18 \ldots \\
\text { FEB } 1997\end{array}$ & -- & -- & -- & -. & -- & -. & -- & -- & -- \\
\hline $04 \ldots$ & 4 & 34 & 13 & -. & 4 & -- & -- & 170 & -- \\
\hline $04 \ldots$ & -- & -. & - & -- & $\ldots$ & -- & -- & -. & -- \\
\hline $13 \ldots$ & 8 & 33 & 18 & -. & 8 & -- & -- & 100 & -. \\
\hline $13 \ldots$ & -. & - & -- & -. & - & -- & -- & $\ldots$ & -. \\
\hline $13 \ldots$ & 10 & 41 & 16 & -- & 9 & -- & - & 100 & -- \\
\hline $14 \ldots$ & -- & -. & -. & -- & -- & -- & -- & -- & -- \\
\hline \multirow{2}{*}{\multicolumn{10}{|c|}{ MAY }} \\
\hline & & & & & & & & & \\
\hline $03 \ldots$ & -- & -- & -- & -- & -. & -- & -- & -- & -- \\
\hline $03 \ldots$ & 14 & 21 & 32 & -- & 11 & -- & -- & 100 & -- \\
\hline $03 \ldots$ & - & -- & -- & -- & - & -- & -- & -- & -- \\
\hline $03 \ldots$ & 5 & 8 & 7 & -- & 4 & -- & -- & 30 & -- \\
\hline $03 \ldots$ & - & -- & -- & -- & - & -- & -- & -- & -- \\
\hline \multicolumn{10}{|l|}{ JUN } \\
\hline $06 \ldots$ & -- & -- & -- & -- & - & -- & -- & -- & -- \\
\hline
\end{tabular}


Table 80. Water-quality data at site 41 (CSW02), July 1995 through June 1997

\begin{tabular}{|c|c|c|c|c|c|c|c|c|c|c|}
\hline DATE & TIME & $\begin{array}{c}\text { RAIN- } \\
\text { FALL } \\
\text { ACCUM } \\
(I N) \\
(00045)\end{array}$ & $\begin{array}{c}\text { DIS- } \\
\text { CHARGE, } \\
\text { INST. } \\
\text { CUBIC } \\
\text { FEET PER } \\
\text { SECOND } \\
(00061)\end{array}$ & $\begin{array}{c}\text { TEMPER- } \\
\text { ATURE } \\
\text { WATER } \\
\text { DEG. C) } \\
(00010)\end{array}$ & $\begin{array}{c}\text { SPE- } \\
\text { CIFIC } \\
\text { CONDUC- } \\
\text { TANCE } \\
\text { LAB } \\
\text { (US/CM) } \\
(90095)\end{array}$ & $\begin{array}{c}\text { SPE- } \\
\text { CIFIC } \\
\text { CONDUC- } \\
\text { TANCE } \\
\text { (US /CM) } \\
(00095\end{array}$ & $\begin{array}{c}\text { PH } \\
\text { WATER } \\
\text { RAW LAB } \\
\text { (STAN- } \\
\text { DARD } \\
\text { UNITS) } \\
(00403 \text { ) }\end{array}$ & $\begin{array}{c}\text { PH } \\
\text { WATER } \\
\text { RAW } \\
\text { FIELD } \\
\text { (STAN- } \\
\text { DARD } \\
\text { UNITS) } \\
(00400)\end{array}$ & $\begin{array}{c}\text { ALKA- } \\
\text { LINITY } \\
\text { LAB } \\
\text { (MG/L AS } \\
\text { CACO3) } \\
(90410)\end{array}$ & $\begin{array}{c}\text { SEDI- } \\
\text { MENT, } \\
\text { SUS- } \\
\text { PENDED } \\
(\mathrm{MG} / \mathrm{L}) \\
(80154)\end{array}$ \\
\hline \multicolumn{11}{|l|}{ AUG 1995} \\
\hline${ }_{\mathrm{OCT}}^{27 \ldots}$ & 0352 & 7.11 & 153 & 23.0 & 30 & 23 & 6.9 & 6.90 & 14 & 425 \\
\hline $04 \ldots$ & 0410 & 2.34 & 0.89 & 19.0 & 146 & 139 & 7.1 & 6.90 & 48 & 54 \\
\hline $04 \ldots$ & 0422 & 2.34 & 0.72 & 19.5 & - & 188 & -- & 6.63 & -- & -- \\
\hline $04 \ldots$ & 0519 & 2.34 & 14 & 20.5 & 24 & 18 & 6.5 & 7.02 & 7.0 & 104 \\
\hline $04 \ldots$ & 0540 & 2.34 & 1.4 & 20.0 & 34 & 27 & 6.8 & 6.73 & 6.0 & 45 \\
\hline $04 \ldots$ & 0821 & 2.34 & 18 & 19.0 & 25 & 20 & 6.6 & 6.73 & 7.0 & 104 \\
\hline $04 \ldots$ & 0936 & 2.34 & 6.6 & 19.0 & 50 & 41 & 6.8 & 6.75 & 10 & 44 \\
\hline $27 \ldots$ & 1953 & 1.34 & 97 & 16.5 & -- & -- & -- & -- & -- & -- \\
\hline \multicolumn{11}{|l|}{ JAN 1996} \\
\hline $24 \ldots$ & 0812 & 0.16 & 0.22 & 11.0 & -- & 166 & -- & 6.82 & -- & 46 \\
\hline \multicolumn{11}{|l|}{ FEB } \\
\hline $20 \ldots$ & 0447 & 0.37 & 0.84 & 12.5 & 265 & 252 & 7.2 & 6.72 & 42 & 58 \\
\hline $20 \ldots$ & 0729 & 0.37 & 0.94 & 10.0 & 66 & 60 & 7.0 & 6.54 & 10 & 27 \\
\hline $20 \ldots$ & 0904 & 0.37 & 0.27 & 10.0 & 74 & 67 & 6.9 & 6.54 & 10 & 25 \\
\hline \multicolumn{11}{|l|}{ MAR } \\
\hline $06 \ldots$ & 0941 & 1.56 & 9.8 & 14.0 & -- & 41 & -- & 6.45 & -- & -- \\
\hline \multicolumn{11}{|l|}{ APR } \\
\hline $03 \ldots$ & 1740 & 0.00 & 0.04 & 14.5 & 169 & 165 & 7.0 & 6.87 & 55 & 35 \\
\hline $26 \ldots$ & 0916 & 0.58 & 8.8 & 15.0 & 132 & 130 & 7.1 & 6.55 & 52 & 306 \\
\hline $26 \ldots$ & 0920 & 0.58 & 8.3 & 16.5 & -- & 75 & -- & 6.63 & -- & -- \\
\hline $26 \ldots$ & 1108 & 0.58 & 3.8 & 16.5 & 22 & 18 & 7.4 & 6.67 & 6.0 & 37 \\
\hline $26 \ldots$ & 1114 & 0.58 & 3.4 & 16.5 & -- & 18 & -- & 6.85 & -- & -- \\
\hline $26 \ldots$ & 1158 & 0.58 & 0.46 & 16.5 & 22 & 26 & 7.2 & 6.68 & 7.0 & 24 \\
\hline \multicolumn{11}{|l|}{ JUL } \\
\hline $30 \ldots$ & 1312 & 0.12 & 0.32 & 27.0 & -- & 29 & -- & 5.94 & -- & -- \\
\hline \multicolumn{11}{|l|}{ AUG } \\
\hline $11 \ldots$ & 1846 & 1.32 & 19 & 22.5 & 46 & 44 & 6.8 & 7.03 & 6.0 & 644 \\
\hline $11 \ldots$ & 2202 & 1.32 & 95 & 22.5 & 25 & 24 & 6.8 & 7.22 & 2.0 & 225 \\
\hline $11 \ldots$ & 2356 & 1.32 & 0.29 & 23.0 & 78 & 75 & 6.8 & 7.10 & 14 & 54 \\
\hline \multicolumn{11}{|l|}{ OCT } \\
\hline $07 \ldots$ & 1728 & 1.45 & 0.24 & 16.5 & 134 & 130 & 6.9 & 6.96 & 36 & 85 \\
\hline $07 \ldots$ & 2253 & 1.45 & 1.9 & 16.5 & 30 & 29 & 6.9 & 7.00 & 7.0 & 50 \\
\hline $08 \ldots$ & 0653 & 1.45 & 0.57 & 16.0 & 79 & 75 & 6.9 & 6.94 & 16 & 53 \\
\hline \multicolumn{11}{|l|}{ JAN 1997} \\
\hline $16 \ldots$ & 0114 & 0.64 & 1.4 & 10.0 & 151 & 142 & 7.2 & 6.96 & 51 & 52 \\
\hline $16 \ldots$ & 0204 & 0.64 & 13 & 10.0 & 27 & 19 & 6.7 & 6.85 & 5.0 & 216 \\
\hline $16 \ldots$ & 0410 & 0.64 & 0.89 & 8.0 & 43 & 36 & 6.8 & 6.66 & 8.0 & 41 \\
\hline $24 \ldots$ & 1118 & 0.25 & 2.6 & 10.5 & -- & 91 & -- & 6.68 & -- & -- \\
\hline \multicolumn{11}{|l|}{ MAY } \\
\hline $07 \ldots$ & 1205 & 0.00 & 0.03 & 15.5 & 165 & 151 & 6.9 & 6.82 & 56 & 7 \\
\hline $08 \ldots$ & 2124 & 0.33 & 2.8 & 16.0 & -- & 160 & -- & 6.85 & -- & -- \\
\hline $08 \ldots$ & 2128 & 0.33 & 2.8 & 16.5 & 130 & 122 & 6.8 & 6.79 & 25 & 220 \\
\hline \multicolumn{11}{|l|}{ JUN } \\
\hline $02 \ldots$ & 1446 & 0.56 & 7.4 & 21.0 & -- & 24 & -- & 6.95 & -- & -- \\
\hline $06 \ldots$ & 0907 & 0.45 & 0.72 & 17.5 & -- & 24 & -- & 6.33 & -- & -- \\
\hline
\end{tabular}


Table 80. Water-quality data at site 41 (CSW02), July 1995 through June 1997-Continued

\begin{tabular}{|c|c|c|c|c|c|c|c|c|c|c|}
\hline DATE & $\begin{array}{c}\text { RESIDUE } \\
\text { TOTAL } \\
\text { AT } 105 \\
\text { DEG. C, } \\
\text { SUS- } \\
\text { PENDED } \\
\text { (MG/L) } \\
(00530) \\
\end{array}$ & $\begin{array}{l}\text { RESIDUE } \\
\text { VOLA- } \\
\text { TILE, } \\
\text { SUS- } \\
\text { PENDED } \\
\text { (MG/L) } \\
(00535) \\
\end{array}$ & $\begin{array}{l}\text { SOLIDS, } \\
\text { RESIDUE } \\
\text { AT } 180 \\
\text { DEG. C } \\
\text { DIS- } \\
\text { SOLVED } \\
\text { (MG/L) } \\
(70300) \\
\end{array}$ & $\begin{array}{c}\text { OXYGEN } \\
\text { DEMAND, } \\
\text { BIO- } \\
\text { CHEMI- } \\
\text { CAL } \\
5 \text { DAY } \\
(\text { MG /L) } \\
(00310) \\
\end{array}$ & $\begin{array}{c}\text { OXYGEN } \\
\text { DEMAND, } \\
\text { CHEM- } \\
\text { ICAL } \\
\text { (HIGH } \\
\text { LEVEL) } \\
\text { (MG/L) } \\
(00340) \\
\end{array}$ & $\begin{array}{c}\text { NITRO- } \\
\text { GEN, AM- } \\
\text { MONIA + } \\
\text { ORGANIC } \\
\text { TOTAL } \\
\text { (MG / L } \\
\text { AS N) } \\
(00625) \\
\end{array}$ & $\begin{array}{c}\text { NITRO- } \\
\text { GEN, } \\
\text { NO2+NO3 } \\
\text { DIS- } \\
\text { SOLVED } \\
\text { (MG/L } \\
\text { AS N) } \\
(00631 \text { ) } \\
\end{array}$ & $\begin{array}{l}\text { NITRO- } \\
\text { GEN, } \\
\text { AMMONIA } \\
\text { DIS- } \\
\text { SOLVED } \\
\text { (MG/L } \\
\text { AS N) } \\
(00608) \\
\end{array}$ & $\begin{array}{l}\text { NITRO- } \\
\text { GEN, } \\
\text { ORGANIC } \\
\text { TOTAL } \\
\text { (MG/L AS } \\
\text { N) } \\
(00605) \\
\end{array}$ & $\begin{array}{l}\text { NITRO- } \\
\text { GEN, } \\
\text { TOTAL } \\
\text { (MG/L } \\
\text { AS N) } \\
(00600)\end{array}$ \\
\hline \multicolumn{11}{|l|}{ AUG 1995} \\
\hline \multicolumn{11}{|l|}{ OCT } \\
\hline $04 \ldots$ & -- & -- & 116 & 6.0 & 15 & 0.31 & 1.10 & 0.150 & 0.16 & 1.4 \\
\hline $04 \ldots$ & - & -- & -- & -. & -- & -- & -- & -- & - & $\ldots$ \\
\hline $04 \ldots$ & -- & -- & 23 & 4.5 & 22 & 0.83 & 0.160 & 0.440 & 0.39 & 0.99 \\
\hline $04 \ldots$ & -- & -- & 33 & 3.8 & 13 & 1.4 & 0.220 & 0.670 & 0.73 & 1.6 \\
\hline $04 \ldots$ & -- & -- & 16 & 6.4 & 24 & 1.6 & 0.160 & 0.430 & 1.2 & 1.8 \\
\hline $04 \ldots$ & - & - & 43 & 9.2 & 18 & 1.7 & 0.370 & 0.660 & 1.0 & 2.1 \\
\hline $\begin{array}{r}27 \ldots \\
\text { JAN } 1996\end{array}$ & -- & -- & -- & -- & -- & -- & -- & -- & -- & -- \\
\hline $24 \ldots$ & -- & -- & -- & -- & -- & -- & - & -- & $\cdots$ & -- \\
\hline \multicolumn{11}{|l|}{ FEB } \\
\hline $20 \ldots$ & 27 & 8 & 224 & 9.0 & 35 & 1.3 & 1.57 & 0.490 & 0.81 & 2.9 \\
\hline $20 \ldots$ & 13 & 6 & 72 & 8.5 & 20 & 0.67 & 0.570 & 0.270 & 0.40 & 1.2 \\
\hline $20 \ldots$ & 15 & 7 & 78 & 2.9 & 20 & 0.66 & 0.510 & 0.230 & 0.43 & 1.2 \\
\hline \multicolumn{11}{|l|}{ MAR } \\
\hline $06 \ldots$ & -- & -- & -- & -- & -- & -- & -- & -- & -- & -- \\
\hline \multicolumn{11}{|l|}{ APR } \\
\hline $03 \ldots$ & 18 & 5 & 105 & $<2.0$ & $<5$ & 0.42 & 1.26 & 0.050 & 0.37 & 1.7 \\
\hline $26 \ldots$ & 296 & 124 & 151 & $>35$ & 170 & 9.8 & 0.870 & 0.780 & 9.0 & 11 \\
\hline $26 \ldots$ & -- & -- & -- & -- & -- & -- & - & -- & - & -- \\
\hline $26 \ldots$ & 30 & 10 & 17 & 12 & 22 & 0.83 & 0.180 & 0.340 & 0.49 & 1.0 \\
\hline $26 \ldots$ & -- & -- & -- & -- & -- & -- & - & - & -- & -- \\
\hline $26 \ldots$ & 10 & 3 & 25 & 13 & 16 & 0.79 & 0.320 & 0.310 & 0.48 & 1.1 \\
\hline \multicolumn{11}{|l|}{ JUL } \\
\hline $30 \ldots$ & - & - & -- & - & -- & -- & - & - & - & -- \\
\hline \multicolumn{11}{|l|}{ AUG } \\
\hline $11 \ldots$ & 608 & 148 & 31 & 20 & 70 & 3.2 & 0.580 & 0.540 & 2.7 & 3.8 \\
\hline $11 \ldots$ & 110 & 24 & 16 & 5.8 & 27 & 1.0 & 0.340 & 0.170 & 0.83 & 1.3 \\
\hline $11 \ldots$ & 21 & 4 & 57 & 7.6 & 21 & 1.0 & 1.56 & 0.080 & 0.92 & 2.6 \\
\hline \multicolumn{11}{|l|}{$\mathrm{OCT}$} \\
\hline $07 \ldots$ & 21 & $<1$ & 101 & 12 & 40 & 1.6 & 1.22 & 0.410 & 1.2 & 2.8 \\
\hline $07 \ldots$ & 40 & 6 & 13 & 6.6 & 18 & 0.69 & 0.250 & 0.100 & 0.59 & 0.94 \\
\hline $08 \ldots$ & 14 & $<1$ & 64 & 5.4 & 28 & 1.0 & 1.19 & 0.070 & 0.93 & 2.2 \\
\hline \multicolumn{11}{|l|}{ JAN 1997} \\
\hline $16 \ldots$ & 7 & 2 & 117 & 3.6 & 11 & 0.34 & 1.18 & 0.070 & 0.27 & 1.5 \\
\hline $16 \ldots$ & 179 & 53 & 17 & 7.2 & 38 & 1.5 & 0.230 & 0.140 & 1.4 & 1.7 \\
\hline $16 \ldots$ & 16 & 4 & 35 & 4.8 & 25 & 0.80 & 0.380 & 0.060 & 0.74 & 1.2 \\
\hline $24 \ldots$ & -- & -- & -- & -- & -- & -- & -- & -- & -- & -- \\
\hline \multicolumn{11}{|l|}{ MAY } \\
\hline $07 \ldots$ & 4 & $<1$ & 137 & $<2.0$ & $<5$ & 0.66 & 1.29 & 0.100 & 0.56 & 2.0 \\
\hline $08 \ldots$ & -- & -- & -- & -- & -- & -- & -- & -- & -- & -- \\
\hline $08 \ldots$ & 204 & 58 & 104 & $>34$ & 120 & 6.1 & 1.80 & 1.60 & 4.5 & 7.9 \\
\hline \multicolumn{11}{|l|}{ JUN } \\
\hline $02 \ldots$ & -- & -- & $\cdots$ & - & - & - & -- & - & -- & -- \\
\hline $06 \ldots$ & -- & -- & -- & -- & -- & -- & -- & -- & -- & -- \\
\hline
\end{tabular}


Table 80. Water-quality data at site 41 (CSW02), July 1995 through June 1997-Continued

\begin{tabular}{|c|c|c|c|c|c|c|c|c|c|c|}
\hline DATE & $\begin{array}{l}\text { PHOS- } \\
\text { PHORUS } \\
\text { TOTAL } \\
\text { (MG/L } \\
\text { AS P) } \\
(00665) \\
\end{array}$ & $\begin{array}{c}\text { PHOS- } \\
\text { PHORUS } \\
\text { ORTHO, } \\
\text { DIS- } \\
\text { SOLVED } \\
\text { (MG/L AS } \\
\text { P) } \\
(00671) \\
\end{array}$ & $\begin{array}{l}\text { OIL AND } \\
\text { GREASE, } \\
\text { TOTAL } \\
\text { RECOV. } \\
\text { GRAVI- } \\
\text { METRIC } \\
\text { (MG/L) } \\
(00556) \\
\end{array}$ & $\begin{array}{c}\text { CARBON, } \\
\text { ORGANIC } \\
\text { TOTAL } \\
\text { (MG/L AS } \\
\text { C) } \\
(00680)\end{array}$ & $\begin{array}{c}\text { STREPTO- } \\
\text { COCCI } \\
\text { FECAL, } \\
\text { (COLS. } \\
\text { PER } \\
100 \mathrm{ML} \text { ) } \\
(31679) \\
\end{array}$ & $\begin{array}{c}\text { COLI- } \\
\text { FORM, } \\
\text { FECAL, } \\
0.45 \\
\text { UM-MF } \\
\text { (COLS. / } \\
100 \mathrm{ML}) \\
(31616) \\
\end{array}$ & $\begin{array}{l}\text { ANTI- } \\
\text { MONY, } \\
\text { TOTAL } \\
\text { (UG/L } \\
\text { AS SB) } \\
(01097)\end{array}$ & $\begin{array}{c}\text { ARSENIC } \\
\text { TOTAL } \\
\text { (UG/L } \\
\text { AS AS) } \\
(01002) \\
\end{array}$ & $\begin{array}{l}\text { BERYL- } \\
\text { LIUM, } \\
\text { TOTAL } \\
\text { RECOV- } \\
\text { ERABLE } \\
\text { (UG/L AS } \\
\text { BE) } \\
(01012) \\
\end{array}$ & $\begin{array}{l}\text { CADMIUM } \\
\text { WATER } \\
\text { UNFLTRD } \\
\text { TOTAL } \\
\text { (UG /L } \\
\text { AS CD) } \\
(01027) \\
\end{array}$ \\
\hline \multicolumn{11}{|l|}{ AUG 1995} \\
\hline \multicolumn{10}{|l|}{ OCT } & -- \\
\hline $04 \ldots$ & 0.190 & 0.130 & -- & 5.8 & -- & -- & $<1$ & 2 & -- & -- \\
\hline $04 \ldots$ & -- & -- & $<1$ & -- & 24000 & 420000 & -- & -- & -- & -- \\
\hline $04 \ldots$ & 0.560 & 0.440 & -- & 6.8 & -- & -- & $<1$ & $<1$ & - & -- \\
\hline $04 \ldots$ & 0.860 & 0.840 & -- & -- & -- & -- & -- & -- & -- & -- \\
\hline $04 \ldots$ & 0.660 & 0.480 & -- & 6.5 & -- & -- & $<1$ & $<1$ & -- & -- \\
\hline $04 \ldots$ & 1.13 & 0.980 & -- & -- & -- & -- & -- & -- & -- & -- \\
\hline \multicolumn{10}{|l|}{ JAN 1996} & -- \\
\hline FEB $24 \ldots$ & -- & -- & $<1$ & -- & K990 & K2 70 & -- & -- & -- & -- \\
\hline $20 \ldots$ & 0.170 & 0.030 & - & 12 & -- & -- & 1 & $<1$ & -- & -- \\
\hline $20 \ldots$ & 0.060 & 0.040 & - & 8.5 & -- & -- & $<1$ & $<1$ & -- & -- \\
\hline $20 \ldots$ & 0.080 & 0.040 & -- & - & 1800 & 1500 & - & -- & - & -- \\
\hline \multicolumn{11}{|l|}{ MAR } \\
\hline $06 \ldots$ & -- & -- & -- & -- & 30000 & K17000 & -- & -- & -- & -- \\
\hline \multicolumn{11}{|l|}{ APR } \\
\hline $26 \ldots$ & 1.58 & 0.660 & 5 & 72 & $\mathrm{~K} 12000$ & 27000 & $<1$ & $<1$ & -- & -- \\
\hline $26 \ldots$ & -- & -- & - & -- & - & -- & -- & -- & -- & -- \\
\hline $26 \ldots$ & 0.180 & 0.130 & $<1$ & 9.2 & K15000 & 26000 & $<1$ & $<1$ & -- & -- \\
\hline $26 \ldots$ & -- & -- & -- & -- & -- & -- & -- & -- & -- & $\cdots$ \\
\hline $26 \ldots$ & 0.190 & 0.140 & -- & -- & $\mathrm{K} 18000$ & 21000 & -- & -- & -- & -- \\
\hline \multicolumn{11}{|l|}{ JUL } \\
\hline $30 \ldots$ & -- & -- & -- & -- & K15000 & 47000 & -- & -- & -- & $\ldots$ \\
\hline \multicolumn{11}{|l|}{ AUG } \\
\hline $11 \ldots$ & 1.03 & 0.090 & -- & 38 & -- & -- & $<1$ & 2 & -- & -- \\
\hline $11 \ldots$ & 0.330 & 0.120 & -- & 8.5 & -- & -- & $<1$ & $<1$ & -- & -- \\
\hline $11 \ldots$ & 0.320 & 0.240 & - - & -- & -- & -- & -- & -- & -- & -- \\
\hline \multicolumn{11}{|l|}{ OCT } \\
\hline $07 \ldots$ & 0.270 & 0.100 & $<1$ & 14 & K11200 & 4300 & $<1$ & $<1$ & -- & -- \\
\hline $07 \ldots$ & 0.160 & 0.036 & -- & 7.2 & -- & -- & $<1$ & $<1$ & -- & -- \\
\hline $08 \ldots$ & 0.280 & 0.140 & -- & -- & -- & -- & -- & -- & -- & -- \\
\hline \multicolumn{11}{|l|}{ JAN 1997} \\
\hline $16 \ldots$ & 0.060 & 0.036 & -- & 2.9 & -- & -- & 1 & $<1$ & -- & -- \\
\hline $16 \ldots$ & 0.330 & 0.038 & -- & 16 & -- & -- & $<1$ & $<1$ & -- & -- \\
\hline $16 \ldots$ & 0.240 & 0.170 & -- & -- & -- & -- & -- & -- & -- & -- \\
\hline $24 \ldots$ & -- & -- & 4 & -- & $\mathrm{K} 1700$ & 2400 & -- & -- & -- & -- \\
\hline \multicolumn{11}{|l|}{ MAY } \\
\hline $07 \ldots$ & 0.060 & 0.020 & 2 & -- & 510 & 2600 & $<1$ & $<1$ & -- & -- \\
\hline $08 \ldots$ & -- & -- & -- & -- & -- & -- & -- & -- & -- & -- \\
\hline $08 \ldots$ & 0.740 & 0.160 & -- & -- & -- & -- & $<1$ & 1 & -- & -- \\
\hline \multicolumn{11}{|l|}{ JUN } \\
\hline $02 \ldots$ & -- & -- & 2 & 12 & 53000 & 36000 & -- & -- & -- & -- \\
\hline $06 \ldots$ & -- & -- & -- & 5.7 & $\mathrm{~K} 18000$ & 31000 & -- & -- & -- & -- \\
\hline
\end{tabular}

K Results based on colony count outside the acceptable range (nonideal colony count). 
Table 80. Water-quality data at site 41 (CSW02), July 1995 through June 1997-Continued

\begin{tabular}{|c|c|c|c|c|c|c|c|c|c|}
\hline DATE & $\begin{array}{c}\text { CHRO- } \\
\text { MIUM, } \\
\text { TOTAL } \\
\text { RECOV- } \\
\text { ERABLE } \\
\text { (UG/L } \\
\text { AS CR) } \\
(01034) \\
\end{array}$ & $\begin{array}{c}\text { COPPER, } \\
\text { TOTAL } \\
\text { RECOV- } \\
\text { ERABLE } \\
\text { (UG/L AS } \\
\text { CU) } \\
(01042) \\
\end{array}$ & $\begin{array}{c}\text { LEAD, } \\
\text { TOTAL } \\
\text { RECOV- } \\
\text { ERABLE } \\
\text { (UG/L } \\
\text { AS PB) } \\
(01051) \\
\end{array}$ & $\begin{array}{c}\text { MERCURY } \\
\text { TOTAL } \\
\text { RECOV- } \\
\text { ERABLE } \\
\text { (UG / L AS } \\
\text { HG) } \\
(71900) \\
\end{array}$ & $\begin{array}{c}\text { NICKEL, } \\
\text { TOTAL } \\
\text { RECOVER- } \\
\text { ABLE } \\
\text { (UG / L } \\
\text { AS NI) } \\
(01067) \\
\end{array}$ & $\begin{array}{c}\text { SELE- } \\
\text { NIUM, } \\
\text { TOTAL } \\
\text { (UG / L } \\
\text { AS SE) } \\
(01147) \\
\end{array}$ & $\begin{array}{c}\text { SILVER, } \\
\text { TOTAL } \\
\text { RECOV- } \\
\text { ERABLE } \\
\text { (UG/L } \\
\text { AS AG) } \\
(01077 \text { ) }\end{array}$ & $\begin{array}{c}\text { ZINC, } \\
\text { TOTAL } \\
\text { RECOV- } \\
\text { ERABLE } \\
\text { (UG /L } \\
\text { AS ZN) } \\
(01092) \\
\end{array}$ & $\begin{array}{c}\text { CYANIDE } \\
\text { TOTAL } \\
\text { (MG / L AS } \\
\text { CN) } \\
(00720)\end{array}$ \\
\hline \multicolumn{10}{|l|}{ AUG 1995} \\
\hline \multicolumn{10}{|l|}{$\mathrm{OCT}$} \\
\hline $04 \ldots$ & 4 & 6 & 6 & - & 2 & -- & -- & 70 & -- \\
\hline $04 \ldots$ & -- & -- & -- & -- & -- & -- & -- & -- & -- \\
\hline $04 \ldots$ & 7 & 10 & 16 & -- & 3 & -- & -- & 100 & -- \\
\hline $04 \ldots$ & -- & -- & -- & -- & -- & -- & -- & -- & -- \\
\hline $04 \ldots$ & 6 & 10 & 13 & -- & -- & -- & -- & 220 & -- \\
\hline $04 \ldots$ & -- & -- & -- & -- & -- & -- & -- & -- & -- \\
\hline $\begin{array}{r}27 \ldots \\
\text { JAN } 1996\end{array}$ & -- & -- & -- & -- & -- & -- & -- & -- & -- \\
\hline \multicolumn{5}{|l|}{ FEB } & -- & -- & -- & -- & -- \\
\hline $20 \ldots$ & 4 & 4 & 6 & -- & 2 & -- & -- & 70 & -- \\
\hline $20 \ldots$ & 4 & 9 & 6 & -- & 2 & -- & -- & 60 & -- \\
\hline $20 \ldots$ & -- & -- & -- & -- & -- & -- & -- & -- & -- \\
\hline \multicolumn{10}{|l|}{ MAR } \\
\hline $06 \ldots$ & -- & -- & -- & -- & -- & -- & -- & -- & -- \\
\hline \multicolumn{10}{|l|}{$A P R$} \\
\hline $03 \ldots$ & 3 & $<1$ & 2 & $<0.10$ & 2 & $<1$ & $<1$ & 40 & $<0.010$ \\
\hline $26 \ldots$ & 15 & 18 & 27 & -- & 6 & -- & -- & 170 & -- \\
\hline $26 \ldots$ & -- & -- & -- & -- & -- & -- & -- & -- & -- \\
\hline $26 \ldots$ & 3 & 5 & 11 & -- & $<1$ & -- & -- & 50 & -- \\
\hline $26 \ldots$ & -- & -- & -- & -- & -- & -- & -- & -- & -- \\
\hline $26 \ldots$ & -- & -- & -- & -- & -- & -- & -- & -- & -- \\
\hline \multicolumn{10}{|l|}{ JUL } \\
\hline $30 \ldots$ & -- & -- & -- & -- & -- & -- & -- & -- & -- \\
\hline \multicolumn{10}{|l|}{ AUG } \\
\hline $11 \ldots$ & 26 & 49 & 74 & -- & 14 & -- & -- & 380 & -- \\
\hline $11 \ldots$ & 7 & 10 & 15 & -- & 3 & -- & -- & 80 & -- \\
\hline $11 \ldots$ & -- & -- & -- & -- & -- & -- & -- & -- & -- \\
\hline \multicolumn{10}{|l|}{$\mathrm{OCT}$} \\
\hline $07 \ldots$ & 5 & 10 & 9 & -- & 3 & -- & -- & 100 & -- \\
\hline $07 \ldots$ & 4 & 13 & 9 & -- & 2 & -- & -- & 100 & -- \\
\hline $08 \ldots$ & -- & -- & -- & -- & -- & -- & -- & -- & -- \\
\hline \multicolumn{10}{|l|}{ JAN 1997} \\
\hline $16 \ldots$ & 3 & 3 & 3 & -- & 1 & -- & -- & 50 & -- \\
\hline $16 \ldots$ & 13 & 25 & 41 & -- & 5 & -- & -- & 180 & -- \\
\hline $16 \ldots$ & -- & -- & -- & -- & -- & -- & -- & -- & -- \\
\hline $24 \ldots$ & -- & -- & -- & -- & -- & -- & -- & -- & -- \\
\hline \multicolumn{10}{|l|}{ MAY } \\
\hline $07 \ldots$ & 1 & $<1$ & 3 & -- & 2 & -- & -- & 30 & -- \\
\hline $08 \ldots$ & -- & -- & -- & -- & -- & -- & -- & -- & -- \\
\hline $08 \ldots$ & 13 & 24 & 31 & -- & 8 & -- & -- & 210 & -- \\
\hline \multicolumn{10}{|l|}{ JUN } \\
\hline $02 \ldots$ & -+ & -- & -- & - & -- & - & -- & -- & -- \\
\hline $06 \ldots$ & -- & -- & -- & -- & -- & -- & -- & -- & -- \\
\hline
\end{tabular}


Table 81. Water-quality data at site 42 (CSW04), July 1995 through June 1997

\begin{tabular}{|c|c|c|c|c|c|c|c|c|c|c|}
\hline DATE & TIME & $\begin{array}{c}\text { RAIN- } \\
\text { FALL } \\
\text { ACCUM } \\
\text { (IN) } \\
(00045)\end{array}$ & $\begin{array}{c}\text { DIS- } \\
\text { CHARGE, } \\
\text { INST. } \\
\text { CUBIC } \\
\text { FEET PER } \\
\text { SECOND } \\
(00061) \\
\end{array}$ & $\begin{array}{c}\text { TEMPER- } \\
\text { ATURE } \\
\text { WATER } \\
\text { DEG. C) } \\
(00010)\end{array}$ & $\begin{array}{c}\text { SPE- } \\
\text { CIFIC } \\
\text { CONDUC- } \\
\text { TANCE } \\
\text { LAB } \\
\text { (US/CM) } \\
(90095)\end{array}$ & $\begin{array}{c}\text { SPE- } \\
\text { CIFIC } \\
\text { CONDUC- } \\
\text { TANCE } \\
\text { (US/CM) } \\
(00095 \\
\end{array}$ & $\begin{array}{c}\text { PH } \\
\text { WATER } \\
\text { RAW LAB } \\
\text { (STAN- } \\
\text { DARD } \\
\text { UNITS) } \\
(00403 \text { ) }\end{array}$ & $\begin{array}{c}\text { PH } \\
\text { WATER } \\
\text { RAW } \\
\text { FIELD } \\
\text { (STAN- } \\
\text { DARD } \\
\text { UNITS) } \\
(00400) \\
\end{array}$ & $\begin{array}{c}\text { ALKA- } \\
\text { LINITY } \\
\text { LAB } \\
\text { (MG/L AS } \\
\text { CACO3) } \\
(90410) \\
\end{array}$ & $\begin{array}{c}\text { SEDI- } \\
\text { MENT, } \\
\text { SUS- } \\
\text { PENDED } \\
\text { (MG /L) } \\
(80154) \\
\end{array}$ \\
\hline \multicolumn{11}{|l|}{ AUG 1995} \\
\hline $\operatorname{OCT}^{27 \ldots}$ & \multicolumn{9}{|c|}{ OCT } & 676 \\
\hline $04 \ldots$ & 0419 & 2.34 & 0.30 & 19.5 & 550 & 32 & 7.6 & 6.80 & 23 & 173 \\
\hline $04 \ldots$ & 0520 & 2.34 & 18 & 20.5 & 66 & 56 & 6.7 & 6.78 & 14 & 431 \\
\hline $04 \ldots$ & 0600 & 2.34 & 4.3 & 20.0 & -- & -- & -- & -- & -- & -- \\
\hline $04 \ldots$ & 0624 & 2.34 & 5.0 & 20.0 & -- & -- & -- & -- & -- & -- \\
\hline $04 \ldots$ & 0643 & 2.34 & 3.4 & 20.0 & 72 & 61 & 6.7 & 7.14 & 13 & 76 \\
\hline $04 \ldots$ & 0846 & 2.34 & 39 & 19.0 & 40 & 32 & 6.6 & 6.80 & 11 & 439 \\
\hline $27 \ldots$ & 2011 & 1.34 & 68 & 16.5 & -. & -- & -- & -- & -- & -- \\
\hline \multicolumn{11}{|l|}{ FEB 1996} \\
\hline $20 \ldots$ & 0452 & 0.37 & 0.70 & 8.5 & 246 & 230 & 7.5 & 6.95 & 50 & 112 \\
\hline $20 \ldots$ & 0732 & 0.37 & 2.0 & 9.0 & 73 & 64 & 7.4 & 6.90 & 13 & 70 \\
\hline $20 \ldots$ & 0827 & 0.37 & 1.1 & 9.0 & -- & 80 & -- & 7.30 & -- & -- \\
\hline $20 \ldots$ & 0834 & 0.37 & 1.0 & 9.0 & 85 & 77 & 7.2 & 6.87 & 13 & 48 \\
\hline $28 \ldots$ & 0920 & 0.10 & 0.46 & 14.0 & -- & 285 & -- & 7.27 & -- & -- \\
\hline $28 \ldots$ & 1016 & 0.10 & 0.90 & 14.5 & -- & -- & -- & -- & -- & -- \\
\hline $28 \ldots$ & 1257 & 0.10 & 0.12 & 14.5 & -- & -- & -- & -- & -- & -- \\
\hline \multicolumn{11}{|l|}{ MAR } \\
\hline $06 \ldots$ & 1010 & 1.56 & 10 & 13.0 & -- & 62 & - & 6.62 & - & -- \\
\hline \multicolumn{11}{|l|}{ APR } \\
\hline $03 \ldots$ & 1645 & 0.00 & 0.02 & 16.5 & 427 & 425 & 7.6 & 7.37 & 132 & 42 \\
\hline \multicolumn{11}{|l|}{ JUN } \\
\hline $07 \ldots$ & 1916 & 0.28 & 15 & 25.0 & -- & 60 & -- & 6.21 & -- & -- \\
\hline $07 \ldots$ & 1920 & 0.28 & 25 & 24.5 & 100 & 86 & 6.9 & 6.64 & 14 & 1390 \\
\hline $07 \ldots$ & 1928 & 0.28 & 22 & 23.5 & -- & 57 & -- & 7.31 & -- & -- \\
\hline \multicolumn{11}{|l|}{ AUG } \\
\hline $11 \ldots$ & 1852 & 1.32 & 9.5 & 25.0 & 56 & 55 & 6.9 & 6.85 & 9.0 & 384 \\
\hline $11 \ldots$ & 2145 & 1.32 & 27 & 23.5 & 50 & 48 & 6.8 & 6.65 & 8.0 & 812 \\
\hline $11 \ldots$ & 2356 & 1.32 & 1.1 & 23.5 & 107 & 105 & 7.1 & 6.85 & 26 & 129 \\
\hline \multicolumn{11}{|l|}{$\mathrm{OCT}$} \\
\hline $07 \ldots$ & 1530 & 1.45 & 0.10 & 13.5 & -- & 275 & -- & 7.71 & - & -- \\
\hline $07 \ldots$ & 1655 & 1.45 & 0.44 & 14.5 & 252 & 243 & 7.4 & 7.35 & 57 & 141 \\
\hline $07 \ldots$ & 2254 & 1.45 & 5.0 & 15.5 & 53 & 45 & 7.0 & 7.17 & 12 & 130 \\
\hline $08 \ldots$ & 0202 & 1.45 & 13 & 15.0 & 64 & 55 & 6.9 & 7.03 & 10 & 136 \\
\hline $08 \ldots$ & 0652 & 1.45 & 2.0 & 15.5 & 95 & 83 & 7.0 & 7.04 & 17 & 59 \\
\hline \multicolumn{11}{|l|}{ JAN 1997} \\
\hline $16 \ldots$ & 0114 & 0.64 & 0.73 & 6.5 & 416 & 418 & 7.5 & 7.34 & 83 & 131 \\
\hline $16 \ldots$ & 0214 & 0.64 & 37 & 8.5 & -- & -- & - & 6.92 & -- & 436 \\
\hline $16 \ldots$ & 0414 & 0.64 & 3.1 & 7.0 & 65 & 58 & 7.0 & 6.82 & 12 & 87 \\
\hline $24 \ldots$ & 1132 & 0.25 & 1.7 & 7.5 & -- & 73 & -- & 6.78 & -- & -- \\
\hline \multicolumn{11}{|l|}{ MAY } \\
\hline $07 \ldots$ & 1240 & 0.00 & 0.02 & 15.0 & 351 & 323 & 7.4 & 6.55 & 133 & 4 \\
\hline $08 \ldots$ & 2137 & 0.33 & 1.7 & 20.0 & -- & 134 & -- & 7.05 & - & - \\
\hline $08 \ldots$ & 2140 & 0.33 & 1.5 & 20.5 & 133 & 123 & 7.0 & 7.07 & 25 & 103 \\
\hline \multicolumn{11}{|l|}{ JUN } \\
\hline $02 \ldots$ & 1501 & 0.56 & 19 & 22.0 & -- & 52 & -- & 7.00 & -- & -- \\
\hline $06 \ldots$ & 0935 & 0.45 & 1.4 & 16.0 & -- & 49 & -- & 6.53 & -- & -- \\
\hline
\end{tabular}


Table 81. Water-quality data at site 42 (CSW04), July 1995 through June 1997-Continued

\begin{tabular}{|c|c|c|c|c|c|c|c|c|c|c|}
\hline DATE & $\begin{array}{c}\text { RESIDUE } \\
\text { TOTAL } \\
\text { AT } 105 \\
\text { DEG. C, } \\
\text { SUS- } \\
\text { PENDED } \\
(\text { MG } / L) \\
(00530) \\
\end{array}$ & $\begin{array}{l}\text { RESIDUE } \\
\text { VOLA- } \\
\text { TILE, } \\
\text { SUS- } \\
\text { PENDED } \\
\text { (MG/L) } \\
(00535) \\
\end{array}$ & $\begin{array}{c}\text { SOLIDS, } \\
\text { RESIDUE } \\
\text { AT } 180 \\
\text { DEG. C } \\
\text { DIS- } \\
\text { SOLVED } \\
(\text { MG } / L) \\
(70300) \\
\end{array}$ & $\begin{array}{c}\text { OXYGEN } \\
\text { DEMAND, } \\
\text { BIO- } \\
\text { CHEMI- } \\
\text { CAL } \\
5 \text { DAY } \\
(\text { MG } / \mathrm{L}) \\
(00310) \\
\end{array}$ & $\begin{array}{c}\text { OXYGEN } \\
\text { DEMAND, } \\
\text { CHEM- } \\
\text { ICAL } \\
\text { (HIGH } \\
\text { LEVEL) } \\
(\text { MG } / \text { L) } \\
(00340) \\
\end{array}$ & $\begin{array}{c}\text { NITRO- } \\
\text { GEN, AM- } \\
\text { MONIA + } \\
\text { ORGANIC } \\
\text { TOTAL } \\
\text { (MG/L } \\
\text { AS N) } \\
(00625) \\
\end{array}$ & $\begin{array}{c}\text { NITRO- } \\
\text { GEN, } \\
\text { NO2+NO3 } \\
\text { DIS- } \\
\text { SOLVED } \\
\text { (MG / L } \\
\text { AS N) } \\
(00631) \\
\end{array}$ & $\begin{array}{l}\text { NITRO- } \\
\text { GEN, } \\
\text { AMMONIA } \\
\text { DIS- } \\
\text { SOLVED } \\
\text { (MG/L } \\
\text { AS N) } \\
(00608) \\
\end{array}$ & $\begin{array}{l}\text { NITRO- } \\
\text { GEN, } \\
\text { ORGANIC } \\
\text { TOTAL } \\
\text { (MG / L AS } \\
\text { N) } \\
(00605) \\
\end{array}$ & $\begin{array}{l}\text { NITRO- } \\
\text { GEN, } \\
\text { TOTAL } \\
\text { (MG/L } \\
\text { AS N) } \\
(00600) \\
\end{array}$ \\
\hline \multicolumn{11}{|l|}{ AUG 1995} \\
\hline${ }_{\text {OCT }}^{27 \ldots}$ & -- & 5 & 25 & 3.4 & 41 & 1.7 & 0.190 & 0.050 & 1.6 & 1.9 \\
\hline $04 \ldots$ & -- & -- & 340 & 13 & 30 & 1.2 & 0.750 & 0.250 & 0.95 & 2.0 \\
\hline $04 \ldots$ & -- & -- & 47 & 7.6 & 50 & 1.6 & 0.250 & 0.400 & 1.2 & 1.8 \\
\hline $04 \ldots$ & -- & -- & -- & -- & -- & -- & -- & -- & -- & -- \\
\hline $04 \ldots$ & -- & -- & -- & -- & -- & -- & -- & -- & -- & -- \\
\hline $04 \ldots$ & -- & -- & 55 & 6.3 & 30 & 1.7 & 0.550 & 0.440 & 1.3 & 2.2 \\
\hline $04 \ldots$ & -- & -- & 29 & 7.2 & 22 & 1.1 & 0.320 & 0.210 & 0.89 & 1.4 \\
\hline $27 \ldots$ & -- & -- & -- & -- & -- & -- & -- & -- & -- & -- \\
\hline \multicolumn{11}{|l|}{ FEB 1996} \\
\hline $20 \ldots$ & 66 & 16 & 182 & 15 & 82 & 6.2 & -- & 1.60 & 4.6 & 6.2 \\
\hline $20 \ldots$ & 46 & 11 & 72 & 8.9 & 29 & 1.3 & 0.750 & 0.370 & 0.93 & 2.0 \\
\hline $20 \ldots$ & -- & -- & -- & -- & -- & -- & -- & -- & -- & -- \\
\hline $20 \ldots$ & 27 & 7 & 80 & 7.5 & 32 & 1.4 & 0.780 & 0.360 & 1.0 & 2.2 \\
\hline $28 \ldots$ & -- & -- & -- & -- & -- & -- & -- & -- & -- & -- \\
\hline $28 \ldots$ & -- & -- & -- & -- & -- & -- & -- & -- & -- & -- \\
\hline $28 \ldots$ & -- & -- & -- & -- & -- & -- & - & -- & -- & -- \\
\hline \multicolumn{11}{|l|}{ MAR } \\
\hline $06 \ldots$ & -- & -- & -- & -- & -- & - & -- & -- & -- & -- \\
\hline \multicolumn{11}{|l|}{ APR } \\
\hline $03 \ldots$ & 12 & 4 & 239 & $<2.0$ & 15 & 0.68 & 0.250 & 0.020 & 0.66 & 0.93 \\
\hline \multicolumn{11}{|l|}{ JUN } \\
\hline $07 \ldots$ & -- & -- & -- & -- & -- & -- & -- & -- & -- & -- \\
\hline $07 \ldots$ & 776 & 100 & 79 & 24 & 84 & 3.7 & 0.910 & 0.500 & 3.2 & 4.6 \\
\hline $07 \ldots$ & -- & -- & -- & -- & -- & -- & -- & -- & -- & -- \\
\hline \multicolumn{11}{|l|}{ AUG } \\
\hline $11 \ldots$ & 290 & 48 & 41 & 15 & 30 & 2.6 & 0.580 & 0.580 & 2.0 & 3.2 \\
\hline $11 \ldots$ & 376 & 44 & 40 & 7.8 & 17 & 1.3 & 0.490 & 0.170 & 1.1 & 1.8 \\
\hline $11 \ldots$ & 74 & 12 & 88 & 7.4 & 37 & 1.3 & 0.810 & 0.110 & 1.2 & 2.1 \\
\hline \multicolumn{11}{|l|}{ OCT } \\
\hline $07 \ldots$ & -- & -- & -- & -- & -- & -- & -- & -- & -- & -- \\
\hline $07 \ldots$ & 88 & 2 & 160 & 18 & 72 & 11 & 1.26 & 5.20 & 5.8 & 12 \\
\hline $07 \ldots$ & 95 & 16 & 28 & 8.7 & 18 & 1.8 & 0.360 & 0.510 & 1.3 & 2.2 \\
\hline $08 \ldots$ & 90 & 8 & 44 & 7.0 & 25 & 2.2 & 0.510 & 0.490 & 1.7 & 2.7 \\
\hline $08 \ldots$ & 23 & $<1$ & 71 & 7.1 & 29 & 4.3 & 0.860 & 0.080 & 4.2 & 5.2 \\
\hline \multicolumn{11}{|l|}{ JAN 1997} \\
\hline $16 \ldots$ & 62 & 12 & 259 & 12 & 30 & 1.3 & 0.930 & 0.200 & 1.1 & 2,2 \\
\hline $16 \ldots$ & -- & -- & -- & -- & -- & 1.8 & 0.300 & 0.160 & 1.6 & 2.1 \\
\hline $16 \ldots$ & 51 & 11 & 58 & 6.3 & 30 & 1.8 & 0.500 & 0.160 & 1.6 & 2.3 \\
\hline $24 \ldots$ & -- & -- & -- & -- & -- & -- & -- & -- & -- & -- \\
\hline \multicolumn{11}{|l|}{ MAY } \\
\hline $07 \ldots$ & 1 & 1 & 239 & $<2.0$ & $<5$ & 0.59 & 0.450 & $<0.015$ & 0.59 & 1.0 \\
\hline $08 \ldots$ & -- & -- & -- & -- & -- & -- & -- & -- & -- & -- \\
\hline $08 \ldots$ & 104 & 24 & 97 & 30 & 89 & 6.1 & 1.66 & 1.50 & 4.6 & 7.8 \\
\hline \multicolumn{11}{|l|}{ JUN } \\
\hline $02 \ldots$ & -- & -- & -- & -- & -- & -- & -- & -- & -- & -- \\
\hline $06 \ldots$ & -- & -- & -- & -- & -- & -- & -- & -- & -- & -- \\
\hline
\end{tabular}


Table 81. Water-quality data at site 42 (CSW04), July 1995 through June 1997-Continued

\begin{tabular}{|c|c|c|c|c|c|c|c|c|c|c|}
\hline DATE & $\begin{array}{c}\text { PHOS- } \\
\text { PHORUS } \\
\text { TOTAL } \\
\text { (MG/L } \\
\text { AS P) } \\
(00665) \\
\end{array}$ & $\begin{array}{c}\text { PHOS- } \\
\text { PHORUS } \\
\text { ORTHO, } \\
\text { DIS- } \\
\text { SOLVED } \\
\text { (MG/L AS } \\
\text { P) } \\
(00671) \\
\end{array}$ & $\begin{array}{c}\text { OIL AND } \\
\text { GREASE, } \\
\text { TOTAL } \\
\text { RECOV. } \\
\text { GRAVI- } \\
\text { METRIC } \\
(\text { MG } / L) \\
(00556) \\
\end{array}$ & $\begin{array}{c}\text { CARBON, } \\
\text { ORGANIC } \\
\text { TOTAL } \\
\text { (MG/L AS } \\
\text { C) } \\
(00680) \\
\end{array}$ & $\begin{array}{c}\text { STREPTO- } \\
\text { COCCI } \\
\text { FECAL, } \\
\text { (COLS. } \\
\text { PER } \\
100 \mathrm{ML} \text { ) } \\
(31679) \\
\end{array}$ & $\begin{array}{c}\text { COLI- } \\
\text { FORM, } \\
\text { FECAL, } \\
0.45 \\
\text { UM-MF } \\
\text { (COLS./ } \\
100 \mathrm{ML} \text { ) } \\
(31616) \\
\end{array}$ & $\begin{array}{l}\text { ANTI- } \\
\text { MONY, } \\
\text { TOTAL } \\
\text { (UG/L } \\
\text { AS SB) } \\
(01097)\end{array}$ & $\begin{array}{c}\text { ARSENIC } \\
\text { TOTAL } \\
\text { (UG/L } \\
\text { AS AS) } \\
(01002) \\
\end{array}$ & $\begin{array}{c}\text { BERYL- } \\
\text { LIUM, } \\
\text { TOTAL } \\
\text { RECOV- } \\
\text { ERABLE } \\
\text { (UG/L AS } \\
\text { BE) } \\
(01012) \\
\end{array}$ & $\begin{array}{l}\text { CADMIUM } \\
\text { WATER } \\
\text { UNFLTRD } \\
\text { TOTAL } \\
\text { (UG/L } \\
\text { AS CD) } \\
\text { (01027) } \\
\end{array}$ \\
\hline \multicolumn{11}{|l|}{ AUG 1995} \\
\hline$\underset{\mathrm{OCT}}{27 \ldots}$ & 0.480 & 0.120 & -- & 10 & -- & -- & 8 & 1 & -- & -- \\
\hline $04 \ldots$ & 0.250 & 0.140 & -- & 11 & -- & -- & $<1$ & $<1$ & -- & -- \\
\hline $04 \ldots$ & 0.420 & 0.140 & -- & 13 & -- & -- & 1 & $<1$ & -- & -- \\
\hline $04 \ldots$ & -- & -- & 1 & -- & 20000 & 29000 & -- & -- & -- & -- \\
\hline $04 \ldots$ & -- & -- & -- & -- & 58000 & 220000 & -- & -- & -- & -- \\
\hline $04 \ldots$ & 0.460 & 0.370 & -- & $\cdots$ & -- & -- & -- & -- & -- & -- \\
\hline $04 \ldots$ & 0.450 & 0.230 & -- & 14 & -- & -- & $<1$ & $<1$ & -- & -- \\
\hline $27 \ldots$ & -- & -- & - & -- & 77000 & K93000 & -- & -- & -- & -- \\
\hline \multicolumn{11}{|l|}{ FEB 1996} \\
\hline $20 \ldots$ & 0.200 & 0.060 & -- & 29 & -- & -- & 2 & $<1$ & -- & -- \\
\hline $20 \ldots$ & 0.150 & 0.080 & -- & 9.2 & -- & -- & $<1$ & $<1$ & -- & -- \\
\hline $20 \ldots$ & -- & -- & -- & - & 4900 & K630 & -- & -- & -- & -- \\
\hline $20 \ldots$ & 0.140 & 0.080 & -- & -- & 3000 & 7600 & -- & -- & -- & -- \\
\hline $28 \ldots$ & -- & -- & 2 & -- & 35000 & $\mathrm{~K} 14000$ & -- & -- & -- & -- \\
\hline $28 \ldots$ & -- & -- & -- & -- & 5700 & 2500 & -- & -- & -- & -- \\
\hline $28 \ldots$ & -- & -- & -- & -- & 3400 & 3900 & -- & -- & -- & -- \\
\hline \multicolumn{11}{|l|}{ MAR } \\
\hline $06 \ldots$ & -- & -- & -- & -- & 22000 & K14000 & -- & -- & -- & -- \\
\hline \multicolumn{11}{|l|}{ APR } \\
\hline JUN $03 \ldots$ & 0.060 & 0.020 & $<1$ & -- & 5900 & 2300 & 4 & $<1$ & $<10$ & $<1$ \\
\hline $07 \ldots$ & -- & -- & -- & -- & -- & -- & -- & -- & -- & - \\
\hline $07 \ldots$ & 0.930 & 0.140 & -- & -- & -- & -- & 5 & 3 & -- & -- \\
\hline $07 \ldots$ & -- & -- & $<1$ & -- & $\mathrm{K} 20000$ & $\mathrm{~K} 15000$ & -- & -- & -- & -- \\
\hline \multicolumn{11}{|l|}{ AUG } \\
\hline $11 \ldots$ & 0.380 & 0.080 & -- & 25 & -- & -- & $<1$ & 4 & -- & -- \\
\hline $11 \ldots$ & 0.540 & 0.150 & -- & 18 & -- & -- & $<1$ & 4 & -- & -- \\
\hline $11 \ldots$ & 0.570 & 0.040 & -- & -- & -- & -- & -- & -- & -- & -- \\
\hline \multicolumn{11}{|l|}{ OCT } \\
\hline $07 \ldots$ & -- & -- & $<1$ & -- & 3500 & K1300 & - & - & -- & -- \\
\hline $07 \ldots$ & 4.60 & 4.80 & 2 & 32 & 24000 & K60000 & $<1$ & 2 & -- & -- \\
\hline $07 \ldots$ & 0.780 & 0.580 & -- & 11 & -- & -- & $<1$ & 2 & -- & -- \\
\hline $08 \ldots$ & 1.36 & 1.08 & -- & 12 & -- & -- & $\ldots$ & -- & - & -- \\
\hline $08 \ldots$ & 1.46 & 1.36 & -- & -- & -- & -- & -- & -- & -- & -- \\
\hline \multicolumn{11}{|l|}{ JAN 1997} \\
\hline $16 \ldots$ & 0.080 & 0.026 & -- & 7.6 & -- & -- & $<1$ & $<1$ & -- & -- \\
\hline $16 \ldots$ & 0.350 & 0.039 & -- & -- & -- & -- & $<1$ & 2 & $\cdots$ & -- \\
\hline $16 \ldots$ & 0.390 & 0.400 & -- & -- & -- & -- & -- & -- & -- & -- \\
\hline $24 \ldots$ & -- & -- & 2 & -- & 2500 & K720 & -- & -- & -- & -- \\
\hline \multicolumn{11}{|l|}{ MAY } \\
\hline $07 \ldots$ & 0.060 & $<0.010$ & 1 & -- & 2400 & K910 & $<1$ & $<1$ & -- & -- \\
\hline $08 \ldots$ & -- & -- & -- & -- & -- & -- & -- & -- & -- & -- \\
\hline $08 \ldots$ & 0.550 & 0.270 & - & -- & -- & -- & $<1$ & 2 & $\sim$ & -- \\
\hline \multicolumn{11}{|l|}{ JUN } \\
\hline $02 \ldots$ & -- & -- & 2 & 44 & 33000 & K13000 & - & -- & -- & -- \\
\hline $06 \ldots$ & -- & -- & -- & 10 & 540000 & $\mathrm{~K} 17000$ & -- & -- & -- & -- \\
\hline
\end{tabular}

K Results based on colony count outside the acceptable range (nonideal colony count). 
Table 81. Water-quality data at site 42 (CSW04), July 1995 through June 1997-Continued

\begin{tabular}{|c|c|c|c|c|c|c|c|c|c|}
\hline DATE & $\begin{array}{c}\text { CHRO- } \\
\text { MIUM, } \\
\text { TOTAL } \\
\text { RECOV- } \\
\text { ERABLE } \\
\text { (UG/L } \\
\text { AS CR) } \\
(01034) \\
\end{array}$ & $\begin{array}{c}\text { COPPER, } \\
\text { TOTAL } \\
\text { RECOV- } \\
\text { ERABLE } \\
\text { (UG/L AS } \\
\text { CU) } \\
(01042) \\
\end{array}$ & $\begin{array}{c}\text { LEAD, } \\
\text { TOTAL } \\
\text { RECOV- } \\
\text { ERABLE } \\
\text { (UG/L } \\
\text { AS PB) } \\
(01051) \\
\end{array}$ & $\begin{array}{c}\text { MERCURY } \\
\text { TOTAL } \\
\text { RECOV- } \\
\text { ERABLE } \\
\text { (UG/L AS } \\
\text { HG) } \\
(71900) \\
\end{array}$ & $\begin{array}{c}\text { NICKEL, } \\
\text { TOTAL } \\
\text { RECOVER- } \\
\text { ABLE } \\
\text { (UG/L } \\
\text { AS NI) } \\
(01067) \\
\end{array}$ & $\begin{array}{c}\text { SELE- } \\
\text { NIUM, } \\
\text { TOTAL } \\
\text { (UG/L } \\
\text { AS SE) } \\
(01147) \\
\end{array}$ & $\begin{array}{c}\text { SILVER, } \\
\text { TOTAL } \\
\text { RECOV- } \\
\text { ERABLE } \\
\text { (UG/L } \\
\text { AS AG) } \\
(01077 \text { ) } \\
\end{array}$ & $\begin{array}{l}\text { ZINC, } \\
\text { TOTAL } \\
\text { RECOV- } \\
\text { ERABLE } \\
\text { (UG/L } \\
\text { AS ZN) } \\
(01092) \\
\end{array}$ & $\begin{array}{c}\text { CYANIDE } \\
\text { TOTAL } \\
\text { (MG/L AS } \\
\text { CN) } \\
(00720) \\
\end{array}$ \\
\hline AUG 1995 & & & & & & & & & \\
\hline${ }_{\text {OCT }}^{27 \ldots}$ & 24 & 33 & 36 & -- & 9 & -- & -- & 80 & -- \\
\hline $04 \ldots$ & 6 & 13 & 6 & -- & 6 & -- & -- & 90 & -- \\
\hline $04 \ldots$ & 19 & 50 & 15 & -- & 7 & -- & -- & 230 & -- \\
\hline $04 \ldots$ & -- & -- & -- & -- & -- & -- & -- & -- & -- \\
\hline $04 \ldots$ & -- & -- & -- & -- & -- & -- & -- & -- & -- \\
\hline $04 \ldots$ & -- & -- & -- & -- & -- & -- & -- & -- & -- \\
\hline $04 \ldots$ & 16 & 27 & 20 & -. & 5 & -- & -- & 90 & -- \\
\hline $\begin{array}{r}27 \ldots \\
\end{array}$ & -- & -- & -- & -- & -- & -- & -- & -- & -- \\
\hline $20 \ldots$ & 8 & 18 & 10 & -- & 8 & -- & -. & 160 & -- \\
\hline $20 \ldots$ & 6 & 23 & 8 & -- & 3 & -- & -- & 70 & -- \\
\hline $20 \ldots$ & -- & -- & -- & -- & -- & -- & -- & -- & -- \\
\hline $20 \ldots$ & -- & -- & -- & -- & -- & -- & -- & -- & -- \\
\hline $28 \ldots$ & -- & -- & -- & -- & -- & -- & -. & -- & -- \\
\hline $28 \ldots$ & -- & -- & -- & -- & -- & -- & -- & -- & -- \\
\hline $28 \ldots$ & -- & -- & -- & -- & -- & -- & -- & -- & -- \\
\hline $\begin{array}{l}\text { MAR } \\
06 \ldots \\
\text { APR }\end{array}$ & -- & -- & -- & -- & -- & -- & -- & -- & -- \\
\hline $03 \ldots$ & 2 & 4 & $<1$ & 0.11 & $<1$ & $<1$ & $<1$ & 50 & $<0.010$ \\
\hline $07 \ldots$ & -- & -- & -. & -- & -- & -- & -- & -- & -- \\
\hline $07 \ldots$ & 68 & 110 & 39 & -- & 24 & -- & -- & 220 & -- \\
\hline $07 \ldots$ & -- & -- & -- & -- & -- & -- & -- & -- & -- \\
\hline AUG & & & & & & & & & \\
\hline $11 \ldots$ & 18 & 21 & 19 & -- & 11 & -- & -- & 150 & -- \\
\hline $11 \ldots$ & 28 & 87 & 14 & -- & 11 & -- & -- & 110 & -- \\
\hline $11 \ldots$ & -- & -- & -- & -- & -- & -- & -- & -- & -- \\
\hline OCT & & & & & & & & & \\
\hline $07 \ldots$ & -- & -- & -- & -- & -- & -- & -- & -- & -- \\
\hline $07 \ldots$ & 10 & 19 & 9 & -- & 7 & -- & -- & 110 & -- \\
\hline $07 \ldots$ & 11 & 7 & 11 & -- & 5 & -- & -- & 100 & -- \\
\hline $08 \ldots$ & -- & -- & -- & -- & -- & -- & -- & -- & -- \\
\hline $08 \ldots$ & -- & -- & -- & -- & -- & -- & -- & -- & -- \\
\hline JAN 1997 & & & & & & & & & \\
\hline $16 \ldots$ & 7 & 13 & 9 & -- & 4 & -- & -- & 100 & -- \\
\hline $16 \ldots$ & 23 & 51 & 28 & -- & 8 & -- & -- & 130 & -- \\
\hline $16 \ldots$ & -- & -- & -- & -- & -- & -- & -- &.- & -- \\
\hline $24 \ldots$ & -- & -- & -- & -- & -- & -- & -- & -- & -- \\
\hline MAY & & & & & & & & & \\
\hline $07 \ldots$ & 2 & 4 & 2 & -- & 4 & -- & -- & 40 & -- \\
\hline $08 \ldots$ & -- & -- & -- & -- & -- & -- & -- & -- & -- \\
\hline $08 \ldots$ & 11 & 23 & 11 & -- & 7 & -- & -- & 120 & -- \\
\hline JUN & & & & & & & & & \\
\hline $\begin{array}{l}02 \ldots \\
06 \ldots\end{array}$ & $\begin{array}{l}-- \\
--\end{array}$ & -- & $\begin{array}{l}-- \\
--\end{array}$ & -- & $\begin{array}{l}-- \\
--\end{array}$ & $\begin{array}{l}-- \\
--\end{array}$ & $\begin{array}{l}-- \\
--\end{array}$ & $\begin{array}{l}-- \\
--\end{array}$ & -- \\
\hline
\end{tabular}


Table 82. Water-quality data at site 43 (CSW07), July 1995 through June 1997

\begin{tabular}{|c|c|c|c|c|c|c|c|c|c|c|}
\hline DATE & TIME & $\begin{array}{c}\text { RAIN- } \\
\text { FALL } \\
\text { ACCUM } \\
(\text { IN) } \\
(00045)\end{array}$ & $\begin{array}{c}\text { DIS- } \\
\text { CHARGE, } \\
\text { INST: } \\
\text { CUBIC } \\
\text { FEET PER } \\
\text { SECOND } \\
(00061)\end{array}$ & $\begin{array}{c}\text { TEMPER- } \\
\text { ATURE } \\
\text { WATER } \\
\text { DEG. C) } \\
(00010)\end{array}$ & $\begin{array}{c}\text { SPE- } \\
\text { CIFIC } \\
\text { CONDUC- } \\
\text { TANCE } \\
\text { LAB } \\
\text { (US/CM) } \\
(90095) \\
\end{array}$ & $\begin{array}{c}\text { SPE- } \\
\text { CIFIC } \\
\text { CONDUC- } \\
\text { TANCE } \\
\text { (US/CM) } \\
(00095 \\
\end{array}$ & $\begin{array}{c}\text { PH } \\
\text { WATER } \\
\text { RAW LAB } \\
\text { (STAN- } \\
\text { DARD } \\
\text { UNITS) } \\
(00403)\end{array}$ & $\begin{array}{c}\text { PH } \\
\text { WATER } \\
\text { RAW } \\
\text { FIELD } \\
\text { (STAN- } \\
\text { DARD } \\
\text { UNITS) } \\
(00400) \\
\end{array}$ & $\begin{array}{c}\text { ALKA- } \\
\text { LINITY } \\
\text { LAB } \\
\text { (MG/L AS } \\
\text { CACO3) } \\
(90410)\end{array}$ & $\begin{array}{c}\text { SEDI- } \\
\text { MENT, } \\
\text { SUS- } \\
\text { PENDED } \\
(\text { MG / L) } \\
(80154)\end{array}$ \\
\hline \multicolumn{11}{|l|}{ JUL 1995} \\
\hline $27 \ldots$ & 1750 & 0.08 & 0.27 & 27.0 & -- & 108 & -- & 7.00 & -- & -- \\
\hline $27 \ldots$ & 1752 & 0.08 & 0.27 & 27.0 & -- & 108 & -- & 7.00 & -- & -- \\
\hline \multicolumn{11}{|l|}{ AUG } \\
\hline${ }_{\text {OCT }}^{27 \ldots}$ & \multicolumn{9}{|c|}{ OCT } & 789 \\
\hline $04 \ldots$ & 0505 & 3.06 & 6.1 & 20.5 & 82 & 73 & 6.9 & 7.14 & 26 & 1060 \\
\hline $04 \ldots$ & 0542 & 3.06 & 11 & 20.0 & 43 & 39 & 6.5 & 6.89 & 17 & 6040 \\
\hline $04 \ldots$ & 0641 & 3.06 & 6.6 & 20.0 & 70 & 61 & 6.6 & 6.86 & 17 & 2350 \\
\hline $04 \ldots$ & 0851 & 3.06 & 33 & 19.0 & 41 & 33 & 6.4 & 6.67 & 16 & 9250 \\
\hline $04 \ldots$ & 0950 & 3.06 & 15 & 19.0 & 55 & 47 & 6.8 & 6.74 & 16 & 2080 \\
\hline \multicolumn{11}{|l|}{ NOV } \\
\hline $11 \ldots$ & 1140 & 1.28 & 13 & 14.0 & -- & 65 & -- & 6.95 & -- & -- \\
\hline $11 \ldots$ & 1500 & 1.28 & 22 & 14.5 & -- & 53 & -- & 6.91 & -- & -- \\
\hline \multicolumn{11}{|l|}{ FEB 1996} \\
\hline $20 \ldots$ & 0656 & 0.34 & 0.80 & 8.5 & 146 & 128 & 7.7 & 7.30 & 39 & 1880 \\
\hline $20 \ldots$ & 0713 & 0.34 & 0.95 & 8.5 & -- & 139 & -- & 7.95 & -- & -- \\
\hline $20 \ldots$ & 0833 & 0.34 & 0.76 & 8.5 & 155 & 134 & 7.7 & 7.00 & 39 & 529 \\
\hline $20 \ldots$ & 1143 & 0.34 & 0.38 & 10.5 & 166 & 148 & 7.6 & 7.00 & 43 & 191 \\
\hline \multicolumn{11}{|l|}{ APR } \\
\hline $03 \ldots$ & 1510 & 0.00 & 0.14 & 18.0 & 158 & 155 & 7.6 & 7.23 & 52 & 104 \\
\hline \multicolumn{11}{|l|}{ JUN } \\
\hline $07 \ldots$ & 1938 & 0.04 & 0.19 & 23.0 & -- & 237 & -- & 7.48 & -- & -- \\
\hline $08 \ldots$ & 1902 & 0.35 & 1.5 & 24.0 & -- & 77 & -- & 7.17 & -- & -- \\
\hline $08 \ldots$ & 1912 & 0.35 & 2.8 & 23.0 & 155 & 145 & 7.4 & 7.08 & 36 & 1840 \\
\hline $08 \ldots$ & 2244 & 0.35 & 0.29 & 21.5 & 109 & 123 & 7.5 & 7.01 & 33 & 95 \\
\hline \multicolumn{11}{|l|}{ AUG } \\
\hline $02 \ldots$ & 2214 & 2.66 & 18 & 23.5 & 244 & 195 & 7.4 & 7.06 & 72 & 19400 \\
\hline $02 \ldots$ & 2232 & 2.66 & 36 & 23.5 & 112 & 100 & 7.1 & 7.31 & 22 & 10300 \\
\hline $02 \ldots$ & 2316 & 2.66 & 14 & 23.0 & 111 & 103 & 7.0 & 7.01 & 13 & 3470 \\
\hline $03 \ldots$ & 0013 & 2.66 & 74 & 22.5 & -- & -- & -- & - & -- & - \\
\hline \multicolumn{11}{|l|}{ OCT } \\
\hline $07 \ldots$ & 1705 & 2.07 & 0.33 & 15.0 & -- & 177 & -- & 7.34 & -- & -- \\
\hline $07 \ldots$ & 1808 & 2.07 & 0.95 & 15.0 & 165 & 164 & 7.2 & 7.14 & 35 & 1100 \\
\hline $07 \ldots$ & 1815 & 2.07 & 0.95 & 15.0 & -- & 164 & -- & 7.33 & -- & 250 \\
\hline $07 \ldots$ & 2354 & 2.07 & 8.4 & 14.5 & 108 & 105 & 6.9 & 7.28 & 17 & 1350 \\
\hline $08 \ldots$ & 0654 & 2.07 & 12 & 15.0 & -- & 53 & -- & 6.88 & -- & -- \\
\hline $08 \ldots$ & 0716 & 2.07 & 9.9 & 15.0 & 60 & 52 & 6.6 & 6.96 & 13 & 305 \\
\hline $08 \ldots$ & 1200 & 2.07 & 2.7 & 16.5 & 82 & 78 & 6.8 & 7.02 & 22 & 107 \\
\hline \multicolumn{11}{|l|}{ JAN 1997} \\
\hline $24 \ldots$ & 1158 & 0.25 & 0.95 & 7.5 & 141 & 138 & 7.1 & 7.22 & 32 & 2190 \\
\hline $24 \ldots$ & 1204 & 0.25 & 0.73 & 7.5 & -- & 123 & -- & 7.16 & -- & -- \\
\hline $25 \ldots$ & 0254 & 0.33 & 6.5 & 5.5 & 94 & 94 & 7.1 & 6.98 & 23 & 2750 \\
\hline $25 \ldots$ & 0518 & 0.33 & 2.4 & 6.5 & 101 & 97 & 7.1 & 7.00 & 24 & 435 \\
\hline $25 \ldots$ & 0923 & 0.33 & 0.87 & 7.0 & -- & -- & -- & -- & -- & -- \\
\hline \multicolumn{11}{|l|}{ MAY } \\
\hline $07 \ldots$ & 1330 & 0.00 & 0.02 & 19.5 & 248 & 231 & 7.6 & 6.65 & 87 & 87 \\
\hline $08 \ldots$ & 2203 & 0.31 & 5.5 & 19.5 & -- & 138 & -- & 7.28 & -- & -- \\
\hline $08 \ldots$ & 2209 & 0.31 & 2.8 & 19.5 & 106 & 99 & 7.2 & 7.22 & 23 & 1390 \\
\hline \multicolumn{11}{|l|}{ JUN } \\
\hline $06 \ldots$ & 0707 & 0.71 & 0.95 & 16.5 & -- & 106 & -- & 6.94 & -- & -- \\
\hline
\end{tabular}


Table 82. Water-quality data at site 43 (CSW07), July 1995 through June 1997-Continued

\begin{tabular}{|c|c|c|c|c|c|c|c|c|c|c|}
\hline DATE & $\begin{array}{c}\text { RESIDUE } \\
\text { TOTAL } \\
\text { AT } 105 \\
\text { DEG. C, } \\
\text { SUS- } \\
\text { PENDED } \\
\text { (MG/L) } \\
(00530) \\
\end{array}$ & $\begin{array}{l}\text { RESIDUE } \\
\text { VOLA- } \\
\text { TILE, } \\
\text { SUS- } \\
\text { PENDED } \\
\text { (MG/L) } \\
(00535)\end{array}$ & $\begin{array}{l}\text { SOLIDS, } \\
\text { RESIDUE } \\
\text { AT } 180 \\
\text { DEG. C } \\
\text { DIS- } \\
\text { SOLVED } \\
(\mathrm{MG} / \mathrm{L}) \\
(70300) \\
\end{array}$ & $\begin{array}{c}\text { OXYGEN } \\
\text { DEMAND, } \\
\text { BIO- } \\
\text { CHEMI- } \\
\text { CAL } \\
5 \text { DAY } \\
(\text { MG/L) } \\
(00310)\end{array}$ & $\begin{array}{c}\text { OXYGEN } \\
\text { DEMAND, } \\
\text { CHEM- } \\
\text { ICAL } \\
\text { (HIGH } \\
\text { LEVEL) } \\
\text { (MG/L) } \\
(00340) \\
\end{array}$ & $\begin{array}{l}\text { NITRO- } \\
\text { GEN, AM- } \\
\text { MONIA + } \\
\text { ORGANIC } \\
\text { TOTAL } \\
\text { (MG/L } \\
\text { AS N) } \\
(00625) \\
\end{array}$ & $\begin{array}{c}\text { NITRO- } \\
\text { GEN, } \\
\text { NO2+NO3 } \\
\text { DIS- } \\
\text { SOLVED } \\
\text { (MG / L } \\
\text { AS N) } \\
(00631) \\
\end{array}$ & $\begin{array}{l}\text { NITRO- } \\
\text { GEN, } \\
\text { AMMONIA } \\
\text { DIS- } \\
\text { SOLVED } \\
\text { (MG /L } \\
\text { AS N) } \\
(00608) \\
\end{array}$ & $\begin{array}{l}\text { NITRO- } \\
\text { GEN, } \\
\text { ORGANIC } \\
\text { TOTAL } \\
\text { (MG / L AS } \\
\text { N) } \\
(00605) \\
\end{array}$ & $\begin{array}{c}\text { NITRO- } \\
\text { GEN, } \\
\text { TOTAL } \\
\text { (MG / L } \\
\text { AS N) } \\
(00600)\end{array}$ \\
\hline \multicolumn{11}{|l|}{ JUL 1995} \\
\hline $27 \ldots$ & -- & -- & -- & -- & -- & -- & -- & -- & -- & -- \\
\hline $27 \ldots$ & -- & -- & -- & -- & -- & -- & -- & -- & -- & -- \\
\hline \multicolumn{11}{|l|}{ AUG } \\
\hline \multicolumn{10}{|l|}{ OCT } & 0.64 \\
\hline $04 \ldots$ & -- & -- & 66 & 10 & 62 & 3.3 & 0.170 & 1.10 & 2.2 & 3.5 \\
\hline $04 \ldots$ & -- & -- & 50 & 6.5 & 330 & 4.6 & 0.160 & 0.120 & 4.5 & 4.8 \\
\hline $04 \ldots$ & -- & -- & 52 & 6.8 & 54 & 2.3 & 0.260 & 0.230 & 2.1 & 2.6 \\
\hline $04 \ldots$ & -- & -- & 45 & 5.2 & 650 & 7.7 & 0.170 & 0.070 & 7.6 & 7.9 \\
\hline $04 \ldots$ & -- & -- & 50 & 4.0 & 51 & 1.8 & 0.160 & 0.120 & 1.7 & 2.0 \\
\hline \multicolumn{11}{|l|}{ NOV } \\
\hline $11 \ldots$ & -- & - & -- & -- & -- & -- & -- & -- & -- & -- \\
\hline $11 \ldots$ & -- & -- & -- & -- & -- & -- & -- & -- & -- & -- \\
\hline \multicolumn{11}{|l|}{ FEB 1996} \\
\hline $20 \ldots$ & 1140 & 125 & 326 & 5.1 & 22 & 1.1 & 0.430 & 0.130 & 0.97 & 1.5 \\
\hline $20 \ldots$ & -- & -- & -- & -- & -- & -- & -- & -- & -- & -- \\
\hline $20 \ldots$ & 368 & 44 & 212 & 4.2 & 22 & 1.0 & 0.490 & 0.130 & 0.87 & 1.5 \\
\hline $20 \ldots$ & 104 & 6 & 170 & 2.8 & 22 & 0.67 & 0.380 & 0.090 & 0.58 & 1.0 \\
\hline \multicolumn{11}{|l|}{ APR } \\
\hline $03 \ldots$ & 17 & 3 & 89 & $<2.0$ & 7 & 0.44 & 0.120 & 0.200 & 0.24 & 0.56 \\
\hline \multicolumn{11}{|l|}{ JUN } \\
\hline $07 \ldots$ & -- & -- & -- & -- & - & -- & -- & -- & -- & -- \\
\hline $08 \ldots$ & -- & -- & -- & -- & -- & -- & -- & -- & -- & -- \\
\hline $08 \ldots$ & 1050 & 132 & 115 & 20 & 74 & 2.8 & 0.380 & 0.180 & 2.6 & 3.2 \\
\hline $08 \ldots$ & 44 & 6 & 77 & 5.8 & 20 & 0.56 & 0.180 & 0.040 & 0.52 & 0.74 \\
\hline \multicolumn{11}{|l|}{ AUG } \\
\hline $02 \ldots$ & 23900 & 2200 & 154 & 15 & 23 & 7.8 & 0.540 & 0.120 & 7.7 & 8.3 \\
\hline $02 \ldots$ & 5290 & 600 & 76 & 11 & 11 & 3.7 & 0.450 & 0.080 & 3.6 & 4.2 \\
\hline $02 \ldots$ & 1050 & 115 & 84 & 8.8 & 16 & 1.8 & 0.550 & 0.080 & 1.7 & 2.3 \\
\hline $03 \ldots$ & -- & -- & -- & -- & -- & 5.4 & - & - & 5.4 & 5.4 \\
\hline \multicolumn{11}{|l|}{ OCT } \\
\hline $07 \ldots$ & -- & -- & -- & -- & -- & -. & -- & -- & - & -- \\
\hline $07 \ldots$ & 248 & 28 & 109 & 7.9 & 29 & 1.4 & 0.200 & 0.220 & 1.2 & 1.6 \\
\hline $07 \ldots$ & -- & -- & -- & -- & -- & -- & -- & -- & - & - \\
\hline $07 \ldots$ & 957 & 83 & 65 & 5.5 & 15 & 1.6 & 0.150 & 0.040 & 1.6 & 1.7 \\
\hline $08 \ldots$ & -- & -- & -- & -- & -- & -- & -- & -- & - & - \\
\hline $08 \ldots$ & 112 & 7 & 52 & 3.1 & 28 & 1.0 & $<0.050$ & 0.026 & 0.97 & 1.0 \\
\hline $08 \ldots$ & 48 & $<1$ & 64 & 2.2 & 18 & 0.89 & $<0.050$ & $<0.015$ & 0.89 & 0.89 \\
\hline \multicolumn{11}{|l|}{ JAN 1997} \\
\hline $24 \ldots$ & 1790 & 236 & 83 & 14 & 120 & 3.1 & 0.950 & 0.270 & 2.8 & 4.1 \\
\hline $24 \ldots$ & -- & -- & -- & -- & -- & -- & -- & -- & -- & -- \\
\hline $25 \ldots$ & 1820 & 220 & 59 & 5.7 & 46 & 0.33 & 0.290 & 0.050 & 0.28 & 0.62 \\
\hline $25 \ldots$ & 212 & 28 & 66 & 3.8 & 25 & 0.76 & 0.210 & 0.022 & 0.74 & 0.97 \\
\hline $25 \ldots$ & -- & -- & -- & -- & -- & -- & -- & -- & -- & -- \\
\hline \multicolumn{11}{|l|}{ MAY } \\
\hline $07 \ldots$ & 90 & 10 & 230 & $<2.0$ & 11 & 1.4 & 0.110 & 0.480 & 0.92 & 1.5 \\
\hline $08 \ldots$ & -- & -- & -- & -- & -- & -- & -- & -- & -- & -- \\
\hline $08 \ldots$ & 1080 & 128 & 79 & 27 & 86 & 3.4 & 1.31 & 0.560 & 2.8 & 4.7 \\
\hline \multicolumn{11}{|l|}{ JUN } \\
\hline $06 \ldots$ & -- & -- & -- & -- & -- & -- & -- & -- & -- & -- \\
\hline
\end{tabular}


Table 82. Water-quality data at site 43 (CSW07), July 1995 through June 1997—Continued

\begin{tabular}{|c|c|c|c|c|c|c|c|c|c|c|}
\hline DATE & $\begin{array}{c}\text { PHOS- } \\
\text { PHORUS } \\
\text { TOTAL } \\
\text { (MG / L } \\
\text { AS P) } \\
(00665) \\
\end{array}$ & $\begin{array}{c}\text { PHOS- } \\
\text { PHORUS } \\
\text { ORTHO, } \\
\text { DIS- } \\
\text { SOLVED } \\
\text { (MG/L AS } \\
\text { P) } \\
(00671) \\
\end{array}$ & $\begin{array}{c}\text { OIL AND } \\
\text { GREASE, } \\
\text { TOTAL } \\
\text { RECOV. } \\
\text { GRAVI- } \\
\text { METRIC } \\
\text { (MG/L) } \\
(00556) \\
\end{array}$ & $\begin{array}{c}\text { CARBON, } \\
\text { ORGANIC } \\
\text { TOTAL } \\
\text { (MG/L AS } \\
\text { C) } \\
(00680) \\
\end{array}$ & $\begin{array}{c}\text { STREPTO- } \\
\text { COCCI } \\
\text { FECAL, } \\
\text { (COLS. } \\
\text { PER } \\
100 \mathrm{ML}) \\
(31679) \\
\end{array}$ & $\begin{array}{c}\text { COLI- } \\
\text { FORM, } \\
\text { FECAL, } \\
0.45 \\
\text { UM-MF } \\
\text { (COLS.) } \\
100 \mathrm{ML}) \\
(31616) \\
\end{array}$ & $\begin{array}{c}\text { ANTI- } \\
\text { MONY, } \\
\text { TOTAL } \\
\text { (UG/L } \\
\text { AS SB) } \\
(01097)\end{array}$ & $\begin{array}{c}\text { ARSENIC } \\
\text { TOTAL } \\
\text { (UG/L } \\
\text { AS AS) } \\
(01002) \\
\end{array}$ & $\begin{array}{c}\text { BERYL- } \\
\text { LIUM, } \\
\text { TOTAL } \\
\text { RECOV- } \\
\text { ERABLE } \\
\text { (UG/L AS } \\
\text { BE) } \\
(01012) \\
\end{array}$ & $\begin{array}{l}\text { CADMIUM } \\
\text { WATER } \\
\text { UNFLTRD } \\
\text { TOTAL } \\
\text { (UG / L } \\
\text { AS CD) } \\
(01027 \text { ) }\end{array}$ \\
\hline \multicolumn{11}{|l|}{ JUL 1995} \\
\hline $27 \ldots$ & -- & -- & $<1$ & -- & -- & -- & -- & -- & -- & -- \\
\hline $27 \ldots$ & -- & -- & -- & -- & 26000 & 92000 & -- & -- & -- & -- \\
\hline \multicolumn{11}{|l|}{ AUG } \\
\hline $27 \ldots$ & 0.600 & 0.050 & -- & 16 & -- & -- & 16 & 3 & -- & -- \\
\hline \multicolumn{11}{|l|}{ OCT } \\
\hline $\begin{array}{l}04 \ldots \\
04 \ldots\end{array}$ & $\begin{array}{l}2.40 \\
7.30\end{array}$ & $\begin{array}{l}0.700 \\
0.070\end{array}$ & $\begin{array}{c}-- \\
1\end{array}$ & $\begin{array}{l}29 \\
16\end{array}$ & $\begin{array}{c}-- \\
41000\end{array}$ & $\begin{array}{c}-- \\
37000\end{array}$ & $\begin{array}{c}2 \\
17\end{array}$ & $\begin{array}{c}6 \\
37\end{array}$ & $\begin{array}{l}-- \\
--\end{array}$ & $\begin{array}{l}-- \\
--\end{array}$ \\
\hline $04 \ldots$ & 2.88 & 0.210 & -- & -- & 52000 & 51000 & -- & -- & -- & -- \\
\hline $04 \ldots$ & 10.5 & 0.060 & -- & 22 & -- & -- & 19 & 38 & -- & -- \\
\hline $04 \ldots$ & 2.43 & 0.160 & -- & -- & -- & -- & -- & -- & -- & -- \\
\hline \multicolumn{11}{|l|}{ NOV } \\
\hline $11 \ldots$ & -- & -- & -- & -- & K11800 & K.7200 & -- & -- & -- & -- \\
\hline $11 \ldots$ & -- & - & -. & -- & K10000 & 28000 & -- & -- & -- & -- \\
\hline \multicolumn{11}{|l|}{ FEB 1996} \\
\hline $20 \ldots$ & 3.90 & 0.040 & -- & 12 & -- & -- & 9 & 17 & -- & -- \\
\hline $20 \ldots$ & -- & -- & $<1$ & -- & 3000 & K810 & -- & -- & -- & $\cdots$ \\
\hline $20 \ldots$ & 0.970 & 0.030 & -- & 12 & -- & -- & 1 & 6 & -- & -- \\
\hline $20 \ldots$ & 0.340 & 0.020 & -- & -- & -- & -. & -- & -- & -- & -- \\
\hline \multicolumn{11}{|l|}{$\mathrm{APR}$} \\
\hline $03 \ldots$ & 0.180 & 0.030 & $<1$ & -- & 80 & 160 & $<1$ & $<1$ & $<10$ & $<1$ \\
\hline \multicolumn{11}{|l|}{ JUN } \\
\hline $07 \ldots$ & -- & -- & $<1$ & 27 & 29000 & K13000 & -- & -- & -- & -- \\
\hline $08 \ldots$ & - & -- & -- & -- & -- & -- & -- & -- & -- & -- \\
\hline $08 \ldots$ & 2.27 & 0.020 & -- & -- & -- & -- & 20 & 10 & -- & -- \\
\hline $08 \ldots$ & 0.110 & 0.020 & -- & -- & - & -- & -- & -- & -- & -- \\
\hline \multicolumn{11}{|l|}{ AUG } \\
\hline $02 \ldots$ & 33.5 & 0.030 & -- & 28 & -- & -- & $<5$ & 59 & -- & -- \\
\hline $02 \ldots$ & 14.8 & 0.030 & -- & 12 & -- & -- & $<1$ & 34 & -- & - \\
\hline $02 \ldots$ & 4.71 & 0.090 & -- & -- & -- & -- & -- & -- & -- & -- \\
\hline $03 \ldots$ & 19.3 & -- & -- & -- & -- & -- & $<5$ & 41 & 710 & -- \\
\hline \multicolumn{11}{|l|}{ OCT } \\
\hline $07 \ldots$ & -- & -- & 1 & -- & 70000 & 33000 & $\ldots$ & -- & -- & -- \\
\hline $07 \ldots$ & 1.02 & 0.030 & -- & 18 & -- & $\cdot-$ & $<1$ & 2 & -- & -- \\
\hline $07 \ldots$ & -- & -- & -- & -- & -- & -- & -- & -- & -- & -- \\
\hline $07 \ldots$ & 2.94 & 0.060 & -- & 16 & -- & -- & $<1$ & 6 & -- & - \\
\hline $08 \ldots$ & -- & -- & -- & -- & 29000 & $\mathrm{~K} 17000$ & -- & -- & -- & -- \\
\hline $08 \ldots$ & 0.660 & 0.040 & -- & -- & 22000 & K17000 & -- & $\rightarrow$ & -- & -- \\
\hline $08 \ldots$ & 0.260 & 0.021 & -- & -- & -- & -- & -- & -- & -- & -- \\
\hline \multicolumn{11}{|l|}{ JAN 1997} \\
\hline $24 \ldots$ & 2.93 & 0.013 & -- & 24 & -- & -- & $<1$ & 19 & -- & -- \\
\hline $24 \ldots$ & -- & -- & 4 & -- & K1900 & $<1000$ & -- & -- & -- & -- \\
\hline $25 \ldots$ & 3.76 & 0.016 & -- & 7.6 & -- & -- & $<1$ & 20 & -- & -- \\
\hline $25 \ldots$ & 0.620 & 0.025 & -- & -- & -- & -- & $<1$ & 7 & -- & -- \\
\hline $25 \ldots$ & -- & -- & -- & -- & K10000 & K1200 & -- & -- & -- & -- \\
\hline \multicolumn{11}{|l|}{ MAY } \\
\hline $07 \ldots$ & 1.23 & 1.23 & 2 & -- & 2200 & $\mathrm{~K} 1200$ & $<1$ & $<1$ & -- & -- \\
\hline $08 \ldots$ & -- & -- & -- & -- & -- & -- & -- & -- & -- & -- \\
\hline $08 \ldots$ & 1.43 & 0.040 & -- & -- & -- & -- & $<1$ & 5 & -- & -- \\
\hline \multicolumn{11}{|l|}{ JUN } \\
\hline $06 \ldots$ & -- & -- & 4 & 20 & K.12000 & K.13000 & -- & -- & -- & - \\
\hline
\end{tabular}

K Results based on colony count outside the acceptable range (nonideal colony count). 
Table 82. Water-quality data at site 43 (CSW07), July 1995 through June 1997-Continued

\begin{tabular}{|c|c|c|c|c|c|c|c|c|c|}
\hline DATE & $\begin{array}{c}\text { CHRO- } \\
\text { MIUM, } \\
\text { TOTAL } \\
\text { RECOV- } \\
\text { ERABLE } \\
\text { (UG/L } \\
\text { AS CR) } \\
(01034) \\
\end{array}$ & $\begin{array}{c}\text { COPPER, } \\
\text { TOTAL } \\
\text { RECOV- } \\
\text { ERABLE } \\
\text { (UG/L AS } \\
\text { CU) } \\
(01042) \\
\end{array}$ & $\begin{array}{l}\text { LEAD, } \\
\text { TOTAL } \\
\text { RECOV- } \\
\text { ERABLE } \\
\text { (UG/L } \\
\text { AS PB) } \\
(01051) \\
\end{array}$ & $\begin{array}{c}\text { MERCURY } \\
\text { TOTAL } \\
\text { RECOV- } \\
\text { ERABLE } \\
\text { (UG/L AS } \\
\text { HG) } \\
(71900) \\
\end{array}$ & $\begin{array}{c}\text { NICKEL, } \\
\text { TOTAL } \\
\text { RECOVER- } \\
\text { ABLE } \\
\text { (UG /L } \\
\text { AS NI) } \\
(01067) \\
\end{array}$ & $\begin{array}{c}\text { SELE- } \\
\text { NIUM, } \\
\text { TOTAL } \\
\text { (UG/L } \\
\text { AS SE) } \\
(01147) \\
\end{array}$ & $\begin{array}{c}\text { SILVER, } \\
\text { TOTAL } \\
\text { RECOV- } \\
\text { ERABLE } \\
\text { (UG/L } \\
\text { AS AG) } \\
(01077 \text { ) }\end{array}$ & $\begin{array}{c}\text { ZINC, } \\
\text { TOTAL } \\
\text { RECOV- } \\
\text { ERABLE } \\
\text { (UG/L } \\
\text { AS ZN) } \\
(01092) \\
\end{array}$ & $\begin{array}{c}\text { CYANIDE } \\
\text { TOTAL } \\
\text { (MG/L AS } \\
\text { CN) } \\
(00720) \\
\end{array}$ \\
\hline \multicolumn{10}{|l|}{ JUL 1995} \\
\hline $27 \ldots$ & -- & -- & -- & -- & -- & -- & -- & -- & -- \\
\hline${ }_{\text {AUG }} 27 \ldots$ & -- & -- & -- & -- & -- & -- & -- & - & -- \\
\hline $\begin{array}{l}27 \ldots \\
\text { OCT }\end{array}$ & 77 & 55 & 9 & $<0.10$ & 35 & -- & -- & 80 & -- \\
\hline $04 \ldots$ & 110 & 94 & 19 & 0.20 & 50 & -- & -- & 180 & -- \\
\hline $04 \ldots$ & 640 & 470 & 85 & 0.50 & 410 & -- & -- & 550 & -- \\
\hline $04 \ldots$ & -- & -- & -- & - & -- & -- & -- & -- & -- \\
\hline $04 \ldots$ & 760 & 870 & 91 & 0.40 & 520 & -- & -- & 770 & -- \\
\hline $04 \ldots$ & -- & -- & -- & -- & -- & -- & -- & -- & -- \\
\hline \multicolumn{10}{|l|}{ NOV } \\
\hline $11 \ldots$ & -- & -- & -- & -- & -- & -- & -- & -- & -- \\
\hline$\underset{F E B}{11 \ldots 96}$ & -- & -- & -- & -- & \multicolumn{2}{|c|}{ FEB 1996} & -- & -- & -- \\
\hline $20 \ldots$ & 93 & 110 & 15 & $<0.10$ & 160 & -- & -- & 200 & -- \\
\hline $20 \ldots$ & -- & -- & - & -- & -- & -- & -- & -- & -- \\
\hline $20 \ldots$ & 35 & 39 & 9 & $<0.10$ & 31 & -- & -- & 90 & -- \\
\hline \multirow{2}{*}{\multicolumn{10}{|c|}{ APR }} \\
\hline & & & & & & & & & \\
\hline$\underset{\text { JUN }}{03 \ldots}$ & 7 & 3 & 1 & $<0.10$ & 4 & $<1$ & $<1$ & 30 & $<0.010$ \\
\hline $07 \ldots$ & -- & -- & -- & -- & -- & -- & -- & -- & -- \\
\hline $08 \ldots$ & -- & -- & -- & - & -- & -- & - & - & -- \\
\hline $08 \ldots$ & 130 & 74 & 17 & 0.10 & 97 & -- & -- & 180 & - \\
\hline $08 \ldots$ & -- & -- & -- & -- & -- & - & -- & -- & -- \\
\hline \multicolumn{10}{|l|}{ AUG } \\
\hline $02 \ldots$ & 1100 & 1200 & 130 & 0.70 & 890 & -- & -- & 1800 & -- \\
\hline $02 \ldots$ & 430 & 510 & 83 & 0.30 & 320 & -- & - & 680 & -- \\
\hline $02 \ldots$ & -- & -- & -- & -- & -- & -- & -- & -- & -- \\
\hline \multirow{2}{*}{\multicolumn{10}{|c|}{ ОСт }} \\
\hline & & & & & & & & & \\
\hline $07 \ldots$ & -- & -- & -- & -- & -- & -. & -- & -- & -- \\
\hline $07 \ldots$ & 27 & 13 & 10 & $<0.10$ & 27 & -- & -- & 140 & -- \\
\hline $07 \ldots$ & -- & - & -- & -- & -- & -- & -- & -- & -- \\
\hline $07 \ldots$ & 98 & 8 & 11 & $<0.10$ & 69 & -- & -- & 200 & -- \\
\hline $08 \ldots$ & -- & -- & -- & -- & -- & -- & -- & -- & -- \\
\hline $08 \ldots$ & -- & -- & -- & -- & -- & -- & - & -- & -- \\
\hline $08 \ldots$ & -- & -- & -- & -- & -- & -- & -- & -- & -- \\
\hline \multicolumn{10}{|l|}{ JAN 1997} \\
\hline $\begin{array}{l}24 \ldots \\
24 \ldots\end{array}$ & 150 & $\begin{array}{l}160 \\
--\end{array}$ & 3 & 0.10 & 87 & -- & $\begin{array}{l}-- \\
--\end{array}$ & $\begin{array}{c}360 \\
--\end{array}$ & -- \\
\hline $25 \ldots$ & 200 & 190 & 130 & 0.10 & 160 & - & - & 250 & -- \\
\hline $25 \ldots$ & 49 & 36 & 19 & $<0.10$ & 27 & -- & -- & 60 & - \\
\hline $25 \ldots$ & -- & -- & -- & -- & - & -- & -- & -- & -- \\
\hline \multicolumn{10}{|l|}{ MAY } \\
\hline $07 \ldots$ & 20 & 15 & 2 & $<0.10$ & 12 & -- & -- & 40 & -- \\
\hline $08 \ldots$ & -- & -- & -- & -- & -- & -- & -- & -- & -- \\
\hline $08 \ldots$ & 62 & 84 & 17 & 0.20 & 45 & - & -- & 240 & -- \\
\hline \multicolumn{10}{|l|}{ JUN } \\
\hline $06 \ldots$ & -- & -- & -- & -- & -- & -- & -- & -- & -- \\
\hline
\end{tabular}


Table 83. Water-quality data at site 44 (CSW10), November 1996 through July 1997

\begin{tabular}{|c|c|c|c|c|c|c|c|c|c|c|}
\hline DATE & TIME & $\begin{array}{c}\text { RAIN- } \\
\text { FALL } \\
\text { ACCUM } \\
(I N) \\
(00045)\end{array}$ & $\begin{array}{c}\text { DIS- } \\
\text { CHARGE, } \\
\text { INST. } \\
\text { CUBIC } \\
\text { FEET PER } \\
\text { SECOND } \\
(00061) \\
\end{array}$ & $\begin{array}{c}\text { TEMPER- } \\
\text { ATURE } \\
\text { WATER } \\
\text { DEG. C) } \\
(00010)\end{array}$ & $\begin{array}{c}\text { SPE- } \\
\text { CIFIC } \\
\text { CONDUC- } \\
\text { TANCE } \\
\text { LAB } \\
\text { (US/CM) } \\
(90095) \\
\end{array}$ & $\begin{array}{c}\text { SPE- } \\
\text { CIFIC } \\
\text { CONDUC- } \\
\text { TANCE } \\
\text { (US /CM) } \\
(00095\end{array}$ & $\begin{array}{c}\text { PH } \\
\text { WATER } \\
\text { RAW LAB } \\
\text { (STAN- } \\
\text { DARD } \\
\text { UNITS) } \\
(00403)\end{array}$ & $\begin{array}{c}\text { PH } \\
\text { WATER } \\
\text { RAW } \\
\text { FIELD } \\
\text { (STAN- } \\
\text { DARD } \\
\text { UNITS) } \\
(00400) \\
\end{array}$ & $\begin{array}{l}\text { ALKA- } \\
\text { LINITY } \\
\text { LAB } \\
\text { (MG/L AS } \\
\text { CACO3) } \\
(90410)\end{array}$ & $\begin{array}{c}\text { SEDI - } \\
\text { MENT, } \\
\text { SUS- } \\
\text { PENDED } \\
(\text { MG / L) } \\
(80154) \\
\end{array}$ \\
\hline \multicolumn{11}{|l|}{ NOV 1996} \\
\hline $30 \ldots$ & 2005 & 0.17 & 18 & 8.0 & 142 & 130 & 7.6 & 7.56 & 52 & 92 \\
\hline \multicolumn{11}{|l|}{ DEC } \\
\hline $01 \ldots$ & 0747 & 1.01 & 47 & 9.5 & 140 & 132 & 7.4 & 7.48 & 41 & 482 \\
\hline $01 \ldots$ & 1027 & 1.01 & 84 & 10.5 & - & 119 & $\ldots$ & 7.32 & - & -- \\
\hline $01 \ldots$ & 1032 & 1.01 & 86 & 10.5 & -- & 120 & -- & 7.21 & -- & 466 \\
\hline $01 \ldots$ & 1102 & 1.01 & 107 & 11.0 & 105 & 95 & 7.4 & 7.16 & 28 & 386 \\
\hline $01 \ldots$ & 1205 & 1.01 & 199 & 11.5 & 117 & 107 & 7.2 & 7.10 & 31 & 871 \\
\hline $01 \ldots$ & 1212 & 1.01 & 213 & 11.5 & - & - & -- & -- & -- & - \\
\hline $01 \ldots$ & 1316 & 1.01 & 305 & 12.0 & 110 & 104 & 7.1 & 7.10 & 27 & 1270 \\
\hline $01 \ldots$ & 1425 & 1.01 & 358 & 12.5 & 100 & 94 & 7.1 & 7.06 & 23 & 1030 \\
\hline \multicolumn{11}{|l|}{ JAN 1997} \\
\hline $09 \ldots$ & 0941 & 1.27 & 492 & 4.5 & 75 & 85 & 6.9 & 7.00 & 16 & 1870 \\
\hline $09 \ldots$ & 1151 & 1.27 & 535 & 5.0 & 72 & 81 & 6.7 & 7.20 & 14 & 939 \\
\hline $10 \ldots$ & 0832 & 1.27 & 65 & 6.5 & 99 & 90 & 7.0 & 7.14 & 26 & 163 \\
\hline \multicolumn{11}{|l|}{ FEB } \\
\hline $13 \ldots$ & 1530 & -- & 21 & 4.5 & -- & 122 & -- & 7.50 & -- & -- \\
\hline $13 \ldots$ & 1632 & -- & 25 & 4.5 & -- & 143 & -- & 7.14 & 42 & 141 \\
\hline $13 \ldots$ & 1824 & -- & 51 & 3.5 & -- & 131 & -- & 7.38 & 36 & 195 \\
\hline $14 \ldots$ & 0520 & - & 269 & 3.0 & 91 & 95 & 7.1 & 7.23 & 21 & 383 \\
\hline $14 \ldots$ & 1038 & -- & 118 & 4.0 & 96 & 106 & 7.2 & 7.00 & 22 & 170 \\
\hline \multicolumn{11}{|l|}{ MAR } \\
\hline $14 \ldots$ & 0612 & 0.61 & 40 & 13.0 & 131 & 122 & 7.5 & 7.61 & 45 & 139 \\
\hline $14 \ldots$ & 1028 & 0.61 & 131 & 13.5 & 114 & 109 & 7.4 & 7.33 & 31 & 465 \\
\hline $14 \ldots$ & 1430 & 0.61 & 70 & 15.0 & 109 & 104 & 7.3 & 7.49 & 30 & 236 \\
\hline \multicolumn{11}{|l|}{$\mathrm{APR}$} \\
\hline $27 \ldots$ & 1916 & 1.16 & 150 & 13.0 & -- & 89 & - & 7.15 & -- & -- \\
\hline $27 \ldots$ & 1923 & 1.16 & 152 & 13.0 & 93 & 99 & 7.1 & 7.18 & 30 & 725 \\
\hline $28 \ldots$ & 0405 & 1.16 & 438 & 12.5 & 75 & 74 & 7.0 & 6.93 & 21 & 504 \\
\hline $28 \ldots$ & 0858 & 1.16 & 138 & 12.5 & 85 & 82 & 7.0 & 7.08 & 25 & 241 \\
\hline \multicolumn{11}{|l|}{ MAY } \\
\hline $07 \ldots$ & 0835 & 0.00 & 19 & 15.0 & 129 & 118 & 7.5 & 6.82 & 47 & 31 \\
\hline \multicolumn{11}{|l|}{ JUL } \\
\hline $23 \ldots$ & 0432 & 4.81 & 232 & 25.5 & 78 & 72 & 6.9 & 6.92 & 16 & 2020 \\
\hline $23 \ldots$ & 0720 & 4.81 & 398 & 24.5 & 59 & 56 & 6.8 & 6.66 & 11 & 2490 \\
\hline $23 \ldots$ & 1714 & 4.81 & 864 & 25.0 & 59 & 22 & 6.7 & 7.13 & 11 & 295 \\
\hline
\end{tabular}


Table 83. Water-quality data at site 44 (CSW10), November 1996 through July 1997-Continued

\begin{tabular}{|c|c|c|c|c|c|c|c|c|c|c|}
\hline DATE & $\begin{array}{c}\text { RESIDUE } \\
\text { TOTAL } \\
\text { AT } 105 \\
\text { DEG. C. } \\
\text { SUS- } \\
\text { PENDED } \\
\text { (MG/L) } \\
(00530) \\
\end{array}$ & $\begin{array}{l}\text { RESIDUE } \\
\text { VOLA- } \\
\text { TILE, } \\
\text { SUS- } \\
\text { PENDED } \\
(\text { MG / L) } \\
(00535) \\
\end{array}$ & $\begin{array}{l}\text { SOLIDS, } \\
\text { RESIDUE } \\
\text { AT } 180 \\
\text { DEG. C } \\
\text { DIS- } \\
\text { SOLVED } \\
(\mathrm{MG} / \mathrm{L}) \\
(70300) \\
\end{array}$ & $\begin{array}{c}\text { OXYGEN } \\
\text { DEMAND, } \\
\text { BIO- } \\
\text { CHEMI- } \\
\text { CAL } \\
5 \text { DAY } \\
(M G / L) \\
(00310) \\
\end{array}$ & $\begin{array}{c}\text { OXYGEN } \\
\text { DEMAND, } \\
\text { CHEM- } \\
\text { ICAL } \\
\text { (HIGH } \\
\text { LEVEL) } \\
\text { (MG/L) } \\
(00340) \\
\end{array}$ & $\begin{array}{c}\text { NITRO- } \\
\text { GEN, AM- } \\
\text { MONIA + } \\
\text { ORGANIC } \\
\text { TOTAL } \\
\text { (MG/L } \\
\text { AS N) } \\
(00625) \\
\end{array}$ & $\begin{array}{c}\text { NITRO- } \\
\text { GEN, } \\
\text { NO2+NO3 } \\
\text { DIS- } \\
\text { SOLVED } \\
\text { (MG / L } \\
\text { AS N) } \\
(00631) \\
\end{array}$ & $\begin{array}{l}\text { NITRO- } \\
\text { GEN, } \\
\text { AMMONIA } \\
\text { DIS- } \\
\text { SOLVED } \\
\text { (MG/L } \\
\text { AS N) } \\
(00608) \\
\end{array}$ & $\begin{array}{l}\text { NITRO- } \\
\text { GEN, } \\
\text { ORGANIC } \\
\text { TOTAL } \\
\text { (MG / L AS } \\
\text { N) } \\
(00605) \\
\end{array}$ & $\begin{array}{c}\text { NITRO- } \\
\text { GEN, } \\
\text { TOTAL } \\
(\text { MG / L } \\
\text { AS N) } \\
(00600) \\
\end{array}$ \\
\hline \multicolumn{11}{|l|}{ NOV 1996} \\
\hline $\mathrm{DEC}^{30 \ldots}$ & 50 & 8 & 103 & 3.1 & 6 & 0.48 & 0.620 & 0.040 & 0.44 & 1.1 \\
\hline $01 \ldots$ & 434 & 60 & 95 & 9.6 & 20 & 1.9 & 0.710 & 0.250 & 1.6 & 2.6 \\
\hline $01 \ldots$ & -- & -- & -- & -- & -- & -- & -- & -- & -- & -- \\
\hline $\begin{array}{l}01 \ldots \\
01 \ldots\end{array}$ & $\begin{array}{r}-- \\
333\end{array}$ & $\begin{array}{l}-- \\
48\end{array}$ & $\begin{array}{l}-- \\
74\end{array}$ & $--\overline{7.7}$ & $\overline{13}$ & $-\overline{1.3}$ & $\overline{0.570}$ & 0.210 & -- & $--\overline{1.9}$ \\
\hline $01 \ldots$ & 748 & 102 & 86 & 13 & 28 & 2.8 & 0.710 & 0.230 & 2.6 & 3.5 \\
\hline $01 \ldots$ & -- & -- & -- & -- & -- & -- & -- & -- & -- & -- \\
\hline $01 \ldots$ & 1050 & 138 & 83 & 15 & 25 & 3.0 & 0.730 & 0.220 & 2.8 & 3.7 \\
\hline $01 \ldots$ & 808 & 102 & 71 & 13 & 25 & 2.0 & 0.710 & 0.220 & 1.8 & 2.7 \\
\hline \multicolumn{11}{|l|}{ JAN 1997} \\
\hline $09 \ldots$ & 1320 & 145 & 48 & 10 & 29 & 2.6 & 0.590 & 0.170 & 2.4 & 3.2 \\
\hline $09 \ldots$ & 508 & 52 & 44 & 7.9 & 21 & 1.8 & 0.600 & 0.120 & 1.7 & 2.4 \\
\hline $10 \ldots$ & 94 & 12 & 74 & 3.8 & 17 & 0.82 & 0.570 & 0.050 & 0.77 & 1.4 \\
\hline \multicolumn{11}{|l|}{ FEB } \\
\hline $13 \ldots$ & -- & -- & -- & -- & -- & -- & -- & -- & -- & -- \\
\hline $13 \ldots$ & 100 & 12 & 87 & 2.7 & 7 & 0.57 & 0.540 & $<0.015$ & 0.57 & 1.1 \\
\hline $13 \ldots$ & 172 & 20 & 81 & 5.7 & 120 & 0.84 & 0.480 & 0.060 & 0.78 & 1.3 \\
\hline $14 \ldots$ & 322 & 40 & 62 & 6.8 & 28 & 1.3 & 0.440 & 0.110 & 1.2 & 1.7 \\
\hline $14 \ldots$ & 128 & 16 & 68 & 5.9 & 21 & 0.93 & 0.490 & 0.070 & 0.86 & 1.4 \\
\hline \multicolumn{11}{|l|}{ MAR } \\
\hline $14 \ldots$ & 117 & 13 & 92 & 4.1 & 12 & 0.73 & 0.580 & 0.050 & 0.68 & 1.3 \\
\hline $14 \ldots$ & 425 & 57 & 72 & 7.1 & 35 & 1.9 & 0.620 & 0.360 & 1.5 & 2.5 \\
\hline $14 \ldots$ & 198 & 25 & 74 & 5.4 & 18 & 1.4 & 0.560 & 0.250 & 1.1 & 2.0 \\
\hline \multicolumn{11}{|l|}{ APR } \\
\hline $27 \ldots$ & -- & -- & -- & -- & -- & -- & -- & -- & -- & -- \\
\hline $27 \ldots$ & 528 & 66 & 77 & 7.6 & 31 & 1.5 & 0.400 & 0.110 & 1.4 & 1.9 \\
\hline $28 \ldots$ & 340 & 44 & 64 & 5.6 & 37 & 1.6 & 0.890 & 0.110 & 1.5 & 2.5 \\
\hline $28 \ldots$ & 161 & 20 & 69 & 3.5 & 28 & 1.0 & 0.550 & 0.040 & 0.96 & 1.5 \\
\hline \multicolumn{11}{|l|}{ MAY } \\
\hline JUL $^{07 \ldots}$ & 17 & 1 & 95 & $<2.0$ & $<5$ & 0.25 & 0.520 & $<0.015$ & 0.25 & 0.77 \\
\hline $23 \ldots$ & 1790 & 230 & 61 & 10 & 25 & 2.9 & 0.510 & 0.430 & 2.5 & 3.4 \\
\hline $23 \ldots$ & 1490 & 210 & 50 & 5.7 & 22 & 2.5 & 0.380 & 0.470 & 2.0 & 2.9 \\
\hline $23 \ldots$ & 188 & 24 & 55 & 4.3 & 22 & 0.97 & 0.660 & 0.140 & 0.83 & 1.6 \\
\hline
\end{tabular}


Table 83. Water-quality data at site 44 (CSW10), November 1996 through July 1997-Continued

\begin{tabular}{|c|c|c|c|c|c|c|c|c|c|c|}
\hline DATE & $\begin{array}{c}\text { PHOS- } \\
\text { PHORUS } \\
\text { TOTAL } \\
\text { (MG/L } \\
\text { AS P) } \\
(00665) \\
\end{array}$ & $\begin{array}{c}\text { PHOS- } \\
\text { PHORUS } \\
\text { ORTHO, } \\
\text { DIS- } \\
\text { SOLVED } \\
\text { (MG / L AS } \\
\text { P) } \\
(00671) \\
\end{array}$ & $\begin{array}{c}\text { OIL AND } \\
\text { GREASE, } \\
\text { TOTAL } \\
\text { RECOV. } \\
\text { GRAVI - } \\
\text { METRIC } \\
\text { (MG / L) } \\
(00556) \\
\end{array}$ & $\begin{array}{c}\text { CARBON, } \\
\text { ORGANIC } \\
\text { TOTAL } \\
\text { (MG/L AS } \\
\text { C) } \\
(00680) \\
\end{array}$ & $\begin{array}{c}\text { STREPTO- } \\
\text { COCCI } \\
\text { FECAL, } \\
\text { (COLS. } \\
\text { PER } \\
100 \mathrm{ML}) \\
(31679) \\
\end{array}$ & $\begin{array}{c}\text { COLI- } \\
\text { FORM, } \\
\text { FECAL, } \\
0.45 \\
\text { UM-MF } \\
\text { (COLS. } \\
\text { 100 ML) } \\
(31616)\end{array}$ & $\begin{array}{l}\text { ANTI- } \\
\text { MONY, } \\
\text { TOTAL } \\
\text { (UG / L } \\
\text { AS SB) } \\
(01097) \\
\end{array}$ & $\begin{array}{c}\text { ARSENIC } \\
\text { TOTAL } \\
\text { (UG/L } \\
\text { AS AS) } \\
(01002) \\
\end{array}$ & $\begin{array}{c}\text { BERYL- } \\
\text { LIUM, } \\
\text { TOTAL } \\
\text { RECOV- } \\
\text { ERABLE } \\
\text { (UG/L AS } \\
\text { BE) } \\
(01012) \\
\end{array}$ & $\begin{array}{l}\text { CADMIUM } \\
\text { WATER } \\
\text { UNFLTRD } \\
\text { TOTAL } \\
\text { (UG/L } \\
\text { AS CD) } \\
(01027) \\
\end{array}$ \\
\hline \multicolumn{11}{|l|}{ NOV 1996} \\
\hline \multicolumn{10}{|l|}{ DEC } & $<1$ \\
\hline $01 \ldots$ & 0.490 & 0.050 & -- & 57 & -- & -- & $<1$ & 2 & $<10$ & $<1$ \\
\hline $01 \ldots$ & -- & -- & -- & -- & 140000 & 20000 & -- & -- & -- & -- \\
\hline $01 \ldots$ & -- & -- & -- & -- & - & -- & -- & -- & -- & -- \\
\hline $01 \ldots$ & 0.380 & 0.043 & -- & 17 & -- & -- & $<1$ & 2 & $<10$ & $<1$ \\
\hline $01 \ldots$ & 0.750 & 0.070 & -- & 21 & -- & -- & $<1$ & 4 & $<10$ & $<1$ \\
\hline $01 \ldots$ & -- & - & -- & -- & 380000 & 51000 & -- & -- & -- & -- \\
\hline $01 \ldots$ & 0.830 & 0.070 & -- & 17 & 420000 & 36000 & $<1$ & 5 & $<10$ & $<1$ \\
\hline $01 \ldots$ & 0.810 & 0.080 & -- & 24 & 370000 & 47000 & $<1$ & 5 & $<10$ & $<1$ \\
\hline \multicolumn{11}{|l|}{ JAN 1997} \\
\hline $09 \ldots$ & 1.02 & 0.034 & -- & 31 & -- & -- & $<1$ & 4 & $<10$ & $<1$ \\
\hline $09 \ldots$ & 0.600 & 0.050 & -- & 24 & 59000 & 29000 & $<1$ & 2 & $<10$ & $<1$ \\
\hline $10 \ldots$ & 0.120 & 0.015 & -- & -- & -- & -- & -- & -- & -- & -- \\
\hline \multicolumn{11}{|l|}{ FEB } \\
\hline $13 \ldots$ & -- & -- & $<1$ & -- & K990 & $\mathrm{K} 450$ & -- & -- & -- & -- \\
\hline $13 \ldots$ & 0.090 & 0.014 & -- & 3.6 & - & -- & $<1$ & $<1$ & $<10$ & 1 \\
\hline $13 \ldots$ & 0.180 & 0.021 & -- & 10 & -- & -- & $<1$ & $<1$ & $<10$ & $<1$ \\
\hline $14 \ldots$ & 0.350 & 0.060 & -- & 13 & - & -- & $<1$ & 1 & $<10$ & $<1$ \\
\hline $14 \ldots$ & 0.200 & 0.043 & -- & -- & $\mathrm{K} 12000$ & K180 & -- & -- & -- & -- \\
\hline \multicolumn{11}{|l|}{ MAR } \\
\hline $14 \ldots$ & 0.180 & 0.017 & -- & 6.8 & -- & -- & $<1$ & $<1$ & $<10$ & $<1$ \\
\hline $14 \ldots$ & 0.590 & 0.110 & - & 16 & 76000 & 30000 & $<1$ & 12 & $<10$ & $<1$ \\
\hline $14 \ldots$ & 0.350 & 0.080 & -- & -- & 60000 & 2200 & -- & -- & -- & -- \\
\hline \multicolumn{11}{|l|}{ APR } \\
\hline $27 \ldots$ & -- & -- & -- & -- & -- & -- & -- & -- & -- & -- \\
\hline $27 \ldots$ & 0.450 & 0.023 & -- & -- & -- & -- & $<1$ & 3 & $<10$ & $<1$ \\
\hline $28 \ldots$ & 0.460 & 0.026 & -- & -- & -- & -- & $<1$ & 2 & $<10$ & $<1$ \\
\hline $28 \ldots$ & 0.200 & 0.014 & -- & -- & -- & -- & -- & -- & -- & -- \\
\hline \multicolumn{11}{|l|}{ MAY } \\
\hline $07 \ldots$ & 0.050 & $<0.010$ & 2 & -- & 560 & 510 & $<1$ & $<1$ & $<10$ & $<1$ \\
\hline \multicolumn{11}{|l|}{ JUL } \\
\hline $23 \ldots$ & 1.18 & $<0.010$ & -- & 23 & -- & -- & 3 & 5 & $<10$ & 1 \\
\hline $23 \ldots$ & 1.09 & $<0.010$ & -- & 11 & -- & -- & $<1$ & 6 & $<10$ & $<1$ \\
\hline $23 \ldots$ & 0.290 & 0.041 & -- & 12 & -- & -- & $<1$ & 1 & $<10$ & $<1$ \\
\hline
\end{tabular}

K Results based on colony count outside the acceptable range (nonideal colony count). 
Table 83. Water-quality data at site 44 (CSW10), November 1996 through July 1997-Continued

\begin{tabular}{|c|c|c|c|c|c|c|c|c|c|}
\hline DATE & $\begin{array}{c}\text { CHRO- } \\
\text { MIUM, } \\
\text { TOTAL } \\
\text { RECOV- } \\
\text { ERABLE } \\
\text { (UG/L } \\
\text { AS CR) } \\
(01034) \\
\end{array}$ & $\begin{array}{c}\text { COPPER, } \\
\text { TOTAL } \\
\text { RECOV- } \\
\text { ERABLE } \\
\text { (UG/L AS } \\
\text { CU) } \\
(01042) \\
\end{array}$ & $\begin{array}{c}\text { LEAD, } \\
\text { TOTAL } \\
\text { RECOV- } \\
\text { ERABLE } \\
\text { (UG/L } \\
\text { AS PB) } \\
(01051) \\
\end{array}$ & $\begin{array}{c}\text { MERCURY } \\
\text { TOTAL } \\
\text { RECOV- } \\
\text { ERABLE } \\
\text { (UG/L AS } \\
\text { HG) } \\
(71900) \\
\end{array}$ & $\begin{array}{c}\text { NICKEL, } \\
\text { TOTAL } \\
\text { RECOVER- } \\
\text { ABLE } \\
\text { (UG/L } \\
\text { AS NI) } \\
(01067) \\
\end{array}$ & $\begin{array}{c}\text { SELE- } \\
\text { NIUM, } \\
\text { TOTAL } \\
\text { (UG/L } \\
\text { AS SE) } \\
(01147 \text { ) }\end{array}$ & $\begin{array}{c}\text { SILVER, } \\
\text { TOTAL } \\
\text { RECOV- } \\
\text { ERABLE } \\
\text { (UG/L } \\
\text { AS AG) } \\
(01077) \\
\end{array}$ & $\begin{array}{c}\text { ZINC, } \\
\text { TOTAL } \\
\text { RECOV- } \\
\text { ERABLE } \\
\text { (UG/L } \\
\text { AS ZN) } \\
(01092) \\
\end{array}$ & $\begin{array}{c}\text { CYANIDE } \\
\text { TOTAL } \\
\text { (MG/L AS } \\
\text { CN) } \\
(00720)\end{array}$ \\
\hline \multicolumn{10}{|l|}{ NOV 1996} \\
\hline \multicolumn{10}{|l|}{ DEC } \\
\hline $01 \ldots$ & 16 & 49 & 9 & $<0.10$ & 6 & $<1$ & $<1$ & 50 & -- \\
\hline $01 \ldots$ & -- & -- & -- & -- & -- & -- & -- & -- & -- \\
\hline $01 \ldots$ & -- & -- & -- & -- & -- & -- & -- & -- & -- \\
\hline $01 \ldots$ & 18 & 35 & 7 & $<0.10$ & 15 & $<1$ & $<1$ & 40 & $<0.010$ \\
\hline $01 \ldots$ & 34 & 87 & 13 & $<0.10$ & 8 & $<1$ & $<1$ & 80 & $<0.010$ \\
\hline $01 \ldots$ & -- & -- & -- & -- & -- & -- & -- & -- & -- \\
\hline $01 \ldots$ & 36 & 96 & 15 & $<0.10$ & 8 & $<1$ & $<1$ & 90 & $<0.010$ \\
\hline $01 \ldots$ & 29 & 78 & 14 & $<0.10$ & 7 & $<1$ & $<1$ & 80 & $<0.010$ \\
\hline \multicolumn{10}{|l|}{ JAN 1997} \\
\hline $09 \ldots$ & 51 & 110 & 17 & 0.10 & 22 & $<1$ & $<1$ & 150 & $<0.010$ \\
\hline $09 \ldots$ & 26 & 52 & 11 & $<0.10$ & 9 & $<1$ & $<1$ & 80 & $<0.010$ \\
\hline $10 \ldots$ & -- & -- & -- & -- & - & -- & -- & -- & -- \\
\hline \multicolumn{10}{|l|}{$\mathrm{FEB}$} \\
\hline $13 \ldots$ & -- & -- & -- & -- & -- & -- & -- & -- & -- \\
\hline $13 \ldots$ & 9 & 20 & 1 & $<0.10$ & 2 & $<1$ & 2 & 50 & $<0.010$ \\
\hline $13 \ldots$ & 28 & 21 & 3 & $<0.10$ & 6 & $<1$ & 5 & 100 & $<0.010$ \\
\hline $14 \ldots$ & 1 & 25 & 6 & $<0.10$ & 5 & $<1$ & 8 & 70 & $<0.010$ \\
\hline $14 \ldots$ & -- & -- & -- & -- & -- & -- & -- & -- & -- \\
\hline \multicolumn{10}{|l|}{ MAR } \\
\hline $14 \ldots$ & 9 & 14 & 4 & $<0.10$ & 3 & $<1$ & $<1$ & 30 & $<0.010$ \\
\hline $14 \ldots$ & 20 & 69 & 20 & 0.10 & 9 & $<1$ & $<1$ & 70 & $<0.010$ \\
\hline $14 \ldots$ & -- & -- & -- & -- & -- & -- & -- & - & - \\
\hline \multicolumn{10}{|l|}{ APR } \\
\hline $27 \ldots$ & -- & -- & -- & -- & -- & -- & -- & -- & -- \\
\hline $27 \ldots$ & 21 & 65 & 9 & $<0.10$ & 9 & $<1$ & $<1$ & 70 & $<0.010$ \\
\hline $28 \ldots$ & 19 & 46 & 8 & $<0.10$ & 8 & $<1$ & $<1$ & 60 & $<0.010$ \\
\hline $28 \ldots$ & -- & -- & -- & - & -- & -- & -- & - & -- \\
\hline \multicolumn{10}{|l|}{ MAY } \\
\hline $07 \ldots$ & 1 & 3 & 1 & $<0.10$ & 2 & $<1$ & $<1$ & 30 & $<0.010$ \\
\hline \multicolumn{10}{|l|}{ JUL } \\
\hline $23 \ldots$ & 69 & 84 & 25 & 0.30 & 25 & $<1$ & $<1$ & 200 & $<0.010$ \\
\hline $23 \ldots$ & 56 & 110 & 10 & 0.40 & 18 & $<1$ & $<1$ & 180 & $<0.010$ \\
\hline $23 \ldots$ & 11 & 19 & 9 & 0.20 & 6 & $<1$ & $<1$ & 50 & $<0.010$ \\
\hline
\end{tabular}


Table 84. Rainfall and streamflow characteristics for the monitored storms at site 33 (CSW08), July 1995 through September 1997

[Peak discharge for event may occur after storm duration ends. Sample types: A - Chemical, nutrients, and metals, B - Organic compounds, C - Volatile compounds, D - Total organic carbon, E - Oil and grease, F - Bacteria]

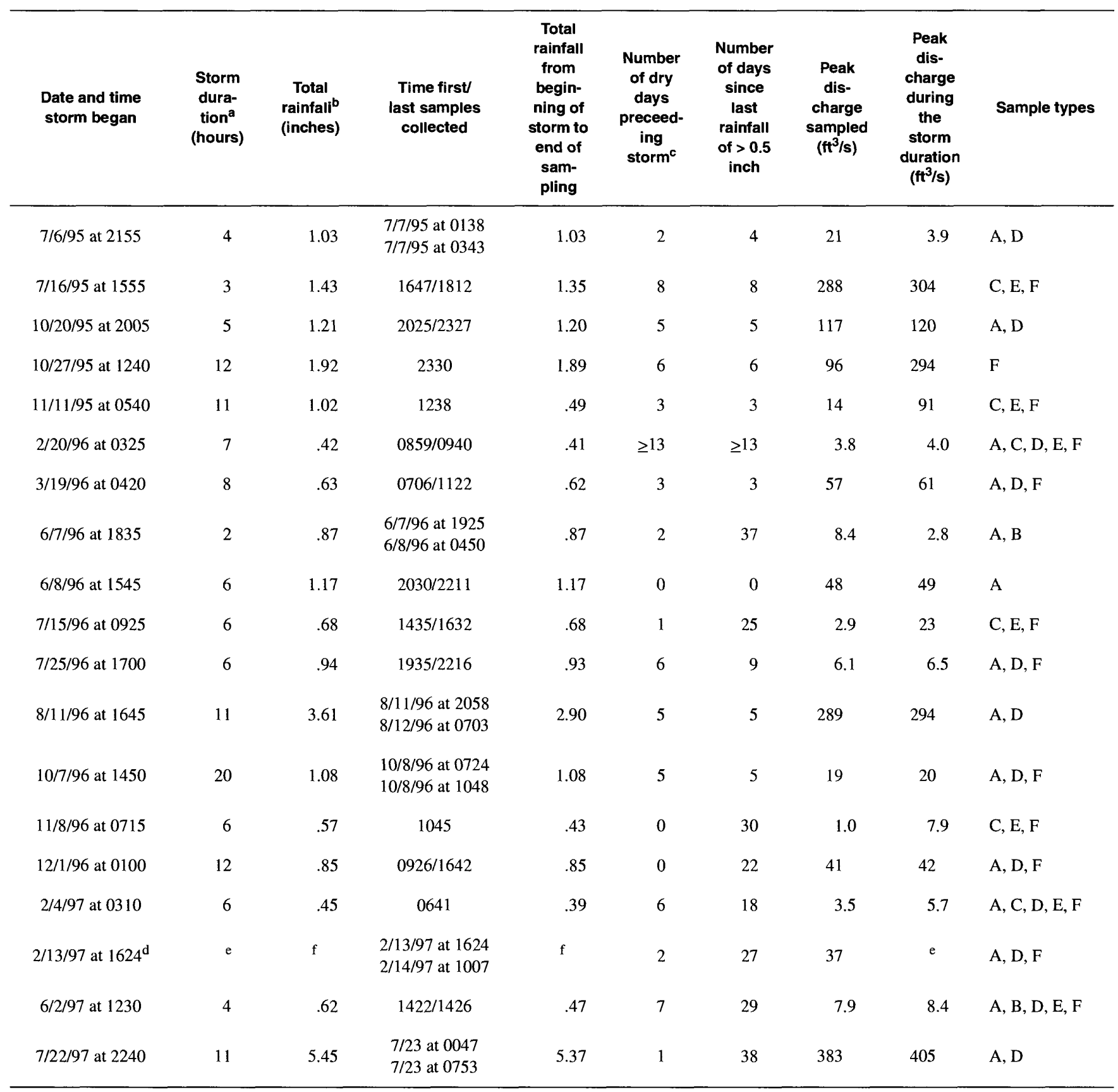

${ }^{a}$ Storm duration is defined as a period when rainfall does not stop for a time period greater than 4 hours.

${ }^{b}$ Rainfall from site 33 (CRN25). Total rainfall is the amount of rain that occurred during the storm duration.

${ }^{\mathrm{c}}$ Number of dry days is defined as days that $\leq 0.10$ inch of rainfall occurred.

${ }^{\mathrm{d}}$ Time first sample collected.

${ }^{\text {e}}$ Storm duration not determined due to the effects of ice storm.

${ }^{f}$ Rainfall totals not available due to the effects of ice storm. 
Table 85. Rainfall and streamflow characteristics for the monitored storms at site 34 (CSW09), July 1995 through September 1997

[Peak discharge for event may occur after storm duration ends. Sample types: A - Chemical, nutrients, and metals, B - Organic compounds, C - Volatile compounds, D - Total organic carbon, E - Oil and grease, F - Bacteria]

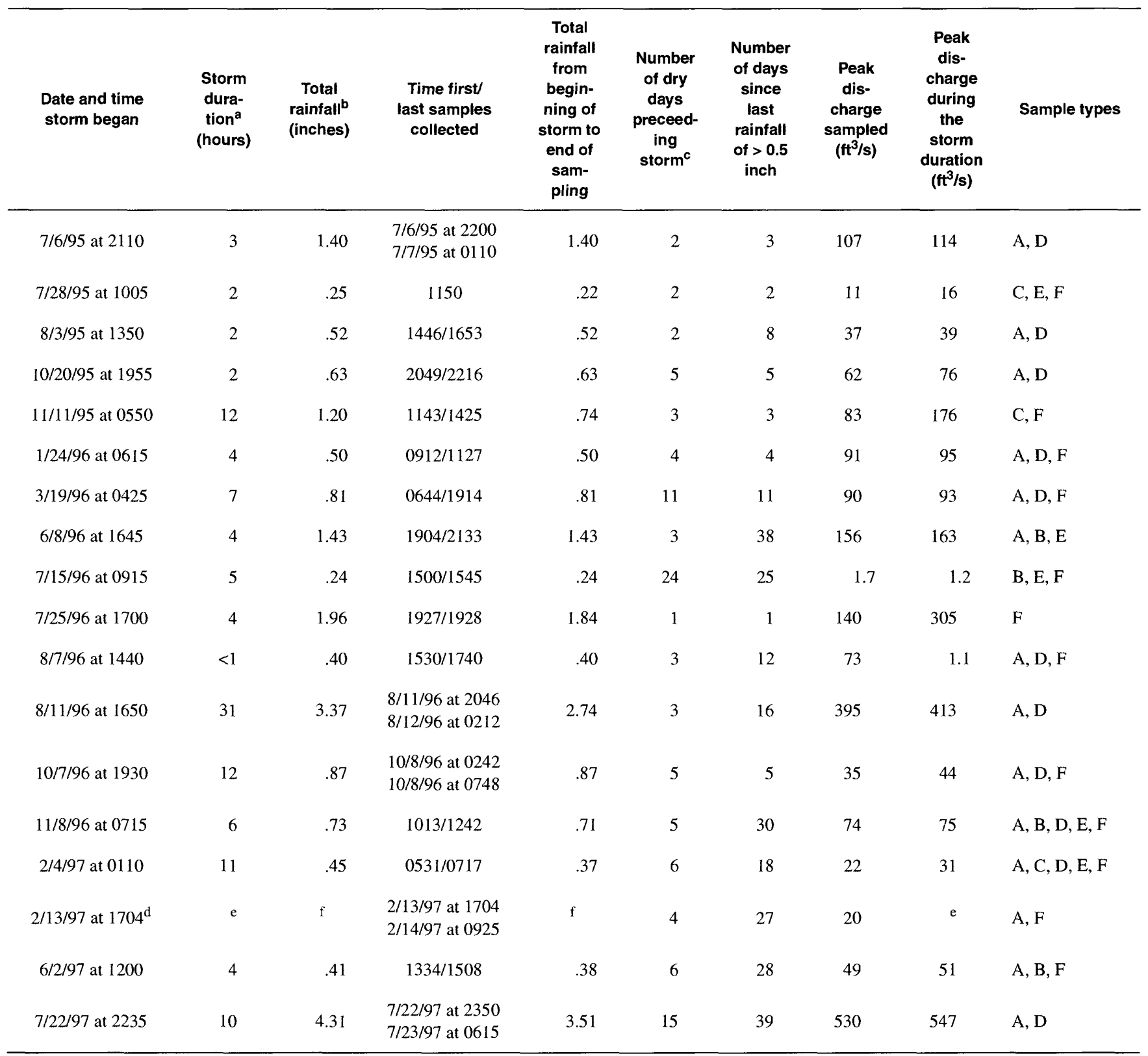

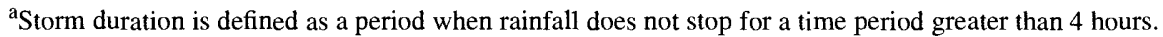

${ }^{b}$ Rainfall from site 34 (CRN24). Total rainfall is the amount of rain that occurred during the storm duration.

${ }^{c}$ Number of dry days is defined as days that $\leq 0.10$ inch of rainfall occurred.

${ }^{\mathrm{d}}$ Time first sample collected.

eStorm duration not determined due to the effects of ice storm.

${ }^{f}$ Rainfall totals not available due to the effects of ice storm. 
Table 86. Rainfall and streamflow characteristics for the monitored storms at site 37 (CSW06), July 1995 through June 1997 [Peak discharge for event may occur after storm duration ends. Sample types: A - Chemical, nutrients, and metals, B - Organic compounds, C - Volatile compounds, D - Total organic carbon, E - Oil and grease, F - Bacteria]

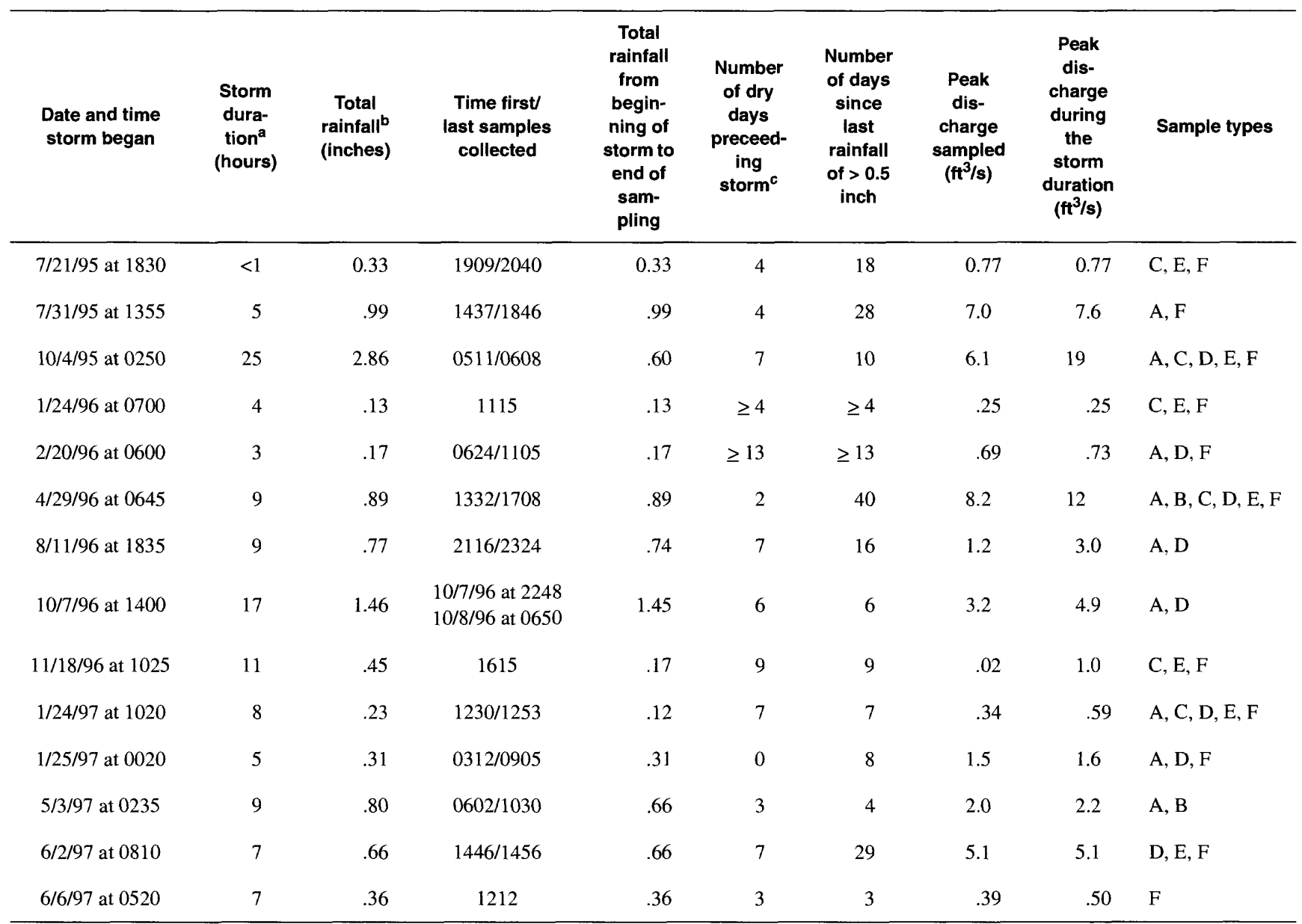

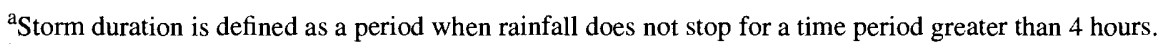

${ }^{b}$ Rainfall from site 37 (CRN28). Total rainfall is the amount of rain that occurred during the storm duration.

${ }^{\mathrm{c}}$ Number of dry days is defined as days that $\leq 0.10$ inch of rainfall occurred. 
Table 87. Rainfall and streamflow characteristics for the monitored storms at site 39 (CSW05), July 1995 through June 1997 [Peak discharge for event may occur after storm duration ends. Sample types: A - Chemical, nutrients, and metals, B - Organic compounds, C - Volatile compounds, D - Total organic carbon, E - Oil and grease, F - Bacteria; --, no data]

\begin{tabular}{|c|c|c|c|c|c|c|c|c|c|}
\hline $\begin{array}{l}\text { Date and time } \\
\text { storm began }\end{array}$ & $\begin{array}{l}\text { Storm } \\
\text { dura- } \\
\text { tion }^{\mathrm{a}} \\
\text { (hours) }\end{array}$ & $\begin{array}{c}\begin{array}{c}\text { Total } \\
\text { rainfallb }\end{array} \\
\text { (inches) }^{\text {inches }}\end{array}$ & $\begin{array}{l}\text { Time first } \\
\text { last samples } \\
\text { collected }\end{array}$ & $\begin{array}{c}\text { Total } \\
\text { rainfall } \\
\text { from } \\
\text { begin- } \\
\text { ning of } \\
\text { storm to } \\
\text { end of } \\
\text { sam- } \\
\text { pling }\end{array}$ & $\begin{array}{l}\text { Number } \\
\text { of dry } \\
\text { days } \\
\text { preceed- } \\
\text { ing } \\
\text { storm }^{c}\end{array}$ & $\begin{array}{l}\text { Number } \\
\text { of days } \\
\text { since } \\
\text { last } \\
\text { rainfall } \\
\text { of }>0.5 \\
\text { inch }\end{array}$ & $\begin{array}{c}\text { Peak } \\
\text { dis- } \\
\text { charge } \\
\text { sampled } \\
\left(\mathbf{f t}^{3} / \mathrm{s}\right)\end{array}$ & $\begin{array}{l}\text { Peak } \\
\text { dis- } \\
\text { charge } \\
\text { during } \\
\text { the } \\
\text { storm } \\
\text { duration } \\
\left(\mathrm{ft}^{3} / \mathrm{s}\right)\end{array}$ & Sample types \\
\hline $7 / 6 / 95$ at 2355 & 2 & 0.39 & $7 / 7 / 95$ at 0156 & 0.38 & 2 & 2 & 2.3 & 2.6 & $\mathrm{C}, \mathrm{E}$ \\
\hline $7 / 21 / 95$ at 1020 & 10 & 1.51 & 1719 & .39 & $\geq 4$ & $\geq 4$ & .04 & 12 & $\mathrm{C}$ \\
\hline $7 / 27 / 95$ at 1625 & 4 & 1.16 & $1528 / 1610$ & -- & 4 & 23 & .24 & 14 & $\mathrm{~F}$ \\
\hline $7 / 31 / 95$ at 1650 & $<1$ & .14 & $1504 / 1545$ & - & 2 & 2 & 6.9 & 7.3 & $\mathrm{~F}$ \\
\hline $10 / 4 / 95$ at 0420 & 9 & 1.97 & $0439 / 0912$ & .92 & 7 & 7 & 3.6 & 4.3 & $\mathrm{~A}, \mathrm{D}, \mathrm{F}$ \\
\hline $11 / 11 / 95$ at 0555 & 11 & 1.13 & 1258 & .74 & 3 & 3 & 2.1 & -- & $\mathrm{F}$ \\
\hline $1 / 24 / 96$ at 0620 & 4 & .24 & $0710 / 1025$ & .24 & 4 & 4 & .95 & 1.3 & $\mathrm{~A}, \mathrm{C}, \mathrm{D}, \mathrm{E}, \mathrm{F}$ \\
\hline $3 / 6 / 96$ at 0350 & 10 & 1.43 & 1118 & 1.37 & $\geq 13$ & $\geq 13$ & .44 & 2.9 & $\mathrm{~F}$ \\
\hline $4 / 26 / 96$ at 0900 & 3 & .45 & $0906 / 1138$ & .45 & 5 & 25 & 1.1 & 1.2 & $\mathrm{~A}, \mathrm{~B}, \mathrm{C}, \mathrm{D}, \mathrm{E}, \mathrm{P}$ \\
\hline $7 / 31 / 96$ at 2205 & 7 & .29 & $2206 / 2306$ & .20 & 2 & 5 & 2.1 & 7.3 & $\mathrm{~A}, \mathrm{D}$ \\
\hline $10 / 7 / 96$ at 1335 & 18 & 1.31 & $\begin{array}{l}10 / 7 / 96 \text { at } 1550 \\
10 / 8 / 96 \text { at } 0638\end{array}$ & 1.27 & 5 & 5 & .82 & 1.6 & $\mathrm{~A}, \mathrm{C}, \mathrm{D}, \mathrm{E}, \mathrm{F}$ \\
\hline $1 / 16 / 97$ at 0050 & 3 & .61 & $0102 / 0405$ & .61 & 6 & 6 & 3.4 & 4.1 & A, D \\
\hline $1 / 24 / 97$ at 1030 & 6 & .28 & 1119 & .10 & 7 & 7 & .61 & .70 & $\mathrm{C}, \mathrm{E}, \mathrm{F}$ \\
\hline $5 / 25 / 97$ at 1430 & 6 & .41 & $1449 / 1540$ & .30 & 21 & 21 & 5.6 & 7.3 & $\mathrm{~A}, \mathrm{~B}, \mathrm{E}, \mathrm{F}$ \\
\hline $6 / 6 / 97$ at 0520 & 8 & .36 & 1102 & .29 & 4 & 4 & .14 & .36 & $\mathrm{D}, \mathrm{F}$ \\
\hline
\end{tabular}

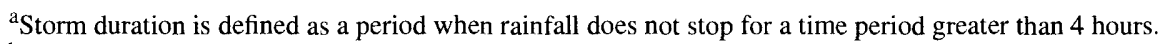

${ }^{b}$ Rainfall from site 15 (CRN03), except events of 7/27/95, 7/31/95, and 11/1 1/95 when rainfall from site 36 (CRN27) was used. Total rainfall is the amount of rain that occurred during the storm duration.

${ }^{c}$ Number of dry days is defined as days that $\leq 0.10$ inch of rainfall occurred. 
Table 88. Rainfall and streamflow characteristics for the monitored storms at site 40 (CSW03), July 1995 through June 1997 [Peak discharge for event may occur after storm duration ends. Sample types: A - Chemical, nutrients, and metals, B - Organic compounds, C - Volatile compounds, D - Total organic carbon, E - Oil and grease, F - Bacteria]

\begin{tabular}{|c|c|c|c|c|c|c|c|c|c|}
\hline $\begin{array}{l}\text { Date and time } \\
\text { storm began }\end{array}$ & $\begin{array}{l}\text { Storm } \\
\text { dura- } \\
\text { tion }^{\text {a }} \\
\text { (hours) }\end{array}$ & $\begin{array}{c}\text { Total } \\
\text { rainfall }^{b} \\
\text { (inches) }^{\text {inches }}\end{array}$ & $\begin{array}{l}\text { Time first/ } \\
\text { last samples } \\
\text { collected }\end{array}$ & $\begin{array}{l}\text { Total } \\
\text { rainfall } \\
\text { from } \\
\text { begin- } \\
\text { ning of } \\
\text { storm to } \\
\text { end of } \\
\text { sam- } \\
\text { pling }\end{array}$ & $\begin{array}{l}\text { Number } \\
\text { of dry } \\
\text { days } \\
\text { preceed- } \\
\text { ing } \\
\text { stormc }\end{array}$ & $\begin{array}{c}\text { Number } \\
\text { of days } \\
\text { since } \\
\text { last } \\
\text { rainfall } \\
\text { of }>0.5 \\
\text { inch }\end{array}$ & $\begin{array}{c}\text { Peak } \\
\text { dis- } \\
\text { charge } \\
\text { sampled } \\
\left(\mathrm{ft}^{3} / \mathrm{s}\right)\end{array}$ & $\begin{array}{l}\text { Peak } \\
\text { dis- } \\
\text { charge } \\
\text { during } \\
\text { the } \\
\text { storm } \\
\text { duration } \\
\left(\mathrm{ft}^{3} / \mathrm{s}\right)\end{array}$ & Sample types \\
\hline $8 / 26 / 95$ at 0345 & 50 & 9.37 & $8 / 27 / 95$ at 0426 & 5.22 & 7 & 7 & 21 & 27 & A, D \\
\hline $10 / 4 / 95$ at 0345 & 15 & 2.28 & $0453 / 0856$ & 1.10 & 7 & 7 & 1.2 & 2.4 & $\mathrm{~A}, \mathrm{C}, \mathrm{D}, \mathrm{E}, \mathrm{F}$ \\
\hline $11 / 11 / 95$ at 0555 & 11 & 1.45 & 1335 & .99 & 3 & 3 & .45 & 1.1 & $\mathrm{~F}$ \\
\hline $3 / 6 / 96$ at 1054 & 10 & 1.23 & 1054 & 1.18 & 14 & $\geq 28$ & .11 & 1.8 & $\mathrm{~F}$ \\
\hline $3 / 27 / 96$ at 1310 & 22 & .55 & $\begin{array}{l}3 / 27 / 96 \text { at } 1845 \\
3 / 28 / 96 \text { at } 1004\end{array}$ & .50 & $\geq 7$ & $\geq 7$ & .02 & .15 & $\mathrm{~A}, \mathrm{D}, \mathrm{F}$ \\
\hline $6 / 7 / 96$ at 1905 & 1 & .37 & $1931 / 1934$ & .34 & 8 & 37 & .15 & .20 & A, B \\
\hline $7 / 23 / 96$ at 1705 & 4 & .23 & 1735 & .01 & 7 & 7 & .01 & .29 & $\mathrm{C}, \mathrm{E}, \mathrm{F}$ \\
\hline $7 / 30 / 96$ at 1140 & 1 & .89 & $1245 / 1310$ & .89 & $\geq 3$ & $\geq 3$ & .18 & .34 & $\mathrm{~A}, \mathrm{D}, \mathrm{F}$ \\
\hline $8 / 11 / 96$ at 1845 & 10 & .70 & $1900 / 1920$ & .09 & 7 & 8 & .34 & .74 & A, D \\
\hline $9 / 11 / 96$ at 1255 & $<1$ & .05 & $1307 / 1317$ & .04 & 0 & 6 & .29 & .45 & $\mathrm{C}, \mathrm{F}$ \\
\hline $10 / 7 / 96$ at 1405 & 18 & 1.60 & $\begin{array}{l}10 / 7 / 96 \text { at } 2210 \\
10 / 8 / 96 \text { at } 0640\end{array}$ & 1.59 & 5 & 5 & .42 & .48 & A, D \\
\hline $11 / 18 / 96$ at 1030 & 10 & .37 & 1616 & .15 & 9 & 9 & .01 & .03 & $\mathrm{C}, \mathrm{E}, \mathrm{F}$ \\
\hline $2 / 4 / 97$ at 0325 & 6 & .28 & $0403 / 1039$ & .28 & 4 & 18 & .02 & .06 & $\mathrm{~A}, \mathrm{D}, \mathrm{F}$ \\
\hline $2 / 13 / 97$ at 1140 & 13 & 1.17 & $1243 / 0902$ & 1.17 & 4 & 27 & .25 & .34 & $\mathrm{~A}, \mathrm{C}, \mathrm{D}, \mathrm{E}, \mathrm{F}$ \\
\hline $5 / 3 / 97$ at 0225 & 10 & .77 & $0552 / 1322$ & .77 & 4 & 4 & .76 & 1.2 & A, B \\
\hline
\end{tabular}

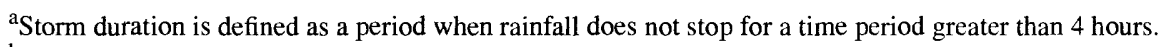

${ }^{b}$ Rainfall from site 29 (CRN20), except event of 7/23/96 when rainfall from site 28 (CRN19) was used. Total rainfall is the amount of rain that occurred during the storm duration.

${ }^{\mathrm{c}}$ Number of dry days is defined as days that $\leq 0.10$ inch of rainfall occurred. 
Table 89. Rainfall and streamflow characteristics for the monitored storms at site 41 (CSW02), July 1995 through June 1997 [Peak discharge for event may occur after storm duration ends. Sample types: A - Chemical, nutrients, and metals, B - Organic compounds, C - Volatile compounds, D - Total organic carbon, E - Oil and grease, F - Bacteria]

\begin{tabular}{|c|c|c|c|c|c|c|c|c|c|}
\hline $\begin{array}{l}\text { Date and time } \\
\text { storm began }\end{array}$ & $\begin{array}{l}\text { Storm } \\
\text { dura- } \\
\text { tion }^{\mathrm{a}} \\
\text { (hours) }\end{array}$ & $\begin{array}{c}\text { Total } \\
\text { rainfall' } \\
\text { (inches) }\end{array}$ & $\begin{array}{l}\text { Time first/ } \\
\text { last samples } \\
\text { collected }\end{array}$ & $\begin{array}{l}\text { Total } \\
\text { rainfall } \\
\text { from } \\
\text { begin- } \\
\text { ning of } \\
\text { storm to } \\
\text { end of } \\
\text { sam- } \\
\text { pling }\end{array}$ & $\begin{array}{l}\text { Number } \\
\text { of dry } \\
\text { days } \\
\text { preceed- } \\
\text { ing } \\
\text { storm }^{c}\end{array}$ & $\begin{array}{c}\text { Number } \\
\text { of days } \\
\text { since } \\
\text { last } \\
\text { rainfall } \\
\text { of }>0.5 \\
\text { inch }\end{array}$ & $\begin{array}{c}\text { Peak } \\
\text { dis- } \\
\text { charge } \\
\text { sampled } \\
\left(\mathrm{ft}^{3} / \mathrm{s}\right)\end{array}$ & $\begin{array}{l}\text { Peak } \\
\text { dis- } \\
\text { charge } \\
\text { during } \\
\text { the } \\
\text { storm } \\
\text { duration } \\
\left(\mathrm{ft}^{3} / \mathrm{s}\right)\end{array}$ & Sample types \\
\hline $8 / 26 / 95$ at 0340 & 49 & 7.11 & $8 / 27 / 95$ at 0352 & 3.25 & 7 & 7 & 153 & 334 & $A, D$ \\
\hline $10 / 4 / 95$ at 0240 & 16 & 2.34 & $0410 / 0936$ & 1.50 & 7 & 7 & 18 & 22 & $\mathrm{~A}, \mathrm{C}, \mathrm{D}, \mathrm{E}, \mathrm{F}$ \\
\hline $10 / 27 / 95$ at 1535 & 6 & 1.34 & 1953 & .87 & 6 & 6 & 97 & 136 & $\mathrm{~F}$ \\
\hline $1 / 24 / 96$ at 0710 & 3 & .16 & 0812 & .03 & 4 & $\geq 10$ & .22 & 1.2 & $\mathrm{C}, \mathrm{E}, \mathrm{F}$ \\
\hline $2 / 20 / 96$ at 0315 & 7 & .37 & $0447 / 0904$ & .35 & $\geq 13$ & $\geq 13$ & .94 & 1.9 & $\mathrm{~A}, \mathrm{D}, \mathrm{F}$ \\
\hline $3 / 6 / 96$ at 0400 & 10 & 1.56 & 0941 & 1.44 & 14 & $\geq 28$ & 9.8 & 34 & $\mathrm{~F}$ \\
\hline $4 / 26 / 96$ at 0910 & 3 & .58 & $0916 / 1158$ & .58 & 5 & 37 & 8.8 & 11 & $\mathrm{~A}, \mathrm{~B}, \mathrm{D}, \mathrm{E}, \mathrm{F}$ \\
\hline $7 / 30 / 96$ at 1140 & 1 & .12 & 1312 & .12 & 4 & 4 & .32 & 29 & $\mathrm{~F}$ \\
\hline $8 / 11 / 96$ at 1840 & 7 & 1.32 & $1846 / 2356$ & 1.30 & 1 & 16 & 95 & 130 & $A, D$ \\
\hline $10 / 7 / 96$ at 1400 & 18 & 1.45 & $\begin{array}{l}10 / 7 / 96 \text { at } 1728 \\
10 / 8 / 96 \text { at } 0653\end{array}$ & 1.37 & 5 & 5 & 1.9 & 6.2 & $\mathrm{~A}, \mathrm{D}, \mathrm{E}, \mathrm{F}$ \\
\hline $1 / 16 / 97$ at 0045 & 4 & .64 & $0114 / 0410$ & .63 & 6 & 7 & 13 & 19 & $\mathrm{~A}, \mathrm{D}, \mathrm{F}$ \\
\hline $1 / 24 / 97$ at 1025 & 6 & .25 & 1118 & .08 & 7 & 7 & 2.6 & 2.6 & $\mathrm{E}, \mathrm{F}$ \\
\hline $5 / 8 / 97$ at 2045 & 3 & .33 & $2124 / 2128$ & .08 & 4 & 4 & 2.8 & 3.0 & $A, B$ \\
\hline $6 / 2 / 97$ at 1425 & 2 & .56 & 1446 & .54 & 7 & 29 & 7.4 & 53 & $\mathrm{D}, \mathrm{E}, \mathrm{F}$ \\
\hline $6 / 6 / 97$ at 0530 & 7 & .45 & 0907 & .31 & 3 & 3 & .72 & 1.5 & $\mathrm{D}, \mathrm{F}$ \\
\hline
\end{tabular}

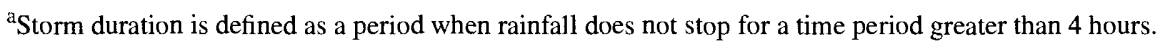

${ }^{b}$ Rainfall from site 22 (CRN12). Total rainfall is the amount of rain that occurred during the storm duration.

${ }^{\mathrm{c}}$ Number of dry days is defined as days that $\leq 0.10$ inch of rainfall occurred. 
Table 90. Rainfall and streamflow characteristics for the monitored storms at site 42 (CSW04), July 1995 through June 1997 [Peak discharge for event may occur after storm duration ends. Sample types: A - Chemical nutrients, and metals, B - Organic compounds, C - Volatile compounds, D - Total organic carbon, E - Oil and grease, F - Bacteria]

\begin{tabular}{|c|c|c|c|c|c|c|c|c|c|}
\hline $\begin{array}{l}\text { Date and time } \\
\text { storm began }\end{array}$ & $\begin{array}{l}\text { Storm } \\
\text { dura- } \\
\text { tion }^{\mathrm{a}} \\
\text { (hours) }\end{array}$ & $\begin{array}{c}\text { Total } \\
\text { rainfall } \\
\text { (inches) }^{\mathbf{b}}\end{array}$ & $\begin{array}{l}\text { Time first/ } \\
\text { last samples } \\
\text { collected }\end{array}$ & $\begin{array}{l}\text { Total } \\
\text { rainfall } \\
\text { from } \\
\text { begin- } \\
\text { ning of } \\
\text { storm to } \\
\text { end of } \\
\text { sam- } \\
\text { pling }\end{array}$ & $\begin{array}{l}\text { Number } \\
\text { of dry } \\
\text { days } \\
\text { preceed- } \\
\text { ing } \\
\text { storm }^{c}\end{array}$ & $\begin{array}{l}\text { Number } \\
\text { of days } \\
\text { since } \\
\text { last } \\
\text { rainfall } \\
\text { of }>0.5 \\
\text { inch }\end{array}$ & $\begin{array}{c}\text { Peak } \\
\text { dis- } \\
\text { charge } \\
\text { sampled } \\
\left(\mathrm{ft}^{3} / \mathrm{s}\right)\end{array}$ & $\begin{array}{c}\text { Peak } \\
\text { dis- } \\
\text { charge } \\
\text { during } \\
\text { the } \\
\text { storm } \\
\text { duration } \\
\left(\mathrm{ft}^{3} / \mathbf{s}\right)\end{array}$ & Sample types \\
\hline $8 / 26 / 95$ at 0340 & 49 & 7.11 & $8 / 27 / 95$ at 0354 & 3.25 & 7 & 7 & 221 & 294 & A, D \\
\hline $10 / 4 / 95$ at 0240 & 16 & 2.34 & $0419 / 0846$ & 1.15 & 7 & 7 & 39 & 57 & $\mathrm{~A}, \mathrm{C}, \mathrm{D}, \mathrm{E}, \mathrm{F}$ \\
\hline $10 / 27 / 95$ at 1535 & 8 & 1.34 & 2011 & 1.12 & 6 & 6 & 68 & 99 & $\mathrm{~F}$ \\
\hline $2 / 20 / 96$ at 0315 & 7 & .37 & $0452 / 0834$ & .34 & $\geq 13$ & $\geq 13$ & 2.0 & 2.1 & $\mathrm{~A}, \mathrm{D}, \mathrm{F}$ \\
\hline $2 / 28 / 96$ at 0845 & 2 & .10 & $0920 / 1257$ & .10 & $\geq 21$ & $\geq 21$ & .90 & .90 & C, E, F \\
\hline $3 / 6 / 96$ at 0400 & 10 & 1.56 & 1010 & 1.47 & 14 & $\geq 28$ & 10 & 44 & $\mathrm{~F}$ \\
\hline $6 / 7 / 96$ at 1905 & 4 & .28 & 1916 & .21 & 8 & 8 & 25 & 29 & $\mathrm{~A}, \mathrm{~B}, \mathrm{C}, \mathrm{E}, \mathrm{F}$ \\
\hline $8 / 11 / 96$ at 1840 & 7 & 1.32 & $1852 / 2356$ & 1.30 & 1 & 16 & 27 & 32 & $A, D$ \\
\hline $10 / 7 / 96$ at 1400 & 18 & 1.45 & $\begin{array}{l}10 / 7 / 96 \text { at } 1530 \\
10 / 8 / 96 \text { at } 0652\end{array}$ & 1.44 & 5 & 5 & 13 & 26 & $\mathrm{~A}, \mathrm{C}, \mathrm{D}, \mathrm{E}, \mathrm{F}$ \\
\hline $1 / 16 / 97$ at 0045 & 4 & .64 & $0114 / 0414$ & .63 & 6 & 7 & 37 & 44 & $A, D$ \\
\hline $1 / 24 / 97$ at 1025 & 6 & .25 & 1132 & .11 & 7 & 7 & 1.7 & 4.6 & $\mathrm{C}, \mathrm{E}, \mathrm{F}$ \\
\hline $5 / 8 / 97$ at 2045 & 3 & .33 & $2137 / 2140$ & .12 & 4 & 4 & 1.7 & 5.0 & A, B \\
\hline $6 / 2 / 97$ at 1425 & 2 & .56 & 1501 & .56 & 7 & 29 & 19 & 49 & $\mathrm{D}, \mathrm{E}, \mathrm{F}$ \\
\hline $6 / 6 / 97$ at 0530 & 7 & .45 & 0935 & .33 & 3 & 3 & 1.4 & 3.7 & $\mathrm{D}, \mathrm{F}$ \\
\hline
\end{tabular}

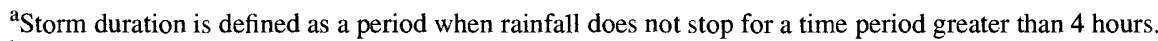

${ }^{b}$ Rainfall from site 22 (CRN12). Total rainfall is the amount of rain that occurred during the storm duration.

${ }^{c}$ Number of dry days is defined as days that $\leq 0.10$ inch of rainfall occurred. 
Table 91. Rainfall and streamflow characteristics for the monitored storms at site 43 (CSWO7), July 1995 through June 1997

[Peak discharge for event may occur after storm duration ends. Sample types: A - Chemical, nutrients, and metals, B - Organic compounds, C - Volatile compounds, D - Total organic carbon, E - Oil and grease, F - Bacteria]

\begin{tabular}{|c|c|c|c|c|c|c|c|c|c|}
\hline $\begin{array}{l}\text { Date and time } \\
\text { storm began }\end{array}$ & $\begin{array}{l}\text { Storm } \\
\text { dura- } \\
\text { tion }^{\mathrm{a}} \\
\text { (hours) }\end{array}$ & $\begin{array}{c}\text { Total } \\
\text { rainfall } \\
\text { (inches) }\end{array}$ & $\begin{array}{l}\text { Time first/ } \\
\text { last samples } \\
\text { collected }\end{array}$ & $\begin{array}{l}\text { Total } \\
\text { rainfall } \\
\text { from } \\
\text { begin- } \\
\text { ning of } \\
\text { storm to } \\
\text { end of } \\
\text { sam- } \\
\text { pling }\end{array}$ & $\begin{array}{c}\text { Number } \\
\text { of dry } \\
\text { days } \\
\text { preceed- } \\
\text { ing } \\
\text { storm }^{c}\end{array}$ & $\begin{array}{c}\text { Number } \\
\text { of days } \\
\text { since } \\
\text { last } \\
\text { rainfall } \\
\text { of }>0.5 \\
\text { inch }\end{array}$ & $\begin{array}{c}\text { Peak } \\
\text { dis- } \\
\text { charge } \\
\text { sampled } \\
\left(\mathrm{ft}^{3} / \mathrm{s}\right)\end{array}$ & 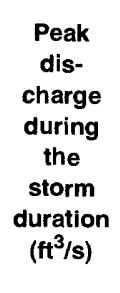 & Sample types \\
\hline $7 / 27 / 95$ at 1555 & 3 & 0.08 & $1750 / 1752$ & 0.04 & 8 & 8 & 0.27 & 0.75 & $\mathrm{C}, \mathrm{E}, \mathrm{F}$ \\
\hline $8 / 26 / 95$ at 0210 & 26 & 7.44 & $8 / 27 / 95$ at 0355 & 4.97 & 7 & 7 & 344 & 354 & A, D \\
\hline $10 / 4 / 95$ at 0405 & 15 & 3.06 & $0505 / 0950$ & 2.12 & 10 & 10 & 33 & 34 & A, C, D, E, F \\
\hline $11 / 11 / 95$ at 0645 & 10 & 1.28 & $1140 / 1500$ & 1.18 & 3 & 3 & 22 & 24 & $\mathrm{~F}$ \\
\hline $2 / 20 / 96$ at 0415 & 8 & .34 & $0656 / 1143$ & .33 & $\geq 13$ & $\geq 13$ & .95 & 1.0 & $\mathrm{~A}, \mathrm{C}, \mathrm{D}, \mathrm{E}, \mathrm{F}$ \\
\hline $6 / 7 / 96$ at 1850 & $<1$ & .04 & 1938 & .04 & $<1$ & 8 & .19 & .21 & $\mathrm{C}, \mathrm{D}, \mathrm{E}, \mathrm{F}$ \\
\hline $6 / 8 / 96$ at 1545 & 5 & .35 & $1902 / 2244$ & .35 & 9 & 9 & 2.8 & 3.1 & A, B \\
\hline $8 / 2 / 96$ at 2125 & 3 & 2.66 & $\begin{array}{l}8 / 2 / 96 \text { at } 2214 \\
8 / 3 / 96 \text { at } 0013\end{array}$ & 2.49 & 7 & 7 & 74 & 74 & A, D \\
\hline $10 / 7 / 96$ at 1520 & 19 & 2.07 & $\begin{array}{l}10 / 7 / 96 \text { at } 1705 \\
10 / 8 / 96 \text { at } 1200\end{array}$ & 2.07 & 6 & 6 & 12 & 29 & $\mathrm{~A}, \mathrm{C}, \mathrm{D}, \mathrm{E}, \mathrm{F}$ \\
\hline $1 / 24 / 97$ at 1115 & 6 & .25 & $1158 / 1204$ & .07 & 7 & 7 & .95 & 1.9 & $\mathrm{~A}, \mathrm{C}, \mathrm{D}, \mathrm{E}, \mathrm{F}$ \\
\hline $1 / 24 / 97$ at 2155 & 7 & .33 & $\begin{array}{l}1 / 25 / 97 \text { at } 0254 \\
1 / 25 / 97 \text { at } 0923\end{array}$ & .33 & 7 & 7 & 6.5 & 7.0 & $\mathrm{~A}, \mathrm{D}, \mathrm{F}$ \\
\hline $5 / 8 / 97$ at 2145 & 1 & .31 & $2203 / 2209$ & .17 & 4 & 4 & 5.5 & 5.5 & A, B \\
\hline $6 / 6 / 97$ at 0525 & 7 & .71 & 0707 & .15 & 16 & 33 & .95 & 4.4 & $\mathrm{D}, \mathrm{E}, \mathrm{F}$ \\
\hline
\end{tabular}

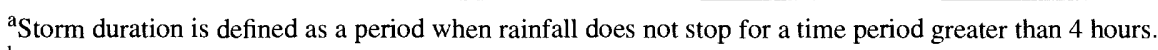

${ }^{b}$ Rainfall from site 19 (CRN08). Total rainfall is the amount of rain that occurred during the storm duration.

${ }^{\mathrm{c}}$ Number of dry days is defined as days that $\leq 0.10$ inch of rainfall occurred. 
Table 92. Rainfall and streamflow characteristics for the monitored storms at site 44 (CSW10), November 1996 through September 1997

[Peak discharge for event may occur after storm duration ends. Sample types: A - Chemical, nutrients, and metals, B - Organic compounds, C - Volatile compounds, D - Total organic carbon, E - Oil and grease, F - Bacteria]

\begin{tabular}{|c|c|c|c|c|c|c|c|c|c|}
\hline $\begin{array}{l}\text { Date and time } \\
\text { storm began }\end{array}$ & $\begin{array}{l}\text { Storm } \\
\text { dura- } \\
\text { tion }{ }^{a} \\
\text { (hours) }\end{array}$ & $\begin{array}{c}\text { Total } \\
\text { rainfall }^{b} \\
\text { (inches) }\end{array}$ & $\begin{array}{l}\text { Time first/ } \\
\text { last samples } \\
\text { collected }\end{array}$ & $\begin{array}{c}\text { Total } \\
\text { rainfall } \\
\text { from } \\
\text { begin- } \\
\text { ning of } \\
\text { storm to } \\
\text { end of } \\
\text { sam- } \\
\text { pling }\end{array}$ & $\begin{array}{l}\text { Number } \\
\text { of dry } \\
\text { days } \\
\text { preceed- } \\
\text { ing } \\
\text { stormc }\end{array}$ & $\begin{array}{c}\text { Number } \\
\text { of days } \\
\text { since } \\
\text { last } \\
\text { rainfall } \\
\text { of }>0.5 \\
\text { inch }\end{array}$ & $\begin{array}{l}\text { Peak } \\
\text { dis- } \\
\text { charge } \\
\text { sampled } \\
\left(\mathrm{ft}^{3} / \mathrm{s}\right)\end{array}$ & $\begin{array}{c}\text { Peak } \\
\text { dis- } \\
\text { charge } \\
\text { during } \\
\text { the } \\
\text { storm } \\
\text { duration } \\
\left(\mathrm{ft}^{3} / \mathrm{s}\right)\end{array}$ & Sample types \\
\hline $11 / 30 / 96$ at 0440 & 14 & 0.17 & 2005 & 0.17 & 8 & $\geq 11$ & 18 & 18 & A, D \\
\hline $12 / 1 / 96$ at 0100 & 18 & 1.01 & $0747 / 1425$ & .99 & 0 & $\geq 11$ & 358 & 375 & $\mathrm{~A}, \mathrm{D}, \mathrm{F}$ \\
\hline $1 / 8 / 97$ at 1955 & 17 & 1.27 & $\begin{array}{c}1 / 9 / 97 \text { at } 0941 \\
1 / 10 / 97 \text { at } 0832\end{array}$ & 1.27 & 2 & 26 & 535 & 542 & $\mathrm{~A}, \mathrm{D}, \mathrm{F}$ \\
\hline $2 / 13 / 97$ at $1530^{\mathrm{d}}$ & $\mathrm{e}$ & $f$ & $\begin{array}{l}2 / 13 / 97 \text { at } 1530 \\
2 / 14 / 97 \text { at } 1038\end{array}$ & f & 2 & 8 & 269 & e & A, C, D, E, F \\
\hline $3 / 13 / 97$ at 1850 & 14 & .61 & $\begin{array}{l}3 / 14 / 97 \text { at } 0612 \\
3 / 14 / 97 \text { at } 1430\end{array}$ & .61 & 7 & 12 & 131 & 135 & $\mathrm{~A}, \mathrm{D}, \mathrm{E}, \mathrm{F}$ \\
\hline $4 / 27 / 97$ at 0030 & 22 & 1.16 & $\begin{array}{l}4 / 27 / 97 \text { at } 1916 \\
4 / 28 / 97 \text { at } 0858\end{array}$ & 1.16 & 3 & 3 & 438 & 164 & $\mathrm{~A}, \mathrm{~B}, \mathrm{E}, \mathrm{F}$ \\
\hline $7 / 22 / 97$ at 2235 & 10 & 4.81 & $\begin{array}{l}7 / 23 / 97 \text { at } 0432 \\
7 / 23 / 97 \text { at } 1714\end{array}$ & 4.81 & 15 & 25 & 864 & 483 & A, D \\
\hline
\end{tabular}

${ }^{\text {a }}$ Storm duration is defined as a period when rainfall does not stop for a time period greater than 4 hours.

${ }^{b}$ Rainfall from site 44 (CRN41). Total rainfall is the amount of rain that occurred during the storm duration.

${ }^{\mathrm{c}}$ Number of dry days is defined as days that $\leq 0.10$ inch of rainfall occurred.

${ }^{\mathrm{d}}$ Time first sample collected.

${ }^{\mathrm{e}}$ Storm duration not determined due to the effects of ice storm.

${ }^{\mathrm{f}}$ Rainfall totals not available due to the effects of ice storm. 